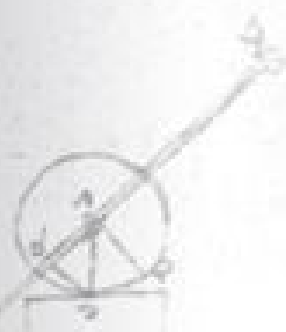

\title{
8.
}

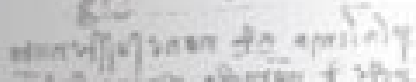

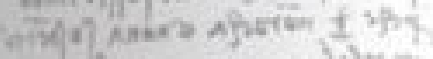

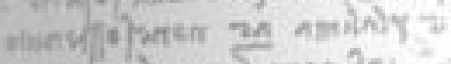

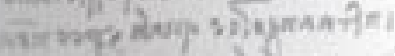

Wo dz atwods?

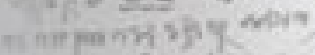

\section{Costruire per temi i paesaggi?}

esiti spaziali della semantica nei parchi tematici europei 
TESI

SCIENZE TeCNOLOGICHE

$-2-$ 


\section{TESI \\ SCIENZE TECNOLOGICHE}

1. Gabriele Paolinelli, La frammentazione del paesaggio periurbano. Criteri progettuali per la riqualificazione della piana di Firenze, 2003 
UNIVERSITÀ DEGLI STUDI DI FIRENZE - FACOLTÀ DI ARCHITETTURA

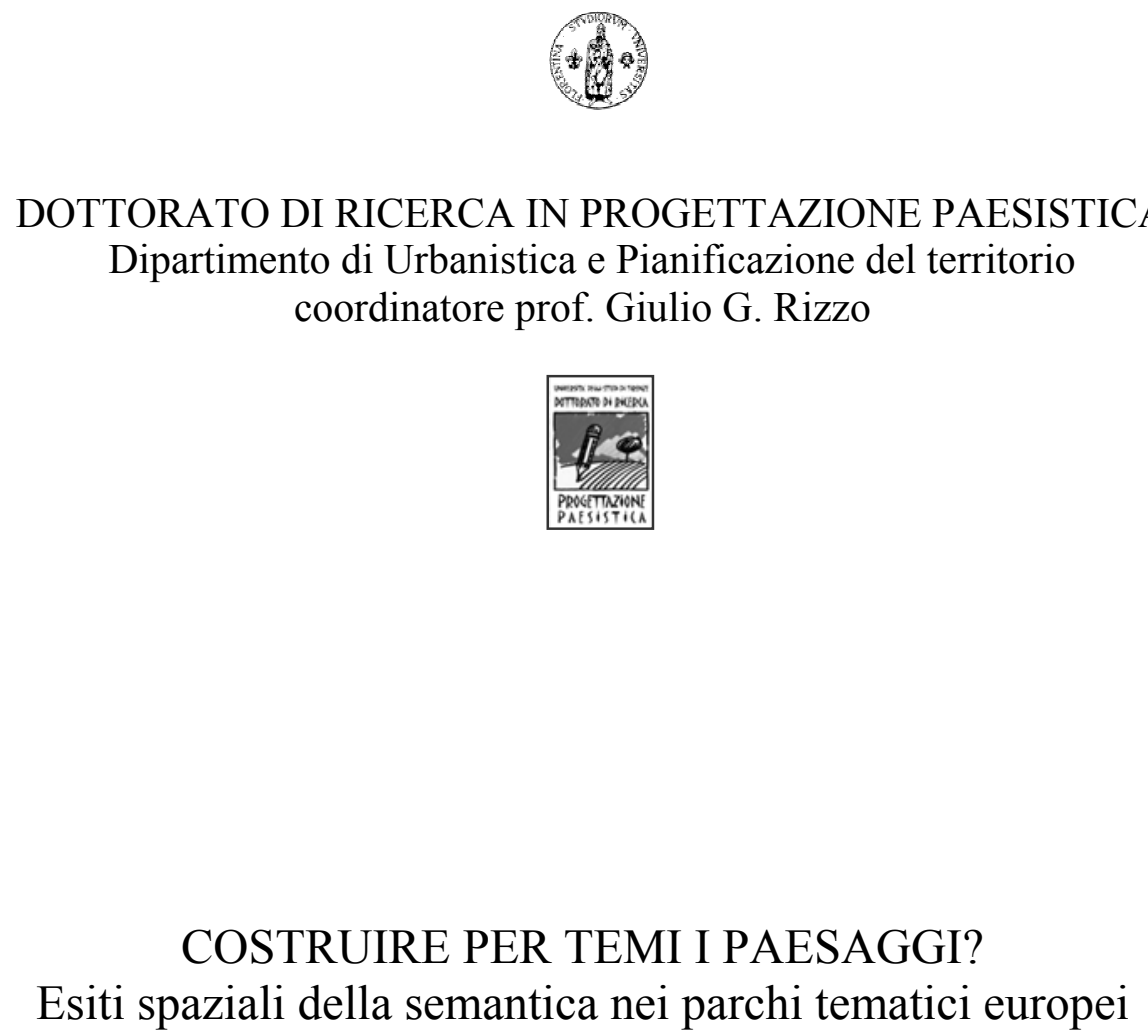

Esiti spaziali della semantica nei parchi tematici europei

Ricerca di Enrica Dall’Ara

Tutor

prof. Guido Ferrara (Università di Firenze)

Co-tutor

prof. Augusto Boggiano (Università di Firenze) 
Costruire per temi i paesaggi? : esiti spaziali della semantica nei parchi tematici europei / Enrica Dall'Ara. - Firenze : Firenze university press, 2004.

(Tesi. Scienze Tecnologiche, 2)

http://digital.casalini.it/8884532353

Stampa a richiesta disponibile su http://epress.unifi.it

ISBN 88-8453-235-3 (online)

ISBN 88-8453-236-1 (print)

712.5 (ed. 20)

Architettura del paesaggio - Giardini e parchi

(C) 2004 Firenze University Press

Università degli Studi di Firenze

Firenze University Press

Borgo Albizi, 28

50122 Firenze, Italy

http://epress.unifi.it/

Printed in Italy 


\section{INDICE}

Introduzione

$\begin{array}{ll}\text { PARTE I - Verso una definizione di parco tematico } & 7\end{array}$

$\begin{array}{lr}1 \text { MATRICI } & 9\end{array}$

1.1 - IL GIARDINO MANIERISTA 11

"L'anello che non tiene" 11

La natura simbolica e il Paesaggio come narrazione $\quad 15$

Paesaggio d'artificio: Il giardino di Pratolino come esito divulgato 20

1.2 - PLEASURE GARDENS, FOLIES, JARDINS SPECTACLES 31

Il Vauxhall di Londra (1661-1859) quale prototipo e sue "esportazioni" in Europa 31

Specificazione del paesaggio 36

Lo spazio creativo 41

1.3 - LE ESPOSIZIONI UNIVERSALI 45

Esperimenti di urbanistica tra utopia ed ecumenismo 45

Giardino e tecnologia $\quad 53$

$\begin{array}{ll}\text { Paesaggi sintetici } & 57\end{array}$

1.4 - GLI AMUSEMENT PARKS AMERICANI 63

Coney Island: parco "pubblico"/ luna park 63

Contesto come piano "0" (per la sovrapposizione di simboli) 68

Il luogo equivalente?

1.5 - MODELLO DISNEYLAND, PAESAGGIO READY-MADE 75

Idee per Disneyland nel retroscena 75

$\begin{array}{ll}\text { Fiction lands } & 78\end{array}$

$\begin{array}{ll}\text { Visioni anticipate } & 81\end{array}$ 
2.1 - LA COSTRUZIONE DEL TEMA: POP-ART, RETORICA POST-MODERN ED ESTRANIAMENTO DAL CONTESTO

2.2 -TEMA come DETERRITORIALIZZAZIONE/DIACRONIZZAZIONE.

Variazioni sul modello Las Vegas

2.3 - TEMA come VALORIZZAZIONE/SINGOLARIZZAZIONE

Il parco diffuso di Tonino Guerra in Romagna

L'Hortus Conclusus di Benevento

I paesaggi di Ian Hamilton Finlay

PARTE II - Analisi simbolo-spaziale e schemi per la progettazione

- ELENCO DEI PARCHI DEL DIVERTIMENTO EUROPEI

- SCHEDA ANALITICA DEI PARCHI DEL DIVERTIMENTO EUROPEI

Risultati della scheda analitica

Parc Asterix, Plailly, France (1989-2002)

Mer de Sable, Ermenonville, France (1962-2002)

Efteling, Kaatsheuvel, Netherlands (1952-2002)

- INTERPRETAZIONE E IMPLICAZIONI PROGETTUALI 179

Direzioni per la progettazione di un parco del divertimento a tema 179

Componenti e composizioni per la progettazione 191

$\begin{array}{ll}\text { Bibliografia generale } & 203\end{array}$

$\begin{array}{ll}\text { Riferimenti iconografici nelle tavole } & 209\end{array}$

Allegati:

- tavole SCHEDA ANALITICA DEI PARCHI DEL DIVERTIMENTO EUROPEI

- tavole ANALISI SIMBOLO-SPAZIALE:

I_1.1 matrice giardino manierista, Pratolino, Firenze (XVI secolo)

I_1.2 matrice pleasure garden, Vauxhall, London (1661-1859)

I_1.3 matrice esposizioni universali,

Midway Pleasance della Chicago Columbian Exposition (1893)

I_1.4 matrice amusement parks americani, Santa Cruz Boardwalk, Santa Cruz, California, U.S.A. (dal 1908)

I 1.5 matrice Disneyland, California, U.S.A. (1955)

I_1.6 Sintesi del rapporto spazio/tema nelle matrici 
II_2.1 Parc Asterix, Plailly, France (1989-2002)

II_2.2 Mer de Sable, Ermenonville, France (1962-2002)

II_2.3 Efteling, Kaatsheuvel, Netherlands (1952-2002)

II_2.4 Sintesi del rapporto spazio/tema nei parchi europei Parc Asterix, Mer de Sable, Efteling

- tavole INTERPRETAZIONE E IMPLICAZIONI PROGETTUALI:

II_3 Direzioni per la progettazione di un parco del divertimento a tema

II_4 Componenti e composizioni per la progettazione 


\section{Prefazione}

Guido Ferrara*

Il progetto degli spazi aperti, per sua natura, ha una duplice valenza: da un lato costituisce il modo con cui l'architettura costruita trova un rapporto e si relaziona al suo intorno e dall'altro possiede un valore in sé, questa volta non solo come tessuto che tiene insieme elementi diversi, ma come trama costituita eminentemente da vuoti, come paesaggio progettato e disegnato in quanto tale e, spesso, come luogo dove é dominante e come tale meritevole di considerazione esplicita la presenza e la riproduzione della vita biologica (non solo vegetale).

Nei casi in cui questo esercizio si colloca entro un'area di pregio, questa responsabilità non può che accentuarsi, soprattutto per il secondo aspetto, e non solo perché gli elementi oggetto di interesse hanno preminenti fondamenti di tipo naturalistico-ambientale e quindi possono essere considerati anche come oggetto di una politica di conservazione e di tutela, non solo perché la grande scala dei fenomeni (per es. laghi, pinete, coste) obbliga necessariamente ad una considerazione dei problemi di insieme, ma anche perché la loro fruizione implicitamente connessa con gli aspetti percettivi e d'uso si può scontrare con inevitabili ancorché non volute trasformazioni, anche provocando indesiderabili processi di degradazione (visiva, ambientale, acustica, ecc.) indotta dalle finalità per cui i visitatori stessi sono stati richiamati.

Perché questo effetto "perverso" si verifichi non é affatto obbligatorio che l'uso delle risorse sia consumistico e distruttivo e quindi contenga già nella sua natura l'ammissione implicita di un degrado annunziato: può essere il prodotto scontato di un utilizzo delle risorse di tipo contemplativo, come nel caso del "pellegrinaggio laico" dei visitatori di un parco (per es. archeologico, naturalistico, storico, paesaggistico) che, nei confronti delle fattezze sensibili del sito che rischiano di essere direttamente compromesse proprio dal grande numero dei visitatori e/o dei parcheggi, dai necessari servizi o dalla loro mancanza, dalla produzione di rifiuti, dal rumore, dal traffico, dal carico ambientale d'insieme, ecc.

Se questa fenomenologia si verifica per un luogo "protetto", figurarsi allora cosa può accadere in un "parco a tema", dove la finalità è principalmente ludico-ricreativa, e per il quale l'attesa di chi lo costruisce è sicuramente indirizzata verso grandi numeri, meglio se a crescita esponenziale nel tempo: quasi una definizione perfettamente contraria a quella di un paesaggio sostenibile.

Tuttavia, il campo della progettazione paesistica sa prendersi la responsabilità del disegno degli spazi aperti anche in questo caso e normalmente è impiegata (nel caso virtuoso) proprio per individuare di volta in volta le modalità più adatte per fare un bilancio preventivo delle molte variabili in gioco, allo scopo proprio di far tornare i conti, quelli responsabili della forma dello spazio, dell'ecologia applicata e sperimentale, dei modi d'uso, delle molteplici azioni mobili nel tempo, ecc. Esiste, naturalmente, anche il caso vizioso, entro cui tutti questi passaggi sono mistificati o affidati a pseudo soluzioni. Ma non c'è proprio nulla di nuovo sotto il sole, dato che simulazioni dello stesso tipo sono riproposte ogni giorno anche in urbanistica, nella geologia ambientale, in medicina, nelle armi di distruzione di massa, ecc.

La considerazione sintetica del paesaggio, inteso come disegno degli spazi di relazione ovvero come "architettura" a grande scala, s'intreccia direttamente con la considerazione dell'intorno immediato degli oggetti edificati, che qualche volta viene nobilitato in "giardino", qualche altra costituisce un sistema più o meno complesso di arredo urbano, di segni, di piazze, di percorsi, di viali alberati, ecc., non come semplice corollario o dilatazione del tema progettuale architettonico, ma come soggetto responsabile di una vera e propria partnership fra spazi costruiti e spazi vuoti, fra architettura e paesaggio, dove i due "opposti" diventano complementari e dialogano fra loro, talvolta con risultati inaspettati di grande 
qualità. Risultati che giovano alla ricerca delle soluzioni più confacenti alla eventuale sensibilità ambientale dell'area, ma che giovano anche all'architettura in quanto tale, perché essa perde così facendo quella definizione astratta e sradicata, da oggetto metafisico posato su un improbabile contesto, e acquista il ruolo cogente di essere proprio là dove doveva essere, come se ci fosse sempre stata, accumulando valore proprio perché riferita ad un orizzonte più ampio e quindi amplificando e completando per quanto possibile il proprio messaggio espressivo.

Esiste anche il caso in cui l'architettura in quanto tale costituisce solo una quinta usata dalla costruzione del paesaggio per ancorare lo spazio, e quindi non importante in sé, ma valida solo come sponda o limite o contenitore di quel sistema dialettico di relazioni che si assume la responsabilità espressiva dell'insieme.

Il tema per sua natura non propone, in altri termini, di disegnare un "verde" che "salvi" o comunque proponga una sorta di indennizzo in conseguenza di qualche errore di progettazione o di concezione (per es., nel nostro caso, il parco a tema) o che si limiti ad episodi di complementarità subalterna, "ornando" di piante (o di arredi, di statue, di fibre ottiche) un luogo marginale per definizione: invece un partner, se deve essere tale, non é né deve essere un servo sciocco, ma é tenuto ad assumere dignità ed autonomia, articolando e dispiegando un ruolo originale e proprio, entro la funzione dialettica e creativa di un'azione a quattro mani. Il "secondo uomo", che normalmente è sotteso nella figura del paesaggista, in questo caso può assumere il ruolo del primo violino, se non addirittura quello del direttore d'orchestra.

Ma qual è il percorso logico che conduce il progettista di paesaggio verso forme inventive e segni ambientali capaci di esprimere l'originalità del nostro momento storico (quello che guarda al futuro), senza una subalternità scontata non solo verso le circostanti architetture ma anche verso le forme e i segni lasciati sul territorio dalle generazioni che ci hanno preceduto? E' una bella domanda, a cui il saggio curato da Enrica Dall'Ara risponde in modo articolato. Ci si è proposto di indagare su un tema ultra moderno (i parchi a tema, appunto) che per la verità è eterno quanto il mondo, dato che l'espressività semantica del giardino che racconta $\mathrm{e}$ che diverte è uno degli scopi non reconditi di molte progettazioni di paesaggio con manifeste finalità estetico-percettive. Ne nasce in primo luogo che il processo attraverso il quale si costruiscono "paesaggi d'artificio", in cui evidentemente è importante soprattutto il ruolo dei vuoti e degli spazi di relazione, ha origini antiche, alcune delle quali particolarmente sperimentate e solide. Il richiamo agli eventi dei pleasure gardens e delle esposizioni universali - paralleli o successivi alla rivoluzione industriale - ricorda che questi precedenti non sono altro che un'anticipazione molto importante delle "tematizzazioni" più recenti $\mathrm{e}$ attuali, a cui la ricerca è dedicata.

Ma poiché prima del progetto bisogna considerare l'esistente, si parte con l'ammissione che quanto nell'ambiente da trasformare si offre alla nostra considerazione merita senza alcun dubbio conoscenza e rispetto, ma certamente non per essere fotocopiato entro un contesto socio-culturale diverso da quello originale (falso paesaggio) o per essere di impedimento a più elevate prestazioni estetiche (vincolo-campana di vetro, a-priori). Peggio: quando le forme e i segni del passato e del presente sono obiettivamente regressivi e dequalificanti (presenza di detrattori, come cave, abbandono, aree relitte), esse obbligano il progettista a concepire delle compensazioni e dei recuperi, ovvero ad inventare il nuovo, decisamente in contraddittorio con le presenze e le motivazioni di basso profilo, operando come fa un chirurgo contro le patologie e gli squilibri indotti. E vale la pena osservare che casi di questo genere sono così frequenti da rappresentare un terreno di sperimentazione progettuale più che stimolante, quale che sia la finalizzazione per cui il progetto di paesaggio trova motivi per nascere, compresi - evidentemente - i parchi a tema.

La risposta fornita in questa sede a questo tipo di sollecitazione è ricca di interessi e assai generosa sul piano del giro d'orizzonte proposto dalla ricerca.

Sotto il profilo del metodo, l'indagine è stata definita innanzitutto per "matrici", finalizzate alla descrizione delle costanti e delle differenze di un certo numero di esempi concreti, con la 
stesura di un primo elenco esemplificativo dei casi più significativi e recenti, affidati a specifiche schede analitiche. Secondariamente, Dall'Ara propone un'analisi per "processi" delle differenti esperienze, accuratamente selezionate e documentate, dove gli aspetti semantici vengono definiti sotto il profilo di "schemi per la progettazione", con conseguente interpretazione dei dati e conseguenti implicazioni progettuali.

Il materiale proposto fornisce quindi stimoli particolarmente interessanti nell'esame dell'analisi semantica e degli schemi per la progettazione, non solo per la ricca documentazione raccolta, ma per il meccanismo dialettico impiegato dalla Dall'Ara per interpretare la costruzione del "tema" a cui i diversi parchi sono appunto finalizzati. Per questo vengono finemente raffrontati esempi di deterritorializzazione/diacronizzazione

e valorizzazione/singolarizzazione, riuscendo a toccare con mano in modo particolarmente convincente un'interpretazione critica delle implicazioni progettuali delle diverse esperienze. Ne nasce che:

- la presentazione delle diverse problematiche è configurata sempre in modo efficace ed incisivo, affidata non solo a formule descrittive, ma anche a schemi interpretativi e bilanci d'insieme che permettono di volta in volta stimolanti comparazioni e raffronti;

- l'approccio specifico ai tematismi della progettazione d'ambiente interagisce a livello dialettico a più scale e con la considerazione contestuale di fattori progettuali diversi e alternativi rispetto a quelli propriamente edilizi, fra cui la luce e il movimento del sole, l'acqua sopra e sotto la terra, il limite bosco-prato, la vedute e le skyline dello sfondo, le piante viventi (esotiche o quelle tipiche della flora endemica)...

- il metodo valutativo appare in buona misura originale e teso più a toccare i "nervi scoperti" del fare paesaggio, che ad argomentare o controdedurre questo o quel tematismo: così facendo, l'analisi svolta appare caricata di termini di riferimento più ampi e strategici del tema indagato e costituisce pertanto un apprezzabile avanzamento critico e teorico.

Ecco perché la ricerca riesce a mettere in evidenza le responsabilità proprie della competenza progettuale in esame, per la quale il paesaggio e la definizione formale e "architettonica" degli spazi aperti riesce a fornire quel contributo formativo e fondativo che appare logico e condivisibile: e infatti quel contributo, quell'esercizio progettuale appare, ora e finalmente, giustificato e necessario, ma a cose fatte è opportuno ricordare che all'inizio del percorso esso poteva non essere preso in considerazione.

* Guido Ferrara, ordinario di Architettura del Paesaggio, Università di Firenze. 


\title{
Introduzione
}

Nella contemporaneità è divenuto ricorrente l'atteggiamento progettuale che procede per assegnazione di temi, nel disegno dei luoghi e delle singole architetture. Recentemente Michael Sorkin, in La tematizzazione della città ${ }^{1} \mathrm{e}$ in Variations on a theme park. The new American city and the end of public space ${ }^{2}$ ha richiamato l'attenzione sul fatto che il modello dei parchi tematici sta dilagando - uscito dai propri tradizionali confini che lo vogliono finalizzato a creare spazi d'eccezione - per investire più genericamente i modi di costruzione dello spazio collettivo. Il che implica sinteticamente: processo di privatizzazione vs costruzione dello spazio pubblico, finalità di omologazione da marchio commerciale vs garanzia della vivacità che è propria dei luoghi plasmati per essere, e dall'essere, spazi di incontro sociale:

\begin{abstract}
"This is the meaning of the theme park, the place that embodies it all, the ageographia, the surveillance and control, the simulations without end. The theme park presents its happy regulated vision of pleasure - all those artfully hoodwinking forms - as a substitute for the democratic public realm, and it does so appealingly by stripping troubled urbanity of its sting, of the presence of the poor, of crime, of dirt, of work. In the "public" spaces of the theme park or the shopping mall, speech itself is restricted: there are no demonstrations in Disneyland. The effort to reclaim the city is the struggle of democracy itself." 3
\end{abstract}

Il problema posto in toni accorati da Michael Sorking sul dilagare della tematizzazione è riconducibile alle riflessioni contemporanee più generali sul rapporto fra globalizzazzione e identità delle comunità locali e dei rispettivi luoghi.

Spaventano le previsioni di Rem Koolhas quando presenta la sua Generic City ${ }^{4}$, che si configura come un'istallazione effimera e a-centrica montata su un suolo occupato da vegetazione ipertrofica, completamente dimentica di qualsiasi regione d'origine o di processi di acclimatazione. Ci si spaventa sopratutto se la si relaziona con l'idea di città quale governata localmente dal proprio genius loci. E nel discorso di Koolhaas è fra le righe che la Generic City condivida qualcosa dei parchi tematici così come ormai li si immagina: incondizionati nella trasformazione, inconsistenti perchè decontestualizzati, alleggeriti da tutto ciò che è identitario ad opera della simulazione, per divenire, parafrasando Koolhaas, "trasparenti come un logo":

\footnotetext{
"The Generic City breaks with this decostructive cycle of dependency: it is mothing but a reflection of present need and present ability. It is the city without history. It is big anough for everibody. It is easy. (...) If it gets too small it just expandes. If it gets old it just self-destructs and renews. It is equally exciting - or unexciting everywhere. It is "superficial" - like a Hollywood studio lot, it can produce a new identity every Monday morning." 5
}

La Generic City è "facile", si espande quando ne ha l'esigenza, si distrugge e si ricostruisce, "è eccitante - o non eccitante - ovunque. E' superficiale - come uno studio di Hollywood, può produrre una nuova identità ogni Lunedì mattina."

Non sembra molto diversa da un parco a tema.

Sempre che sia possibile figurarsi l'impianto urbanistico della Generic City, la città di Koolhaas richiama in qualche strana maniera la Ville Radiose di le Corbusier, se si assume che i nodi dell'intermodalità -gli aeroporti- sostituiscano le unità di abitazione e che le

\footnotetext{
${ }^{1}$ Michael Sorkin , La tematizzazione della città, in "Lotus" n. 109, Il nuovo mondo allegorico. Sui parchi tematici, Electa, Milano 2001.

${ }^{2}$ Michael SORKIN (a cura di), Variations on a theme park. The new American city and the and of public space, Hill and Wang, New York 1999.

${ }^{3}$ MichaEl SORKIN (a cura di), Op.cit., Hill and Wang, New York 1999, pagg. 1239-1267.

${ }^{4}$ Rem KoolhaAs, The Genric City, in S,M,L,XL, Taschen, Germany 1997.

${ }^{5}$ Rem KoolhaAs, Op.cit, in $S, M, L, X L$, Taschen, Germany 1997, pag. 1250.
} 
"generiche" e nude praterie su cui si collocano queste ultime siano spazi brulicanti di istallazioni e attività. Generic City è un modello urbanistico non territoriale e non identitario. La città Cyburbia, presentata da Michael Sorkin nell'introduzione a Variations on a theme park, indagando la realtà americana, ha aspetti analoghi a Generic City. Si costruisce secondo le stesse logiche che regolano il sistema televisivo, per cui ogni elemento appartiene ad una rete ed ha al suo interno un uguale valore. E' la connessione ad attribuire un senso allo spazio. Alla città storica, fisicamente spaziale e fenomenologia della relazione sociale, "in cui l'ordine è una funzione della prossimità", si contrappone uno spazio invisibile, solo concettuale, scevro sia dalla geografia fisica sia da quella culturale: "La nuova città sostituisce l'anomalia e il diletto di tali luoghi [le città tradizionali] con un particolare universale (...). Ossessionata dal principio della produzione e del prezzo, la nuova città è poco più che uno sciame di bit urbani, buttando a mare la visione fisica del complesso, sacrificando l'idea di città come posto della comunità e delle relazioni umane".

Cyburbia è solo comunicazione (persuasione) e replicabilità.

In La tematizzazione della città Sorkin prelude semplicemente: "Ai nostri giorni possiamo osservare il diffondersi, su scala mondiale, di un nuovo fenomeno: la tematizzazione." Subito a seguito della premessa egli affronta il discorso in termini di analisi degli aspetti positivi e negativi dei parchi tematici. Così facendo rivela implicitamente come essi siano 1'"origine" dell'idea di tematizzazione e come quindi la tematizzazione contenga, in forma più o meno esplicita, quella di fruizione nel tempo libero, di intrattenimento, di divertimento. Il parco tematico appare attualmente costituire un particolare modello utopico di costruzione spaziale e trasformazione territoriale.

Anche Francoise Choay in Allegoria del patrimonio ${ }^{6}$ senza parlare espressamente di tematizzazione, fa una critica all'impresa culturale che nei processi di riqualificazione e restauro del patrimonio artistico, falsifica, commercializza, vende il bene in forme di visita più simili a un intrettenimento che a un modo di conoscenza. Il bene artistico viene snaturato a questi scopi.

La diffusione della progettazione intesa come tematizzazione rende interessante e utile ragionare sull'oggetto parco tematico che ne rappresenta l'esempio più puro, per comprenderlo.

Sul parco tematico il dibattito è fermo ad un empasse sintetizzato da una provocazione, in forma di quesito aperto, di Isabelle Auricoste: "parchi o utopie noiose?" 7 Il problema è nuovamente affrontato da Patrizia Mello nel capitolo Luoghi di evasione: parchi o utopie mortali? interno a Metamorfosi dello Spazio. Annotazioni sul divenire metropolitano ${ }^{8}$.

La domanda fa riferimento alla contraddizione per cui il divertimento risulta possibile - per definizione- solo in luoghi che presentano un margine di incertezza e di libertà, mentre i parchi del divertimento contemporanei sembrano esasperare controllo e pianificazione di tutti gli aspetti in gioco.

Altri, oltre ad Isabelle Auricoste, hanno segnalato questo nodo - Michael Sorkin ${ }^{9}$, Miles Orvell ${ }^{10}$ relativamente al paesaggio americano, e Marc Augé ${ }^{11}$ - senza fare seguire alle

\footnotetext{
${ }^{6}$ FrANCOISE CHOAY , L'allegoria del patrimonio, a cura di Ernesto d'Alfonso e Ilaria Valente, Officina Edizioni, 1995.

${ }^{7}$ Provocazione espressa in IsABelle AuRicoste , Parchi o utopie mortali?, “ Ottagono” n. 99, Intrattenimento, 1991, pagg. 16-30, e in ISABELle AURICOSTE, I parchi ricreativi in Europa: il divertimento e l'altrove, in Monique Mosser, Georges Teyssot, L'Architettura dei giardini d'Occidente- Dal Rinascimento al Novecento, Electa, Milano 1990, pagg. 479-490.

${ }^{8}$ Patrizia Mello, Metamorfosi dello Spazio. Annotazioni sul divenire metropolitano, Bollati Boringhieri, Torino 2002, pagg. 22-37.

${ }^{9}$ In Michael Sorkin (a cura di), Op. cit., Hill and Wang, New York 1999 e in Michael Sorkin, Op. cit., in "Lotus" n. 109, Il nuovo mondo allegorico. Sui parchi tematici, Electa, Milano 2001.

${ }^{10}$ in Miles ORVELL, The real thing. Imitation and authenticyty in American culture, 1880-1940, The University of North Carolina Press, Chapel Hill \& London 1989.

${ }^{11}$ In MARC AugÉ, Disneyland e altri nonluoghi, Bollati Boringhieri, Torino 2002.
} 
constatazioni indicazioni propositive per la soluzione del problema. L'Auricoste offre comunque uno spiraglio:

\begin{abstract}
"Allo stato attuale, tuttavia, razionalizzazione economica e buone intenzioni dei pianificatori sembrano pesare ancora troppo per potere essere efficacemente contrastate, e la sopravvivenza del divertimento si affida soprattutto alle doti inventive di progettisti e visitatori. (...) L'evoluzione o, al contrario, la scomparsa dell'attuale modello dipendono dunque essenzialmente dalla capacità di offrire nuove soluzioni ad una siutuazione in apparenza senza sblocco. L'analisi delle innumerevoli modalità assunte nel corso del tempo dal divertimento fa pensare che l'invenzione resti sempre possibile in questo settore che si direbbe alimentato da una capacità illimitata di rinnovamento. Non c'è infatti sistema, neppure il più perfetto, che non presenti quella minima incrinatura che basta ad aprire la via al divertimento." 12
\end{abstract}

E' abbastanza chiaro che la paesaggista consideri quale modello attuale quello costituito da Disneyland (California, 1955) che si presenta esportabile ovunque, perfettamente controllato e pianificato, così come controllato e pianificato risulta il comportamento dei suoi visitatori.

La definizione di divertimento sottolineata dall'Auricoste già anticipa come difficilmente il modello del parco tematico possa venire proposto come forma urbana tout court, in quanto la sua caratteristica sostanziale è l'essere luogo che si contrappone allo spazio civico ordinario, aspetto che confina il parco e lo individua come spazio d'eccezione.

"Divertire (divertere) non significa forse distogliere, allontanare dall'uso comune, così come d'altronde distrarre (distrahere) rinvia all'azione di tirare in senso inverso? (...) sono al tempo stesso le metafore in termini di esperienza concreta che connettono l'altrove con la nozione di territorio situato. Il senso di estraneità risiede nella conoscenza vissuta delle diversità e dei confini tra due territori." $" 13$

Dare in una qualche misura scioglimento all'empasse descritto è l'obiettivo della presente ricerca.

Essa intende individuare direzioni progettuali, se esistono, che consentano allo stesso tempo di avere un paesaggio complessivo (dentro e fuori del parco a tema) identitario e un parco nel senso proprio del termine, che consenta un divertimento reale, grazie e nonostante un processo di tematizzazione. Questo senza che il progetto finisca con il realizzare un'utopia noiosa.

Tematizzazione e divertimento sono termini associati a paesaggi suis generis, soprattutto perchè richiamano rapidamente alla mente la prepotenza di interessi economici e marchi commerciali.

In realtà entrambi (tematizzazione e divertimento) sono intrinsecamente legati ai concetti - e alla storia - del giardino e del parco. Ragionare sui parchi tematici significa interrogarsi sul rapporto fra elemento naturale, architettura e simbolo (in maniera potenziata rispetto a quanto si potrebbe fare riguardo a parchi "semplici"): si tratta di una questione di architettura del paesaggio nel suo senso più proprio e nelle sua forme più estreme.

Sembra quindi che possa essere carico di conseguenze significative guardare a questo oggetto "parco tematico" con lo sguardo del paesaggista e domandarsi di quale paesaggio si tratti, cosa comporti progettarlo, se sia possibile conferire identità e qualità a questi luoghi.

$\mathrm{Nel}$ cercare le risposte ci si avvia lungo un percorso suggestivo, diretto a recuperare le componenti di memoria (densa) dei parchi del divertimento, ovvero le esperienze maturate a livello internazionale che ne hanno formato il significato contemporaneo e che qui chiameremo Matrici a esprimere la loro azione di imprinting.

Le Matrici vengono rintracciate visualizzando le componenti dei parchi tematici di oggi che ognuno conosce: affluenza di massa, elevata infrastrutturazione dei trasporti, la presenza di rides e attrazioni, ma soprattutto di un apparato da fiaba e d'emozione, sotto l'egida della comunicazione. Si può sintetizzare l'elenco utilizzando una sola espressione assunta da

\footnotetext{
12 Isabelle Auricoste, Op.cit., in Monique Mosser, Georges Teyssot, L'Architettura dei giardini d'Occidente- Dal Rinascimento al Novecento, Electa, Milano 1990, pag. 490.

${ }^{13}$ Isabelle Auricoste, Op.cit., in MoniQue Mosser, Georges Teyssot, L'Architettura dei giardini d'Occidente- Dal Rinascimento al Novecento, Electa, Milano 1990, pag. 479.
} 
Gregory Beck ${ }^{14}$ : architettura dell'esperienza. Il fine di quest'ultima consiste nel "creare irresistibili luoghi-eventi, mescolando design ambientale, tecnologia mediatica e racconto." In particolare in Europa la storia dei parchi del divertimento si interseca con quella della nascita dei parchi pubblici:

“diffusi per l'intera Europa, questi giardini [i pleasure gardens di origine Londinese del XVIII sec.], specializzati nell'assicurare il divertimento ai propri frequentatori, sono un genere la cui storia corre parallela a quella dei giardini pubblici; anzi può accadere a volte che si configurino come porzioni specializzate inserite al loro interno. la contiguità ideologica fra pleasure garden e il giardino pubblico inteso nella sua forma più piena deve essere sentita anche in origine, se già nel Settecento molti di questi pleasure garden sono chiamati " giardini pubblici". D'altra parte molti sono i punti di contatto: il medesimo pubblico, il solido legame con la società urbana e le sue mode. Ma insieme hanno un'identità ben precisa (...)". ${ }^{15}$

E risulta pertanto intrinsecamente legata all'arte dei giardini.

Considerazione che nell'apparente ovvietà non è priva di implicazioni, da un punto di vista progettuale.

L'origine dei parchi tematici sembra in particolare congiunta ai momenti speciali in cui il giardino ospita un "surplus" di simbolizzazione ("surplus" rispetto al contenuto simbolico comunque intrinseco al concetto stesso di giardino); ai momenti in cui il simbolo è sia sintomo sia strumento di una volontà di accesso a nuove forme di conoscenza, attraverso uno sguardo divertito e stupito. Non è un caso che questi luoghi siano progettati in epoche di transizione, di crisi culturale, dandovi espressione. In tali periodi il divertimento inteso nell'accezione di Isabelle Auricoste è senza dubbio sentito come urgenza, coincide con l'evasione, assume contenuti frivoli, disimpegnati e genera forme aperte, disinibite, fantastiche - si ricorre al fantastico soprattutto quando il reale non è convincente.

Il percorso che ricerca le origini del parco tematico conduce al giardino manierista, ai pleasure gardens, alle folies, ai jardins spectacles, alle esposizioni universali, agli amusement park americani, a Disneyland, idee di luoghi che la ricerca vuole indagare con attenzione ai contesti che li generano, ai modi specifici di costruzione del paesaggio, e operando un'analisi sugli esiti che tali modi raggiungono, affinché sia possibile costituire un apparato critico per la comprensione e la valutazione dei parchi ricreativi contemporanei: occorre avere alcuni giudizi che consentano di procedere nelle fasi di approfondimento (PARTE II della ricerca) con una chiave interpretativa a disposizione.

L'intuizione alla base della ricerca è che forse l'empasse messo il luce da Isabelle Auricoste può essere in qualche misura superato da un'accorta progettazione del paesaggio: è infatti nel rapporto fra natura-simbolo-genius loci che può crearsi uno spazio non totalmente codificato (un punto di sorpresa), nelle varietà di combinazioni di presenza e negazione, collaborazione e predominio di un elemento rispetto all'altro. Il modello americano Disney, che appare tanto perfetto quanto "sterile", potrebbe non essere - storicamente non è - l'unica fonte a cui attingere. Per questo la ricerca tenta, nella PARTE I, la definizione di parco tematico attraverso il recupero delle matrici del parco del divertimento e parallelamente attraverso un ragionamento sui processi di tematizzazione e sulle loro finalità, interrogandosi sul rapporto fra tema e aura dei luoghi coinvolti.

La seconda parte della ricerca intende fotografare, per quanto possibile, la situazione europea, cercando di leggere nelle realizzazioni contemporanee di parchi del divertimento le matrici prevalenti, la presenza o meno del tema, il ruolo del tema e l'avvenuta o meno progettazione del paesaggio, e scegliere all'interno del quadro alcuni esempi di interesse da sottoporre ad un'analisi di maggiore dettaglio che suggerisca indicazioni per le progettazioni future.

\footnotetext{
${ }^{14}$ BECK GREGORY, Un atlante dei parchi tematici, in "Lotus" n. 109, il nuovo mondo allegorico. Sui parchi tematici, Electa, Milano 2001, pag. 104.

${ }^{15}$ Franco PANZINI, Per i piaceri del popolo. L'evoluzione del giardino pubblico in Europa dalle origini al XX secolo, Zanichelli, Bologna 1997, pag. 99.
} 


\section{DIAGRAMMA DELLA STRUTTURA DELLA RICERCA E DEL VOLUME}

PARTE I - verso una definizione di parco tematico

\section{PARCO TEMATICO}

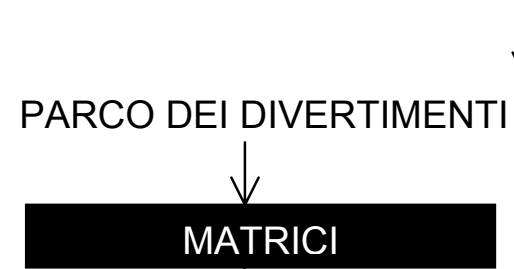

$\vee$

$\downarrow$

Il giardino manierista

Pleasure gardens, folies, jardins spectacles

Le Esposizioni universali

Gli Amusement parks americani

Il modello Disneyland<smiles></smiles>

\section{TEMA}

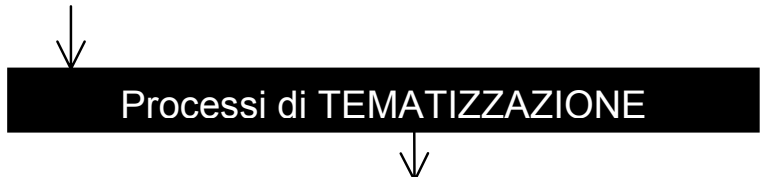

COSTRUZIONE DEL TEMA

FINALITÀ/ESITO DEL TEMA:

- deterritorializzazione

- singolarizzazione

L'indagine su ciascuna MATRICE è articolata in tre parti

( ognuna corrispondente ad un paragrafo; è possibile quindi una LETTURA

TRASVERSALE: i diversi contesti, i diversi modi di costruzione del paesaggio, i diversi esiti)

- CONTESTO/PREMESSE

- MODI DI COSTRUZIONE DEL PAESAGGIO

- ESITI/IMPLICAZIONI<smiles>[AlH2]</smiles>

Il giardino manierista

"L'anello che non tiene" (CONTESTO/PREMESSE)

La natura simbolica e il Paesaggio come narrazione (MODI DI COSTRUZIONE DEL PAESAGGIO)

Paesaggio d'artificio: Il giardino di Pratolino come esito divulgato (ESITI/IMPLICAZIONI)

Pleasure gardens, folies, jardins spectacles

Il Vauxhall di Londra (1661-1859) quale prototipo e sue "esportazioni" (CONTESTO/PREMESSE)

Specificazione del paesaggio (MODI DI COSTRUZIONE DEL PAESAGGIO)

Lo spazio creativo (ESITI/IMPLICAZIONI)

Le Esposizioni universali

Esperimenti di urbanistica tra utopia ed ecumenismo (CONTESTO/PREMESSE)

Giardino e tecnologia (MODI DI COSTRUZIONE DEL PAESAGGIO)

Paesaggi sintetici (ESITI/IMPLICAZIONI)

Gli Amusement parks americani

Coney Island: parco "pubblico"/luna park (CONTESTO/PREMESSE)

Contesto come piano "0" (per la sovrapposizione di simboli) (MODI DI COSTRUZIONE DEL

PAESAGGIO)

Il luogo equivalente? (ESITI/IMPLICAZIONI)

Il modello Disneyland

Idee per Disneyland nel retroscena (CONTESTO/PREMESSE)

Fiction Lands (MODI DI COSTRUZIONE DEL PAESAGGIO)

Visioni anticipate (ESITI/IMPLICAZIONI) 
PARTE II - analisi simbolo-spaziale e schemi per la progettazione

\section{ELENCO DEI PARCHI DEI DIVERTIMENTI EUROPEI}

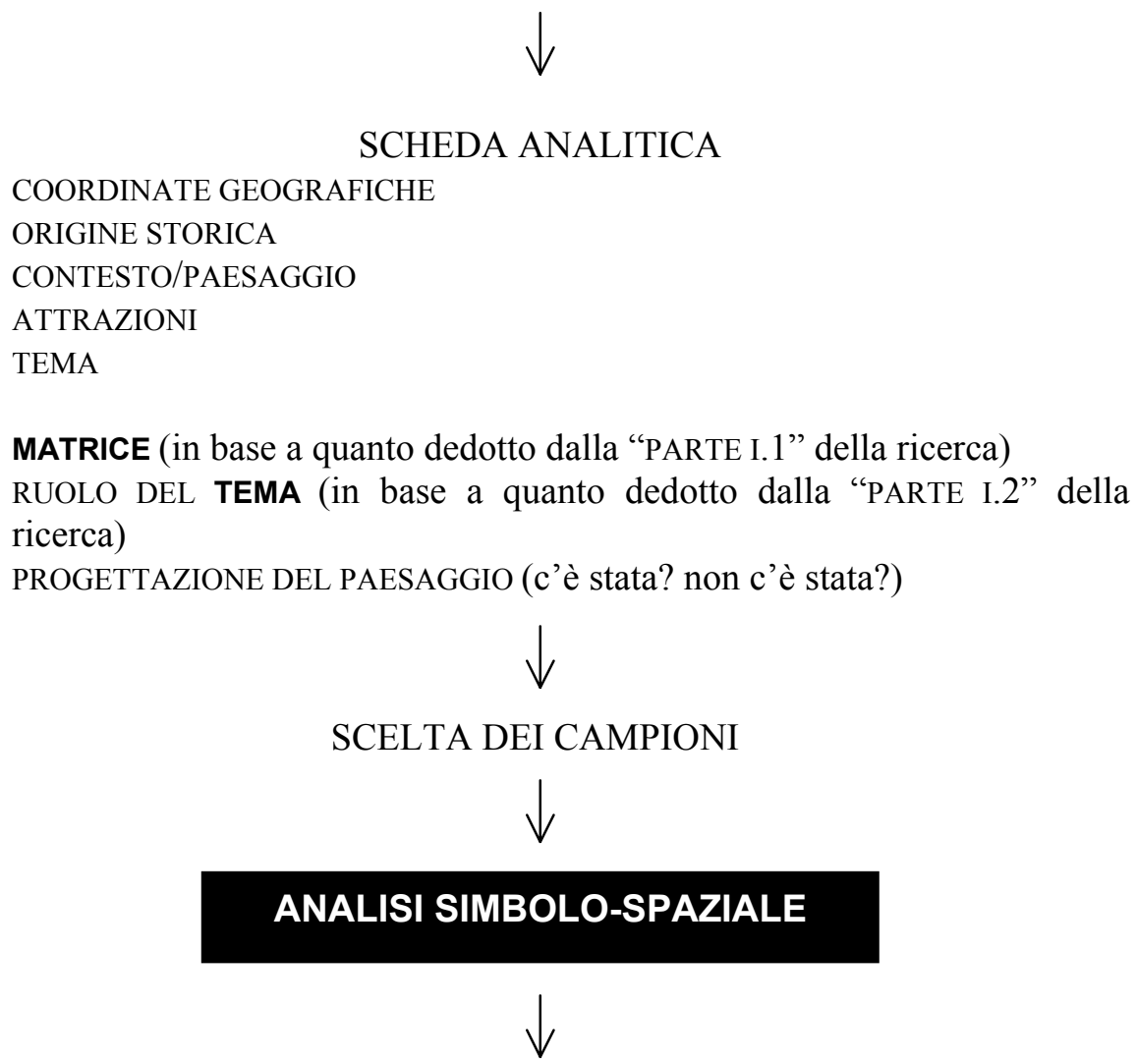

IMPLICAZIONI PROGETTUALI 
PARTE I - Verso una definizione di parco tematico 


\section{MATRICI}

È possibile definire il parco tematico - in maniera sintetica - come un parco che si struttura intorno ad un tema portante. Fermandosi a questa accezione il numero di situazioni e spazi considerabili parchi tematici diverrebbe enorme ed esisterebbero parchi tematici in tutte le epoche: potrebbero dirsi tali la Villa di Adriano a Tivoli (come luogo per ricreare ambienti visti durante i viaggi dell'imperatore), i giardini cinesi (quali miniature di paesaggi di natura selvaggia) ecc..

Peraltro il concetto di parco tematico richiama l'idea di parco aperto al pubblico e di parco quale spettacolo rivolto ad uno spettatore, realizzato non perché vi viene riconosciuto un valore intrinseco, ontologico, ma per essere visto, ammirato, e per divertire.

Così il termine è difficilmente dissociabile da modelli determinati: i pleasure gardens, le folies, i jardins spectacle, così come essi si vennero a definire dal sedicesimo al diciannovesimo secolo, e gli amusement parks americani.

E' indispensabile inoltre considerare il rapporto avvenuto dalla seconda metà dell'ottocento fra parchi del divertimento ed esposizioni universali, non solo perché i loro eventi spesso si intersecano, ma anche perché parchi tematici ed esposizioni universali condividono i problemi logistici, e le relative soluzioni, legati all'affluenza di un pubblico di massa.

Il capitolo intende esplorare i precedenti storici che hanno contribuito alla formazione del concetto moderno di parco tematico, che è possibile per semplicità definire inaugurato "a metà degli anni cinquanta a Disneyland, in California", dove "più che un semplice luogo di divertimento animato dai suoi personaggi, Walt Disney inventò un modo del tutto nuovo di vivere storie di fantasia nella realtà del tempo e dello spazio. Il progetto, la pianificazione e la tecnologia furono messi al servizio di obiettivi teatrali: l'architettura aveva insomma la sua sceneggiatura" .

\footnotetext{
${ }^{1}$ GREGORY BECK in Un atlante dei parchi tematici, in "Lotus" n. 109, il nuovo mondo allegorico. Sui parchi tematici, Electa, Milano 2001, pagg. 104-117.
} 


\section{1 - IL GIARDINO MANIERISTA}

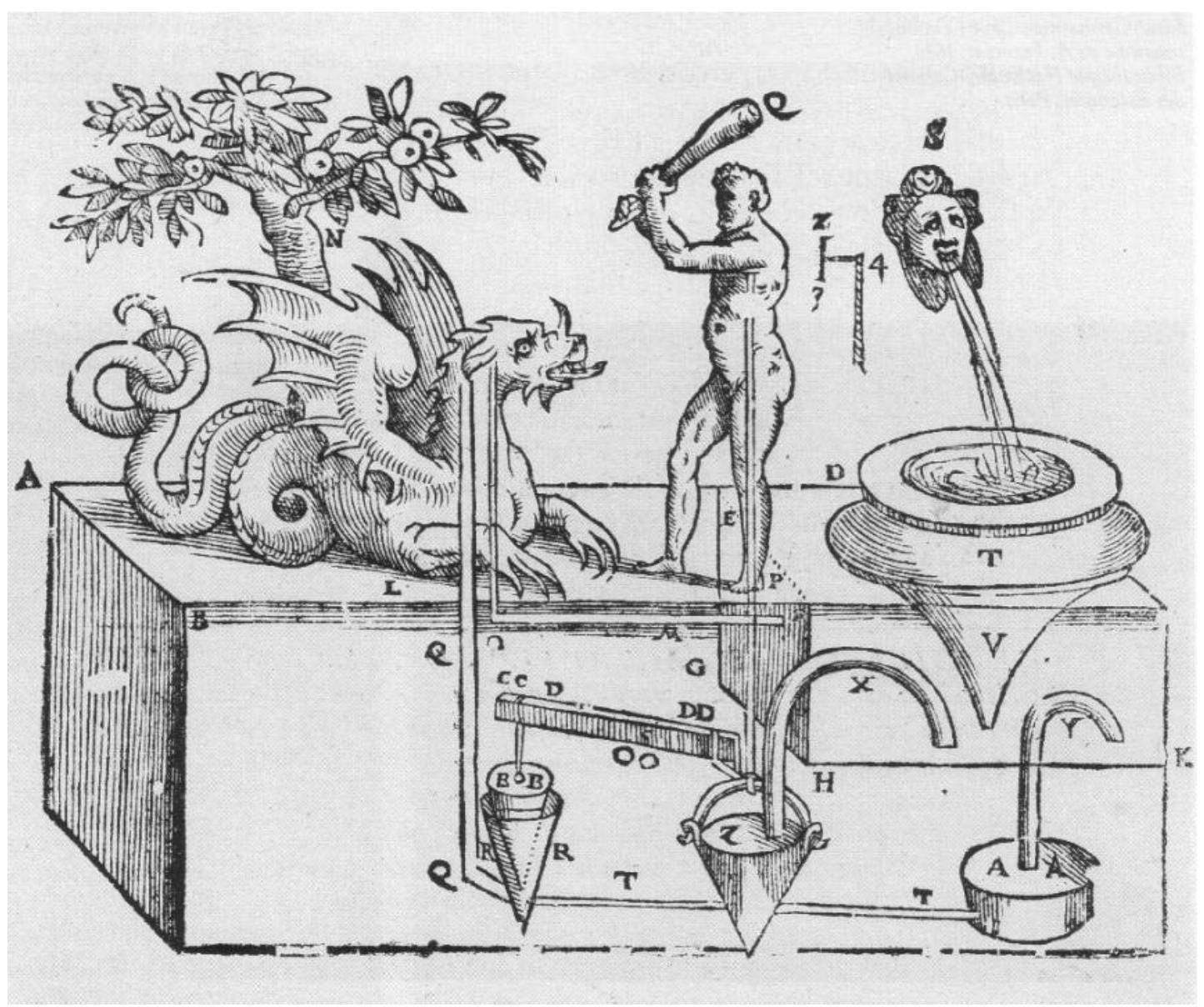

Fig. 1 Far che con un dracone, che stia a guardia dei pomi d'oro, combatta un Ercole. Progetto di teatro meccanico, incisione di G.B. Aleotti, 1589.

\section{“L’ANELLO CHE NON TIENE"}

L'idea di parco-giardino come luogo in cui è consentito l'affaccio ad un mondo completamente altro, diverso (origine del divertimento nell'accezione sottolineata da Isabelle Auricoste ${ }^{1}$ ) può essere fatta risalire al periodo manierista e al il proliferare delle sue Wunderkammern. Marcello Fagiolo parla del parco manierista del Sacro Bosco di Bomarzo in termini di "antimondo che affonda le radici nell'aldilà" e "contestazione dei mondi tradizionali” specificando:

"Aldilà della storia classica (...). Aldilà dello spazio occidentale con l'ansia di entrare in sintonia con mondi esotici. Aldilà dello spazio prospettico. Aldilà dell'umano con l'esaltazione della pazzia d'Orlando e del mostruoso (...)" 2

\footnotetext{
${ }^{1}$ ISABELlE AURICOSTE, I parchi ricreativi in Europa: il divertimento e l'altrove, in MONIQUE MOSSER, GEORGES TEYssot, L'Architettura dei giardini d'Occidente- Dal Rinascimento al Novecento, Electa, Milano 1990, pagg. 479-490.

${ }^{2}$ Marcello Fagiolo, Le due anime nelle ville della Tuscia, in Vezzosi Alessandro (a cura di), Il giardino d'Europa. Pratolino come modello nella cultura europea, Mazzotta, Firenze 1986.
} 
Luigi Zangheri definisce il giardino manierista come luogo che sembra voler mostrare "un volto insolito immediatamente sensibile e frequentabile":

"Nel Cinquecento, il collezionismo ed il mecenatismo dei principi corrosero la compattezza dei concetti della fisica aristotelica. La Gran Macchina Cosmica, già laboriosamente decifrata nei suoi rapporti uomo-natura, nei principi delle "simmetrie" e delle affinità stabilite su base geometrica e razionale, perdette progressivamente l'impronta di un sereno classicismo per far posto ad una serie di incertezze sul ruolo dell'uomo nella natura. Gli interessi alchemici, la curiosità per i reperti naturali mostruosi, l'interesse per i dati "favolosi" che pervenivano dalle terre scoperte da poco, condizionarono tutta la cultura del Cinquecento. Lo stesso giardino si aprì a mille curiosità esibendole pubblicamente, e in maniera sistematica, quasi a voler mostrare, nell'artificio delle citazioni dirette dalla natura (acque, minerali, animali, piante), un volto insolito immediatamente sensibile e frequentabile" ${ }^{\prime 3}$.

Zangheri evidenzia nel brano citato un altro aspetto: all'epoca la natura si presenta frequentemente costituita di elementi non armonici, mostruosi, "sbagliati", come presentato anche da Fausto Testa che sottolinea come essa "non è più stabile specchio di una norma, ma fascinosa e terrifica presenza di una plurima, dilatata varietà di fenomeni, (...) inesauribile serbatoio di nuovi esseri e di nuove forme" .

La crisi del Cinquecento è epocale e consiste nell'intuizione di un confine slabbrato fra ragione e follia, regola decifrabile e mistero. Si percepisce lo "sbaglio di Natura,/ il punto morto del mondo, l'anello che non tiene,/ il filo da disbrogliare che finalmente (...) metta/ nel mezzo di una verità." 5

Lo sbaglio di natura è costituito dall'anomalia, dal bizzarro, dal prodigioso, dal mostruoso naturale che diviene oggetto di collezione e osservazione. E' possibile parlare ancora, paradossalmente, di un atteggiamento artistico di carattere mimetico nei riguardi della natura, solo a patto di considerare come nel XVI secolo lo sbaglio di natura appaia essere quasi rappresentativo della natura stessa:

"L'intervento attivo dell'operatore demiurgico dell'officina naturale è peraltro compatibile con la vitalistica fluidità della struttura, fisica ed epistemologica, dell'universo "animistico" del "500: un universo instabile ( o destabilizzato) che , avendo smarrito la legalità "convenzionale" della fisica aristotelica appare ormai "regredito" alla magmatica indeterminatezza di un work-in-progress incolmabile che non conosce più un canone di leggi ma solo una frequenza statistica di deboli consuetudini, non regole ma regolarità, e che si riproduce piuttosto per via di combinazioni e metamorfosi provocate da centri di iniziativa molteplici e spesso antagonisti. In questo universo del possibile ogni essere è dunque intrinsecamente prodigioso nella misura in cui non appartiene alla costanza di una serie (...) ma viene suscitato ogni volta nella sua inedita singolarità (...). Il prodigium, il monstrum, possono così essere reintegrati alle procedure ufficiali della natura non già nella veste eccentrica della deroga, ma come luoghi privilegiati di verifica e di sovradeterminazione visibile della fortunosa ars combinatoria $(\ldots)^{, 6}$

Il potere immaginifico del prodigium e del monstrum è tale da interpellare l'artista su quali siano le proprie armi nell'archetipica sfida che vede in campo natura e artificio, Dio e artista. Gli strumenti più consoni appaiono essere la fantasia - come dato soggettivo e come elemento in grado di competere con il potere creativo della natura ${ }^{7}$ - e la tecnologia

\footnotetext{
${ }^{3}$ LuIgi ZANGHeri, Naturalia $e$ curiosa nei giardini del Cinquecento, in MONIQUE Mosser, GeORGES TeYsSOT, L'Architettura dei giardini d'Occidente- Dal Rinascimento al Novecento, Electa, Milano, 1990, pag. 55.

${ }^{4}$ FAUSto Testa, Spazio e allegoria nel giardino manierista, La Nuova Italia, Firenze 1991, pag. 1.

${ }^{5} \mathrm{Da}$ I limoni di Eugenio Montale. "Vedi, in questi silenzi in cui le cose/ s'abbandonano e sembrano vicine/ a tradire il loro ultimo segreto,/ talora ci si aspetta/ di scoprire uno sbaglio di Natura,/ il punto morto del mondo, l'anello che non tiene,/ il filo da disbrogliare che finalmente ci metta/ nel mezzo di una verità/.

Lo sguardo fruga d'intorno,/ la mente indaga accorda disunisce/ nel profumo che dilaga/ quando il giorno più languisce./ Sono i silenzi in cui si vede/ in ogni ombra umana che si allontana/ qualche disturbata Divinità."

6 Alessandro Rinaldi, La ricerca della "terza natura": artificialia e naturalia nel giardino toscano del Cinquecento, in MARCEllo FAGIOLO, Natura e artificio. L'ordine rustico, le fontane, gli automi nella cultura del Manierismo europeo, Officina Edizioni, Roma 1997, pagg. 155-156.

${ }^{7}$ Riguardo al legame fra percezione della natura multiforme e fantasia o creatività è interessante la specificazione di Maurizio Calvesi. Egli nella sua recente indagine sull'iconografia del Sacro Bosco di Bomarzo, individua in Vinicio Orsini, signore e ideatore del giardino, un "nascente gusto della dismisura, non morale ma artistica e immaginativa, con le dimensioni colossali della scultura. (...) In realtà quegli eccessi (...) sembrano invece
} 
(nell'arte dei giardini) e il virtuosismo (nelle arti figurative), come mezzi materiali di riproduzione della sua complessità. Il ricorso a questi strumenti non si pone solo in termini di sfida nei confronti della natura, ma anche in termini di possibilità di accesso alla conoscenza.

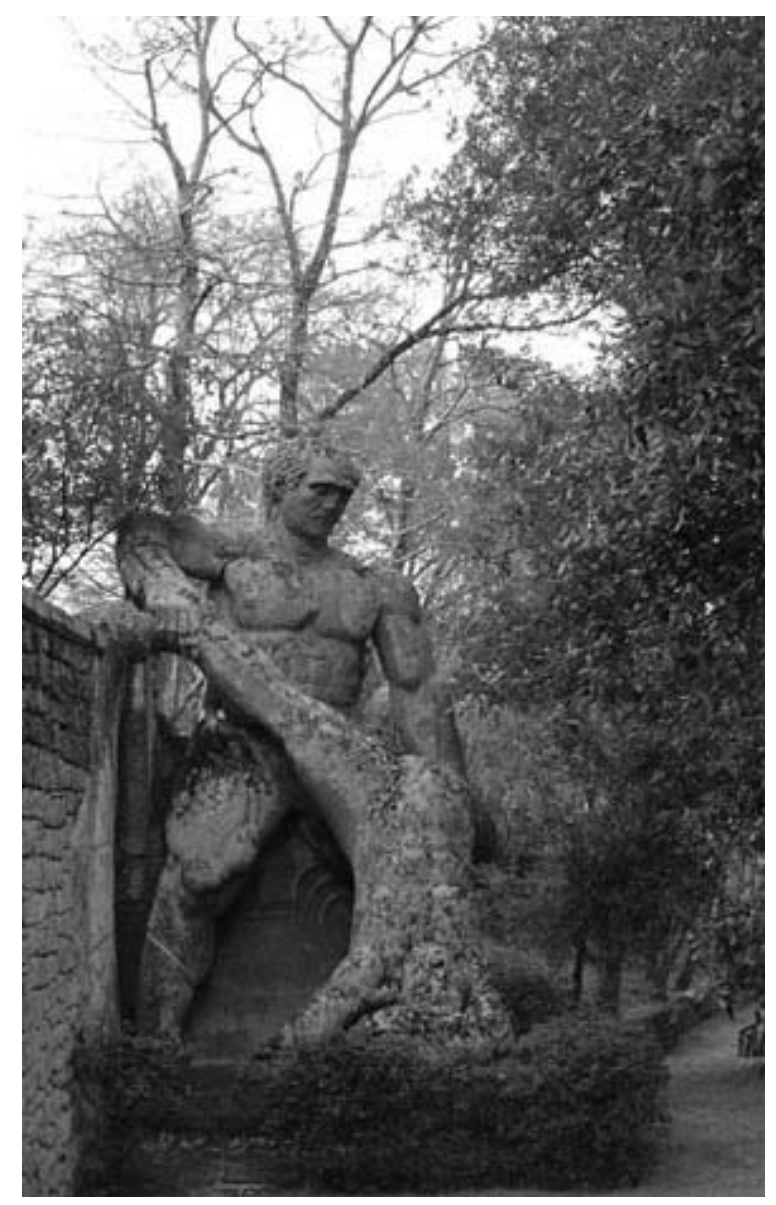

Fig. 2: Scultura al Sacro Bosco di Bomarzo, secondo Maurizio Calvesi rappresenta la furia di Orlando. La composizione dei due corpi gioca sul ribaltamento e allo stesso tempo sulla tensione forza-debolezza, soliditàinstabilità, con valore quasi metaforico della condizione umana.

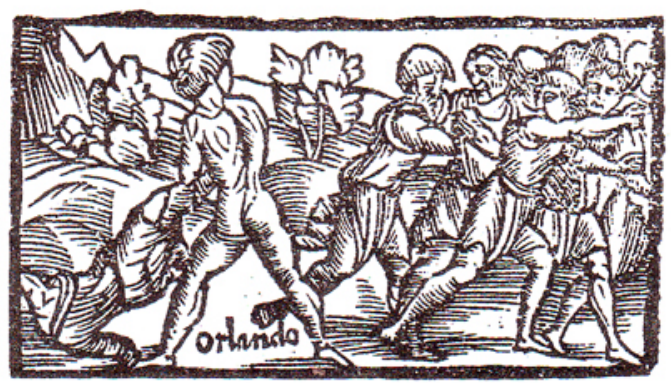

Fig. 3 Orlando in preda alla follia fa strage di nemici (dall'ed. dell'Orlando Furioso dello Zoppino).

esplodere ormai nella sua fantasia, come per rivalsa, o in un ossessivo ritorno della memoria, nei termini di quello sbalorditivo grottesco" in MAURIZIo CALVeSI, Gli incantesimi di Bomarzo. Il Sacro Bosco tra arte e letteratura, Bompiani, Milano 2000, pag. 164. Si rimanda inoltre nuovamente ad Alessandro Rinaldi: “ (...) Le scienze della natura del '500 si impegnano nella laboriosa decifrazione del "Gran Testo del Mondo e della farraginosa scrittura, traccia cifrata attraverso cui risalire alle interiora naturae, alle pulsioni profonde che animano la materia e il cui possesso/conoscenza coincide con il potere faustiano di assumerne tutta l'efficacia creativa", in ALESSANDRO Rinaldi, Op.cit., in MarCello FAgIOLO, Natura e artificio. L'ordine rustico, le fontane, gli automi nella cultura del Manierismo europeo, Officina Edizioni, Roma 1997, pag. 157. 
Esiste una novità rispetto al secolo precedente, riguardo a quest'ultimo punto: la ricerca scientifica va acquisendo nel '500 strumenti di indagine propri, divenendo sempre più autonoma rispetto ai processi artistici. L'arte (in senso lato e quindi anche l'arte dei giardini) conseguentemente perde il fine di comprendere, mediante la sua riproduzione, la struttura cosmica e può abbandonare il linguaggio normato della proporzione rinascimentale. Non è casuale che nelle forme artistiche delle epoche di transizione - ne è un esempio la letteratura romana negli anni che preludono la caduta dell'impero - siano ricorrenti espressionismo, ridondanza, declinazioni dello stile in maniera, come anche figure fantastiche, metamorfosi. Sono elementi che denunciano l'urgenza di una più profonda conoscenza della condizione umana e dell'universo a cui questa si rapporta, sintomi del desiderio di essere immessi "nel mezzo di una verità".

Marcello Fagiolo delinea l'istinto umano all'artificio riconducendosi al mito di Prometeo e di Caino (Caino significa homo faber, egli infatti è agricoltore e fondatore di città) e sottolineando come all'interno di questi miti, il ricorso alla tecnica abbia la doppia valenza di gesto orgoglioso d'opposizione alla divinità-natura e di tentativo verso la redenzione, ovvero di tensione sempre verso la conoscenza:

\begin{abstract}
"Per spiegare e colmare l'abisso tra Dio e il Mondo, tra il Bene e il Male, gli gnostici avevano sentito l'esigenza di introdurre creature intermedie, gli Eoni. L'ultimo di questi spiriti discendenti da Dio s'era ribellato a Dio per desiderio smodato di conoscenza, e cadendo in basso aveva generato il mondo. (...) L'ansia della conoscenza e il conseguente bisogno del lavoro ci sembrano elementi fondamentali della leggenda e delle religioni relative all'idea della tecnica e del progresso. In questa luce l'Arte e la Tecnica discendono da un nodo indissolubile di Bene e male, di Caduta e Salvezza. La mitologia classica e la tradizione cristiana concordano nell'individuare le radici della storia umana in queste fondamentali strutture antropologiche della Conoscenza e della Creatività: in questo senso ci sembra che la leggende di Caino si saldi col mito di Prometeo.",
\end{abstract}

L'autore aggiunge, facendo riferimento al mito di Prometeo come presentato nel Prometeo di Eschilo:

"La tecnica era vista come luce per uscire dalle tenebre, come principio di formazione per superare lo stadio di informità delle grotte."9

Il concetto di fantasia come strumento cognitivo introduce al ruolo dell'immagine: fantasia, sogno, follia - che compaiono come componenti-temi essenziali della letteratura del '500 (con riferimento speciale alle opere dell'Ariosto e del Tasso) e prendono forma nel giardino e nelle arti figurative - sono quei particolari stati di coscienza che maggiormente rapportano alle cose mediante immagini. Il modo in cui la fantasia consente la conoscenza risulta sostanzialmente essere una rappresentazione.

Riccardo Pacciani ${ }^{10}$ spiega il significato degli attributi con cui la rappresentazione viene definita nel Sofista di Platone, icastica e fantastica: il primo termine è riferibile esclusivamente all'imitazione che conserva inalterate sia le proporzioni sia i colori dell'originale (la rappresentazione costituisce una "copia" esatta), il secondo a tutti i rimanenti generi. Egli evidenzia inoltre come fra ' 400 e ' 500 la rappresentazione prospettica - che traduce spazi ed oggetti in dimensioni apparenti e sarebbe quindi per Platone fantastica - sia considerata comunque icastica e come fantastiche fossero solo la rappresentazione di cose inesistenti. La considerazione ci rivela quanto il concetto di imitazione della natura, anche nell'accezione di restituzione "fedele", fosse ritenuta comprensiva, nell'epoca

\footnotetext{
${ }^{8}$ Marcello Fagiolo, Strutture antropologiche dell'artificio: il mito del Demiurgo e la sfida di Caino e di Prometeo, in Marcello Fagiolo, Natura e artificio. L'ordine rustico, le fontane, gli automi nella cultura del Manierismo europeo, Officina Edizioni, Roma 1997, pag. 7.

${ }^{9}$ Marcello Fagiolo, Op.cit., in Marcello Fagiolo, Natura e artificio. L'ordine rustico, le fontane, gli automi nella cultura del Manierismo europeo, Officina Edizioni, Roma 1997, pag. 11.

${ }^{10}$ Riccardo PaCciani, Aspetti dell'imitazione dalla natura fra Quattrocento e Cinquecento, in MarCELLO FAGIOLO, Natura e artificio. L'ordine rustico, le fontane, gli automi nella cultura del Manierismo europeo, Officina Edizioni, Roma 1997, pag. 17.
} 
manierista, anche delle deformazioni soggettive dovute allo sguardo dell'osservatore e quanto spazio si aprisse pian piano alla libertà di invenzione nell'arte.

Tecnica e fantasia si associano l'una all'impegno, l'altra alla leggerezza: la loro simbiosi ha il potere di assottigliare i confini fra arte e ludus. Gli intenti cognitivi sconfinano nella ricerca del piacere. All'interno di questa compagine culturale il giardino ed il parco vengono confermati come luoghi delle più complesse combinazioni di natura e artificio. Per la prima volta in forma così esplicita essi divengono gli spazi privilegiati del ludus.

"Tavola sinottica di artificialia e naturalia, scenario d'illusione in cui il teatro degli elementi collude con lo psicodramma degli archetipi, " kermesse tecnologica" (Conforti) di automi, macchine idrauliche, congegni meccanici, (...) recinto di bestiari vivi o figurati, ricostruzione scalare del mondo attraverso l'evocazione abbreviata dei suoi luoghi geografici, il giardino si qualifica (...) come l'officina "immaginale" in cui l'eccipiente del ludus può promuovere, in vitro, un'estrema, capziosa sintesi tra arte e scienza, tra arte e natura". ${ }^{11}$

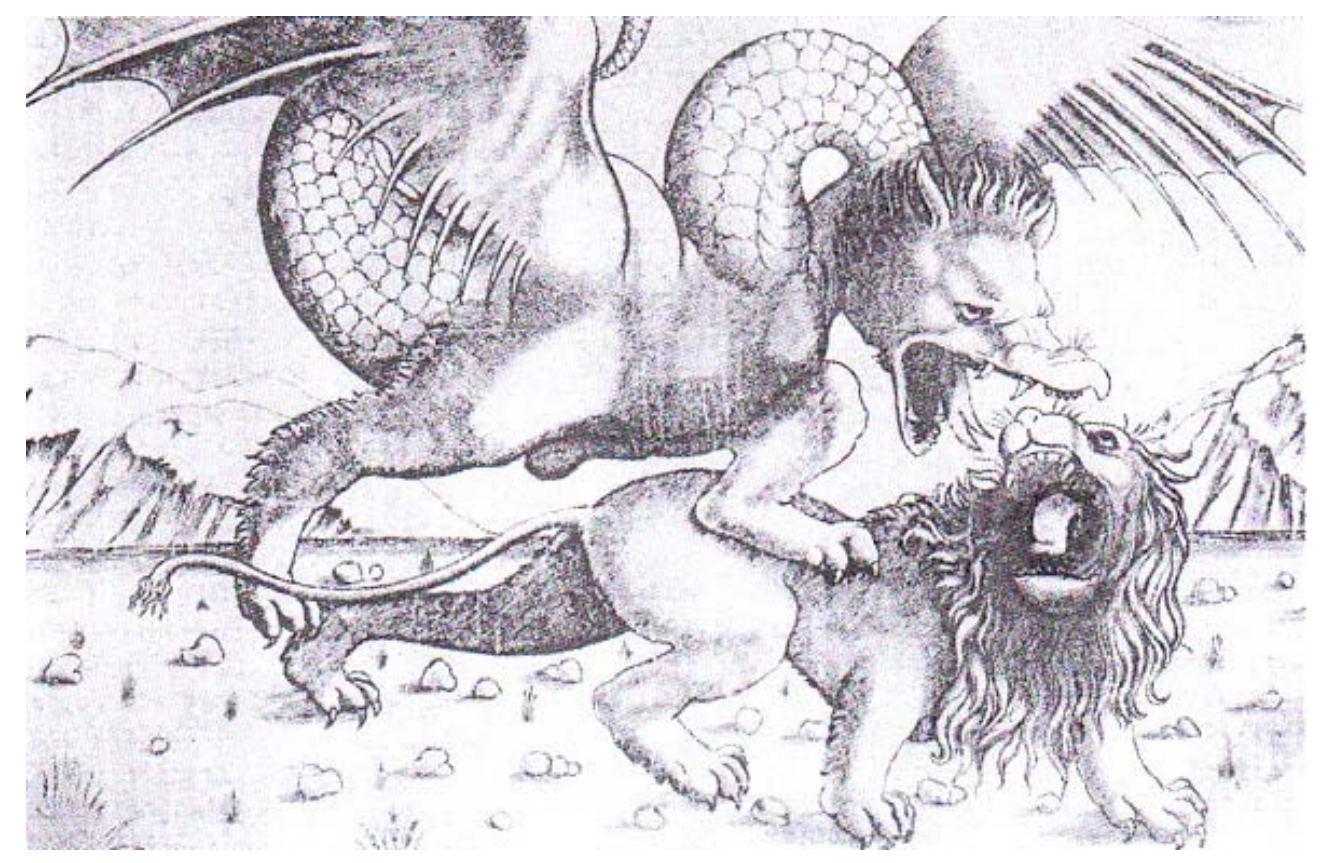

Fig. 4 Drago in lotta con un leone. Incisione del 1500-1505 circa. L'idea di movimento della composizione e l'aspetto del leone appaiono di grande realismo. Anche il drago sembra reale per come vengono rappresentati i suoi muscoli e l'atto dell'aggressione. Ma il drago è un essere della fantasia. L'immagine denuncia come nel '500 reale e fantastico si confondano.

\section{LA NATURA SIMBOLICA E IL PAESAGGIO COME NARRAZIONE}

Perduta la sua giustificazione in quanto materializzazione di una regola universale che lo identifica come microcosmo, il giardino assume significato dalla presenza di uno spettatore. Ovvero in relazione ad un individuale, particolare soggetto che dall'esperienza offerta dal giardino tragga sia piacere sia conoscenza, veicolati dall'emozione della meraviglia. Può essere pertanto riferito al giardino il discorso con cui Fausto Testa evidenzia il valore esperianzale dell' architettura del '500:

“ Non più volto a dare traduzione formale a strutture dall'universale valore metafisico, il linguaggio dell'architettura si apre a nuovi problematici valori, può acquistare una nuova flessibilità linguistica, si impegna in un incessante sperimentare, dove l'assolutezza delle forme classiche, da cui si parte, viene declinata in una

\footnotetext{
11 AlESSANDRO RINALDI, La ricerca della "terza natura": artificialia e naturalia nel giardino toscano del Cinquecento, in Marcello Fagiolo, Natura e artificio. L'ordine rustico, le fontane, gli automi nella cultura del Manierismo europeo, Officina Edizioni, Roma 1997, pag. 164.
} 
polifonia semantica in cui trovano espressione anche contenuti emotivo-psicologici connessi con la soggettività dell'artista, e dove si disloca una fruizione che implica una partecipazione fisico- emotiva dell'osservatore."12

Il parco manierista è strutturato come un percorso fra simboli che si svolgono in narrazione. Simboli possono definirsi gli apparati iconografici dei gruppi scultorei, delle architetture.

Il ricorso al simbolo è consequenziale al fatto che la natura stessa viene percepita come elemento culturale. Alessandro Rinaldi parla di una "natura simbolica", definita simultaneamente dalla propria oggettività e dalle rappresentazioni, descrizioni o "favole" che le sono state riferite: notitia e res congiunte restituiscono alla collettività un patrimonio di immagini.

"Accanto alla griglia delle segnature naturali sta poi tutto il patrimonio delle "notitiae" e delle "favole" accumulatesi sul supporto della cosa e ormai indistinguibili dal nucleo in re intorno a cui si sono venute sedimentando per attrazione."13

Nel parco manierista la narrazione è complessa nella misura in cui natura e arte hanno significato in riferimento reciproco. L'esempio più chiaro è costituito dal Sacro Bosco di Bomarzo: le sculture acquistano la magia di esseri straordinari dall'essere collocati all'interno del bosco che richiama il topos letterario della "selva incantata" presente nell'opera del Boiardo e soprattutto in quella dell'Ariosto: secondo la lettura dell'iconografia di Bomarzo di Maurizio Calvesi, il termine sacro, attributo del bosco di Bomarzo $^{14}$, significa proprio magico, stregato ${ }^{15}$. Sarebbe appropriato anche viceversa affermare che il Bosco è incantato in virtù della presenza delle figure fantastiche e mitologiche che lo abitano:

"Ciò che stabilisce immediatamente un nesso tra il Sacro Bosco e l'Hipnerotomachia, è senza dubbio, come suggerisce il Bredekamp, il concretarsi dei fantasmi della fantasia in "visioni naturali di un regno sospeso tra la realtà e il sogno", nonché l'aspetto del paesaggio, che in apertura del Sogno Di Polifilo si annuncia con una selva abitata dall'incantesimo di Circe e percorsa da un ruscello". ${ }^{16}$

La morfologia e la vegetazione del parco partecipano alla creazione del racconto. Calvesi individua due percorsi narrativi all'interno del Sacro Bosco.

Il primo materializza nello spazio del giardino il percorso di Polifilo dell'Hipnerotomachia di Francesco Colonna, metafora delle vicende personali di Vicino Orsini ${ }^{17}$ colpito in quegli anni dalla morte della moglie. Ha come momento iniziale il tempietto che richiama due costruzioni del Sogno di Polifilo :

"Il primo edificio è il luogo dove Polia si manifesta a Polifilo, gratificandolo del primo bacio, e secondo la nostra ipotesi corrisponde al tempio di coronamento del santuario predestino: quello cioè che è collocato al di sopra dei terrazzamenti, in modo analogo al tempietto di Bomarzo.

Il secondo è eretto intorno al sacro fonte di Venere, nell'isola dell'amore; qui la dea appare a Polifilo e a Polia, consacrando la loro unione." 18

Il racconto prosegue per le sculture disposte lungo il ruscello: la Fontana di Pegaso e il moncone di colonna inclinato - iconografie che riportano al mito di Pegaso, in grado di arrestare con il battito degli zoccoli i terremoti- poi il Ninfeo e le statue delle tre Grazie,

\footnotetext{
${ }^{12}$ FAUSTO TeStA, Spazio e allegoria nel giardino manierista, La Nuova Italia, Firenze 1991, pag. 27.

${ }^{13}$ AlESSANDRo RinAlDI, Ibidem.

${ }^{14} \mathrm{~L}$ 'iscrizione incisa in una pietra vicina al tempietto del parco di Bomarzo ammonisce:

CEDAN ET MENPHI OGNI ALTRA MARAVIGLIA

CH'EBBE GIÀ IL MONDO IN PREGIO AL SACRO BOSCHO

CHE SOL SE STESSO E NULL'ALTRO ASSOMIGLIA.

${ }^{15}$ Si rimanda a MAURIzio CALVesi, Gli incantesimi di Bomarzo. Il Sacro Bosco tra arte e letteratura, Bompiani,

Milano 2000. In particolare ai paragrafi Il “Sacro Bosco”, pagg. 183- 185, e La selva incantata dal Boiardo a

Bernardo Tasso, pagg. 209-212.

${ }^{16}$ Maurizio Calvesi, Op.cit., Bompiani, Milano 2000, pag. 124.

${ }^{17}$ Signore di Bomarzo, ideatore del Sacro Bosco.

${ }^{18}$ Maurizio Calvesi, Op.cit., Bompiani, Milano 2000, pag. 123.
} 
davanti ad esso la fontana a forma di imbarcazione che "sembra librarsi sul pendio come su dei flutti"19. Calvesi riporta l'immagine di Bredekamp, che nuovamente identifica l'osmosi fra la struttura del giardino e lo sviluppo dei simboli realizzato dal succedersi dei gruppi scultorei: non solo il bosco è una "selva incantata", ma il prato vicino al ruscello può trasfigurarsi in mare da navigare per giungere all'isola Citera, isola dell'Amore, che nel parco di Bomarzo è la radura, che segue la fontana-nave, in cui sono collocati il teatro e il sacrario di Venere.

L'andamento del terreno, il ritmo fra parti a bosco e prati aperti, la vegetazione, supportano la narrazione, attribuiscono significato alle scene e assorbono a loro volta i significati di architettura e scultura. Gli elementi naturali del parco sono un medium simbolico di cucitura fra gli eventi.

Il secondo percorso, realizzato successivamente, invera una selva incantata dai toni differenti ${ }^{20}$ - cupi e infernali, trasmessi dalle statue di Plutone, di Proserpina, dai grandi vasi in serie con iscrizioni, che corrispondono all'immagine di vasi funerari. Esso infatti è impregnato delle suggestioni letterarie dell'Adamigi di Bernardo Tasso e della Gerusalemme Liberata di Torquato Tasso cui alla "selva delle meraviglie" - rappresentata dalla selva del castello di Atlante nel Boiardo e nell'Ariosto, luogo in cui i prigionieri del mago erano trattenuti in piaceri e delizie - si affianca la "selva perigliosa" di Oronte, caratterizzata da orrori e spavento. E' sempre Calvesi a descrivere come i caratteri dello stile letterario e dell'iconografia letteraria vengono tradotti in paesaggio, confermando l'idea che la natura sia simbolica:

“(...) dal grottesco- comico dell'Ariosto (e di Giulio Romano), Vicino sembra deviare -(...)- verso una forma di grottesco che non dissimula del tutto un'oscura inquietudine, cercando in essa il proprio appagamento espressivo ed estetico. Egli sfiora soltanto il comico, schivandolo, e infondendo l'inverosimile e l'irreale di una forza plastica di realtà, che si nutre del contatto con i chiaroscuri della natura.

Anche nel Sacro Bosco, come a mezza via tra il grottesco e un'arcaica forma di Sublime, quest'ultimo sentore già si respira. Le colossali sculture drammatizzano il circostante ambiente naturale, cui appartengono per la loro rude e porosa materia di roccia. Entrano in simbiosi con la vegetazione che le avvolge, comunicandole la loro conturbante energia, mescolandosi ad essa in agguati di ombre e rumori di acque, in scrosci di luce, assedi di arbusti intricati o sorprese di radure. Il visitatore, come voleva la convenzione letteraria della "prova" e come vorrà la logica del Sublime, si confronta alle loro sovrastanti dimensioni e, sia pure giocosamente, con la loro inusitata e spaventevole fisionomia." 21

I percorsi narrativi e la natura simbolica oltrepassano i confini del parco. Sono fili che legano fatti ed eventi d'attualità dell'epoca, uscendo quindi a costituire una rete di simboli, di connessioni culturali a scala territoriale: il legame di primo grado è istituito con la Villa di Vinicio Orsini a Bomarzo, non solo presente in quanto visibile dal parco, ma presente in quanto parte della narrazione, "castello di Atlante" nella selva incantata, come lui stesso definisce la sua sede di Bomarzo in una lettera del 1573 all'amico Drouet. Tramite il sacro Bosco l'intera Bomarzo è investita di significato, emanando la sua energia di luogo magico e "per incantamenti spensierato" sui suoi intorni ma anche nei territori in cui Vinicio aveva legami di amicizia e culturali.

Il fatto, sottolineato da Calvesi, che nel parco di frequente sia possibile leggere l'influenza, in termini di suggestione iconografica, delle rappresentazioni tenutesi a Firenze nel dicembre del 1565 in occasione di una grande festa, offre l'idea del moltiplicarsi di echi nel territorio. L'incisione del territorio operata dai significati del parco privato non è una prerogativa solo del Sacro Bosco di Bomarzo, anche perché corrisponde alla volontà di esprimere il potere signorile e quindi ad una realtà diffusa, nel ' 500 .

\footnotetext{
${ }^{19}$ La frase è di Bredekamp, citata in MAURIZIO CALVESI, Op.cit., Bompiani, Milano 2000, pag. 128.

${ }^{20} \mathrm{Si}$ fa sempre riferimento all'analisi di Maurizio Calvesi, dell'opera citata.

${ }^{21}$ Maurizio CAlvesi, Op.cit., Bompiani, Milano 2000, pag. 215.
} 


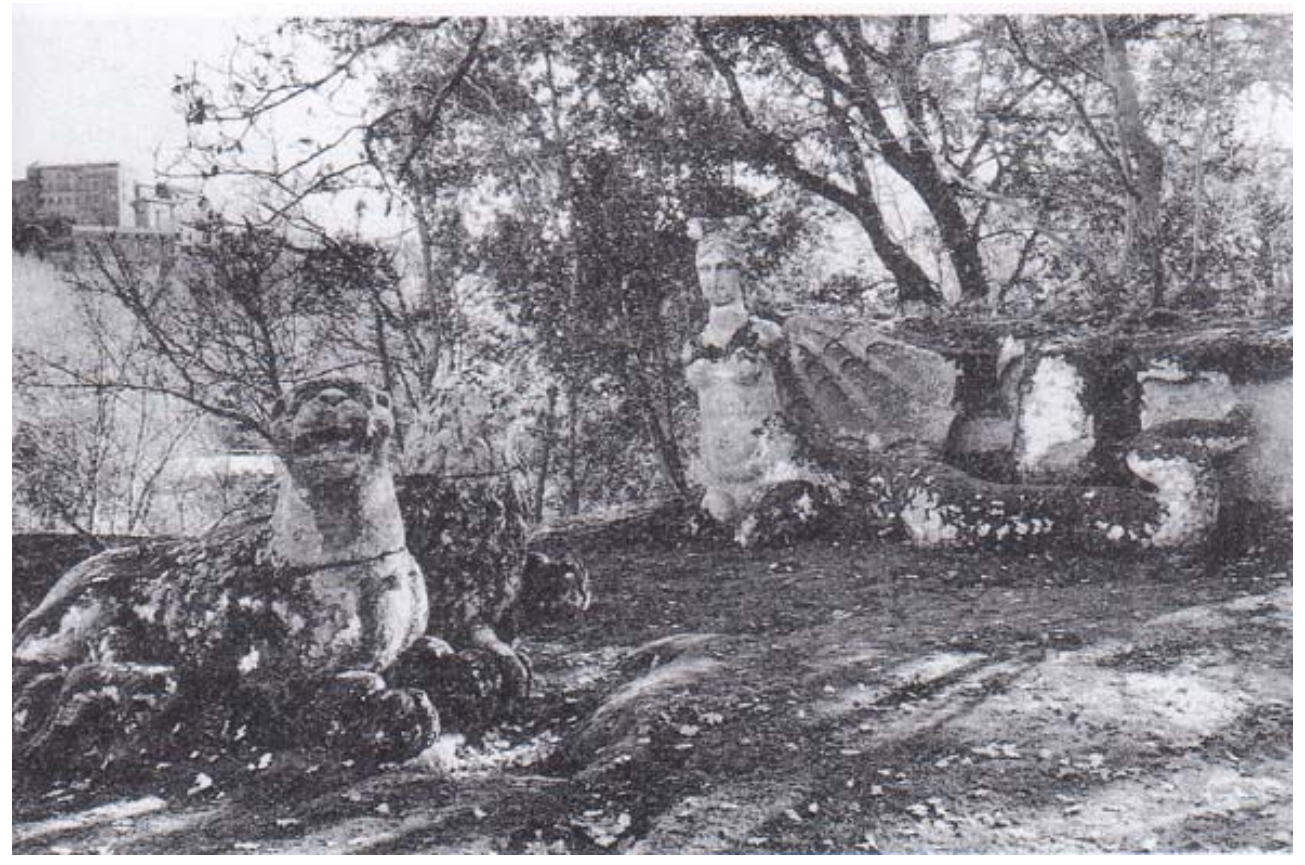

Fig. 5 Leoni e arpie al Sacro Bosco di Bomarzo. Sullo sfondo è visibile Bomarzo ed il palazzo signorile: Il parco è culturalmente e fisicamente legato al proprio territorio.

Marcello Fagiolo ${ }^{22}$ propone una lettura simbolica dell'architettura e degli apparati iconografici della Villa Farnese a Caprarola, individuando una continuità interno-esterno che non si limita ad investire i giardini segreti del palazzo, ma che sembra dilatarsi nel contesto urbano. Si percepisce un itinerario narrativo con inizio e fine indefiniti, di scala territoriale, con un fulcro invece ben evidente al centro dell'architettura, nello spazio circolare interno della villa, costituito dal cortile e dal criptoportico anulare sottostante su cui si affacciano i locali di servizio, "un vero e proprio regno infero di Bacco",

"L'aura mitica dei sotterranei si prolunga nel cortile circolare del Palazzo. Uno spazio che, derivando dal prototipo raffaellesco di Villa Madama, può essere ricollegato allegoricamente al mito conio delle origini del sottostante Lago di Vico: secondo una tradizione riferita da Servio il lago si sarebbe formato quando Ercole avrebbe estratto con forza l'asta di ferro da lui stesso conficcata nel suo viterbese. In effetti al centro del cortile è infisso, se così si può dire, il grande pilastro a fungo."23

L'architettura collabora con la natura e vi si confonde: entrambe hanno carattere di simbolo trovano compiutezza non più nell'essere proporzione, quanto nell'essere comunicazioneespressione - ed il simbolo ribadisce il potere del genius loci.

L'Appennino del parco di Pratolino, realizzato dal Giambologna nel 1580, è l'esempio maggiormente sintetico di natura simbolica e architettura simbolica come vie di rimando ai miti territoriali: la figura di vecchio rappresenta la catena montuosa degli Appennini, concreziona in roccia mediante metamorfosi affacciato su uno specchio d'acqua, che può assumere il significato di lago creatosi per il disgelo - il vecchio sembra anche, infatti, irrigidito dal rigore invernale dei ghiaccioli che lo bloccano. L'acqua è l'emblema dell'intero parco ripresentandosi, pervasivamente, sotto forma di fontana, di vivaio, di forza motrice per gli automi, di energia che genera particolarità geologiche all'interno delle grotte interne alla statua stessa, e dei sotterranei della Villa. Viene raccolta a monte in un ampio bacino, poi incanalata all'interno in una rete idrica che sfrutta l'andamento in pendenza del terreno.

${ }^{22}$ Marcello Fagiolo, Caprarola: la montagna sacra, la "grotta dell'orrore" e $i$ miti acquatici, in VeZzosi ALESSANDRo (a cura di), Il giardino d'Europa. Pratolino come modello nella cultura europea, Mazzotta, Firenze 1986, pagg. 74-76.

${ }^{23}$ Marcello Fagiolo, Op. cit., in Vezzosi Alessandro (a cura di), Il giardino d'Europa. Pratolino come modello nella cultura europea, Mazzotta, Firenze 1986, pag. 75. 


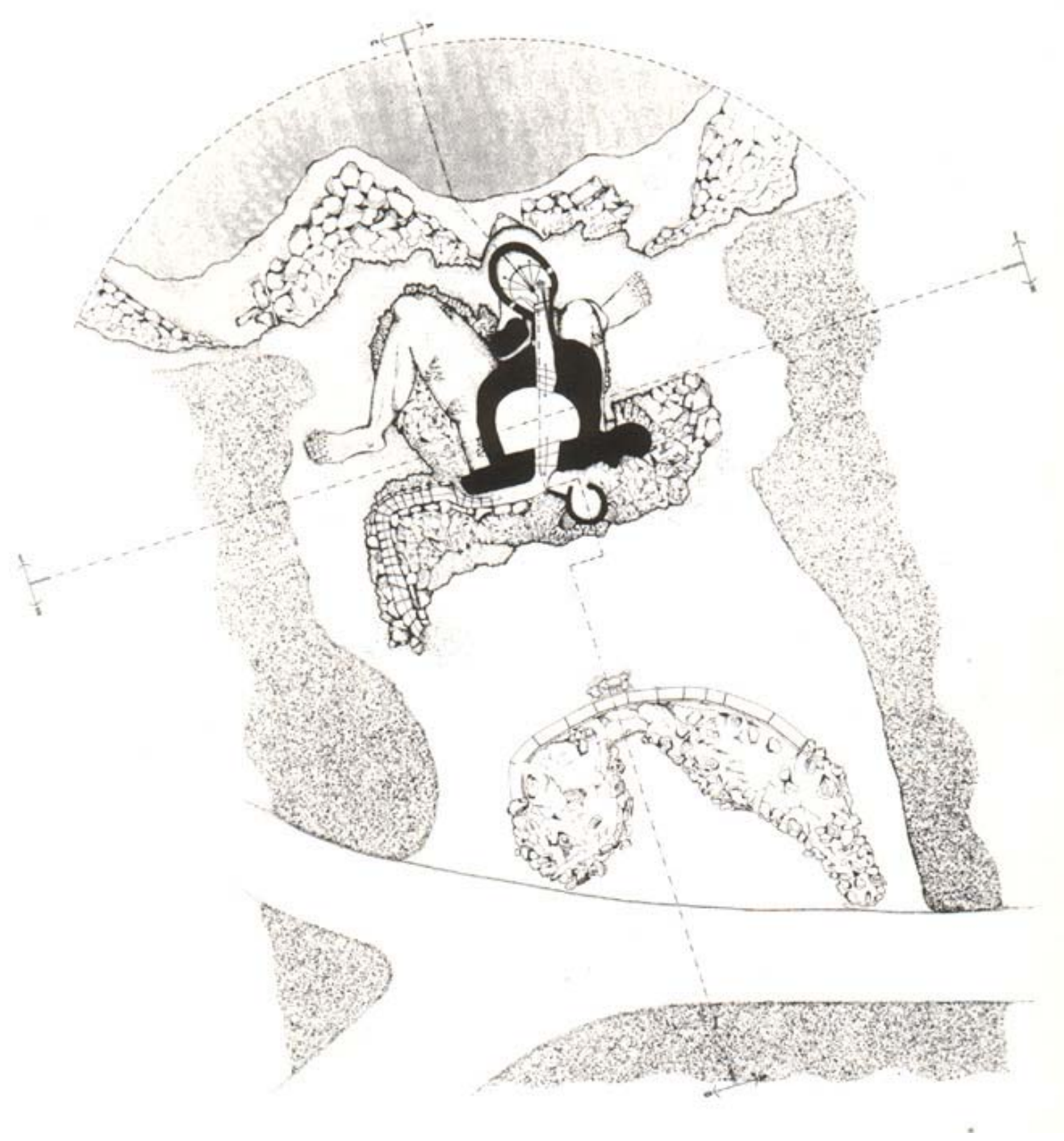

Fig. 6 planimetria dell' Appennino del Giambologna a Pratolino (1580): allo stesso tempo è scultura, architettura e simbolo complesso che evoca il genius loci.

La natura simbolica coinvolge sia l'intelletto - la ratio, si è detto infatti come essa sia simbolica in virtù di connotati culturali, attribuiti dalle notitiae inerenti - sia i sensi, rendendola "disponibile" al duplice scopo di raggiungere piacere e conoscenza. L'aspetto interessante è che risulta semplicistico pensare che i sensi generano il piacere e l'intelletto conduce alla conoscenza. Anzi sembra quasi di potere capovolgere il discorso in maniera opportuna e dire che Vinicio Orsini al Sacro Bosco e Francesco I a Pratolino si siano compiaciuti delle sottili, cerebrali, connessioni con cui hanno legato pezzo con pezzo le architetture, le sculture e gli spazi dei propri parchi e che abbiano ricercato, mediante le sollecitazioni sensoriali - visive, uditive, tattili, olfattive - indotte dai loro stessi artefatti (suoni, apparizioni, dismisure, concrezioni in statue e grotte, getti d'acqua....), un affaccio sul mistero, un'insenatura più profonda nelle interiorae naturae.

Luigi Zangheri riferendosi alla grotta del giardino di Boboli del Bountalenti descrive il ruolo dell'acqua come cosa vivificante, indispensabile per un' "architettura delle metamorfosi" 24 . L'acqua stimola i cinque sensi:

"L'acqua, come materia capace di vivificare le composizioni decorative, e fino le tessere dei rivestimenti parietali, sgorgava dalle mammelle delle capre e dagli zufoli dei suonatori, saliva nei bacini delle fonti marmoree solleticando e suscitando emozioni nel senso dell'udito, dopo aver coinvolto quelli della vista e del tatto. Anche

\footnotetext{
${ }^{24}$ Luigi Zangheri assume l'espressione dall'Heikamp.
} 
l'odorato poteva essere interessato in occasioni particolari e raffinate quando i Medici facevano "spruzzare acque odorosissime" dagli zampilli delle fontane."25

L'esperienza sensoriale si presenta voluttuosa e totalizzante: il giardino è sensuale.

Ma la presenza della "naturalità" nel parco, che caratterizza Pratolino ed è riscontrabile anche a Bomarzo, consente anche momenti contemplativi in cui l'emozione è controllata. Le sollecitazioni sensoriali e la stravaganza delle forme non sono un continuum: lo spazio di Bomarzo è fluido ed il parco, se da un lato è un medium simbolico, a brani è anche uno spazio riposante: nel giardino manierista c'è spazio "a sufficienza", elusa ogni congestione: l'epoca non ha ancora abbandonato completamente, sul piano etico, il senso dell'opportunità di rimanere nella mediocritas, ovvero in uno stato di equilibrio rispetto agli stravolgimenti che inducono le passioni. Conseguentemente il giardino non cessa di essere un locus amoenus, nonostante sia anche un luogo di interlocuzione con esseri misteriosi.

Si può dire del paesaggio di Pratolino e Bomarzo quanto è riferito da Calvesi ai paesaggi letterari di Bernardo Tasso:

"Il soprannaturale di Bernardo Tasso, molto spesso, non è che una fantastica proiezione, più lirica che epica, di fenomeni naturali; ovvero della natura nei suoi aspetti più minacciosi, nelle sue forze scatenate con maggior violenza, nei miraggi delle sue forme più oscure, in aspetti spaventosi tuttavia come stagionalmente reversibili e pronti a mutarsi in panorami rasserenanti, che tornano a proporre il tradizionale locus amoenus." 26

\section{PAESAGgio D’ARTIFICIO: IL GIARDINO DI PRATOLINO COME ESITO DIVULGATO}

Nel parco di Francesco I de Medici - Principe Reggente dal 1564 al 1574, poi Granduca di Toscana - a Pratolino, la natura simbolica trova una controparte nella tecnologia, che si impone quale strumento umano in grado di riprodurre i processi naturali e allo stesso tempo di ricreare il mistero di questi. Pratolino è l'espressione massima delle capacità intellettuali e degli interessi per l'alchimia e le "arti meccaniche" del suo signore ${ }^{27}$, doti che influenzano la cultura del " 500 a livello europeo, come sottolineato soprattutto all'interno dei contributi pubblicati in occasione della XVI Esposizione del Consiglio d'Europa dedicata a "Firenze e la Toscana nell'Europa del XVI secolo" (1986).

La tecnologia sta al giardino come le forze occulte stanno alla natura: è il mezzo per animare le cose, per offrire ad esse un quid che le vivifichi. (Non è un caso che nel Purgatorio Dante caratterizzi i personaggi che incontra descrivendo un loro gesto particolare, quotidiano ad esprimere una condizione umana vicina a quella della vita terrena, non trasfigurata dalla santità - nel Paradiso - e neppure stravolta e deformata dalla pena). La Ri-creazione del movimento è il tentativo ultimo di imitazione della natura.

"Sulla capacità dell'artifex demiurgico di mettere in opera prodigi, si gioca dunque la prova decisiva dell'emulazione tra arte e natura: e poiché il postulato della superiorità di quest'ultima risiede nel "moto intrinseco" che essa imprime alle proprie creature, il tema centrale della sfida ruoterà proprio intorno alla riproduzione artificiale del movimento., 28

Nel mito di Pigmalione, che rappresenta l'azione demiurgica dell'arte, è eluso il problema di cosa generi il movimento: la statua scolpita da Pigamlione prende vita direttamente

\footnotetext{
${ }^{25}$ Luigi ZANGHeri, Tra abilità e tecnologia. l'acqua nelle grotte del manierismo, in AA.VV., Arte delle Grotte, Sagep Editrice, Genova 1987, pag. 9.

${ }^{26}$ Maurizio Calvesi, Op. cit., Bompiani, Milano 2000, pag. 212.

Vincenzo Cazzato parla del giardino (a Pratolino e a Bomarzo) come "luogo della "remissio" e della "pax" spirituale", in MARCELLO FAGIOLO, Le due anime nelle ville della Tuscia, in VEZzosi AlESSANDRO ( a cura di ), Il giardino d'Europa. Pratolino come modello nella cultura europea, Mazzotta, Firenze 1986, pag. 69.

${ }_{27}$ Luigi Zangheri cita come momenti in cui è stato sottolineato il ruolo di mecenate scientifico avuto da Francesco I, come personalità che ha influenzato la cultura europea del XVI secolo, il testo

${ }^{28}$ AlesSANDRO RinALDI, Ibidem.
} 
dall'amore sensuale del suo autore, scaturito a sua volta dalla bellezza perfetta delle forme della scultura, che supera quella riscontrabile in natura.

"Ma un giorno con arte felice e meravigliosa, si mise a scolpire dell'avorio bianco come neve e gli dette forma di donna, così bella, che nessuna può nascere più bella. E concepì amore per la sua opera. L'aspetto è quello di una fanciulla vera, e diresti che è viva e che, se non fosse così timida, vorrebbe muoversi. ${ }^{, 29}$

Amata, la statua apre gli occhi e proprio nel compiere questo gesto partecipa della vita e della natura, vedendo l'uomo e il cielo con il medesimo sguardo. ${ }^{30}$

Ad imprime il movimento agli automi di Pratolino è l'energia idraulica, anche se il motore appare l'aria, illusionisticamente, per l'essere i gesti accompagnati dal suono, come evidenzia Alessandro Rinaldi. Il ricorso alla risorsa naturale ribadisce il dominio dell'uomo sulla natura, coerentemente al compito affidatogli da Dio, secondo le parole della Genesi, ma è un richiamo anche all'atto prometeico. Viceversa il livello simbolico in cui l'acqua si manifesta evidenzia come essa abbia un contenuto sacro.

Se le forze naturali sono occulte, la tecnologia viene occultata. Essa è invisibile al visitatore cui è dato di vedere il solo apparato iconografico del complesso scultoreo che "veste" il meccanismo ed è quindi condotto a pensare ad un processo alchemico piuttosto che ad uno tecnologico. Allo spettatore elemento naturale ed elemento artificiale del parco appaiono condividere la medesima sfera ontologica e la narrazione si avvale dei simboli dell'uno e dell'altro collaboranti.

"La frammentazione ordinata delle acque e del suono secondo scansioni compatibili e funzionali alla sceneggiatura del "teatro automatico" segna la completa reinscrizione dell'acqua nella "disciplina" del racconto scenico e quindi l'epilogo del processo di decantazione formale dell'elemento che passa ora da cosa a segmento della narrazione". ${ }^{31}$

La tecnologia, riproducendo gesti e azioni, consente di inserire nel parco-giardino degli accadimenti - qualcosa in più rispetto a semplici immagini - teatralizzando lo spazio e preludendo all'estetica barocca.

La conseguenza è che il parco è uno strumento nelle mani del signore per la sua personale iniziazione alla conoscenza ${ }^{32}$, ma allo stesso tempo è modo di conferma della gerarchia sociale: il nobile manifesta il proprio sapere, l'abilità gestionale ed il potere economico. Il visitatore di più modeste origini ed istruzione - a cui occasionalmente il parco viene aperto si sente stupito e anche instabilizzato dalle forme estetiche grottesche e sproporzionate (Bomarzo) o dall'innovazione tecnologica che oltrepassa la sua capacità di comprensione (Pratolino) $)^{33}$. Il ruolo egemone del signore è manifesto, ad un livello diverso, anche ai suoi

${ }^{29}$ Publio Ovidio Nasone, Metamorfosi, Einaudi, Torino 1994, Piero Bernardini Marzolla a cura di), pagg. 399401.

30 Si riporta l'espressione di Ovidio "(...) timidumque ad lumina lumen attollens, pariter cum caelo vidit amantem” (e levando timidamente gli occhi verso la luce, vede, assieme al cielo, l'amante).

${ }^{31}$ Alessandro Rinaldi, Op. cit. in MARCEllo FAgiolo, Natura e artificio. L'ordine rustico, le fontane, gli automi nella cultura del Manierismo europeo, Officina Edizioni, Roma 1997, pag. 169.

${ }^{32}$ Sull'interpretazione dei parchi di Pratolino e di Bomarzo come percorsi di iniziazione si sofferma Paola Maresca nel testo Paola Maresca, Boschi sacri e giardini incantati, Angelo Pontecorboli Editore, Firenze 1997.

${ }^{33}$ Un episodio che contribuisce a dare l'idea di quale energia venisse spesso dispiegata nel ' 500 per confondere e divertire gli ospiti del signore, cosa che aveva soprattutto la capacità di ribadirne il potere, è riportato da Paola Maresca in PAOla MARESCA, Boschi sacri e giardini incantati, Angelo Pontecorboli Editore, Firenze 1997, pagg. 65-66. Si tratta di un banchetto notturno avvenuto agli orti Oricellai a Firenze al tempo di Francesco I, alla presenza del Granduca e a quella di suoi amici, occasione in cui, per l'intrattenimento dei convitati, era stato messo in scena uno spettacolo con ospite un "negromante". Il falso mago dispone gli invitati in circolo attorno ad una buca precedentemente predisposta e fa accomodare il Duca al centro, in modo e luogo che egli "non si facesse male alcuno". Seguono invocazioni del mago a richiamo di diavoli, che si fanno sentire con rumori spaventosi: "con cotanto strepitio, e rimbombo orrendo e spaventoso; parendo esse veramente un proprio inferno, e che tutto il modo si volesse rovinare. Udivansi infinite voci, e lamenti, ululati strani, stridore di denti, battere palme a palme, scuotere catene di ferro, pianti sospiri e singhiozzi, e infinite fiamme di fuoco, le quali scaturivano da tutte le parti, uscendo fuora da molti buchi, fatti ad arte meravigliosa rasente il terreno del prato 
pari, nobili di altre casate e regioni invitati nel parco per cerimonie ed occasioni politiche, che condividono con il signore l'appartenenza ad una koinè culturale, che va alimentandosi di apparati iconografici, trasposizioni figurative dei paesaggi e degli avvenimenti della letteratura cavalleresca dell'epoca. Essi sono in grado di decifrare i simboli e cogliere contemporaneamente contenuto narrativo, abilità organizzativa-costruttiva, spessore culturale. Calvesi a proposito della genesi del Sacro Bosco di Bomarzo riporta una lettera di Vicino Orsini ad Alessandro Farnese, del 1561, che denunzia chiaramente come il giardino induca effetti diversi a seconda del livello culturale del proprio pubblico:

"Io sto tuttavia intorno al mio boschetto per vedere sello posso far vedere meraviglioso a Lei come a molti balordi che vi vengono, ma questo non avverrà perché la maraviglia nascendo da l'ignorantia non può cadere in lei". ${ }^{34}$

Alessandro Rinaldi ci descrive la condizione psicologica in cui si viene a trovare il visitatore all'interno del parco di Pratolino e le implicazioni di questa:

"Catturato dalla "macchinazione totale" del giardino, sospinto dagli "impulsi" programmati dei giochi d'acqua e degli automatismi prossemici indotti dalla sapiente impaginazione psicologica dei percorsi, sottoposto ad una calcolata progressione di chocs percettivi, il visitatore viene inesorabilmente (...) svuotato di ogni possibilità di iniziativa autonoma e costretto infine ad "abbandonarsi totalmente alla discrezione de' Fontanieri" cioè al capriccio dispotico del Principe che esse veicolano. L'incontro con l'automa segnerà il compimento della vicissitudine onirica, la conclusione insieme del processo di iniziazione all'universo del ludus e dell'itinerario pedagogico verso lo spossessamento di se'. Il simulacro meccanico docile alla fascinazione del canto offrirà allora al visitatore lo specchio beffardo della sua nuova identità reificata.",35

A Pratolino la tecnologia intende spaesare, divertire consentendo giochi e scherzi. La finalità è evidente se si legge la descrizione della Grotta del Satiro e della Grotta di Cupido dal "Ristretto delle Cose più notabili della Città di Firenze" nell'edizione del 1719:

“(...) si vede una piccola Grotticella e di difuori, con una sorgente d'acqua freschissima, che esce per una botte di marmo e da un fianco, che tiene in mano un Satirino di bronzo. Quest'acqua è di qualità buona per bevere, e dandosene a gustare a i Forestieri, questi intanto vengono bagnati di zampilli d'acqua, che fortiscono di dentro alla Grotticella e di difuori. Più oltre avanzandosi, vi è una Grotta di figura tonda, detta di Cupido, per esservi la sua statuetta di bronzo in testa alla medesima, che per ingegnoso artifizio d'acqua si volta in giro, e tramanda acqua a' riguardanti. Questa grotta è tutta inganni, come lo è lo gentilesco Simulacro; perché quelli, che v'entrano dentro, non se ne accorgendo, si trovano bagnati, così nell'entrarvi, che nel sedervi (...).,36

E' tanto esplicito il fatto che l' "artifizio" è un gioco, che l' emigrazione oltralpe degli ingegneri degli automi di Pratolino, appare quale episodio antesignano della nascita dell' "industria" di attrazioni per parchi (nel XIX secolo), a questo si deve il successo dei teatri di automi, imitati in tutta Europa: nel giardino di Hellbrunn, di Massimiliano II al Neugebaude di Vienna, di Rodolfo II a Praga, di Richmond in Galles, di Enrico IV a Saint-Germain-enLaye e a Fontainebleau. Luigi Zangheri parla di "un'esportazione di tecnologia" 37 e fornisce

intorno al Circolo, a quali abbrugiavano fin l'erbe che vi erano. Onde vedendo ciò il Negromante, terminò di dar fuoco alla mina. E percuotendo il suolo con un piede, cenno, per che aprissero il Catenaccio, se n'è andaron tutti sottosopra precipitando giusto nella buca (...). da C. MALESPINI, Novelle scelte, Lanciano 1915.

Il racconto prosegue con i diavoli che si avvicinano tutto attorno alla buca, sempre con orrendi rumori. Poi scompaiono all'improvviso, tutto si placa e arrivano fanciulle seminude che conducono il granduca e gli invitati al banchetto e a rinfrescarsi. Colpisce nella descrizione come il ludus - gli ospiti in realtà sono portati a credere che gli accadimenti siano veri - consista nell'incutere stupore misto alla paura di correre un pericolo ("pareva che tutto il mondo si volesse rovinare"), mentre la verità e che ogni cosa corrisponde ad un piano e non può succedere niente a nessuno.

${ }^{34}$ Maurizio Calvesi, Op. cit., Bompiani, Milano 2000, pag. 212.

${ }^{35}$ Alessandro Rinaldi, Op. cit., in MARCEllo FAgiOLO, Natura e artificio. L'ordine rustico, le fontane, gli automi nella cultura del Manierismo europeo, Officina Edizioni, Roma 1997, pag. 172.

${ }^{36}$ Documento riportato in Alessandro Vezzosi (a cura di), Il concerto di statue, Alinea, Firenze 1986, pag. 92.

${ }^{37}$ In ZANGHeri LuIgI, Suggestioni e fortuna dei teatrini d'automi. Pratolino come una Broadway manierista, in "Quaderni di teatro", Vii, 25, 1984, pagg. 78-84. 
una mappa geografica dei contatti e degli scambi culturali della famiglia De' Medici nel XVI secolo, a cui si deve questa "esportazione".

L'occasione principale per la discussione e il riconoscimento del ruolo del parco nell'influenzare l'arte dei giardini delle epoche successive, in Europa, è specialmente il Convegno tenutosi a Firenze nel 1986: Il giardino D'Europa. Pratolino come modello nella cultura europea, nell' anno in cui Firenze è città europea della cultura. All'esportazione delle tecniche di Pratolino si associa anche, conseguentemente, la diffusione del concetto stesso di giardino come luogo informato da immagini, "personaggi", rappresentazioni, esasperando il contenuto simbolico-allegorico comunque implicito nell'arte stessa dei giardini, ai fini della teatralizzazione, tanto che il parco è stato definito "una Broadway manierista" 38 .

Alessandro Vezzosi riporta le parole di De' Vieri $(. . .)^{39}$ in Pratolino d'Europa, degli antichi e dei moderni :

"Finalmente se gli antichi ritrovarono gli ingegnosi strumenti, de' quali si servivano, o in su le guerre, o nelle muraglie, o nelle opere di medicina, o in altre professioni. [...] Et in Pratolino, perché quelle statue si voltino, suonino, gettino acqua, sono tanti, \& tanti artifizij stupendi in luoghi occulti, che gli vedesse tutti insieme, se n'andrebbe in estasi."

Luigi Zangheri scrive:

"Pratolino significò un nuovo modo di concepire l'arte dei giardini non solo per la Firenze del XVI secolo ma per tutta la cultura europea, anticipando persino temi che avrebbero avuto compiuta definizione nel XVIII e XIX secolo. (...) A Pratolino il giardino fu inteso per la prima volta come parco (...). A Pratolino la tazza delle fontane fu sostituita da bacini, piscine e gamberane, un dedalo di grotte prese il posto di nicchie e della più modesta grotta rinascimentale. Dal piacere suscitato dall'armonia bloccata in un'immagine tagliata nel verde si passò alla meraviglia solleticata dal moto degli automi che offrivano scene e musiche nel verde." 40

"La "meraviglia" e " il grandissimo stupore" provato dai visitatori del parco erano dati anche dalle "opere meravigliose", dagli "stupendi artifici" dagli "ingegni magnifici" che vi avevano realizzato Bonaventura da Orvieto, Goceramo da Parma, Tommaso Francini, Maestro Lazzaro delle Fontane. Organi idraulici, macchine eroniane simulanti il canto degli uccelli, scherzi d'acqua e tanti teatrini d'automi mossi dall'acqua formavano un complesso fiabesco che era applaudito non solo dagli ospiti illustri invitati dal principe, ma anche dai popolani ammessi nel recinto del Parco solo in occasione di feste particolari.",

John Dixon Hunt al capitolo The theaters, gardens, and Gardens Theaters del suo Gardens and the Picturesque. Studies in the History of Landscape Architecture chiarisce il legame culturale esistente fra i pleasure gardens, in particolare il Vauxhall di Londra, del XVII e XVIII secolo, e i giardini manieristi, citando Pratolino e i suoi visitatori illustri - Evelyn e William Kent - che ne diffondono le suggestioni oltralpe. Il legame culturale messo in luce definisce ulteriormente il giardino manierista quale giardino dello spettacolo e del gioco, finalizzati al divertimento.

Operando un salto temporale più ardito ancora, è possibile notare come gli automi manieristi siano gli antesignani dei contemporanei robots. Walt Disney è appassionato studioso e produttore di audioanimatronici in quanto gli consentono di liberare i suoi personaggi dalla bidimensionalità. Suoi primi esperimenti sono la miniatura The dancing man e il quartet of mechanical singers che costituisce parte di una scena all'interno di una vecchia bottega di barbiere.

\footnotetext{
${ }^{38}$ In ZANGHERI LuIGI, Op.cit., in "Quaderni di teatro", Vii, 25, 1984.

${ }^{39}$ Alessandro Vezzosi, Pratolino d'Europa”, degli antichi e dei moderni, in Alessandro Vezzosi (a cura di), Il giardino d'Europa. Pratolino come modello nella cultura europea, Mazzotta, Firenze 1986, pag. 24.

${ }^{40}$ Luigi Zangheri, Lo splendore di Pratolino e Francesco I de' Medici, in Alessandro VeZzosi (a cura di), Il giardino d'Europa. Pratolino come modello nella cultura europea, Mazzotta, Firenze 1986, pag. 15.

${ }^{41}$ Luigi Zangheri, Op.cit., in Alessandro Vezzosi (a cura di), Il giardino d'Europa. Pratolino come modello nella cultura europea, Mazzotta, Firenze 1986, pag. 16-17.
} 

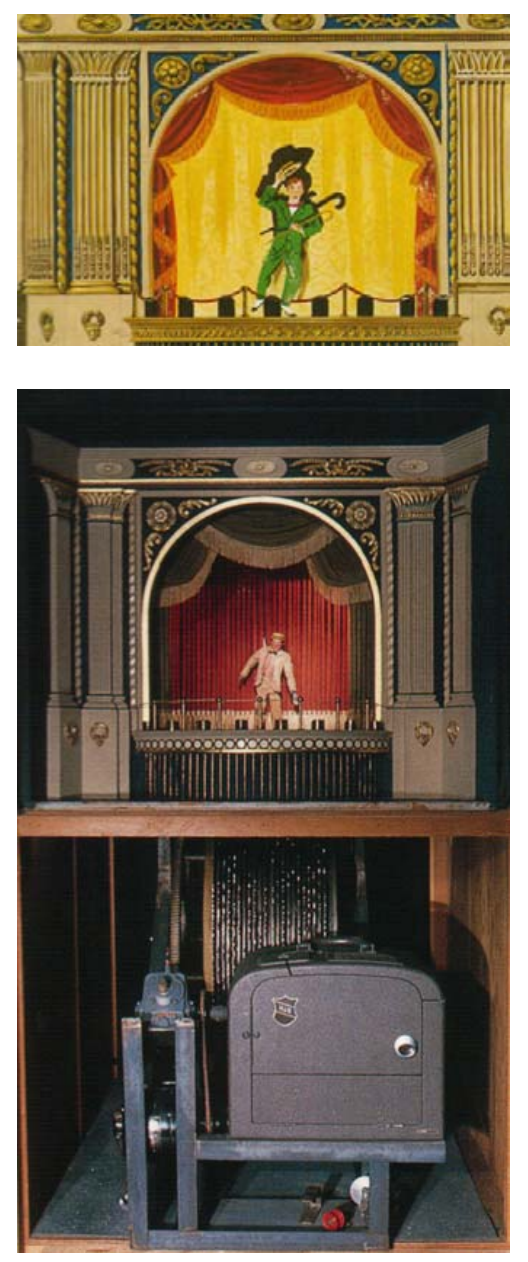

Fig. 7 Dancing man 1949 (studio Disney), scena teatrale in miniatura- disegno di ideazione di Ken Anderson (in alto) e realizzazione. Gli antesignani dei robot degli odierni parchi dei divertimenti possono essere individuati negli automi del XVI secolo.

Ma la perfezione nell'imitazione viene raggiunta in seguito, a Disneyland, con la realizzazione dei Pirati dei Carabi e della Casa Stregata.

Ancora una volta, come nei giardini manieristi, sono le caverne - dei Pirati dei Caraibi - ed i piani interrati - della Casa stregata - ad ingoiare il visitatore in luoghi stravaganti e concretissimi nello stesso momento. Nei Pirati dei Carabi lo spazio è senza fine, la luce cancella o sfonda le pareti e le scene di saccheggio e feste grottesche degli pirati-automi hanno gesti e suoni del tutto verosimili. Il paesaggio è costruito tutto dalla luce che mette a fuoco solo l'uomo (i pirati-automi), le sue case, i suoi oggetti. Fuori da questo fuoco è il nulla che trasfigura la reale architettura delle caverne - comunissime stanze. La Casa Stregata (Hauted Mansion) viene descritta da Umberto Eco:

"Si passa attraverso un cimitero abbandonato dove mani ossute di scheletro sollevano dall'interno le pietre tombali, si attraversa una collina allietata da un sabba completo di streghe e folletti, si sorvola un salone dalla mensa imbandita popolato di fantasmi trasparenti che danzano in abiti ottocenteschi mente convitati diafani, a tratti vanificantisi nell'aria, presenziano al banchetto di un sovrano barbarico, si viene accarezzati da ragnatele, ci si specchia in cristalli sulla cui superficie appare, dietro alle nostre spalle, una figura verdastra, si incontrano candelieri vaganti....(...). Come nei nuovissimi film dell'orrore, non c'è distacco, non si assiste all'orrore altrui, si è dentro l'orrore per sinestesia totale (...)."42

\footnotetext{
${ }^{42}$ Umberto Eco, La città degli automi, in Umberto Eco, Dalla periferia dell'impero. Cronache da un nuovo medioevo, Bompiani, Milano 2003, pagg. 57-58.
} 
Quello che a Disneyland è l'energia elettrica a Pratolino è energia idraulica, e quello che a Disneyland è la metamorfosi dell'architettura in set cinematografico a Pratolino (e a Boboli per opera del Buontalenti) è la metamorfosi dell'architettura in grotta.

Per entrambi i contesti è valida l'osservazione che Eco riferisce all'uso delle tecniche audioanimatroniche di Disneyland :

"Il piacere dell'imitazione, lo sapevano già gli antichi, è uno dei più connaturati all'animo umano, ma qui oltre che a godere una imitazione perfetta si gode la persuasione che l'imitazione abbia raggiunto il proprio culmine e che di qui in avanti la realtà le sarà sempre inferiore." 43

Esiste forse una differenza ontologica fra automi e robots, suggerita in nota a La ricerca della "terza natura" da Alessandro Rinaldi, a proposito del significati diversi assunti nel tempo dagli automi:

"Nel secolo successivo [al XIV] l'automa dimette ogni residuale connotazione magica per essere pienamente integrato, come una sorta di esemplare paradigma, alla struttura meccanica della natura; da interlocutore sia pure allarmante, dell'uomo, si trasforma così in un suo equivalente, e quindi potenziale sostituto (...)". ${ }^{4}$

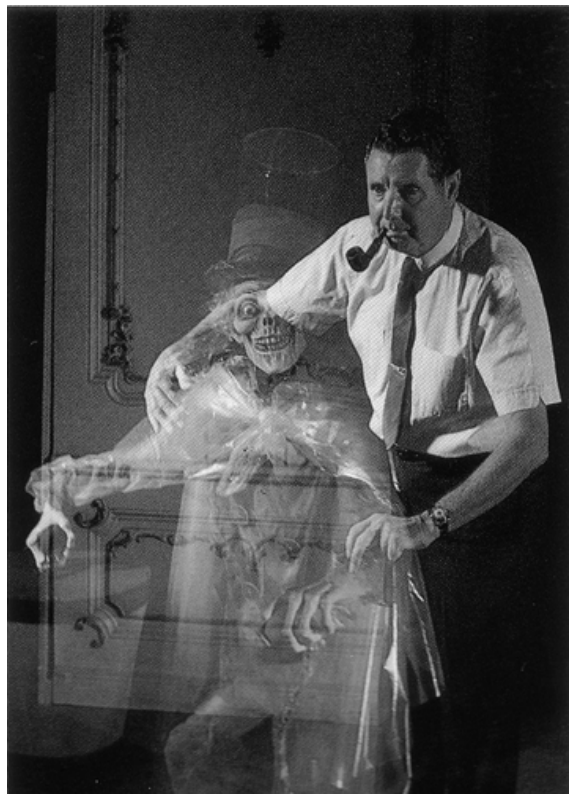

Fig. 8 Prove di effetti speciali per la Haunted Mansion di Disneyland. La "realizzazione" del fantasma è un altro esempio di copia-simulacro dell'uomo.

L'ingerenza della tecnologia negli spazi del giardino, la volontà di scomporre e ricomporre gli elementi della natura, di "manipolazione" dei loro contenuti simbolici, dettano a Pratolino precisi rapporti spaziali fra le parti che compongono il parco, con esiti diversi rispetto a quanto avviene nel giardino di Bomarzo, per il quale si è parlato di medium simbolico costituito dagli elementi naturali e morfologici a fluidificare in passaggio fra scena e scena. (A Pratolino il medium simbolico è costituito forse dall'acqua che scompare e ricompare in mille forme, ma non è leggibile un racconto unitario).

\footnotetext{
${ }^{43}$ UMBERTO ECO, Ibidem.

44 Alessandro Rinaldi, Op. cit., in Marcello Fagiolo, Natura e artificio. L'ordine rustico, le fontane, gli automi nella cultura del Manierismo europeo, Officina Edizioni, Roma 1997, pag. 175.
} 


\section{Pratolinum Magni Ducis Hetruriæ.}

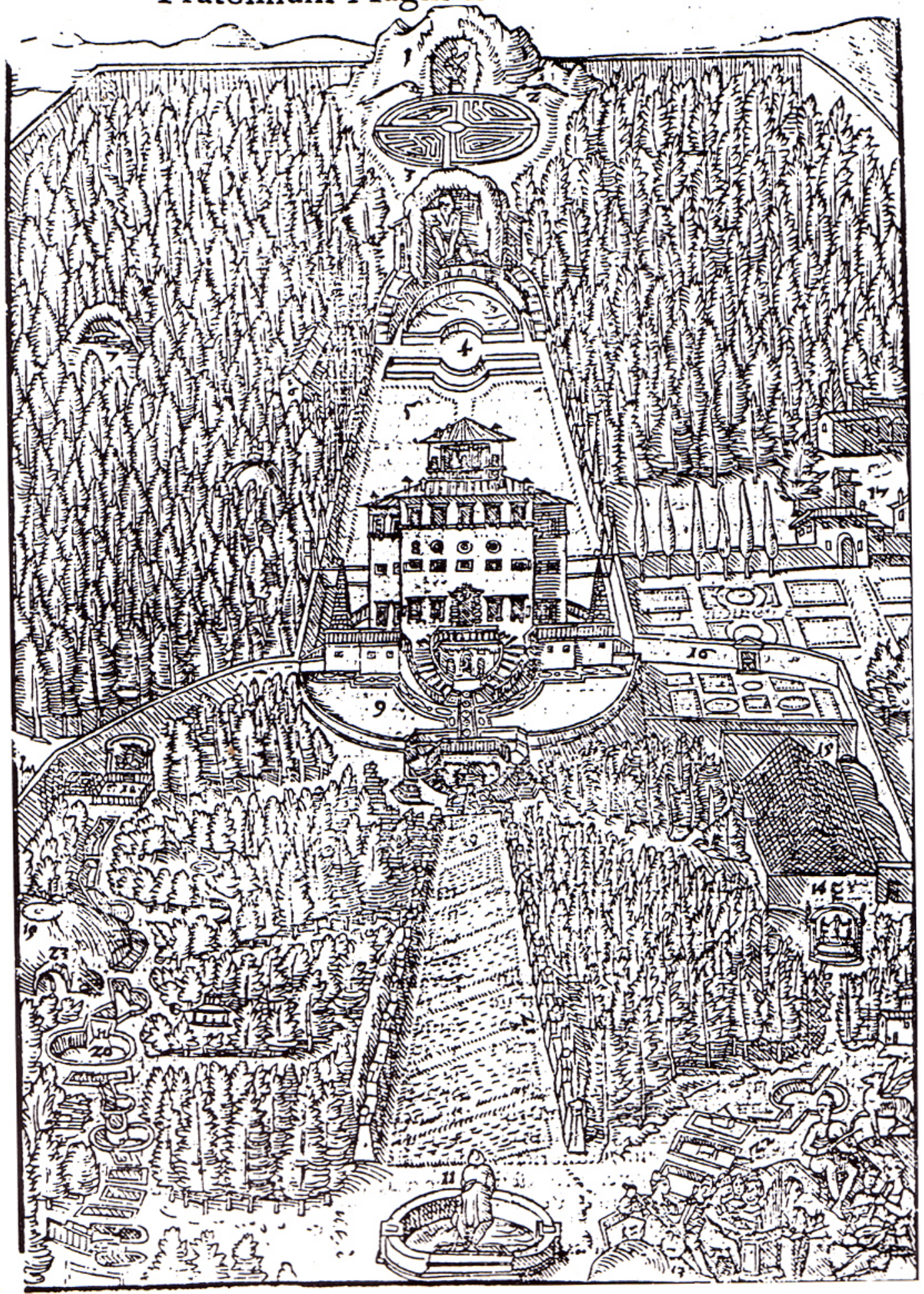

Fig. 9 "Veduta dell'intero parco di Pratolino da sud", anteriore al 1590, in " S. Vitale, " Ad Annales Sardiniae", Firenze 1639.

A Pratolino c'è un'alternanza di concentrazione e dilatazione, data dalla diversa dimensione delle cose e dal diverso grado della loro simbolizzazione. Esistono grandi campiture senza apparati iconografici e viceversa picchi in cui le immagini si addensano, si moltiplicano gli oggetti da cui lo sguardo viene attirato. Fra la statua della Appennino e la Villa a Sud c'è un 
prato, ovvero c'è una porzione adeguata di spazio libero in grado di assorbire le dimensioni gigantesche della statua da un lato, e di creare dall'altro una pausa fra la visione delle grotte nei sotterranei della villa e le grotte dentro l'architettura dell'Appennino. Le grotte costituiscono infatti grumi di simboli: la scala degli automi al loro interno è piccola, la natura compare in forme miniaturizzate, in concrezioni, in frammenti di rocce ricomposte artificialmente, in conchiglie, in madreperle, in ritagli di luce, in spruzzi d'acqua, in continue e variegate sollecitazioni dei sensi:

"Dirimpetto dunque alla portata dinanzi di Tramontana della Villa, vi è uno spaziosissimo Prato di figura semiovale chiuso per di sotto da una gran cancellata di ferro, tramezzata da dei pilastri incrociati di grottesco, che fa prospettiva all'entratura della Villa, e al gran Vivaio, che è pella sua sommità: salendo dolcemente dai lati è chiuso, o per meglio dire ornato da folti Abeti, e da Statue, e Aguglie con discreta distanza poste. Il gran Monte Appennino è significato per lo Gigante, che a sedere di pietra rustica fatto si vede, sopra il Vivaio, ove in larga copia per una ganascia del Drago, che è sotto il medesimo Gigante, si versa l'acqua nel Vivaio, la qual figura è tanta mole, che se ella fusse ritta in piedi, sarebbe circa a trentasei braccia, ed è tutta vota, essendovi nel corpo della medesima, una piccola stanza ornata di grottesca con spugne, nicchi, e madreperle, e con diversi scherzi d'acqua." 45

Segnato dal Viale degli Zampilli che ricalca l'asse di simmetria della Villa e sale a darvi accesso, il bosco dall'aspetto selvicultural ${ }^{46}$ ha il medesimo ruolo di sfondo ampio: è una superficie omogenea su cui si collocano eventi straordinari, ma spazialmente limitati, puntuali che fagocitano il visitatore intrattenendolo con giochi e ricchezza di immagini: sono il Bagno della Maschera, la Fonte Rossa o dei Galletti, la Grotta di Cupido con i sui scherzi d'acqua, la Vasca della Ranocchia, le Fonti del Satiro e di Baccola a ovest, o l' "arbore praticabile" (la Fontana della Rovere), il Concerto di Statue a monte delle Gamberaie, il Monte Parnaso, la Girandola, il Contadino a Est.

La stessa acqua ricorre all'interno del parco a scale opposte: a grandi superficie nelle vasche ampie del Vivaio dell'Appennino, delle Gamberane e dei Vivai più a Sud nei pressi della Fontana della Lavandaia, a quinta continua ed estesa nei getti del Viale degli Zampilli; allo stesso tempo in piccoli spruzzi, in gocce sulle rocce all'interno delle grotte, imponendosi quale elemento di scansione ritmica dell'intero spazio del parco e riproponendo il respiro ora concitato ora ampio che anche gli altri elementi del parco intendono realizzare.

Anche Alessandro Taglioni descrive il diverso rapporto di scala fra gli elementi di Pratolino, come caratterizzante il paesaggio e quale espediente per conservare un'atmosfera in bilico fra reale e fantastico:

"Il fine è (...) l'attuazione di un sogno fantastico e questa visione onirica è avvalorata dalla dimensione dei personaggi che popolano il luogo, nelle scale diverse che essi assumono, e che destabilizzano la percezione del reale $(\ldots) .{ }^{.47}$

\section{RIFERIMENTI BIBLIOGRAFICI}

ACIDINI LUCHINAT CRISTINA, L’Appennino del Giambologna: uomo , grotta, palazzo parte I, in AA.VV., Arte delle Grotte, Sagep Editrice, Genova 1987, pagg. 95- 100.

AURICOSTE ISABELLE, I parchi ricreativi in Europa: il divertimento e l'altrove, in MONIQUE Mosser GeOrges Teyssot, L'Architettura dei giardini d'Occidente- Dal Rinascimento al Novecento, Electa, Milano 1990, pagg. 479-490.

\footnotetext{
${ }^{45}$ Dal Distretto delle cose più notabili della Città di Firenze, nell'edizione del 1719, riportato in ALESSANDRO Vezzosi (a cura di), Il concerto di statue, Alinea, Firenze 1986, pag. 90-91.

${ }^{46} \mathrm{Nel}$ componimento poetico Vaghezze sopra Pratolino di Raffaello Gualterotti, dedicate al granduca Francesco e riportato in TAgLiolini Alessandro, Storia del giardino italiano, La casa Usher, Firenze 1988, pag. 167, il paesaggio di Pratolino viene connotato dal "bel verde selvaggio":

“(...) La natural bellezza,/ Che'l Lauro, e '1 Mirto havea l'Abete, e'l Faggio/ E'l bel verde selvaggio/ E'l bel verde fiorito, altra vaghezza (...)".

${ }^{47}$ Tagliolini Alessandro, Storia del giardino italiano, La casa Usher, Firenze 1988, pag. 168.
} 
Bonito Oliva AchiLle, L'ideologia del Traditore - Arte, maniera, manierismo, Electa, Milano 1998.

CAlvesi Maurizio, Gli incantesimi di Bomarzo. Il Sacro Bosco tra arte e letteratura, Bompiani, Milano 2000.

ECo UMBerto, Dalla periferia dell'impero. Cronache da un nuovo medioevo, Bompiani, Milano 2003.

EISNER D. MiCHAEL (foreword by), Imagineering. A Behind the Dreams Look at Making the Magic Real, Disney Editions, New York 1996.

Maling Karal Ann, Designing Disney's Theme Parks: The Architecture of Reassurance, Flammarion, Paris 1998.

MARESCA PAOLA, Boschi sacri e giardini incantati, Angelo Pontecorboli Editore, Firenze1997.

PACCIANI RICCARDO, Aspetti dell 'imitazione della natura fra quattrocento e Cinquecento, in MARCELlo FAGIOLO, Natura e artificio, Officina Edizioni, Roma 1997.

PANZINI Franco, Per i piaceri del popolo. L'evoluzione del giardino pubblico in Europa dalle origini al XX secolo, Zanichelli, Bologna 1997.

PUPPI Lionello, Natura, artificio, inganno. Il giardino in Italia nel Cinquecento: temi e problemi, in MONIQUE MOSSER, GEORGES TEYSSOT, L'Architettura dei giardini d'Occidente- Dal Rinascimento al Novecento, Electa, Milano 1990.

RINALDI ALESSANDRO, La ricerca della "terza natura": artificialia e naturalia nel giardino toscano del Cinquecento, in MARCELlo FAGIOLO, Natura e artificio. L'ordine rustico, le fontane, gli automi nella cultura del Manierismo europeo, Officina Edizioni, Roma 1997.

TAGLIOLINI AlESSANDRO, Storia del giardino italiano, La casa Usher, Firenze 1988.

TeSSARI RoBerto, Il mercato delle Maschere, in Roberto Alonge, Guido DAVICO BONINO (diretta da), Storia del teatro moderno e contemporaneo, volume primo, La nascita del teatro moderno. Cinquecento- Seicento, Einaudi, Torino, 2000, pag. 119.

TESTA FAUSTO, Spazio e allegoria nel giardino manierista, La Nuova Italia, Firenze 1991.

VÉRIN HÉLÈN, La tecnologia nel parco: ingegneri e giardinieri nella Francia del Seicento, in Monique Mosser, GeOrges Teyssot, L'Architettura dei giardini d'Occidente- Dal Rinascimento al Novecento, Electa, Milano1990, pagg. 131-142.

VÉRIN HÉLÈN, La tecnologia nel parco: ingegneri e giardinieri nella Francia del Seicento, in Monique Mosser, GeOrges Teyssot, L'Architettura dei giardini d'Occidente- Dal Rinascimento al Novecento, Electa, Milano1990, pagg. 131-142.

VEZZOSI AlesSANDRO (a cura di), Il concerto di statue, Alinea, Firenze 1986.

Vezzosi Alessandro (a cura di), Il giardino d'Europa. Pratolino come modello nella cultura europea, Mazzotta, Firenze 1986.

ZANGHERI LUIGI, Pratolino, il giardino delle meraviglie, Firenze 1987.

ZANGHERI LUIGI, Naturalia $e$ curiosa nei giardini del Cinquecento, in MONIQUE Mosser, GEORgES TEYSSOT, L'Architettura dei giardini d'Occidente- Dal Rinascimento al Novecento, Electa, Milano 1990, pagg. 55-64.

ZANGHERI LuIGI, Suggestioni e fortuna dei teatrini d'automi. Pratolino come una Broadway manierista, in "Quaderni di teatro", Vii, 25, 1984, pagg. 78-84.

ZANGHERI LUIGI, Tra abilità e tecnologia. l'acqua nelle grotte del manierismo, in AA.VV., Arte delle Grotte, Sagep Editrice, Genova 1987, pagg. 9- 14.

\section{RIFERIMENTI ICONOGRAFICI}

Fig. 1: ZANGHERI LUIGI, Naturalia e curiosa nei giardini del Cinquecento, in MONIQUE Mosser, GeOrges Teyssot, L'Architettura dei giardini d'Occidente- Dal Rinascimento al Novecento, Electa, Milano 1990, pag. 57.

Fig. 2: fotografia di Enrica Dall'Ara. 
Fig. 3: Guglielmino Salvatore, Grosser Hermann, Il sistema letterario. Guida alla storia letteraria e all'analisi testuale. Quattrocento e Cinquecento, Principato, Milano 1987, pag. 1072.

Fig. 4: Calvesi Maurizio, Gli incantesimi di Bomarzo. Il Sacro Bosco tra arte e letteratura, Bompiani, Milano 2000, tavola 21.

Fig. 5: foto Vasari in CALVesI MAURIZIO, Gli incantesimi di Bomarzo. Il Sacro Bosco tra arte e letteratura, Bompiani, Milano 2000, tavola 29.

Fig. 6: rilievo quotato e materico dell'Appennino, in VEZzosi ALESSANDRO (a cura di), $I l$ concerto di statue, Alinea, Firenze 1986, pag. 72.

Fig. 7: fotografia del robot Dancing Man in EISNER D. MiCHAEL (foreword by), Imagineering. A Begind the Dreams Look at Making the Magic Real, Disney Editions, New York 1996, pag.119; disegno di Ken Anderson in MALING KARAL AnN, Designing Disney's Theme Parks: The Architecture of Reassurance, Flammarion, Paris 1998, pag. 48.

Fig. 8: EISNER D. MICHAEL (foreword by), Imagineering. A Behind the Dreams Look at Making the Magic Real, Disney Editions, New York 1996, pag. 123.

Fig. 9: VeZzosi AlesSANDRo (a cura di), Il giardino d'Europa. Pratolino come modello nella cultura europea, Mazzotta, Firenze 1986, pag. 104. 


\section{2 - PLEASURE GARDENS, FOLIES, JARDINS SPECTACLES}

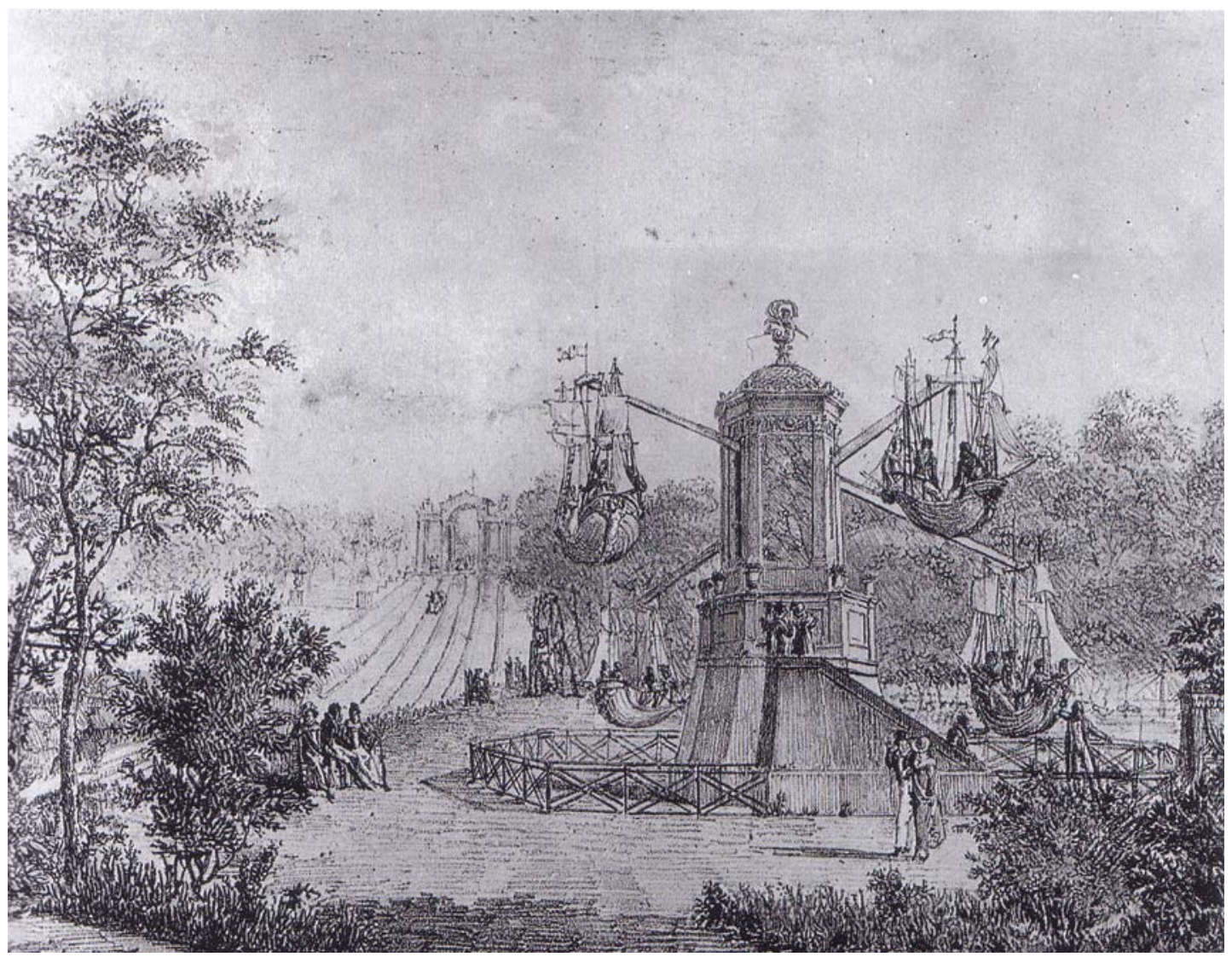

Fig. 1 Torre di Eolo. Passeggiata aerea a Tivoli, Parigi, litografia di C. Motte, post 1800. Musée Carnevalet, Paris. Sullo sfondo sono visibili le montagne russe.

\section{IL VAUXHALL DI LONDRA (1661-1859) QUALE PROTOTIPO E SUE “ESPORTAZIONI” IN EUROPA}

Precursori dei moderni parchi del divertimento possono intendersi i pleasure gardens di matrice Londinese: la Londra del XVIII sec. appare costellata di giardini suburbani aperti al pubblico - una delle principali attrazioni della città - nati come folies lungo la riva del Tamigi.

"Si tratta di luoghi di ritrovo, in cui il giardino funge da spazio contenitore di attività teatrali e ludiche (...). Si configurano anzi quali parchi pubblici specializzati, a sostanziare il legame indissolubile fra giardino, spettacolo, volontà di divertimento $(\ldots)^{\text {"1 }}$.

Lo spettacolo all'interno dei pleasure gardens consiste sia in perfomances, sia nella proposizione di ambienti esotici mediante esposizioni botaniche e sperimentazioni stilistiche nelle architetture nel parco.

Il più famoso è Vauxhall (1661 - 1859), ed esercita un tale fascino da imporsi come "prototipo" d'esportazione a livello internazionale - anche New York possiede nel XIX

\footnotetext{
${ }^{1}$ Franco Panzini a proposito dei giardini ricreativi, in FrAnCo PANZINI, Per i piaceri del popolo. L'evoluzione del giardino pubblico in Europa dalle origini al XX secolo, Zanichelli, Bologna, 1997, pag. 98.
} 
secolo un Vauxhall - e divenire nome comune per designare i parchi che sovrappongono al giardino elementi specializzati finalizzati al divertimento. Definiscono il giardino musica, danza, balli in maschera, spettacoli pirotecnici, esibizioni d'acrobati, voli in pallone, trasfigurazioni notturne attraverso giochi d'illuminazione. Franco Panzini restituisce l'immagine del parco riportando le descrizioni letterarie di scrittori illustri quali John Evelyn, Jonathan Swift, Charles Disckens, da cui emerge come il parco venga apprezzato per la piacevolezza dell'ambiente rurale e le possibilità di incontro che lo rendono luogo prediletto per artisti e letterati.

Vauxhall subisce cambiamenti di carattere e di pubblico nel corso del tempo: nel XVII secolo è conosciuto come Spring Garden e ad attrarre sono la "grazia"2 del paesaggio e la presenza di un'acqua curativa; in seguito, soprattutto sotto la gestione di Jonathan Tyers, che ha inizio nel 1728, aumentano gli spettacoli proposti, gli intrattenimenti serali: più in generale il parco assume l'aspetto di spazio teatrale, per le architetture e $i$ panorami allestiti. Vauxhall nel XVIII secolo è un giardino per il pubblico alla moda, asseconda il gusto crescente per l'esotico e per le composizioni eclettiche, così come per i ritrovi serali, con cene e balli che avvengono sotto lo sfavillio delle luci. E' fra la fine del XVIII secolo e gli inizi del XIX che Vauxhall "va gradualmente mutando il suo carattere per divenire più un moderno parco dei divertimenti che uno spazio in cui la buona società si ritrova"”. Il mutamento è dovuto al fatto che in quegli anni diventano frequenti le esibizioni di cantanti lirici famosi all'epoca; vengono inoltre introdotti spettacoli pirotecnici, arrivano a Vauxhall la funambola M.me Saqui già celebre e il domatore di Leoni Van-Ambrungh. Sono eventi che attirano il pubblico popolare.

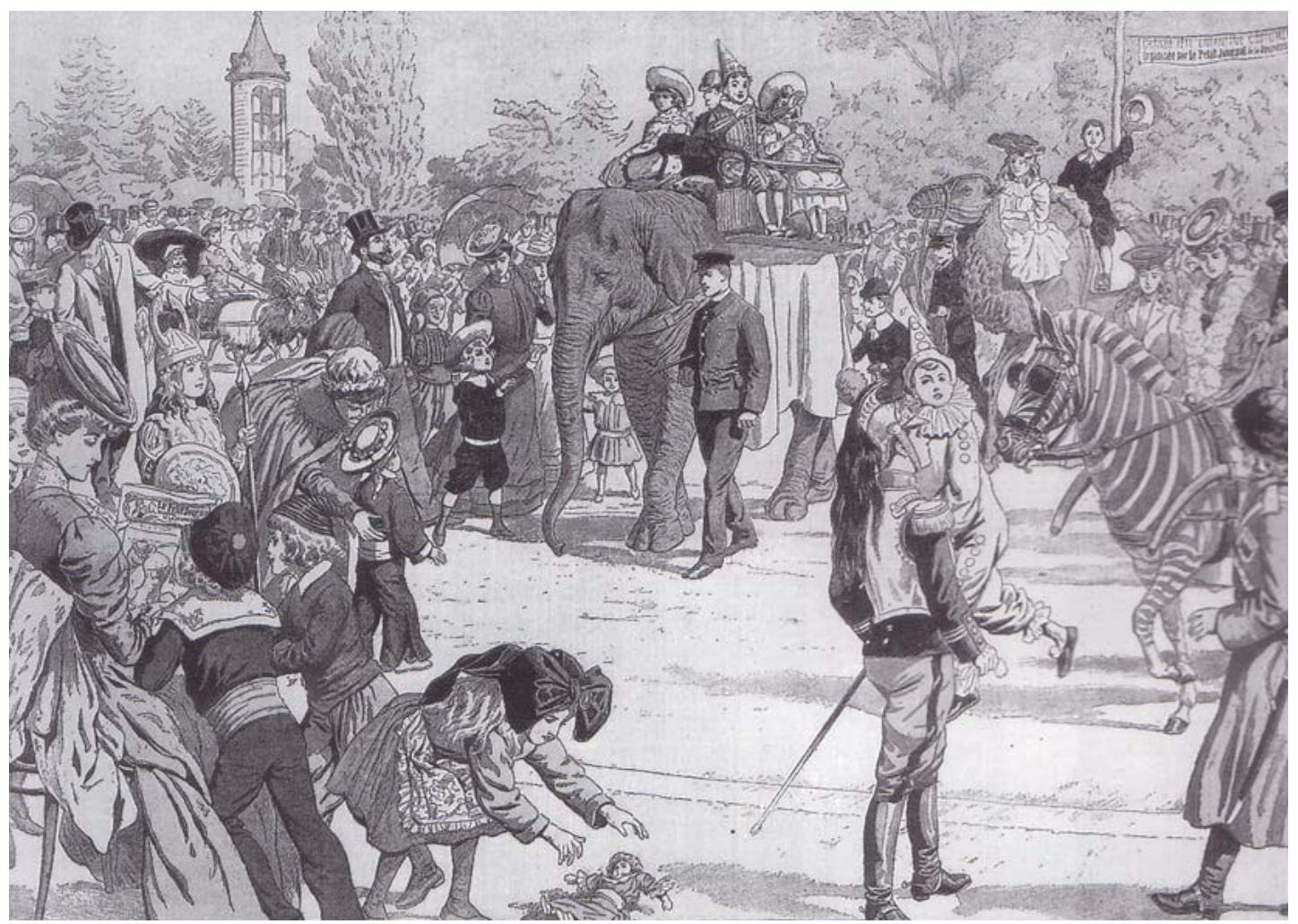

Fig. 2 Festa in costume per bambini, al Jardin d'Acclimatation di Parigi. Musée Carnevalet, Paris.

\footnotetext{
${ }^{2}$ John Evelyn visita il giardino nel 1661 e commenta "Sono andato a vedere il nuovo Spring Garden a Lambeth, un giardino composto con molta grazia", commento riportato in E.S. DE BEER (a cura di), The Diary of John Evelyn, Oxford University Press, London 1959, citato poi in Franco PANZINI, Op.cit, Zanichelli, Bologna, 1997, pag. 101.

${ }^{3}$ Franco PanZini, Op. cit., Zanichelli, Bologna, 1997, pag. 104.
} 
Il popolo è attirato dalla grandiosità degli spettacoli e soprattutto dagli elementi inconsueti, strani, dall'atmosfera tragicomica del circo. L' esotico costituisce un elemento di grande fascino, da conoscere e con cui divertirsi allo stesso tempo.

Il Jardin d'Acclimatation al Bois de Boulogne di Parigi inaugurato da Napoleone III nel 1860 nasce esponendo piante, animali e uccelli esotici in una compagine parigina di aristocrazia avvezza ai viaggi, reali o immaginari, interessata di scienza ed etnologia. Dal 1900 diviene giardino di piacere per le famiglie e ospita attività artistiche e culturali, concerti, circhi, conferenze sui processi di acclimatazione, sulla medicina, sui viaggi. Ha il potere immaginifico di un serraglio imperiale, quale quello documentato da Salimbene da Parma (1221-1288) nella sua Cronica, al seguito di Federico II, che materializzava il potere derivato dal dominio sulla scienza e dal dominio territoriale, spettacolarizzandolo e rendendolo sperimentabile pubblicamente.

Nel 1874 Il teatro delle pantomime del Tivoli di Copenaghen viene ricostruito in stile cinese e denominato Chinese Peacock per la forma del suo sipario. Nel 1888 il parco ospita The Nordic Exhibition of Industry Agricolture and Art all'interno di padiglioni, realizzati per l'occasione, in stili architettonici esotici costruiti con tecniche locali, nel 1900 viene eretta la Torre cinese.

In Francia il giardino per il divertimento del XVIII secolo, ha volti diversi e distinti, che si confondono successivamente negli anni che seguono la Rivoluzione del 1789. In apertura del suo Folies, Tivolis et Attractions. Les Primiers parcs de loisir parisiens, Gilles-Antoine Langlois riassume l'intero percorso di genesi dei parchi dei divertimenti parigini sintetizzandone le componenti eterogenee, le une legati ai modi della festa dell'aristocrazia, le altre alle fiere e ai luoghi delle feste popolari:

"Ils ne sont pas une création du pouvoir, ils n'ont pas cherché à correspondre à un besoin, et s'ils sont le fruit d'un hasard historique assez heureux, la plupart de leurs activités existaient déjà. Mais elles étaient dispersées, dan les foires, lors des fêtes aristocratiques ou populaires, dans la trame même des jardins et des décors de la fin du XVIII siècle. Ils constituent la rencontre de ces éléments, sans doute la première incarnation de ce mélange des classes sociales et de leurs symboles que la Révolution appela « Egalité » ». ${ }^{4}$

In seguito alla rivoluzione si ritrovano in luoghi unici le componenti che durante il corso del XIX secolo erano invece « disperse » in contesti differenti e specialmente nelle folies e nelle fiere popolari.

Le folies sono giardini privati, caratterizzati dalla stravaganza e dall'eclettismo degli stili e sorgono negli intorni di Parigi negli anni settanta del XVIII secolo, in concomitanza con il diffondersi dello stile anglo-cinese e più in generale nel fermento di rielaborazione del pittoresco di matrice inglese in territorio francese. Le folies si impongono quali esempi estremi del nuovo gusto, che rivendica i diritti dell'originalità, spinta fino alla stravaganza, nella progettazione del giardino. Il Jardin Monceau (1773), disegnato da Carmontelle, ne è un manifesto, commentato fra l'altro dallo stesso Carmontelle nel suo Jardin Monceau, près del Paris (1779): il paesaggista definisce la propria opera "terra di illusioni" e "una semplice fantasia contenente tutti i tempi e tutti i luoghi" - le espressioni sono celeberrime e dichiarano la volontà di effettuare una ricognizione di quanto la storia e la geografia rendono disponibile a livello iconografico, volontà alimentata dal desiderio di divertirsi nel comporre e scomporre arbitrariamente, a fini puramente scenografici.

Per descrivere il Jardin Monceau, John Dixon Hunt premette che Carmontelle è anche scenografo e disegnatore di trasparent panoramas, ovvero di sequenze di scene dipinte su carta trasparente illuminate dal retro con luce di candela, che si svolgono girando una manovella.

\footnotetext{
${ }^{4}$ Gilles-Antolne Langlois, Folies Tivolis et attractions. Les premieres parcs de loisirs parisiens, Délégation à l'action artistique de la ville de Paris, Paris 1991, pag. 25.
} 
"[The transparent panoramas] obviously allowed a much greater freedom of invention, including multiplicity of scenes, than most actual gardens ever would. (...) The jardin Monceau elaborately re-created in three dimensions and full scale the experience of these transparencies". 5

Hunt ci informa in questo modo sia del fatto che il parco è concepito come una progressione di scene differenti, sia che ad improntarlo è la libertà di invenzione: sono aperte le porte agli accostamenti più arditi, nelle singole forme dell'architettura, fabriques, come nell'allestimento di interi ambienti esotici e improbabili.

$\mathrm{Si}$ affida la descrizione del Jardin Monceau a Langlois, che ne sa restituire la leggerezza, dovuta, all'interno del giardino, a qualcosa di frivolo nei contenuti e nell'aspetto, che si intravede nel gioco dei colori, negli ornati a cui richiamano le fabriques, nell'alternanza di grandi campiture e oggetti "traforati":

“C'est particulièrement flagrant à Monceau, l'ancienne folie de Chartres entourée d'un parc où plusieurs trames de spectacle étaient immédiatement visibles; l'une en couleurs (...) ; l'autre en matières (les sols : " déserts », prairies, cultures, sous-bois), enfin la grande diversité des fabriques (moulin hollandais, ruines romaines, château gothique, minaret turc, tente tartare, jeu de bague chinois, etc.), représentations animales vivantes (moutons, vaches, dromadaires) et humaines, sculptées» ${ }^{6}$

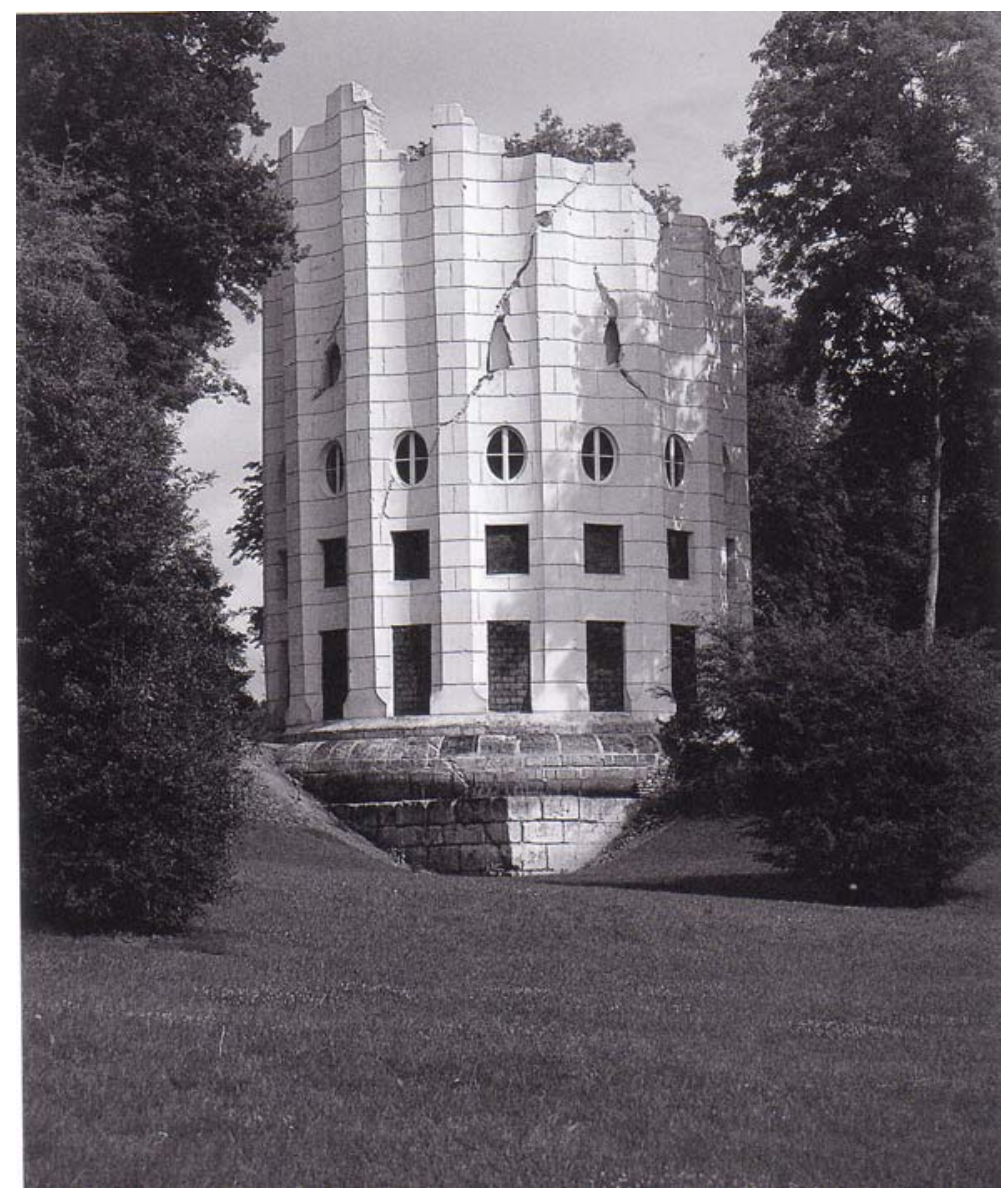

Fig. 3 Colonna in rovina al Désert de Retz. Le sue dimensioni gigantesche e assolutamente improbabili hanno il potere di spaesare il visitatore. L'espediente della variazione di scala rispetto al "reale" per stupire è proprio anche del giardino manierista e diviene ricorrente nella progettazione dei parchi del divertimento.

Il Désert de Retz (1774), di proprietà del Baron de Monville, è un altro esempio noto di folie. Alcuni suoi elementi lo avvicinano all'estetica dei giardini del periodo manierista: il

\footnotetext{
${ }^{5}$ Dixon John Hunt The Picturesque Garden in Europe, Thames and Hudson, London 2002, pagg. 120-121.

${ }^{6}$ Gilles-Antoine Langlois, Folies Tivolis et attractions. Les premieres parcs de loisirs parisiens, Délégation à

l'action artistique de la ville de Paris, Paris 1991, pag. 30.
} 
ruolo della grotta come luogo di accesso al meraviglioso, dato dal connubio di natura e artificio composti per l'esibizione davanti al proprio visitatore; e il ricorso alla variazione di scala come strumento per far sentire l'osservatore in una condizione di instabilità, di smarrimento. Al Dèsert de Retz una grotta si presenta quale ingresso al parco provenendo dalla foresta reale di Marly, sorvegliata da satiri che portano fiaccole. La grotta, luogo stretto e chiuso, immette nel paesaggio del parco: una distesa ariosa di prati e boschi, costellati di architetture esotiche o richiamanti mondi antichi, che si impone, varcata la grotta, quale quadro improvviso. C'è la rovina di una colonna gigantesca, eccentricità inquietante su cui si sono soffermati Monique Mosser ${ }^{7}$ e J. Dixon Hunt.

Hunt scrive:

"But the pièces de résistance was the colossal Broken Column, striking above all for being so unlike the usual miniaturized fabriques of most gardens, it suggest the sublime dimensions both in time (when had it been erected?) and space (it must once have been immense - where did it come from?). ${ }^{8}$

Altre folies sono il parco di Méréville, le Petit Trianon a Versaille, la Folie-Marbeuf e la folie del finanziere Simon-Charles Boutin che sarà il celeberrimo Tivoli di Parigi, dopo la confisca del parco durante la rivoluzione.

L'eclettismo della folie di Boutin è dato in primo luogo dalla tematizzatone dei giardini che si aprono lungo il viale centrale che dà ossatura al parco: si incontrano un giardino italiano, un giardino francese, un giardino inglese, il giardino olandese, il giardino italiano, anche un orto, serre, un vivaio, una stalla.

\begin{abstract}
"Boutin semble d'ailleurs aimer les images, tout en les maniant avec discrétion : à l'opposé de la maison, après avoir longé les parterres et traversé un axe qui va du pavillon du gladiateur à la grande cascade, on bute finalement sur un tombeau.

Tivoli est dessiné en croix : un axe va de folie au tombeau, l'autre d'une île au cœur d'une "nature » à une cascade traversant des vergers. L'on passe continuellement d'un ordre à un désordre, d'axes nettement indiqués à de sinueuses allée. ${ }^{9}$
\end{abstract}

Il giardino inglese è un inserto di natura irregolare, declinazione della wilderness all'interno del giardino ed interpreta in forme originali il mito della natura selvaggia di J. J. Rousseau. Boutin con il suo giardino sembra quasi volere stabilire un confronto, divertito e possibilista, fra diversi valori spaziali e culturali-il rigore geometrico che si impone come dichiarazione di dominio sulla natura, la produttività espressa dagli orti, la conoscenza scientifica (botanica) rappresentata dalle serre e dal vivaio.

Le folies compaiono quali espressioni artistiche colte, dei proprietari e dei paesaggisti che ne seguono la progettazione.

Le fiere popolari offrono altri generi di divertimento che vengono riversati all'interno delle folies in seguito alla confisca e alla nuova gestione pubblica di queste.

E' sempre a fornire in breve l'idea dell'atmosfera che si viene a creare nella coesistenza del mondo della folie e di quello della fiera. Il caos del mercato, del gioco spontaneo, dello spettacolo di strada viene ospitato da un paesaggio studiato e costruito con attenzione ad ogni vista prospettica, perfettamente (e raffinatamente) determinato nella disposizione delle architetture, nell'uso dei colori ecc.

"Les nostalgiques [della rivoluzione] voulaient y voir l'invasion par le peuple des jardins de la caste possédante ; l'homme de la rue trouvait sans doute un peu extravagants ces jardins-spectacles au décor neuf, sorti des mains de plus grands talents d'Europe : architectes, paysagistes, peintres... Mais les uns y retrouvaient l'enchantement des folies, les autres la folie enchantée de la foire. $\rangle^{10}$

\footnotetext{
${ }^{7}$ In MosSER MONIQUe, Le architetture paradossali ovvero piccolo trattato sulle "fabriques" in MONIQUE Mosser, Georges Teyssot, L'Architettura dei giardini d'Occidente- Dal Rinascimento al Novecento, Electa, Milano1990, pagg. 259-276.

${ }^{8}$ Dixon John HunT, Op.cit., Thames and Hudson, London 2002, pagg. 125.

${ }^{9}$ Gilles-Antoine Langlois, Op.cit., Délégation à l'action artistique de la ville de Paris, Paris 1991, pag. 88.

${ }^{10}$ Gilles-Antoine Langlois, Op.cit., Délégation à l'action artistique de la ville de Paris, Paris 1991, pag. 25.
} 
La «follia incantata della fiera » risiede negli spettacoli di saltimbanchi, di marionette, di giocolieri, funamboli, acrobati, commedianti, prestigiatori e si riversa non solo nelle raffinate folies, ma anche nei giardini pubblici a pagamento che nascono alla fine del XVIII secolo e vengono nominati jardins spectacles- o anche jardin-foire, proprio perché le attrazioni richiamano le fiere: impresari vi organizzano feste con artisti ambulanti, balli e spettacoli pirotecnici, .

"Comment définir ces jardins? Au vrai ce ne semblent guère que des enclos assez vastes, en lisière de Paris, propres à servir de pistes de danse, et de plates-formes pour les jeux pyrotechniques $(\ldots) »^{11}$.

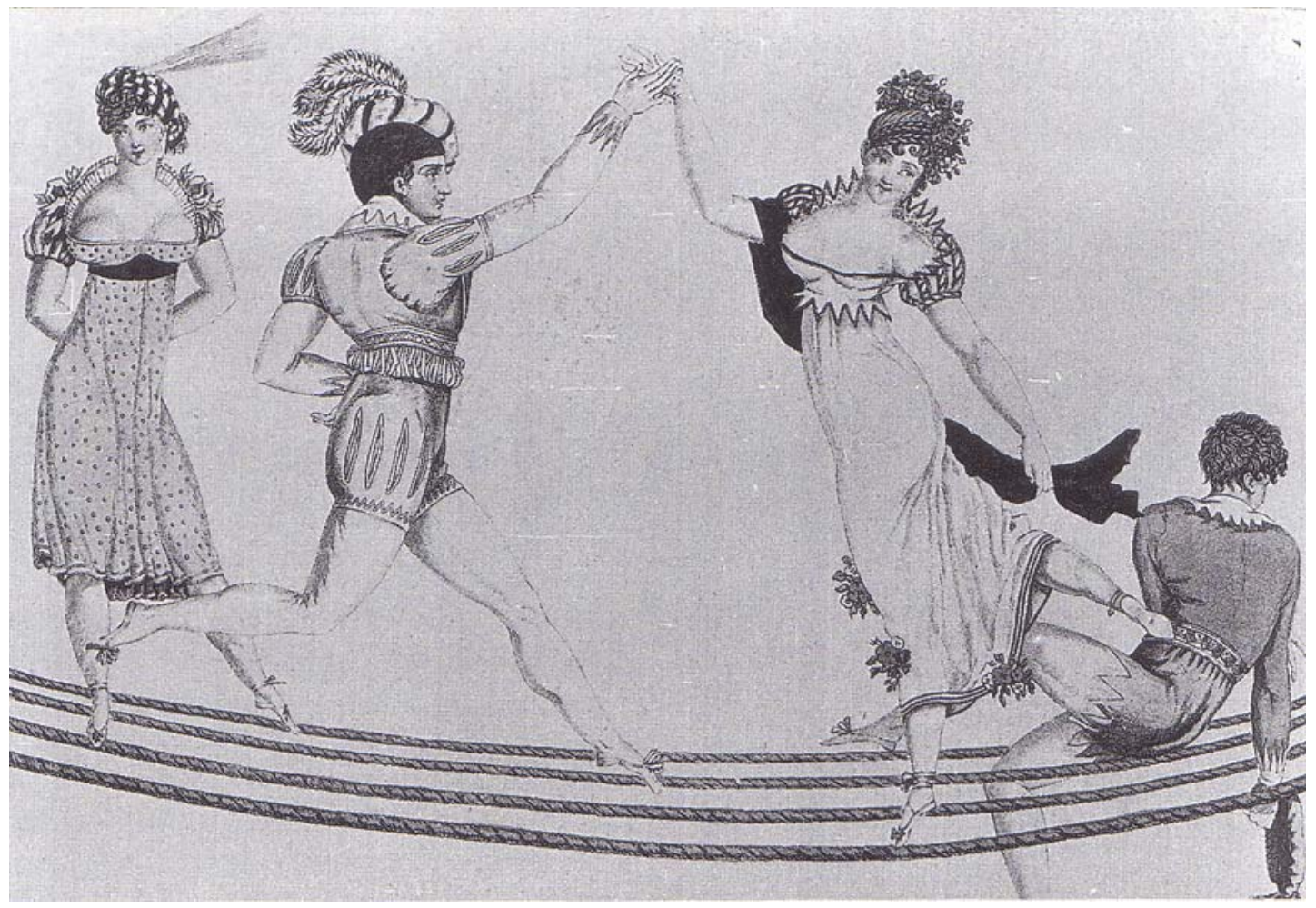

Fig. 4 Il funambolo Forioso e un ballo popolare sotto il Consolato, 1810 circa.

\section{SPECIFICAZIONE DEL PAESAGGIO}

I pleasure gardens sono costruiti seguendo un "determinato" processo di trasformazione spaziale (i modelli compositivi invece dipendono dalla compagine culturale specifica): nascono normalmente in luoghi già apprezzati per le qualità del paesaggio naturale - quiete $\mathrm{e}$ ombra di ambienti rurali, presenza di fonti d'acqua termale e curativa - o in giardini di pregio urbani e extraurbani. In sovrapposizione - comunque a partire da - questo layaut esistente, viene costruito l'apparato che specializza, specifica la ricreazione. Si operano, dove assenti, gli interventi atti alla "fondazione" del luogo: la materializzazione dei confini con apertura di limitati ingressi, riproponendo elementi propri degli hortus conclusus medievali (per la separazione netta dello spazio ludico e ricreativo), oppure la creazione di viali comodi per il passeggio da cui si aprono prospettive scenografiche.

Non si parte con la creazione di una tabula rasa (punto di inzio per i parchi Disney), ma si plasma a ulteriori usi un determinato contesto.

Il Tivoli di Copenhagen sorge ad opera del militare Georges Carstensen che nel 1842 affitta un terreno sul terrapieno esterno lungo le mura della città e fonda una società di gestione. Lo

\footnotetext{
${ }^{11}$ Gilles-Antoine Langlois, Op.cit., Délégation à l'action artistique de la ville de Paris, Paris 1991, pag. 28.
} 
schema planimetrico segue la geometria stellare del terrapieno: un viale alberato ribadisce il particolare profilo a zig zag, su ogni lato si aprono le attrazioni - il teatro per i concerti è realizzato sfruttando come cavea il pendio del terrapieno.

Il Bakken sorge, antichissimo, nel 1583 nei pressi di Copenaghen, in seguito alla scoperta di una fonte termale in un luogo naturale fuori della città, presenza che fa prediligere l'area per trascorrervi i giorni di vacanza. Attirati dall'afflusso di "turisti" si radunano artisti itineranti stanziati in accampamenti, trasformati in seguito in insediamento stabile, concentrato su una collinetta ( in danese "bakken") vicina alla fonte.

Emblematico è Il Vauxhall di Londra. Vauxhall conserva, nonostante le modifiche che subisce nel tempo mirate a farne un giardino sempre ricco di proposte nuove per il pubblico, una duplice anima: uno spazio definito dalle architetture - peristili ed esedre - ed un paesaggio campestre, interno al giardino, ma omogeneo con l'intorno, la campagna a sud del Tamigi che si raggiunge soprattutto in barca, attraversando il fiume. Nel 1728 le attrazioni e l'apparato architettonico vengono potenziate da Jonathan Tyers, che affitta la tenuta, amplia le aree alberate, realizza boschetti in sostituzione dei frutteti, attento a creare al loro interno effetti teatrali.

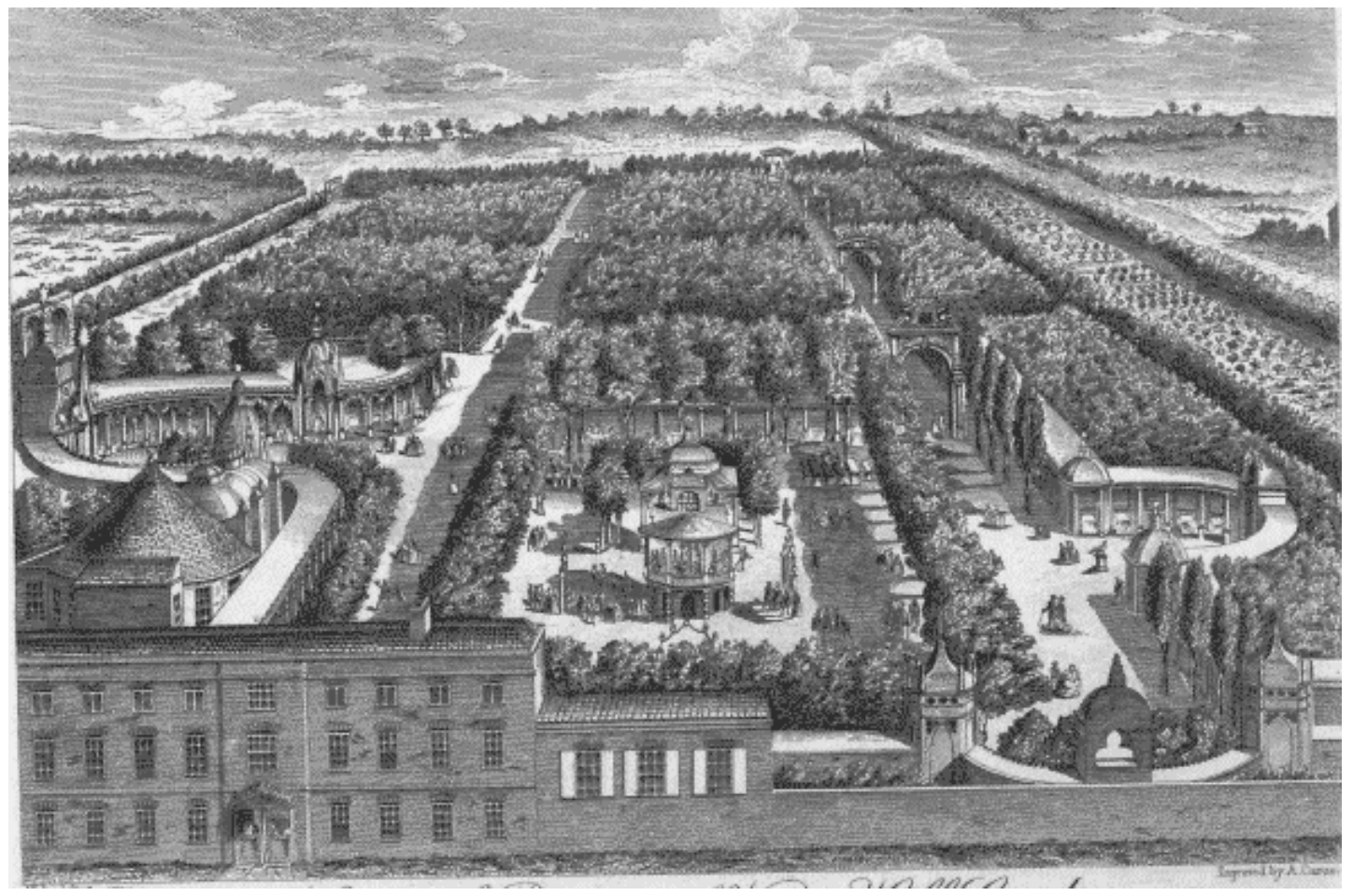

Fig 5 Vauxhall gardens: la prospettiva esprime perfettamente il graduale passaggio dall'architettura alla "natura", dalla città alla campagna. La serie di archi lungo il South Walk (a destra) accentua questo andamento. Gli edifici sul fronte sembrano mura di città ed il parco sconfina verso l'ambiente rurale sullo sfondo.

In una veduta di Vauxhall del 1751 (fig. 5), dagli edifici di ingresso la prospettiva si apre seguendo gli assi longitudinali - il Great Walk e il South Walk scandito da archi in serie - che organizzano il giardino dandogli un senso estremo di direzione. E' come se l'occhio stesse percorrendo una strada pubblica che progressivamente si immette nella campagna, varcata la cortina muraria in primo piano, i viali del giardino sembrano aperti e sfociano in un paesaggio vago sullo sfondo. I settori a bosco in qualche modo ricalcano la maglia dei campi, visibili, oltre la siepe di confine, sulla destra. La parte prospiciente l'ingresso ha margini più solidi e definiti, tutta racchiusa da porticati e da esedre in cui è possibile sedersi e mangiare guardando gli spettacoli che avvengono nel prato centrale, dove è l'Orchestra. Da un lato, oltre i portici, emerge la sala da musica chiamata Rotunda. 
Osservando la planimetria di Vauxhall del 1826 (fig. 6) l'aspetto bifronte del giardino è molto chiaro, anche se si legge la presenza di nuove costruzioni a chiusura delle prospettive lungo i viali. L' impianto planimetrico ad opera di Tyers conserva il carattere del luogo, carattere di transizione fra architettura e natura, che è sia un fatto fisico, di composizione spaziale, sia metafora dell'abbandono della città verso spazi al di fuori della giurisdizione di questa, conferendo al parco il ruolo di frontiera:

"La frontiera rappresenta (...) la fine della terra, il limite ultimo oltre il quale avventurarsi significava andare al di là della superstizione, contro il volere degli dèi, oltre il giusto e il consentito, verso l'inconoscibile che ne avrebbe scatenato l'invidia. Varcare la frontiera, significa inoltrarsi dentro un territorio fatto di terre aspre, dure difficili, abitato da mostri pericolosi contro cui dover combattere". ${ }^{12}$

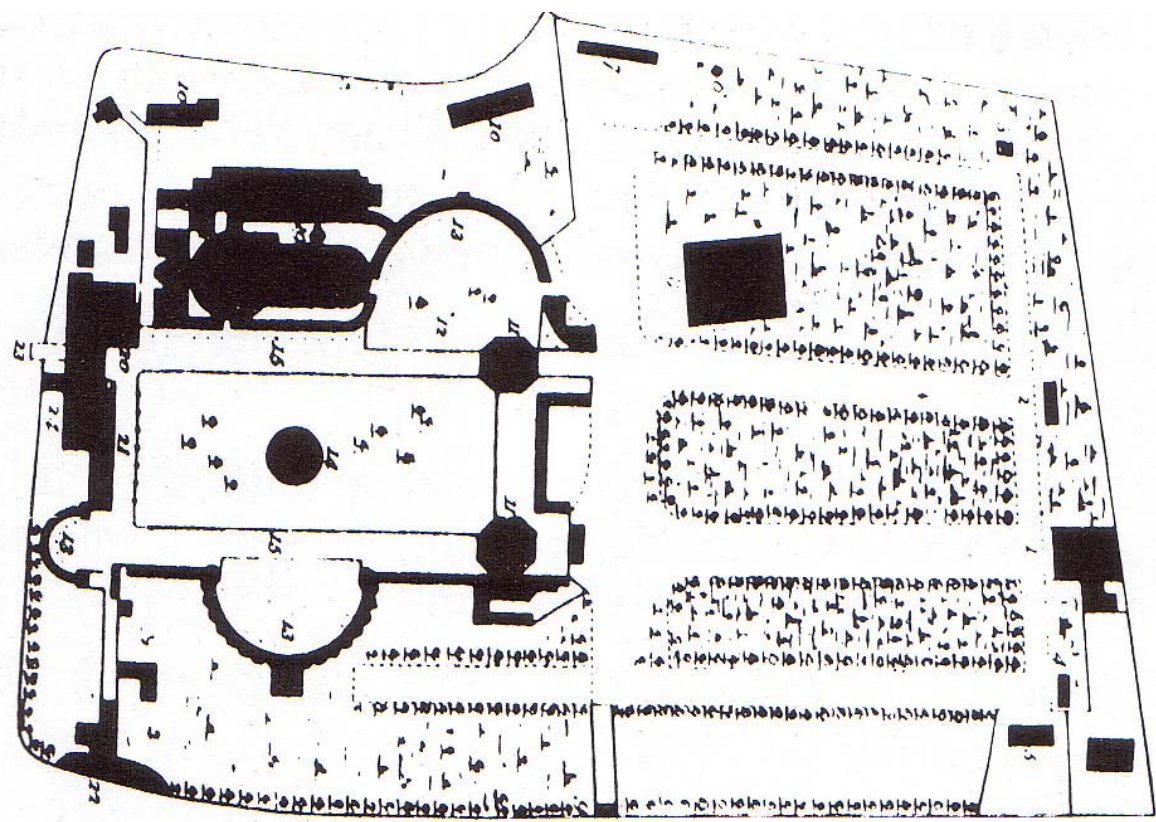

Fig. 6 Pianta di Vauxhall del 1826: sono leggibili i due diversi volti del giardino, che ripropone al suo interno il confine fra natura e architettura, in qualche modo fra città e paesaggio della campagna.

Proprio l'essere frontiera rende Vauxhall tanto rinomato. Gli intrattenimenti organizzati da Tiyer, "serviti" dall'architettura che li asseconda, sono anch'essi un modo di costruzione della frontiera:

“Intrattenere" significa all'origine proprio questo, tenere separato, in mezzo, quindi "creare uno spazio liminale nel quale le perfomances possano aver luogo". In questo senso il teatro, inteso sia come luogo fisico sia come luogo figurato, è uno spazio di confine, posto in mezzo a tutto il resto come spazio dialettico aperto contemporaneamente sull'interno e sull'esterno, sul reale come sull'immaginario."13

Il parco replica al suo interno il confine fra città e campagna e fra architettura e natura, confine costituito dal viale trasversale che, nella planimetria del 1826, conduce dalla Chinese Entrance alla Trasparency. L'asse inizia e termina nell'ambiente rurale esterno. E' come se il paesaggio della campagna incontrasse in corrispondenza del Vauxhall Garden un accidente, una variazione di stato operata dagli spettacoli allestiti e dall'intersezione con uno spazio teatrale costruito dall'architettura.

Isabelle Auricoste caratterizza $i$ giardini di piacere come luoghi extraterritoriali in senso figurato, ovvero luoghi al di fuori delle logiche che presiedono al territorio-città:

\footnotetext{
${ }^{12}$ Piero Zanini, Significati del confine. I limiti naturali, storici, mentali, Mondadori, Milano 1997, pagg. 10-11.

${ }^{13}$ Piero Zanini, Op.cit, Mondadori, Milano 1997, pagg. 146-147.
} 
"Nei jardins de plaisir, giardini privati aperti al pubblico, è ancora una volta un'area alberata a dare corpo a una nuova forma di luogo di piacere, ragioni di lucro e frammenti di natura in città coniugandosi, anche in queste operazioni, in un invito al viaggio immaginario. Grazie all'illimitata licenza di cui gode il proprietario lottizzatore, cie accede a questi luoghi può praticarvi una libertà di costumi e di opinione veramente eccezionale. (...) la formula originale di questi luoghi di piacere, prodotto dello spirito d'iniziativa degli speculatori, ha imposto luoghi aperti in cui il pubblico può sottrarsi ai vincoli collettivi dello spazio civico."14

La specificazione del paesaggio avviene in relazione alla concezione del giardino come luogo di intrattenimento. Il visitatore deve prendere parte attiva e, affinché questo sia garantito, deve essere sottoposto a suadenti sollecitazioni. E' chiara la discendenza dei pleasure gardens dal giardino manierista italiano, come evidenziato da John Dixon Hunt:

"For the Italian garden that most influenced France and England was one [the exedra] where the visitor was no longer a passive spectator (...). The expectation of a fine garden, whatever the formal means by which the effects were achieved, was that it work upon its visitors, involving him often insidiously as a participant in its dramas, which were presented to him as he explored its spaces by a variety of statue, inscriptions and (above all) hydraulically controlled automata.",15

John Dixon Hunt propone questa considerazione presentando il ruolo dell'elemento architettonico dell'exedra all'interno del giardino (architettura abilmente utilizzata al Vauxhall Garden di Londra) che mediante il suo "ambiguous status" è in grado di conferire un carattere teatrale:

“(...) exedra seats were both vantage points to watch other events and stages where visitors themselves were made to feel they constituted the garden dramas". ${ }^{16}$

Sollecitazione massima sul visitatore è interpellarlo continuamente sul fatto che le cose che egli vede siano vere o immaginarie. Le architetture, il sistema dei percorsi, le statue, le fontane sono realizzate in maniera tale che resti in bilico il rapporto fra paesaggio reale e scenografia. Meglio ancora: il paesaggio è costituito contemporaneamente da spazi físici e illusori, da oggetti propri e simulati (la componente esotica all'interno dei pleasure gardens è prediletta perchè contiene in sé queste dualità. Essa e' rappresentata da vegetazione $\mathrm{e}$ architetture che realmente sono vegetazione e architetture, ma che è assolutamente inverosimile si possano trovare in quel contesto).

"Confusion of art and reality, a staple feature alike of garden and theatre, were further pursued at Vauxhall in tableaux or cinematic illusions, like the famous cascade which Tyers ${ }^{17}$ created to rival Garrick's at Drury Lane. It displayed a water mill, miller's house, and a foaming waterfall, made of tin. Its attraction (...) was the strong illusion of reality (...) contrived out of the palpably unreal: the "switching-on" of this mechanism was actually announced by warning bell to give people time to convene from all the parts of the garden."18

Monique Mosser parla di "architetture paradossali" a proposito delle fabriques ${ }^{19}$. Introduce il discorso riportando un brano dalla Théorie des jardins (1776) del francese Jean-Marie Morel. Il brano esprime in maniera chiara come l'architettura d'effetto sia collocata e dimensionata in funzione del paesaggio esistente:

\footnotetext{
${ }^{14}$ Auricoste IsABelle, I parchi ricreativi in Europa: il divertimento e l'altrove, in MONIQUE MOSSER, GEORGES Teyssot, L'Architettura dei giardini d'Occidente- Dal Rinascimento al Novecento, Electa, Milano 1990, pag. 482.

15 John Hunt Dixon, The theatres, gardens, and Gardens Theatres, in John Dixon Hunt, Gardens and the Picturesque, mitt Press, Cambridge (Mass.) 1992, pag. 54.

${ }^{16}$ John Hunt Dixon, Op.cit. , in John Dixon Hunt, Gardens and the Picturesque. Studies in the Hostory of Landscape Architecture, mit Press, Cambridge (Mass.) 1992, pagg. 53-54.

17 I Tyers sono proprietari di Vauxhall dal 1730 al 1792. Jonathan Tyers è proprietario negli anni trenta del XVIII sec. ed è colui che conferisce al giardino l'aspetto che noi oggi conosciamo.

18 John Hunt Dixon, Op.cit. , in John Dixon Hunt, Gardens and the Picturesque Studies in the History of Landscape Architecture, mit Press, Cambridge (Mass.) 1992, pag. 56.

${ }^{19}$ Mosser MONIQue, Le architetture paradossali ovvero piccolo trattato sulle "fabriques" in MONIQUE Mosser, GeORges Teyssot, L'Architettura dei giardini d'Occidente- Dal Rinascimento al Novecento, Electa, Milano1990, pagg. 259-276.
} 
“E' soprattutto questo rapporto fra carattere [dell'edificio] e sito che io chiamo proporzione nell'arte dei giardini. Quando avrò illustrato, quando avrò parlato dell'ubicazione che conviene ai vari tipi di edificio, del loro carattere, forma e volume, come pure dello stile, del colore che li armonizzerà con il paesaggio in cui si trovano, avrò esaurito il mio compito. (...) Le costruzioni considerate da questo punto di vista sono quelle che in pittura vengono chiamate fabriques, espressione di cui mi servirò per designare tutti gli edifici di effetto e tutte le costruzioni che l'opera dell'uomo aggiunge alla natura per l'abbellimento dei giardini (...).,20

A seguito la Mosser ci dice del valore simbolico delle fabriques come elementi per cui "il giardino diventa enciclopedia: percorrendolo si sfogliano diversi capitoli del libro del mondo"21. Specificazione del paesaggio quindi come veicolo per il divertimento che si accompagna, nella tematizzione di minuscoli paesaggi d'altri luoghi e d'altri tempi ${ }^{22}$, alla classificazione e alla ricomposizione in nuovi stili.

La Mosser cita il principe di Ligne "uno degli spiriti illuminati più rappresentativi della fine del XVIII secolo" il quale "considera l'arte delle fabrique come una sorta di "macchina da guerra anticlassica",'23 e richiama l'attenzione sul valore dell'originalità rispetto all'atteggiamento pedissequo dei canoni classici, che porta, sempre secondo il principe, ad esiti più noiosi. L'ecclettismo e l'originalità vengono contrapposti al classicismo per desiderio di novità. (Analogamente il simbolismo dell'architettura postmoderna - a cui ricorrono gli imprenditori dei parchi tematici contemporanei, soprattutto la Walt Disney Company - denuncia l'esigenza di un'architettura maggiormente comunicativa e popolare, in opposizione all'estetica del modernismo).

Se il giardino manierista era concepito come una collezione, quello delle fabriques ha il valore di enciclopedia, e se l'Heikamp definisce le grotte manieriste "architettura della metamorfosi", la Mosser definisce le fabriques "architettura sperimentale", "a metà fra il modello e la realtà" ${ }^{24}$. I giardini manieristi come i pleasure gardens, le folies, i jardins spectacles, sono sintomi di crisi epocali. Mostrano nella loro stranezza - deformità, esotismo, sproporzione di scala - il desiderio di esplorare nuove possibilità formali e altri accessi alla conoscenza. ${ }^{25}$

“ Il giardino dei divertimenti, così come appare in Europa a partire dal Settecento, conserva ancora la vocazione e la capacità di far oltrepassare a coloro che vi passeggiano la soglia di un altro mondo e calpestare il suolo di uno spazio consacrato alla meraviglia." 26

20 J.-M. Morel, Théorie des jardins, Paris 1776, citato in Mosser MoniQue, Op. cit., in MoniQue Mosser, Georges Teyssot, L'Architettura dei giardini d'Occidente- Dal Rinascimento al Novecento, Electa, Milano1990, pag. 259.

${ }^{21}$ Mosser MONIQUe, Ibidem.

${ }^{22}$ Sempre in riferimento alle fabriques: "Che si tratti di un antico tholos, di un ponte cinese o di un torrione gotico, essi vengono percepiti come oggetti emblematici, evocatori di una contrada lontana o di un'epoca passata.", Mosser Monique, Op.cit., in Monique Mosser, Georges Teyssot, L'Architettura dei giardini d'Occidente- Dal Rinascimento al Novecento, Electa, Milano1990, pagg. 259.

${ }^{23}$ Mosser Monique, Op.cit., in Monique Mosser, Georges Teyssot, L'Architettura dei giardini d'OccidenteDal Rinascimento al Novecento, Electa, Milano1990, pagg. 261.

${ }^{24}$ Mosser Monique, Op.cit., in Monique Mosser, GeOrges Teyssot, L'Architettura dei giardini d'OccidenteDal Rinascimento al Novecento, Electa, Milano1990, pagg. 261.

${ }^{25}$ Al proposito si confronti ancora una volta Monique Mosser: "Labirinto erotico, percorso iniziatici: nello spirito degli appassionati dei giardini, come in quello degli artisti, l'unica regola delle fabriques sta nella folle libertà del'immaginazione. Tradizionalmente si è insistito soprattutto sul lato attraente di queste architetture del divertimento, di queste "folies". (...) Solo più raramente se ne è sottolineata l'inquietante stranezza (...). Ora questa mancanza di misura è sintomatica degli immensi interrogativi che assillano gli uomini della fine del XVIII secolo." In Mosser Monique, Op.cit., in Monique Mosser, Georges Teyssot, L'Architettura dei giardini d'Occidente- Dal Rinascimento al Novecento, Electa, Milano1990, pagg. 259-276.

${ }^{26}$ IsABelle Auricoste, Parchi o utopie mortali?, “ Ottagono” n. 99, Intrattenimento, 1991, pag. 16. 


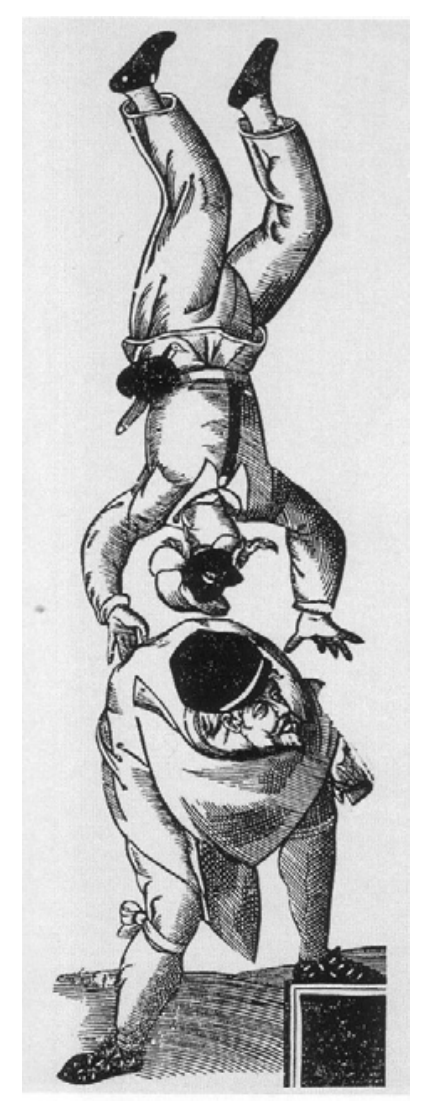

Fig. 7 Francatrippa e Capitan Coccodrillo in un'azione acrobatica, incisione, secolo XVII, particolare.

\section{LO SPAZIO CREATIVO}

Nei pleasure gardens si rintracciano intersezioni con le arti figurative e con il teatro propri delle epoche "della crisi", dell'incrinazione o instabilità culturale, in cui svago e urgenza di conoscenza si incontrano di frequente nei medesimi spazi, per cui il momento della ricreazione diviene anche occasione di creazione.

Questo costituisce una matrice - per il parco a tema - di segno contrario rispetto al modello Disneyland (Anaheim, California, U.S.A., 1955) che realizza il parco come portato dell'invenzione artistica, offerta quale ready-made alla fruizione del pubblico. Quest'ultimo viene avviluppato dal prodotto dell'arte di Walt Disney, creazione di origine cinematografica, con una richiesta di fiducia sulla propria qualità. La giostra di tazzine del "Paese delle meraviglie" a Fantasyland gira dopo che le immagini del cartone animato di Disney sono state associate alla fiaba diventandone identificative: l'operazione di fantasia è già conclusa, avvenuta a monte nell'ideazione dell'artista. Salire sulla giostra richiede semplicemente empatia con l'immagine, cui consegue l'assimilazione delle regole che sottendono il gioco dispiegato all'interno del parco. Fuori del "Disney way of thinking" è impensabile riuscire ad operare un'immediata sostituzione iconografica personale ${ }^{27}$.

\footnotetext{
${ }^{27}$ Sottolinea Isabelle Auricoste a proposito dei parchi tematici successivi agli anni '50 - quindi al Disneyland californiano (1955): "Il funzionamento è basato su una partecipazione passiva (...). L'adesione spontanea all'immaginario del parco che questi temi suscitano è rafforazata dalla presenza di un personale numeroso, altamente specializzato e presente ovunque". IsABelle Auricoste, Parchi o utopie mortali?, "Ottagono" n. 99, Intrattenimento, 1991, pag. 18.
} 
Le descrizioni di Vauxhall a Londra ci prospettano una situazione assolutamente diversa ${ }^{28}$ :

“ Templi , saloni, panorami e fontane brillavano scintillanti davanti ai nostri occhi; la bellezza delle cantanti ed il contegno elegante dei gentiluomini catturavano i nostri cuori, mentre alcune centinaia di lampade abbagliavano $\mathrm{i}$ nostri sensi; una tazza o due di punch disorientavano i nostri cervelli. Ed eravamo felici."

"Quando consideravo il profumo dei sentieri e delle pergole, misto al coro degli uccelli che cantavano sugli alberi, e l'umanità che libera vagava sotto le loro ombre, non potevo che guardare quel sito come una sorta di paradiso maomettano. Sir Roger mi disse che il luogo gli ricordava un boschetto situato vicino alla sua casa di campagna (...). E qui emise un sospiro profondo e stava lasciandosi andare a uno stato meditativo, quando una ragazza in maschera, che gli giunse da dietro, lo toccò gentilmente sulla spalla, per chiedergli se voleva bere una bottiglia di idromele con lei." 29

Il giardino costituisce il luogo della libertà di trasgredire da cui scaturisce il divertimento, recuperando la forza del significato etimologico della parola:

“ L'altrove è sempre un paradiso. Lasciare il paesaggio quotidiano è di per sé un'esperienza concreta di spaesamento. Il desiderio di sperimentare la dualità dell'esistenza, che è una delle costanti della storia dell'umanità, ci apre un mondo diverso dal nostro, sede di ogni possibile. In questo senso, il nesso fra giardini e paradiso non riproduce soltanto un luogo comune universale (...) ma riconosce altresì nei giardini il luogo privilegiato di un fecondo accostamento tra l'universo del desiderio e quello dell'esperienza.

(...)

Il qui e l'altrove sono territori di diversa giurisdizione, rappresentano due modi di essere in perenne tensione fra loro. Stranamente, i termini stessi che designano il piacere derivante da questa tensione non esprimono l'appagamento di un desiderio ma indicano sempre un'azione. Divertire (divertere) non significa forse distogliere, allontanare dall'uso comune, così come d'altronde distrarre (distrahere) rinvia all'azione di tirare in senso inverso? (...) sono al tempo stesso le metafore in termini di esperienza concreta che connettono l'altrove con la nozione di territorio situato. Il senso di estraneità risiede nella conoscenza vissuta delle diversità e dei confini tra due territori." 30

L'altrove è uno spazio che consente quel grado di libertà necessario all'immaginazione, che è condizione per l'intuizione, al contatto con qualcosa che divarica rispetto alla situazione reale. E' una fessura fra le tende sul sipario: l'invenzione in cui competono gli imprenditori che gestiscono i giardini di piacere porta alla costruzione di apparati rappresentativi a loro volta catalizzatori di altre immagini nello spettatore, per l'occasione di viaggio che viene loro offerta, venduta. L'estraniazione interrompe il rapporto con il già dato e immette nel desiderio della novità che avrà la sua propria rappresentazione, frutto di un processo creativo in tensione verso altre forme di conoscenza.

Gli elementi che caratterizzano i pleasure gardens - soprattutto il Vauxhall di Londra - e che li connotano come Theatre gardens secondo la definizione di Jhon Dixon Hunt, costituiscono il motivo del loro successo: i pleasure gardens si impongono a un esteso e differenziato pubblico come un altrove, democratizzando l'arte dei giardini. ${ }^{31}$

"It is this ineluctable theatricality of Vauxhall and Ranelagh that was so fascinating at the time and is interesting now. That these gardens maintained close connections with the legitimate theatre and benefited from an exchange

${ }^{28}$ In Charls Dickens, Sketches by Boz, Oxford University Press, Londra, 1957, pagg. 126-129, cit. in FranCO PANZINI, Per i piaceri del popolo. L'evoluzione del giardino pubblico in Europa dalle origini al XX secolo, Zanichelli, Bologna, 1997, pag. 105.

${ }^{29}$ In D.F. Bond (a cura di), The Spectator, Clarendon Press, Oxford, 1965, vol. III, pagg. 438-439, cit. in Franco PANZINI, Per i piaceri del popolo. L'evoluzione del giardino pubblico in Europa dalle origini al XX secolo, Zanichelli, Bologna 1997, pag. 102.

${ }^{30}$ ISABELlE AURICOSTE, I parchi ricreativi in Europa: il divertimento e l'altrove, in MONIQUE MOSSER, GEORGES TEYSSOT, L'Architettura dei giardini d'Occidente- Dal Rinascimento al Novecento, Electa, Milano 1990, pag. 479.

${ }^{31}$ L'espressione è assunta sempre da J. Dixon Hunt, il quale riferendosi a Jonhatan Tyers, proprietario e manager del Vauxhall di Londra, afferma: "He democratised garden art, at a time when it was still largely a rich family's pastime, and he maintained its links with theatre at a time when " capability" Brown's designs were eliminating not only "readability" from a landscape but various theatrical forms and opportunities as well", in JOHN HUNT Dixon, Op.cit. , in John DixOn Hunt, Gardens and the Picturesque. Studies in the History of Landscape Architecture, mit Press, Cambridge (Mass.) 1992, pag. 71. 
of personnel is not, however, any satisfactory explanation of the phenomenon. There was in increasingly larger sections of society (...) a taste for theatricality which discovered at places like Vauxhall and Ranelagh increased scope for both fantasy and role playing. These garden Theatres readily accommodated the fete campetre and the masquerade, both of which were naturalized in England in the eighteenth century. The essence of masquerade, of course, was the chance to disguise one's everyday self and play with and in new, temporary rules". 32

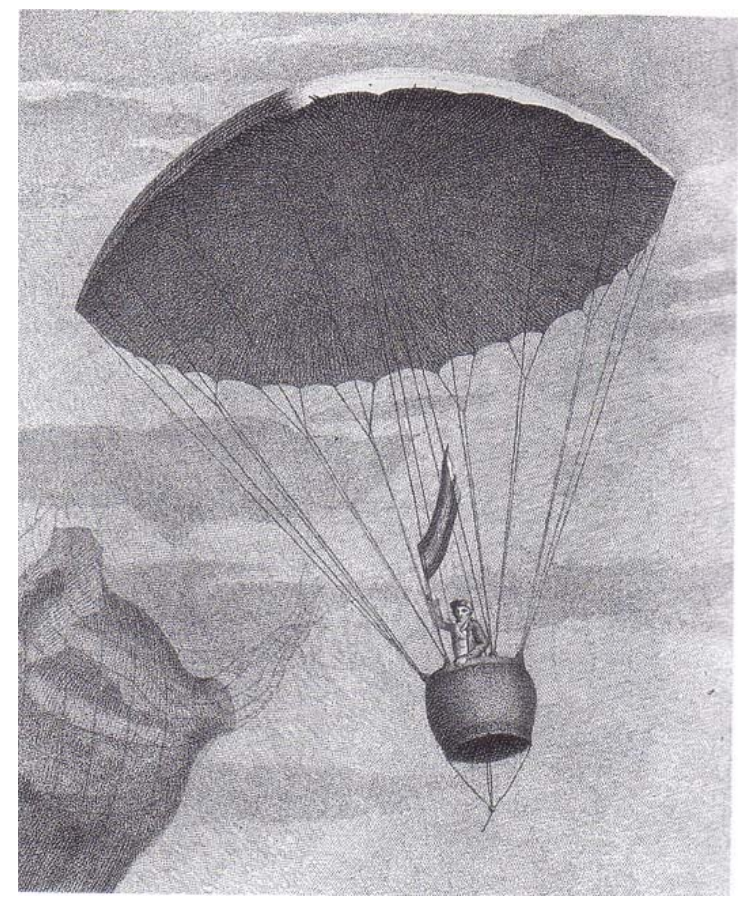

Fig. 8 L'arenauta Garnerin a Monceau. L'immagine può essere vista come un'icona del desiderio di libertà e di distacco dalle situazioni ordinarie che sono l'anima dei parchi del divertimento del Settecento e dell'Ottocento.

\section{RIFERIMENTI BIBLIOGRAFICI}

AURICOSTE ISABELLE, I parchi ricreativi in Europa: il divertimento e l'altrove, in MONIQUE Mosser, GeORges TEYSSOT, L'Architettura dei giardini d'Occidente- Dal Rinascimento al Novecento, Electa, Milano 1990.

AURICOSTE ISABELle, Parchi o utopie mortali?, "Ottagono" n. 99, Intrattenimento, 1991, pagg. 16-30.

HUNT DiXON JoHn, The Picturesque Garden in Europe, Thames and Hudson, London 2002. Hunt Dixon John, The theatres, gardens, and Gardens Theatres, in JOHN DIXON HunT, Gardens and the Picturesque, The MIT Press, Cambridge (Mass.) 1992, pagg. 49-73.

HUNT DiXON JOHN, Vauxhall and London Garden Theatres, Chadwick-Healey in association with the Consortium for Drama and Media in Higher Educations, Cambridge 1985.

Hunt Dixon John, Willis Peter (edited by), The Genius of the Place. The English Landscape Garden 1620-1820, The MIT Press, Cambridge (Mass.) 1997.

LANGLOIS GILlES-ANTOINE, Folies Tivolis et attractions. Les premiers parcs de loisirs parisiens, Délégation à l'action artistique de la ville de Paris, Paris 1991.

MOSSER MONIQUE, Le architetture paradossali ovvero piccolo trattato sulle "fabriques" in Monique Mosser, GeOrges TeYssot, L'Architettura dei giardini d'Occidente- Dal Rinascimento al Novecento, Electa, Milano1990, pagg. 259-276.

\footnotetext{
${ }^{32}$ John Hunt Dixon, Op.cit. , in John Dixon Hunt, Gardens and the Picturesque. Studies in the Hostory of Landscape Architecture, mit Press, Cambridge (Mass.) 1992, pag. 71.
} 
PANZINI FRANCO, Per i piaceri del popolo. L'evoluzione del giardino pubblico in Europa dalle origini al XX secolo, Zanichelli, Bologna 1997.

ROLAND Michel MARIANNE, Scenografia e prospettiva nei giardini francesi del Settecento, in Monique Mosser, GeORges Teyssot, L'Architettura dei giardini d'Occidente- Dal Rinascimento al Novecento, Electa, Milano1990, pagg. 239-248.

TESSARi RoBerto, Il mercato delle Maschere, in Roberto Alonge, Guido Davico BONINO ( diretta da ), Storia del teatro moderno e contemporaneo, volume primo, La nascita del teatro moderno. Cinquecento- Seicento, Einaudi, Torino, 2000, pag. 119.

VERIN HELEN, La tecnologia nel parco: ingegneri e giardinieri nella Francia del Seicento, in Monique Mosser, Georges Teyssot, L'Architettura dei giardini d'Occidente- Dal Rinascimento al Novecento, Electa, Milano1990, pagg. 131-142.

Wylson ANTONY \& PATRICIA, Theme Parks, Leisure Centres, Zoos and Aquaria, Longman, 1994, pagg. 3-9.

Siti web:

Le Jardin d'Acclimatation, in www.jardindacclimatattion.fr/pages/histoireJardin

The story of Bakken, in www.bakken.dk/engelsk/htm/sider/history

www. tivoligardens.com

www.cichw.net/pmvaux $2 . h t m l$

www.vauxhallandkennington.org.uk/sgdetail.shtml

\section{RIFERIMENTI ICONOGRAFICI}

Fig. 1: AURICOSTE ISABELLE, I parchi ricreativi in Europa: il divertimento e l'altrove, in Monique Mosser, GeORges Teyssot, L'Architettura dei giardini d'Occidente- Dal Rinascimento al Novecento, Electa, Milano 1990, pag. 483.

Fig. 2: LANGLOIS GILleS-ANTOINE, Folies Tivolis et attractions. Les premiers parcs de loisirs parisiens, Délégation à l'action artistique de la Ville de Paris, Paris 1991, pag. 191 tavola 198.

Fig. 3: Hunt DiXon JoHn, The Picturesque Garden in Europe, Thames and Hudson, London 2002, pag. 124.

Fig. 4: LANGlois Gilles-AntoINE, Folies Tivolis et attractions. Les premiers parcs de loisirs parisiens, Délégation à l'action artistique de la Ville de Paris, Paris 1991, pag. 41 tavola 241.

Fig. 5: PANZINI FRANCO, Per i piaceri del popolo. L'evoluzione del giardino pubblico in Europa dalle origini al XX secolo, Zanichelli, Bologna 1997, pag. 101.

Fig. 6 : PANZINI FRANCO, Per i piaceri del popolo. L'evoluzione del giardino pubblico in Europa dalle origini al XX secolo, Zanichelli, Bologna 1997, pag. 103.

Fig. 7: TESSARI ROBERTO, Il mercato delle Maschere, in ROBERTO AlONGE, GUIDO DAVICO BONINO ( diretta da ), Storia del teatro moderno e contemporaneo, volume primo, La nascita del teatro moderno. Cinquecento- Seicento, Einaudi, Torino, 2000, illustrazione 41.

Fig. 8: LANGloIS GILleS-AntoINE, Folies Tivolis et attractions. Les premiers parcs de loisirs parisiens, Délégation à l'action artistique de la Ville de Paris, Paris 1991, pag. 142 tavola 78 . 


\section{3 - LE ESPOSIZIONI UNIVERSALI}

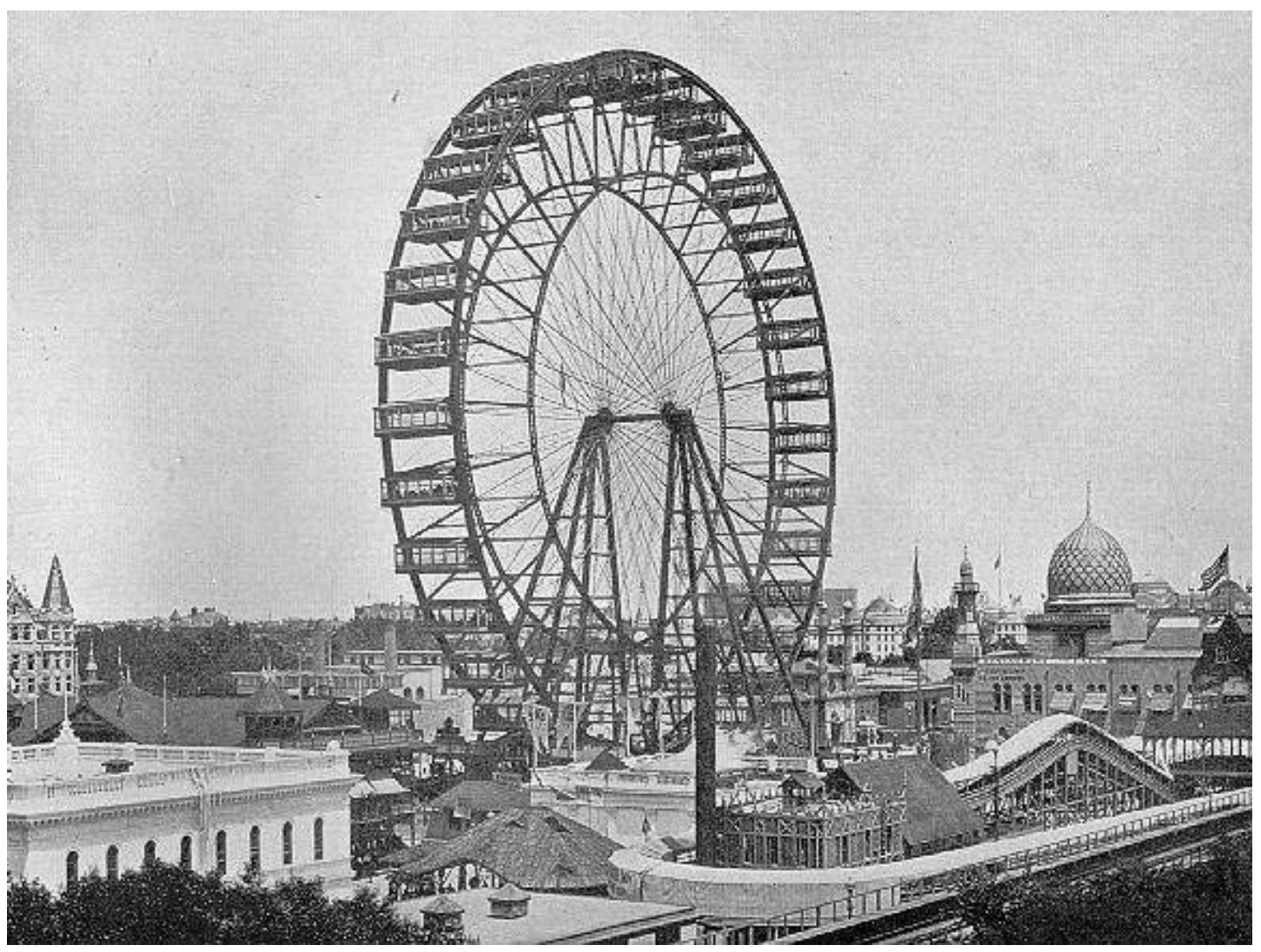

Fig. 1 Veduta del Midway Plaisance, area dei divertimenti della Chicago Columbian Exposition (1893). In primo piano è la Ferris Wheel, ruota panoramica progettata dall'ingegnere Ferris, esposta per la prima volta in occasione dell'esposizione.

\section{ESPERIMENTI DI URBANISTICA TRA UTOPIA ED ECUMENISMO}

Le Esposizioni Universali, dal XIX sec. in avanti, rendono possibile il confronto di una molteplicità di culture e dei loro "prodotti", in un unico luogo. Non solo. Diventano occasioni per la sperimentazione di modelli urbanistici, costituendo miniature di città, innanzitutto perché hanno l'esigenza di trovare una soluzione, a scala ridotta e con ingenti finanziamenti a disposizione, ai medesimi problemi presentati dalle realtà metropolitane: difficoltà logistiche legate all'affluenza di massa, gestione dei trasporti, attribuzione di ruolo allo spazio pubblico, alla ricreazione, al commercio, organizzazione (separazione e coesione) di molteplici funzioni. In generale in occasione delle esposizioni universali viene dispiegata una forza economica e creativa enorme, indirizzata alla definizione e all'esposizione di ciò che è qualità: dei prodotti dell'industria, dei giardini, delle architetture.

E' proprio il binomio qualità/parco, difficilmente realizzabile nelle situazioni urbane "reali" (come se la fiera costituisse un'apparizione!) che interessa agli imprenditori degli amusement park americani e che sembra essere soprattutto l'imprinting esercitato dalle esposizioni su Walt Disney, quando inizia a pensare alla realizzazione di un parco a tema sui suoi personaggi animati:

"But it might also be argued that in creating his theme-park empire, Walt Disney was a child of broader circumstances, building upon generic conditions and aspirations. Rather than the experience of any particular fair, 
it was the fair experience itself, with its amusements strips, disciplined crowds, and park-like settings, that supplied the necessary nurture. American expositions provided visions of higher life set amid heavenly landscapes."

La prima Esposizione Internazionale è la Great Exposition of Works of Industry of All Nations, del 1851, a Londra. Erik Mattie nel suo World's Fair esplicita il movente che inaugura la serie di eventi delle Esposizioni: la volontà di costituire una comunità internazionale fondata su una condivisa civiltà industriale, e di definire il ruolo delle singole nazioni all' interno di questa comunità:

"The first international world exhibition was born out of the hope that all the peoples of the worlds might live in harmony, and, more prosaically, out of the desire to tap new potential markets for British products. (...) The belief in technology and human ability was immense, and it was envisaged that the selection of goods and affordable products on display from all corners of the world would stimulate trade and industry."2

La crescente industrializzazione e la necessità di far fronte alle sue conseguenze costituiscono la caratteristica del XIX secolo. Essa induce all'elaborazione di modelli utopici e sperimentali che rappresentino soluzioni:

"Durante il XIX secolo, lo sforzo dell'industria di affrontare i suoi problemi assunse molte forme, dai modelli di fabbrica, di ferrovia, e dei relativi insediamenti, al progetto di comunità utopiche, intese come prototipi di un qualche futuro Stato illuminato". 3

L'exploit degli eventi fieristici avviene in concomitanza con lo svilupparsi delle teorie urbanistiche di Furier espresse in Le nouveau monde industrielle (1829) e dei due modelli europei di garden city proposti da Arturo Soria y Mata e Ebenezer Howard. Questi ultimi hanno alla base della determinazione della struttura della città le infrastrutture dei trasporti: il primo modello ha forma lineare, il secondo, come rappresentato nel saggio Tomorrow: $A$ Peaceful Path to Real Reform (1898), ha forma radiocentrica, definita e contenuta dimensionalmente dalla linea ferroviaria (Fig. 2).

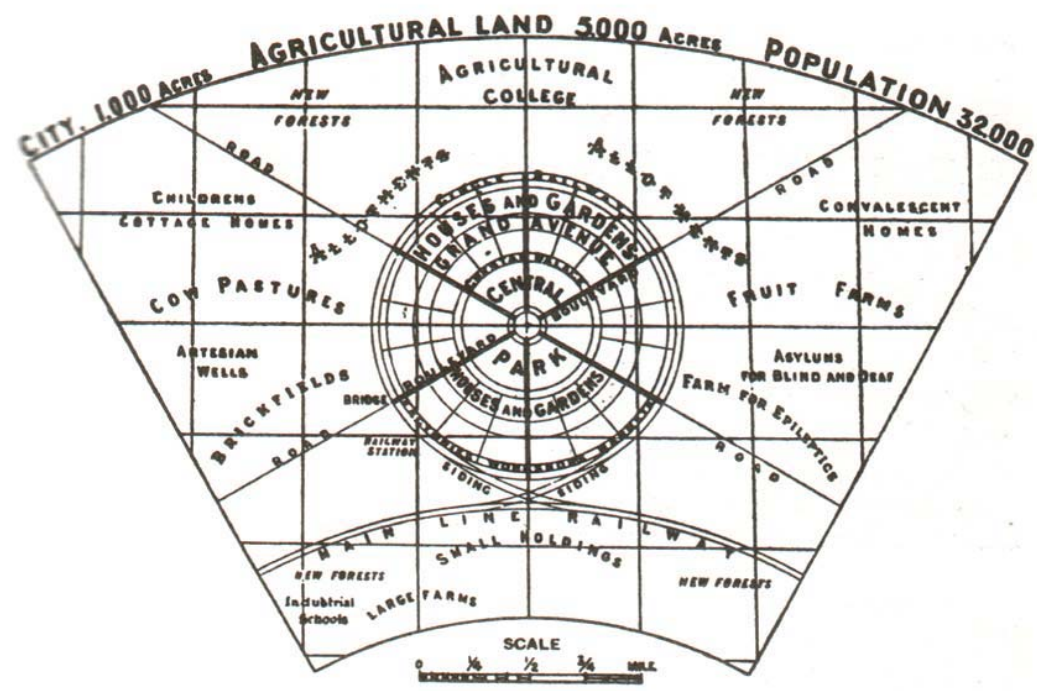

Fig. 2 E. Howard, Rurisville, schema di città-giardino da Tomorrow, 1898.

\footnotetext{
${ }^{1}$ Harris NeIL, Expository expositions. Preparing for the theme parks, in Maling KARAL Ann, Designing Disney's Theme Parks: The Architecture of Reassurance, Flammarion, Paris 1998, pag. 19.

${ }^{2}$ ERIK MatTIE, World's Fair, Princeton Architectural Press, New York City 1998, pag. 12.

${ }^{3}$ KenNeth Frampton, Storia dell'architettura moderna, Zanichelli, Bologna 1998, pag. 13.
} 
Quest'ultimo modello influenzerà enormemente i pianificatori delle successive fiere internazionali e ispirerà l'organizzazione planimetrica di Disneyland (California - 1955): l'idea della Garden City radiocentrica risulta affascinante perché promette ordine e controllo sulle parti, grazie ad un'esplicita zonizzazione e alla propria dimensione ridotta, presentando le istanze di efficienza e produttività, che si affiancano all'idea di abitare in un giardino (scrive Kenneth Frampton mettendo a confronto la proposta di Howard con quella di Soria y Mata: "Là dove la versione inglese era autosufficiente, limitata e provinciale, il modello spagnolo è regionale, indeterminato e continentale" ${ }^{\natural 4}$.

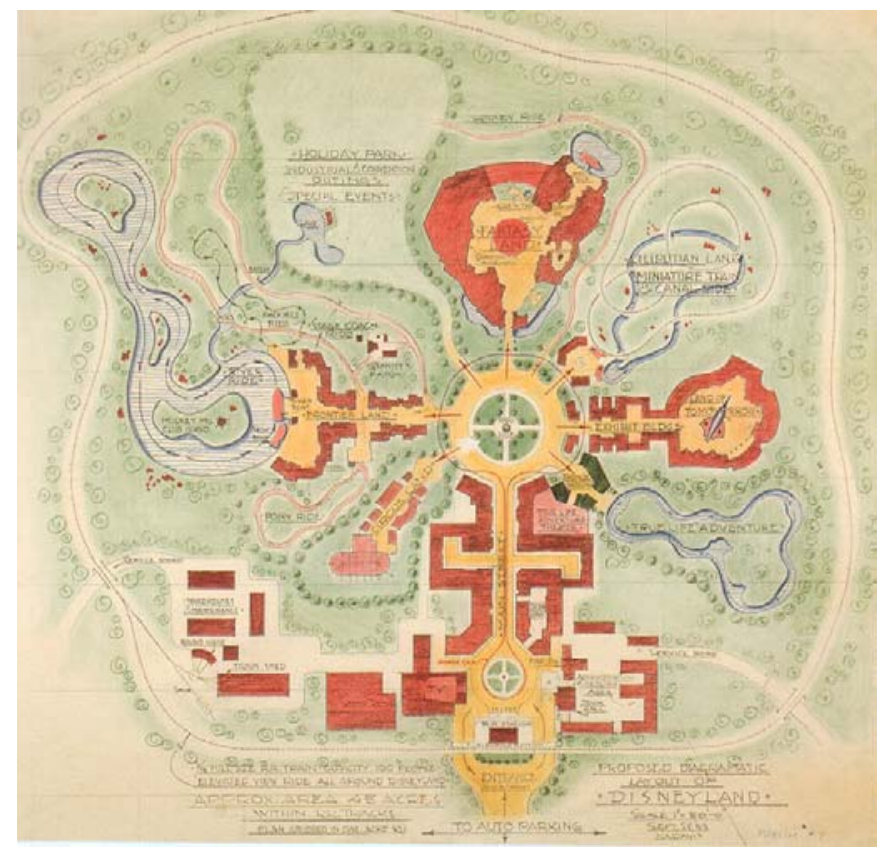

Fig. 3 Planimetria di Disneyland utilizzata da Walt Disney e Herbert Ryman come base di riferimento per la prima prospettiva a volo d'uccello di descrizione del parco in corso di progettazione. Si leggono gli elementi di somiglianza con l'impianto planimetrico della New York Wold's Fair (1939) e il modello della Garden City di E. Howard.

La New York World's Fair del 1939, in particolare, presenta un impianto che deriva dalla Garden City di Howard (si noti che entrambe le "città" hanno titoli/temi similari nel riferimento ad un ideale futuro, rispettivamente "Building the World of Tomorrow" e "Garden Cities of Tomorrow", nella versione del 1902). Il paesaggio è caratterizzato dalla chiarezza formale data da un polo centrale concepito come landmark e costituito dalle architetture emblema dell'esposizione- è il Theme Center con il Trylon e lo Perisphere, epitomi del tema "Building the World of Tomorrow" - e dalle arterie stradali disposte a raggiera, che suddividono il parco dell'esposizione in settori (Transportation, Comunications and Business Systems, Production and Distribution, Food, Community Interest, Government, Amusement Area). Anche i settori vengono tematizzati e questo costituisce una novità carica di suggestione.

"But an absolutely new feature of the 1939 Fair was called for by its almost unprecedented magnitude (...) This feature was the system of zoning by exhibit categories."

La tematizzazione è rafforzata dall'utilizzo di colori differenti nelle architetture lungo le diverse arterie. L'idea di connotare l'esposizione attraverso l'uso del colore viene già proposta in occasione della Chicago Columbian Exposition (1893), pensata con un bianco

\footnotetext{
${ }^{4}$ Kenneth Frampton, Op. cit., Zanichelli, Bologna 1998, pag. 20.

${ }^{5}$ Stanley Appelbaum, The New York World's Fair 1939/1940, Dover Publications, New York 1977, pagg. xiiixiv.
} 
imperante ad esaltazione del classicismo delle architetture, a New York il bianco è utilizzato solo nel Theme Center che è il fulcro, il tema assoluto. Gli altri colori classificano le declinazioni del tema e costituiscono un sistema di orientamento nella fruizione della varie aree (sono simbolo e rispondono ad una precisa funzione). Rivelano il livello ideale di esistenza di questa città/esposizione, temporanea e sperimentale, facendola assomigliare ad un diagramma concettuale.

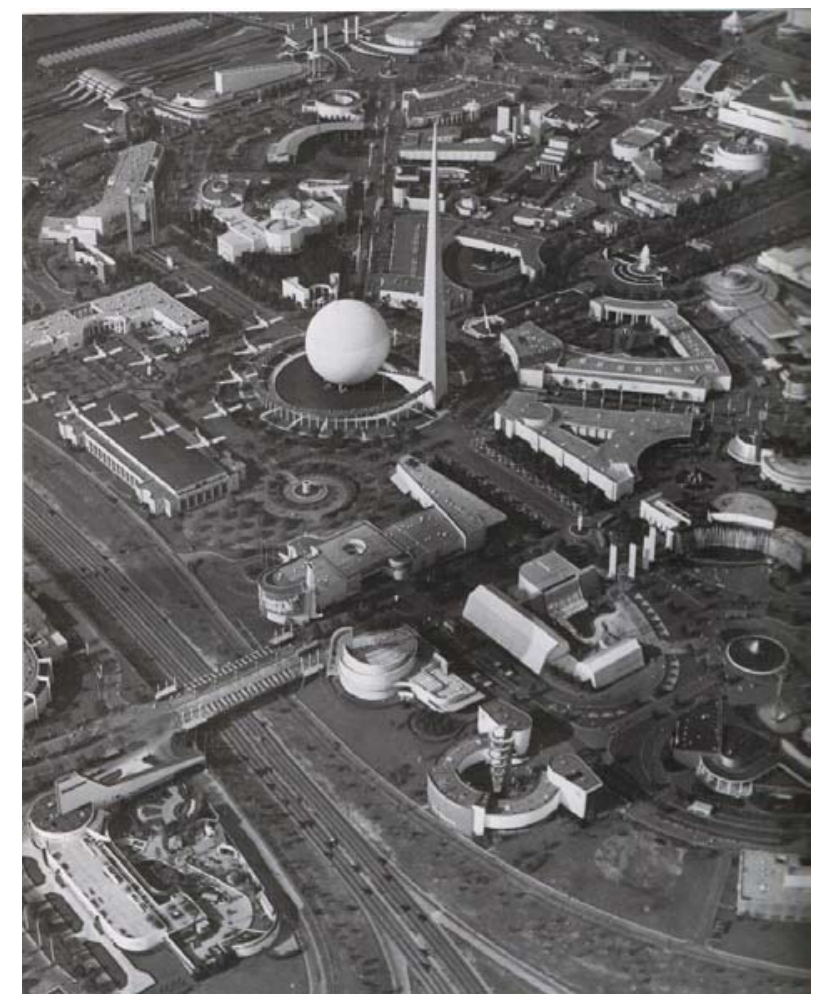

Fig. 4 Il Theme Center della New York World's Fair (1939) visto dall'alto: è visibile l'impianto radiocentrico dell'Esposizione, che riprende il modello della Garden City di Howard.

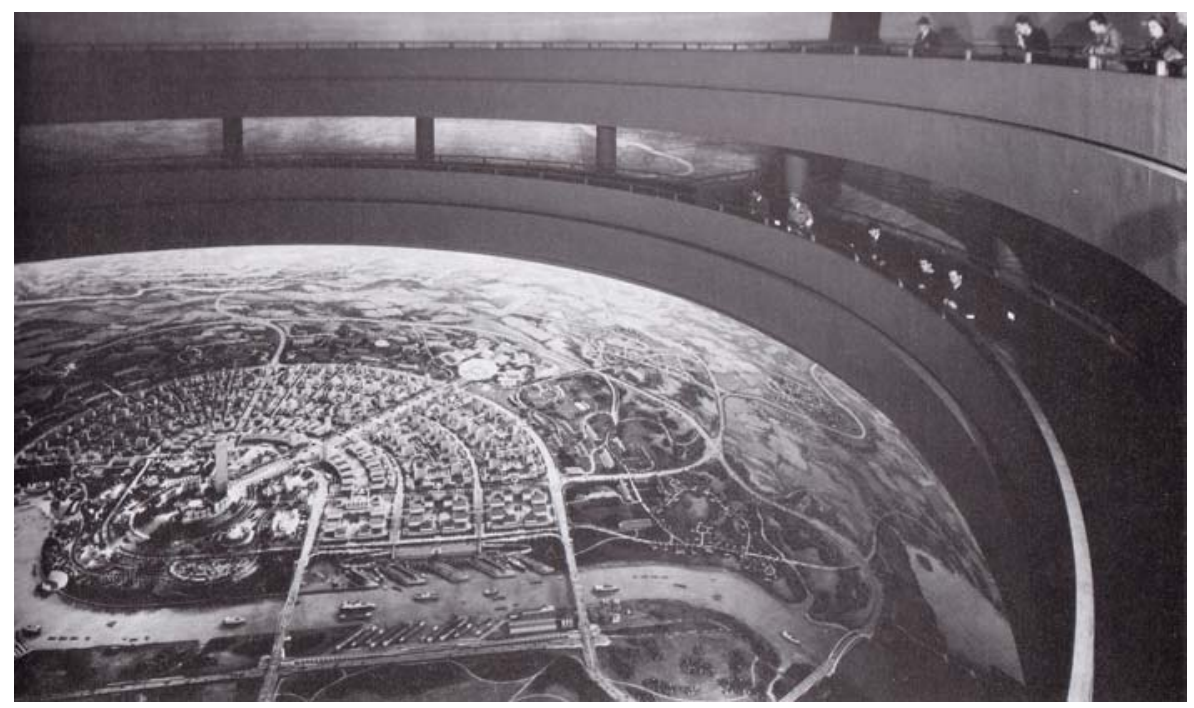

Fig. 5 Democracy, diorama al Theme Centre della New York World's Fair del 1939, il cui tema è "Tomorrow: A Peaceful Path to Real Reform". Il diorama è allestito all'interno dello Perisphere, fulcro dell'esposizione. La planimetria dell'esposizione concretizza il modello urbano esposto nel diorama. (Fotografia di Richard Wurts). 
La New York World's Fair del 1939 espone nel Theme Center un ampio diorama, un modello di complesso urbano ed extraurbano per il futuro che sembra essere collaudato nell'impianto planimetrico stesso dell'esposizione, operati gli adeguamenti del caso. Il cuore della fiera contiene il proprio DNA e lo espone didatticamente ai visitatori, esperienza che Stanley Appelbaun descrive a commento di un'immagine visionaria del fotografo Richard Wurts $^{6}$ (Fig. 5):

"Visitors rode part way up the Trylon on what was then the world's highest escalator, then entered the Perishere (interior design by Henry Dreyfuss), stepping onto one of two moving rings, from which they viewed the vast diorama of Democracy, a planned urban and exurban complex of the future. The accompanying musical score by William Grant Still was conducted by André Kostelanetz and the narration spoken by the popular newscaster $\mathrm{H}$. V. Kalternborn. At the end of the six-minute show, film projections presented several groups of happy farmers and workers. Afterwards, the visitors walked back to ground level via ramp called Helicline."

Nello spazio esterno dello Pherisphere The Astronomer, statua dello svedese Carl Milles, è un uomo che guarda verso l'alto - si immagina che osservi il cielo per trarne auspici per il futuro - arcaico nel suo gesto e sacrale. Anch'egli dichiara l'idealismo dell'esposizione, tendendo un filo, nell'iper-tecnologica e iper-scientifica atmosfera dell'evento, a ricreare un contatto fra destino umano e misteri della natura.

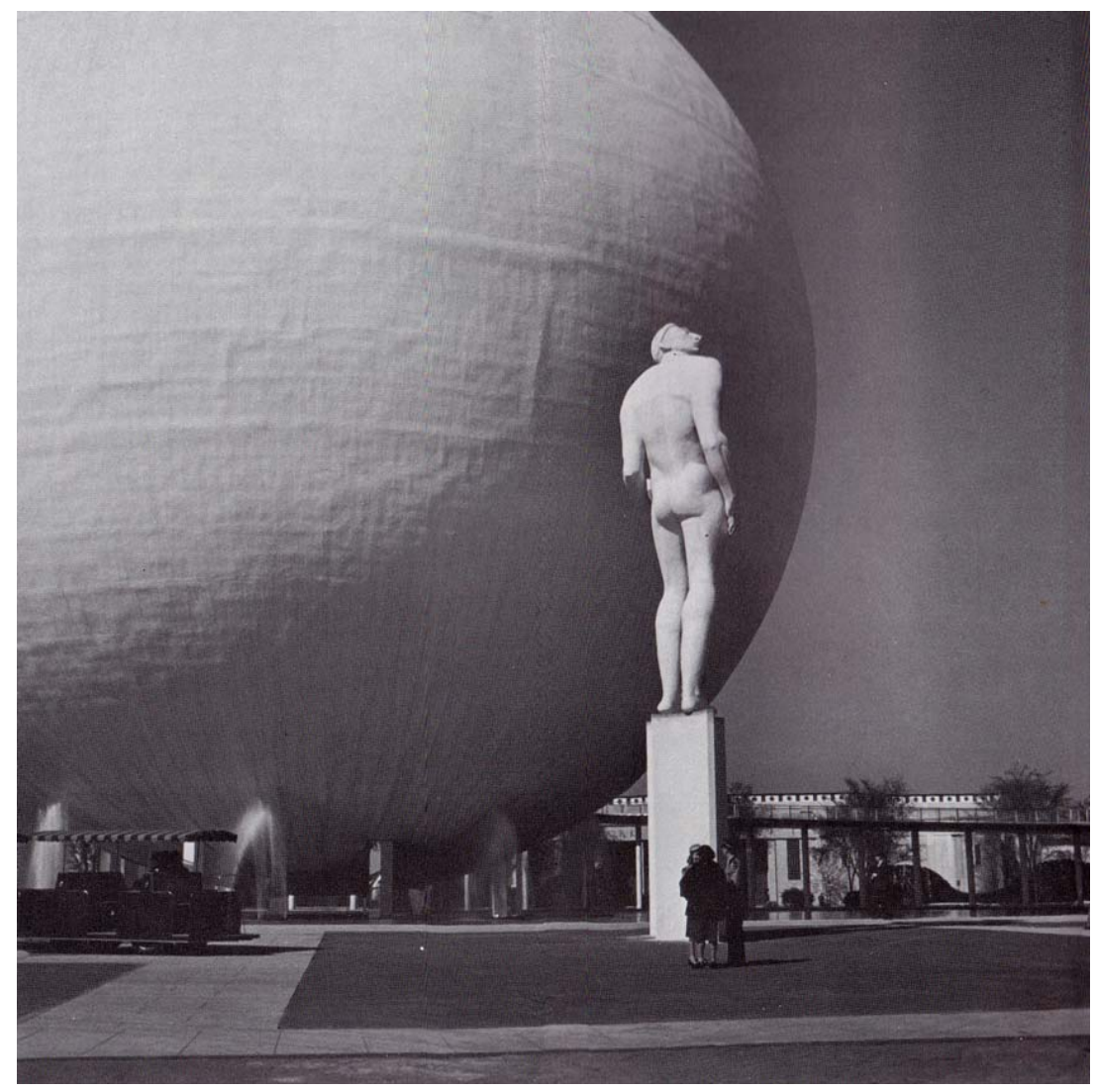

Fig. 6 The Astronomer di Carl Milles vicino allo Perisphere (Fotografia di Richard Wurts).

Anche le singole architetture delle esposizioni universali, per forma e contenuti assunti, dichiarano l'eccezionalità dell'evento fieristico. Si porta l'esempio del padiglione principale dell'Esposizione Universale di Parigi del $1867^{7}$ che ha sede a Champ de Mars : è di forma

\footnotetext{
${ }^{6}$ Stanley Appelbaum, The New York World's Fair 1939/1940, Dover Publications, New York 1977, pag. 3.

${ }^{7}$ Nelle esposizioni universali successive ospitate da Parigi - 1878, 1889, 1900, 1937 - la pianificazione degli spazi della fiera, in funzione della necessità di avere a disposizione spazi maggiormente ampi, è più fortemente legata ai luoghi della città e alla ridefinizione del loro ruolo: con l'Esposizione del 1878 realizzata a Champ de
} 
ovale e organizzato per sale concentriche (ognuna delle quali destinata all'esposizione di un tipo di prodotto), la geometria rappresenta gli emisferi australe e boreale, oggettivando l'universalità della manifestazione. Oltre alla forma, sono le dimensioni enormi della struttura (in parte dettate dalla volontà di allestire la fiera tutta su un unico livello per dare pari opportunità commerciali a tutti gli espositori) a sradicarla completamente dal tessuto urbano circostante, ripreso solamente dagli allineamenti degli assi dell'ellisse, che intercettano arterie cittadine. Intorno alla corte centrale Le Play, ideatore dell'impianto del padiglione, suggerisce la disposizione di una istallazione tematica La storia del Lavoro, lungo la Colisée du Travail, che dichiara il valore simbolico attribuito all'architettura:

"Thus the Paris fair had an idealistic agenda in addition to its more prosaic commercial goals" 8

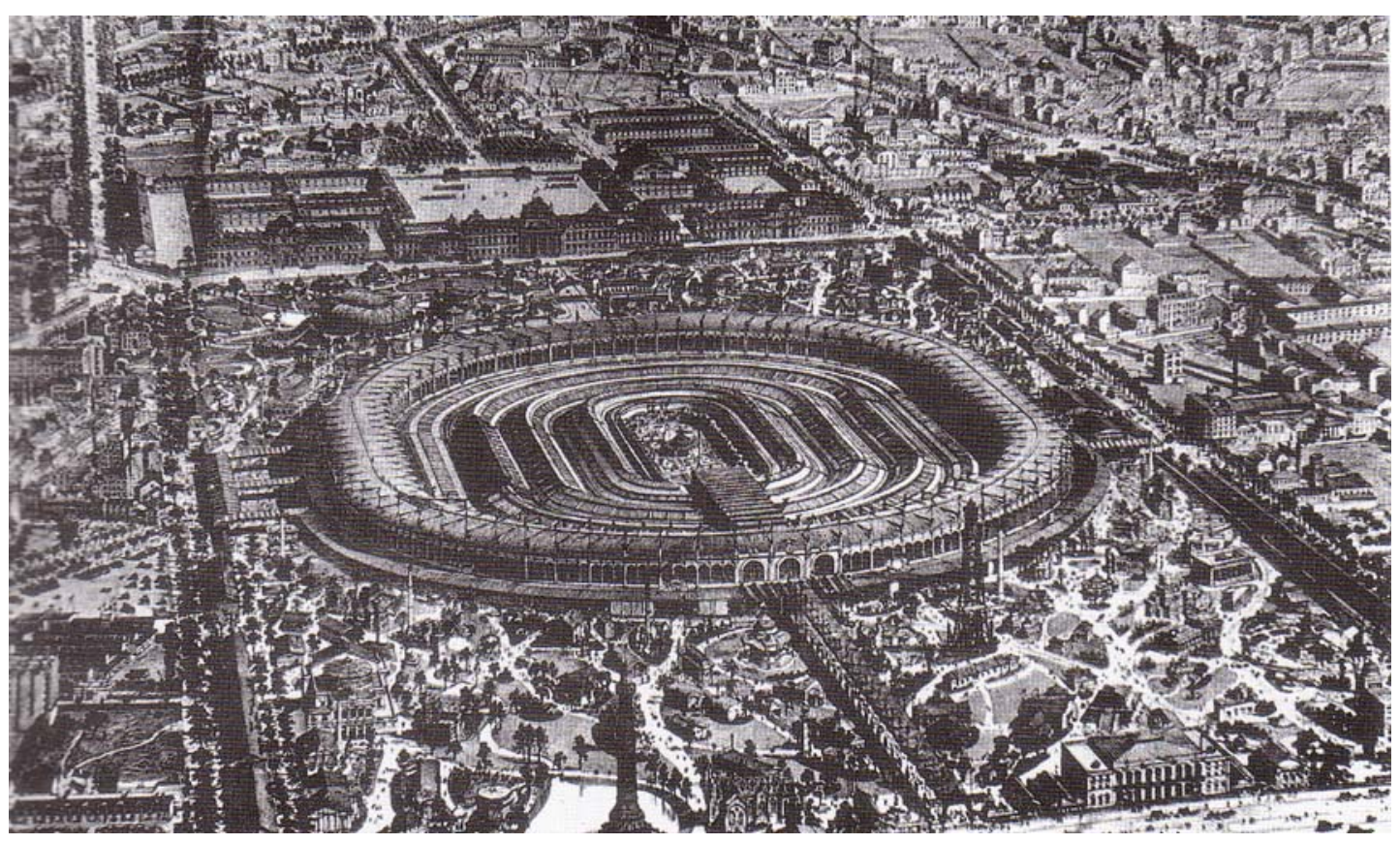

Fig. 7 Exposition Universelle - Parigi (1867) - vista a volo d'uccello: le dimensioni enormi del padiglione principale e il valore simbolico della sua pianta esprimono l'eccezionalità dell'evento. L'architettura non intende rapportarsi con il contesto, aspetto ordinario della città, ma fornire un'immagine ideale di Parigi al mondo.

Il valore simbolico non resta confinato nel luogo e nel momento dell'esposizione, ma ha potere sul contesto, la Parigi del XIX secolo - ed è in un modo sottile una ricezione di imput immateriali, ideali, dal contesto, dalla realtà urbana parigina in trasformazione. Questo aspetto è reso perfettamente da Philippe Hamon che riporta nel suo Esposizioni un testo di Victor Fournel tratto da Paris nouveau et Paris futur, del 1865, quale descrizione letteraria di una città futura, del 1965, che anticipa l'assetto urbanistico e architettonico dell'esposizione di Parigi del 1867, la città "vicina, per il suo aspetto panottico, a certi falansteri fourieristi"'. L'intuizione di Fournel dei mutamenti futuri, ma già in corso, ha una prima concretizzazione nell'evento dell'esposizione, che si impone a sua volta quale catalizzatore di reazioni. La descrizione ironizza sulla volontà della città di imporsi quale exemplum.

Mars e contemporaneamente a Trocadero sull'altro lato della Senna, ad interessare anche il ponte e gli spazi giardino affacciati sui due argini, è manifesta la portata dell'operazione come momento di ridefinizione delle polarità urbane).

${ }^{8}$ ERIK MatTIE, World's Fair, Princeton Architectural Press, New York City 1998, pag. 24.

${ }^{9}$ Philippe Hamon, Esposizioni: letteratura e architettura nel XIX secolo, Ed. It. Maurizio Giuffredi (a cura di), CLUEB, Bologna 1995, pag. 100. 
"Coloro che venivano dalla provincia e gli stranieri non avevano più bisogno di guide per visitare Parigi; bastava che seguissero il boulevard che si trovava di fronte a loro uscendo dall'albergo; la sera si trovavano al punto di partenza dopo aver visto approfonditamente tutte le curiosità del primo cerchio [...]. L'indomani, ricominciavano dal cerchio successivo [...]. Si era raggiunto così lo scopo perseguito da così lungo tempo: quello di fare di Parigi un oggetto di lusso e di curiosità piuttosto che d'uso, una città d'esposizione."

"I cinquanta boulevard che si irradiavano dal centro alla circonferenza portavano i nomi delle principali città francesi; e le cinquanta porte corrispondenti quello dei dipartimenti di cui ognuna di queste città era il capoluogo [...], in modo che la conoscenza della geografia e della storia aiutava ad orientarsi a Parigi, così come una passeggiata a Parigi era una lezione di storia e geografia [...]. Parigi era come una grande tavola mnemotecnica, sincronica e cronologica". ${ }^{10}$

Anche Langlois, in riferimento all'organizzazione delle Esposizioni Universali tenutesi a Parigi, esprime l'importanza dei simboli (dell'immagine) con cui la città ospite dell'esposizione si propone al mondo intero. Per Langlois non è la realtà del paese ad essere esposta ma la sua rappresentazione:

“(...) Bien conscients qu'il s'agit d'une représentation du pays et non de sa réalité, ces meme hommes sont toujours fascinés par l'emploi de la "magie" technologique, assez spectaculaire pour fournir à l'Expo son cœur, à la France nouvelle (industrielle) son image. A cet égard, la Tour Eiffel est la plus réussie des attractions. »"

Non è un caso che i padiglioni principali, realizzati dalle città in cui viene organizzata l'esposizione, presentino un contenuto ideologico intrinseco alla forma stessa dell'architettura - i modelli compositivi cui si ricorre di frequente sono quelli delle cattedrali a navate multiple e transetto (per fare solo alcuni esempi: Cristal Palace di Londra- 1851, Main Building di Philadelphia-1876, Main Building di Malbourne- 1880/1881), e la pianta centrale (ad esempio del padiglione principale di Parigi- 1867, della Rotunda di Vienna1873). All'idealismo della forma si accompagna lo sperimentalismo tecnologico che crea una "magie tecnhnologique". Idealismo e sperimentalismo tecnologico sono entrambi espressione di potere.

Come anticipato, la concretizzazione delle teorie utopiche ha come sfida prioritaria la risoluzione di problemi logistici, in particolare di quelli legati alla mobilità. E' soprattutto l'aspetto a cui guardarono, prendendone esempio, gli investitori e proprietari dei parchi dei divertimenti nel XIX e XX secolo. Fra l'altro le Fiere non solo avevano una propria organizzazione dei trasporti ma di frequente avevano come oggetto di esposizione i mezzi e le tecnologie per il trasporto. Per la Centennial Exposition di Philandelphia (1876), ad esempio, viene realizzata una linea ferroviaria che porta i visitatori fino al parco sede della fiera e un'altra sopraelevata per gli spostamenti all'interno, un prototipo delle monorail delle fiere successive, sistema riproposto da Walt Disney nel suo Disneyland (California, 1955). Michael Sorkin ${ }^{12}$ mette in luce che l'importanza della struttura dei trasporti costituisce comune denominatore fra parchi tematici ed esposizioni, e parla di un "eccesso di capacità trasportativa":

"Si potrebbe dire che, essendo questi dei luoghi in cui si raccolgono persone da tutto il mondo, la loro condizione sia simboleggiata da un eccesso di "capacità trasportativa"."

Un'altra componente importante delle Esposizioni Universali è la presenza al loro interno di aree destinate solo al divertimento, e/o di momenti, specialmente serali, vocati esclusivamente allo spettacolo e alla festa. Questi spazi o momenti si contrappongono ai programmi ordinari della fiera per essere una declinazione informale e disimpegnata di quanto già rende le fiere divertenti: il costituire una novità nel panorama locale, l'offrire la

\footnotetext{
10 Fournel Victor, Paris nouveau et Paris futur, cit. in ${ }^{10}$ PhilipPe Hamon, Esposizioni: letteratura e architettura nel XIX secolo, Ed. It. Maurizio Giuffredi (a cura di), CLUEB, Bologna 1995, pag. 100-101.

${ }^{11}$ Gilles-Antoine Langlois, Folies Tivolis et attractions. Les premieres parcs de loisirs parisiens, Délégation à l'action artistique de la ville de Paris, Paris 1991, pag. 54.

12 Michael Sorkin, La tematizzazione della città, in "Lotus" n. 109, , Il nuovo mondo allegorico. Sui parchi tematici, Electa, Milano 2001, pag. 7.
} 
possibilità di incontri, il consentire di conoscere quello "che si dice e che si fa" nel mondo ecc.

Sulla necessità di avere all'interno dell'esposizione settori adibiti a parchi del divertimento veri e propri si sofferma Neil Harris. Nel discorso di Harris emerge anche come il parco dei divertimenti produca un indotto nel contesto urbano adiacente o collaudi attrazioni esportabili in parchi dei divertimenti stabili:

"Close by their cultivated urban parks, sometimes even within them, lay concentrated groupings of exotic restaurants, thrilling rides, illusionistic concessions, and alluring performers. Variously termed Midway, Pike, Zone, Pan, Warpath, Pay Streak, or Gay Way, these clusters of concessions contributed mightily to the success of their larger hosts. (...)

There many "dignified reasons" why an international exhibition should host an amusement district, Frank Morton Todd declared in his history of San Francisco's 1915 Panama-Pacific Exposition: precedent, a need for recreation, the exhibition of exotica. "But we may as well admit the main reason, which is that people want to have some fun, and there is not reason why they shouldn't have it to the profit of the undertaking".

Tested by market demand and precisely measured by gate receipts, these attractions could then be transferred from an exposition to a permanent amusement park."13

Longlois attribuisce invece al momento del divertimento all'interno dell'esposizione un ruolo più incisivo, che quello di rispondere semplicemente ad un desiderata del pubblico - $\mathrm{o}$ meglio dà una lettura del fenomeno con una sfumatura diversa, mettendo in relazione il divertimento con il successo dell'evento fieristico in generale e di conseguenza con l’immagine della città. Il suo discorso segue infatti quello già citato precedentemente:

"Enfin le succès des expositions dépend de l'adhésion du public; et le public ne vient que se s'il mise en scène, spectacle avec deus ex machina. Croire dans les progrès des sciences et des arts ne va pas, chacun le sent, sans un besoin fou d'utopie : donner envie d'aller à l'Expo, ce sera récupérer les vieillies recettes de Torré ou Garnerin, faire à la technique un habit de lumière. ${ }^{14}$

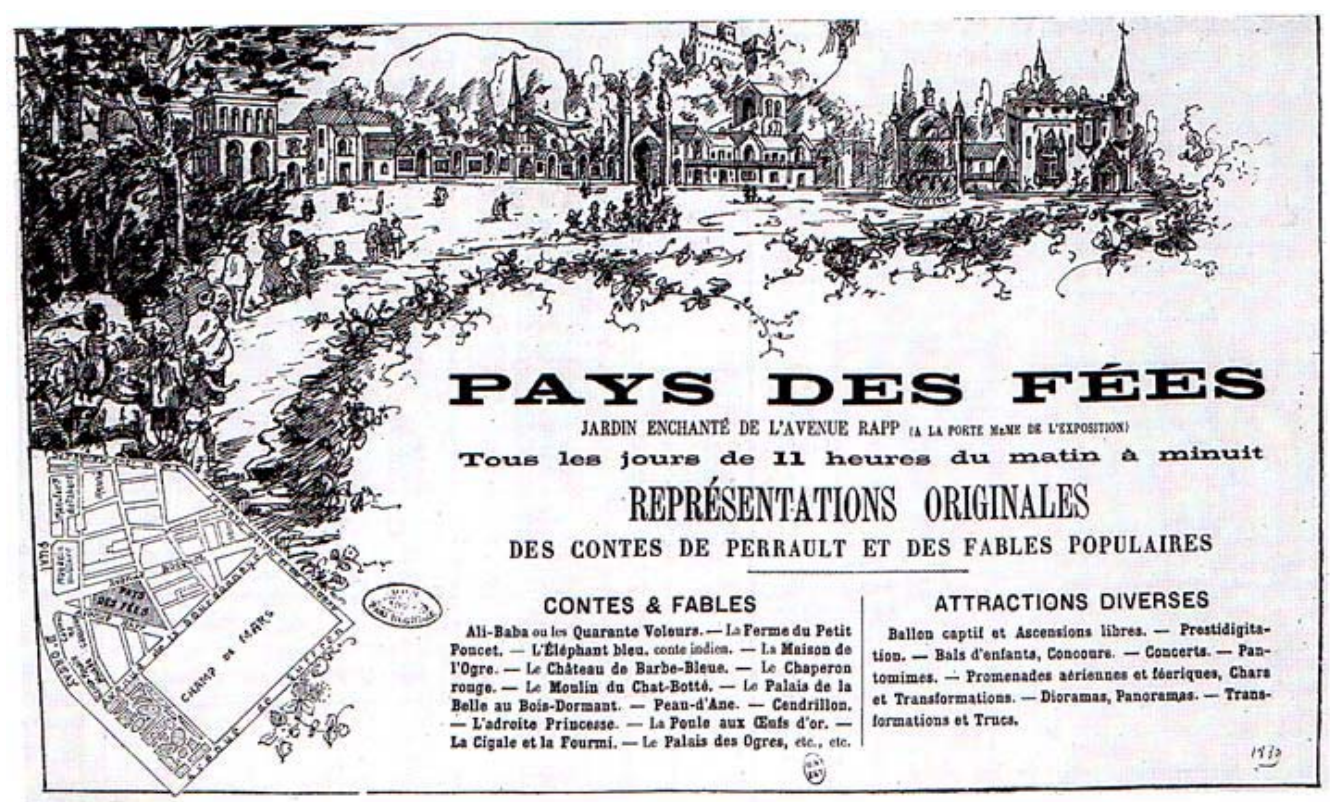

Fig. 8 manifesto del Pays des Fées - parco dei divertimenti dell'Esposizione Universale di Parigi del 1889. Pubblicizza la rappresentazione di racconti di Perrault e di fiabe popolari. Il sottotitolo definisce il Pays des Fées giardino incantato, all'Evenue Rapp.

\footnotetext{
13 Neil Harris, Op.cit., in Maling Karal Ann, Designing Disney's Theme Parks: The Architecture of Reassurance, Flammarion, Paris 1998, pag. 21.

${ }^{14}$ Gilles-Antoine Langlois, Ibidem.
} 


\section{GIARDINO E TECNOLOGIA}

Dato il contenuto idealistico delle Esposizioni Universali, dichiarato spesso dai loro temi/slogan - The Great Exhibition of the Works of Industry of All Nations (London 1851), Building the World of Tomorrow; For Peace and Freedom (New York 1939) - è facile pensare che nella compagine dell'esposizione si ricorra ancora una volta all'idea di giardino come paradiso, e quindi al giardino quale componente essenziale di qualsiasi luogo che intenda rappresentare la tensione verso la perfezione. Per questo, oltre che per la ragione pratica dettata dalla necessità di avere ingenti spazi aperti a disposizione, le fiere vengono ospitate da parchi, reinterpretati e riqualificati per l'occasione. l'intento è concretizzabile grazie a quantità di denaro eccezionali se confrontate con quelle normalmente (anche all'epoca) disponibili per la realizzazione e manutenzione dei parchi pubblici.

Le fiere divengono, secondo un'espressione di Neil Harris, "giganteschi parchi esse stesse, allestimenti controllati che proiettano visioni utopiche dello spazio e della storia":

"In any event, the construction and maintenance of city parks were expensive, and taxpayers frequently abandoned their early, costly commitments; many cities never put ambitious plans into effect. It often took the presence of a great event, like an exposition, to galvanize energies and stimulate political leadership to action. In their wake the expositions in Chicago, Buffalo, St. Luis, Atlanta, Nashville, and elsewhere left a string of altereted urban parks, newly landscaped or much enlarged, either for purposes of receiving the fairs in the first place or to return the grounds to public use after the expositions had closed.

From one angle, the expositions were giant parks themselves, supervised settings that projected utopian visions of space and history." 15

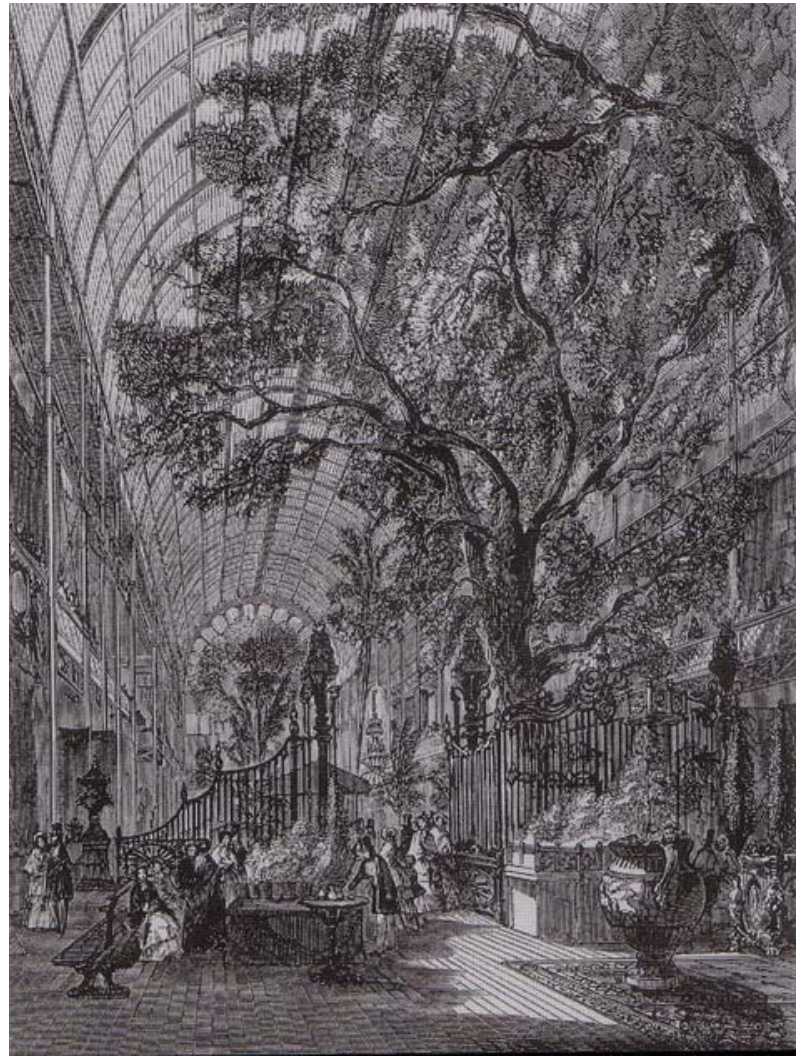

Fig. 9 Crystal Palace come un "giardino per le macchine".

Si è detto come la società dell'era industriale tenti di formulare risposte in grado di contrastare o cambiare i connotati all'urbanizzazione crescente e disperante delle grandi città. Le proposte per la soluzione dei problemi, sull'esempio dell'opera di Hausmann nella

${ }^{15}$ NeIL HARRIS, Ibidem. 
metropoli parigina, vertono su interventi di regolarizzazione come quello progettato da Ildefonso Cerdà per l'espansione di Barcellona (1858) e da Daniel H. Burnham per Chicago. All'interno delle maglie regolari i pianificazioni cercano di inserire quante più aree verdi possibili, a riavvicinare nuovamente l'uomo metropolitano alla natura (ricostruita ora con le forme cartesiane del classicismo francese ora con quelle pittoresche di origine inglese e di successiva assunzione americana ${ }^{16}$ ), per individuare tutte le combinazioni possibili di intersezione fra modernità e condizione edenica.

A scala architettonica e non urbana, il padiglione di Joseph Paxton per l'Esposizione Internazionale di Londra del 1851 intende realizzare proprio una sorta di Eden del progresso, utilizzando a fini espressivi e di comunicazione i due elementi che caratterizzano in qualche modo l'epoca: da una parte la tecnologia del vetro e dell'acciaio, che dilata le possibilità espressive dell'architettura, dall'altra il giardino di piante esotiche, simbolo del potere coloniale, di una geografia dai confini dilatati e allo stesso tempo prossimi. Il tutto studiato per esporre i prodotti della civiltà delle macchine dando la suggestione di un'avvenuta riconciliazione fra uomo dell'era industriale e natura, all'interno di uno spazio ecumenico.

Michael Sorkin definisce il Cristal Palace "riconciliazione climatizzata di Arcadia e industria, un giardino per le macchine":

"Finally the Palace depicted paradise. Not only was it laid out like a cathedral, with nave and transept, but it was also the largest greenhouse ever built, its interior filled with greenery as well as goods, a climate-controlled reconciliation of Arcadia and industry, a garden for machines." 17

Avere l'illusione di questa riconciliazione sarà fondamentale anche all'interno dei parchi tematici della Disney del divertimento, per la conservazione del loro carattere di luogo spensierato: l'Eden deve essere protetto dal peccato originale che la tecnologia in qualche modo rappresenta, pena la sua stessa negazione.

In generale sull'attribuzione di significato e bellezza al giardino si basa quindi la possibilità di una "redenzione". ${ }^{18}$

Recuperando le considerazioni dei Philippe Hamon sulle proprietà dell'esposizione, e sulla concezione dello spazio e dell'arte nel secolo XIX in generale - di cui il Cristal Palace è emblema riconosciuto - la "redenzione" avviene soprattutto in termini estetici ed è basata sulla visione, sullo spettacolo, in quanto la società del tempo è "caratterizzata dall'esercizio euforico della vista e dalla possibilità di abitare un mondo che la vetrina, il telescopio, il diorama, l'oblò, il portello di treno, o la semplice finestra trasformano in spettacoli giustapposti, in collezione da gestire con gli sguardi e i percorsi" $"$.

Un esempio di costruzione dei luoghi che sembra mirare alla "redenzione" nei termini detti di connubio fra tecnologia e natura - è dato dal progetto del paesaggista Frederick Law Olmsted per l'impianto planimetrico della World's Columbian Exposition di Chicago, del 1893. La stazione ferroviaria che la serve immette in una piazza ampia- la Court of Honoroccupata quasi interamente da un "lago" di forma regolare, uno specchio piatto delimitato da edifici di minimale classicismo (accentuato dal colore bianco uniformato, da cui l'appellativo di White City) su tre lati. La piazza ha la stessa ampiezza del fascio di binari ferroviari, come in proiezione verso il lago Michigan. E' uno spazio proporzionatamente controllato, che ricompone tutte le articolazioni-divagazioni dell'intorno in una condizione di quiete opposta all'atmosfera da bazar di Midway Plaesance, amusement park

\footnotetext{
${ }^{16} \mathrm{Si}$ fa riferimento in particolare modo al Central Park di New York (1869) e all'espansione di Chicago Riverside (1869) di Frederick Law Olmested.

${ }^{17}$ Michael Sorkin, See you in Disneyland, in Michael Sorkin (a cura di), Variations on a theme park. The new American city and the and of public space, Hill and Wang, New York 1999, pagg. 209-210.

${ }^{18}$ Il critico Richard Ingersoll ha in questi anni sottolineato l'urgenza contemporanea di concepire la progettazione del paesaggio e del giardino come opera di redenzione, in qualche misura come risarcimento per il disastro ambientale. In particolare si rimanda al testo: RICHARD INGERSOLL, Sprawlscape: il paesaggio come redenzione, prefazione di A. DE Rossi, et al., Linee nel paesaggio, UTET, Torino 1999.

${ }^{19}$ PhilipPe Hamon, Op.cit., Ed. It. Maurizio Giuffredi (a cura di), CLUEB, Bologna 1995, pag. 90.
} 
dell'Esposizione, e al carattere pittoresco del Wooded Island, isola circondata da un lago dalle forme pseudo-naturali immediatamente a Nord della Court of Honor.

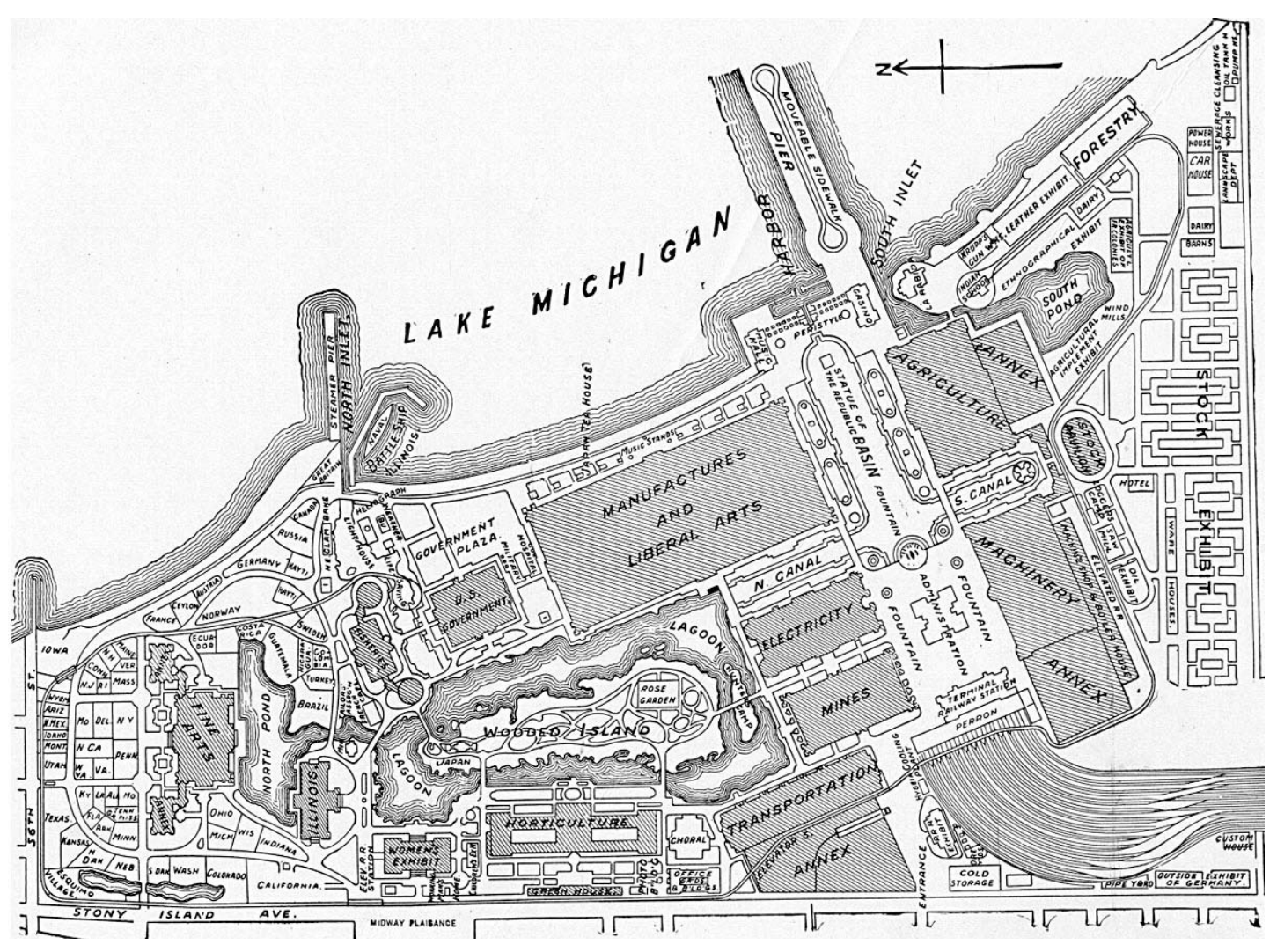

Fig. 10 Planimetria della Chicago Columbian Exposition del 1893. La Court of Hounor ha la stessa ampiezza del fascio di binari ferroviari, come in proiezione, verso il lago Michigan.
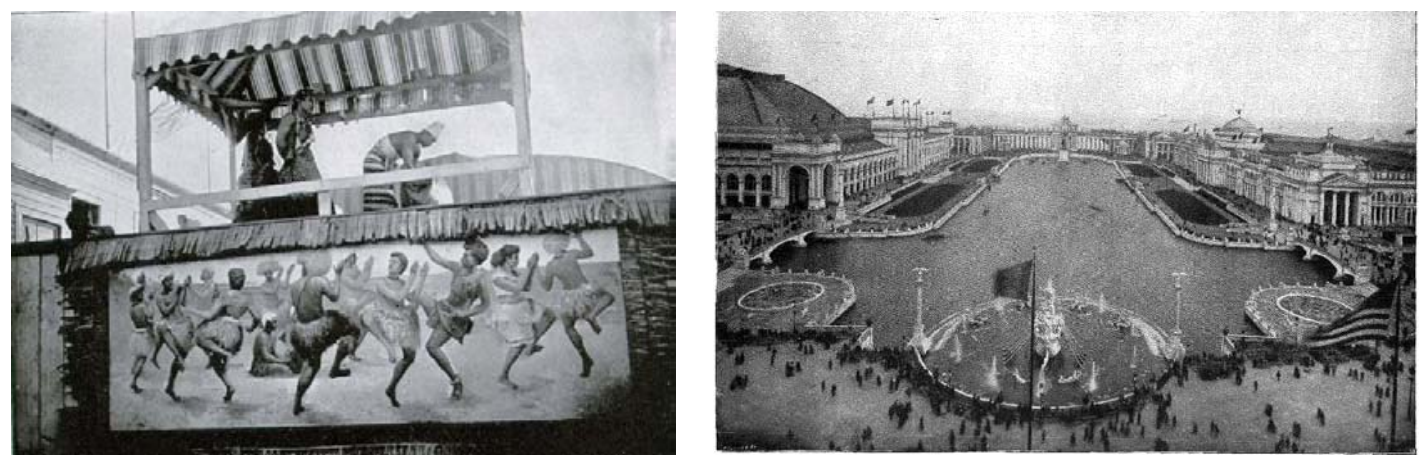

Fig. 11 Luoghi con carattere opposto, della Chicago Columbian Exposition del 1893: danzatori samoani al Midway Pleasance (in alto), parco dei divertimenti dell'esposizione, e veduta della Court of Honor dalla stazione ferroviaria (in basso).

Questa lotta per una "riconciliazione" - che contiene come detto l'idea di spettacolo - appare ancora più determinata, parlando sempre della città di Chicago, se si considera che i parchi dell'esposizione del 1893 saranno parte, secondo la proposta del Plan of Chicago pubblicato da Daniel H. Burnham ed Edward H. Bennett nel 1909, del Chicago Park System (Fig. 12), a proposito del quale Ignasi de Solà Morales scrive:

“( ...) Potremmo vedere così che il piano inteso come architettura della città è inseparabile dal piano inteso come parco urbano. In un certo senso il grande progetto della dinamica metropoli vicino al lago Michigan è, nell'insieme, paragonabile al progetto di un immenso parco, alla maniera di Versailles, nel quale si inseriscano episodi urbani, monumentali, viari, di giardini e parchi di scala minore. Il principio di analogia proprio 
dell'estetica Beaux-Arts è portato ai limiti estremi, di modo che i parchi e i giardini della città sono immagini ridotte, realizzate secondo lo stesso modello del progetto globale per la grande metropoli senza limiti. (...) Il giardino e il parco nati con il modello Beaux Arts diventano in questo modo il più potente strumento di controllo moderno del fenomeno metropolitano." 20

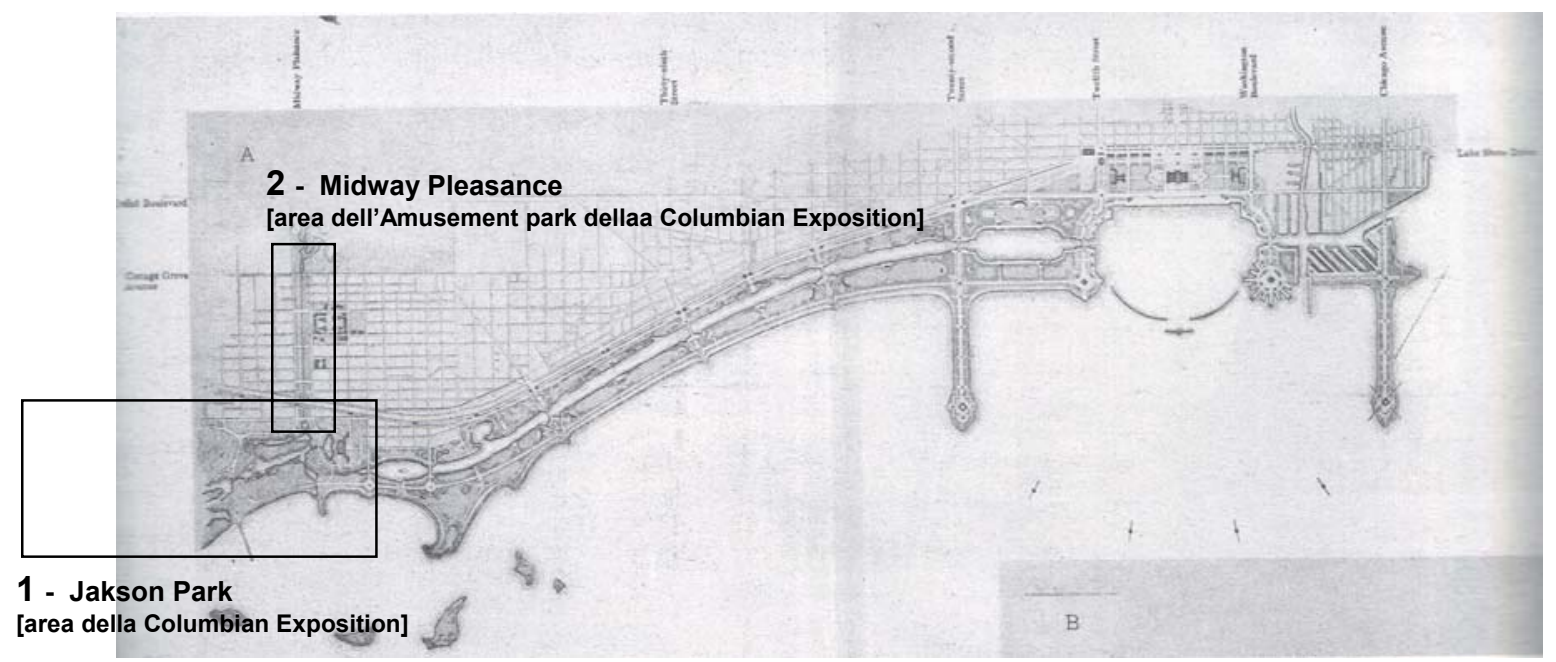

Fig. 12 Planimetria del Chicago Park System del 1909 con evidenziati l'area dell'esposizione del 1893 e il Midway Pleasance, sede dell'amusement park dell'esposizione, parco di connessione fra il parco lungo il lago Michigan e l'odierno Washington Park.

Nella planimetria del parco previsto lungo la riva del lago Michigan è leggibile come sia quest'ultimo ad "originare" un lago o canale artificiale, centro del parco lineare che da Nord prosegue fino all'area dell'Esposizione e si collega al lago che circonda l'isola Wooded Island con una diramazione d'acqua che percorre l'asse del Midway sino a raggiungere l'odierno Washington Park (quest'ultima connessione d'acqua è già presente nel Plan of The Chiacago South Park Commission, 1871, Olmsted, Vaux \&Co.; South Park è il nome del parco, pensato come unitario, costituito dal Jackson Park, area dell'Esposizione del 1993, dal Midway Plaisance e dal South Open Ground, oggi Washington Park).

La composizione sembra istituire una metafora: la natura si insinua nell'area metropolitana, totalmente trasformata in parco, cambiando aspetto, sempre in bilico fra rigore geometrico e forme pseudo-naturali (calcolo e spontaneità). Si deve immaginare questa vena d'acqua vitalizzatrice attraversare la metropoli industriale per eccellenza dell'America degli inizi del XX secolo, avendo in mente che sono gli anni della sua ricostruzione dopo il terremoto del 1871, in cui sono attivi gli esponenti della "Scuola di Chicago" che la caratterizzano la città mediante le proprie architetture con cui perseguono un purismo estetico dell'innovazione strutturale-tecnologica.

La dialettica fra tecnologia e giardino è visibile anche al Fairmount Park, sede della Centennial Exposition di Philadelphia del 1876, parco che ha come cuore un lago e la Lawsnowne Valley, alimentati dal fiume vicino, lo Schuylkill River (Fig. 13).

Il lago progettato e realizzato per l'occasione - unitamente a tutte le altre componenti per la sistemazione dell'area ai fini dell'esposizione, ad opera dell'architetto paesaggista Herman Joseph Schwarzmann -sembra al contrario preesistente rispetto agli assi viari che strutturano l'impianto, per 1' "autonomia" della sua ubicazione e della sua morfologia. Gli assi continuano la maglia del tessuto urbano adiacente, mentre l'acqua all'interno del parco si rapporta con il fiume ed il suo ambito organizzando, non la localizzazione dei padiglioni principali, ma la parte di fiera costituita da architetture tematiche puntuali sparse nel paesaggio. Questo assetto suggerisce l'idea di una collaborazione fra tecnologia e natura: il

${ }^{20}$ Ignasi de Solà Morales, Il giardino Beaux - Arts, in Monique Mosser, Georges Teyssot, L'Architettura dei giardini d'Occidente- Dal Rinascimento al Novecento, Electa, Milano 1990, pagg. 399 e 404. 
parco, coerentemente con l'idea di Frederick Law Olmested, è lo spazio principe delle attività sia ricreative sia formative, nel contatto con la natura: non c'è strappo fra metropoli e ambiente "naturale".

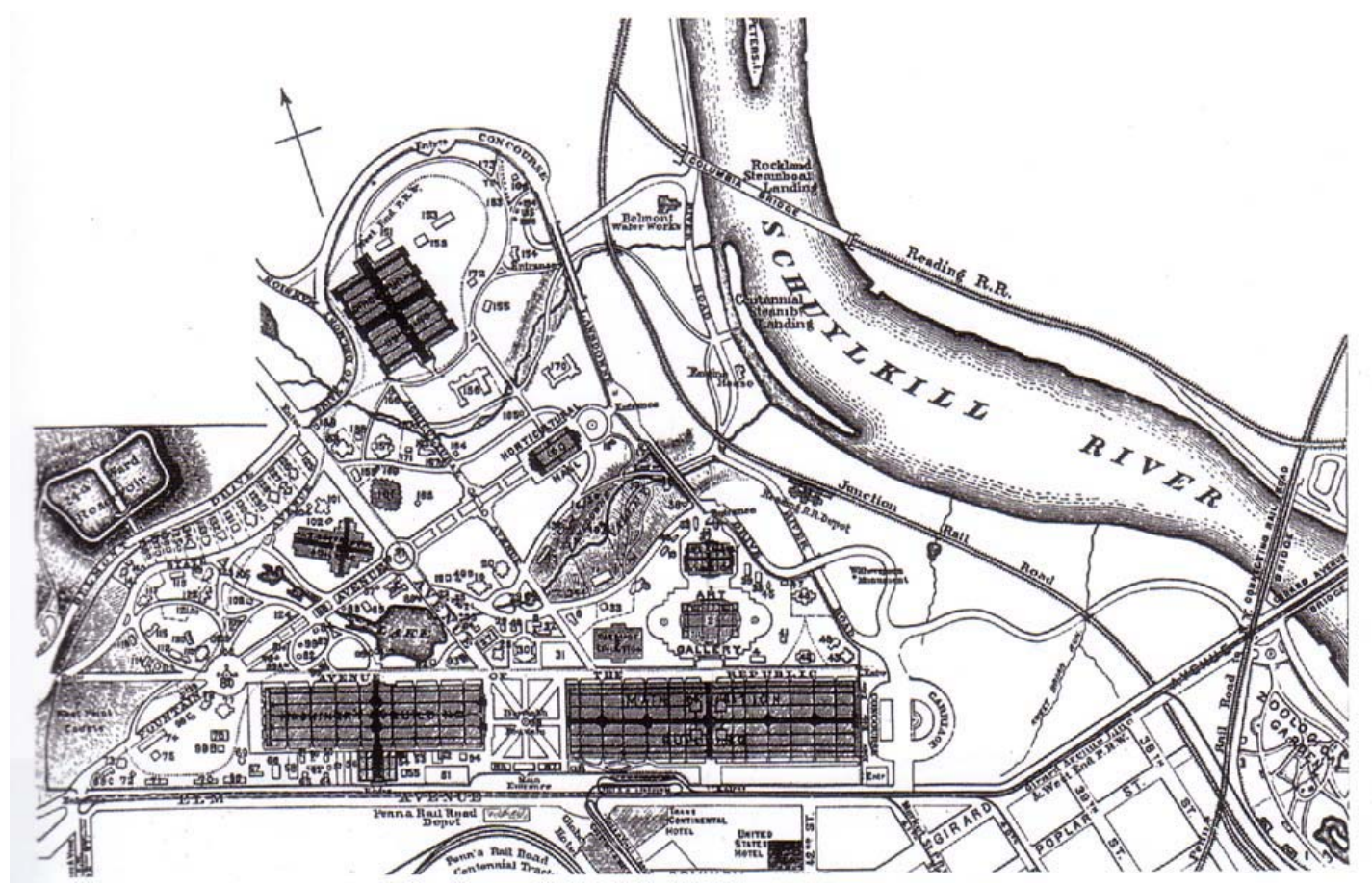

Fig. 13 Planimetria della Philadelphia Centennial Exposition del 1876. Il paesaggio "naturale" dell'esposizione si rapporta con l'ambito fluviale, gli assi viari e i padiglioni assecondano la geometria del tessuto urbano, come a tentare una conciliazione fra città industriale e natura.

\section{PAESAGGI SINTETICI}

Francoise Choay concisamente caratterizza il XIX secolo nel suo The Modern City: planning in the 19th Century (1969):

"[Con] lo sviluppo di mezzi di comunicazione sempre più astratti, la continuità del tipo consueto di comunicazione è sostituita da nuovi sistemi che continuano a perfezionarsi per tutto il XIX secolo, consentendo una crescente mobilità alla popolazione e fornendo un'informazione sincronizzata in modo più preciso con il ritmo in accelerazione della storia. Ferrovia, stampa quotidiana e telegrafo soppianteranno gradualmente lo spazio nel suo precedente ruolo informativo."21

Nell'esperienza delle esposizioni universali lo spazio non viene propriamente sostituito, viene piuttosto contratto, senza peraltro che sia annullata la sensazione di compiere un viaggio fisico. Marc Augè considera comunque il viaggio come un muoversi fra nonluoghi, in quanto per l'autore "lo spazio come pratica dei luoghi e non del luogo deriva (...) da un doppio spostamento: del viaggiatore, certo, ma parallelamente anche dei paesaggi di cui egli ha sempre delle visioni parziali, delle "istantanee", sommate alla rinfusa nella sua memoria" 22 . Secondo questa concezione la visita alle esposizioni dovrebbe essere un viaggio in un nonluogo dal segno negativo potenziato: alle esposizioni accade che in uno spazio contenuto siano accostate architetture, giardini, interi ambienti, tematizzati dalla specifica cultura che rappresentano. Vengono ricostruiti emblemi in grado di evocare l'intero mondo oppure, come nel caso dell' Esposizione Internazionale di Parigi del 1889 e della sua Tour

\footnotetext{
${ }^{21}$ Citazione in KenNeth Frampton , Storia dell'architettura moderna, Zanichelli, Bologna 1998, pag. 11.

${ }^{22}$ MARC AugÉ , Nonluoghi, Elèuthera, Milano 1993, pag. 80.
} 
Eiffel sono le architetture stesse della fiera ad assurgere a simbolo permanente in una geografia urbana che propone una sintesi in una sola città universale, mediante la coesione di frammenti/ architetture ${ }^{23}$ di città territorialmente distanti.

Sintesi che secondo Hamon è accompagnata da un'ulteriore sintesi: quella operata dai cataloghi dell'esposizione, dai commenti delle riviste di moda, dai racconti, sempre all'interno di un'atmosfera di divertimento, leggera, estetizzante:

"Chi dice esposizione dice preminenza dello sguardo, dei suoi specifici piaceri e dispiaceri, dice spazio architettonico pianificato leggibilmente per un percorso più o meno vincolante, dice presentazione razionale di collezioni di oggetti, dice pratiche istituzionali e sociali ritualizzate, e dice anche ostentazione di un sapere e quindi esercizio accompagnatorio di un linguaggio esplicativo, da un parte (l'esposizione spiega), designativo e descrittivo dall'altra (l'esposizione mette in mostra oggetti etichettati e designati). Non si dà monumento senza memorandum, senza guida di percorso. Non si dà buona esposizione senza catalogo."24

La visita all'esposizione avviene comunque mediante movimento: la peculiarità di questo viaggio è che il paesaggio cambia rapidamente per lo spazio ristretto che intercorre fra un ambiente-tema e l'altro e a volte anche in forma ancora più accelerata, per l'utilizzo di mezzi di trasporto veloci come la monorail presente all'esposizione di Philadelphia (1876).

Il binomio linea ferroviaria/ambiente esotico istaura una "dialettica fra apparenza e realtà", fra distanza e prossimità come sintetizzato da Michael Sorkin:

"Those tropical landscape (...) helped to invent the idea of simulated travel, initiating the great touristic dialectic of appearance and reality. The decline in popularity of these environments toward the end of the century was the result of the spread of railways, which made actual exotic travel possible.

This dislocation is central. Whatever its other meanings, the theme park rhapsodises on the relationship between transportation and geography. The winter garden evokes distance, the railroad proximity.,"25

La rete dei trasporti è inoltre l'elemento materiale in grado di legare la fiera al proprio territorio, al contesto urbano che la supporta. La visione accelerata dovuta alla velocità dei mezzi, che estremizza la sintesi dei paesaggi visibili, costituisce una novità rispetto a quanto poteva avvenire nella fruizione dei parchi eclettici, delle folies francesi del XVIII secolo, anch'essi sintetici in un loro specifico modo. L'alta velocità della visione enfatizza una differenza esistente comunque fra il paesaggio dell'esposizione ed il giardino eclettico: in quest'ultimo l'uomo partecipa del paesaggio, è al suo interno - anche nelle corrispondenti rappresentazioni pittoriche - ed in questa sua posizione rispetto al luogo consiste il sublime, "che è (ef)fusione e "possesso" dello spettatore dentro e attraverso lo spettacolo"; l'esposizione è al contrario "giustapposizione e di stanziamento di oggetti e di spettacoli, creazione di un discontinuo (...).",26

Numerosi parchi dei divertimenti ripropongono un paesaggio similmente sintetico, i cui elementi non sono ne' il progresso ne' l'arte, ma una sorta di loro surrogato.

L'esempio che soprattutto costituisce riferimento è il Midway Plaisance, amusemenet park della World's Columbian Exposition di Chicago del 1893 (Fig. 14), in cui ad essere tematizzati in rappresentanza di diverse nazioni e civiltà dal mondo non sono semplicemente le architetture - e non lo sono ai fini delle presentazione delle arti elevate o dei prodotti industriali - ma i modi dell'incontro e dell'intrattenimento. Il Midway si configura come una variegata scenografia di supporto degli spettacoli etnici programmati, costituito di Tarkish Village, German Villange, Japanese Bazar, Persian Theatre, ecc. e della Street of Cairo, la cui fama sarà celeberrima e che verrà riprodotta all'interno del Luna Park (1903) di Coney

\footnotetext{
${ }^{23}$ Frammenti accostati sono sia i padiglioni nazionali (folies) isolati nel parco (a Philadephia nel 1876, ai giardini al Trocadero di Parigi nel 1878, per esempio), sia le facciate in serie lungo Rue des Nations sempre a Parigi nel 1878.

${ }^{24}$ PhilipPe Hamon, Op.cit., Ed. It. Maurizio Giuffredi (a cura di), CLUEB, Bologna 1995, pag. 15.

${ }^{25}$ Michael Sorkin, See you in Disneyland, in Michael Sorkin (a cura di), Variations on a theme park. The new American city and the and of public space, Hill and Wang, New York 1999, pag. 210.

${ }^{26}$ PhilipPe Hamon, Op.cit., Ed. It. Maurizio Giuffredi (a cura di), CLUEB, Bologna 1995, pag. 98.
} 
Island, a New York. Il lotto è edificato in maniera tale da costruire al suo interno rapporti spaziali e architetture tipiche di una strada del Cairo, animata dal transito di persone in costume e da cammelli e dromedari.

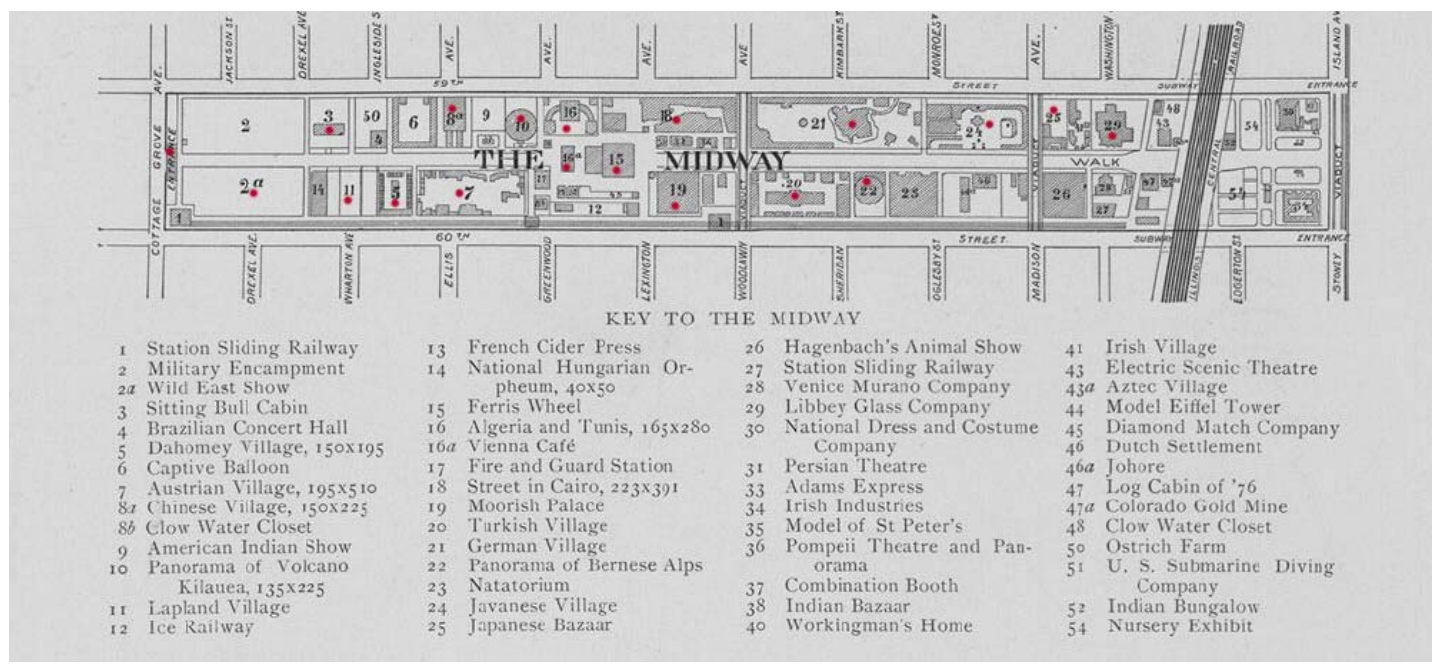

Fig. 14 Planimetria del Midway Pleasance: ogni "lotto" appare organizzato secondo un diverso schema urbanistico, i vari paesi allestiscono il loro spazio di esibizione secondo le composizioni spaziali e architettoniche proprie.

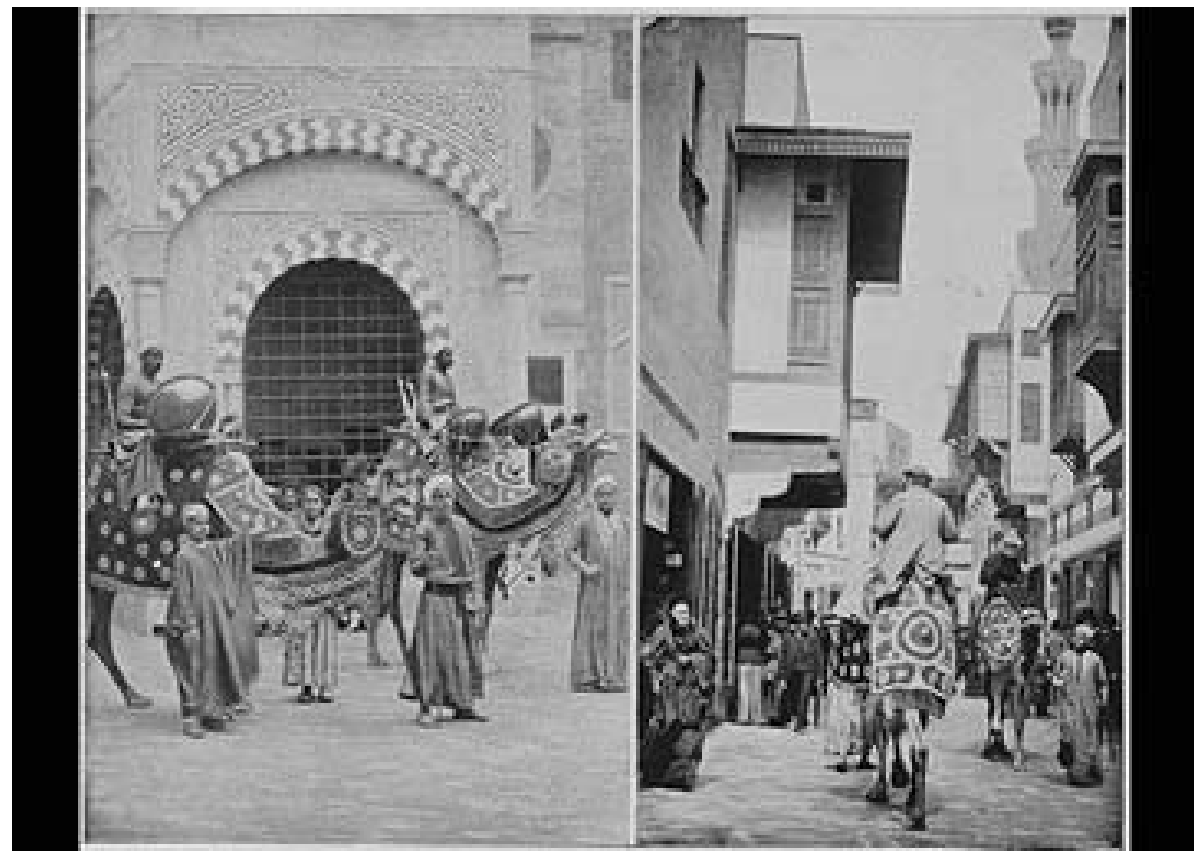

Fig. 15 Immagini della Street of Cairo al Midway Plaesance.

Gli amusement park americani che sorgeranno successivamente al 1893tenteranno di ricreare lo spirito carnevalesco del Midway. In generale essi attingeranno dalle fiere internazionali tecnologie e soluzioni per il trasporto dei visitatori e allo tesso tempo acquisteranno o copieranno le attrazioni presenti. La ruota panoramica del Midway, chiamata Ferris Wheel perché progettata dall'ingegnere Ferris, diventerà attrazione comune alle fiere successive $\mathrm{e}$ nei parchi dei Divertimenti; l'Iron Tower, uno degli emblemi del West Brighton di Coney Island, viene comprata e importata dalla Centennial Exposition di Philadelphia.... Gli esempi sono numerosissimi. 
Le Fiere internazionali fungono da espositori anche per l'industria dei divertimenti. Ma nell'assumere i prodotti delle esposizioni, gli amusement park alleggeriranno il loro peso eliminando i contenuti ideali e progressisti alla base delle esposizioni - costituirà eccezione il parco Disneyland (1955) intriso dell'idealismo del suo autore Walt Disney.

\section{RIFERIMENTI BIBLIOGRAFICI}

Appelbaum Stanley, The New York World's Fair 1939/1940, Dover Publications, New York 1977.

AUGÉ MARC, Nonluoghi, Elèuthera, Milano 1993.

BECK GREGORY, Un atlante dei parchi tematici, in "Lotus" n. 109, 2001, pagg. 104-115.

De SolÀ Morales Ignasi, Il giardino Beaux -Arts, in Monique Mosser, Georges TEYSSOT, L'Architettura dei giardini d'Occidente- Dal Rinascimento al Novecento, Electa, Milano 1990, pagg. 395- 404.

EISNER D. MICHAEL (foreword by), Imagineering. A Behind the Dreams Look at Making the Magic Real, Disney Editions, New York 1996.

FRAMPTON KENNETH, Storia dell'architettura moderna, Zanichelli, Bologna 1998.

HAMON PHILIPPE, Esposizioni: letteratura e architettura nel XIX secolo, Ed. It. Maurizio Giuffredi (a cura di), CLUEB, Bologna 1995.

HARRIS NeIL, Expository expositions. Preparing for the theme parks, in MALING KARAL AnN, Designing Disney's Theme Parks: The Architecture of Reassurance, Flammarion, Paris 1998, pagg. 19-27.

KoOlhaAs Rem AND MAU B., La città generica, in $S, M, L, X L$, (new edition) Taschen, Germany 1997.

LANGloIS GiLles-AnTOINE, Folies Tivolis et attractions. Les premieres parcs de loisirs parisiens, Délégation à l'action artistique de la ville de Paris, Paris 1991.

MATTIE ERIK, World's Fair, Princeton Architectural Press, New York City 1998.

ORVELl Miles, The real thing and the Machine-made World, in MiLES ORVELL, The real thing. Imitation and Authenticity in America Culture, 1880-1940., The University of North Carolina Press, U.S.A. 1989, pag. 157-197.

SORKIN MiCHAEL, La tematizzazione della città, in "Lotus" n. 109, , Il nuovo mondo allegorico. Sui parchi tematici, Electa, Milano 2001, pagg. 7-17.

Siti web:

A History of the fair in www. xroads.virginia.edu/ MA96/WCE/title

Interactive Guide to the World's Columbian Exposition in

www.users.vnet.net/schulman/Columbian/columbian.html

The Chicago Columbian Exposition of 1893 in

www.park.org/Pavilions/WorldExpositions/chicago.text.html

The Chicago Columbian Exposition in www.chicagohs.org/history/expo.html

The World's Columbian Exposition - Chicago 1893 in

www.chicagohs.org/AOTM/May98/may98fact3

The World's Columbian Exposition in 1893 in www.columbus.gl.iit.edu/

\section{RIFERIMENTI ICONOGRAFICI}

Figg. 1, 10, 11, 14-15: in http://columbus.gl.iit.edu/

Fig. 2: FRAMPTON KENNETH, Storia dell'architettura moderna, Zanichelli, Bologna 1998, pag. 21, illustrazione 15 .

Fig. 3: EISNER D. MiCHAEL (foreword by), Imagineering. A Bihind the Dreams Look at Making the Magic Real, Disney Editions, New York 1996, pag. 15. 
Figg. 4-6: ApPelbaum Stanley, The New York World's Fair 1939/1940, Dover Publications, New York 1977, pag. 46, 3,4.

Fig. 7: MATTIE ERIK, World's Fair, Princeton Architectural Press, New York City 1998, pag.17.

Fig. 8: LANGLOIS GILleS-AntoIne, Folies Tivolis et attractions. Les premieres parcs de loisirs parisiens, Délégation à l'action artistique de la ville de Paris, Paris 1991, pag. 199 tavola 179.

Fig. 9 : MATTIE ERIK, World's Fair, Princeton Architectural Press, New York City 1998, pag.14.

Fig. 12: De SolÀ Morales Ignasi, Il giardino Beaux -Arts, in Monique Mosser, GEORGES TEYSSOT, L'Architettura dei giardini d'Occidente- Dal Rinascimento al Novecento, Electa, Milano 1990, pag. 399.

Fig. 13: MATTIE ERIK, World's Fair, Princeton Architectural Press, New York City 1998, pag. 33 . 


\section{4 - GLI AMUSEMENT PARKS AMERICANI}

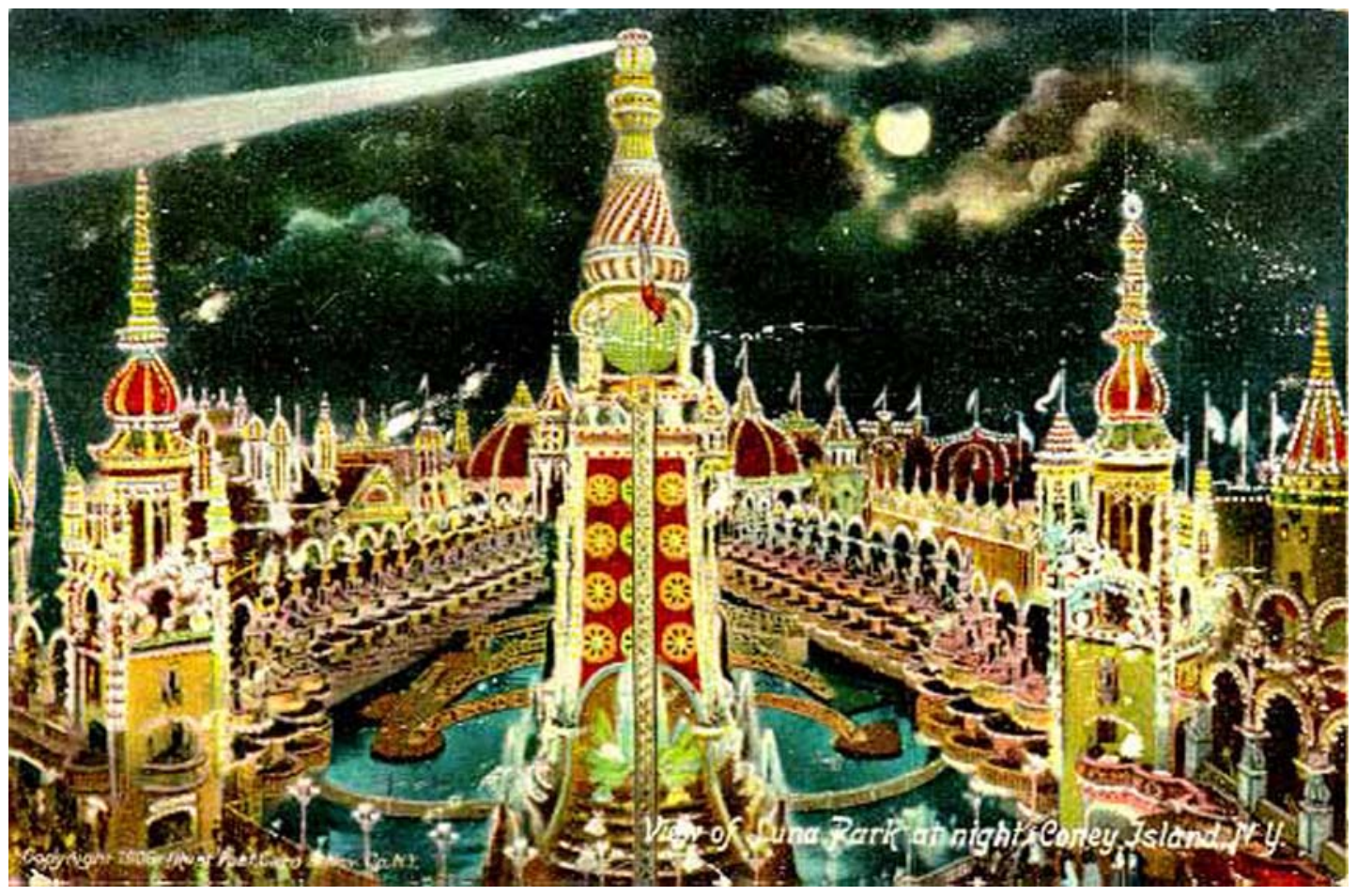

Fig. 1 Luna Park (1903) a Coney Island - cartolina dell'epoca che ne racconta la stravaganza, il "lusso" delle architetture, dell'illuminazione, il paesaggio onirico.

\section{CONEY ISLAND: PARCO “PUBBLICO”/ LUNA PARK}

Coney Island, sulla costa atlantica a nove miglia di Manhattan è, durante il XIX secolo e i primi decenni del XX secolo, il parco "pubblico" dei Newyorkesi (intendendo con il termine pubblico, non un regime di proprietà, ma una connotazione sociale: tutte le classi e le etnie vi trovano oggetti di desiderio per il loro tempo di vacanza) segnando un momento di passaggio culturale, sociale ed economico. Si impone come esteso amusement park richiedendo una ri-definizione, mediante il potere della sua eccezionalità, sia degli attributi spaziali associati in quella compagine al concetto di parco, sia del ruolo sociale dello spazio pubblico. Il fenomeno fa moltissimo rumore a tutti i livelli e la critica di costume e di arte, nell'osservare i sui sviluppi, si sente fortemente interpellata.

La prossimità e l'isolamento rispetto alla metropoli di New York sono la premessa per Coney Island, la rendono fin dai primi decenni dell' 800 luogo di attrazione, con il sorgere del primo Hotel, il Coney Island House (1829), di ristoranti, birrerie, bagni.

Prossimità e isolamento sono alla base della costruzione di quel particolare tipo di waterfront che Rem Koolhas, dopo più di un secolo, alla voce Quarters della sua Generic City ${ }^{l}$, teorizzerà come necessaria controparte per ogni città, ovvero un confine dove essa incontra "altre condizioni". E "la vicinanza della possibilità d'evasione è la miglior garanzia per il divertimento"

\footnotetext{
${ }^{1}$ Rem KoolhaAs, The Generic City, S,M,L,XL, Taschen, 1997, pag. 1257.

${ }^{2}$ Si è già parlato del rapporto fra svago e spazio di "frontiera" a proposito dei pleure gardens del XIX secolo, recuperando alcune suggestioni sul concetto di confine di Piero Zanini.
} 
"Each Generic City has a waterfront, not necessarily with water - it can also be with desert, for instance - but at least an edge where it meets another conditions, as if a position of near escape is the best guarantee for its enjoyment." 3

A Coney Islands queste altre condizioni non sono affatto, come Frederick Law Olmsted ${ }^{4}$ invece propone negli stessi anni per la stessa città di New York, una natura ritrovata che interrompa un contesto esasperatamente urbanizzato - studiata e potenziata nelle sue componenti estetiche e disponibile a qualsiasi attività - e una ricreazione che sia elevazione verso un rafforzato senso civico e verso un raffinato gusto del bello.

Al contrario le altre condizioni sono: una natura primitiva come quella che si trova nel punto di contatto fra terra e acqua, liceità di comportamento e stravaganza dell'architettura. Questi ingredienti sono finalizzati ad un divertimento inteso semplicemente come piacere, e sono improntati allo spirito carnevalesco-orgiastico di origine europea, che caratterizza i pleasure gardens del XVIII secolo. In questo consiste lo scandalo.

Nel 1860 la reputazione di Coney Island è pessima. E' il tempo delle trasformazioni operate dal politico della downtown New York Mike Norton, che di fatto lo rende un luogo d'incontro per uomini di malaffare.

Durante gli anni '70 del XIX secolo le cose cominciano a cambiare per l'azione di due imprenditori, Mckane e Feltman. Feltaman, iniziata la sua attività molti anni prima, con l'invenzione dell' hot dog e la sua vendita in un chiosco ambulante, subaffitta una porzione di terreno e vi costruisce una serie di ristoranti e birrerie-giardino ("beer gardens shaded by maple trees" $)$, ed il famoso Iron Pier che ospita 1200 bagni-armadietti, giochi e stands culinari, una sala da ballo e una piazza per 5000 spettatori - i numeri danno l'idea della furia imprenditoriale di cui Coney Island è frutto.

Il riferimento principale in territorio americano per gli imprenditori che investono su Coney Island è il Midway Pleasance, parco dei divertimenti (parte, ma a margine) della Chicago Columbian Exposition del 1893:

"It stimulated a new industry of travelling carnival shows and new attractions for popular resorts, It suggested an entirely different model of democratic urban recreation from either Central Park or the White City, a park designed not according to the civic values of cultural elites but according to the commercial values of entrepreneurs determined to attract a mass audience. The new "amusement parks" which emerged at the turn of the century would be the results - parks which, as their name suggests, sought frankly to entertain rather than to uplift". 6

Finita la Guerra Civile, la volontà di trasformare Norton's Point (così chiamato per essere stato sotto la "giurisdizione" di Mike Norton) in una località d'attrazione, porta all'incremento delle infrastrutture di collegamento con la metropoli - aumenta il numero di battelli a vapore e di linee ferroviarie e viene realizzata l'Ocean Parkway (1976), superstrada arredata (landscaped) con alberature ed aree ad arbusti, che connette direttamente con il Brooklyn's Prospect park - e ad investire nella costruzione di nuovi alberghi lungo costa. Sorgono in questo modo, sulla parte occidentale di Coney Island, tre località: West Brighton, Brigthon Beach, e Manhattan Beach. Manhattan Beach è realizzata dal ricco banchiere e magnate ferroviario Austin Corbin il quale costruisce la New York \& Manhattan Beach Railway (1876-77), accorciando le distanze esistenti fra il centro di Manahattan e la spiaggia dei divertimenti:

\footnotetext{
${ }^{3}$ Rem KoolhaAs, Op. cit., in O.M.A., Rem KoolhaAs, Bruce Mau, S,M,L,XL, Taschen, 1997, pag. $1257 .$.

${ }^{4}$ Riguardo al rapporto fra la cultura dominante americana nel XIX secolo e l'opera di Frederick Law Olmsted si rimanda la testo JOHN F. KASSON, Amusing the million. Coney Island at the turn of the Century, Hill \& Wang, New York 1978. Al suo interno l'autore - con toni a tratti eccessivi - evidenzia la svolta effettuata da Coney Islands, sottolineando la contrapposizione fra l'amusement park, il Central Park di New York (1863) e la Chicago Columbian Exposition (1893), contemporanei.

${ }^{5}$ JEFFREY STANTON, Early Coney Islands History (1609-1880), www.naid.sppsr.ucla.edu/coneyisland/histart.htm.

${ }^{6}$ JoHn F. KASSON, Amusing the million. Coney Island at the turn of the Century, Hill \& Wang, New York 1978. pagg. 26-27.
} 
“(...) So that New York nabobs could travel in an hour from midtown Manhattan directly to his stylish new hotels". 7

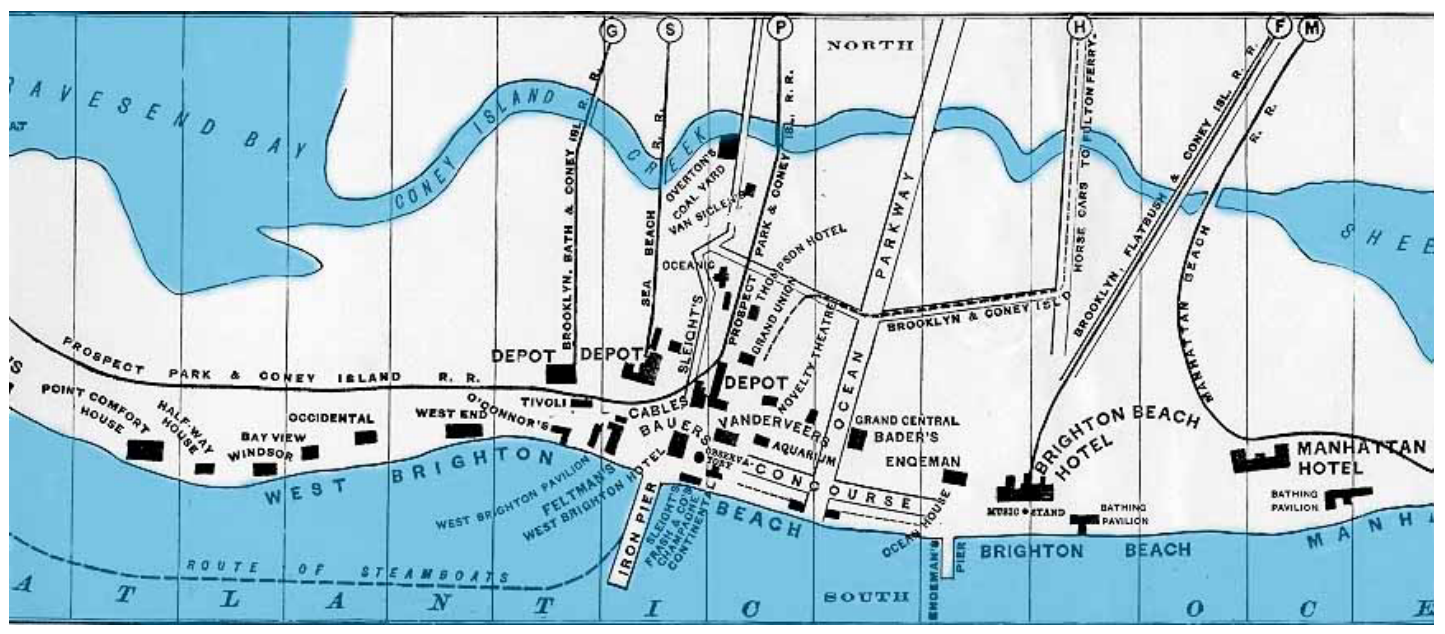

Fig. 2 Rete dei trasporti di Coney Island. West Brighton, Brigthon Beach, e Manhattan Beach non sono collegate fra loro, ma ognuna è connessa direttamente con il downtown di New York (ogni imprenditore investe per avere un accesso riservato ai propri complessi ricreativi).

I tre luoghi si caratterizzarono in maniera differente: Manhattan Beach segue il tentativo di offrire una nuova immagine di rispettabilità puntando a divenire una meta per l'elite, Brighton Beach, collegata a New York mediante una linea ferroviaria privata, ospita servizi rivolti alla classe media e alle sue famiglie (anche perché maggiormente vicina all'area più degenerata) e West Brighton costituisce "la parte per il tutto" di Coney Island, rappresentandola con la sua Iron Tower (struttura con un ascensore a vapore importata dalla Philandelphia Centennial Exposition del 1876 che consente una vista panoramica dell'intorno) e il suo Elephant Hotel (1882).

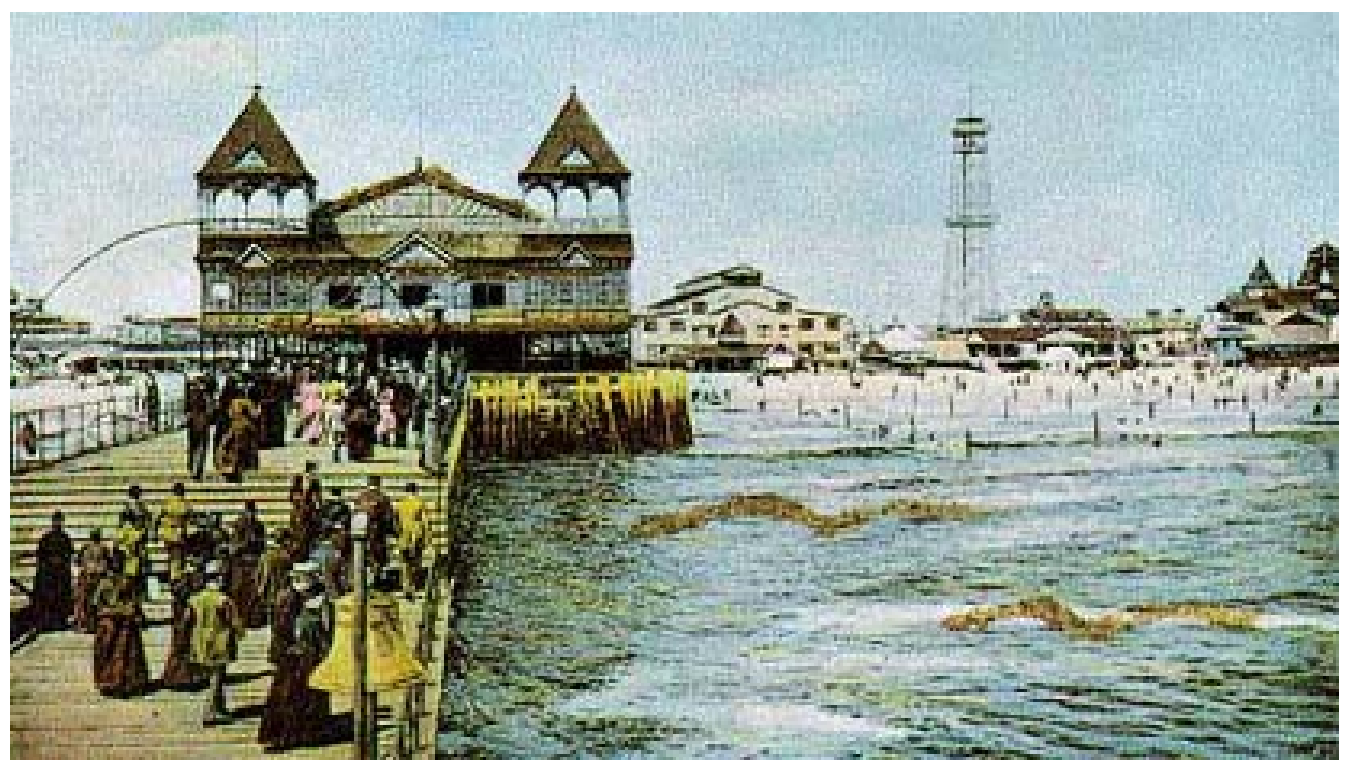

Fig. 3 L'Iron Pier al West Brighton di Coney Island. Sullo sfondo è l'Iron Tower, importato dalla Philadelphia Centennial Exposition del 1876.

\footnotetext{
${ }^{7}$ John F. KASSON, Op. cit., Hill \& Wang, New York 1978. pag. 31.
} 
Nel 1895 apre a Coney Island il primo parco dei divertimenti delimitato e chiuso, il Sea Lion Park. Il tema è acquatico: si offrono spettacoli con quaranta leoni marini ammaestrati e water rides. Segue la costruzione di Steeplechase Park nel 1897, su un territorio di 15 acri, racchiuso da un circuito per la corsa dei cavalli (steeplechase), resa più pericolosa ed entusiasmante per la notevole pendenza del percorso. Il proprietario-realizzatore di Steeplechase Park, George C. Tilyou, visita la Chicago Columbian Exposition del 1893 e, fallito il tentativo di acquistare la Ferris Wheel $^{8}$, ne ordina una di nuova costruzione e di maggiore dimensione. Nel 1901 visita la Pan-American Exposition a Buffalo (New York) e importa nel suo parco un'attrazione presentata alla fiera, il Cyclorama "A trip on the Moon", in cui gli spettatori entrano in un'astronave per un viaggio immaginario sulla luna ed abbandonata l'astronave la esplorano. Tilyou porta a Steeplechase anche i due progettisti del Cyclorama Thompson e Dundy, i quali in seguito comprano il Sea Lion Park e lo trasformano in un nuovo parco, il Luna Park.

Diversificandosi da Steeplechase Park, che mira a creare un'atmosfera da brivido mediante l'avanguardia delle tecnologie delle proprie rides, attinte dalle esposizioni nazionali e internazionali del periodo, Luna Park fonda il proprio appeal sull'essere una splendida fiera dell'esotico. E' una “dream city", città-sogno:

“As about 45,000 men, women, and children streamed toward Luna Park on opening night in May 1903, according to one reporter, they stopped, "rubber their eyes and stood in wonder and pinched themselves". They stood at the imposing gates of what appeared to be a dream city, a mysterious palace of play. Once inside, they discovered, in the words of a second journalist, "an enchanted, storybook land of trellises, columns, domes, minarets, lagoons, and lofty aerial flights (...)"

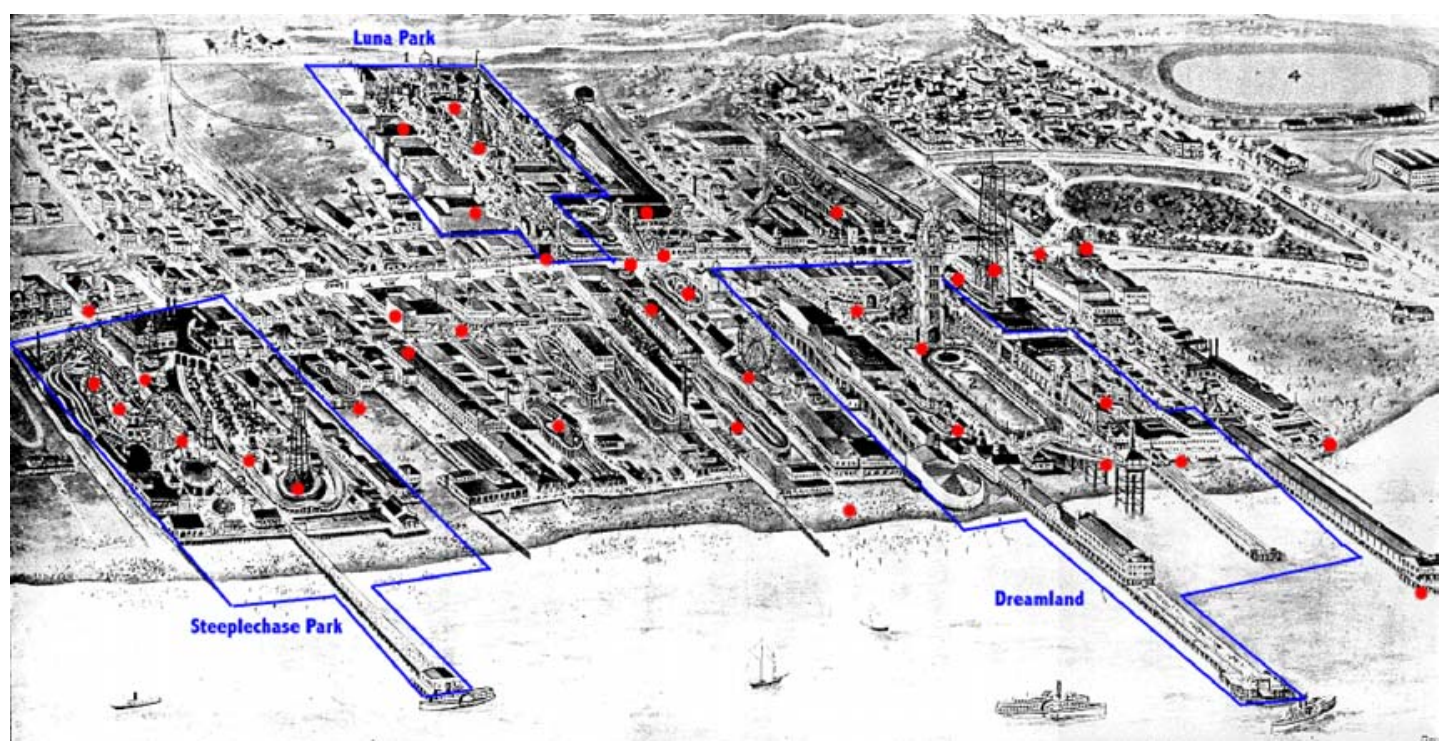

Fig. 4 Coney Island nel 1906. Sono evidenziati i tre grandi parchi dei divertimenti: Steeplechase Park ( 1897), Luna Park (1903) e Dreamland (1904).

Sulla scia di Luna Park, nel 1903, un gruppo di politici guidati dal senatore e manager di teatro William H. Reynolds, realizza il terzo parco di Coney Island, Dreamland, il cui emblema resta la Beacon Tower (reinterpretazione della torre Giralda di Siviglia) famosa per la sua straordinaria illuminazione notturna.

\footnotetext{
${ }^{8}$ La Ferris Wheel è la famosa ruota panoramica, che prende nome dall'ingegnere Ferris, a cui si deve il suo studio e la sua messa a punto. Dopo la sua "apparizione" alla Columbian Exposition del 1893 diviene attrazione ricorrente nei parchi dei divertimenti.

9 John F. KASSON, Op. cit., Hill \& Wang, New York 1978. pag. 63.

Kasson riporta parte dei testi da " The New York Times", 17 May 1903; Albert Bigelow Paine, The New Coney Island, "Century”, 68 (August 1904): 535.
} 
In particolare Frederic Thomson comprende il ruolo dell'architettura in funzione del divertimento di massa, come scenografia in grado di accompagnare sensazioni e comportamenti all'interno del parco. I visitatori, per la crescente immigrazione a New York di questi anni, sono di origini e culture diverse (poco integrate rispetto alla genteel culture imperante in città) e cercano in Coney Island uno statuto etico/estetico maggiormente variegato e possibilista.

Il "destino" di Coney Island ne dichiara la consistenza effimera e ne amplifica l'aura. Non rimane nulla: nel 1911 scaturisce un incendio all'interno di Dreamland - dovuto al troppo spinto livello di sofisticazione - che rade al suolo il parco. Quanto a Luna Park, morto nel 1907 il responsabile delle finanze Skip Dundy, iniziano difficoltà personali e professionali per Thompson, che nel 1912 dichiara bancarotta. Thomson muore nel 1919, facendo seguire per Luna Park periodi di minore splendore. Negli agli anni ' 40 il parco subisce una serie di incendi, in seguito ai quali viene sostituito da un parcheggio e da lottizzazioni edilizie. Steeplechase Park, primo ad essere costruito, è l'ultimo a scomparire.

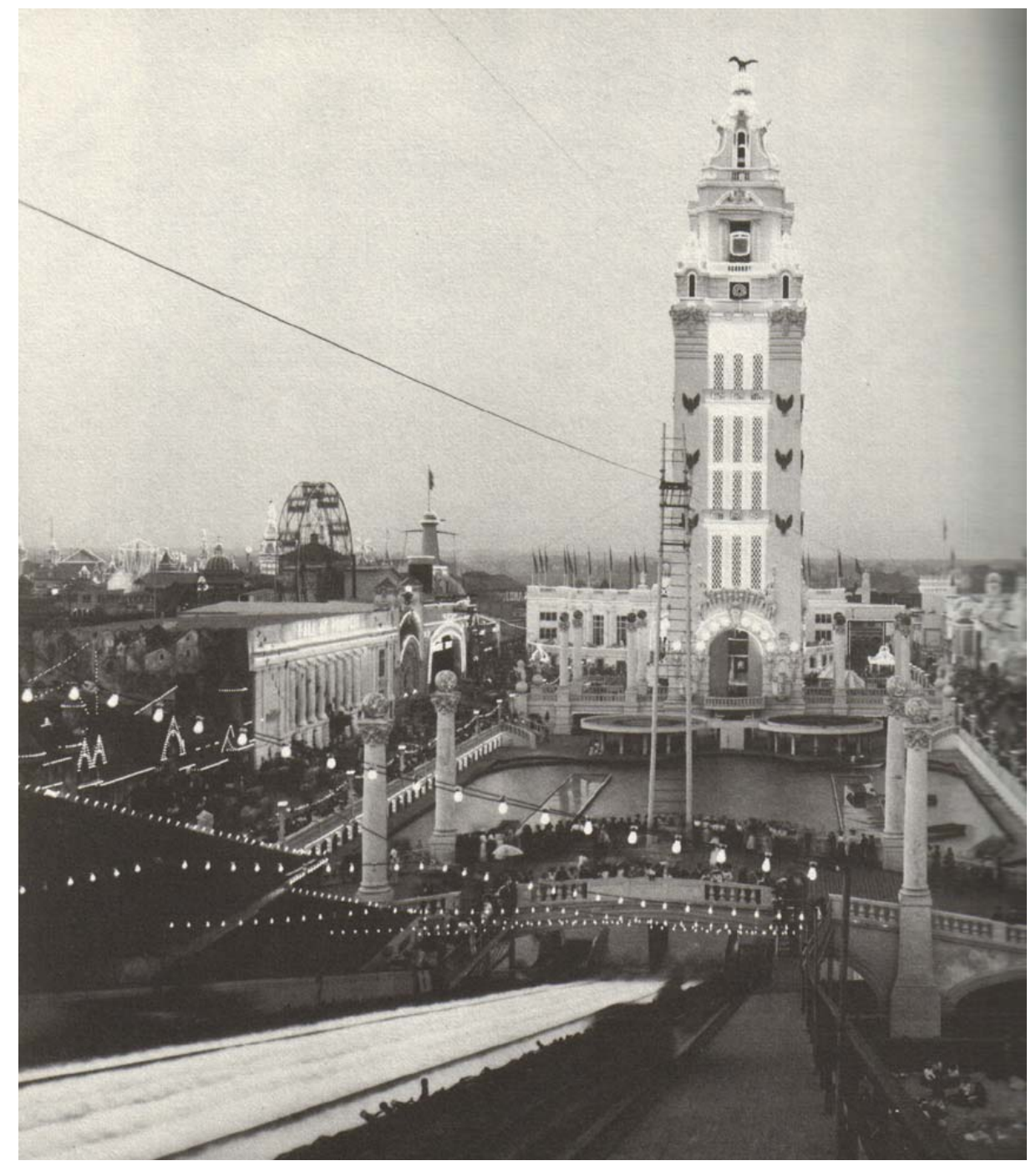

Fig. 5 Dreamland nel 1905. 


\section{CONTESTO COME PIANO “0” (PER LA SOVRAPPOSIZIONE DI SIMBOLI)}

L'Elephant Hotel a Coney Island segnala un nuovo connubio fra architettura e simbolo, quest'ultimo si impone come alterazione dei dati reali del paesaggio, operando salti dimensionali e geografici:

\footnotetext{
"This was the Elephant, an immense sculpture made out of wood and covered with tin, with spiral staircases in its hind legs, a diorama and a cigar store in its front legs, a shopping mall and guest rooms in its body, and an observatory in its heads."
}

L'attenzione del visitatore viene attirata non solo dalla presenza di un elefante sulla costa atlantica, ma dal fatto che l'elefante in realtà sia un grattacielo. Il meccanismo è analogo a quello realizzato dalla statua/architettura dell'Appennino del Parco di Pratolino (XVI sec.), in cui l'immagine antropomorfica che rappresenta il gruppo montuoso appenninico evocando un paesaggio che è "genius loci", è anche palazzo e grotta, e concentra, nella complessità della costruzione iconografica e architettonica, molteplici significati in un singolo punto capace di emanare la propria influenza sui luoghi circostanti.

"Il paperino di Long Island" (negozio di papere) preso come esempio di architettura-simbolo da Robert Venturi, ai fini della sua analisi e "proposizione" del paesaggio dello Strip di Las Vegas, non è qualcosa di molto diverso .

Quello che accomuna le tre "sculture" è il loro ruolo di oggetti comunicativi, ruolo che svincola la forma dalla funzione, l'aspetto esterno dall'interno. Per il suo simbolismo l'Elephant Hotel si pone totalmente al di fuori dei canoni del movimento moderno, embrionali negli anni della sua realizzazione e germinanti nelle architetture della Court of Honour della Chicago Columbian Exposition ${ }^{11}$. Sottolinea Venturi:

"La contraddizione fra esterno ed interno era comune in architettura prima del Movimento Moderno, particolarmente nell'architettura urbana e monumentale. Le cupole barocche erano simboli e allo stesso tempo costruzioni spaziali ed erano più grandi in scala e più alte all'esterno rispetto all'interno allo scopo di dominare il loro intorno urbano e comunicare il loro messaggio simbolico". ${ }^{12}$

Quello che, al contrario, diversifica l'Appennino del Giambologna dall' Elephant Hotel e dal "Paperino di Long Island" è il suo essere parte di un percorso narrativo. A Coney Island come a Las Vegas le architetture-simbolo non sono in relazione fra loro, ma si accostano in competizione reciproca. Il Luna Park di Coney Island, durante la sua stagione di apertura, presenta accostati una città di Venezia completa di gondole, un giardino giapponese, un villaggio Irlandese, uno Eschimese, un teatro Cinese, e l'anno successivo vi aggiunge il Durbar di Delhi. Non intercorre fra i vari episodi tanto spazio quanto si è sottolineato esistere nelle folies francesi del XVIII secolo, spazio che connotava le fabriques come eventi che si incontrano lungo un itinerario e, in una percezione statica, da fermi, quali punti eccentrici inquadrati in un paesaggio campestre, secondo i principi dell'estetica del pittoresco.

A Coney Island la "natura" è assente, è nello sfondo, nello spazio adiacente su cui si affaccia l'urbanizzazione densissima, costituita dalla rete dei trasporti e dalle attrazioni, ma non all'interno.

\footnotetext{
${ }^{10}$ JoHn F. Kasson, Op. cit., Hill \& Wang, New York 1978. pag. 33.

11 ed in generale della città di Chicago in ricostruzione dopo il terremoto del...ad opera degli esponenti della cosiddetta scuola di Chicago, Adler e Sullivan.

12 Robert Venturi, Denise Scott Brown, Stephen Izenuor, Imparando da Las Vegas. Il simbolismo dimenticato della forma architettonica, CLUVA Editrice, 1985, pag. 27.
} 

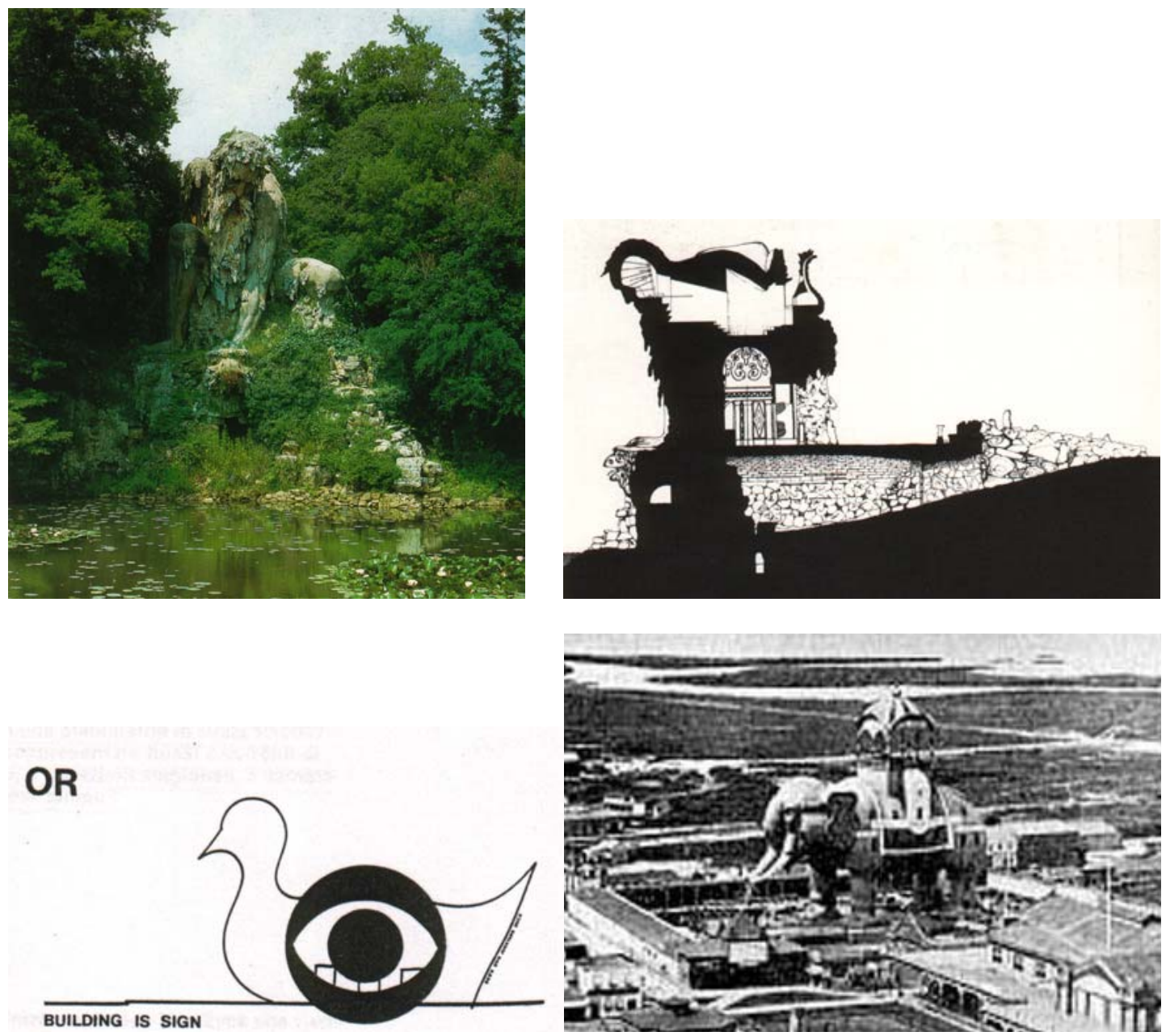

Fig. 6 Architetture- simbolo: L'Appennino del Giambologna nel giardino di Pratolino (XVI sec.), fotografia e sezione - diagramma di Robert Venturi per visualizzare il concetto di architettura-simbolo astraendo dalla forma del "Paperino di Long Island" - L’Elephant Hotel di Coney Island (1876).

Coney Island esaspera l'idea di luogo costruito per sovrapposizione di icone, affastellate in uno spazio circoscritto, i cui connotati originali dati dalla struttura fisica del territorio sono quasi del tutto ininfluenti (fungendo da precursore delle modalità di costruzione dei parchi dei divertimenti successivi). Riguardo a Coney Island si tratta dell' icona di quanto archetipicamente associabile al piacere: esotico, erotico, ricchezza, mistero, fantasia.

In questo senso Coney Island costituisce la faccia opposta, suggerita dal Midway Pleasance, della White City della Chicago Columbian Exposition (1893) i cui artisti e architetti, unitamente a Frederick Law Olmested che ne cura il piano, si ispirano ai canoni del rinascimento italiano, prefigurandone uno nascituro d'oltreocano: compostezza, misura, pulizia formale.

John F. Kasson, nel riportare un commento di uno dei direttori di Dreamland sulle sorti subite dal parco, informa della consapevolezza che, una volta stabilito l'obiettivo di coinvolgere un pubblico di massa, nella progettazione degli spazi è necessario utilizzare elementi vernacolari:

"The promoters of Dreamland sought to appeal to a highly developed sense of the artistic but it did not take theme long to discover that Coney Island was scarcely the place for that sort of thing. Architectural and decorative beauty were virtually lost upon the great majority of visitors $(. .). "{ }^{13}$

\footnotetext{
${ }^{13}$ George F. Dobson, quoted in Pilat and Ranson, "Sodom", pag. 172, cit. in JOHN F. KASSON, Op. cit., Hill \& Wang, New York 1978, pag. 110.
} 
Nonostante il divario esistente fra i concetti di costruzione del paesaggio che informano il Central Park di New York e le logiche che presiedono allo sviluppo di Coney Island, c'è forse un aspetto che avvicina i due mondi: entrambi in qualche maniera ruotano attorno al problema della condivisione di valori a livello collettivo. Il primo vi aspira esplicitamente, ${ }^{14}$ il secondo agevola di fatto la trasformazione dei valori etici della cultura vittoriana in nuovi più duttili, agevolando l'integrazione fra la differenti etnie-civiltà della metropoli di New York.

Ma un rovescio della medaglia rivela l'immagine di ulteriori codici e di un annoiato conformismo, che segnano il declino di Coney Island. I suoi imprenditori non riescono a fare fronte alla richiesta concitata di novità, sempre più difficile da soddisfare: la cultura di massa "promossa" dai parchi dei divertimenti estende negli anni '20 il suo potere e trova nuovi oggetti di distrazione ovvero il consumismo generalizzato anche nella vita quotidiana - e non più confinato negli spazi per lo svago- e la televisione.

C'è un altro aspetto concomitante: l'incremento dei collegamenti fra il centro di New York e Coney Island, su cui continuamente gli imprenditori di quest'ultima investono, abbrevia troppo quelle distanze che consentivano di percepire un passaggio di stato nell'entrare a Coney Island.

" The extension of the subway from New York to Coney Island in 1920 made the resort more accessible than ever before, but it reduced the element of contrast, the distinctive sense of entering a special realm operating under its own laws. The experience of the city and the experience of the resort increasingly blurred. Coney declined most markedly in the power to astound, and cultural critics stopped paying attention. A harbinger of the new mass culture, Coney Island lost its distinctiveness by the very triumph of its values."15

\section{IL LUOGO EQUIVALENTE?}

Negli amusement park americani, non si riscontra un particolare studio prospettico che relazioni scena con scena. Sorti spesso per costruzioni successive nel tempo di edifici ed attrazioni, come avviene nei centri fieristici e nelle stesse città, casualmente o secondo le logiche commerciali che regolano acquisizioni e affitti di porzioni di suolo, gli amusement park non presentano né coerenti (né coesive) tematizzazioni, né un voluto assetto spaziale, ma al contrario una miscellanea di richiami. In questo modo lo spazio è equivalente, nel senso che al suo interno ci si muove random in tutte le direzioni, privi di un itinerario stabilito. Il sistema orientativo è dato dalle infrastrutture della mobilità che rendono obbligate le modalità di accesso, e dai simboli, i soli ad essere sottoposti a una gerarchizzazione, che si impone su una base indifferenziata.

Coney Island è stata esattamente questo modello di amusement park, modello che si riscontra ovunque il parco sia pensato in forma di luna-park, fatto di molteplici temi, a volte assegnati alle singole attrazioni, senza costruire ampi ambienti con simboli omogenei.

Negli amusements park il contesto funge da pretesto, in realtà la fiera (permanente) potrebbe quasi essere localizzata in qualsiasi posto nella medesima maniera: gli amusement park ereditano i caratteri insediativi degli spettacoli itineranti. C'è un altro aspetto che può aiutare a comprendere il perché di questa "equivalenza" dei luoghi ospiti. Mara Memo, nel rilevare una sostanziale differenza fra Disneyland e Coney Island - l'una dà forma al

\footnotetext{
14 "Landscape architecture, as Olmsted conceived its broad scope and mission, had to reflect as well as shape collective values; for among the profession's loftier aims was to create tranquil landscapes in public domain, where people of diverse backgrounds could relax, intermingle, and develop a sense of community and cooperation, thereby furthering the progress of civilization." In PeTER WalKer, Melanie Simo, Invisible Gardens. The search for Modernism in the American Landscape, The MIT Press, Cambridge, Massachusetts 1996, pag.11.

${ }^{15}$ JoHn F. KASSON, Op. cit., Hill \& Wang, New York 1978, pag. 112.
} 
fantastico, nell'altra "il grande assente è l'inedito" 16 - parla, riguardo al parco newyorkese, di "ampliamento dei modi dell'urbanity"17. Se si considera che i luoghi degli amusement park sono soprattutto periurbani o extraurbani e rappresentano quindi proprio il territorio di limite fra città e paesaggio naturale (o rurale), se non un territorio assolutamente altro rispetto all'urbano, che potrebbe costituire un momento di contatto con la natura, l' "ampliare i modi dell'urbano" fa pensare ad un'indifferenza pressoché assoluta nei confronti del carattere del luogo. L'esigenza che muove la costruzione babelica di Coney Island sfrutta il potere di attrazione dell'elemento naturale - il mare - per poi prescinderne e duplicare la realtà metropolitana, cambiandola di segno:

"Le scene della città riprodotta parlavano con immagini popolari ma reali, l'eccentricità degli spettacoli proponeva numeri da circo e scene di tribù africane perfettamente ricostruite per introdurre l'elemento esotico, tutto lo svolgersi della dimensione effimera del parco contribuiva comunque ad ampliare i modi dell'urbanity e del consumo, offrendo un'esperienza diversa rispetto a quella quotidiana. La city costituiva un prodotto del consumo che si collocava a metà tra una messa in scena e una partecipazione diretta, dove però la scenografia di fondo usava maggiormente modelli presi dalla realtà." 18

Paradossalmente, il paesaggio preesistente, naturale o artificiale, sul quale viene "montato" il luna park è in realtà indispensabile per l'immagine del luogo. Diviene lo sfondo che, unitamente alle attrazioni/simbolo (quando ne esistono di non anonime in grado di imporsi come landmark) rende riconoscibili e prossimi i contesti culturali specifici. E' l'esistenza contemporanea dell'Elephant Hotel e dell'Oceano Atlantico a fornirci le coordinate geografiche per fissare Coney Island in un punto esatto della mente e dello spazio.

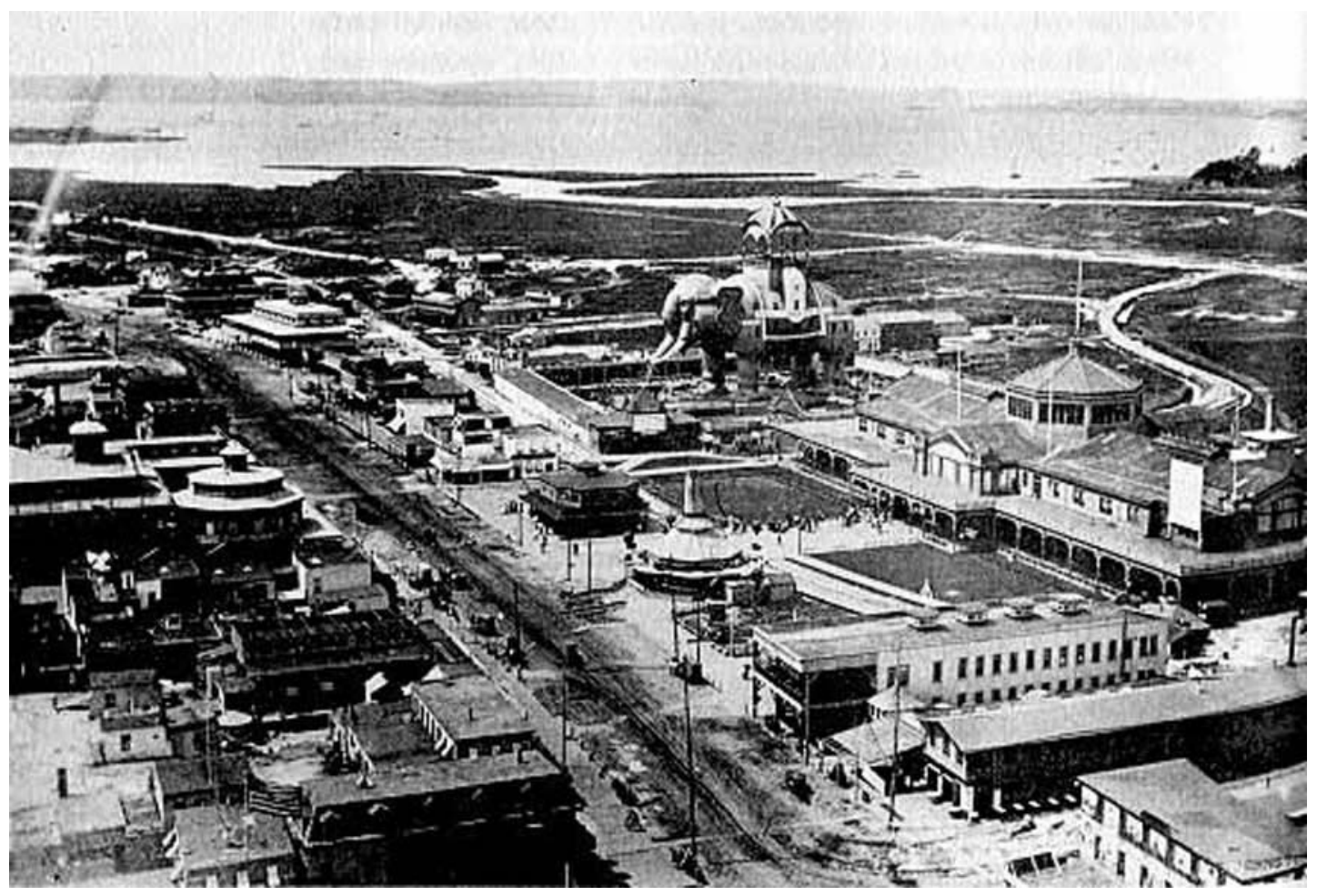

Fig. 7 Coney Island in una fotografia della fine del XIX sec.: l'Elephant Hotel come architettura-simbolo e la costa atlantica come paesaggio primigenio collaborano nel conferimento di identità al luogo.

\footnotetext{
${ }^{16}$ Mara Mero, Parco tematico. Iperreale fantastico commerciale walt disney of course!, in PAOLO DeSIDERI E MAssimo IlARDi (a cura di), Attraversamenti. I nuovi territori dello spazio pubblico, Costa \& Nolan, Genova 1997, pag. 124.

${ }^{17}$ Mara Mero, Op. cit., in PaOlo Desideri e Massimo Ilardi (a cura di), Attraversamenti. I nuovi territori dello spazio pubblico, Costa \& Nolan, Genova 1997, pag. 124.

${ }^{18}$ MARA MERO, Ibidem.
} 


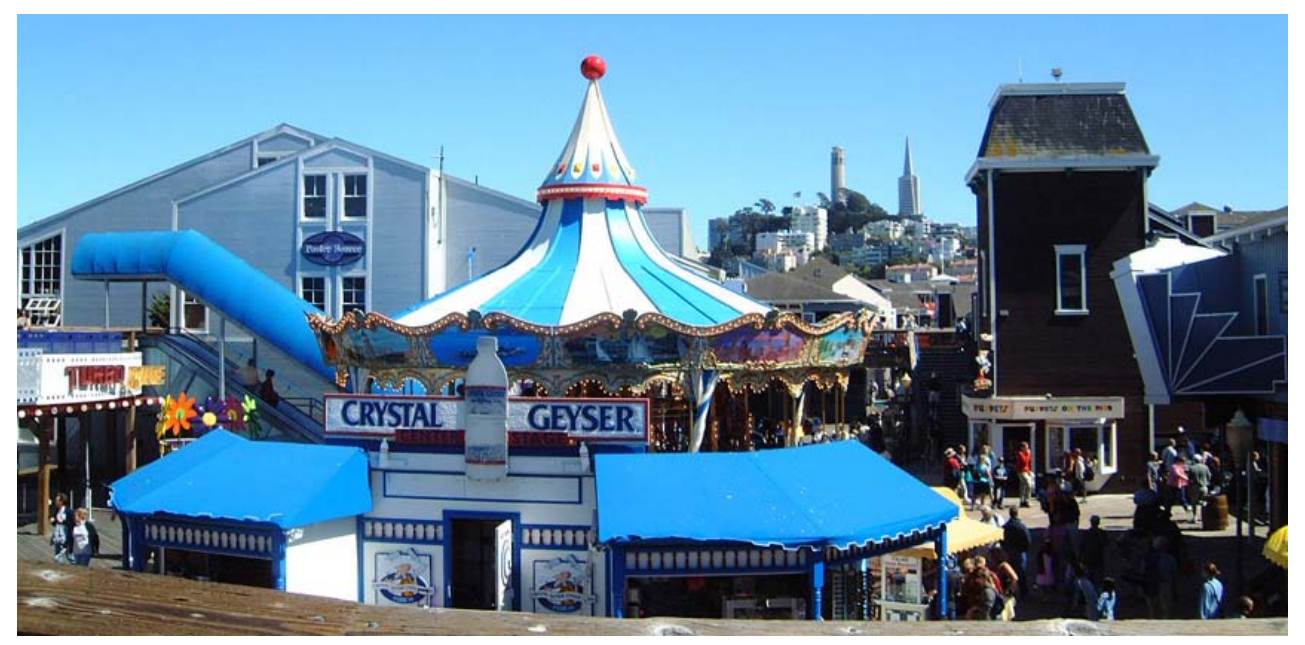

Fig. 8 Centro commerciale al Pier 39 di Fisherman's Warf a San Francisco ( California U.S.A.), sullo sfondo lo skyline della città.

Allo stesso modo 1'Isola di Alcatraz nella baia di San Francisco, visibile al termine della promenade, fornisce una caratterizzazione al centro commerciale del Pier 39 di Fisherman's Warf. Lo alleggerisce di peso, svela la consistenza effimera di una fiera traboccante di insegne e architettura kitch, con la forza (eterna, non culturale, non epocale) dell'elemento naturale. Viceversa i grattacieli del Financial District, in direzione opposta, dichiarano l'appartenenza alla particolarità della città che ribadisce i propri valori urbani, a memento.

Un rapporto analogo fra parco e contesto avviene alla Santa Cruz Boardwalk a Santa Cruz (California), in cui il paesaggio contratto e "stordente" del complesso dei divertimenti è affacciato sulla spiaggia ampia e riposante.

L'aspetto da istallazioni temporanee ed extraterritoriali degli amusement park americani (ma anche di numerosissimi piers commerciali inglesi) domanda una cornice autonoma e distaccata, una neutralità che li contrasti. Questo avviene a Santa Cruz, dove, volendo, si può girare lo guardo verso l'oceano e i promontori costieri che sembrano ancora selvaggi e lasciare letteralmente alle proprie spalle la Boardwalk, "pulendo" la vista di tutto.

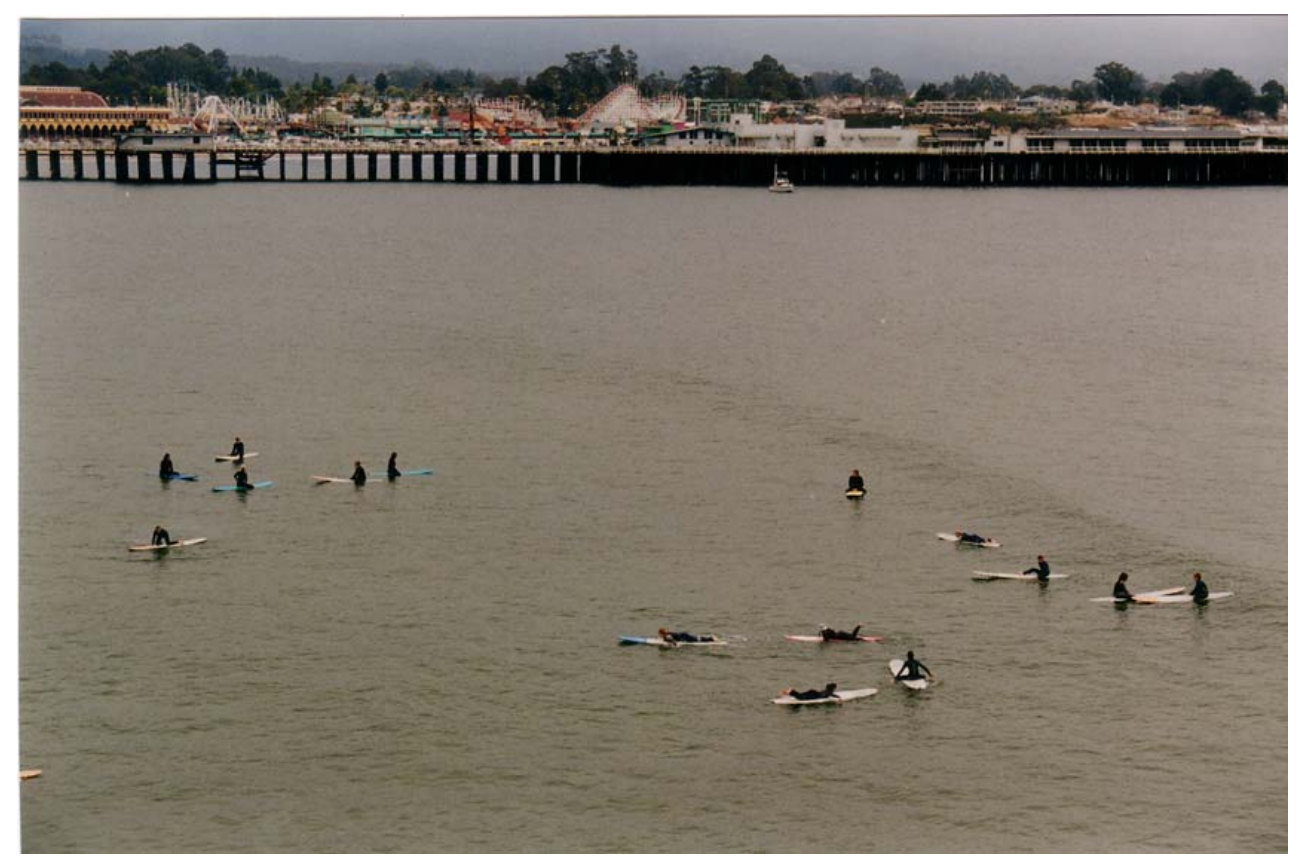

Fig. 9 Santa Cruz Boardwalk (California) nello sfondo. 
Per gli amusement park si rendono necessari lo scorcio, la presa di contatto improvvisa, così come, all'opposto, l'accompagnamento, la presenza a lato prolungata e costante della natura, ovvero una possibilità di risarcimento, almeno sul piano estetico, del probabile depauperamento ecologico e dell' "inquinamento" visivo; elementi che sono anche un modo per dare malinconia al divertimento, una pacificazione finale, qualcosa che ammorsi la festa al luogo, approfondisca la sensazione, elimini quella leggerezza che spesso è solo chiasso.

Gli amusement park che, anche senza consapevolezza, sfruttano questo gioco di contrasto, appaiono divenire nuovo paesaggio, immaginifico.

\section{RIFERIMENTI BIBLIOGRAFICI}

Auricoste ISABelle, Parchi o utopie mortali?, "Ottagono" n. 99, Intrattenimento, 1991, pagg. 16-30.

BECK GREGORY, Un atlante dei parchi tematici, in "Lotus" n. 109, il nuovo mondo allegorico. Sui parchi tematici, Electa, Milano 2001, pagg. 104-117.

Cerver Francisco AsEnsio, Landscape of Recreation II: Amusement Parks, (World of Environmental Design, V. 6), Watson-Guptill Pubns, Slipcase edition 1998.

Cranz GAlen, Il parco della riforma negli Stati Uniti, in MoniQue Mosser, Georges TEYSSOT, L'Architettura dei giardini d'Occidente- Dal Rinascimento al Novecento, Electa, Milano 1990, pagg. 462- 465.

ECO UMBERTO, Travels in Hyperreality, Harvest/HBJ Book, United State of America 1990.

Howett CATHERIN, Attrezzature ricreative negli Stati uniti (1930-1940), in MONIQUE Mosser, Georges Teyssot, L'Architettura dei giardini d'Occidente- Dal Rinascimento al Novecento, Electa, Milano 1990, pagg. 506-508.

KASSON F. JOHN, Amusing the million. Coney Island at the turn of the Century, Hill \& Wang, New York 1978.

KoolhaAs Rem, The Generic City, S,M,L,XL, Taschen, Germany 1997, pagg. 1239-1267.

MERo Mara, Parco tematico. Iperreale fantastico commerciale walt disney of course!, in Desideri PaOlo E IlaRdi MASSIMO (a cura di), Attraversamenti. I nuovi territori dello spazio pubblico, Costa \& Nolan, Genova 1997, pagg. 120-148.

PONTE ALESSANDRA, Il parco pubblico in Gran Bretagna e negli Stati Uniti. Dal genius loci al "genio della civilizzazione", in MONIQUE MOSSER, GEORGES TEYSSOT, L'Architettura dei giardini d'Occidente- Dal Rinascimento al Novecento, Electa, Milano 1990, pagg. 369382.

SORKIN MiCHAEL, La tematizzazione della città, in "Lotus" n. 109, , Il nuovo mondo allegorico. Sui parchi tematici, Electa, Milano 2001, pagg. 7-17.

STANTON JEFFREY, Early Coney Island History (1609-1880), Early Coney Island History (1881-1903), Amusement Industry, Coney Island - Rides \& Shows List, Coney Island Transportation, in www.naid.sppsr.ucla.edu/coneyisland/histart.htm

Walker Peter, Simo Melanie, Invisible Gardens. The search for Modernism in the American Landscape, The MIT Press, Cambridge, Massachusetts 1996.

Wylson Anthony \& PAtricia, Theme Parks, Leisure Centres, Zoos and Aquaria, Longman, 1994.

\section{RIFERIMENTI ICONOGRAFICI}

Fig. 1: KASSON F. JOHN, Amusing the million. Coney Island at the turn of the Century, Hill \& Wang, New York 1978, pag 27. 
Fig. 2-4: STANTON JEFFREY, Early Coney Island History (1609-1880), Early Coney Island History (1881-1903), Amusement Industry, Coney Island - Rides \& Shows List, Coney Island Transportation, in www.naid.sppsr.ucla.edu/coneyisland/histart.htm.

Fig. 5: rielaborazione di Enrica Dall'Ara di foto Samuel H. Gottscho, KASSON F. JOHN, Amusing the million. Coney Island at the turn of the Century, Hill \& Wang, New York 1978, pag 84 .

Fig. 6: fotografia e disegno statua dell'Appennino in VEZZOSI ALESSANDRO (a cura di), $I l$ concerto di statue, Alinea, Firenze 1986, pag. 52 e pag. 74-illustrazione 45; diagramma di Robert Venturi in VENTURI ROBERT, SCOTT BROWN DENISE, IZENUOR STEPHEN, Imparando da Las Vegas. Il simbolismo dimenticato della forma architettonica, CLUVA editrice, 1985, pag. 28- illustrazione 23; foto dell'Elephant Hotel di Coney Island particolare da KASSON F. JOHn, Amusing the million. Coney Island at the turn of the Century, Hill \& Wang, New York 1978, pag 32.

Fig. 7: KASSON F. JOHN, Amusing the million. Coney Island at the turn of the Century, Hill \& Wang, New York 1978, pag 32.

Fig. 8- 9: fotografie di Enrica Dall'Ara.

Siti Web:

www.underthewalk.com/history.html 


\section{5 - MODELLO DISNEYLAND, PAESAGGIO READY-MADE}

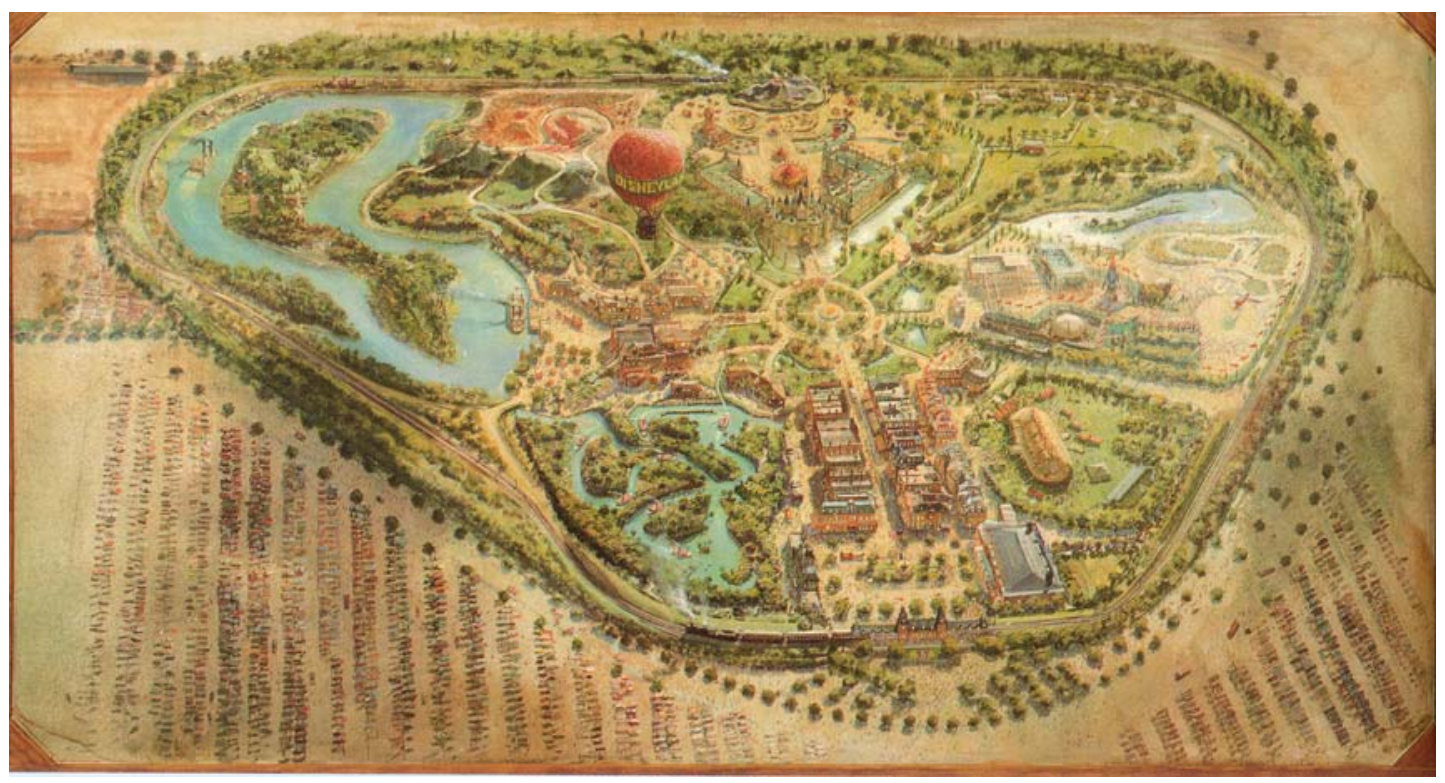

Fig. 1 Progetto per Disneyland ad Anaheim, California (1955), prospettiva a volo d'uccello con cui Walt Disney presenta al pubblico la sua idea per la costruzione del parco- disegno di Peter Ellenshaw.

Non è chiaro. Contrariamente alla volontà di Walt Disney di creare un ambiente semplice e rassicurante, protetto rispetto ai rischi che comportano complessità e contraddizioni, il prototipo Disneyland (Anaheim, California, U.S.A. 1955) appare essere tutto ed il suo contrario. Converte in tre dimensioni i disegni bidimensionali dei cartoni animati del suo autore. Intende inverare un mondo che non esiste in nessun luogo ed in nessun tempo nel momento stesso in cui si impone come modello reale, concreto, sociale, iconografico, di urbanizzazione. E' paesaggio culturale specifico della California del dopo seconda guerra mondiale, erede della vicina Los Angeles, sua costola e controfaccia, così come è utopia esportabile (esportata), senza adeguamenti o traduzioni, in qualsiasi territorio.

\section{IDEE PER DISNEYLAND NEL RETROSCENA}

L'origine del parco è l'arte cinematografica di Walt Disney: un modo personale di percepire lo spazio e un corpo di immagini che tenta di diventare luogo, materializzandosi, e assumendo come strumenti della traslazione, da pellicola ad architettura, alcuni precisi modelli che il contesto culturale contemporaneo mette a disposizione del suo autore. La prima idea di Walt Disney è "Kiddieland", nel 1951, in reazione alla noia suscitata negli anni '30 da visite a comuni amusement park accompagnando la figlia (incipit "ufficiale" ricorrente nella letteratura sul fenomeno Disney ed esplicita dichiarazione/propaganda di intenti), un parco didattico per bambini sulla storia americana. Si prefigura inoltre uno spettacolo itinerante "Disneylandia", consistente nella presentazione di scene animate tratte dalla storia e dal folclore americani.

Questi pensieri iniziali trovano una traduzione nei disegni per "Disneylandia", parco da realizzarsi nello spazio esterno al Walt Disney Studio in Burbunk quale set cinematografico con ruolo operativo, contemporaneamente luogo in cui la favola accade ed in cui il pubblico affascinato dai personaggi Disney può realmente incontrare Micky \& Co. Planimetria e schizzi del parco per il Burbank Studio sono interpretabili solo conoscendo i retroscena culturali e le passioni personali di Walt Disney (Fig. 2). 
Linea di forza è infatti una linea ferroviaria lungo la quale, creando un itinerario, sono disposti gli ambienti tematici. In quegli anni in America l'interesse per le tecnologie dei trasporti è molto diffuso, anche declinato nella forma della riproduzione e del collezionismo di modelli a scala ridotta. Nel 1948 Walt Disney visita la Chicago Railroad Fair - che si tiene negli spazi che nel 1933 avevano ospitato la Century of Progress Exposition - in occasione del centenario dell'ingresso nella città della prima locomotiva a vapore. L'allestimento dell'esposizione simula l'esperienza stessa del viaggiare, strategia ricorrente nelle fiere internazionali, resa maggiormente forte per il fatto che il movimento, per quanto contratto e non geografico, è fisico:

"World's Fairs had long exhibit artefacts from foreign cultures in picturesque "villages". In fact, the Railroad Fair occupied the portion of the old Century of Progress site once devoted to the Alpine, Hawaiian, and Spanish Villages. What was different about the 1948 railroad extravaganza was the coherence and concentration of the experience, the sensation of having taken the train on a whirlwind journey trough most the nation's beauty spots in a single day."

La ferrovia attraversa, sulla carta, i prototipi storici New town, Old town, Granny's Farm trasposizione dal set di So Dear to My Heart, film Disney del 1949, "Indian Village", confina un grande lago e la Bird Island, temi poi realizzati nel 1955, reinterpretati, in Frontierland e Adventurland a Disneyland in Anaheim (affiancato a questo nocciolo, all'ingresso, è un gruppo di attrazioni maggiormente "libere" - Fairgrounds, Carnival, Carousel, Picnic area- che non troveranno nessuna localizzazione nell'effettiva Disneyland).

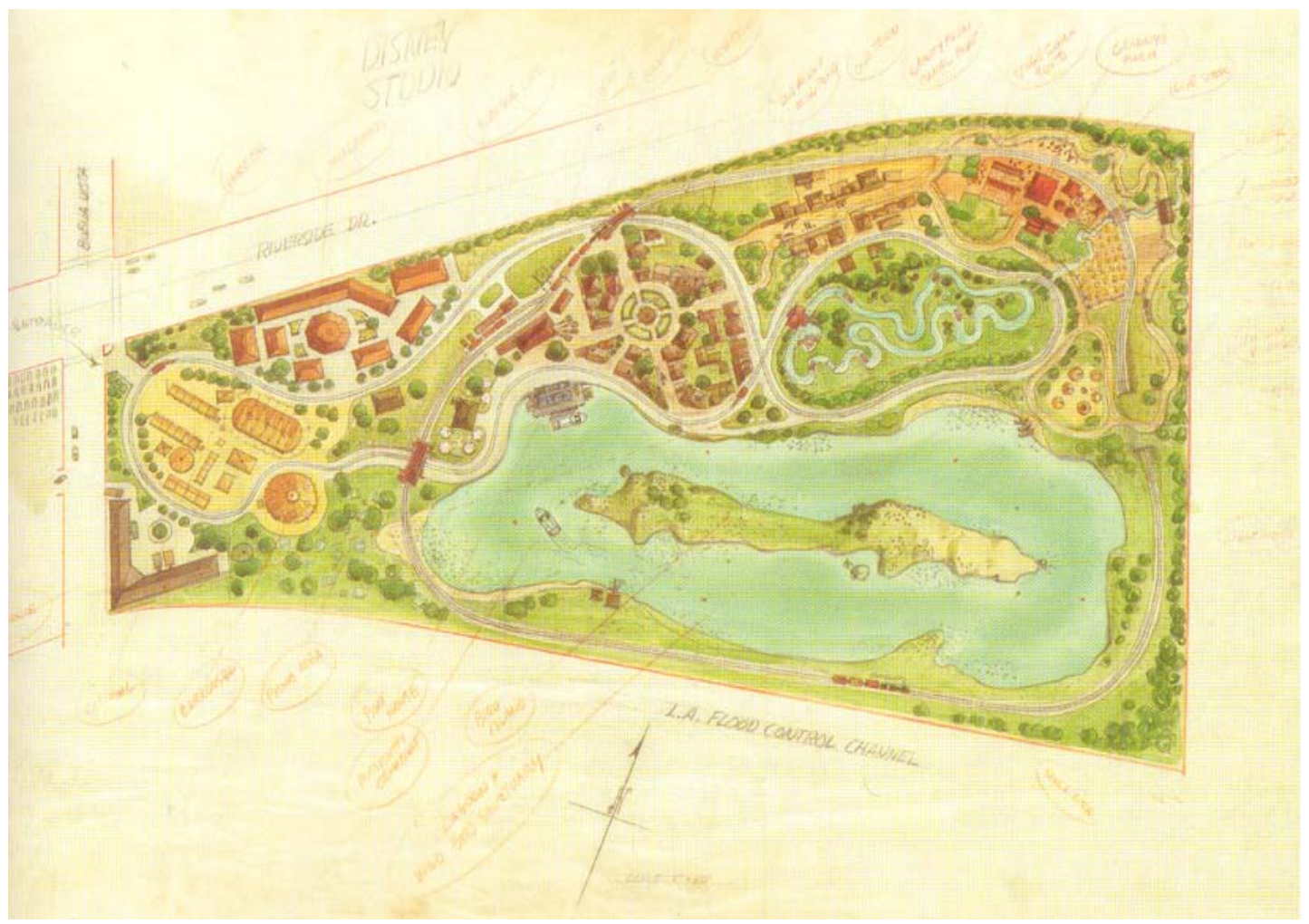

Fig. 2 Planimetria per un parco da localizzarsi a Burbunk nei pressi del Disney Studio, con annotazioni a penna probabilmente di Walt Disney. Disegno di Harper Goff (1951).

Altro motivo di fascino per Walt Disney sono le miniatures ovvero ambienti in scala ridotta (casa per le bambole) completi di ogni dettaglio, camere da guardare voyeristicamente, senza potervi avere accesso, un mondo lillipuziano perfetto quale sono state le Thorne Room

\footnotetext{
${ }^{1}$ Karal Ann Maling, Imagineering the Disney theme parks, in Karal Ann Maling (a cura di), Designing Disney's Theme Parks: The Architecture of Reassurance, Flammarion, Paris 1998, pag. 45.
} 
Miniatures - trentadue scatole aperte sul lato frontale riproducenti camere arredate in stile inglese e della prima America - presentate alla Golden Gate International Exposition di San Francisco del 1939, che Disney visita. Karal Ann Marling sottolinea la sfumatura peculiare del futuro così come presentato all’ Esposizione di San Francisco:

"This fair was less determinedly futuristic in style than its East Coast counterpart [the New York's World Fair, 1939]. A strange mixture of Latin American and vaguely Pacific motifs, with overtones of Art Deco streamlining, the architecture is best described as festive or fantastic. Turrets, castles, palaces, and towers sprouted everywhere. (...) San Francisco's tomorrow was, by and large, a reckless near future, premised on the homely verities of today. It was a domestic tomorrow of suburbs and ranch style homes."

Non è quindi un fatto casuale che Disney nel 1949 lavori alla progettazione di scene per Disneylandia, rooms con piccoli personaggi meccanici che danzano o cantano - Dancing Man e una bottega di barbiere con un gruppo di quattro abitués che formano un quartetto versioni "scultoree" dei musicals disegnati dei sui cartoni.

Fiere, railroad, miniatures.... costituiscono un insieme di possibilità di costruzione dello spazio. Sono forme e immagini semplici, con concetti spaziali associati: rispettivamente un territorio sufficientemente vasto per fondare una città in miniatura, compendio dello spazio pubblico molto "maneggevole"; una linea in movimento quale tessuto connettivo ed idea di progresso; un contenitore aperto - sipario, schermo televisivo - più piccolo del vero in cui sia possibile mostrare - show - un completo, esaustivo, microcosmo.

Queste forme sono a disposizione per la concretizzazione di un'utopia che si alimenta della fantasia dell'artista, di alcune istanze proprie del modernismo, di altre che cominciano all'epoca a contrapporsi a quest'ultimo, della volontà di affermare una identità californiana, del West America, diversa da quella dell'Est, capace di ottimismo, spontaneità, divertimento.

“(...) Modernism produced another version of the city, one more central to Disney’s American imaginings. The movement for garden cities, expostulated by Englishman Ebenezer Howard in his 1902 screed Garden Cities of Tomorrow, stands in approximately the same relationship to le Corbusier's Cartesian fantasies as English landscape gardening did to French in the eighteenth century. (...) Both, though, were versions of the pastoral, embracing the idea that the renaturalization of the "denaturated" city would strip it of its dread, that the reversion to the natural would have a salutary effect on human nature itself.

The garden city is the physical paradigm that presages Disney space, the park in the theme park."3

Michael Sorkin ${ }^{4}$ individua nella volontà di fuga rispetto alla congestione metropolitana, nell'organizzazione dei trasporti e della mobilità come base della forma della città, nella pianta a struttura radiale, nella scala a misura di pedone, gli elementi comuni di Disneyland e della garden city di Howard. Sottolinea come la tecnologia ed il modello della garden city fossero protagoniste in connubio alle fiere internazionali degli anni '30, la Century of Progress Exposition di Chicago del 1933 e la New York World's Fair del 1939, plasmate sulle idee di ordine, movimento e giardino.

Si aggiunge la volontà di Disney di promuovere un modello di urbanizzazione in grado di opporsi all'alienazione indotta dalle metropoli industriali, il riscontrato fallimento di luoghi per la ricreazione quali il Coney Island di New York, che presentano i medesimi problemi congestione, sporcizia, pericolosità, caoticità - degli spazi urbani.

"Disneyland offered a California-based critique not only of specific easter models but also of certain flaws in America civilation that eastern cities had helped to create. Coney Island's New York, in the eyes of Disney, was perhaps no more than the most extreme case of a broad national malaise: people had lost all sense of spontaneity and play. (...) Disney was intended as an antidote to the perceived urban malaise of the day. In being transported to some not very well defined golden age -perhaps the period 1900-1910 as presented along Main Street U.S.A., or the pioneer era suggests in Frontierland - and in touring "lands" devoted to fantasy, adventure, and the future,

\footnotetext{
${ }^{2}$ Karal Ann Maling, Op.cit., Flammarion, Paris 1998, pag. 36.

${ }^{3}$ Michael Sorkin, See you in Disneyland, in Michael Sorkin (a cura di), Variations on a theme park. The new American city and the and of public space, Hill and Wang, New York 1999, pag. 212.

${ }^{4}$ Ibidem.
} 
visitors could escape their unnatural present day care, "drop their defences", and "become more like themselves"'. 5

Con questo intento Disney sceglie - in seguito alla proposta presentatagli dallo Stanford Institut of Reserach, incaricato dello studio dell'opportuna localizzazione di Disneyland e sfumata l'idea di realizzarlo nelle vicinanze dello studio in Burbunk viste le dimensioni che il progetto, così come ideato, comincia a richiedere - un terreno nella periferia rurale di Los Angeles, in Orange County, dove è in corso la progettazione della Santa Ana Freeway ed il clima appare particolarmente mite ed idoneo alla vita all'aperto, non ventoso come nelle zone costiere.

Qui tabula rasa, poi il regno della magia e della fantasia: uno spazio camminabile (con le automobili relegate fuori del confine del parco) e comodo come era lo studio in Burbunk, che,m sfruttando tecnolgie e materiali d'avanguardia, dava il senso caldo degli ambienti domestici, con finiture dal sapore artigianale - come avevano le miniatures di Miss Thorne all'esposizione di San Francisco; i protagonisti Disney a passeggio; controllo dei dettagli e spaziale, chiaro sistema di orientamento.

\section{FICTION LANDS}

La struttura di Disneyland ad Anaheim risulta essere concisa. Dopo il varco d'ingresso immediatamente si percorre Main Street U.S.A., asse centrale di simmetria. La strada appartiene ad una città americana di inizio ' 900 proponendo la memoria nostalgica dell'Old time America. Il motivo è analogo al tema del vicino parco Knott's Berry Farm - più antico, risalente al 1920 - il cui proprietario, ristoratore, inizialmente per intrattenere i clienti in fila per gustare le specialità culinarie della moglie, aveva allestito una Ghost Town traslocando dai luoghi originali di provenienza, o riproducendoli, edifici appartenenti all'antica storia del West. Ghost Town restituisce qualcosa di autentico. Main Street U.S.A. è un disegno di facciate - studiate nello stile e nei colori secondo i valori dell'illustrazione e non dell'architettura - che si articola anche, stranamente, in profondità, consentendo di entrare attraverso le porte e curiosare all'interno di nuove, in scala reale, Miss Thorne's rooms.

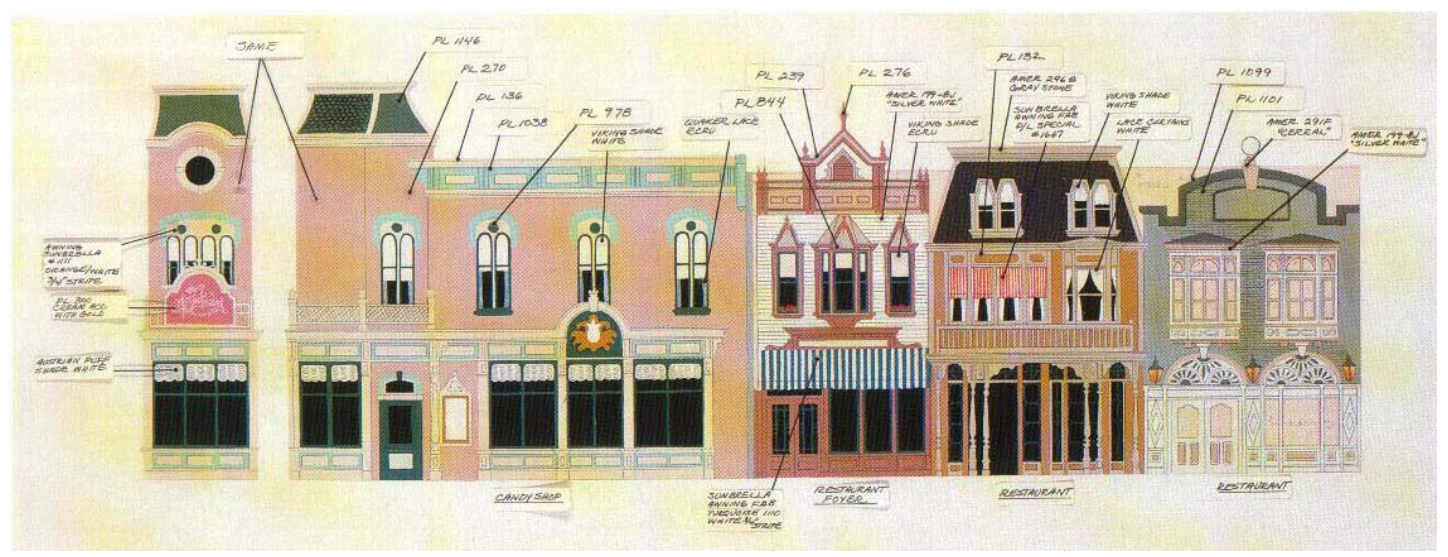

Fig. 3 Studio di prospetto per la Main Street U.S.A. a Disneyland, che ripropone una tipica strada americana dell'inizio '900. La scala degli edifici e l'uso del colore la rendono allo stesso tempo domestica ed irreale.

\footnotetext{
5 John M. Findlay, Disneyland: The happiest Place on Earth, in Magic Lands, Western City scapes and America Culture after 1940, University of California Press, Berkeley 1993, pag. 67.
} 
A dire il vero la scala non è precisamente reale. Le proporzioni di Main Street U.S.A. sono prospetticamente corrette, realizzate diminuendo il rapporto di scala rispetto alle dimensioni reali - 90 percento al piano terra, 80 al primo piano, dal 60 al 70 perento al secondo piano.

La via è un modellino curatissimo, eluso il rischio di qualsiasi associazione con uno spazio pubblico reale, mediante l'uso dei colori e lo studio dimensionale. Non ha il carattere della strada, sembra piuttosto di essere in un interno domestico. Questo dà il "benvenuto".

Sullo sfondo è il castello della Bella Addormentata che fa rimbalzare in un'ulteriore dimensione, quella fantastica, nella forma del miraggio o del sogno. Al termine di Main Street U.S.A. e antistante il castello c'è il grande spazio dello Hub, cuore del parco e stanza dei bottoni (telecomando):

"The Hub also diagrammed the concepts of choice and change. Like a Wednesday-night viewer, the tourist standing in the Hub at Disneyland was presented with a whole range of possibilities. Like an impatient viewer in front the set, the tourist could switch from one channel-oops! Land- to another in just a few steps. "I'm tired of museums and fairs where you have to walk your legs off," Walt insisted (...). This was not a linear, museum-like experience, one era shown after another, ad infinitum. (...)

The Hub is a giant TV set, showing pictures of what can be found at every side, previews of next week's show, or this afternoon's destination. "Don't touch that dial!" the announcer warns. Please do, says Disneyland. The circle inscribed at the centre of the park is the locus of change from Snow white's descent into a kind of cartoon nightmare to mark Twain's-eye-view of life on the nineteenth-century Mississippi, from a cruise up the crocodileinfested Amazon to a voyage to a distant star. Go ahead. Touch that dial!" 6

Gli ambienti a cui si accede dallo Hub sono, nella configurazione attuale (nel 1955 solo lo Hub metteva in relazione Frontierland, Adventurland, Fantasiland) collegati circolarmente, contigui senza presentare momenti di transizione. Non è necessario percorrere un' interdistanza, un viaggio minimo che sottolinei confini spaziali e temporali. E' solo un cambio di scena fra tema e tema. Si potrebbe parlare di Jumpcut urbanism ${ }^{7}$ alla scala ed ad opera di un trenino. Karal Ann Malin parla di discontinous narrative ${ }^{8}$ sottolineando come sia proprio il processo di tematizzazione, l'esistenza di un racconto, seppure articolato in più paragrafi, l'elemento che differenzia Disneyland dagli amusement parks americani che lo hanno preceduto.

"As if making movies, the designers of Disneyland stressed the transition from scene to scene. Rather than clash, the ideas built into the various zones or rides presented "an orderly sequence of messages".,"

Il racconto è in grado di potenziare la capacità dello spazio senza che si abbia la percezione di un costipamento, della "speculazione" che sfrutta il suolo moltiplicandone i piani. Il livello terra è di dimensioni contenute, ma vi si aprono molteplici divagazioni: itinerari interni alle architetture, nel sottosuolo, "in aria", non più da intraprendere a piedi ma su monorotaie, treni....ad una velocità accelerata attraverso spazi trasfigurati dalla scenografia. Buio, luci, musiche e rumori rendono impossibile riconoscere la struttura dell'involucro, la realtà degli spostamenti, la loro durata. E' la continuità del tema a fluidificare il passaggio fra il parco all'aperto e gli ambienti nascosti, fra passeggiata e rides.

\footnotetext{
${ }^{6}$ Karal Ann Maling, Op.cit., Flammarion, Paris 1998, pagg. 74-75.

${ }^{7}$ Il concetto è assunto da RiCHARD INGERSOLL, Jumpcut urbanism, "Casabella", n. 597-598, Il disegno degli spazi aperti, 1993, pagg. 52-57. Richard Ingersoll evidenzia una modalità di percezione dello spazio urbano che porta a quello che definisce Jumpcut urbanism, dovuta alla visione dall'automobile in movimento, fatta di "imprecisioni ed interruzioni", che "ricompone frammenti temporali attraverso un processo di montaggio" nel quale viene annullata l'unità di tempo e di spazio del teatro classico. "L'unità spazio-temporale condivisa dal palcoscenico prospettico e dallo spazio pubblico figurato è stata definitivamente sconvolta dall'introduzione dell'automobile.(...). In nota R. Ingersoll spiega: "Jumpcut" è termine di origine cinematografica: indica un improvviso stacco da un fotogramma all'altro, diversi fra loro per soggetto, per profondità o per senso".

${ }^{8}$ KARAL ANN Maling, Ibidem.

${ }^{9}$ John M. FindLAY, Op.cit., University of California Press, Berkeley 1993, pag. 69.
} 

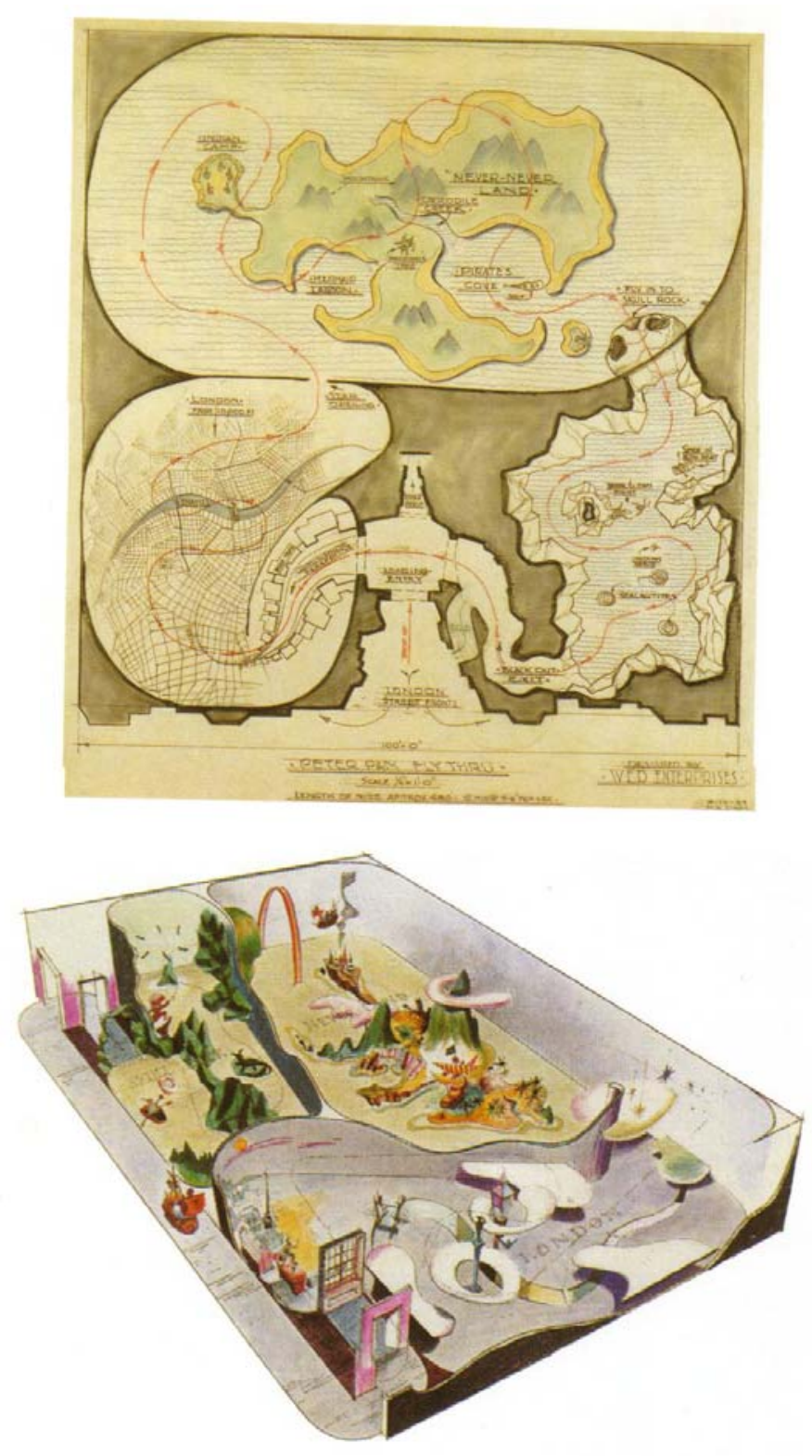

Fig. 4. Progetto per il Peter Pan Fly - planimetria e spaccato prospettico. Un accurato studio dimensionale e prospettico e l'utilizzo della luce consentono la creazione di spazi illusionistici e dilatati: tutto è simulazione per annullare la realtà dell'architettura all'interno della quale si svolge il "volo".

$\mathrm{Ci}$ si muove in uno spazio meandritico con ingresso al piano zero, livello semplice e razionale da cui parte l'avventura. Per capire meglio come funziona questo meccanismo è utile analizzare i disegni preparatori per il Peter Pan Fly: si entra in una casa di London Street, si sale a bordo di una nave in miniatura su rotaia, si parte. Si attraversa un corridoio che è, nella finzione, la strada da cui si è entrati, allestita in maniera tale da simulare l'inizio di un volo mediante una diminishing perspective (indicazione nel disegno) che fa vedere $\mathrm{i}$ tetti delle case sempre più distanti fino ad immettere in un ambiente il cui pavimento è una Londra minuscola (vista da 10000 piedi di distanza) su cui "si vola" sinuosamente, morbidamente e così via....per i luoghi conosciuti della fiaba (Fig. 4). 


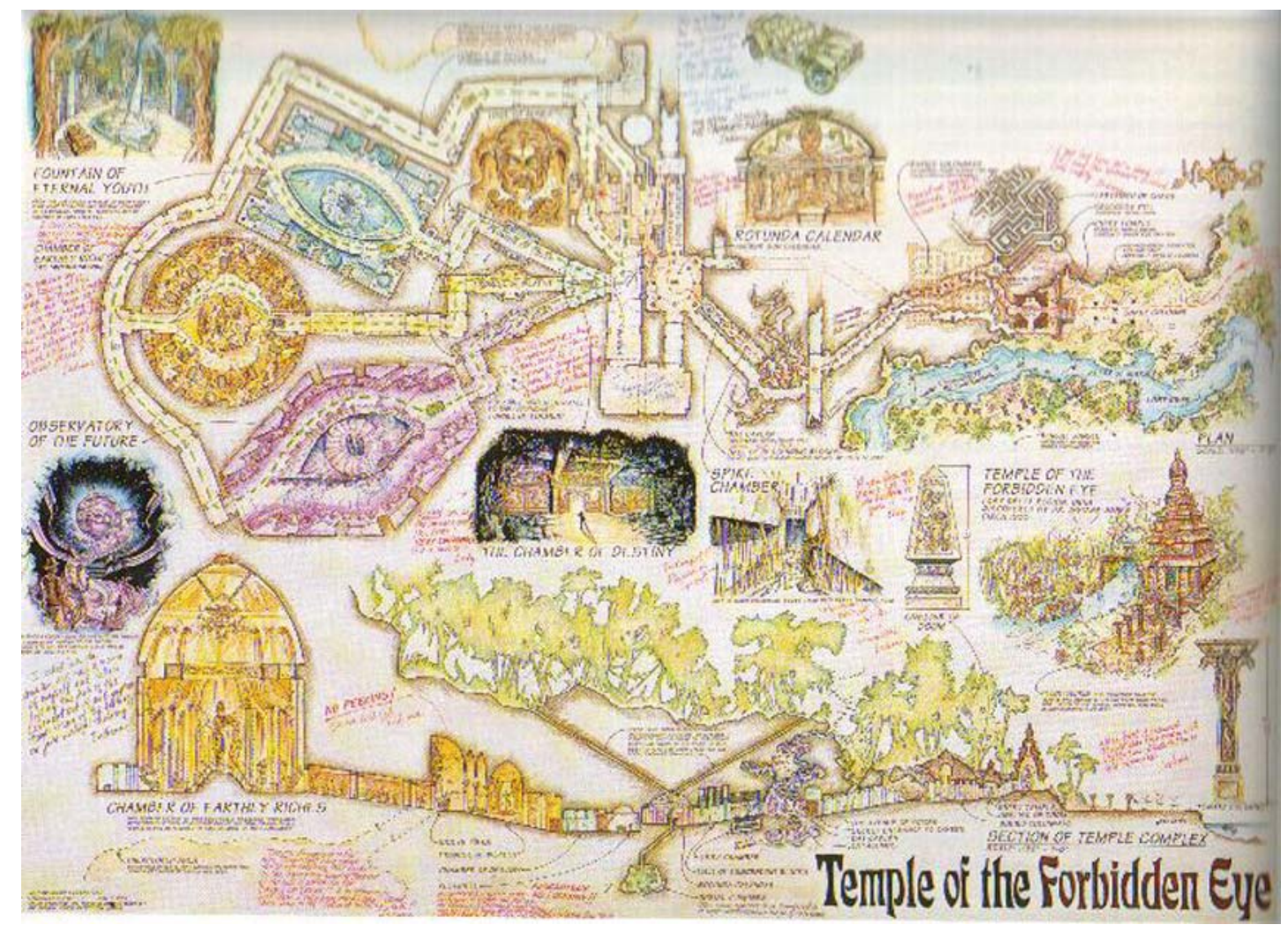

Fig. 5 progetto per il Temple of Forbidden Eye, all'interno dell'attrazione Indiana Jones. L'artcolazione degli spazi in planimetria ed in sezione consente di sfruttare al massimo lo spazio disponibile. Il piano terra del parco immette in "divagazioni" meandritiche in cui il racconto si svolge facendo perdere il senso del tempo e dello spazio.

\section{VISIONI ANTICIPATE}

Disneylandia è, prima ancora che il parco di Anaheim venga realizzato, anche un programma televisivo. Nella ricerca di finanziamenti per la costruzione di Disneyland Walt Disney fa un accordo con la $\mathrm{ABC}$ - American Broadcasting Company - con il quale , in cambio di 5 milioni di dollari da utilizzare per la propria impresa, egli si impegna a produrre una serie televisiva settimanale. Il programma inizia un anno prima dell'inaugurazione di Disneyland, nella primavera del 1954. E' un mezzo di propaganda fondamentale per il futuro successo del parco. L'esperienza dei visitatori non è così anticipata semplicemente dalla "confidenza" con le immagini dei cartoni animati di Disney ma anche dalla presentazione del parco stesso, mediante la quale si annulla ogni dubbio sugli aspetti pratici di un'eventuale giornata a Disneyland e si creano al contrario puntualissime aspettative al riguardo.

Disney, nella sua pretesa di conoscere cosa la gente desideri realmente essere e di fare in modo che lo possa essere all'interno del parco, trova i mezzi per trasformare immagini e modi in modelli:

"Like other aspects of material culture in the Far West, the theme park was both ahead of its time, in its innovations on the landscape, and perfectly in step with its time, in the values and assumptions built into the park. It was different enough to be novel, but not so unusual that those who Paid to visit it or who wish to adapt its innovations to other places and contexts found it strange or intimidating."10

\footnotetext{
${ }^{10}$ John M. FindLAY, Op. cit., University of California Press, Berkeley 1993, pag. 56.
} 
Di più. Il Disney way of thinking non solo risponde ai gusti e allo stile di vita della classe media, ma ha il potere di plasmarli - condizionamento sia estetico sia sociale - assottigliando lo strato protettivo che isola Disneyland, permettendo un trade off pervasivo sull'esterno.

Marc Augé è impressionato dal potere di questa anticipazione della visione, come aspetto inseparabile dalle componenti materiali del parco, nella comprensione del paesaggio Disneyland:

"Quando si arriva a Disneyland dalla strada (un amico aveva accettato di portarci e di venirci a prendere in serata) l'emozione nasce in primo luogo dal paesaggio. In lontananza all'improvviso, come sorto dall'orizzonte ma già vicino (esperienza visiva analoga a quella che permette di scoprire con un solo colpo d'occhio il Mont- Saint Michel o la cattedrale di Chartres), il castello della Bella Addormentata si staglia sul cielo, con le sue torri e le sue cupole, simile, stranamente simile alle foto viste sui giornali e alle immagini della televisione. Era senza dubbio questo il primo piacere di Disneyland: ci si offriva uno spettacolo del tutto simile a quello che ci era stato annunciato. (...) Qui, senza dubbio (ci riflettei in seguito), si trovava la chiave di un mistero che mi colpì immediatamente: perché c'erano tante famiglie americane che visitavano il parco, quando evidentemente avevano già visitato quelli simili d'Oltreatlantico? Ebbene, per l'appunto, esse vi ritrovavano quello che conoscevano già. Gustavano i piaceri della verifica, le gioie del riconoscimento."11

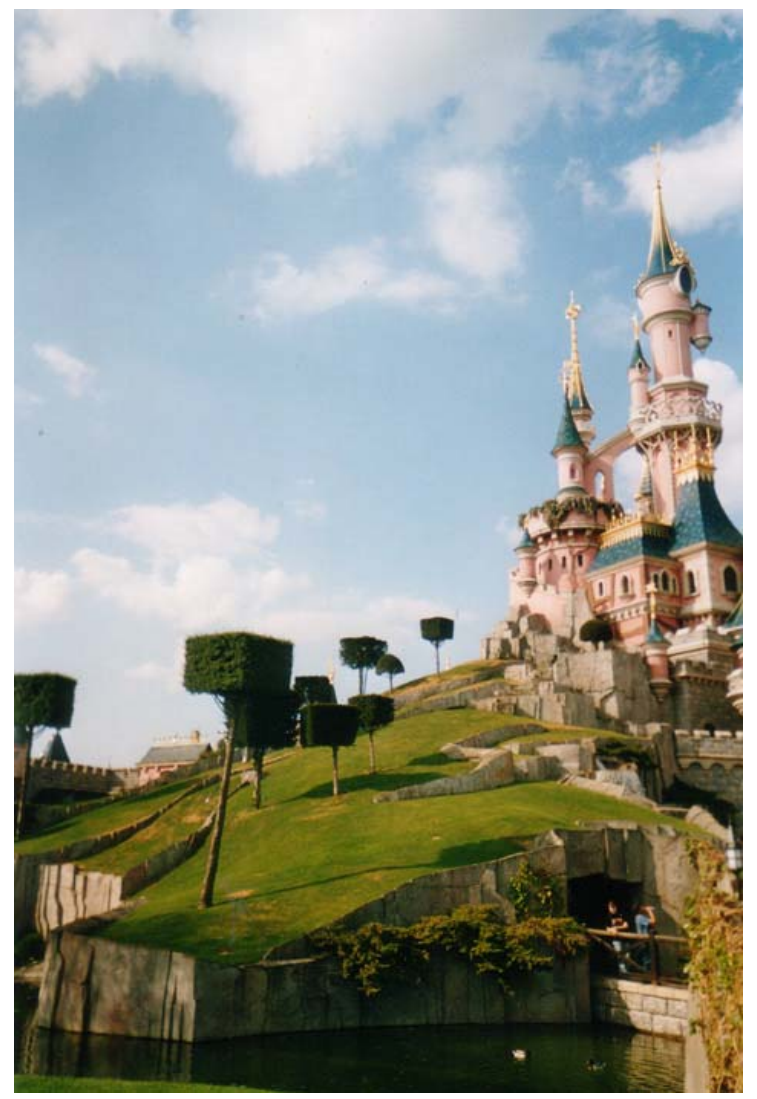

Fig. 6: il castello di Disneyland Paris, Paris, France (1992), quale "visione anticipata".

Il piacere del riconoscimento di cui parla Augé non è un'emozione scontata. Anzi richiede empatia nei confronti di un paesaggio figurativo così deterministicamente dato. L'intenzionale eliminazione di qualsiasi casualità, nell'organizzazione ma soprattutto nelle situazioni spaziali, che rende il parco un'esperienza lontana dalle ambiguità e contrasti dello spazio urbano, paradossalmente bandisce dal momento ricreativo quella spontaneità - di cui Disney lamenta l'impossibilità nelle metropoli americane - che appare invece maggiormente

\footnotetext{
${ }^{11}$ La descrizione di Augé si riferisce alla "copia" parigina (1992) del modello "originale" californiano (1955), in Marc AugÉ, Un etnologo a Disneyland, in MARC AugÉ, Disneyland e altri nonluoghi, Bollati Boringhieri, Torino 2002, pag. 18.
} 
riscontrabile proprio dove sia le architetture sia gli incontri sono spontanei, regolati da volontà eterogenee $\mathrm{o}$ incontrollati, ovvero nelle città reali.

Tematizzazione e anticipazione si rafforzano a vicenda riassumendosi quasi in un unico fenomeno: il tema è il mondo Disney, e tutto ciò che con firma Disney viene presentato dai media culturali rende leggibile l'ambiente del parco, lo informa. I personaggi e le scenografie del parco non significherebbero nulla se non fossero già fornite di senso in anticipo, nel pensiero del visitatore. Il tema coincide con l'identità del paesaggio ed il tema è un insieme di immagini fornite di un significato unico, genericamente noto.

"Disney indeed encouraged little that be considered casual. It meant to attract costumers for an entire day of amusement, during which they never become distracted or lost or bored. It proposed to dispense with the spatial disorganization, "surly attendants", unkempt grounds, and " carny show' hard sell", that reportedly prevailed at conventional amusement parks. Most important, Disneyland subordinated the identities of individual rides, exhibits, at others features to the larger, thematically coordinated environment. The result, Hill ${ }^{12}$ summarized, was "less an amusement park than a state of mind." 13

Ne Il paesaggio introvabile il geografo Raffestin, definendo come paesaggio il pensiero inerente un territorio, avvisa su quanto siano rilevanti le implicazioni del processo di pianificazione che a partire da un immagine (paesaggio nella mente) costruisce un territorio, il quale non viene quindi ad essere determinato da azioni spontanee di una comunità che utilizza un suolo ai fini della propria sussistenza, ricreazione, identificazione....

"Una volta il territorio era il prodotto organico della vita quotidiana, non era pianificato come oggi. Non significa che non esistevano delle norme e delle regole ma voglio dire che il disegno non esisteva prima di fare il territorio. Esistevano delle pratiche la combinazione delle quali produceva un territorio. Oggi, il territorio è progettato, disegnato e pianificato. Allora significa che il territorio prima di essere costruito è già un'immagine." ${ }^{4}$

Non è difficile intuire di quale consistenza possa essere un territorio costruito seguendo disegni che sono cartoni animati e fumetti, una visione che parte dalla fantasia del singolo e diviene patrimonio iconografico di massa, ed in cui le "pratiche" si riassumono nell'occupazione del tempo libero in divertimento e consumo di prodotti-marchio, pilotati da un status già ovvio e quasi, per questo, inconsapevole.

\begin{abstract}
"Reassurance and control (...) are not quality readily associated with the Modernist canon. At least not when control is placed in the service of reassurance. Making someone feel good is what greeting cards do, what the worst kind of kitsch does. But not art. Great art never tells you to have a nice day. (...) "If picture-postcard art moves people, then I like it" Walt told his eldest daughter in 1957. "If I'm corny, then millions of people in this country must be corny, too." (...) he did not believe for a moment that that art- his art, the picture-postcards kind - was obliged to be disturbing, challenging, unsettling." 15
\end{abstract}

Non esiste all'interno del parco, si può dire, alcun territorio. L'architettura è smontabile ed eliminati arredi e scenografie quello che resta è un lago artificiale e forse qualcosa della vegetazione esotica di Adventureland, scenario non molto diverso dalla tabula rasa che ha preceduto Disneyland nel 1955. Di territorio, invece, è possibile parlare riguardo all'esterno immediato su cui Disneyland ha potere reale di modificazione.

Quando infatti iniziano i lavori per la costruzione di Disney in Orange County, Anaheim è una piccola città nel paesaggio rurale di campi ad aranci, nella periferia di Los Angeles. La presenza di Disneyland non solo induce una progressiva urbanizzazione dell'Orange County ma rende progressivamente il parco stesso e Anaheim, trasformata alla stregua dei maggiori centri di affari, centri in grado di spezzare l'indifferenziazione dello sprawlscape di frangia di Los Angeles. Ponendosi come cesura (luogo segregato) - evidentissima se si osservano le

\footnotetext{
${ }^{12}$ Galdwin Hill, reporter del "New York Times", il discorso è tratto da "New York Times", Feb. 2, 1958, Oct. 4, 1959.

${ }^{13}$ JOHN M. FinDLAY, Op. cit., University of California Press, Berkeley 1993, pagg. 65-66.

${ }^{14}$ Claude Raffestin, Il paesaggio introvabile, in PaOlo CAStelnOvi (a cura di), "Il valore del paesaggio", Contributi al seminario internazionale, Torino, 9 giugno 2000, pagg. 25-36.

${ }^{15}$ Karal AnN Maling, Op.cit., Flammarion, Paris 1998, pagg. 83.
} 
fotografie del luogo risalenti al tempo in cui sono in corso gli interventi preliminari di "fondazione" del parco- esercita una forza coesiva enorme sul suo intorno. Los Angeles ha in Disneyland una controparte, la quale pur avendo segno contrario rispetto alla megalopoli californiana, frequentemente, nel tempo, corre il rischio di presentarne i medesimi problemi.

\section{RIFERIMENTI BIBLIOGRAFICI}

ABRAMS JANET , intervista a Michael Eisner, in "Ottagono", n. 99, giugno 1991, Intrattenimento, pagg. $38-72$.

AugÉ MARC, Disneyland e altri nonluoghi, Bollati Boringhieri, Torino 2002.

Auricoste IsABelle, Parchi o Utopie mortali?, in "Ottagono", n. 99, giugno 1991, Intrattenimento, pagg. 15-30.

AURICOSTE, ISABELLE, I parchi ricreativi in Europa: il divertimento e l'altrove, in MONique Mosser, GeOrges TeYssot, L'architettura dei giardini d'Occidente, Electa, Milano 1990, pagg. 491-502.

Chiaramente Giovanni, Dall'apparenza alla visione, in "Ottagono", n. 99, giugno 1991, Intrattenimento, pagg. 31-37.

ECo UMBerto, Dalla periferia dell'impero. Cronache da un nuovo medioevo, Bompiani, Milano 2003.

ECO UMBERTO, Travels in Hyperreality, Harcourt Brace Jovanovich, U.S.A. 1990.

EISNER D. MiCHAEL (foreword by), Imagineering. A Behind the Dreams Look at Making the Magic Real, Disney Editions, New York 1996.

Findlay John M., Disneyland: The happiest Place on Earth, in Magic Lands, Western City scapes and America Culture after 1940, University of California Press, Berkeley 1993, pagg. 52-116.

HOWETt CATHERIN, Attrezzature ricreative negli Stati uniti (1930-1940), in MONIQUE Mosser GeOrges Teyssot, L'architettura dei giardini d'Occidente, Electa, Milano 1990, pp. 491-502.

HunT JOHN DIXON, "Ut pictura poesis: il giardino e il pittoresco in Inghilterra (1710-1750), in MONIQUe Mosser, GeORges TEYssot, L'architettura dei giardini d'Occidente, Electa, Milano 1990, pagg. 227-237.

INGERSOLL RICHARD, Jampcut Urbanism, in "Casabella" n. 597-598, Electa, Milano1993, pagg. 96-97

Koening David \& Laura, Mouse under Glass: Secrets of Disney Animation \& Theme Parks, Bonaventure, 1997.

Maling Karal Ann, Designing Disney's Theme Parks: The Architecture of Reassurance, Flammarion, Paris 1998.

MERo MARA, Parco tematico. Iperreale fantastico commerciale walt disney of course!, in Desideri PaOlo E IlARDi MASSIMO (a cura di), Attraversamenti. I nuovi territori dello spazio pubblico, Costa \& Nolan, Genova 1997, pagg. 120-148.

RAFFESTIN CLAUDE, Il paesaggio introvabile, in PAOLO CASTELNOVI (a cura di), "Il valore del paesaggio", Contributi al seminario internazionale, Torino, 9 giugno 2000.

SANGUANINI BRUNO, Effetto benessere \& qualità della vita. A DisneyWorld, EuroDisney, Gardaland e altrove, in MINARDI EVERARDO, LUSETTI MARIALUISA (a cura di), I parchi del divertimento nella società del Loisir, Franco Angeli, Milano 1998, pagg. 86-127.

SOJA EDWARD W., Inside Exopolis: Scenes from Orange County, in MICHAEL SORKIN (a cura di), Variations on a theme park. The new American City and the end of public space, Hill and Wang, New York 1999, pagg. 94- 122.

SORKIN Michael, See you in Disneyland, in SORKIN MiChAel (a cura di), Variations on a theme park. The new American city and the end of public space, Hill and Wang, New York 1999, pagg. 205-232.

VARCELlONI MATTEO, Eterotopie della Compensazione. I parchi a tema Disney e le loro strutture alberghiere, in "Domus", n. 787, 1996, pagg. 35-39. 
Von Moos Stanislaus, La "sindrome di Disney", in "Domus", n. 787, novembre 1996, pagg. 4-8.

Wylson ANTHONY \& PATRICIA, Theme Parks, Leisure Centres, Zoos and Aquaria, Longman, 1994.

\section{RIFERIMENTI ICONOGRAFICI}

Fig. 1 : EISNER D. MICHAEL (foreword by), Imagineering. A Behind the Dreams Look at Making the Magic Real, Disney Editions, New York 1996, pag. 18.

Fig. 2: Maling Karal AnN, Designing Disney's Theme Parks: The Architecture of Reassurance, Flammarion, Paris 1998, pag. 51- illustrazione 31.

Fig. 3: Maling Karal AnN, Designing Disney's Theme Parks: The Architecture of Reassurance, Flammarion, Paris 1998, pag. 87- illustrazione 74.

Fig. 4: Maling Karal AnN, Designing Disney's Theme Parks: The Architecture of Reassurance, Flammarion, Paris 1998, pag. 63- illustrazioni 43-44.

Fig. 5: Maling Karal AnN, Designing Disney's Theme Parks: The Architecture of Reassurance, Flammarion, Paris 1998, pag. 116- illustrazione 111.

Fig. 6: fotografia di Enrica Dall'Ara. 


\section{TEMATIZZAZIONE E $A U R A$}

La scelta di assegnare un tema ad un parco o a un paesaggio può seguire intenti differenti. Spesso è legata alla volontà di valorizzazione, di un patrimonio naturale e/o culturale, a cui si accompagna il fine di intrattenere i suoi "fruitori". Per questo il campo della tematizzazione è sia espandibile alla dimensione della città e del territorio, sia contraibile alla dimensione della singola architettura. Come esemplificato da Francoise Choay in L'allegoria del patrimonio $^{l}$, all'intento di valorizzazione fanno seguito operazioni di conservazione e restauro, di inquadramento scenografico, di animazione, di modernizzazione, di conversione in moneta del valore in oggetto, per cui questo ultimo viene segnalato, comunicato, ostentato con i mezzi dell'impresa culturale e ai fini del turismo culturale. In tale direzione la tematizzazione è una modalità di coinvolgimento di un pubblico di massa, poiché ha il potere di semplificare il processo di comprensione: porta alla distrazione nella fruizione, come intesa da Benjamin in L'opera d'arte nell'epoca della sua riproducibilità tecnica ${ }^{2}$. La distrazione è un'esigenza propria del momento ricreativo e quindi anche del muoversi e vivere all'interno di un parco. Allo stesso tempo per il suo carattere di sottolineatura la tematizzazione altera il modo di percepire la realtà.

Si tenta di comprendere i mezzi e i modi di costruzione del tema e ci si interroga sulla capacità del tema di manifestare l'aura di un luogo - come ad esempio avviene nelle creazioni di Ian Hamilton Finlay, le cui istallazioni trasfigurano il paesaggio mediante evocazioni letterarie e pittoriche lo trasferiscono nei domini dell'arte - o di sottrarla, nel segno del "più visibile del visibile, l'oscenità di quello che non racchiude più un segreto ed è completamente solubile in informazione e comunicazione."3

Si vuole capire quale rapporto può esistere fra tematizzazione ed identità dei luoghi e fra tematizzazione e divertimento: è intuitivo pensare che assegnare un tema ad un luogo possa essere un'operazione che consente il divertimento al suo interno, se il tema è di fantasia, esotico, se immette in una dimensione diversa dal solito; ma è anche facile pensare che il tema possa divenire un modo per "costringere" il visitatore all'interno di uno spazio completamente codificato e persuasivo, mettendolo nell'impossibilità di divertirsi.

Questa doppia possibilità di tema "divertente" e tema "noioso" come entra in relazione con lo spazio e con la struttura fisica del luogo?

Il tema appare essere tanto più divertente quanto più consente un estraniamento dal contesto: è utile scoprire quali siano i modi di estraniamento dal contesto che amplificano, e non impoveriscono, i significati del paesaggio in cui il tema si inserisce.

Walter Benjamin pone l'accento sul fatto che l'aura dell'opera d'arte è legata all'hic et nunc, "alla sua esistenza unica e irripetibile nel luogo in cui si trova"4. Qualcosa di simile è possibile dire per l'aura di un paesaggio, anche se per il paesaggio il nunc è dilatato a comprendere la memoria e le aspettative.

\footnotetext{
${ }^{1}$ FrANCOISE CHOAY , L'allegoria del patrimonio, a cura di Ernesto d'Alfonso e Ilaria Valente, Officina Edizioni, 1995.

${ }^{2}$ Walter Benjamin, L'opera d'arte nell'epoca della sua riproducibilità tecnica, Einaudi, Torino 1966 e 1991.

${ }^{3}$ Jean Baudrillard.

${ }^{4}$ Walter Benjamin, Op.cit., Einaudi, Torino 1991, pag. 22.
} 


\section{1 - LA COSTRUZIONE DEL TEMA: POP-ART, RETORICA POST- $M O D E R N$ ED ESTRANIAMENTO DAL CONTESTO}

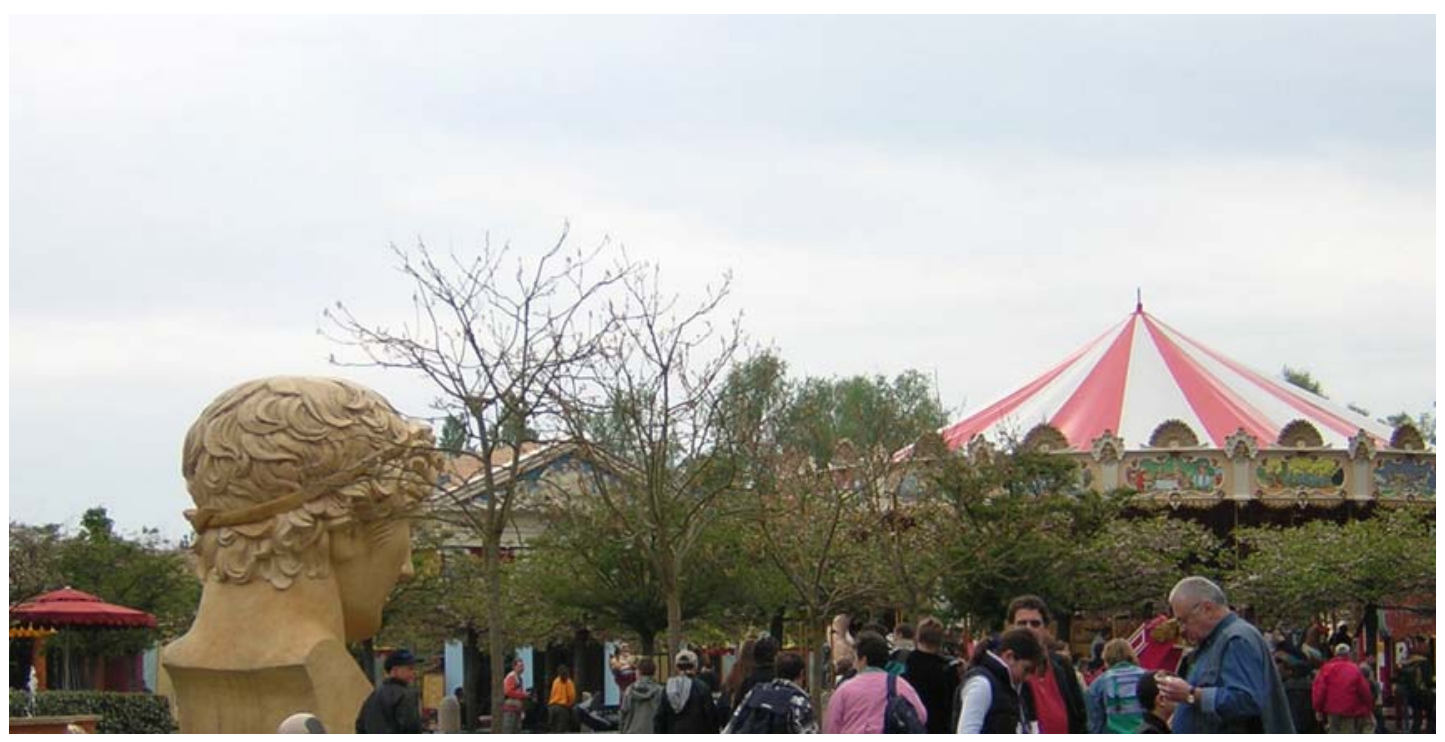

Fig. 1 Parc Asterix, Plailly, France (1989). La grande testa pseudo-scultura romana è una figura retorica - è sia citazione, sia metonimia - e contrasta iconograficamente con il Carrousel de César di cui si vede la copertura sullo sfondo.

All'interno del giardino manierista, dei pleasure gardens di matrice inglese, come dei parchi tematici contemporanei, l'esplorazione dello spazio da parte del visitatore è guidata. Gli strumenti a cui si fa ricorso in questa operazione di pilotaggio dei modi di fruizione del parco, sono i medesimi che sottendono qualsiasi processo narrativo, ovvero una successione di immagini/ simboli correlati da un percorso/struttura: il parco a tema è un luogo imperniato sul linguaggio. E siccome "il discorso" che si svolge al suo interno è finalizzato ad operare nel visitatore una dissociazione rispetto all'ordinarietà dell'esperienza quotidiana, nel linguaggio si fa un abile uso delle forme retoriche più suadenti, divertenti e destabilizzanti: la metonimia (la presentazione di una parte per il tutto), la citazione, l'enfasi, l'ossimoro (accostamento dei contrari) ecc., che consentono fra l'altro alterazioni e divagazioni dalla linearità dell'itinerario concettuale e/o spaziale.

L'utilizzo di queste figure retoriche all'interno dei parchi del divertimento non è affatto casuale: è interessante notare come esse siano riconducibili agli "aspetti della fantasia" presentati da Bruno Munari in Fantasia ${ }^{l}$.

Prima di parlare dei vari aspetti, Munari dà una definizione di fantasia, mettendola in relazione con i concetti di invenzione, creatività, immaginazione:

"La fantasia è la facoltà più libera delle altre, essa infatti può anche non tenere conto della realizzabilità o del funzionamento di ciò che ha pensato. E' libera di pensare qualunque cosa, anche la più assurda, incredibile, impossibile." 2

Mentre l'invenzione è una fantasia finalizzata "ad uso pratico", non preoccupata del lato estetico, la creatività è progetto, un "uso finalizzato della fantasia (...) e dell'invenzione, in modo globale" ${ }^{3}$. L'immaginazione è la rappresentazione della fantasia.

\footnotetext{
${ }^{1}$ Bruno Munari, Fantasia, Universale Laterza, prima ed. 1977, Roma-Bari 1998.

2 Bruno MunARI, Op.cit., Universale Laterza, prima ed. 1977, Roma-Bari 1998, pag. 21.

${ }^{3}$ Bruno Munari, Ibidem.
} 
Gli aspetti della fantasia sono tutti dipendenti dall'estraniamento dal contesto e dal cambiamento rispetto alla norma:

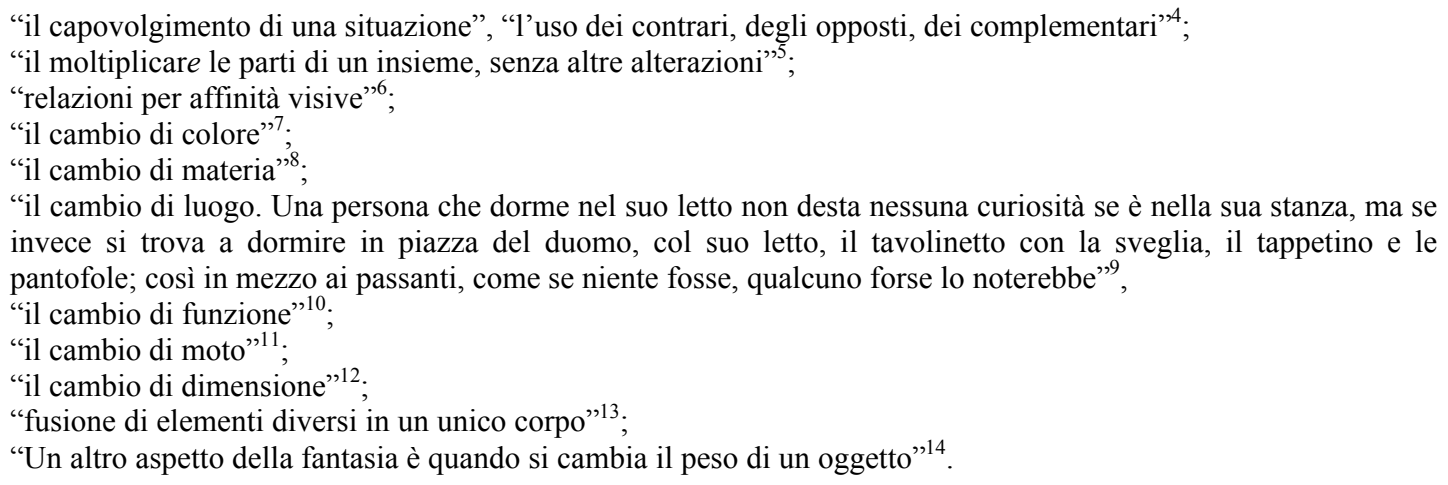

Si è detto dell'accostamento dei contrari nel giardino manierista di Pratolino (ad esempio nella compresenza degli automi minuscoli e dell'Appennino gigante, dell'acqua quale elemento tecnologico e dell' acqua quale elemento simbolico ecc.), del potere destabilizzante della dimensione impossibile della Colonna in rovina alla folie Desert de Retz (secolo XVIII), del continuo " cambio di luogo" alle esposizioni universali, in cui architetture e paesaggi da tutto il mondo si trovano affiancati in uno spazio esiguo. Costantemente nel tempo i luoghi vocati al divertimento ricorrono agli espedienti elencati da Bruno Munari, per esplicitare che si tratta di mondi della fantasia.

L'estraniamento dal contesto è l'elemento cardine della poetica della Pop-Art, che si sviluppa dalla fine degli anni ' 50 . Ne è consapevole Munari quando sceglie in prevalenza fotografie di quadri surrealisti, dadaisti e pop, ad illustrazione dei vari "aspetti della fantasia". La Pop-Art parte dall'oggetto già visto, conosciuto , esperienziato quotidianamente, il ready-made, ri-proposto mediante "cambio di luogo", "cambio di colore", ecc.. I prodotti della cultura di massa divengono oggetti d'arte che la cultura di massa può comprendere, appunto per la familiarità che intercorre nei confronti dell'oggetto.

L'oggetto così riproposto rimane ambiguo: è vero o falso?

All'interno del discorso sul "cambio di funzione", Munari inserisce una sorta di racconto dal titolo "Vero o fantastico?" che come un carosello svolge una serie di immagini e di azioni relazionati ad oggetti la cui funzione non corrisponde alla forma, forma che a sua volta è coerente con la funzione di altri oggetti che presentano però una forma incoerente alla propria funzione, e così via....senza tregua di immagini sbilanciate:

"Si apre la cassaforte ed ecco il bar illuminato con le sue mille bottiglie di Whisky Bernardoni, di Vodka Buganza e altre specialità pregiate. Beviamo. Evviva. Apriamo un armadio veneziano del Settecento ed ecco la televisione che si accende automaticamente. Da un secchiello per il ghiaccio a forma di foot-ball prendiamo, con la pinzetta a forma di zampe di gallina, un cubetto di ghiaccio. Si intende che la pinzetta è ( o sembra) d'oro. Da un paralume fatto di carta da musica e sostenuto da un lungo flauto nero, scende una luce dorata ( le lampadine a forma di candela restano nascoste). Con un cavatappi a forma di porco apriamo una bottiglia di Barolo Gazzoni mentre posiamo la cenere della sigaretta in un portacenere a forma di mano femminile, di ceramica dorata. (...)" ${ }^{15}$

\footnotetext{
${ }^{4}$ Bruno MunARI, Op.cit., Universale Laterza, prima ed. 1977, Roma-Bari 1998, pag. 38.

${ }^{5}$ Bruno Munari, Op.cit., Universale Laterza, prima ed. 1977, Roma-Bari 1998, pag. 49.

${ }^{6}$ BRunO MunARI, Op.cit., Universale Laterza, prima ed. 1977, Roma-Bari 1998, pag. 63.

${ }^{7}$ Bruno MunARI, Op.cit., Universale Laterza, prima ed. 1977, Roma-Bari 1998, pag. 68.

${ }^{8}$ BRuno MunARI, Op.cit., Universale Laterza, prima ed. 1977, Roma-Bari 1998, pag. 71.

${ }^{9}$ BRUno MunARI, Op.cit., Universale Laterza, prima ed. 1977, Roma-Bari 199, pag. 79.

${ }^{10}$ Bruno MunARI, Op.cit., Universale Laterza, prima ed. 1977, Roma-Bari 1998, pag. 83.

${ }^{11}$ Bruno MunARI, Op.cit., Universale Laterza, prima ed. 1977, Roma-Bari 1998, pag. 86.

${ }^{12}$ Bruno Munari, Op.cit., Universale Laterza, prima ed. 1977, Roma-Bari 1998, pag. 91.

${ }^{13}$ BRuno Munari, Op.cit., Universale Laterza, prima ed. 1977, Roma-Bari 1998, pag. 107.

${ }^{14}$ BRunO MunARI, Op.cit., Universale Laterza, prima ed. 1977, Roma-Bari 1998, pag. 113.

${ }^{15}$ Bruno MunARI, Op.cit., Universale Laterza, prima ed. 1977, Roma-Bari 1998, pag. 84.
} 
Il racconto continua analogamente ancora a lungo e se alla lettura ogni oggetto sembra estraniato, è altrettanto vero che la vita quotidiana è fatta di frequente di gesti analoghi con cui si manipolando oggetti dello stesso genere. L' "estraniamento" dell'oggetto porta ironia, demistificazione, presa di coscienza, che sono sempre aspetti legati al divertimento. Per questo i parchi del divertimento presentano oggetti estraniati, non molto diversamente da quanto compie la Pop-Art. Gli oggetti del racconto di Munari non sono affatto dissimili da quelli di Meret Oppenheim, o di Man Ray o di Claes Oldemburg.

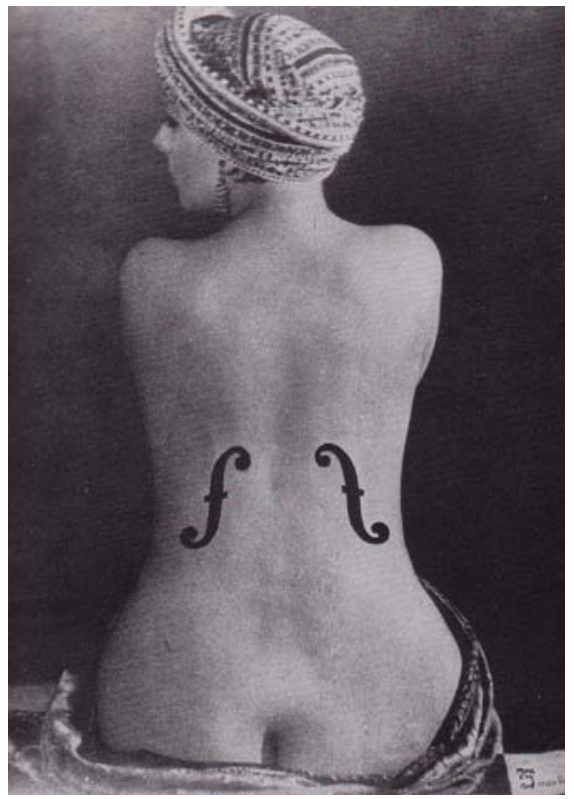

Fig. 1 “Affinità visive": Violon d'Ingres, Man Ray (1924) - la schiena di una donna evoca un violoncello, sono sufficienti pochi segni grafici per evidenziarlo.

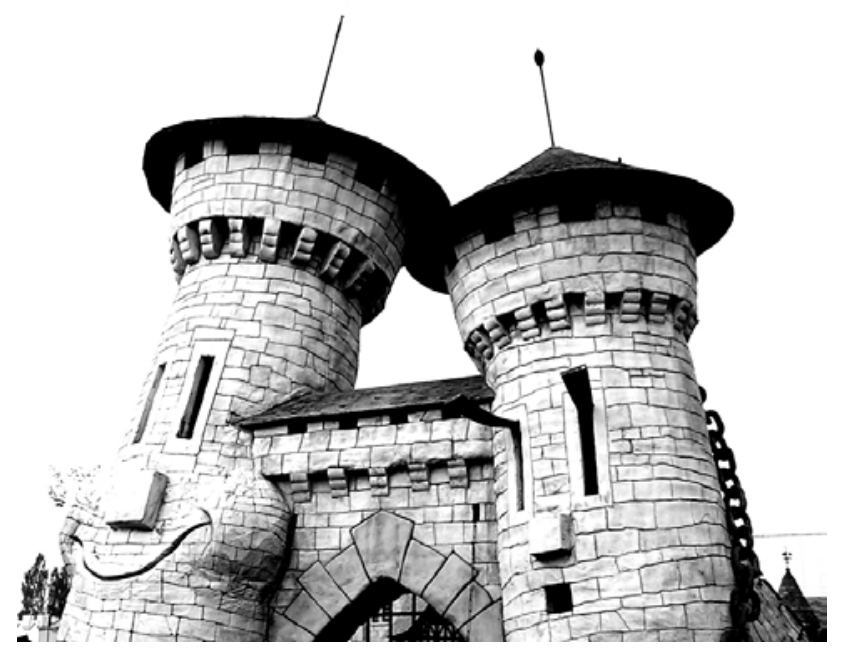

Fig. 2 "Affinità visive" : castello a Parc Asterix, Plailly, France.

Non sono diversi neppure dalle tazzine del Paese delle Meraviglie a Fantasyland a Disneyland (California), che sono i sedili di una giostra, e le rispettive variazioni sul tema sparse in tanti parchi del divertimento, o dallo Snoopy sdraiato sul tetto di un negozio al parco Kontt Barry Farm (California) che è una lampada per illuminare l'ingresso (e ribadire il nuovo tema del vecchissimo parco risalente al 1920).

La Pop -Art richiede la presenza di un'osservatore, non come accessoria, ma come parte del processo di straniamento degli oggetti, che si basa sulla percezione: Roland Barthes a 
proposito della Pop-Art parla di "messa in scena" - nuovamente ci si trova di fronte, nel comprendere i riferimenti che stanno dietro alla semantica e all'estetica dei parchi tematici, all'idea del teatro:

“(...) Il pop è un'Arte perché, nel momento stesso in cui sembra rinunciare ad ogni significato, accettando di riprodurre solo le cose nella loro banalità, mette in scena, usando certi procedimenti che gli sono propri e che costituiscono uno stile, un oggetto che non è la cosa e neppure il suo senso, ma che è: il suo significante ; o meglio il Significante. (...) Del resto, se l'opera dell'artista pop rientra nella sfera dell'Arte non è solamente perché l'artista mette in scena il significante, ma anche perché quell'opera è guardata (e non più soltanto vista): il pop può ben spersonalizzare il mondo. Appiattire gli oggetti, disumanizzare le immagini, sostituire l'artigianato tradizionale della tela con un macchinismo: alla fine rimane sempre "un po' di soggetto". Quale soggetto? Quello che guarda, in mancanza di quello che fa. Certo si può fabbricare una macchina, ma chi la contempla non è una macchina: è uno che desidera, che ha paura, che si delizia, che si annoia, ecc."16

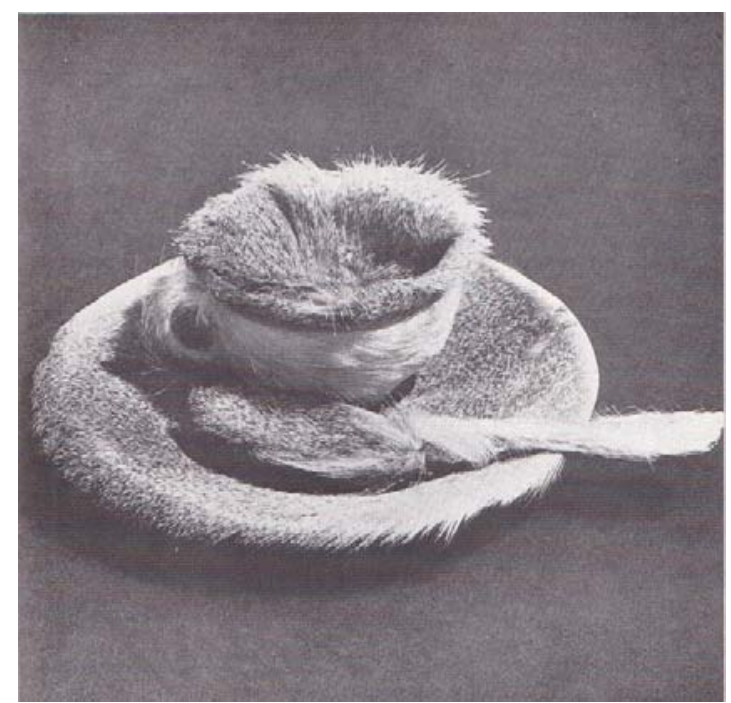

Fig. 3 Oggetto senza titolo, Meret Oppenheim (1936). Museum of Modern Art di New Tork

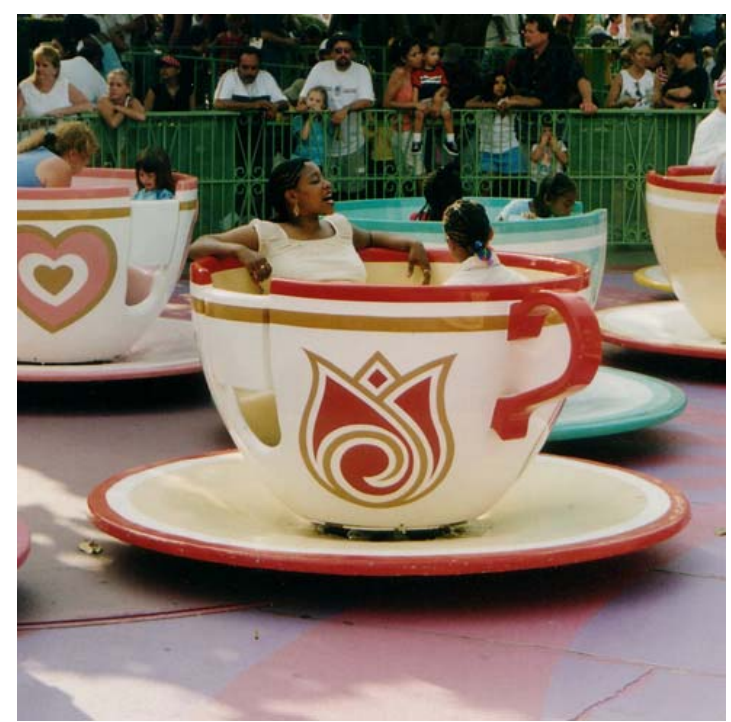

Fig. 4 tazzina di Alice nel Paese delle Meraviglie a Fantasyland, Disneyland, California, U.S.A. (1955)

\footnotetext{
${ }^{16}$ Roland Barthes, L'arte, questa vecchia cosa, in Pop Art. Evoluzione di una generazione, catalogo, Electa, Mialno 1980, cit. in LEA Vergine, L'arte in trincea, lessico delle tendenze artistiche 1960-1990, Skira, Milano 1999, pag. 73 .
} 
La disinibizione nell'assunzione degli oggetti per manipolarli e per metterli in relazione l'uno con l'altro a fini evocativi, in una certa misura accomuna Pop-Art e Postmodern. In entrambe le correnti l'originalità si esprime più nel sottoporre a variazione il materiale già disponibile, piuttosto che nell'invenzione ex-novo.

Nel contrapporre l'architettura "eroica e originale" del movimento moderno a quella " brutta e ordinaria" del postmoderno, che ha valore di simbolo e si presenta quindi arricchita di significato, anche Robert Venturi, architetto fra i più importanti esponenti e teorizzatori dell'architettura postmoderna, collega il Postmoderno alla Pop-Art, parlando proprio di estraniamento dal contesto:

“Una certa ricchezza può derivare dall'architettura convenzionale. Per 300 anni l'architettura europea è consistita in variazioni sulla regola classica: un ricco conformismo. Questa la si può ottenere per mezzo di un aggiustamento della scala o del contesto di elementi famigliari e convenzionali, al fine di innescare insoliti significati. Gli artisti Pop usarono insolite giustapposizioni degli oggetti di ogni giorno in vivaci giochi ricchi di tensioni tra vecchie e nuove associazioni di idee, per ironizzare sull'interdipendenza quotidiana fra contesto e significato, offrendoci una nuova interpretazione degli oggetti del XX secolo. L'oggetto familiare che sia leggermente estraniato dal suo contesto possiede un potere strano e rivelatore."17

Sia Bruno Munari che Robert Venturi evidenziano il ruolo fondamentale che assume la variazione su cose già conosciute:

"Il prodotto della fantasia, come quello della creatività e della invenzione, nasce da relazioni che il pensiero fa con ciò che conosce". ${ }^{18}$

(Presentate queste sottolineature, da ora in poi si esclude che la causa per cui i parchi tematici rischiano di essere "utopie noiose", luoghi in cui la fantasia è totalmente impoverita, sia la famigliarità con le immagini e con il tema dispiegati al loro interno.)

Anche Lea Vergine nel definire il Postmoderno evidenzia componenti che denunciano una certa analogia con la Pop-Art, le idee di remake e di copia :

"Per la pittura e la scultura, il "dopo -moderno" ha il senso di un ritorno all'ordine, al quadro, alla pittura di narrazione, sia pur rivisitata da taluni con ironia e disincanto. Non è più un passato su cui ci si interroga, si rifà semplicemente: si va dal revival al remake, dalla regressione alla copia (...)."19

Achille Bonito Oliva ${ }^{20}$ parla, in relazione all'utilizzo delle immagini nell'arte manierista di "un'economia dello spreco, dell'accumulo e dell'ecclettismo capaci di fondare uno stile che lavora sulla frantumazione." ${ }^{21} \mathrm{La}$ considerazione è ugualmente riferibile agli amusement park americani sorti dal XIX secolo in poi, ed è perfetta se volta alla descrizione del paesaggio di Las Vegas indagato, nel rapporto fra semantica ed estetica, da Robert Venturi ${ }^{22}$. Se nell'arte il ripiegare sulla ridondanza dell'immagine è sintomo, nelle varie epoche in cui si presenta, di sfiducia nel suo valore progettuale e manifesta la ricerca di un piacere consolatorio per la propria perduta positività, nella costruzione del parco-paesaggio è, quasi in senso opposto, volontà di sperimentazione tecnologica e di superamento del dato naturale/reale, che si accompagna all'ottimismo sulla possibilità di inverare l'impossibile grazie al supporto della tecnologia - sfida sottesa dagli automi del giardino di Pratolino (XVI sec.), dai roller coaster ( XIX sec.), dai paesaggi virtuali della contemporaneità.

\footnotetext{
17 Robert Venturi, Denise Scott Brown, Stephen Izenuor, Imparando da Las Vegas. Il simbolismo dimenticato della forma architettonica, CLUVA editrice, 1985, pag. 93.

${ }^{18}$ BRUnO Munari, Op. cit., Universale Laterza, prima ed. 1977, Roma-Bari 1998, pag. 29.

${ }^{19}$ Lea Vergine, Op. cit., Skira, Milano 1999, pag. 237.

${ }^{20} \mathrm{Il}$ critico Achille Bonito Oliva è stato attento osservatore e teorico delle correnti artistiche del Postmodern.

${ }^{21}$ AChille Bonito Oliva, L'ideologia del Traditore - Arte, maniera, manierismo, Electa, Milano, 1998, pag. 10.

22 si rimanda ai testi Robert Venturi, Denise Scott Brown, Izenuor, S., Imparando da Las Vegas. Il simbolismo dimenticato della forma architettonica, CLUVA editrice e ROBERT VENTURI, Complessità $e$ contraddizioni nell'architettura, Dedalo, Bari, 1980.
} 
"In qualche modo l'immagine produce un senso all'insensatezza della realtà e mette in scena la passione, anzi la crea a partire dall'esemplarità della propria presenza. La consapevolezza di questa disparità produce nell'artista uno stato di esaltazione che ha portato verso l'intensa idea di un'onnipotenza creativa che si sviluppa sempre più ossessivamente mediante la sperimentazione di nuove tecniche e di nuovi materiali.",23

Il piacere di vedere la natura superata dalla tecnologia o dall'artificio, se si vuole recuperare un termine che rimanda all'arte dei giardini, è probabilmente archetipico nell'uomo. ${ }^{24}$

L'utilizzo che gli architetti del Postmodern fanno della tecnologia è del tutto differente rispetto a quanto proposto dal movimento moderno: abbandonata l'idea che il valore estetico sia intrinseco al processo tecnologico stesso, il quale data una funzione determina la forma, l'architettura postmoderna non associa alla tecnologia razionalità e standardizzazione. Piuttosto nella compagine culturale del Postmodern la tecnologia diventa uno strumento al servizio del superamento - quanto a perfezione nella riproduzione - del dato autentico, e parallelamente è uno strumento per la costruzione di simboli, ovvero per la costruzione di temi.

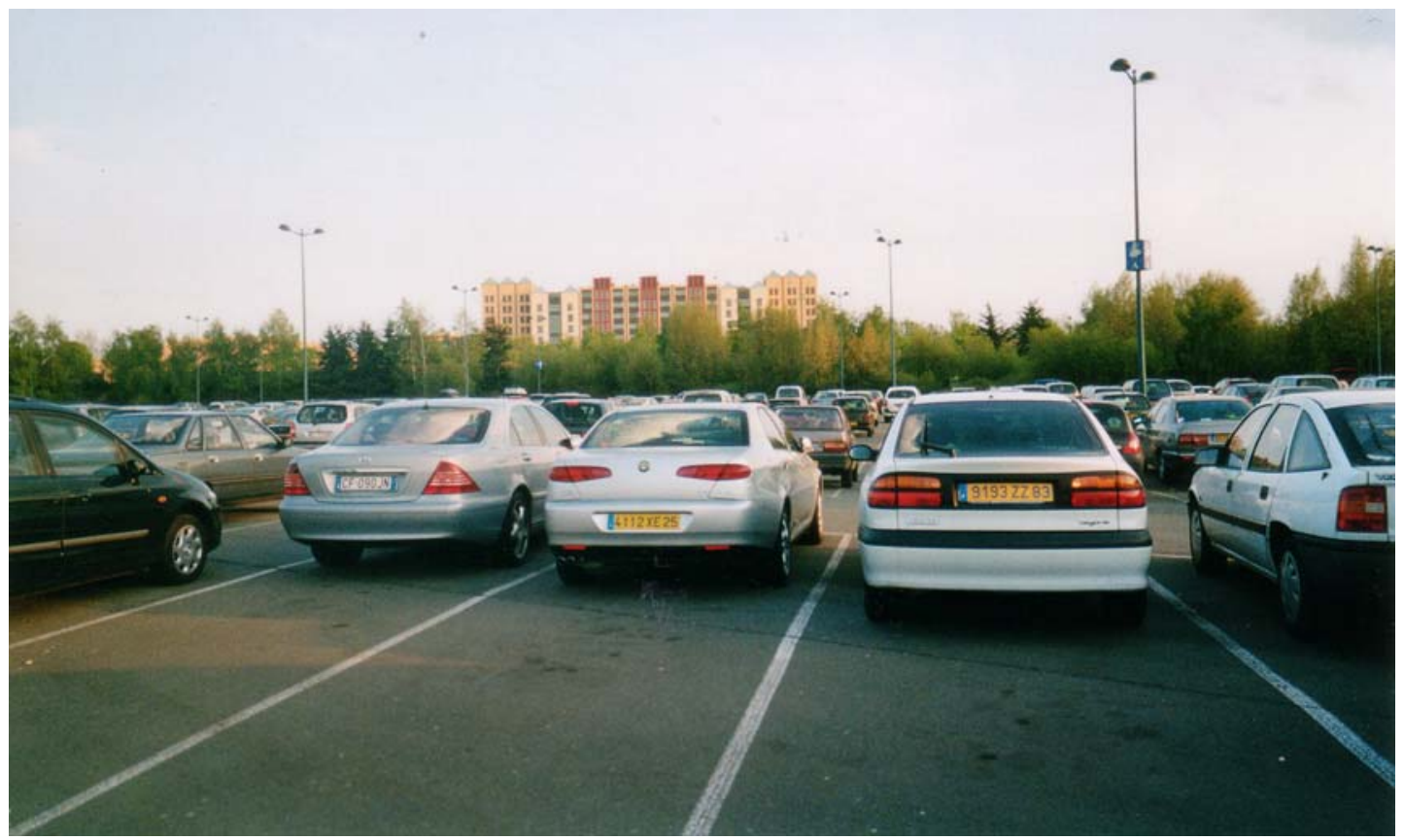

Fig. 5: parcheggi del Disneyland Paris, Paris, France. Sullo sfondo il New York Hotel del Disneyland Resort, di Michael Graves, quale architettura allusiva.

Non è un caso che la Disney si avvalga di architetti che ricorrono agli aspetti tecnologici in maniera "diseconomica" esasperandoli a fini espressivi come avviene nel Guggenheim di Bilbao - ed egualmente al Walt Disney Concert Hall - di Frank O. Gehry, oppure che li applicano in decorazioni-simboli dal sapore artigianale, come fa Arata Isozaki nel Team Disney Building (Orlando, Florida, 1990) e Michael Graves nello Swan Hotel, nel Dolphin Hotel, e nell'Office Building di Burbank (California, 1986) in cui i sette nani in dimensioni colossali sono posti quali cariatidi.

Anche Anthony a Patricia Wylson mettono in relazione parchi tematici e architettura del movimento postmoderno, parlando di ecletic and evocative ${ }^{25}$, ad introduzione di Theme Parks, Leisure Centres, Zoos and Aquaria ${ }^{26}$ :

\footnotetext{
${ }^{23}$ Achille Bonito Oliva, Op. cit., Electa, Milano, 1998, pag. 17.

24 Per un discorso inerente le conseguenze dell' "istinto" alla riproduzione e superamento dell'originale naturale (o artistico) si rimanda al testo UMBERTO ECO, Travels in Hyperreality, Harcourt Brace Jovanovich, U.S.A. 1990.

${ }^{25}$ The ecletic and evocative è il titolo del primo paragrafo del testo citato.

${ }^{26}$ Antony \& PAtricia Wylson, Theme Parks, Leisure Centres, Zoos and Aquaria, Longman, 1994.
} 
"In the years following World War II, contrary to the purist attitude encouraged by the modern movement (to dissociate from historic styles), the commercial appeal and esteem status conveyed by eclectic designed continued.

Revivalism and stylistic pluralism are now playing a significant part in contemporary architecture. (...) The desire to communicate diverse cultures or visual images of other countries, cultures or history, either as a caricature in a theme park or re-created in a live museum, is a justifiable indulgence in historic simulation.,27

Evocativo ed eclettico non sono concetti molto distanti da quelli di inclusivo e allusivo proposti da Robert Venturi in Imparando da Las Vegas. Il simbolismo dimenticato della forma architettonica:

"allusione e commento sul passato o sul presente o sui nostri grandi luoghi comuni o vecchi cliché, nonché inclusione dell'ambiente quotidiano, sacro e profano: questo è ciò che manca all'architettura moderna dei nostri giorni",28

Robert Venturi all'interno del suo più esteso ragionamento sul ruolo delle insegne, dell'araldica, della fisiognomica in architettura, presenta come nuove idee di architettura il duck (papera- scultura) e lo shed (capannone) decorato, così commentato da Maurizio Sabini (Fig. 6):

"Lo shed decorato è un modo di intendere l'architettura più rivolto all'idea e all'immagine, alla tematicità che ogni opera dovrebbe sviluppare e all'attento controllo della percezione che di essa avrà il suo fruitore. L'idea dello shed decorato ha richiesto, ovviamente, a Robert Venturi e a Scott Brown un'attenzione tutta particolare sul ruolo del simbolo in architettura, in quanto strumento primo di comunicazione, esplicita, ambigua e ironica, per un'architettura di rappresentazione, ordinaria, convenzionale e, in ultima analisi, anche economica." 29
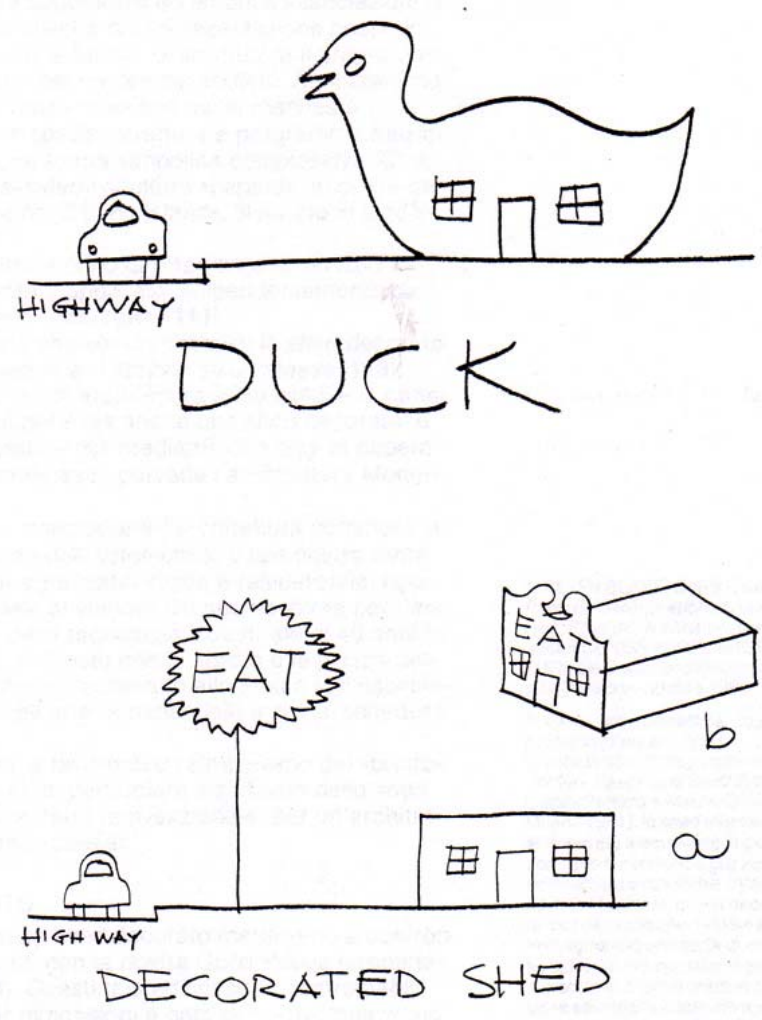

${ }^{27}$ ANTONY \& PATRICIA Wylson, Op. cit., Longman, 1994, pag. 1.

28 Robert Venturi, Denise Scott Brown, Stephen IzenUor, Imparando da Las Vegas. Il simbolismo dimenticato della forma architettonica, CLUVA editrice, 1985, pag. 65.

${ }^{29}$ Robert Venturi, Denise Scott Brown, Stephen Izenuor, Op.cit., CLUVA editrice, 1985, pag. 76. 
Fig. 6 Schizzo per la rappresentazione di due idee di architettura: il duck (papera- scultura) e lo shed decorato, entrambi esempi di architettura di allusione e comunicazione: nel primo il messaggio è dato dall'immagine che diventa forma dell'architettura, nel secondo dall'insegna sovrapposta come decorazione alla facciata.

Il simbolo/tema esplicita l'intenzionalità che sottende il progetto, cosa che orgogliosamente Robert Venturi dichiara, nel prendere come esempio di shed decorato la sua Guild House:

"la presunzione dell" "ordine gigante" sul fronte, la composizione simmetrica, da "palazzo", con i suoi tre piani monumentali (così come i suoi sei piani reali), conclusa alla sommità con un pezzo di scultura - o di quasiscultura - "allude" a qualcosa di eroico e di originale. E' vero che in questo caso la facciata eroica e originale è in qualche modo ironica, ma è questa giustapposizione di simboli contrastanti - l'applicazione di un ordine di simboli su di un altro - che costituisce per noi lo shed decorato. Questo è ciò che rende la Guild House uno shed decorato da un architetto - e non un' architettura senza architetti. ${ }^{30}$

L'ultima frase denuncia che l'utilizzo del simbolo in architettura è un modo per fare realmente progettazione. Un'idea analoga risulta da un'intervista del $1991^{31}$ a Michael Eisner, presidente e direttore esecutivo della Walt Disney Company. Egli interrogato sul significato della parola tema, afferma: "Si tratta soltanto di un eufemismo per definire un progetto. (...) Tema è sinonimo di progettazione". Il tema è più propriamente l'enfatizzazione del progetto, è un progetto non nascosto e non taciuto, al contrario è un progetto fondato sull'eloquenza, tanto più efficace quanto più pervasiva.

\section{RIFERIMENTI BIBLIOGRAFICI}

AUGÉ MARC, Nonluoghi, Elèuthera, Milano 1993.

BARTHES ROLAND, Miti d'oggi, Einaudi, Torino 1974.

BAUMAN ZYGMUNT, La società dell'incertezza, Il Mulino, Bologna 1999.

BECK GREGORY, Un atlante dei parchi tematici, in "Lotus" n. 109, Il nuovo mondo allegorico. Sui parchi tematici, Electa, Milano 2001, pagg. 104-117.

BENJAMIN WALTER, L'opera d'arte nell'epoca della sua riproducibilità tecnica, Einaudi, Torino 1966 e 1991.

BONAZZI GABRIELE (a cura di), L'estetica nel pensiero contemporaneo (dal romanticismo al surrealismo), G. D’Anna, Firenze 1980.

BONITO OliVA ACHILle, L'ideologia del Traditore - Arte, maniera, manierismo, Electa, Milano, 1998.

CHOAY FRANCOISE, L'allegoria del patrimonio, a cura di Ernesto d'Alfonso e Ilaria Valente, Officina Edizioni, 1995.

Desideri PaOlo, Ilardi Massimo (a cura di), Attraversamenti. I nuovi territori dello spazio pubblico, Costa \& Nolan, Genova 1997.

ECO UMBerto, Dalla periferia dell'impero. Cronache da un nuovo medioevo, Bompiani, Milano 2003.

ECO UMBERTO, Travels in Hyperreality, Harcourt Brace Jovanovich, U.S.A. 1990.

KracAuer SiEgFried, Montagne russe - l'illusione metropolitana, in "Casabella", n. 673/674, U.S.A., Architettura come spettacolo, Electa, Milano dicembre 1999- gennaio 2000. LYOTARD JEAN-FRANCOIS, la condizione postmoderna, Feltrinelli, Milano 1981.

MotT TONy, Giardini e fotografia, in MONIQUe MOSSER, GEORGES TEYssot, L'architettura dei giardini d'Occidente, Electa, Milano 1990, pagg. 491-502.

MUNARI BRUNO, Fantasia, Universale Laterza, prima ed. 1977, Roma-Bari 1998,

NiCOLIN PIERLUigI, Architettura "light", in "Lotus" n. 105, 2000

OCKMAN JOAN E ADAMS NiCHOLAS, Forme dello spettacolo, in "Casabella", n. 673/674, Architettura come spettacolo, Electa, Milano dicembre 1999- gennaio 2000, pagg. 4-7.

\footnotetext{
${ }^{30}$ Robert Venturi, Denise Scott Brown, S. IZenuor, Op. cit., CLUVA editrice, pag. 75.

${ }^{31}$ JANHET ABRAMS, Intervista a Michael Eisner. Nuovi progetti della Walt Disney Company, in “Ottagono” n. 99, Intrattenimento, 1991, pagg. 39-72.
} 
OCKMAN JOAN E AdAMS Nicholas, La città delle insegne: Times Square, in "Casabella", n. 673/674, Architettura come spettacolo, Electa, Milano dicembre 1999- gennaio 2000, pagg. 24-31.

Patrizia Mello, Lo spazio come incidente di percorso, in Patrizia Mello, Metamorfosi dello Spazio. Annotazioni sul divenire metropolitano, Bollati Boringhieri, Torino 2002, pagg. 67-105.

PATRIZIA Mello, Luoghi di Evasione: parchi o utopie mortali?, in PATRIZIA Mello, Metamorfosi dello Spazio. Annotazioni sul divenire metropolitano, Bollati Boringhieri, Torino 2002, pagg. 22-37.

RAJCHMAN JOHN, Effetto Bilbao, in "Casabella", n. 673/674, Architettura come spettacolo, Electa, Milano dicembre 1999- gennaio 2000.

SORKIN MICHAEL, La tematizzazione della città, in "Lotus" n. 109, Il nuovo mondo allegorico. Sui parchi tematici, Electa, Milano 2001, pagg. 7-17.

VENTURI ROBERT, SCOTt BROWN DENISE, IZENUOR STEPHEN, Imparando da Las Vegas. Il simbolismo dimenticato della forma architettonica, CLUVA editrice, 1985.

VENTURI ROBERT, Complessità e contraddizioni nell'architettura, Dedalo, Bari 1980.

Venturi RoBert, ScotT Brown Denise, Las Vegas postclassica, in "Domus", n. 787, novembre 1996, pagg. 9-10.

VERGINE LEA, L'arte in trincea, lessico delle tendenze artistiche 1960-1990, Skira, Milano 1999.

Siti web:

Post modern, in www. Francescomorante.it/pag_3/318.

\section{RIFERIMENTI ICONOGRAFICI}

Fig. 1, 3, 5-6 : fotografia di Enrica Dall'Ara.

Fig. 2: MunARI BRUNO, Fantasia, Universale Laterza, prima ed. 1997, Roma-Bari 1998, pag. 67.

Fig. 4: MunARI BRUNO, Fantasia, Universale Laterza, prima ed. 1997, Roma-Bari 1998, pag. 73 ,

Fig. 7: VentURi RoBert, SCOTT BRown Denise, Las Vegas postclassica, in "Domus", n. 787, novembre 1996, pag. 70, illustrazione 113. 


\section{2 -TEMA come \\ DETERRITORIALIZZAZIONE/DIACRONIZZAZIONE. Variazioni sul modello Las Vegas}

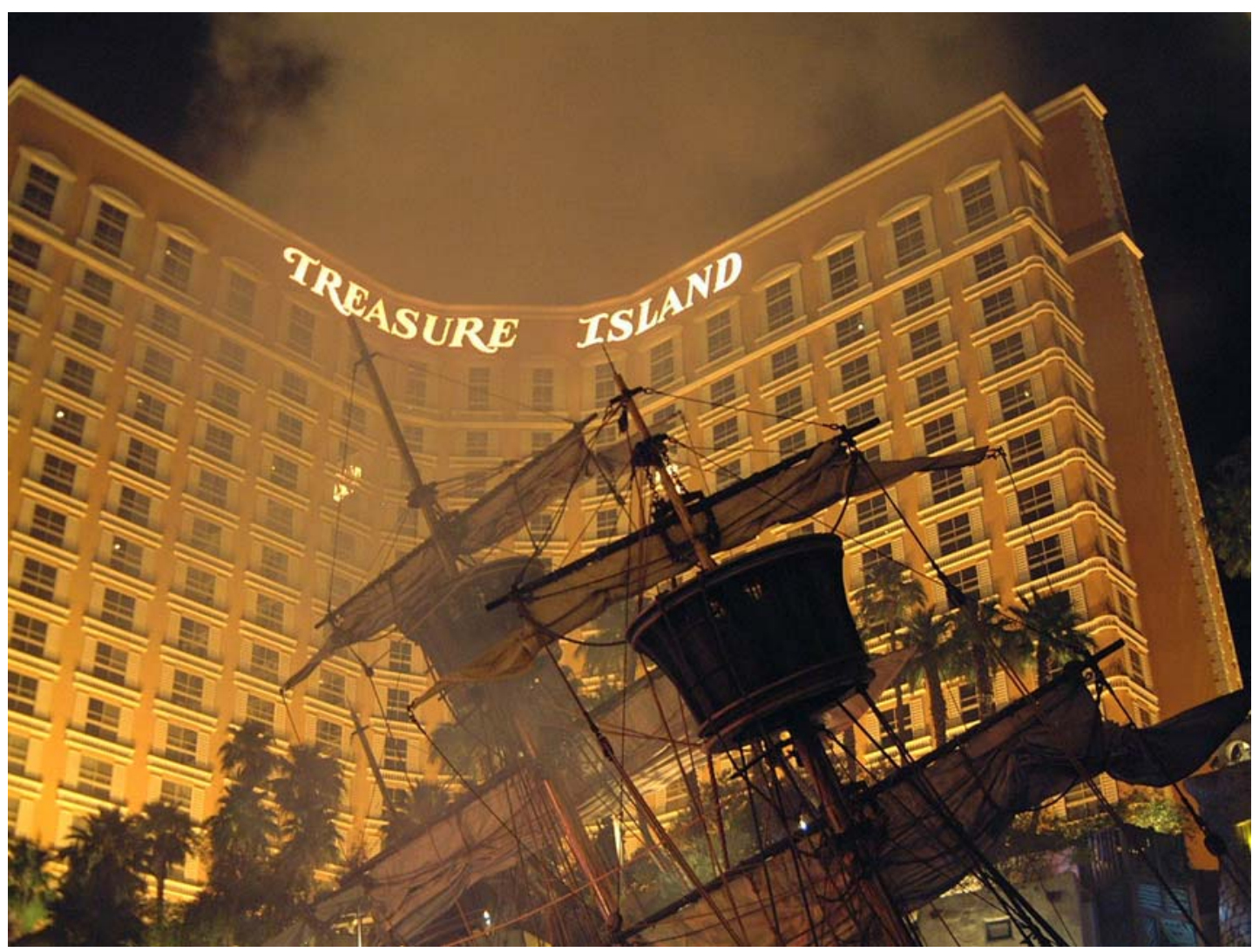

Fig. 1 Las Vegas: la nave affonda colpita dai pirati (spettacolo del Treasure Island nel paesaggio di un paese caraibico ricreato nella piazza antistante L'hotel-casinò, visibile sullo fondo).

Di frequente la tematizzazione è un mezzo per annullare le specificità geografiche e temporali di un luogo-avvenimento, processo che si può definire di deterritorializzazione e di diacronizzazione, intendendo con i due termini rispettivamente, sradicamento dal territorio e attualizzazione di elementi di epoche temporalmente slegate, passate e future.

La Strip di Las Vegas è probabilmente l'esempio più rilevante di luogo determinato da oggetti-marchio e da falsi d'architetture, che riassume tutti $i$ tempi nell'unità di spazio e tutti i luoghi nell'unità di tempo (la giornata della visita). Circondata dal deserto del Nevada, al confine con la California, sembra essere il sogno di un folle, costituita di frammenti di tutto ovunque. Forse si può parlare di Las Vegas come "non luogo" nei sensi riproposti da Patrizia Mello ${ }^{1}$ in riferimento alla definizione di Marc Augé2:

“Il non luogo è un tipo di spazio non identitario ne' relazionale ne' storico".

\footnotetext{
${ }^{1}$ Cfr. Patrizia Mello, Luoghi di Evasione: parchi o utopie mortali?, in Patrizia Mello, Metamorfosi dello Spazio. Annotazioni sul divenire metropolitano, Bollati Boringhieri, Torino 2002, pagg. 22-37. e PATRIzIA Mello, Lo spazio come incidente di percorso, in PATRIZIA Mello, Op. cit., Bollati Boringhieri, Torino 2002, pagg. 67105.

${ }^{2}$ Espresso in MARC AUGÉ, Nonluoghi, Eleuthera, Milano 1993.
} 
Attualmente Las Vegas è una città vera e propria, come ricordato da Umberto Eco in La città degli automi $i^{3}$, con una downtown e una periferia, con aree residenziali, attività commercialiricreative, attività direzionali. La sua trasformazione avvenuta dagli anni '60 ad oggi è "fotografata" dallo stesso Robert Venturi ${ }^{4}$ che rileva le modifiche spaziali-urbanistiche subite dalla Strip. La Strip oggi ha un nuovo nome, Las Vegas Boulevard: sostanzialmente quello che prima era una striscia di casinò e alberghi ai lati di una strada che nasceva e finiva nel deserto, con parcheggi sul fronte e sul retro degli edifici, è ora un viale con aree pedonali a contatto con giardini e piazze a tema, che hanno sostituito i parcheggi per offrire la possibilità di un passeggio per un comune shopping. Sui lati del viale l'urbanizzazione è dilatata lungo arterie ortogonali all' "antico" Strip. Las Vegas adesso si presenta come una normale cittadina con un grande centro commerciale/parco tematico diviso a sua volte in aree tematiche, sotto controllo e quindi per famiglie. Questa è un'idea ormai condivisa e già segnalata dalle guide turistiche. Ma anche in questa veste domestica e borghese Las Vegas non smette di indurre a credere che l'imitazione consente di fare a meno dell'esperienza autentica con un luogo o con l'arte.

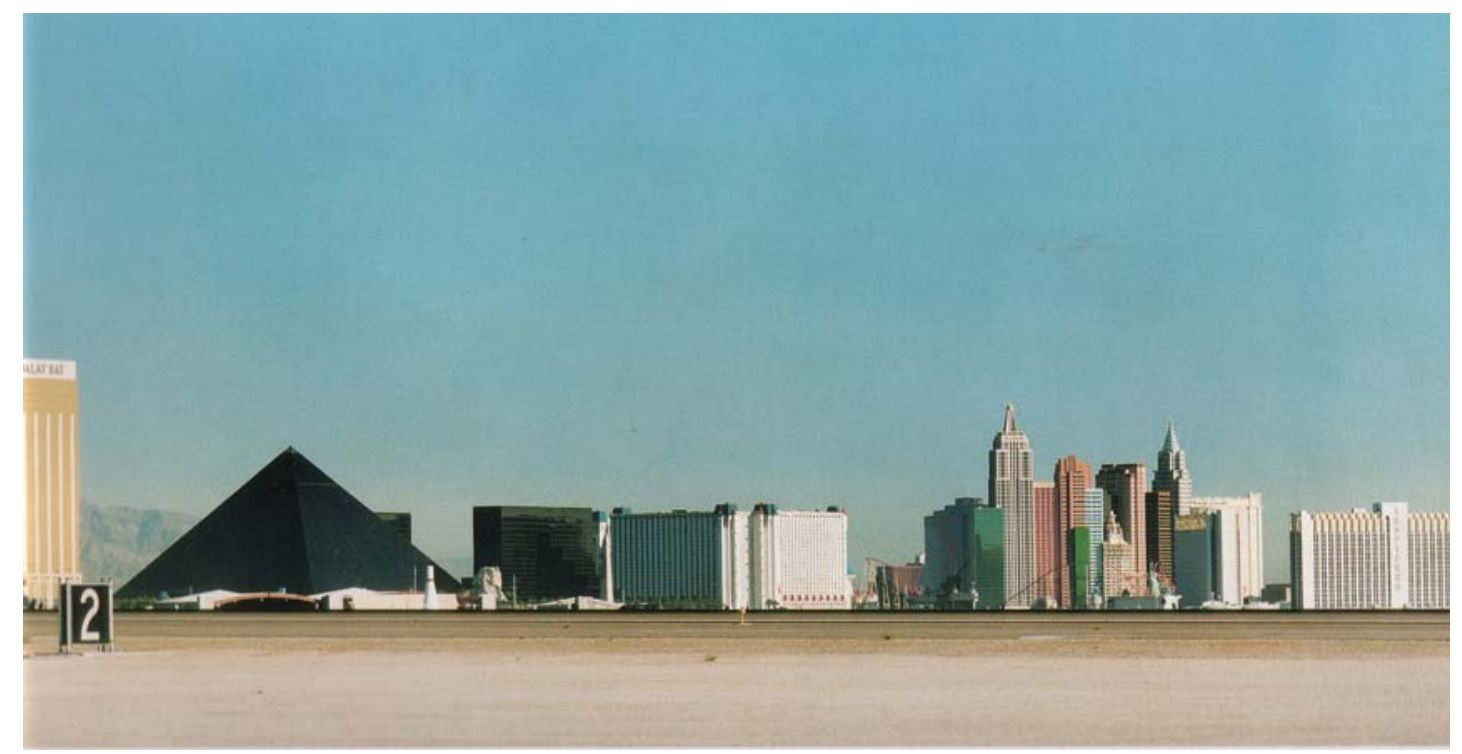

Fig. 2 veduta di las Vegas, dall'aereoporto, 1998 - foto Robert Polidori. Sono visibili la Piramide di Luxor, il castello Excalibur, e New York.

Il messaggio di Las Vegas è identico a quello di Disneyland in California.

Infatti Adventurland, Frontierland, e soprattutto Main Street U.S.A. di Disneyland, si presentano come appetibili sostituti di un rapporto con territori esotici e i loro paesaggi naturali e con la storia dell' Old America, reale, non priva di criticità.

Umberto Eco, dal suo Viaggio nell'Iperrealtà, ci riporta le finalità del ricorso ora al vero e ora al falso, a seconda dei casi:

"(...) una volta che "il tutto falso" è ammesso, occorre che per essere goduto esso sembri tutto vero (...). E se nei musei delle cere, la cera non è carne, a Disneyland, quando sono in gioco delle rocce, roccia è, e se si parla d'acqua è acqua, e un baobab è un baobab. Quando c'è il falso, ippopotami, dinosauri, serpenti di mare, questo non tanto avviene perché non sarebbe possibile avere l'equivalente vero, ma perché si desidera che il pubblico ammiri la perfezione del falso, e la sua docilità al programma. In questo senso Disneyland non solo produce illusione ma - nel confessarla - ne stimola il desiderio: un coccodrillo vero lo si trova anche al giardino zoologico, e di solito sonnecchia e si nasconde, mentre Disneyland ci dice che la natura falsificata risponde molto

\footnotetext{
${ }^{3}$ Umberto Eco, La città degli automi, in Umberto Eco, Dalla periferia dell'impero. Cronache da un nuovo medioevo, Bompiani, Milano 2003, pagg. 51-60.

${ }^{4}$ Robert Venturi , Denise Scott Brown, Las Vegas postclassica, in "Domus”, n. 787, Milano 1996.
} 
di più alle nostre esigenze di sogno ad occhi aperti. (...) Disneyland ci dice che la tecnica ci può dare più natura della realtà." 5

I moventi per la deterritorializzazione "di modello americano" non sono comprensibili se non ragionando sul concetto americano di real thing come fanno Miles Orvell ${ }^{6}$, Jean Baudrillard in America (1986) e Umberto Eco in Travels in Hiperreality (1986), lungo percorsi di studio della cultura popolare americana.

Real thing è una perifrasi iperbolica che sta per "la miglior cosa", "il meglio", e significa letteralmente "la cosa vera". Sintetizzando le considerazioni di Eco e Orvell, sembra di poter affermare che la cosa vera, così come la miglior cosa, sia in realtà una cosa del tutto falsa: essa però riesce, nel suo essere simulazione, a superare per alcuni aspetti il suo corrispettivo originale (dal brano su Disneyland di Eco si evince proprio questo).

"Ecco la ragione del nostro viaggio nell'iperrealtà, alla ricerca dei casi in cui l'immaginazione americana vuole la cosa vera e per farlo deve realizzare il falso assoluto; e dove i confini fra il gioco e l'illusione si confondono, il museo d'arte viene contaminato dal baraccone delle meraviglie, e la menzogna viene goduta in una situazione di "pieno" di "horror vacui"",?

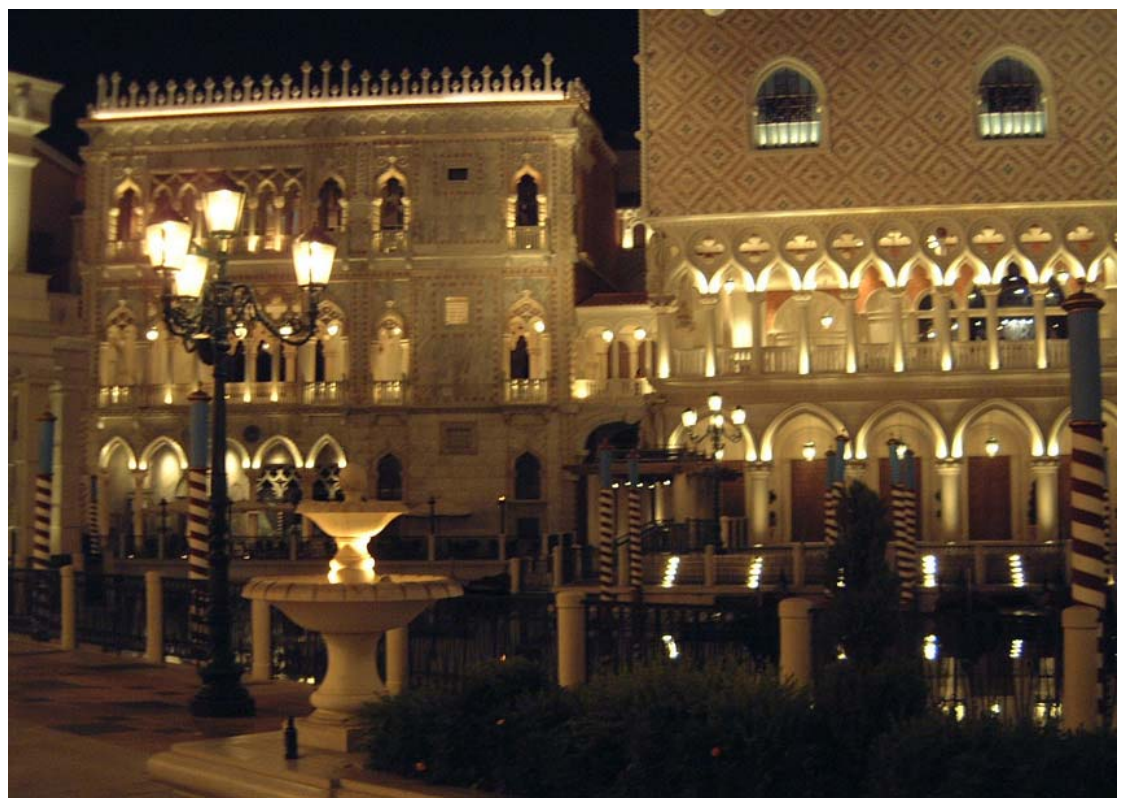

Fig.3 Venezia antistante il Venice Hotel, Las Vegas, U.S.A. (esistono molte altre repliche di Venezia nel mondo fra cui per fare solo alcuni esempi: Venezia a Italia in Miniatura, Rimini, Italia;Venezia a Epcot World, Florida, U.S.A.).

Questo pensiero americano non è privo di implicazioni, se rivolto alla progettazione del paesaggio: non si sa che fine possa subire il genius loci, una volta che la copia è ritenuta altrettanto vera dell'originale, anzi ancora più vera dell'originale, se cioè il paesaggio può essere completamente slegato dal suo territorio.

Umberto Eco lascia il discorso aperto in virtù di un dubbio:

"Se l'America è quella del Guggenheim Museum o dei nuovi grattacieli di Manhattan, allora Disneyland è un'eccezione curiosa (...). Ma se l'America è quella che abbiamo visto nel corso del nostro viaggio, allora

\footnotetext{
${ }^{5}$ Umberto Eco, Op.cit., Bompiani, Milano 2003, pagg. 55-56.

${ }^{6}$ In Miles OrVell, After the machine. Visual arts and the erasing of cultural boundaries, University Press of Mississippi, USA 1995, e in ORVELL MILES, The real thing. Imitation and authenticity in American culture, 18801940, The University of North Carolina Press, Chapel Hill \& London 1989.

${ }^{7}$ UMBerto ECO, Op. cit., Bompiani, Milano 2003, pagg. 18.
} 
Disneyland ne è la Cappella Sistina e gli iperrealisti delle gallerie sono solo dei timidi voyeurs di un immenso e continuo "oggetto trovato".,"

Nel caso la seconda ipotesi fosse quella esatta per cui Disneyland rappresenta l'anima dell'America, sia Las Vegas sia Disneyland risulterebbero pienamente rispettose e rispondenti al proprio genius loci (a patto comunque di accettare che il genius loci americano sia quello del "1492 in avanti" e non uno spirito indigeno nomade che lascia il deserto com'è e si ciba della sua vegetazione spontanea senza pretendere di farne il migliore dei mondi possibili). Ad ogni modo non tutto il territorio è l'America e l'esportazione dei suoi modelli si scontra con altri genius loci.

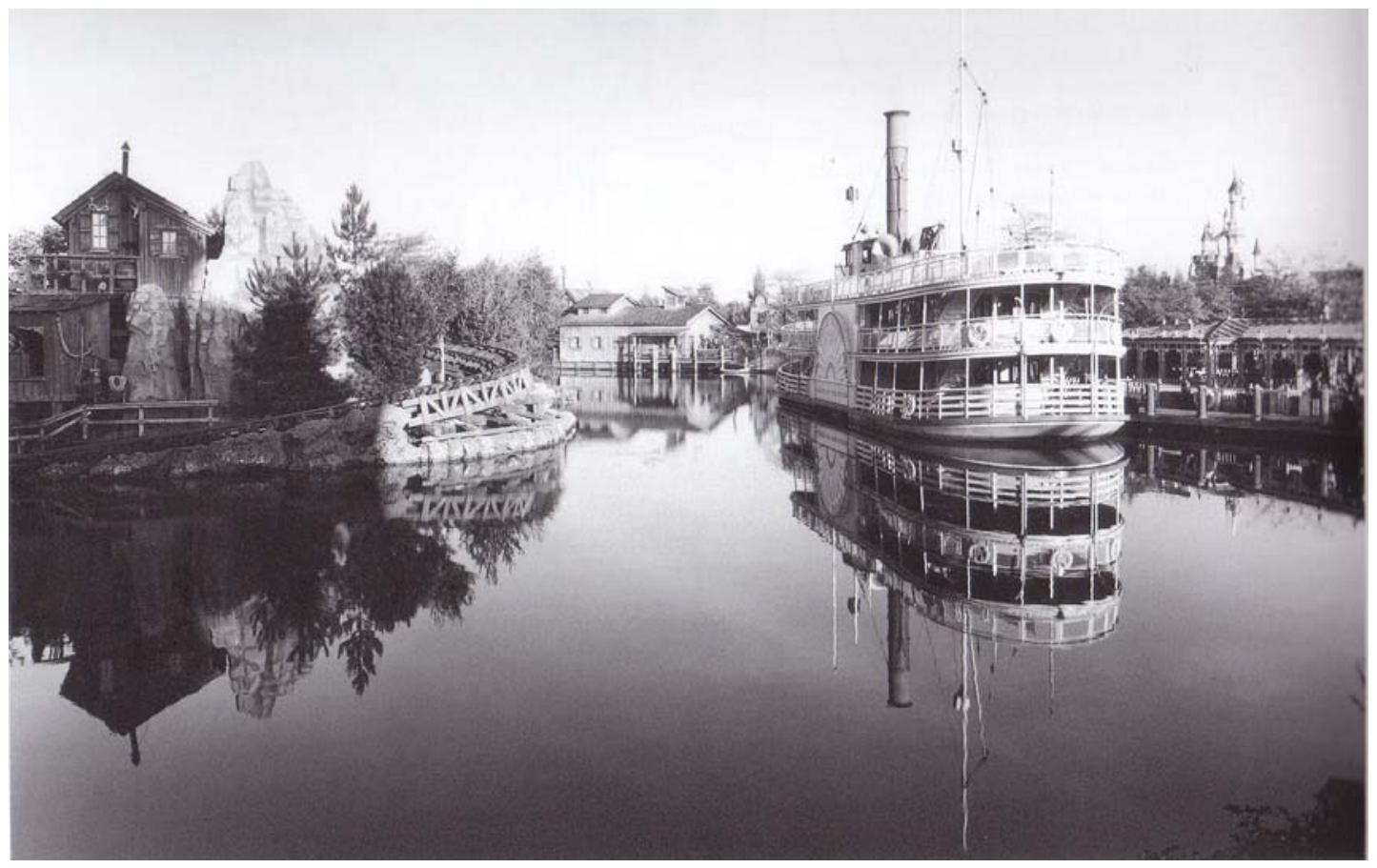

Fig. 4: Frontierland a Disneyland Paris, Marne-la- Valléè, France. Simulacro di un paesaggio lungo il Mississippi. Foto Catherin Wagner.

L'allarme è forte se si pensa, come fa Michael Sorkin ${ }^{9}$, che la tematizzazione nel senso di deterritorializzazione si sta espandendo diventando idea urbana, mentre l'allarme si smorza se il tema resta confinato, quale prerogativa di un parco.

Le Esposizioni universali del XIX secolo abituano il pubblico a muoversi in ambienti tematizzati che raccontano di territori lontani e non rapidamente raggiungibili, nonostante la tecnologia dei trasporti già all'epoca faccia presagire un accorciamento temporale, reale, delle distanze fra i luoghi. Si è detto dei paesaggi sintetici delle Esposizioni.

$\mathrm{Ma}$ in occasione della fiera le culture coinvolte, sia nell'esposizione delle arti e delle tecniche, sia nell'intrattenimento dei visitatori all'interno degli amusement area, sono portate ad esprimere le proprie specificità "dal vero": lo sforzo è presentare l'aura di un popolo, ed è proprio nell'offrire la possibilità di contatto con questa aura che risiede la straordinarietà dell'evento. Il problema del rapporto fra tematizzazione e perdita di aura sorge se l'esperienza di una cosa è basata esclusivamente sulla relazione con una copia e se questa esperienza è sentita come sufficiente.

Quella operata dalle Esposizioni universali non è propriamente una deterritorializzazione, è piuttosto un'astrazione dall'ordinarietà del contesto: la città che ospita l'Esposizione

\footnotetext{
${ }^{8}$ Umberto Eco, Op.cit., Bompiani, Milano 2003, pag. 60.

${ }^{9}$ Michael Sorkin, La tematizzazione della città, in "Lotus" n. 109, Il nuovo mondo allegorico. Sui parchi tematici, Electa, Milano 2001.
} 
ribadisce energicamente il valore del proprio genius loci per offrire un'immagine magnifica al mondo che la osserva (analogamente nel giardino manierista il genius loci è evocato per manifestare il potere del signore e la bellezza dei suoi possedimenti). Le Esposizioni promuovono l'apertura verso mondi diversi per estendere le conoscenze, consentire la circolazione delle idee, ma non si slegano in maniera assoluta dal territorio che le ospita e che viene trasformato all'occasione per essere adeguato all'evento.

Al contrario, al riguardo dei contemporanei parchi tematici sembra spesso di poter condividere le parole di Isabelle Auricoste, la quale mette bene in chiaro l'assoluta differenza che esiste fra il giardino come luogo radicato nel proprio contesto ed il parco tematico come spazio deterritorializzato:

"La disposizione del verde, la scelta di essenze esotiche e decorative, la qualità delle attrazioni, l'attenzione con cui viene predisposta la planimetria, elementi che costituiscono tradizionalmente mezzi e materiali dei giardini, non contribuiscono affatto ad assimilare i parchi per il tempo libero alla grande famiglia dei giardini. L'apparente somoglianza nasconde appena una differenza radicale. Giardini e parchi sono composizioni architettoniche che hanno mantenuto, fino ai nostri giorni e a prescindere dal loro stile, una relazione complementare con il territorio che li ospita. Luoghi di eccezione in quello stesso territorio, nel senso latino di lucus, la loro esistenza e le loro attrazioni non possono concepirsi al di fuori del sistema duale formato dal territorio e dal parco. Nessun giardino potrebbe esistere altrove, se non nel territorio in cui si trova e da cui dipende. Si tratta di uno spazio materiale, di cui suolo, clima topografia, e referenze culturali locali costituiscono determinanti essenziali. Gli attuali parchi del tempo libero al contrario, non devono nulla al sito in cui sono allestiti, considerato come semplice supporto. Sono delle organizzazioni "fuori suolo", nel senso dell'agricoltura fuori terra, la cui concezione si basa su un'analisi razionale del potenziale bacino di visitatori e su una logica deliberatamente universalista, trasportabile e capace di ubiquità, poiché applica dei dati antropologici elementari."10

La tematizzazione come processo che rende lo spazio "universalista, trasportabile e capace di ubiquità" fa aumentare i rischi sottolineati da Raffestin ne Il paesaggio introvabile ${ }^{I I}$, di creare paesaggi a prescindere dal territorio, senza che di fatto esista un territorio.

Lo spazio è correlato al tempo. Dove lo spazio è contratto anche il tempo lo è, e viceversa. Se il tema è "storico" o pseudo-"storico", oltre che geografico, una visita al parco restituisce le suggestioni di altre civilta" e possiamo essere con un salto velocissimo da macchina del tempo nel passato più remoto e nel futuro più lontano, diacronicamente. Michael Sorkin parla di "creazione di una temporalità mistificata e forzata" 12 . Anche Sorkin riconduce l'esperienza del parco a tema a quella più generale della cultura postmoderna:

“Oggi in seguito alla rivoluzione elettronica e all'avanzare della virtualità, la nozione tradizionale di tempo viene profondamente intaccata, e lo spazio e il tempo sono sempre più svincolati tra loro. Il postmoderno si è spesso dedicato a rappresentare il tempo passato in uno spazio svincolato dal tempo reale. Nel parco tematico questo aspetto è dominante: lo spazio è svincolato dal tempo e viceversa". ${ }^{13}$

Sarebbe forse sufficiente, per salvaguardare l'aura del vero, pur trastullandosi con la sua copia, posizionare in un qualche punto una "scala metrica", ovvero un oggetto o un accidente che manifesti l'inganno e sveli che non si tratta dell'originale, che è qualcosa di manchevole, di incompleto, di inesatto.

Nei parchi tematici miniature - come sono, per fare esempi europei, l'Italia in Miniatura, Minimundus a Klagenfurt (Austria) Portugal do pequenitos in Coimbra ecc.- il tema è deterritorializzante, ma la scala ridotta in cui sono riprodotti tutti gli oggetti esplicita che il mondo rappresentato è un giocattolo. (Nessuno si accontenta di vedere Venezia all'Italia in Miniatura, mentre è più facile essere appagati dalla Venezia di Las Vegas che è in dimensioni reali.)

\footnotetext{
${ }^{10}$ IsABelle Auricoste, Parchi o utopie mortali?, “ Ottagono” n. 99, Intrattenimento, 1991, pagg. 19-20.

${ }^{11}$ Claude Raffestin, Il paesaggio introvabile, in PAOlo CAStelnovi (a cura di), "Il valore del paesaggio", Contributi al seminario internazionale, Torino 9 giugno 2000.

${ }^{12}$ Michael Sorkin, La tematizzazione della città, in "Lotus" n. 109, Il nuovo mondo allegorico. Sui parchi tematici, Electa, Milano 2001, pag. 9.

${ }^{13}$ Michael SORKIN, Ibidem.
} 


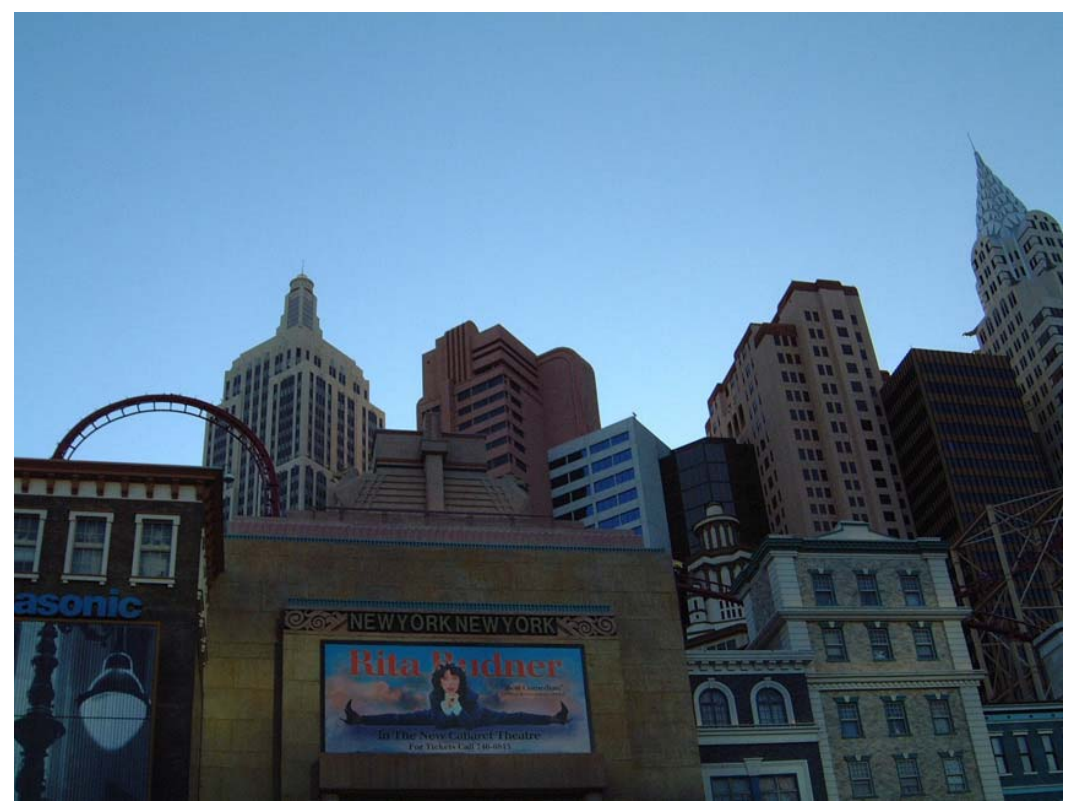

Fig. 5 New York a Las Vegas.

Deterritorializzazione e diacronizzazione sono cause e fini per i quali il parco a tema spesso si pone come spazio di cesura in un paesaggio ordinario, quasi che per la sua eccezionalità fosse condizione necessaria e sufficiente la scissione dal contesto.

Le motivazioni però non sono convincenti in quanto il tema-simbolo può da solo, se progettato in maniera adeguata, consentire il salto, 1' "affaccio", l'astrazione, il sogno e trasfigurare il paesaggio esistente fino a renderlo irriconoscibile. Il giardino manierista e i pleasure gardens costituiscono la "prova" che la tabula rasa disneyana non è l'unico strumento per dare la sensazione ad un visitatore di essere in un luogo che non è nessun altro luogo (se non è nessun altro luogo non è chiaro, fra l'altro, perché Disneyland debba essere ovunque $\left.{ }^{14}\right)$.

L'astrazione dal contesto può consistere in un fatto percettivo, dovuto ai significati immessi dai simboli, mentre il territorio può rimanere intatto (accogliendo le divagazioni dovute alle attività del tempo libero) e lo spazio essere un elemento materiale e contingente:

"Credo che una geografia dell'espressione debba occuparsi di "spazi" come quelli in cui affiorano contraddizioni e conflitti latenti. Virtuali o fisici che siano questi spazi hanno in comune la caratteristica di essere spazi-eventi locali, biforcazioni di un labirinto senza codici ne' mappe, perciò potenziali "ingressi al mondo" nel senso di Merleau-Ponty, passaggi dalla "deterritorializzazione assoluta" alla riterritorializzazione" di Deleuze, che è concettualizzazione e non semplice ricodificazione." 15

\footnotetext{
${ }^{14} \mathrm{Si}$ confronti Miles Orvell a proposito di Disneyland e le sue possibili repliche: "Perhaps it is just here that we draw the line between Disney and " high art" - in Disney's intolerance for ambiguità or irony, its need to fill the empty spaces with something, some recognizable signifier". In any case, the newly constructed Disneyland is surely symptomatic of convergence of spheres, if not an erasure of boundaries between the playful, historically allusive tendencies of post-modern art and the playful, thematically allusive nature of Disney."

"Experiencing Disneyland in Europe surely contextualizes the experience in way that is quite different from America's theme parks: in the United Sates, Disneyland serves the function, among other things, of an escape into a realm of imagined European cultured - the castles, the European fairy tales, figures of royalty, the ersatz European restaurants, etc. That corresponding function could hardly be served in France, within a stone's throw of chateaux of the Loire Valley." in Understanding Disneyland, in MILES ORVELL, After the machine. Visual arts and the erasing of cultural boundaries, University Press of Mississippi, USA 1995, pagg. 151-152, 158.

${ }^{15}$ GiUSEPPE Dematteis , Un modem per Estia. Riflessioni sulla "geografia dell'espressione", cit. in PATRIZIA Mello, Lo spazio come incidente di percorso, in Patrizia Mello, Metamorfosi dello Spazio. Annotazioni sul divenire metropolitano, Bollati Boringhieri, Torino 2002, pag.76.
} 


\section{RIFERIMENTI BIBLIOGRAFICI}

AUgÉ MARC, Nonluoghi, Elèuthera, Milano 1993.

BECK GREGORY, Un atlante dei parchi tematici, in "Lotus" n. 109, il nuovo mondo allegorico. Sui parchi tematici, Electa, Milano 2001, pagg. 104-117.

BENJAMIN WALTER, L'opera d'arte nell'epoca della sua riproducibilità tecnica, Einaudi, 1966 e 1991.

BONAZZI GABRIELE (a cura di), L'estetica nel pensiero contemporaneo (dal romanticismo al surrealismo), G. D'Anna, Firenze 1980.

CHOAY FRANCOISE, L'allegoria del patrimonio, a cura di Ernesto d'Alfonso e Ilaria Valente, Officina Edizioni, 1995.

ECo UMBerto, Dalla periferia dell'impero. Cronache da un nuovo medioevo, Bompiani, Milano 2003.

JACKSON JOHN B., Architettura vernacolare, in "Casabella" n. 680, Electa, Milano 2000.

JACKSON JOHN B., Discovering the Vernacular Landscape, Yale University Press, 1984

KraCAUER SiEgFried, Montagne russe - l'illusione metropolitana, in "Casabella", n. 673/674, Architettura come spettacolo, Electa, Milano dicembre 1999- gennaio 2000.

LYOTARD JEAN-FRANCOIS, la condizione postmoderna, Feltrinelli, Milano 1981

NiCOLIN PIERLUiGI, Architettura "light", in "Lotus" n. 105, 2000.

OCKMAN J. E ADAMS, N., Forme dello spettacolo, in "Casabella", n. 673/674, Architettura come spettacolo, Electa, Milano, dicembre 1999- gennaio 2000.

OCKMAN J. E ADAMS, N., La città delle insegne: Times Square, in "Casabella", n. 673/674, Architettura come spettacolo, Electa, Milano dicembre 1999- gennaio 2000.

ORVELl Miles, After the machine. Visual arts and the erasing of cultural boundaries, University Press of Mississippi, USA 1995.

ORVELl MiLES, The real thing. Imitation and authenticity in American culture, 1880-1940, The University of North Carolina Press, Chapel Hill \& London 1989.

Patrizia Mello, Lo spazio come incidente di percorso, in Patrizia Mello, Metamorfosi dello Spazio. Annotazioni sul divenire metropolitano, Bollati Boringhieri, Torino 2002, pagg. 67-105.

PATRIZIA Mello, Luoghi di Evasione: parchi o utopie mortali?, in PATRIZIA Mello, Metamorfosi dello Spazio. Annotazioni sul divenire metropolitano, Bollati Boringhieri, Torino 2002, pagg. 22-37.

RAFFESTIN ClAUde, Il paesaggio introvabile, in PAOlo CASTELnOVI (a cura di), "Il valore del paesaggio", Contributi al seminario internazionale, Torino, 9 giugno 2000

RAJCHMAN JOHN, Effetto Bilbao, in "Casabella", n. 673/674, Architettura come spettacolo, Electa, Milano dicembre 1999- gennaio 2000

SORKIN MICHAEL, La tematizzazione della città, in "Lotus" n. 109, Il nuovo mondo allegorico. Sui parchi tematici, Electa, Milano 2001, pagg. 7-17.

VENTURI ROBERT, Complessità e contraddizioni nell'architettura, Dedalo, Bari, 1980

VeNTURI RoBert, SCOTt BRown DENISE, IZENUOR STEPHEN, Imparando da Las Vegas. Il simbolismo dimenticato della forma architettonica, CLUVA editrice, 1985.

Venturi RoBert, ScotT BRown Denise, Las Vegas postclassica, in "Domus", n. 787, Milano 1996.

\section{RIFERIMENTI ICONOGRAFICI}

Fig. 1: fotografia di Enrica Dall'Ara.

Fig. 2: "Casabella", n. 673/674, Architettura come spettacolo, Electa, Milano dicembre 1999- gennaio 2000, pag. 7 .

Figg. 3, 5 : fotografie di Enrica Dall'Ara.

Fig. 4: Maling Karal AnN, Designing Disney's Theme Parks: The Architecture of Reassurance, Flammarion, Paris 1998, pag. 106, illustrazione 94. 


\section{3 - TEMA COME VALORIZZAZIONE/SINGOLARIZZAZIONE}

Anche l'inserimento dell'oggetto artistico è una modalità di tematizzazione. In particolare l'arte allestita nel paesaggio ha la capacità di porsi quale medium fra il paesaggio fisico ed il paesaggio culturale, reinterpretando le componenti di memoria e conferendo nuovi significati.

Si portano alcuni esempi di azione artistica nel paesaggio - Il parco diffuso di Tonino Guerra in Romagna, L'Hortus conclusus a Benevento, i giardini di Ian Hamilton Finlay, in cui il tema si pone quale affermazione della passione per un luogo specifico.

Nel parco di Tonino Guerra allo spazio viene affidato un racconto fatto di memoria "o meglio dell'ECO prolungata del passato nella coscienza, che determina anche l'incertezza delle frontiere tra la memoria e il SOGNO", racconto fatto quindi anche di fantasia. Memoria e fantasia danno un ritmo complesso al tempo e allo spazio, con continui passaggi dal vicino al lontano, e viceversa: il paesaggio assorbe la presenza di altre terre e civiltà per cui l'hic et nunc si affaccia sull'universale.

Nell'Hortus Conclusus la nuova galleria di ingresso al giardino viene creata quale un'anticamera necessaria alla scoperta delle opere dello scultore della Transavanguardia Mimmo Paladino che si riveleranno, per le forme sintetiche, quasi archetipiche e slegate dal contesto. Allo stesso tempo tali forme saranno evocatrici di una civiltà antica, quella dei Sanniti che fondarono Benevento, animate da una fantasia mitologica, ponendosi quindi lungo un tracciato di continuità culturale.

L'arte dei giardini di Ian Hamilton Finlay parte da evocazioni letterarie, storiche, filosofiche che gli elementi di un paesaggio dato suscitano nell'artista, il quale materializza l'evocazione in oggetti puntuali disposti a loro volta all'interno di quel paesaggio. Il giardino è quindi il paesaggio tradotto in arte ed è tematico perché caricato di simboli, che non gli sono intrinsechi, ma gli vengono assegnati dall'artista, in seguito ad un'idea che rimanda ad altro: altri luoghi, altre opere d'arte, altri momenti storici, altri uomini. Il paesaggio esistente è un'immagine da cui ne seguono nuove, nella mente dell'artista, e nuove ancora nell'osservatore che guarda il paesaggio in cui è l'opera dell'artista.

Gli esempi non sono parchi del divertimento nel senso comune del termine- lo sono in qualche modo però se si intende come divertimento la diversione dal contesto quotidiano.

Si intende comprendere il processo artistico sul paesaggio quale modo di attribuzione del tema, per vedere se esso può offrire "suggerimenti utili" anche per la progettazione dei parchi del divertimento quali normalmente intesi.

Si fa questo anche per provocazione. Per risollevare velatamente l'antica questione del rapporto - Possibile? Impossibile? - fra fruizione di massa, quale quella dei parchi tematici, e presenza dell'arte: Walt Disney pare abbia rinunciato consapevolmente a concepire la propria arte, di disegnatore di cartoni animati e di progettista di parchi, quale arte elevata, anche in seguito all'insuccesso di critica del proprio film Fantasia. Si è detto come i giardini manieristi costituissero un'esperienza diversa per i nobili colti, amici dei proprietari, e per i popolani a cui occasionalmente venivano aperte le porte. Si è parlato di come le raffinatissime folies parigine del XVIII secolo fossero trasformate, in seguito alla loro confisca in tempi di Rivoluzione, dall'ospitare al loro interno, nel paesaggio studiato come un quadro, le attrazioni proprie delle fiere popolari, caotiche ed informali.

Walter Benjamin ${ }^{2}$ afferma che la massa è distratta e nella distrazione risiede la possibilità che essa assimili l'arte.

\footnotetext{
1 JaCQues Le Goff, presentazione in Tonino Guerra, Dizionario fantastico, Pietroneno Capitani Editore, Rimini 2000, pag. 10.

${ }^{2}$ Walter Benjamin, L'opera d'arte nell'epoca della sua riproducibilità tecnica, Einaudi, Torino 1966 e 1991.
} 

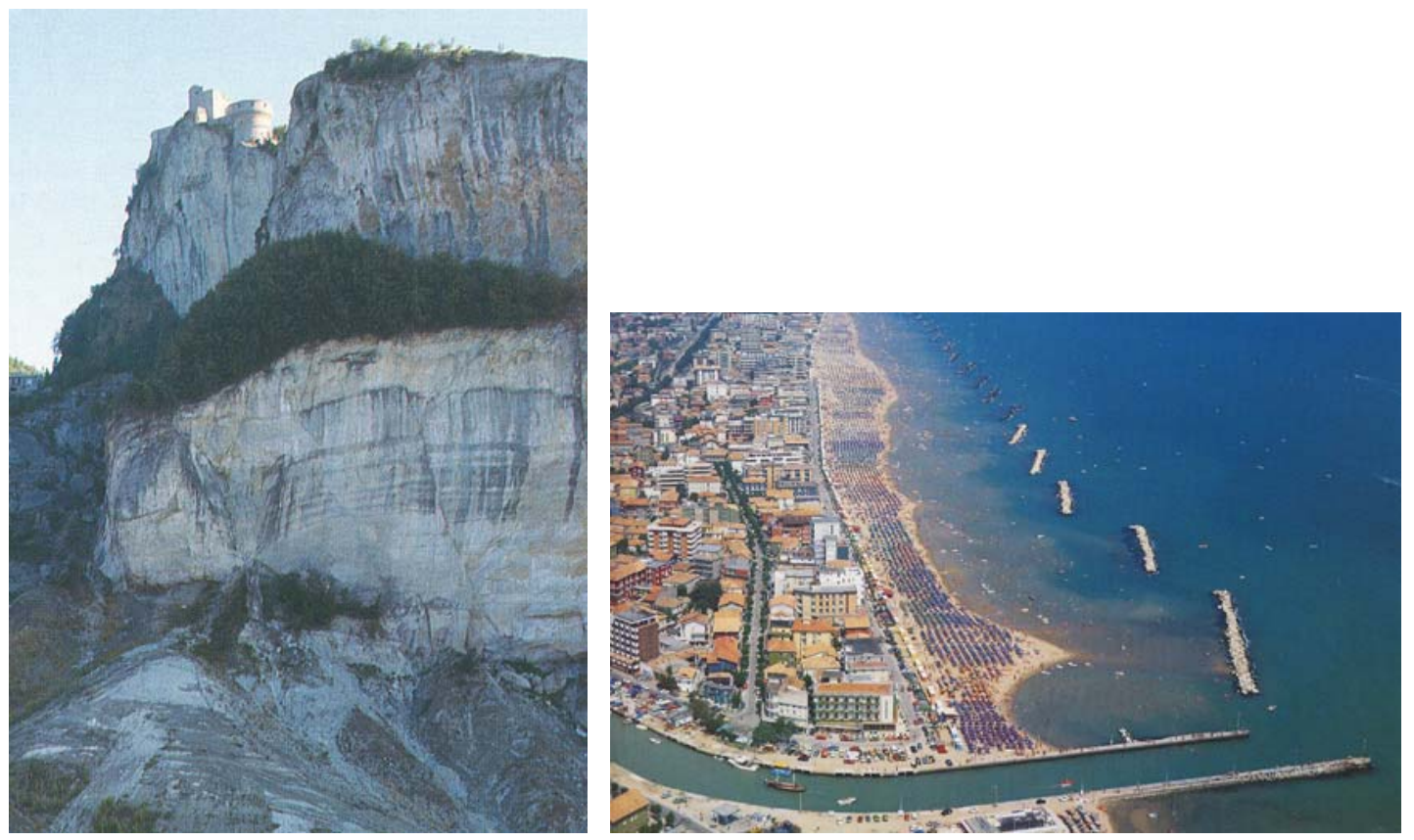

Fig. 1 Paesaggio della Valmarecchia: San Leo. Fig. 2 Costa romagnola nei pressi di Bellaria.

Il territorio su cui si estende il parco diffuso realizzato da Tonino Guerra, scenografo e poeta originario di Santarcangelo (Rimini), va dalla costa adriatica romagnola (Cervia) fino all'estremità della Valmarecchia, in provincia di Pesaro, nel cuore della regione storica del Montefeltro.

Si tratta di una terra carica di contraddizioni che nel tempo hanno generato una dialettica di situazioni oggi coesistenti in un improbabile equilibrio.

Da un lato ci sono i paesaggi storici delle vallate, con le loro rocche e pievi antichissime (la Madonna di Saiano, San Leo, Verucchio, Maioletto, Torriana e Montebello, Pennabilli...), le fughe d'occhio del Rinascimento con la luce di Piero della Francesca celebrata, tra gli altri, da Ezra Pound, dall'Alta Valmarecchia fino al tempio malatestiano di Rimini, gli ambienti naturali caratteristici, i massi erratici di calcare su cui sono sorte San Marino e le altre cittadelle fortificate che dai crinali vicini appaiono come un esercito con le lance puntate verso il mare, i boschi di querce e il letto enorme di ghiaia bianca della "Marecchia argentina", come la vedeva Giovanni Pascoli.

Dall'altro queste valli rimaste al margine della storia, con i loro personaggi straniti da cui Tonino Guerra ha tratto ispirazione, sfociano su una costa che ha fatto del divertimento e dello spettacolo la struttura delle sue città, mangiandosi le dune costiere e sostituendole con una conurbazione metropolitana tentacolare.

Le realizzazioni recenti di Tonino Guerra, sparse in questo territorio, vogliono richiamare interesse su "luoghi minimi, con il fascino della memoria e dell'abbandono" 3 . Il valore di muoversi nelle brevi distanze consiste nella possibilità di incontrare la propria storia : nei termini di presa di coscienza del radicamento dovuto alla famigliarità con un paesaggio singolare e contemporaneamente dell' appartenenza all'universale per cui "Vicino a casa improvvisamente vi potete perdere perché il viaggio non si ferma dove arrivate ma continua dentro la vostra memoria." E' possibile trovare i "depositi di immagini che ci ha consegnato l'umanità. Possiamo imbatterci in apparizioni fissate da quando eravamo primitivi."

Nelle opere letterarie di Tonino Guerra, apparizione, interesse, concentrazione sono associati e costruiscono i momenti in cui l'aspetto fantastico avvicina ad una verità.

\footnotetext{
${ }^{3}$ Tonino Guerra, cit. in Rita Giannini, Tonino Guerra e la sua valle, Raffaelli Editore, Rimini 1998.
} 
Ricorrono spesso situazioni in cui improvvisamente un luogo è invaso da un'unica cosa. Essa fa scomparire, oppure trasfigura, gli elementi che lo identificano, perché ne impedisce la vista o anche perché il suo avvento è straordinario e attira tutta l'attenzione.

L'immagine è coperta da una sensazione esclusiva:

“ (...)Vedo solo bianco. Neve, nevica, nevicando questo pulviscolo nell'aria e io che cammino, felicità più completa." 4

"Poi sono arrivate tutte quelle farfalle bianche/ che venivano dal fiume/ e si sono posate su tutto, e la roba lì attorno ha preso il colore del latte: porte,/ finestre , muri e noi che eravamo a sedere/ sembravamo dei bambocci di gesso". 5

Analogamente la fioritura eccezionale del racconto L 'inverno fiorito è presenza continua in fondo alle strade. Dopo una notte in cui è piovuto e soffiato il vento, "lei si affaccia alla finestra e vede che tutta la campagna fino al mare è bianca come se avesse nevicato."

Il film Amarcord ${ }^{7}$ di Federico Fellini, il cui soggetto è ideato da Tonino Guerra, inizia con una danza nell'aria delle "manine", i pollini dei pioppi, che viaggiano sui tetti delle case, scendono per le strade del Borgo -arriveranno al mare- mentre le persone, nella meraviglia, si scompongono tentando di afferrarle. Successivamente una nebbia "che ha cancellato tutto attorno a lui" disorienta il nonno, fra i protagonisti, davanti alla propria casa e alimenta un monologo nello smarrimento crescente: "Non sono più da nessuna parte". "Ma dove sono? Se la morte è così non è un bel lavoro ${ }^{8}$... finito tutto!(...)".

Queste visioni totalizzanti stravolgono l'ordinarietà mediante uno svago o un sentimento intensi, dovuti al mutamento subito dallo spazio intorno. La descrizione è in bilico fra assolutezza dell'immagine e chiarezza del dettaglio, proponendo comunque una ricomposizione forte attorno all'idea centrale: " $\mathrm{Ci}$ sono dei momenti in cui il corpo fa attenzione a tutto: gli occhi vedono un fiore rosso, le mani sentono il calore di una stoffa, la bocca ritrova un vecchio sapore. E così uno si sente frantumato. Si potrebbe ricomporre con un grosso dolore, concentrarsi insomma attorno ad un interesse unico. Quando mi capita, io guardo il sole e così raduno il mio corpo attorno ad un dolore accecante"9

Negli allestimenti sul territorio la ricerca dell'assolutezza si affievolisce. La sottolineatura è operata inserendo forme decorative che informano i luoghi di figuratività, offrendo una realtà ricreata, ridata, tramite la sovrapposizione, da parte dell'artista, del suo stile - per questo è possibile una loro lettura come episodi di un parco unico sotto il "timbro"10 di Tonino Guerra.

\footnotetext{
${ }^{4}$ Tonino Guerra, Dizionario fantastico, Pietroneno Capitani, Rimini 2000, pag. 68.

${ }^{5}$ Tonino Guerra, Il viaggio, Maggioli Editore, Rimini 1986, pagg. 25-26. L'immagine dello sciame di farfalle si ritrova anche in Tonino Guerra, Dizionario fantastico, Pietroneno Capitani, Rimini 2000, pag. 66.

${ }^{6}$ Tonino Guerra, Il Polverone, Maggioli Editore, Rimini 1992, pagg. 9-11.

7 Il titolo è in dialetto romagnolo e significa "Mi ricordo".

8 "Non è un bel lavoro" è un'altra espressione dialettale romagnola, per significare " non è una bella cosa".

9 Tonino Guerra, Dizionario fantastico, Pietroneno Capitani, Rimini 2000, pagg. 83-84.

${ }^{10}$ Dalla dichiarazione di poetica dell'autore in occasione di un'intervista di Giancarlo Papi per l'apertura dell'esposizione Con la poesia alle spalle, Rimini 15 dicembre 2001-19 gennaio 2002, Galleria Fabjbasaglia.
} 

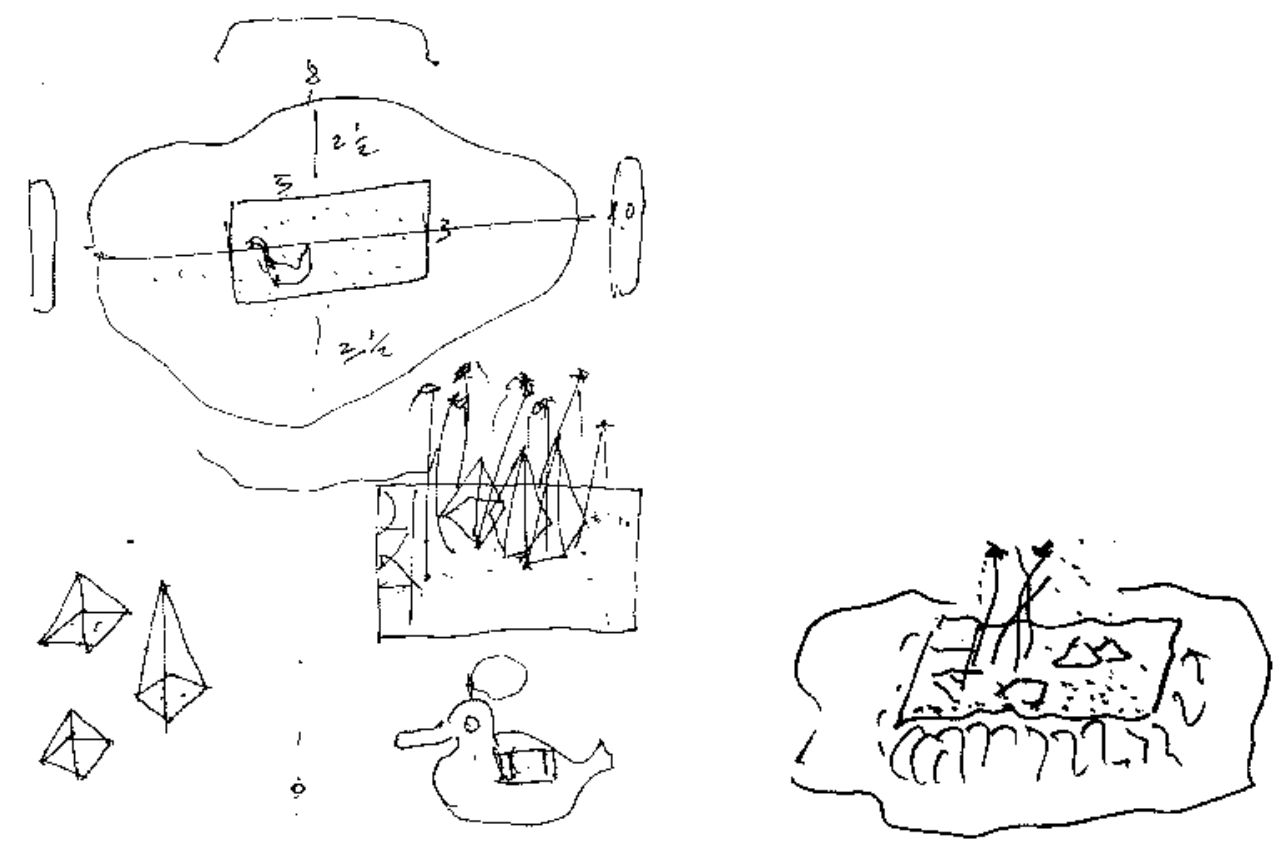

Fig. 3 Schizzi di Tonino Guerra per la fontana Il Tappeto sospeso a Cervia.

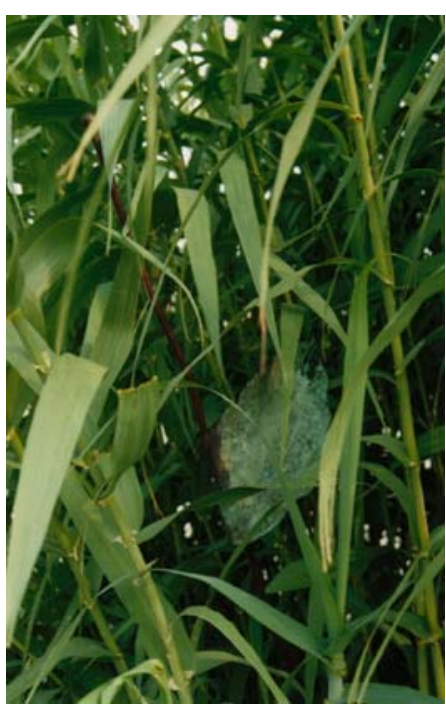

Fig. 4 Foglia di rame nel canneto.

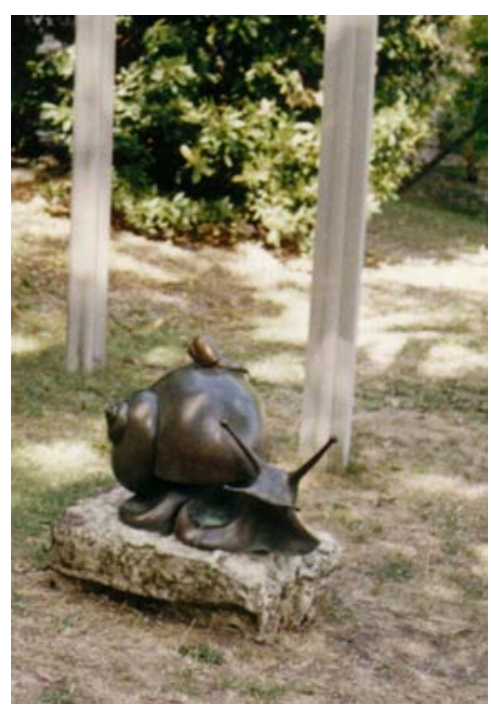

Fig. 5 Lumaca di bronzo.

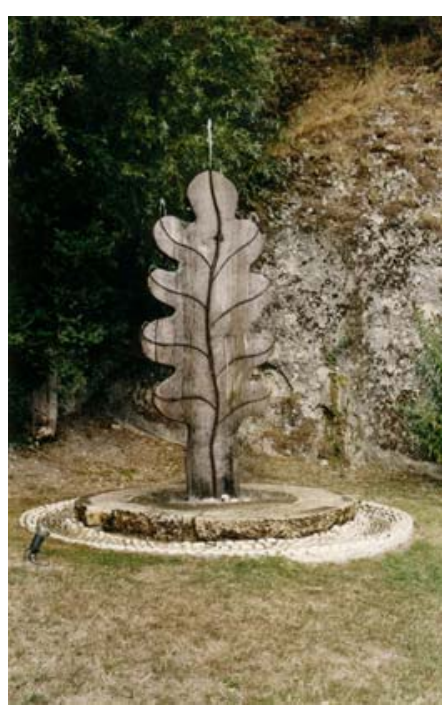

Fig. 6 Fontana La Voce della foglia.

$\mathrm{Ci}$ sono tanti oggetti parlanti. La natura è scelta a raccontare se stessa esponendosi: nelle mostre museografiche spesso convivono gli oggetti reali e la loro rappresentazione. Così a Cervia, nel giardino Il Tappeto sospeso, un canneto di ceramica e rame è cresciuto in un minuscolo "lago" ed un altro, naturale, nel prato accanto. Se ci si avvicina a questo si scopre anche qui, fra le foglie, qualche fusto di ferro, sparso.

A Pennabilli nell' Orto dei Frutti dimenticati - realizzato dove era un orto conventuale - una lumaca di bronzo della dimensione di un gatto abita Il bosco incantato, un labirinto di steli di pietra serena, mentre dopo la pioggia sicuramente qualche lumaca viva si potrà vedere lungo $\mathrm{i}$ muretti e altre sono in rilievo sulla Porticciola delle lumache realizzata da Aldo Rontini, ceramista faentino.

Così nello stesso orto vivono le foglie dei tanti alberi da frutto esposti, una foglia di legno alta tre metri che versa acqua sulla pietra di un antico mulino - la fontana La Voce della foglia una sagoma di foglia di dimensioni analoghe, realizzata a fil di ferro e mimetizzata contro $i$ 
muschi della parete rocciosa, e le foglie scritte del mese di Ottobre su una delle targhe in ceramica in un vecchio lavatoio - Le parole dei mesi: OTTOBRE $i$ tappeti di foglie secche sotto $i$ piedi. Alla roccia che delimita l'orto stanno appesi nidi a casetta di legno colorati, per gli uccelli, mentre due colombi di bronzo proiettano la loro ombra su un disco bianco, a terra, disegnando i volti di Federico Fellini e Giulietta Masina - La Meridiana dell'incontro.

Una griglia di ferro tridimensionale a forma di elefante struttura una pianta rampicante: è un prototipo per i successivo allestimento, a Perticara (Pesaro), di uno Zoo verde di animali giganteschi. Ci sono un altro elefante, una giraffa, una tartaruga, un rinoceronte.

A Torriana la Fontana dell'Albero dell'acqua è un albero con il tronco di legno ed i rami effimeri disegnati da getti d'acqua.

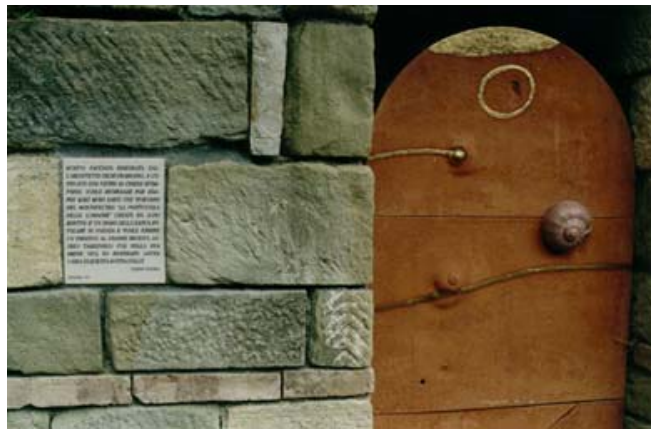

Fig. 7 Porticciola delle lumache.

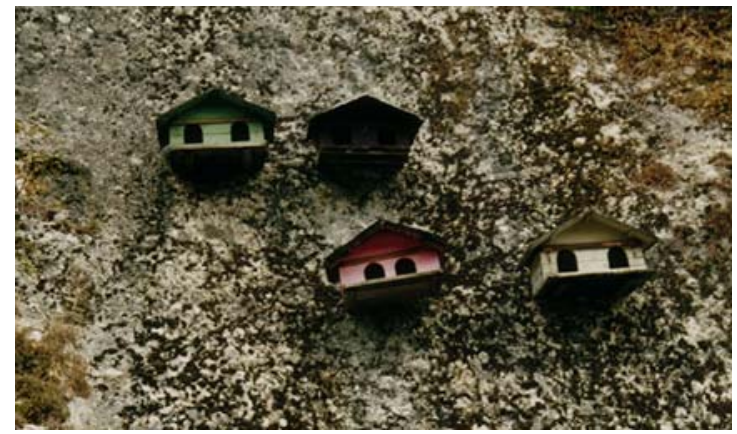

Fig. 8 Nidi a casetta di legno colorato.

Il risultato è un senso ambiguo fra reale e surreale per cui le cose possono acquistare tutte le dimensioni ed essere fatte di tutti i materiali senza che muti la loro sostanza, ma amplificando i rimandi fantastici contenuti nel loro nome. In questo modo anche il racconto si amplifica, trasforma la natura in personaggio recitante simultaneamente molte eco, a chi, in particolare, conosca l'opera poetica dell'autore.

Le piante di rosmarino a Cervia:

“(...) Petrèlla Guidi par léu/ l'era un udòur ad smaroin/ ch u t butéva la tèsta indrì."

ed i ciottoli ordinati a bordo d'acqua:

"U i era te fiom dal béusi 'd sabia/ in dò ch'u s'avdéva in qua e in là/ di sas e alòura Rico u i amucéva s'una cana/ te mèz, che i féva péna si stéva smanèd." 12

Gli esemplari di alberi da frutto "dimenticati" a Pennabilli, costituiscono un orto botanico di piccole dimensioni che presenta le piante tipiche di un'agricoltura pre-industriale.

Eppure non sono solo materia didattica o una possibilità di ricordo, ma soprattutto materia poetica:

"In tutta la valle del Marecchia ne parlavano. E cioè da San Leo a Gradara, da Verucchio a Montebello e a tutta la zona che qualcuno vorrebbe chiamare il trapezio Medievale. I vecchi dicevano che una fioritura d'inverno così totale e completa l'avevano avuta soltanto alla fine dell'altro secolo. Eravamo soltanto a metà gennaio e il sole sbriciolava la terra. Ciliegi meli e tutti gli alberi da frutto erano carichi di fiori e di api golose. Al paese si vedevano gli alberi fioriti in fondo a tutte le strade e la gente parlava d'affari ma ogni tanto accennava a questa primavera precoce..."13

\footnotetext{
11 "Petrella Guidi per lui/ era un odore di rosmarino/ così forte che ti buttava la testa indietro." da ToNINO GuERrA, Il Viaggio, Maggioli Editore, Rimini 1986, pagg. 30-31.

12 “ C'erano nel fiume delle buche di sabbia/ dove si vedevano qua e là/ dei sassi e allora Rico li ammucchiava nel mezzo/ con una canna perché gli facevano pena se stavano isolati." da TONINO GUERRA, Il Viaggio, Maggioli Editore, Rimini 1986, pagg. 28-29.

${ }^{13}$ Tonino GuERra, Il ciliegio fiorito, in Il polverone, Maggioli Editore, Rimini 1992, pag. 9.
} 


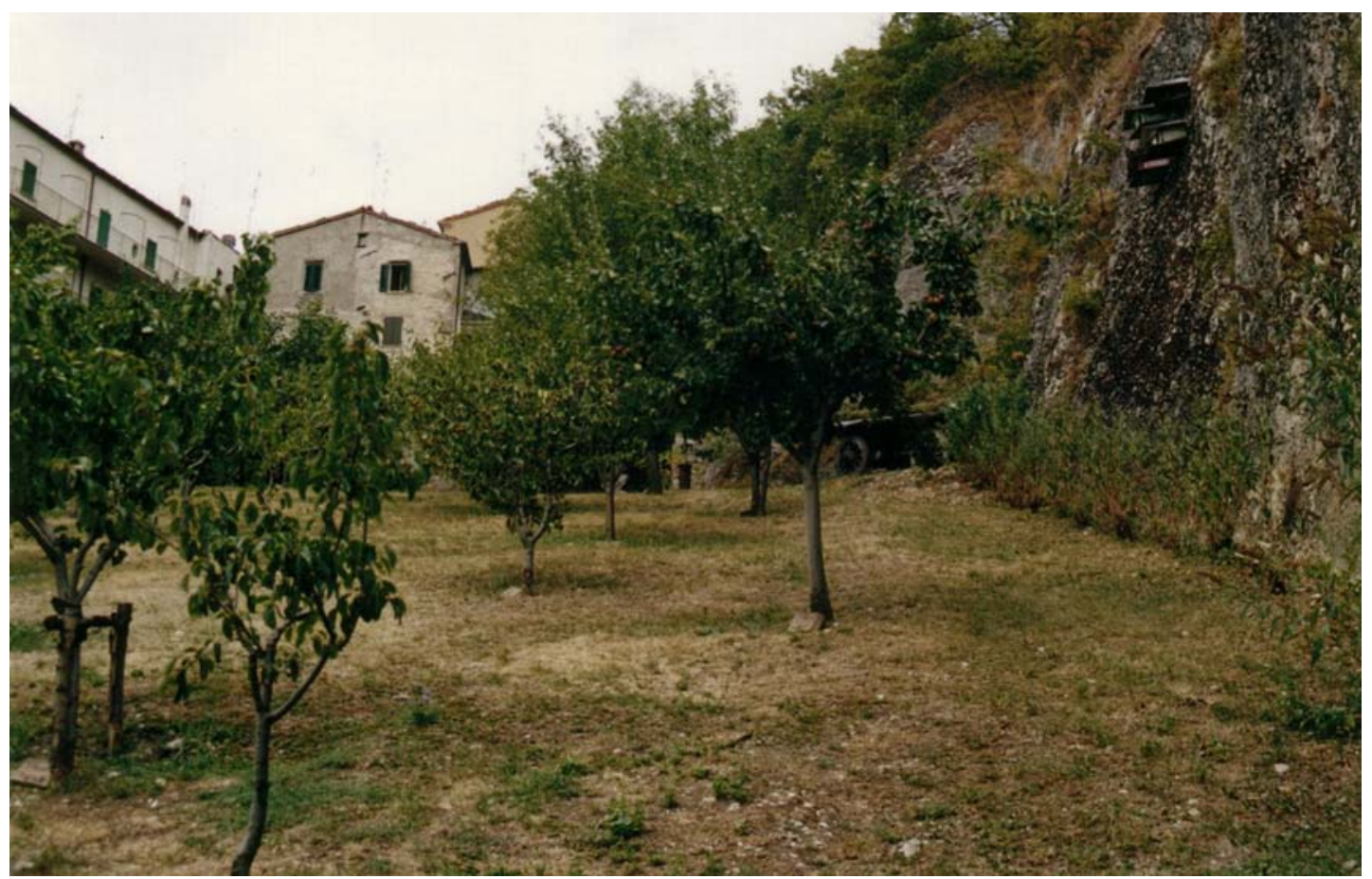

Fig. 9 Alberi da frutto ne L'orto dei frutti dimenticati a Pennabilli.

“(...)Così ho cominciato a capire che stavo andando al paese./ Infatti c'erano gli ulivi e anche molti mandorli nei campi./ Ulivi e mandorli grossi come cominciano a vedersi solo a un centinaio di chilometri dal mio paese. (...) Probabilmente quindi andavo verso il mio paese./ Siccome non ho la certezza di essere in nessun posto (...)"14

Sono anche gli oggetti di Il ciliegio in fiore ${ }^{15}$, Al fòi de zris - Le foglie del ciliegio ${ }^{16}$, L'amòur - Il gelso ${ }^{17}$.

Materia letteraria e materia del giardino coincidono: naturale/meraviglioso, memoria/presenza, esotico-orientale/locale. Sintetico è il tappeto volante della fontana a Cervia, realizzato a mosaico - come un oggetto d'arte bizantina della vicina Ravenna e dell'antico oriente - sul quale si sollevano dall'acqua due cumuli di sale a ricordare la Cervia delle saline e la funzione originaria di magazzini del sale degli edifici che delimitano la piazza.

L'assonanza fra i giardini di Tonino Guerra e i paesaggi scritti delle sue opere esiste non solo perché gli elementi della narrazione sono gli stessi, ma anche per il fatto che in entrambi il timbro è simultaneamente di gravità e leggerezza :

“(...) Ci sono dei giorni che questi alberi si caricano di tanti pezzetti di stoffa così da sembrare fioriti specialmente negli anni delle guerre e delle pestilenze.

L'albero più amato è un grande caragac. Senza foglie tutto l'anno perché strappate dalle mani avide di grazie per far posto ai pezzi di stoffa, l'albero si alza in una gola di pochi metri in mezzo a due montagne che dividono il deserto dai giardini di albicocchi. Una è rossa e l'altra è bianca. Tutte le sere nasce un vento risucchiato oltre la gola delle foglie ancora calde degli albicocchi e dai frutti gialli di sole.

I pellegrini che arrivano a chiedere la grazia, scrivono il loro desiderio sul pezzetto di stoffa che appendono ai rami del grande caragac. Così quando di notte il vento scuote le fettucce, le parole scritte si tramutano magicamente in suoni che si ascoltano a chilometri di distanza: se le sente anche chi le ha scritte questo vuol dire che il desiderio è accolto. (...) Per due notti si alzò appena un vento leggero e a valle arrivarono delle parole dilatate nell'aria. Erano parole sciolte che appartenevano un po' a tutte le scritte. Quindi un groviglio di pensieri

\footnotetext{
${ }^{14}$ Tonino Guerra, Treno, in Dizionario fantastico, Pietroneno Capitani, Rimini 2000, pag. 163.

${ }^{15}$ TONINO GUERRA, Il polverone, Maggioli Editore, Rimini 1992, pagg. 9-11.

${ }^{16}$ TONINO GUERRA, Il libro delle chiese abbandonate, Maggioli Editore, Rimini, 1988, pagg. 83-85.

${ }^{17}$ Tonino Guerra, Op. cit., Maggioli Editore, Rimini, 1988, pagg. 109-111.
} 
confusi. Ma poi una notte si alzò vento grosso che scosse violentemente i rami del caragac agitando le banderuole di panno che schioccarono come fruste protese verso i giardini." ${ }^{18}$

" In una delle tante favole raccontate in un villaggio ai confini con il Turkmenistan, si parlava sempre di un giardino sonoro che incantava i cammellieri e deviava il corso delle carovane cariche di seta. (...) Un giovane si mise in cerca di questo giardino. L'unica cosa che trovò fu una foresta pietrificata. Cento alberi di duro marmo rugoso e verdastro con solchi come fessure gelate. (...) Battendo i tronchi il giovane si accorse che mandavano un suono duro senza nessuna vibrazione. Con un coltello spinse a lungo in una fessura che era chiusa da un materiale friabile e polveroso. D'improvviso fu investito da un gettito d'acqua. Il tronco era pieno di acqua limpidissima e fresca. (...) Ma un giorno l'acqua finì e la piccola foresta pietrificata rimase con gli alberi vuoti d'acqua. Così una notte di vento gli alberi suonarono e tutti capirono che quel bosco era il famoso giardino sonoro di cui parlavano le favole." $" 19$

Nel giardino di Cervia la gravità è nella collina con i blocchi di pietra squadrata che costituiscono la lunga panca, nei due massi lungo il sentiero d'assi di legno e nella semisfera di pietra con inciso il messaggio-premessa dell'autore, nella forma chiusa della fontana e del sentiero. La leggerezza è negli spruzzi d'acqua dal canneto di ceramica e rame, nell'acqua che apparentemente solleva il tappeto volante, nell'alternarsi d'erba e legno a terra, nella presenza delle cavolaie attorno ai cespugli di lavanda: le stesse farfalle, probabilmente, del prodigio raccontato alla voce farfalla in Dizionario Fantastico e del ricordo di Rico in $\mathrm{Il}$ Viaggio.

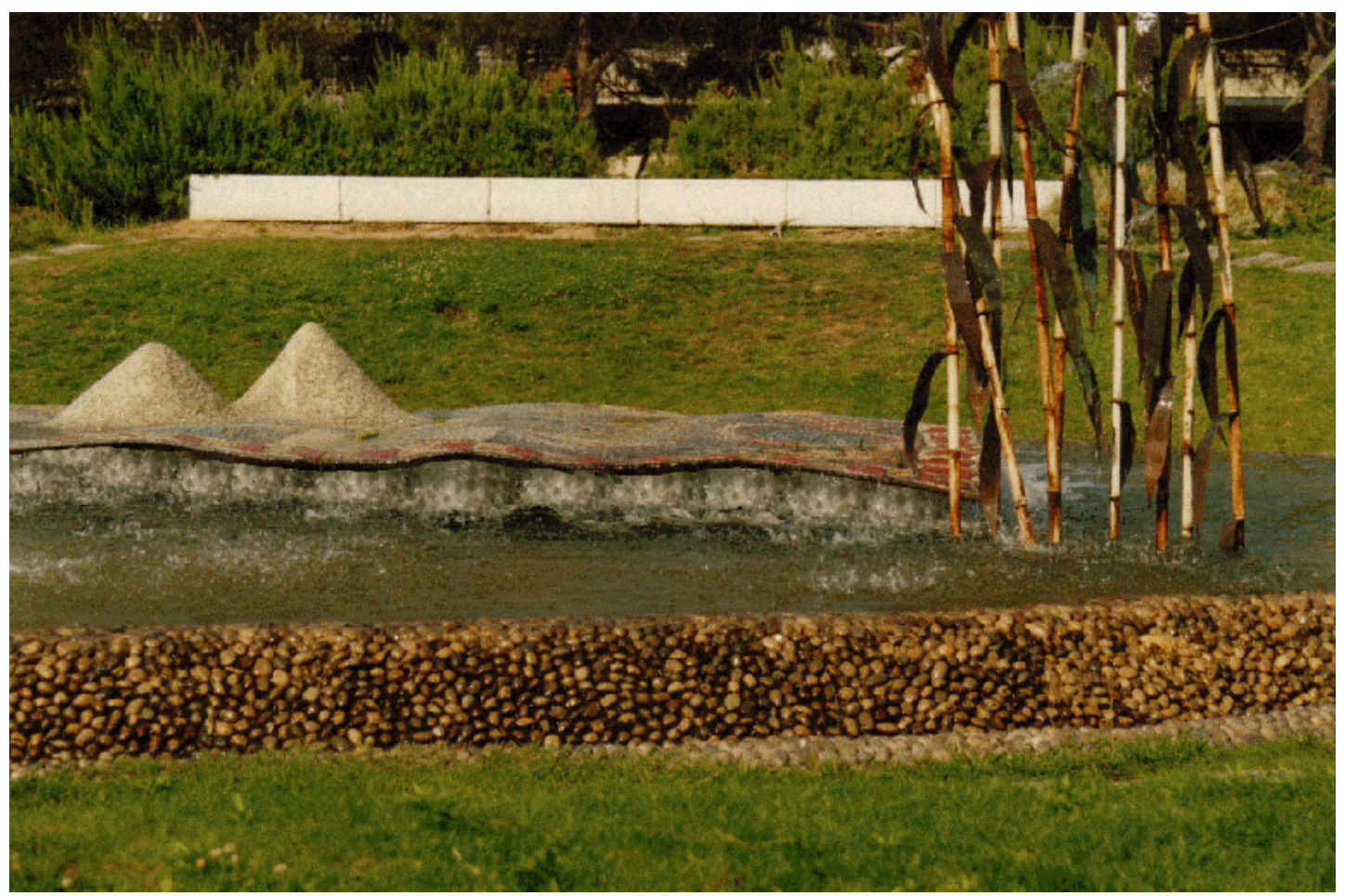

Fig. 10 Il Tappeto sospeso a Cervia.

\footnotetext{
${ }^{18}$ TONINO GUERRA, L'albero nero, in Il polverone, Maggioli Editore, Rimini 1992, pagg. 12-14.

${ }^{19}$ Tonino Guerra, Il giardino sonoro, in Il polverone, Maggioli Editore, Rimini 1992, pagg. 70-71.
} 


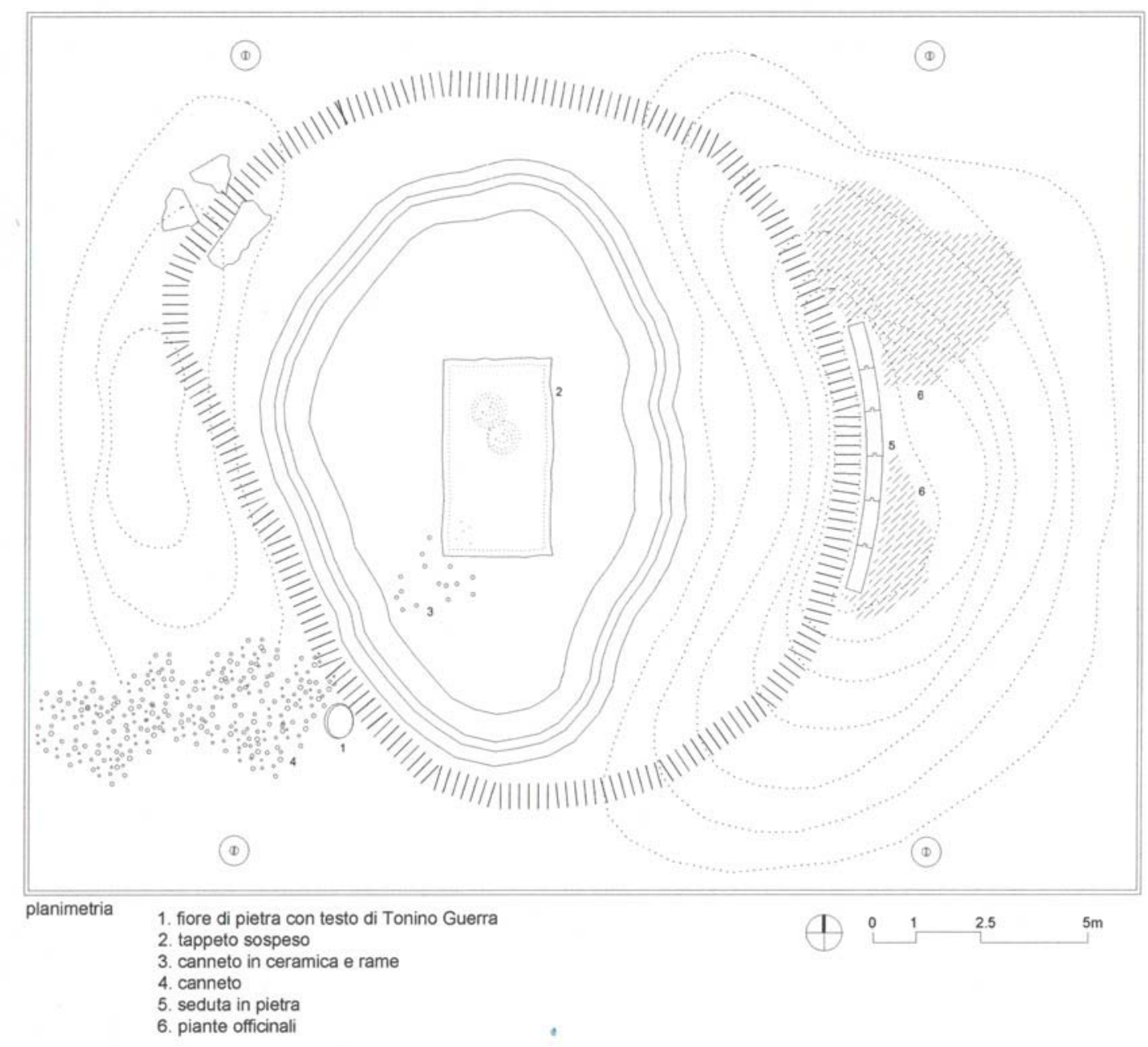

Fig. 11 "Il Tappeto sospeso" a Cervia (RA) - planimetria: 1. "Fiore di pietra" con iscrizione di Tonino Guerra, 2. tappeto a mosaico, 3. canneto di ceramica e rame, 4. canneto, 5. panca in pietra, 6. piante officinali

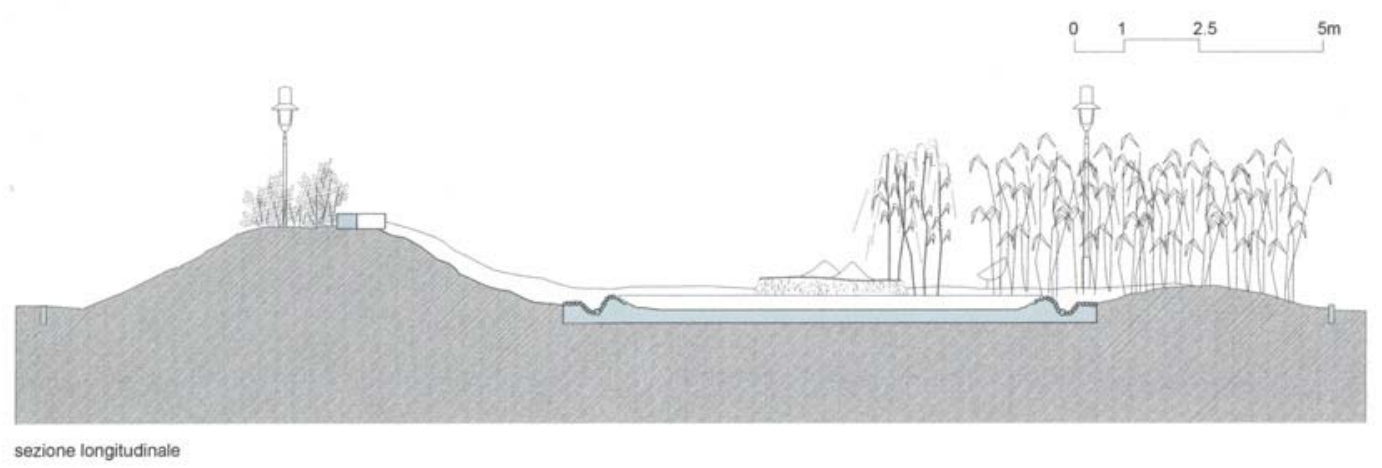

Fig. 12 “Il Tappeto sospeso” a Cervia - sezione.

Nell'Orto dei frutti dimenticati a Pennabilli, la gravità del bronzo, del legno e della pietra delle sculture è negata dalla composizione degli elementi nello spazio, che muove per aggregazione di punti a costituire nebulose: l'insieme delle steli de Il bosco incantato, la distribuzione seriale degli alberi da frutto, l'ammucchiarsi delle formelle appese a un muro con le madonne "raccolte" dai cippi abbandonati. Le raffigurazioni dell' Arco delle favole per gli occhi dell'infanzia che immette nel bosco incantato, delle formelle sacre, delle istallazioni 
ed il loro decorativismo orientale-popolare, costituiscono un ulteriore elemento smaterializzante, riducendo la percezione del volume degli oggetti che rivestono.

Lieve è il carattere fantastico di tutti gli eventi/presenze dell'Orto, che sono addirittura a volte - è il caso dei volti di Fellini e della Masina e della meridiana in cui a segnare l'ora è il corpo dello stesso visitatore - semplici ombre. Inoltre leggerezza e gravità stanno in un rapporto ambiguo nella volontà di memoria che rappresenta l'intento esplicito dell'Orto dei frutti dimenticati e nell'inserimento delle due meridiane, per cui il tempo diviene componente del giardino ai pesi diversi del passato e del presente.

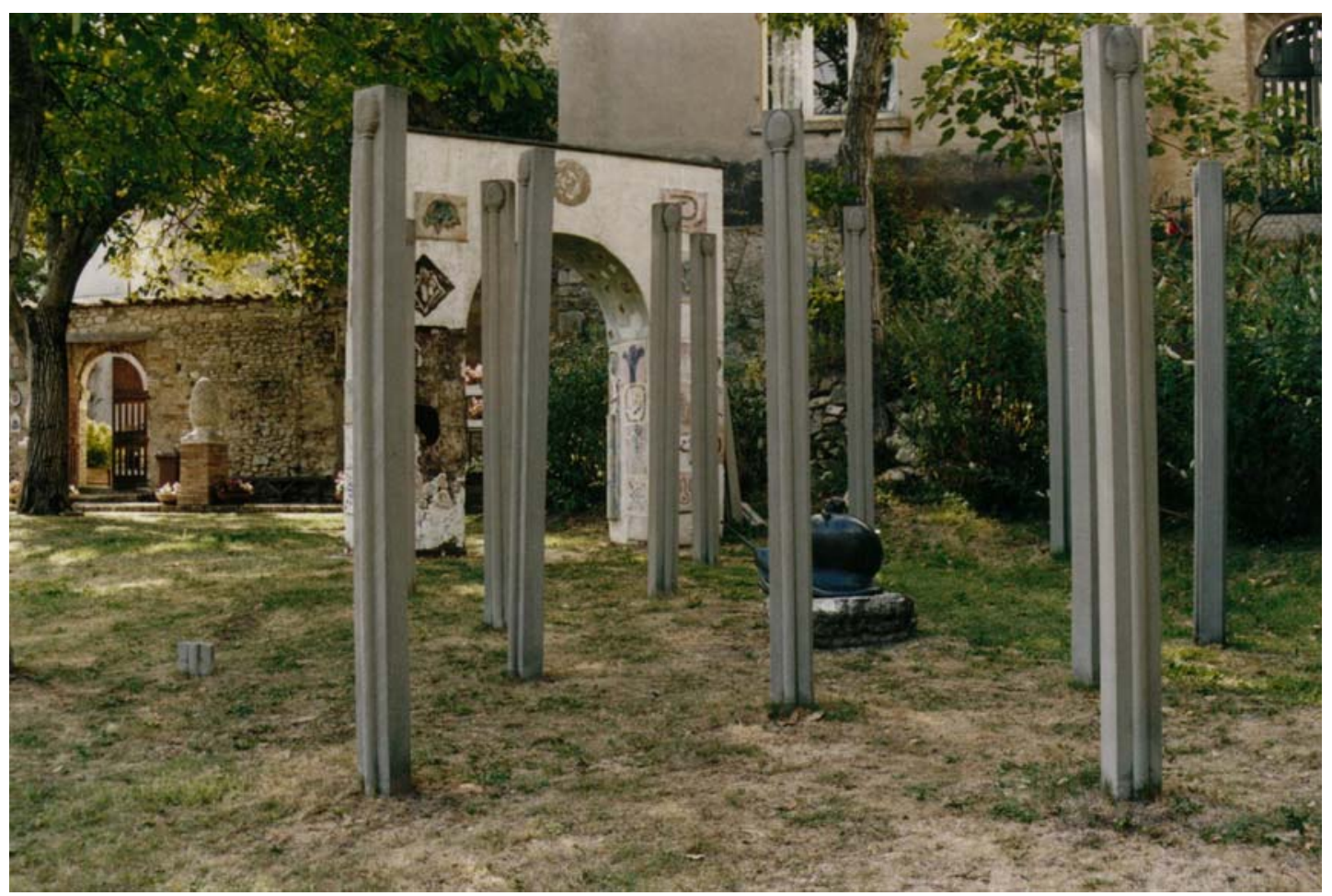

Fig. 13 L'orto dei frutti dimenticati a Pennabilli: labirinto di steli e Arco delle favole per gli occhi dell'infanzia.

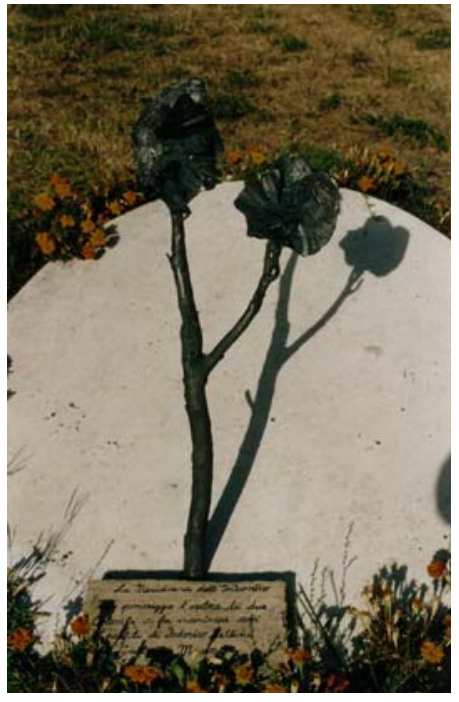

Fig. 14 La meridiana dell'incontro.

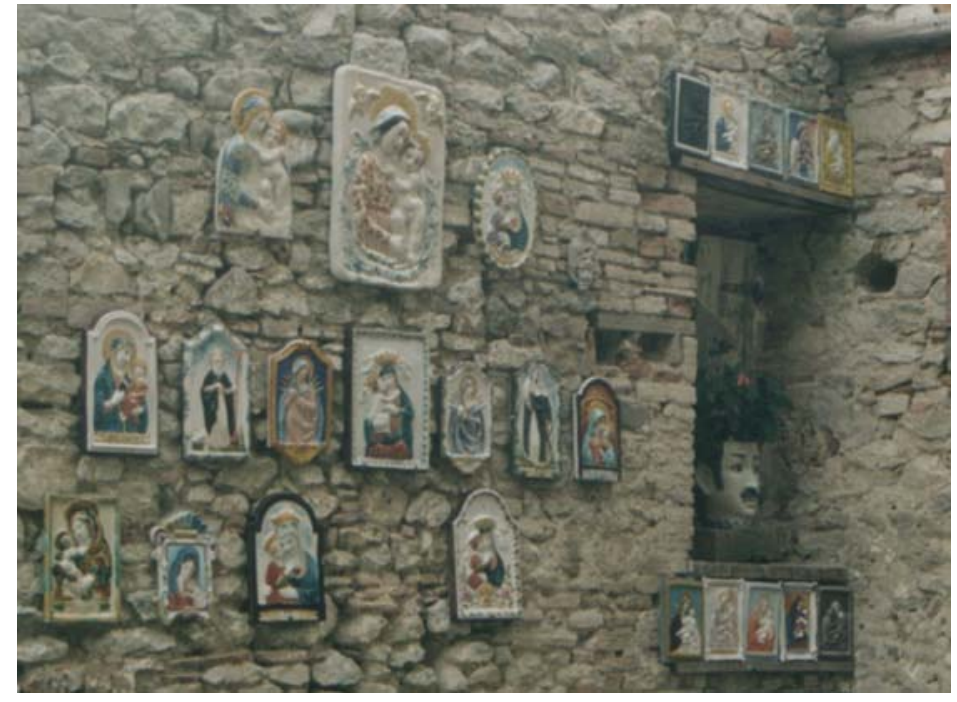

Fig. 15 Formelle in ceramica Le madonne abbandonate.

Avviene qualcosa di simile, nonostante siano diverse le culture di riferimento - da un lato la cultura classica, dall'altro il rapporto con la tradizione popolare - al legame fra reale e 
simbolico, fra natura ed evocazione letteraria, che Ian Hamilton Finlay istaura con le sue opere. Tonino Guerra firma messaggi scritti su pietra e ceramica: non sono solo semplici chiarimenti dell'autore a prologo della visione, ma operano un trasferimento dalle cose alla cultura dietro le cose. Impediscono la distrazione, guidano l'assegnazione di significati diminuendo i gradi di libertà dell'immaginazione. Eppure se per Finlay - in Silence per la foresta di Dean, A Remembrance of Annette a Monaco, paesaggio firmato Hodler a Furka Pass in Svizzera, I sing for the Muses And for Myself a Stockwood Park a Luton in Inghilterra- l'iscrizione ha potere di trasformazione sul paesaggio mutandone l'estetica e il contenuto, per cui il rapporto fra lettere, materie e ambiente circostante è quello proprio della poesia concreta, nei giardini di Tonino Guerra la parola scritta non diviene paesaggio e dichiara più esplicitamente il ruolo di filtro: il giardino viene annunciato e immediatamente interpretato. L'autore racconta il giardino che a sua volta manifesta l'autore e ha la sua voce. L'Orto dei frutti dimenticati di Pennabilli fa parte di un itinerario tematico più ampio I luoghi dell'anima, in cui il discorso prosegue, con un linguaggio maggiormente popolare, fino a $\mathrm{Il}$ Santuario dei pensieri.

Si incontrano La strada delle meridiane in cui sono esposte altre sei meridiane artistiche appese agli edifici del borgo, l'Angelo coi baffi all'interno di una piccola chiesa - un dittico di Luigi Poiaghi affiancato dalla poesia omonima di Tonino Guerra, con coreografia di uccelli imbalsamati e riproduzione dei cinguettii - e totem lungo il percorso.

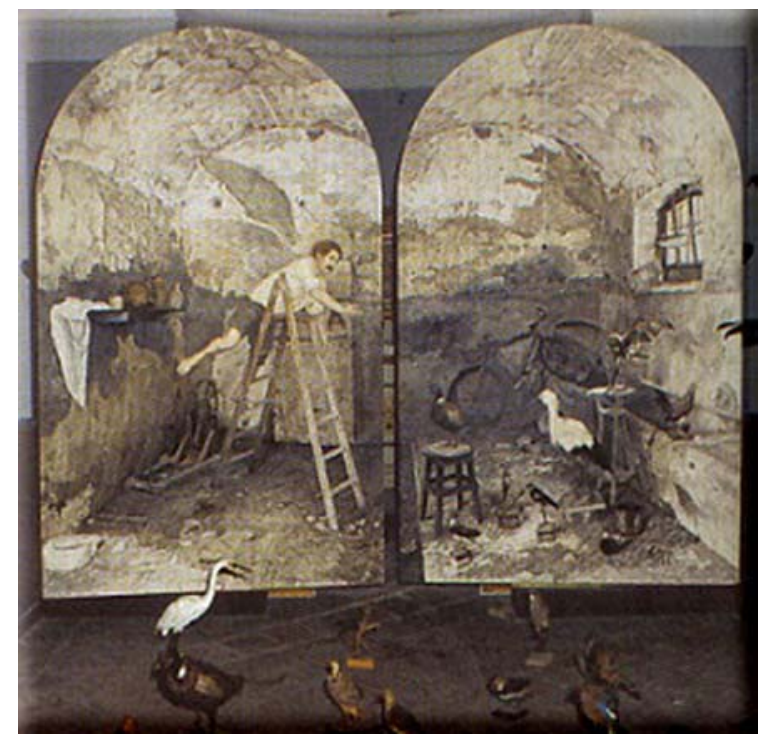

Fig. 16 L'Angelo coi baffi.

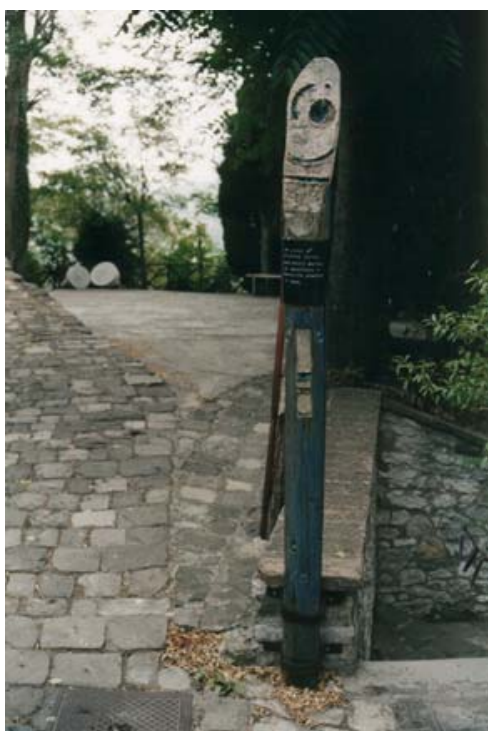

Fig. 17 Totem.

Il Santuario dei pensieri trasforma, mediante un restauro ideato dall'autore, i muri di un'antica abitazione del guasto malatestiano in un giardino raccolto .

Nel prato sono disposte sette pietre implicitamente fitomorfe, disposte secondo linee "magiche" spiegate in un'iscrizione. Il loro rapporto con la natura è nascosto ed esse assomigliano solo vagamente a cose già viste: un tulipano, una foglia. Lo spazio ospita poche cose e nessuna decorazione.

Le parole di Tonino Guerra invitano, prima di entrare, altre parole - quelle del visitatore - da rivelare ai “sette confessori muti". Poi l'autore esce di scena aspettandosi il silenzio, ma rimanendo comunque, nel rito, un celebrante:

" Mi nascosi dietro ad un muretto e cercai di raccogliere il silenzio come si prende un uccello in trappola. Una volta registrato il silenzio, sapevo che in quel silenzio poteva inserirsi un grido o la risata o la sillaba mistica di un 
Dio purchessia che fa vibrare il nulla e ripete l'atto della creazione, con la miseria degli uomini e il gelo e il fuoco e l'acqua e tutte le cose piene di sostanza sonora". ${ }^{20}$

Il timbro si alleggerisce al fine di una narrazione aperta il cui strumento sono i vuoti fra le pietre.

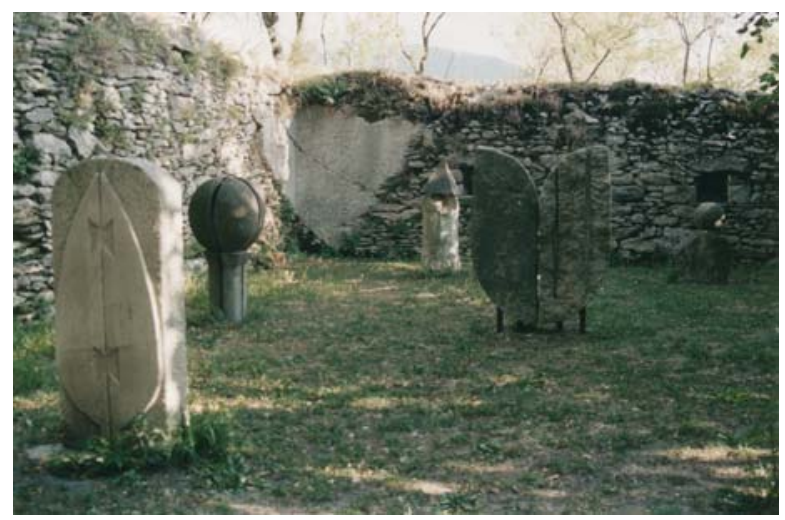

Fig. 18 Il santuario dei pensieri.

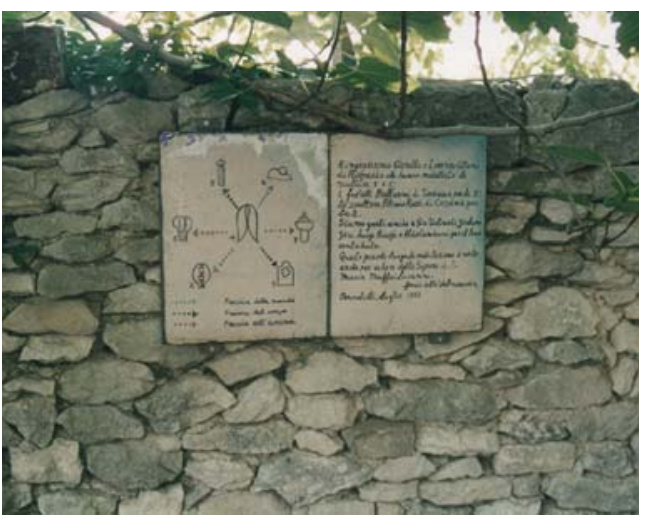

Fig. 19 Il santuario dei pensieri: iscrizione.

A Bascio il silenzio è costruito diversamente, nel Giardino pietrificato: ai piedi della torre malatestiana sono disposti i tappeti del ceramista Giovanni Urbinati, fra le pieghe del prato, a memoria dei grandi personaggi della storia e dell'arte che sono passati nel territorio vicino, Giotto, Dante, Buonconte da Montefeltro, Uguccione della Faggiola, Matteo da Bascio, Ezra Pound, Fanina dei Borboni.

Tonino Guerra esprime il ricordo come fosse una favola semplice, comunicabile a tutti, su cui si potrebbe anche chiaccherare. Non si crea però nessun rumore perché il racconto avviene a livello del terreno, fra piramidi e chiese minuscole, negli interstizi, lungo segni orizzontali che non costituiscono né massa né volume da consentirgli di entrare in vibrazione contrappuntistica con la torre eretta contro la vallata.

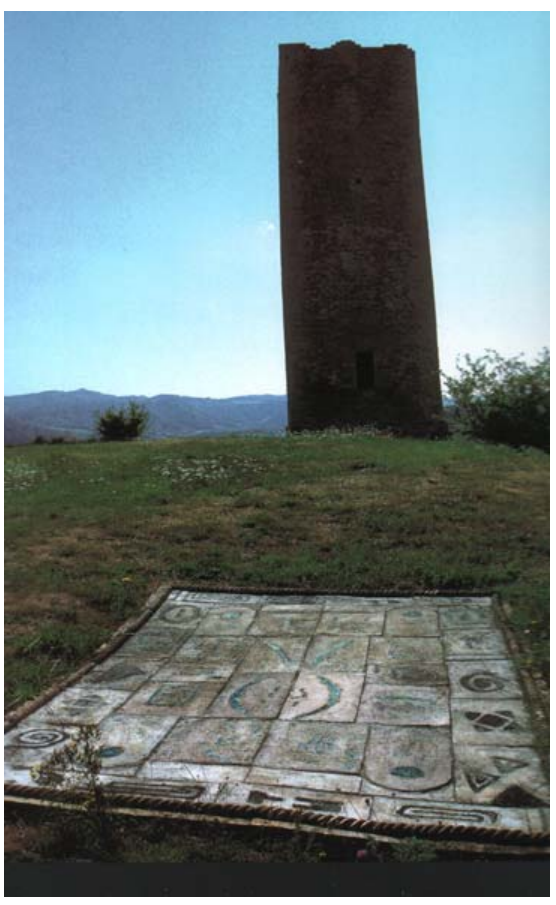

Fig. 20 Tappeto dei pensieri chiari.

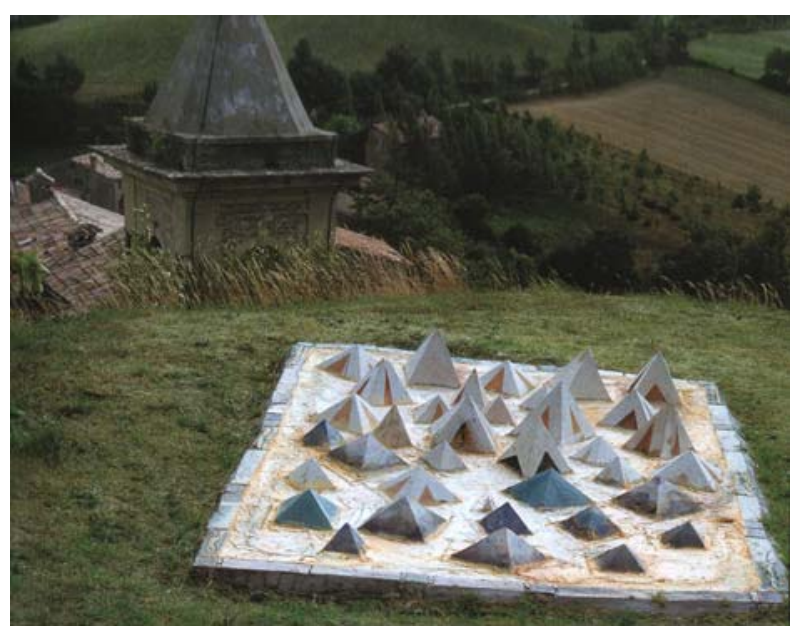

Fig. 21 Tappeto delle Piramidi.

\footnotetext{
${ }^{20}$ Tonino Guerra, Silenzio, in Dizionario fantastico, Pietroneno Capitani, Rimini 2000, pagg. 147-148.
} 


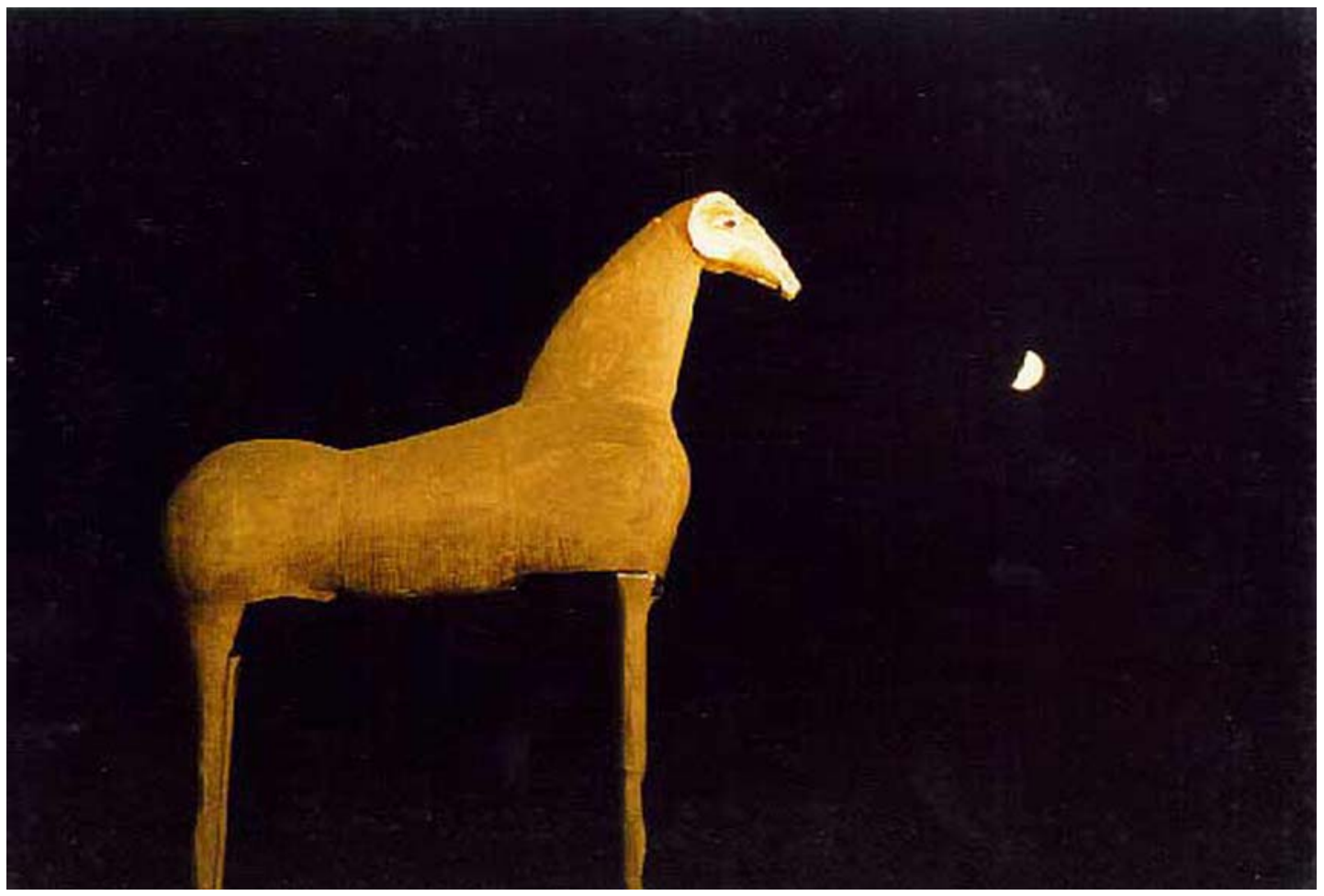

Fig. 22 Il cavallo.

All'Artista Mimmo Paladino era stato chiesto di realizzare un gruppo scultoreo per la propria città, Benevento.

Egli appartiene, con Sandro Chia, Francesco Clemente, Enzo Cucchi, Nicola De Maria, alla corrente, riconducibile al movimento Post-Modern, che il critico Bonito Oliva definisce sulla rivista "Flash Art" Transavanguardia, nel Novembre del 1979, significando un riscontrato allontanamento dal darwinismo linguistico delle avanguardie del secondo dopoguerra nell'assunzione di un " atteggiamento nomade di reversibilità di tutti i linguaggi del passato" 21 . A questa labilità del discorso, guidato da un "pensiero debole" quale proposto, in Italia, dai filosofi Gianni Vattimo e Pier Aldo Rovatti, si affianca un orientamento verso soluzioni neo-espressioniste, a causa di una razionalità non cartesiana, che cede spazio alla tensione, alla fisicità, alla "primarietà",22.

L'occasione per l'esposizione delle opere di Mimmo Paladino a Benevento è offerta dal restauro più ampio, di cui è curatore l'architetto Palmieri, legato al recupero funzionale dell'ex-convento di San Domenico utilizzato precedentemente come sede del Tribunale e attualmente sede amministrativa della nuova Università di Benevento.

L'artista invita l'architetto Roberto Serino alla realizzazione, per ideazione congiunta, di un luogo il cui "tema architettonico è quello della ridefinizione dei "margini" entro i quali mettere in scena la rappresentazione dell'arte": lo spazio che nel Seicento era un orto pensile diviene hortus conclusus.

\footnotetext{
${ }^{21}$ Achille Bonito Oliva (a cura di), Avanguardia Transavanguardia, Electa, Milano 1982, cit. in LEA VERGINE, L'arte in trincea - Lessico delle tendenze artistiche 1960- 1990, Skira, Milano 1996, pag. 245.

${ }^{22}$ Il concetto di primarietà è ripreso da Flavio CAROLI, I Mostri di una domenica tedesca, in La giovane pittura in Germania, catalogo, Galleria d'Arte Moderna, Bologna, 1982, e da Flavio CAROLI, Il Magico primario, in Magico primario, Fabbri, Milano 1982, cit. in LEA VergINE, L'arte in trincea - Lessico delle tendenze artistiche 1960- 1990, Skira, Milano, 1996, pagg. 246-247.
} 
La formazione del temenos ${ }^{23}$ - all'interno è uno spazio ieratico in cui le sculture dell'artista sono sia genitrici sia sacerdotesse - è perseguita, su un lato, mediante la costruzione di una doppia parete che costituisce una galleria, continuazione di un vecchio passaggio di accesso, recuperato.

Il recinto è immaginato come spazio percorribile: dalla prospettiva che apre sul giardino le figure vengono anticipate ma subito negate per l'itinerario che devia. La galleria ha funzione di limbo. Intende realizzare un luogo di distacco che consenta di lasciare la contingenza della città. Attraverso di essa l'orto stesso si allontana e non è possibile percepire nessun traguardo oltre il blu assorbente della parete di fronte, da cui si riversa acqua come da una fonte sacra.

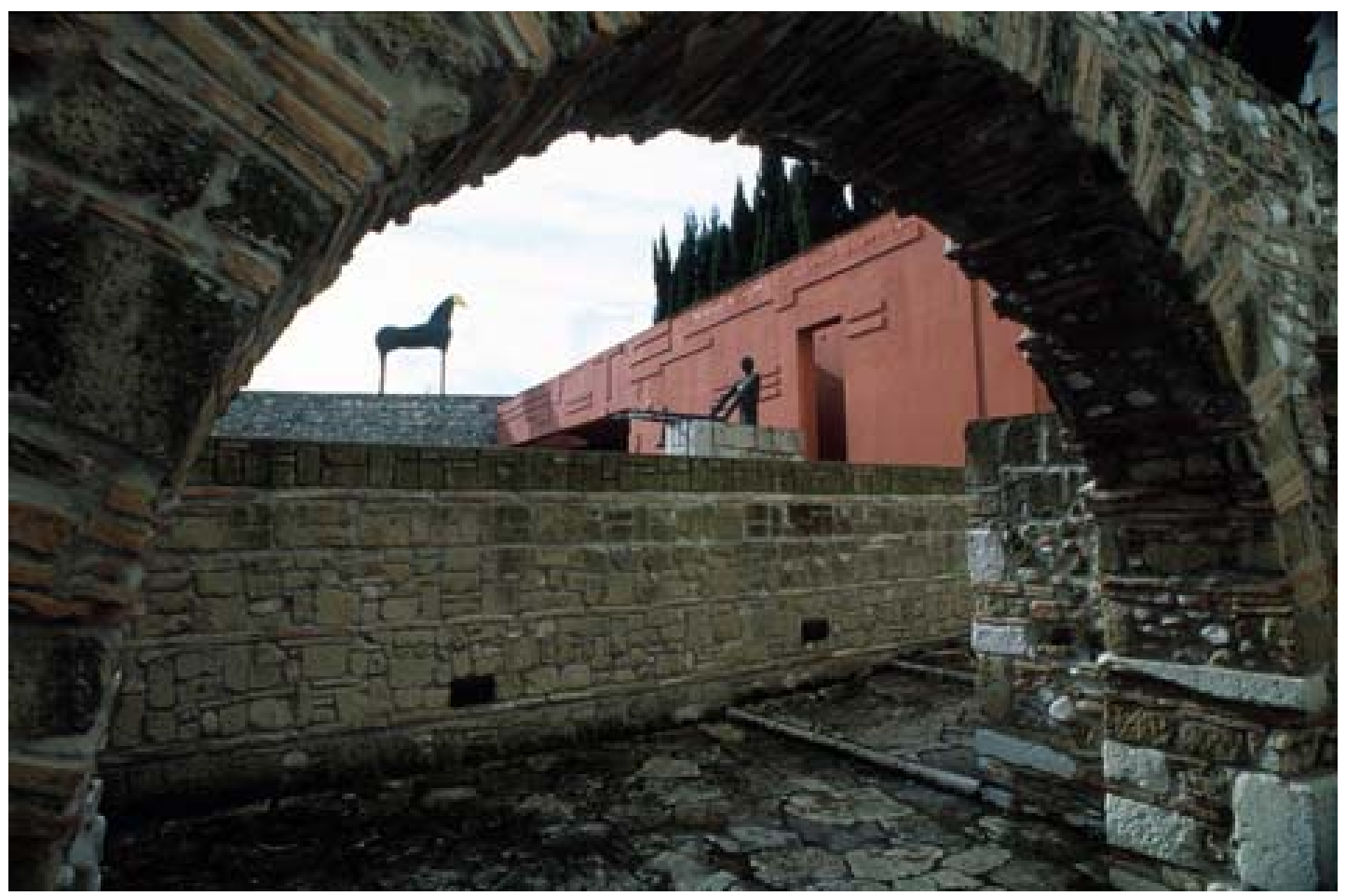

Fig. 23 Prospettiva che anticipa le figure del giardino.

Una finestra fa riapparire, transitando, l'interno, senza consentire l'accesso.

La scultura che si oppone al passaggio assume il compito di allungare il periodo della percorrenza, avvisando che gli eventi successivi avranno un carattere di alterità: questo fornisce giustificazione all'estraneazione temporale.

Ulteriormente è possibile, tramite le scale che conducono ad un giardino degli odori, indugiare per altri spazi.

L'ideazione sembra muovere attorno alla volontà di creare un'anti-camera necessaria alla scoperta delle opere di Paladino che si riveleranno, per le forme sintetiche, quasi archetipiche e slegate dal contesto. Allo stesso tempo saranno evocatrici di una civiltà antica - quella dei Sanniti che fondarono Benevento - animate da una fantasia mitologica, lungo un tracciato di continuità culturale. ${ }^{24}$

\footnotetext{
${ }^{23}$ Il termine viene riferito ad un altro progetto di Roberto Serino - la chiesa, non realizzata, per Gibellina - in Benedetta Gravagnuolo, Architetture scolpite con la luce: opere di Roberto Serino, "Controspazio", n. 6, Gangemi Editore, Roma 1994, pagg. 34-46.

24 "Dal Neo-espressionismo la Transavangurdia prende il concetto di immagine ridotta ai suoi elementi espressivi fondamentali, realizzati con tratti violenti e colori accesi. Ma contemporaneamente se ne distacca per una visione meno drammatica ma più solare. In particolare per quel gusto tutto mediterraneo di un'affabulazione sensuale che si ritrova soprattutto in Paladino. (...) Nella Transavanguardia troviamo infatti il ricorso alla memoria e alla citazione, con la scelta optata verso la tradizione delle Avanguardie storiche. Memoria e citazione che rimangono però legati esclusivamente allo stile. I riferimenti poetici, specie in Paladino sono ben altri. Essi riguardano la
} 


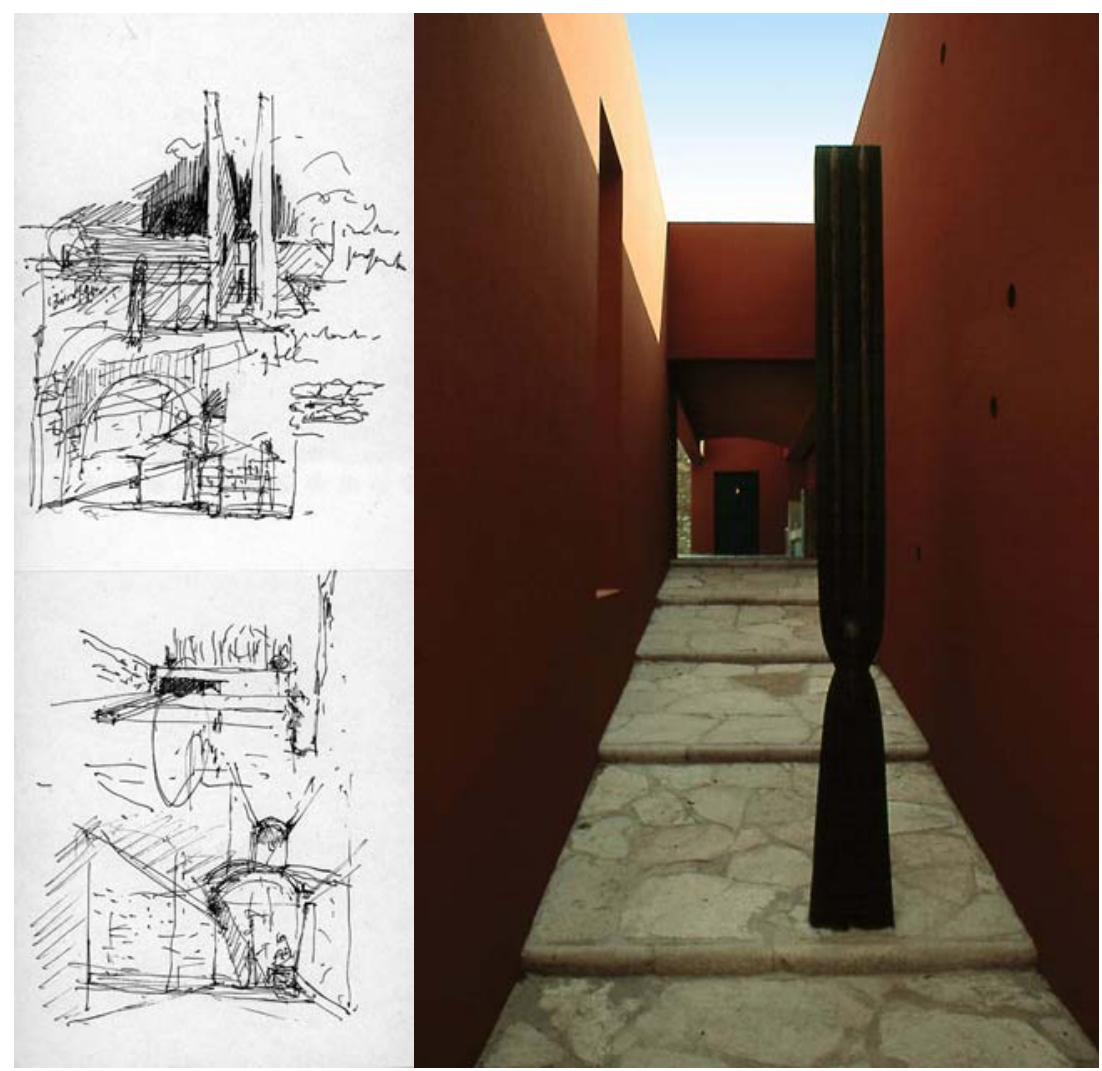

Fig. 24 Schizzi per la galleria. Fig. 25 La galleria.

Dopo il preludio della galleria l'accesso avviene improvviso, lateralmente, mediante un portale ampio, che immette nella piazza dei "Rimandi". E' una superficie di pietra ad opus incertum, dal sapore antichissimo, con una sensazione quasi tattile, rugosa, variabile nella tessitura, mentre lo sguardo gira a indagarla nella sua interezza. Su questa superficie poggiano, ruotando con movenze lentissime - in realtà è l'osservatore a spostarsi nell'esplorazione - un disco al centro, una conchiglia, una campana, una testa equina, un lungo teschio di bucranio ${ }^{25}$. Il rapporto con un ambiente consueto è sensibile solo nel giardino, progettato da Palmieri, sul quale sono presenti i tetti di altri edifici, ed in qualche modo richiamato da un ombrello rovesciato a terra ai piedi di un albero che spacca, esile, la crosta di pietra.

Il cavallo rivolto oltre il confine dell'orto, a vedetta, proietta verso cose assenti all'interno, stabilendo un potere sulla città invisibile. Unitamente ai colori primari del rosso e del blu cobalto contro lo sfondo di cipressi, la sua maschera d'oro e la sagoma nera ritagliata definiscono un'iconografia bidimensionale e statica, quasi fosse una raffigurazione arcaica di sovranità. La statua oggettiva la poetica di Paladino:

riscoperta della memoria "profonda", quella dove, con spirito quasi metafisico, le forme restano fisse in istanti senza tempo. Devitalizzati dalle tensioni contingenti, per mostrare l'essenza delle cose, le forme stesse si riducono a quel nocciolo duro che più duramente e lentamente il tempo riesce a scalfire.

(nell'opera di Paladino) ricorrono immagini che rimandano ad un universo arcano e primitivo, dove le forme sono tradotte in segni eleganti e semplificati.” Da Mimmo Paladino, in www. Francescomorante.it/pag_3/318a.

${ }^{25}$ Le statue dell'Hortus Conclusus non hanno un titolo assegnato dall'autore coerentemente alla volontà di identificare l'intero spazio come opera unica. I nomi utilizzati hanno solo un valore descrittivo- identificativo. 
“(...) Ogni mio lavoro è sempre e totalmente in equilibrio, nel senso che il movimento non è mai dichiarato (...). Io mi considero un artista sospeso ad un filo: posso cadere da un momento all'altro, ma non voglio cadere da nessuna delle due parti. ${ }^{26}$

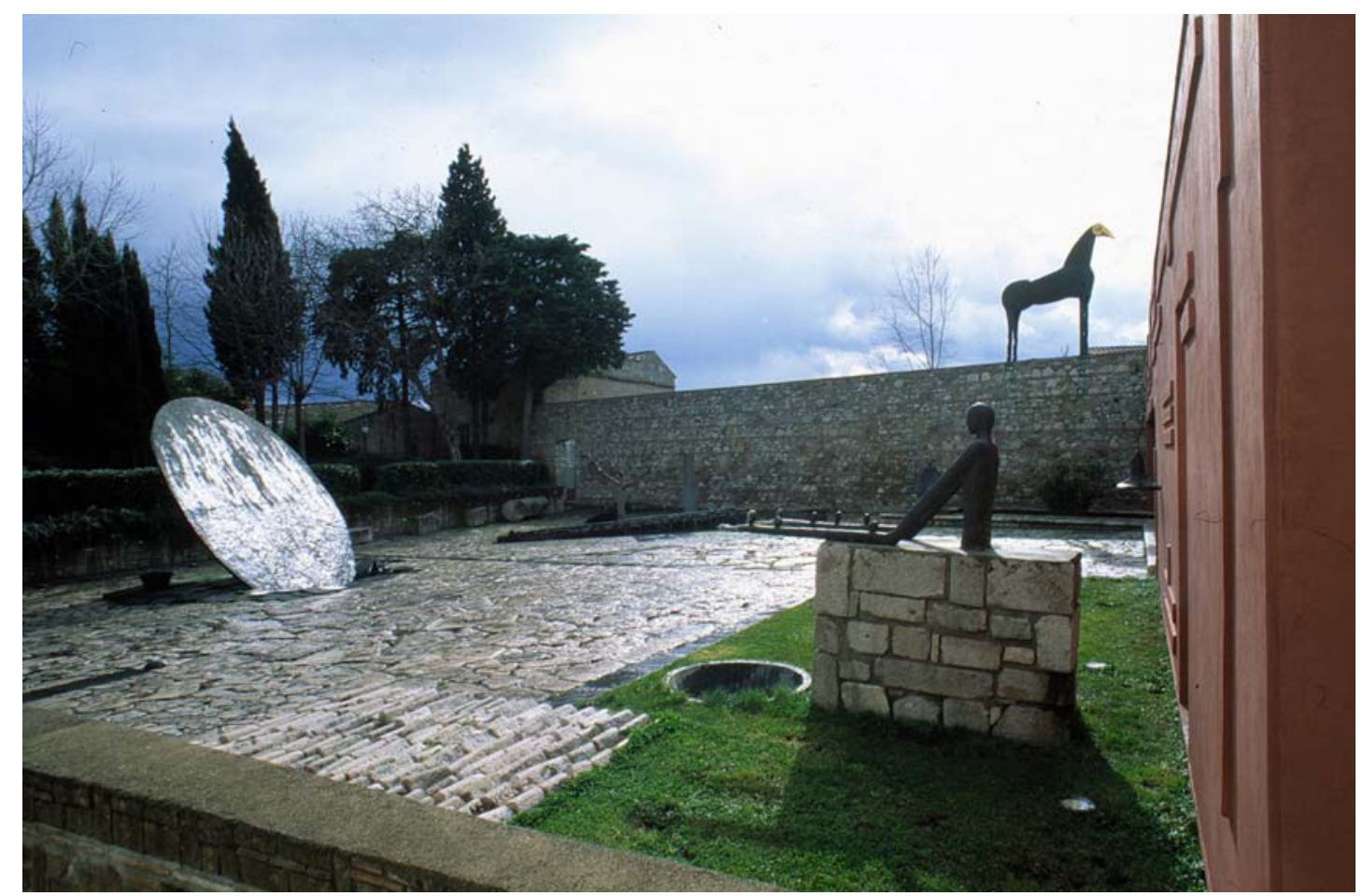

Fig. 26 Interno dell'Hortus conclusus.

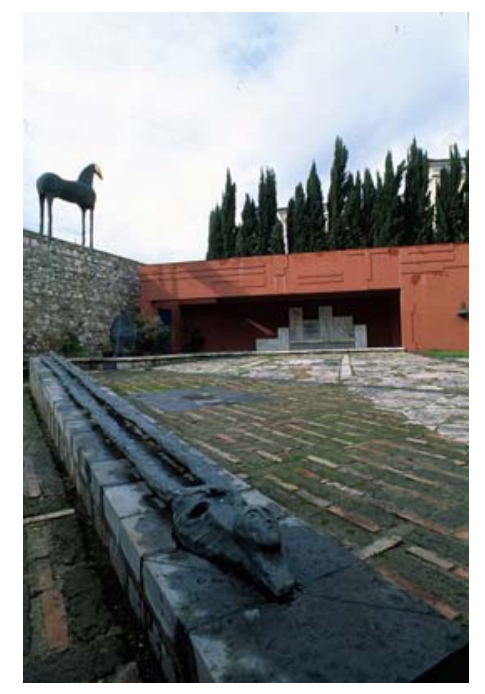

Fig. 27 Interno dell'Hortus conclusus. Fig. 28 Acqua dal blu cobalto.

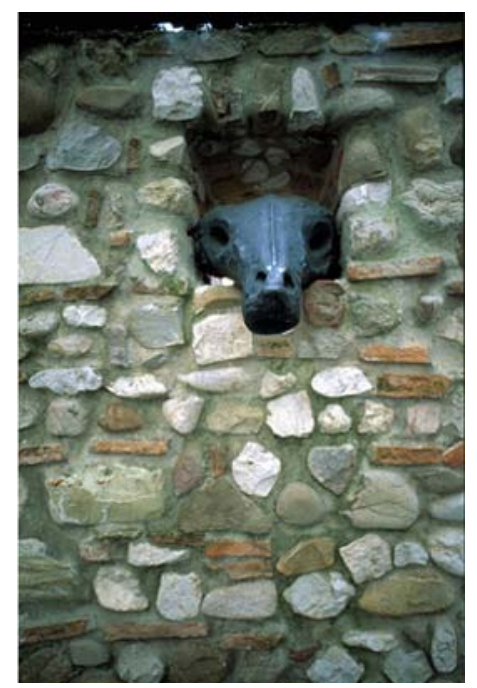

Fig. 29 Figura nell'Hortus conclusus.

Osservandole nei rimandi coreografici, le sculture di Paladino potrebbero raccontarsi qualcosa come le fanciulle de "Il Marinaio" 27 di Fernando Pessoa.

Anche lo spazio dell'Hortus sembra volere ospitare un "dramma statico in un quadro"28 conferendo un significato intenso al suo essere conclusus.

\footnotetext{
${ }^{26}$ Da Intervista con Michele Bonuomo, in Terrae motus, catalogo, Electa, Napoli 1984, cit. in LEA VERGINE, L'arte in trincea - Lessico delle tendenze artistiche 1960- 1990, Skira, Milano 1996, pag. 250.

${ }^{27}$ Fernando PeSsoa, Il marinaio, Einaudi, Torino 1988.
} 
Ogni elemento attribuisce fissità.

Seppure l'acqua sgorghi ovunque, non c'è trasformazione, in quanto la terra immediatamente la inghiotte, scivolata sul disco. Dal muro blu della galleria si stende, piatta, in un rettangolo fermo su cui si duplica il profilo immobile del cavallo. Una figura stabilizza i gomiti di braccia troppo lunghe su un piano di pietra, per lasciarsi cadere acqua dalle mani, senza un gesto, mentre nell'erba si dispongono colonne in serie orizzontale, impossibilitate al crollo. Semplicemente l'acqua itera l'esistenza di queste divinità.

Se sia fisica oppure onirica non è chiaro e si creano le componenti di cui sono costituiti i miti: realtà energiche conosciute mediante una fantasia primitiva.

Oppure è il sogno che si materializza. ${ }^{29}$

"PRIMA VEGLIATRICE: L'ora non è mai suonata.

SECONDA VEGLIATRICE: Non potevamo sentirla, non ci sono orologi qui vicino. Tra poco dovrebbe essere giorno.

TERZA VEGLIATRICE: No, l'orizzonte è buio.

PRIMA VEGLIATRICE: Non desiderate, sorelle mie, che ci intratteniamo raccontando quel che siamo state? E' bello ed e' sempre falso.

SECONDA VEGLIATRICE: No, non ne parliamo. E poi, siamo state qualcosa?

PRIMA VEGLIATRICE: Forse. Non lo so. Ma ad ogni modo è sempre bello parlare del passato. Le ore sono colate e noi abbiamo serbato il silenzio. Io , da parte mia, sono rimasta a guardare la fiamma di quella candela. A volte trema, a volte diventa più gialla, a volte impallidisce. Non so perché succeda questo. Ma forse che noi sappiamo, sorelle mie, perché le cose succedono?"30

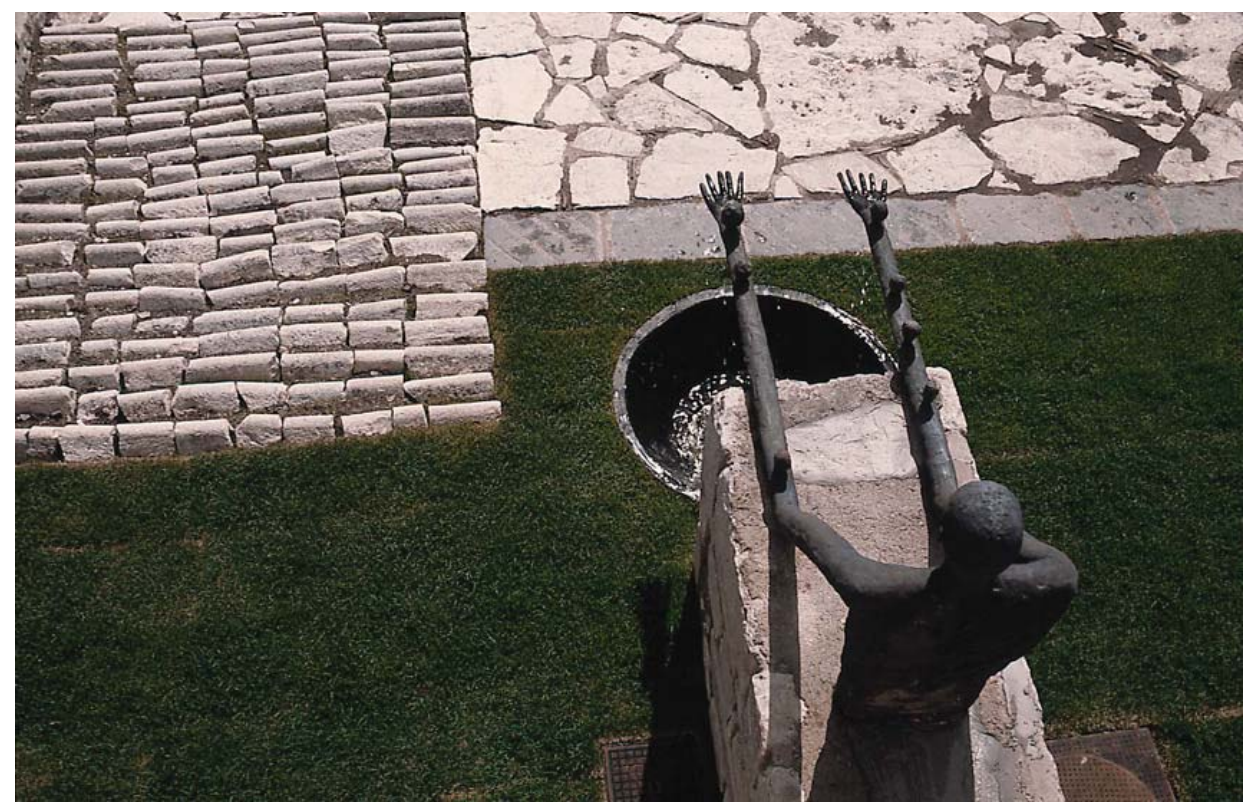

Fig. 30 Figura con lunghe braccia dalle cui mani scivola acqua - Tessitura a terra di colonne e pietra.

\footnotetext{
${ }^{28}$ Così Ferdinando Pessoa definisce Il marinaio. Il titolo originale dell'opera è O Marinheiro. Drama estatico em um quadro.

${ }_{29}$ Il particolare rapporto fra realtà inconscia e realtà materica proprio della Transavanguardia è delineato in maniera lucidissima da Giulio Carlo Argan: "La Transavanguardia rivendica la parte del corpo anche come veicolo di energie dell'inconscio. Le stesse immagini conservano una pesantezza fisica, rimangono impastate con la materia del colore. Non trascorrono con la labilità propria delle immagini della televisione o della pubblicità, ristagnano e costringono a certi tempi di osservazione. La natura di quelle immagini è ambigua: da un lato sono appesantite dalla materia pittorica, dall'altra inconsistenti come nomi, quasi si volesse provare visivamente il nesso lacaniano di inconscio e linguaggio. Il ristagno è come una ripetizione e con la ripetizione l'immagine si altera e corrompe, diventa abituale, la si riconosce senza neppure guardarla, ma proprio perciò finisce per assumere un senso vagamente mitico. Infine ci si accorge che ha una sua vitalità soltanto fisiologica, ma tenace (...).", in Achille Bonito Oliva ( a cura di ), Avanguardia Transavanguardia, Electa, Milano, 1982, cit. in LEA VERGINE, L'arte in trincea - Lessico delle tendenze artistiche 1960-1990, Skira, Milano 1996, pag. 243.

${ }^{30}$ Da Fernando Pessoa, Il marinaio, Einaudi, Torino 1988, pag. 5.
} 


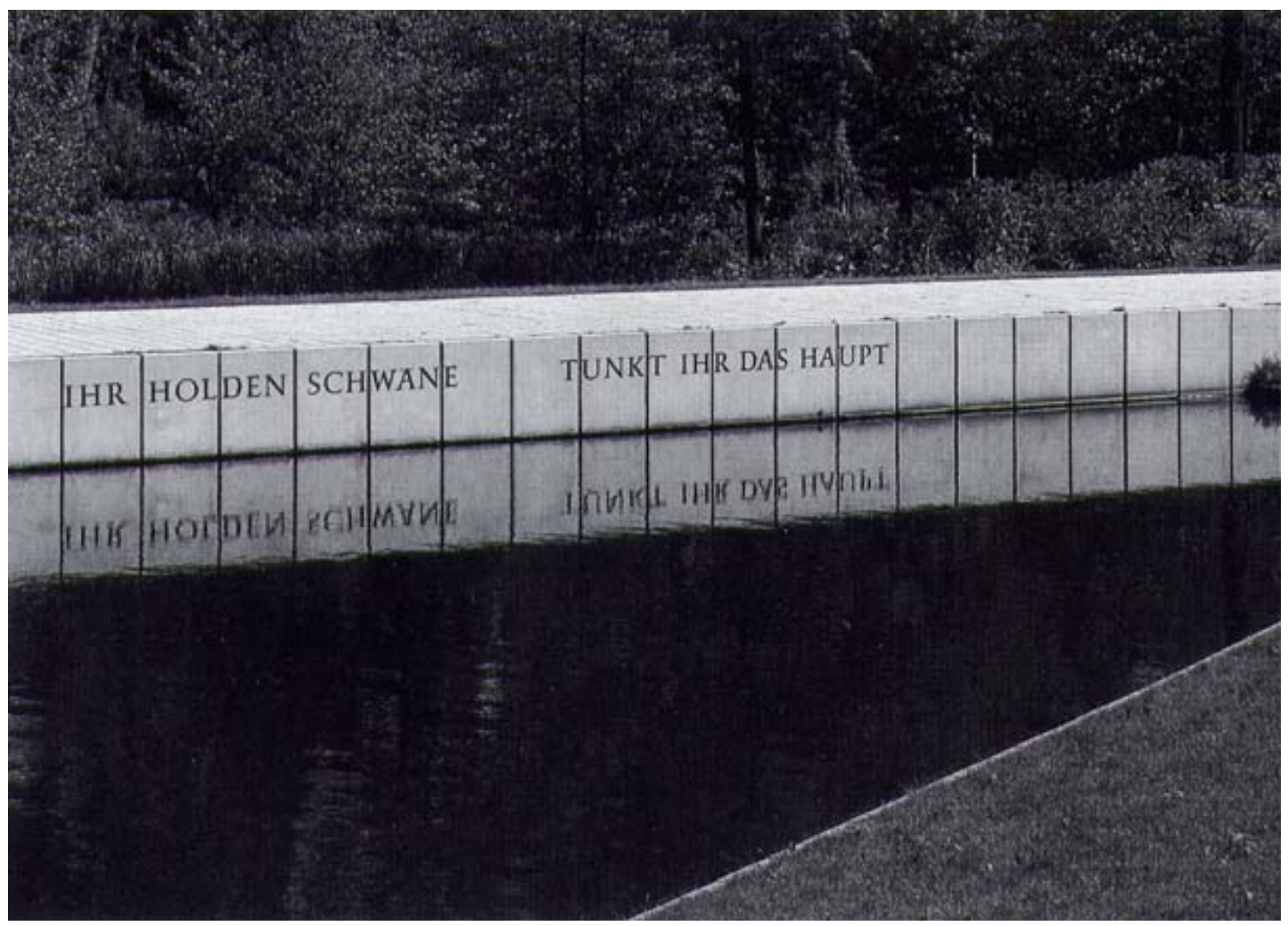

Fig. 31 Schlosspark, Grevenbroich (1995) - A didascalia del progetto delle iscrizioni lungo il percorso, si legge: "Two lines from Holderling Halfte Des Lebens are inscribed at the side of a path where it borders a lake. They are animated by the presence of real swans", a dichiarare il rapporto fra paesaggio ideale e paesaggio reale.

Si sceglie di parlare dei giardini di Ian Hamilton Finlay, per il ruolo che il simbolo assume nella creazione e nella percezione dei paesaggi dell'artista, e per lo stimolo offerto da una frase di Stephen Bann che definisce l'autore divertente. L'aggettivo, in relazione all'artista, sembra strano, probabilmente non è il primo attributo che viene in mente, dato il significato comune del termine e il tono dotto, erudito della poetica di Finlay. Ma lungo il percorso della ricerca la parola divertente ha recuperato un'accezione più complessa: divertente è qualcosa che consente una percezione diversa, nuova, che trasforma al visione.

"Ciò che colpisce di più del lavoro di Ian Hamilton Finlay, è che lui non si scusa mai per il proprio status culturale. E' divertente senza essere superficiale, sottile senza stare nell'ombra. Finlay pone ancora una volta il progetto paesaggistico al centro della ricerca creativa." ${ }^{31}$

Finaly è uno degli esponenti della "poesia concreta", nata alla fine degli anni '50 con la rivista "Noigrandes" brasiliana, il cui sviluppo in Inghilterra si deve al lavoro editoriale della Wild Hawthorn Press, fondata da Ian Hamilton Finlay e Jessie McGuffie, nel 1961.

La poesia concreta attribuisce alle parole, che sono suono - se pronunciate - e significato, un valore ulteriore dato dalla loro posizione nello spazio, cosicché scompare il confine fra pagina scritta e quadro. La composizione di poesia concreta propone un messaggio non dissociabile dalla sua collocazione: il ritmo temporale con cui si leggono le parole, il respiro che la poesia impone fra le immagini, viene concretizzato nello spazio, cessa di consistere nel semplice andare a capo, nell'interpunzione, per occupare liberamente la pagina.

\footnotetext{
${ }^{31}$ StePhen BAnN, L'Arcadia di Finlay, “Lotus”, n. 88, Electa, Milano 1996, pag. 92.
} 
"I precedenti si chiamano Apollinaire, Mallarmé, Kassak, Breton, Tzara, Picabia, Van Doesburg, Balla, Morgenstern ecc." 32

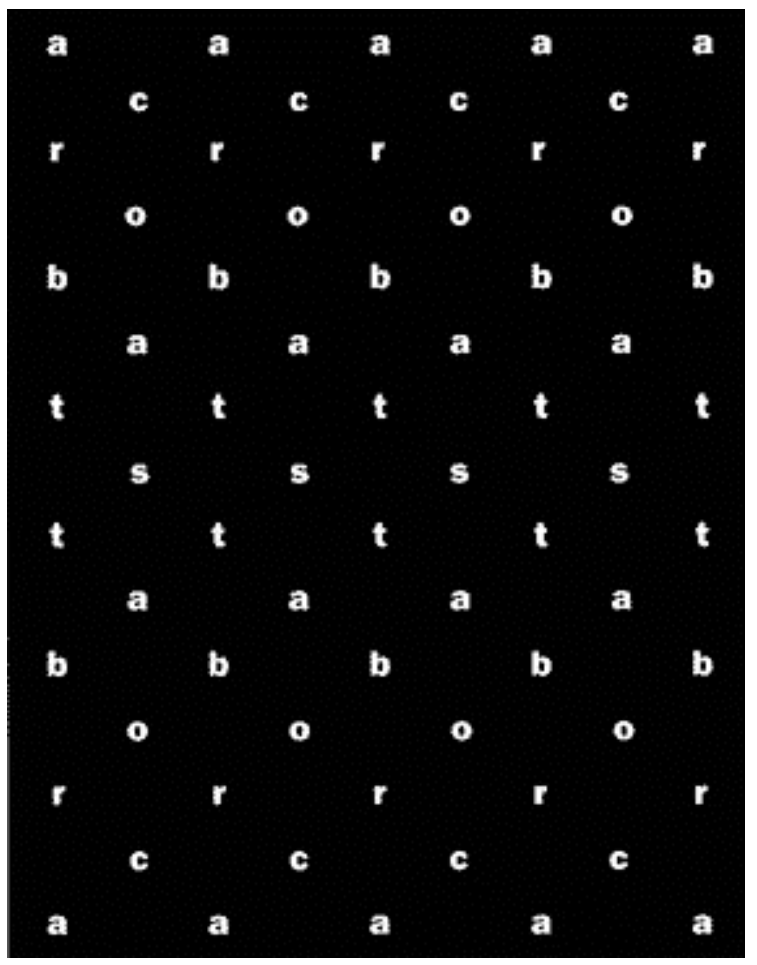

Fig. 32 Poster poem: Acrobats di Ian Hamlton Finlay

Max Bense precisa, nel 1966 su un numero unico della rivista genovese "Modulo", come nella "poesia concreta":

“ Non la giustapposizione delle parole nella mente, ma il loro intreccio nella percezione è (...) il principio costruttivo"33

Se lo spazio fra le parole diventa tridimensionale ed inoltre non è più un vuoto ma un pieno articolato di materiali differenti e aventi significato già in se stessi, quale è un giardino, il discorso viene a complicarsi sensibilmente. Si moltiplicano i segni e i significati. Durante un'intervista del 1996 in cui viene posta la domanda se l'arte dei giardini sia in qualche modo per l'autore un'estensione della propria poesia concreta, Finlay risponde:

"Some works I would do outside would be what you would consider a broadening of it, and other works derive more from the classical traditions. But at the beginning it was clear to me that concrete poetry was peculiarly suited for using in public settings. (...) I would have liked to do it - I used to have dreams about doing big concrete poems.." 34

L'estensione della poesia concreta nello spazio del giardino approssimativamente ha inizio nel 1966, quando Finlay si trasferisce con la moglie nella regione delle Pentland Hills in Scozia e incomincia ad allestire nel suo giardino meridiane, pietre con iscrizioni...La meridiana è un tema ricorrente, quale oggetto misterioso che ha il potere di stabilire un legame fra la dinamicità del tempo e la fissità dello spazio. La meridiana The Canterbury Sundial (1972) tracciata su una panca alla University of Kent, interpretando l'espressione di

\footnotetext{
${ }^{32}$ LEA Vergine, L'arte in trincea - Lessico delle tendenze artistiche 1960- 1990, Skira, Milano 1996, pag. 111.

33 Cit. in Anty Pansera, Maurizio Vitta, Guida all'Arte contemporanea, Marietti, Casale Monferrato 1986, pag. 182.

34 Da un'intervista a Finlay, condotta da Nagy Rashwan il 12 Aprile 1996, a Little Sparta (Stonypath), Dunsyre, Lanark, Scotland, in http://jacketmagazine.com/15/rash-iv-finlay.html.
} 
Stephen Bann, è veicolo per intercettare il genius loci del luogo, evocato dall'iscrizione SEA - LAND/ a lato dei tracciati della meridiana:

“(...) la meridiana rimane aperta ai raggi del sole, e nella sua intersezione di linee, celebra l'incrociarsi di "terra" e "mare" in un sito in cui la costa dell'East Kent è spesso visibile all'imbocco della Stour Valley.",35

La meridiana immette all'interno del giardino, nella posizione solitaria in cui la panca è collocata, la vastità dello spazio esterno, l'immagine aperta, fino alla costa, di un paesaggio allo stesso tempo dato e infinito. Paesaggio che è anche reale - in quanto esistente, di cui è possibile avere esperienza - e immaginato, perché assente nel punto in cui è la panca, richiamato alla mente dalle parole scritte. Finlay crea un rapporto prossimità-distanza, presenza-assenza, reale-immaginario che individua uno spazio per la percezione creativa dell'osservatore, il quale sente in modo personale l'oscillazione fra i termini dei binomi, inserendosi con un proprio ritmo nella contemplazione del paesaggio. Finlay è consapevole della presenza dell'osservatore, quale agente finale nel processo di modificazione del paesaggio che egli, in qualità d'artista, intraprende per primo con l'inserzione dell'elemento culturale. La "proposta per Furka Pass, Svizzera 1987" è una dichiarazione di poetica come lo sono in generale le "proposte", pubblicazioni a tiratura limitata, edite da The Wild Hawthorn Press, con cui Finlay illustra e descrive in anteprima i concetti e i risultati dei progetti.
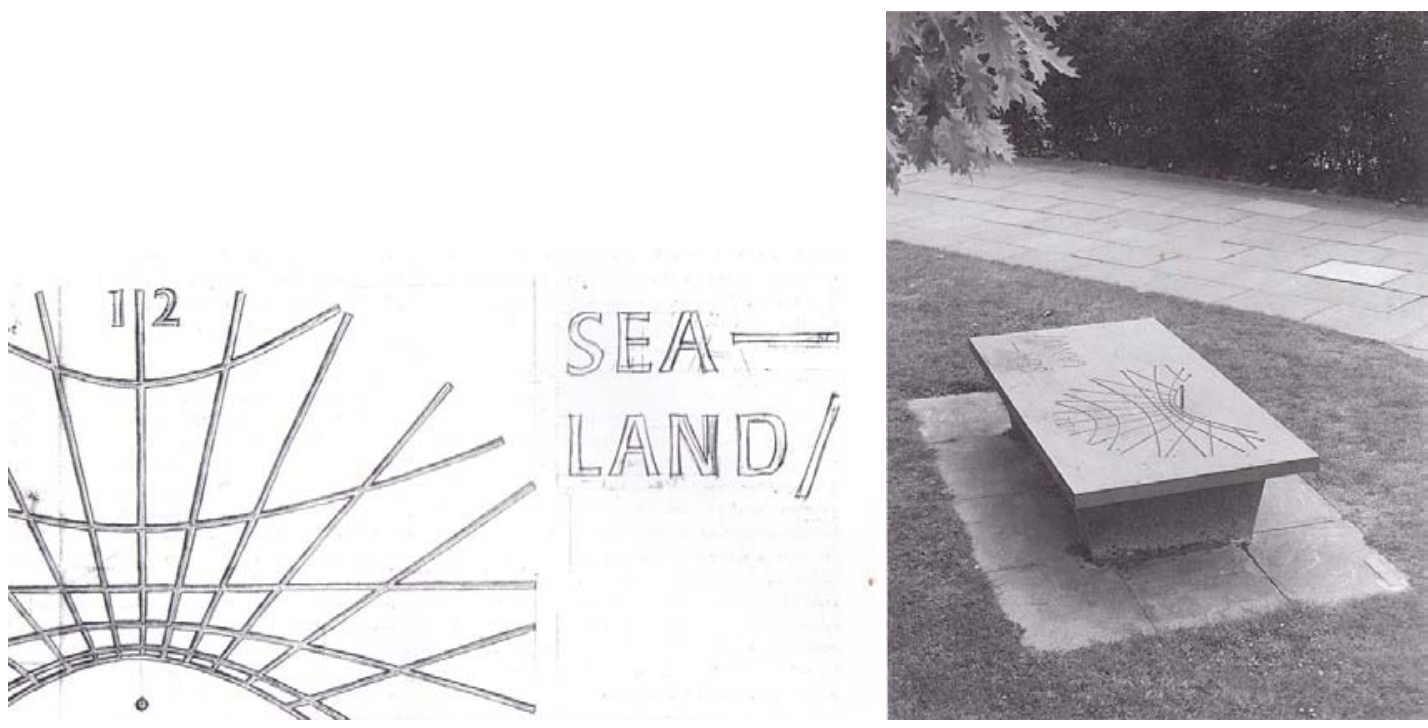

Fig. 33 The Canterbury Sandial (1972) - particolare dell'iscrizione dal disegno di Michael Harvey e foto Werner J. Hannappel.

La proposta Furka Pass consiste in "La firma dell'artista svizzero Hodler, iscritta su una pietra, (...) fotografata nel paesaggio alpino di Furka Pass."

Il fatto che l'opera artistica risieda nella fotografia finale e non nel paesaggio reale rivela come Finlay si collochi all'interno della temperie culturale del paesaggismo inglese del diciottesimo secolo, operando una sua attualizzazione, nel concepire il vedutismo pittorico quale ispirazione per la trasformazione concreta del paesaggio e nel riprodurre infine, nuovamente, quest'ultimo in forma pittorica, in un ciclo determinato totalmente dalla visione dell'osservatore e dal valore estetico di tale visione. Non è un caso che all'interno delle "proposte" siano presentati disegni di paesaggio sullo stile del pittore Claude Lorrain, citato nelle didascalie che terminano con un esplicito omaggio: "after Claude Lorrain (Liber Veritatis, 1649).

${ }^{35}$ STEPHEn BAnN, Vivi ignorato: l'arte dei giardini di Ian Hamilton Finlay, "Lotus", n. 88, Electa, Milano 1996, pag. 96. 

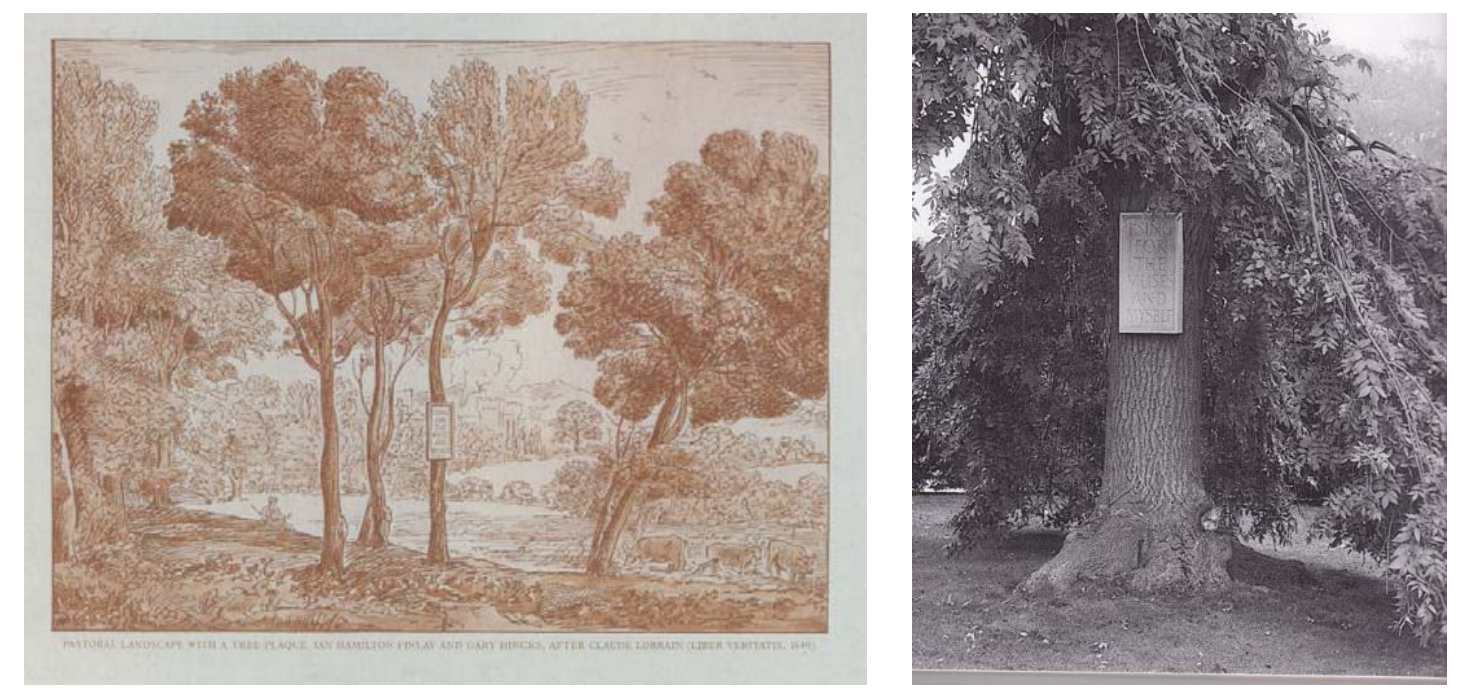

Fig. 34 Stockwood Park, Luton England (1991), “I Sing For The Muses And Myself” - disegno con didascalia: PASTORAL LANDSCAPE WITH A TRE-PLAQUE. JAN HAMILTON FINLAY E GARY HINCKS, AFTER CLAUDE LORRAIN (LIBER VERITATIS, 1649) e rielaborazione da una fotografia di Werner J. Hannappel.

La fotografia svolge un ruolo analogo a quello del dipinto. Finlay sottolinea come essa sia parte del processo artistico, rifiutando il concetto che la fotografia si ponga quale duplicazione oggettiva del reale:

“ Non è una duplicazione, ma un'articolazione del mondo, e quindi intrattiene con esso un rapporto simbolico. (...) Benché una fotografia sia sempre di qualcosa, il che induce a sospettare un rapporto diretto tra oggetto e rappresentazione, non mostra il mondo qual è ma quale noi lo vediamo."36

La firma su pietra Hodler a Furka Pass, trasforma la veduta del paesaggio da quel punto in un opera di Hodler (il testo della "proposta" inizia proprio con il titolo "From a point of view"), cosicché nonostante le modifiche che il paesaggio subisce con il passare del tempo, quel paesaggio rimarrà sempre stigmatizzato dal sigillo inserito dall'artista, e dalla fotografia che ne paralizza un istante e un'inquadratura precisi.

In questo modo Finlay vuole attribuire un'aura ad un luogo, di ordine superiore, ovvero realizzata dal filtro dell'arte che si sovrappone all'aura di un paesaggio già esistente. Nonostante questa azione di trasformazione e sottolineatura:

"Egli provoca il coinvolgimento dell'osservatore che, contemplando la firma, la veduta e la storia, si forma una concezione dell'interazione fra natura e arte, una concezione che poggia sulla conoscenza della tradizione e l'applicazione della convenzione. Eppure il risultato è tuttaltro che convenzionale o fisso. Nel misurare i complessi percorsi di riferimento implicati nell'opera - percorsi che vanno da Hodler a Finlay, da Finlay allo spettatore, - dallo spettatore a Hodler e Finlay - l'osservatore acquista nuove capacità di penetrazione del tradizionale all'interno dell'attuale." ${ }^{37}$

\footnotetext{
${ }^{36}$ Da Una proposta per il Furka Pass, Svizzera, 1987, elaborata da Ian Hamilton Finlay e illustrata da Kathleen Lindsley. Edizione di 200 esemplari pubblicata da The Wild Hawthorn Press, Little Sparta, Dunsyre, Lanark, Scozia. Testo di Wouter Weijers, trad. it. dell'autrice.

${ }^{37}$ Ibidem.
} 


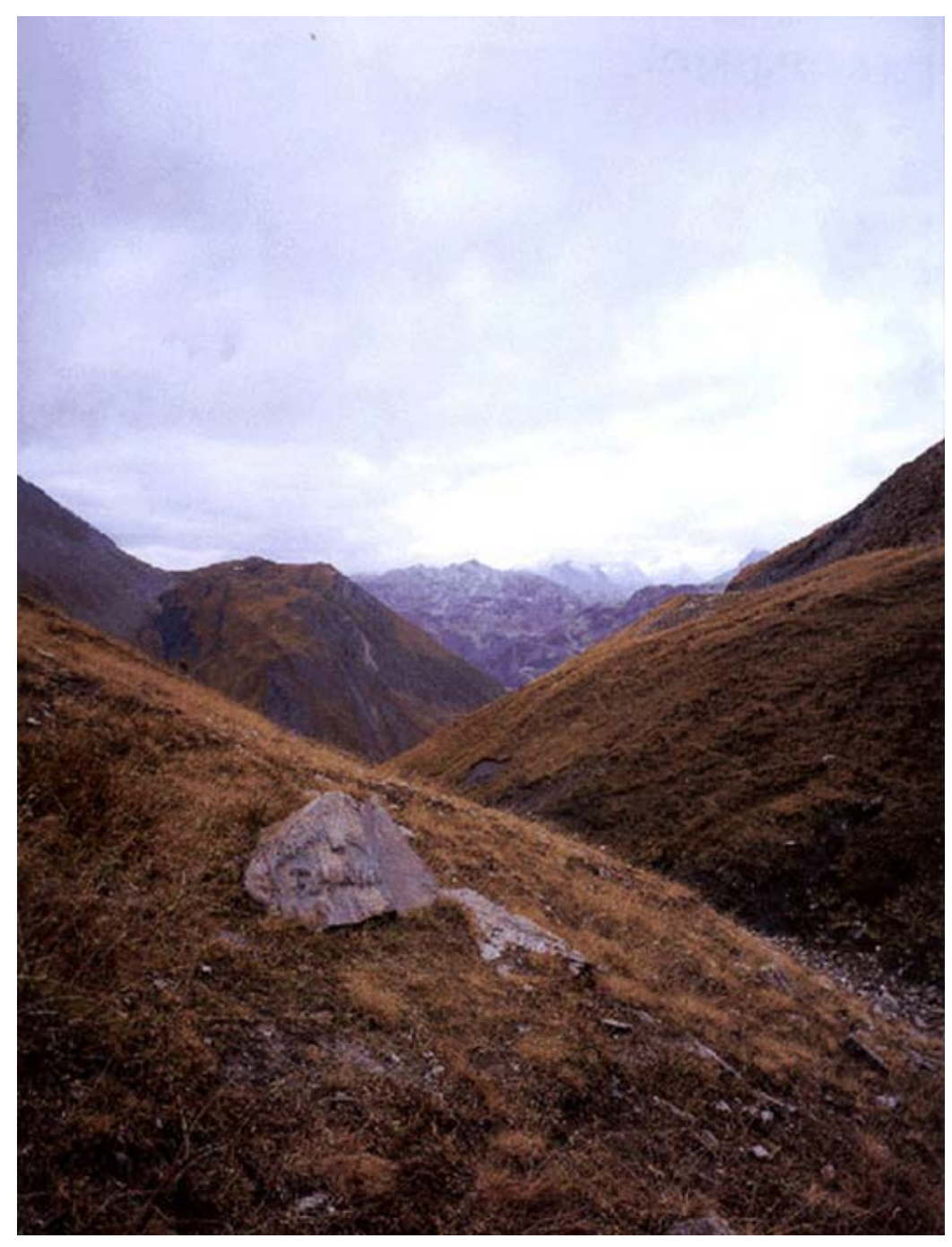

Fig. 35 Furka Pass, Switzerland (1987) .

C'è una spiegazione di Finlay sul rapporto possibile fra messaggio culturale dell'artista e il visitatore del giardino: le iscrizioni possono essere "a) guardate, b) lette, c) ignorate), d) lette e meditate. Così come uno vuole durante la giornata." ${ }^{38}$

L'affermazione esplicita che esistono diversi gradi di consapevolezza (e di interesse) rispetto ai significati che l'arte introduce nel giardino. L'artista si ritira dal luogo e consente che ognuno assuma da esso quanto e quello che vuole, secondo il proprio respiro che si va ad intersecare con quello avuto dall'autore nel comporre i segni nello spazio. Il fatto che le parole possano essere guardate dice del loro valore estetico, a prescindere dal contenuto, dato dalla proporzione delle lettere, dal carattere tipografico, come anche dalla luce o dalla piante che vi si intrecciano. In questo modo Finlay si rivolge anche al fruitore "distratto" di cui parla Walter Benjamin, che intende "ignorare" le parole ed il loro contenuto - o semplicemente che non possiede le chiavi per una loro lettura - ma si "abitua" alla loro estetica "attraverso sguardi occasionali", muovendosi all'interno del giardino lungo i percorsi e fra le istallazioni.

Anche il giardino del Sacro Bosco di Bomarzo, realizzato da Vinicio Orsini (XVI secolo) comunicava a livelli diversi in relazione al patrimonio culturale del suo visitatore: è possibile non comprendere i riferimenti culturali iconografici ma essere sensibili alla sproporzione

${ }^{38}$ In Isotta CorteSI, Op. cit., Federico Motta, Milano 2000, pag. 168. Isotta Cortesi traduce il testo di Ian Hamilton Finlay, in JoHn BREADSLEY, Earthworks and Beyond, cit., pag. 75. 
delle statue, alle loro espressioni nei volti e nelle pose. Lo stesso avviene per l'arte di Finlay Se si conosce la poesia di Holderling, di cui sono citati due versi sulle sponde del lago dello Schlosspark (1994-1995), il pensiero si inoltra nei rimandi; altrimenti le parole "Voi amabili cigni", "Voi immergete la testa" richiamano più semplicemente un'immagine, confermata dalla presenza di cigni nel lago, mentre i caratteri tipografici delle lettere incise conferiscono un'aria solenne alla quiete, e scandiscono la superficie dell'acqua ferma.

La pergola in legno Inter Artes Et Naturam che lungo il percorso dello Schlosspark subisce una metamorfosi in direzione di una crescente rusticità delle sue parti, è una metafora del rapporto fra artificio e natura, quali elementi indissociabili del giardino-paesaggio, ma è anche una dimostrazione di come la forma possa esprimere un significato che viene colto mediante la percezione visiva.

E' chiaro che l'arcadica compostezza dei giardini di Finlay è in realtà tutta costruita mediante il ricorso all'enfasi, ovvero alla sottolineatura, all'esagerazione, all'amplificazione, che viene però ricomposta in equilibrio, secondo le regole dell'estetica classica, che Finaly vuole attualizzare. Enfatica è la panca per The Leasowes, Dudley, England (1992)

“ Una panchina di pietra, con iscritta la descrizione di Dodsley dell'ambiente circostante com'era all'epoca di Shenstone." 39

riportante le seguenti parole:

“Qui il sentiero inizia gradualmente a salire sotto un abisso d'ombra, al cui fianco c'è un esile gorgogliante ruscello che forma minuscoli promontori, scorre su ciottoli o precipita in piccole cascate, il tutto in segreto, ammaestrato a mormorare molto gradevolmente."

La preoccupazione di Finlay per The Leasowes, un giardino inglese storico del diciottesimo secolo, realizzato dal poeta William Shenstone, è di restituire al luogo l'aura di cui godeva ai tempi di Shenstone, difficilmente rintracciabile nell'aspetto contemporaneo del parco. Finlay teme che il giardino non narri sufficientemente il proprio antico splendore e ne affida il racconto ad una panca, vicina al ruscello citato nella descrizione. Mediante la suggestione letteraria il paesaggio dell'intorno subisce un incantesimo e torna ad essere - ad apparire quello di un tempo.

In questo senso l'arte di Finaly è divertente: nel catalizzare la metamorfosi della percezione. Di metamorfosi parla consapevolmente Wouter Weijer (dando voce a Finaly) nella "Proposta per Furka Pass":

"Tale esperienza determina il modo con cui vedremo questo paesaggio alpino, e questa metamorfosi, a sua volta, ci trascina in affascinanti meditazioni sulla linea di confine tra natura arte".

La metamorfosi è ancora più ammaliante se avviene con ironia: allo Stockwood Park, Luton, England (1991), un muro è incastonato di otto lapidi ispirate alle metamorfosi di Ovidio, la prima costituisce il titolo della serie: " The Errata of Ovid" - sono errata corrige immaginati scherzosi e di fresco potere iconografico: "for "DAPHNE' read "LAUREL", "for 'PHILOMENA' read 'NIGHTINGALE' ecc.

\footnotetext{
${ }^{39}$ Da Una proposta per The Leasowes, Ian Hamilton Finaly, Nicholas Sloan, 1992, trad. it. dell'autrice.
} 


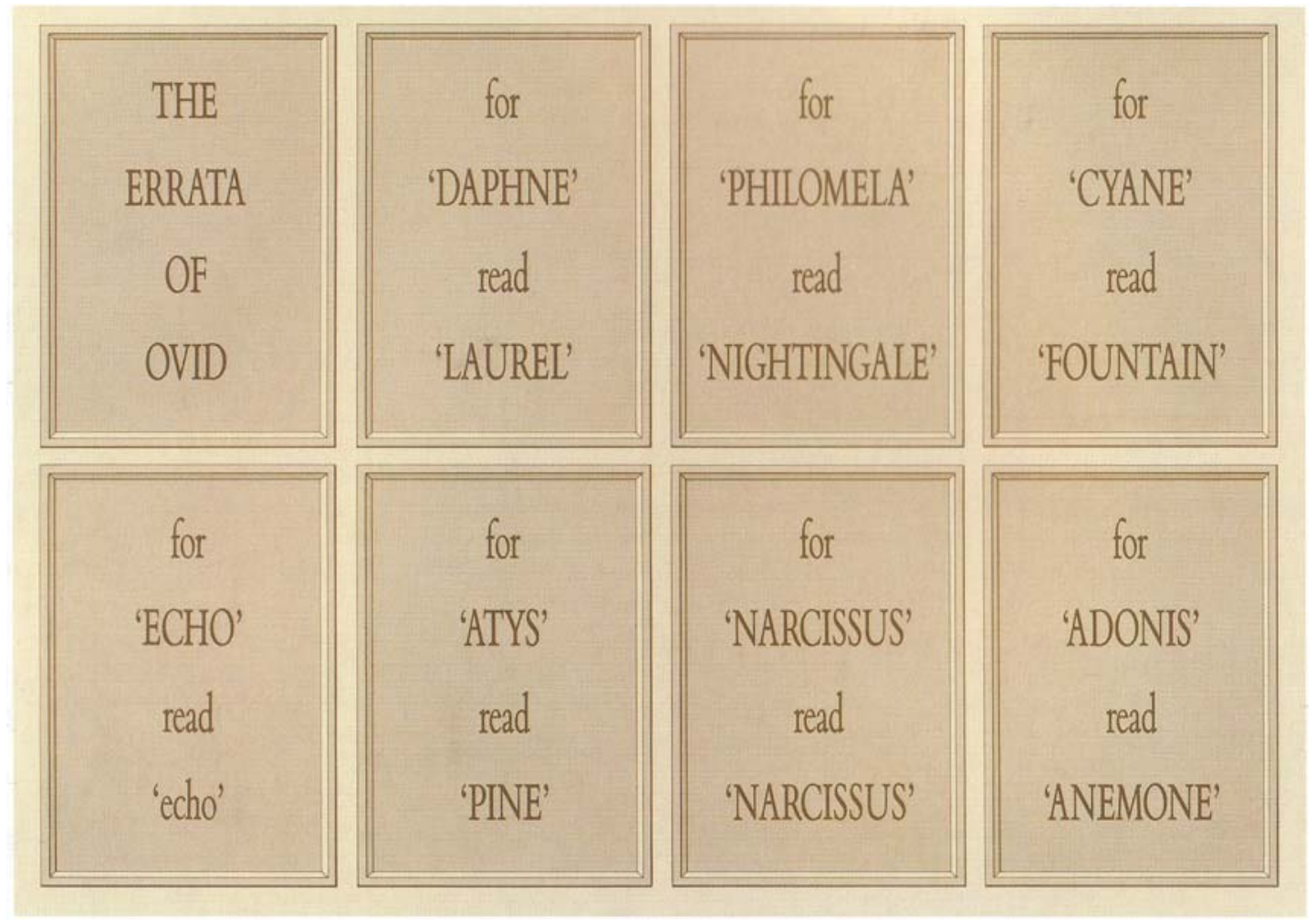

Fig. 36 Le iscrizioni tratte dalle Metamorfosi di Ovidio per Stockwood Park, Luton, England (1991).

Le Errata trasformano i personaggi dei miti in elementi del giardino alloro, usignolo, fontana, eco, pino, narciso, anemone, in stretta attinenza con il racconto di Ovidio e giocando sull'idea della metamorfosi.

Il tono ironico allo Stockwood Park è presente anche nel gruppo di pietre disposte nel prato a suggerire l'immagine di un gregge, una delle pietre porta un'iscrizione di un testo che dà la definizione pitagorica di "gregge".

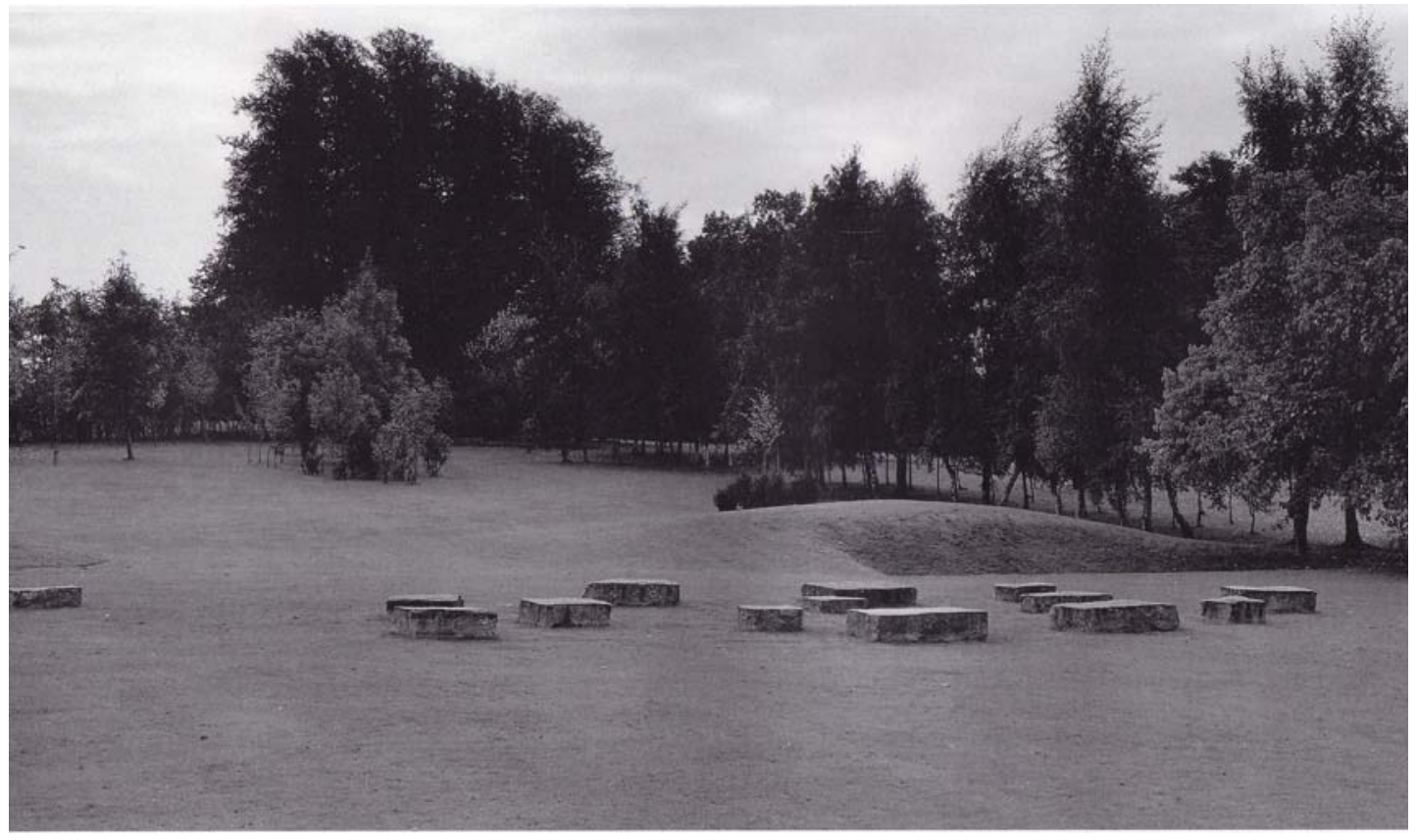

Fig. 37 "Gregge" di pietre a Stockwood Park, Luton, England (1991). 


\section{RIFERIMENTI BIBLIOGRAFICI}

Per Il parco diffuso di Tonino Guerra in Romagna:

GIANNINI RITA, GUERRA TONINO, URBINATI GIOVANNI , Il giardino pietrificato, Ramberti Arti Grafiche, Rimini.

GIANNINI RITA, Tonino Guerra e la sua valle, Raffaelli Editore, Rimini 1998.

Guerra TONINO, Con la poesia alle spalle, Edizioni Tipertì - Galleria Fabjbasaglia, Rimini 2001.

Guerra Tonino, Dizionario fantastico, Pietroneno Capitani, Rimini 2000.

GUERRA TONINO, Il libro delle chiese abbandonate, Maggioli Editore, Rimini 1988.

Guerra Tonino, Il Polverone, Maggioli Editore, Rimini 1992.

GUERRA TONINO, Il Viaggio, Maggioli Editore, Rimini 1986.

Il tappeto sospeso - fontana monumentale, catalogo, Maurizio Montanari, Ravenna 1997.

LOCATELli ViTTORIO, Le architetture parlanti di Tonino Guerra, "АNAГKH", 4, Alinea Editrice, Firenze 1993.

PANZINI FRANCO (a cura di), Giardini di Tonino Guerra - Pennabilli (Pesaro) in Giardini delle Marche, Federico Motta, Milano 1998.

SLEITER Rossella, L'orto di Tonino, "Il Venerdì di Repubblica", 14 luglio 1989.

Siti web:

I luoghi dell'anima in WWW.montefeltro.net/pennabilli.htm

Per L'Hortus Conclusus a Benevento:

Bonito Oliva ACHILle (a cura di), Avanguardia Transavanguardia, Electa, Milano 1982.

CAPPIEllo Vito, Architettura contemporanea in Campania, "D'A", 13, pag. 34 e pagg. 3638.

CROISET PierRe Alain (a cura di), "Casabella", numero monografico Il disegno degli spazi aperti, 597-598, 1993, Milano, pag. 44.

Gravagnuolo BenedetTA, Architetture scolpite con la luce, "Controspazio", n. 6, Gangemi Editore, Roma 1994, pagg. 34-36.

Gravagnuolo BenedeTtA, Hortus Infinitus, "Architettura e Arte”, n. 3, 1998, pagg. 5861.

Hortus conclusus. Almanacco dell'Architettura Italiana, Electa, Milano 1993, pagg. 62-63.

LIAO ALICE, Hortus Conclusus, in "www. Lightforum.com/magazine", 2000-2001.

NeONATO FRANCESCA, Un recinto sacro, “Acer", n. 6, Il Verde Editoriale, Milano 2001, pagg.32-35.

PANSERA ANTY, VITTA MAURIZIO, Guida all'Arte contemporanea, Marietti, Casale Monferrato 1986.

VERGINE LEA, L'arte in trincea - Lessico delle tendenze artistiche 1960- 1990, Skira, Milano 1996.

Siti web:

Hortus Conclusus, in www. Comune .benevento.it/Turismo/ Hortus.

Hortus Conclusus, in www. Francescomorante.it/pag_3/318ab.

Mimmo Paladino, in www. Francescomorante.it/pag_3/318a.

Post modern, in www. Francescomorante.it/pag 3/318.

Per I paesaggi di Ian Hamilton Finlay:

BANN STEPHEN, Giardino e arti visive. Arcadia, postclassico e "land art", in Mosser MONIQUe, TEYSSOT GEORGES, L'architettura dei giardini d'Occidente, Electa, Milano 1990, pagg. 491-502. 
BANN STEPHEN, I giardini di Ian Hamilton Finlay, in Mosser MoniQue, TeYssot GEORGES, L'architettura dei giardini d'Occidente, Electa, Milano 1990, pagg. 518-520.

BANN STEPHEN, Vivi ignorato: l'arte dei giardini di Ian Hamilton Finlay, "Lotus", n. 88, Electa, Milano 1996, pagg. 94-131.

BANN StePHEN, L'Arcadia di Finlay, "Lotus”, n. 88, Electa, Milano 1996, pagg. 92-93.

CORTESI IsOTTA, Il parco pubblico_ paesaggi 1985-2000, Federico Motta, Milano 2000, pagg. 164-169.

Donin GiamPiero, Parchi, Biblioteca del Cenide, Cannitello (RC) 1999, pagg.141-142.

Siti Web:

http://stuartcollection.ucsd.edu/finlay/unda6.gif

http://www.dundee.ac.uk/transcript/volume2/issue2_1/finlay.htm

http://stuartcollection.ucsd.edu/finlay/bio.html

http://jacketmagazine.com/15/rash-iv-finlay.html

\section{RIFERIMENTI ICONOGRAFICI}

Fig. 1: rielaborazione di Enrica Dall'Ara di foto Pubbliaerfoto in TURRI EUGENIO (a cura di), Adriatico mare d'Europa - La geografia e la storia, Arti grafiche Amilcare Pizzi S.p.A., Cinisello Balsamo (Milano)1999, pag. 129.

Fig. 2: rielaborazione di Enrica Dall'Ara di fotografia in AA.VV., La Valle del Marecchia, Regione Emilia Romagna, Grafiche Zanini, Bologna 1988, pag. 68.

Fig. 3: rielaborazione di Enrica Dall'Ara da Il tappeto sospeso - fontana monumentale, catalogo, Maurizio Montanari, Ravenna 1997.

Figg. 4-10: fotografie di Enrica Dall'Ara.

Figg. 11,12: rielaborazione degli elaborati grafici di progetto a cura di Enrica Dall'Ara.

Figg. 13-15: fotografie di Enrica Dall'Ara

Fig. 16: da WWW.montefeltro.net/pennabilli.htm

Figg. 17-19: fotografie di Enrica Dall'Ara

Figg. 20-21: foto Luciano Liuzzi, in GIANNINI RITA, GUERRA TONINO, URBINATI GIOVANNI Il giardino pietrificato, Ramberti Arti Grafiche, Rimini.

Fig. 22: foto Le Pera, da Hortus Conclusus, www. Comune.benevento.it/Turismo/ Hortus.

Figg. 23- 30: per gentile concessione di Roberto Serino.

Fig. 31: CORTESI ISOTTA, Il parco pubblico - paesaggi 1985-2000, Federico Motta, Milano 2000, pag. 167.

Fig. 32: http://www.dundee.ac.uk/transcript/volume2/issue2_1/finlay.htm

Figg. 33, 34, 36, 37: BANN STEPHEN, Vivi ignorato: l'arte dei giardini di Ian Hamilton

Finlay, "Lotus", n. 88, Electa, Milano 1996, pag. 94-131.

Fig. 35: http://www-personal.umich.edu/ ezakem/HA/5.htm. 
PARTE II -

Analisi simbolo-spaziale e schemi per la progettazione 


\section{- ELENCO DEI PARCHI DEL DIVERTIMENTO EUROPEI}

Ad introduzione della presente ricerca si è motivato l'interesse per i parchi del divertimento tematici: la provocazione di Isabelle Auricoste - "parchi o utopie noiose?" - in riferimento ai parchi ricreativi contemporanei, chiede oltre che una risposta una soluzione in termini di individuazione di criteri progettuali.

Il binomio divertimento-tema amplifica la complessità della risposta, in quanto esistono modi di attribuzione del tema che catalizzano il divertimento e altri che lo ostacolano: ad inibirlo sono soprattutto quei modi per cui il tema diventa sinonimo di pianificazione e controllo totali dell'esperienza consentita al visitatore del parco, per cui il tema è un'immagine assoluta, immodificabile e non reinterpretabile.

I parchi ricreativi sono un insieme ampio, all'interno del quale è possibile individuare più categorie. Le classificazioni riscontrabili in letteratura e le classificazioni ufficiose ricavabili dai discorsi comuni e dalle pubblicità inerenti l'argomento sono diverse. Per fare solo alcuni esempi: il sito italiano www.parksmania.it, che censisce e descrive i parchi ricreativi sul territorio internazionale individua le tipologie parco meccanico (prevalentemente costituito di rides e attrazioni high-tech di velocità e brivido), acquatico, vita marina, tematico, didattico, indoor (si tratta di strutture al chiuso, architetture del divertimento, non propriamente di parchi), children playground (derivazioni dei comuni parchi giochi per bambini), family park (parchi con attrazioni per tutta la famiglia), faunistico, miniature; Marialuisa Lusetti ${ }^{1}$ distingue in parchi di vita marina, parchi faunistici e zoosafari, parchi d'attrazione (sono quelli che si associano comunemente all'espressione parco dei divertimenti, "appartengono alla tradizione dei luna-park itineranti di importazione americana"2), parchi in miniatura, parchi acquatici, parchi a tema. Mariano Sartore propone un'articolazione che distingue due famiglie principali parchi naturalistico-faunistici e parchi

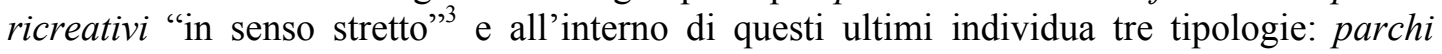
d'attrazione, parchi tematici, parchi acquatici.

Comunque si intenda fare distinzioni tassonomiche, è chiaro che vengono compresi con il termine ricreativi parchi del divertimento non tematici e parchi tematici che non intendono essere per il divertimento. Nell'analisi e nel censimento seguenti questi ultimi vengono tralasciati, proprio perché al loro riguardo non si pone il problema -o non si pone in termini sostanziali- del rapporto fra parco-divertimento-tema, che è l'oggetto perno della ricerca.

Il comune denominatore dei parchi censiti è il loisir, il divertimento.

Si operano quindi delle scelte: i parchi faunistici e i parchi didattico-scientifici, i parchi archeologici e i parchi d'arte - che possono considerarsi tematici - vengono compresi solo se costituiscono una parte specializzata all'interno di complessi per il divertimento, o se sono strutturati essi stessi in maniera esplicita come parchi del divertimento.

Sono esclusi inoltre i parchi acquatici, in quanto ritenuti un caso troppo particolare: essi generalmente non presentano quella complessità di contenuti, di programmi e di composizione spaziale, rilevata come caratteristica dei parchi del divertimento lungo il percorso di individuazione delle matrici della Parte I della ricerca, possono essere considerati "evoluzioni tecnologiche della piscina più che del parco vero e proprio"

\footnotetext{
${ }^{1}$ MARIALUISA LuSETTI, Verso la città del quaternario: i parchi ricreativi-tematici come metafora di una società leisure oriented, in EVERARDO MINARDI, MARIALUISA LUSETTI (a cura di), I parchi del divertimento nella società del Loisir, Franco Angeli, Milano 1998, pagg. 27-55.

2 Marialuisa Lusetti, Op. cit., in Everardo Minardi, Marialuisa Lusetti (a cura di), I parchi del divertimento nella società del Loisir, Franco Angeli, Milano 1998, pag. 35.

${ }^{3}$ MARIAno SARTORE, Tipologie, geografia e problematiche dei parchi ricreativi in Italia, in "Paesaggio Urbano", n. 5, Parchi di divertimento, Maggioli Editore, Rimini 1993, pag. 25.

4 Marialuisa lusetti, Op. cit., in Everardo Minardi, Marialuisa Lusetti (a cura di), I parchi del divertimento nella società del Loisir, Franco Angeli, Milano 1998, pag. 33.
} 
stati inclusi solo i parchi acquatici che realizzano ambientazioni tematiche forti o che sono parte di parchi che offrono anche attrazioni di altro genere.

L'elenco comprende quindi i parchi del divertimento -tematici e non tematici- rilevati in Europa, intercettati sia tramite ricerca bibliografica, sia consultando siti web specifici sui parchi del divertimento (esistono degli elenchi di parchi dei divertimenti pubblicati su internet da organizzazioni del settore, da appassionati in materia, da aziende per il turismo di livello nazionale e internazionale).

Non tutte le nazioni considerate per la stesura dell'elenco fanno parte della Comunità Europea: si sono considerate europee in senso lato tutte le nazioni menzionate all'interno degli elenchi di "Parchi Europei" incontrati nel corso del censimento.

[Ad ogni parco dell'elenco è affiancato il sito web ufficiale e fanno seguito gli specifici riferimenti bibliografici e i siti web consultati].

\section{Austria}

- Familienland Erlebnispark - Mühlau (www.wiener-prater.at/)

- Minimundus - Klagenfurt

Tosetto Danilo, Parchi Ricreativi nel Mondo, Vol. 2 , Facto, Padova 1987, pagg. 196- 203.

- Prater Park - Vienna (www.wiener-prater.at/)

- Styrassic - Bad Gleichenberg (www.styrassicpark.at/index4_5.htm)

- Tirol fun - Leutasch (www.tirolfun.com/)

\section{Belgium}

- Bellewaerde - Westoek (www.sixflagseurope.com/e/bwp/index.html)

- Bobbejaanland - Lichtaart (www.bobbejaanland.be/)

Tosetto Danilo, Parchi Ricreativi nel Mondo, Vol. 5 , Facto, Padova 1990, pagg. 210- 221.

- Boudewijnpark - Brugges (www.boudewijnpark.be/)

- Brupark - Brusselles (www.bruparck.com/)

www.minieurope.com/index.htm www.atomium.be/ -

- Monde Sauvage Safari Deignè - Aywaille (http://www.mondesauvage.be/)

http://www.aywaille.be/fr/tourisme/sitetouriste/safari.htm 
- Plopsaland - Adinkerke-De Panne (www.plopsaland.be/)

- Six Flags Belgium (ex- Walibi) - Wavre

Tosetto Danilo, Parchi Ricreativi nel Mondo, Vol. 2 , Facto, Padova 1987, pagg 6475.

- Telecoo - Stavelot (www.telecoo.be/html/acc_telecoo.html)

www.telecoo.be $/ \mathrm{html} / \mathrm{acc}$ telecoo.html

\section{Cyprum}

- Lucky Star Park (www.luckystarpark.com.cy/)

- Waterworld - (www.waterworldwaterpark.com/ Agia Napa)

\section{Denmark}

http://www.tuttinsieme.it/tutti/tut/eur/denmark/placestosee.htm http://w1.1585.telia.com/ u197401534/Links.htm http://themeparkcity.com/EURO_dane.htm

- Bakken - Klampenborg (www.bakken.dk/)

- BonBon-Land - Holme-Olstrup (www.bonbonland.dk/)

- Djurs Sommerland - Nimtofte (www.djurssommerland.dk/)

- Ebeltoft - Ebeltoft (www.ebeltoft-familiepark.dk/) www.familieparken.dk www.archipelago.nu/SKARGARD/ENGELSKA/DENMARK/Ebeltoft.

- Fantasy world - Ringsted (www.fantasy-world.dk/)

www.danimarca.dt.dk/barn.html

- Fårup Sommerland - Saltum (www.faarupsommerland.dk/)

- Kattegatcentret - Greena

- Knuthenborg - Greena

- Legoland - Billund (www.lego.com/legoland/billund/defaultus.asp)

Tosetto Danilo, Parchi Ricreativi nel Mondo, Vol. 2 , Facto, Padova 1987, pagg. 204- 215.

- Nordsjaellands Sommerpark - Graested

- Sommerland Sjælland - Nykøbing (www.sommerlandsj.dk/index_03_2.php) 
- Sommerland Syd - Tinglev (www.sommerland-syd.dk)

- Tivoli - Copenhagen (www.tivoli.dk/composite-351.htm)

Wylson ANTHONy \& PATRICIA, Theme Parks, Leisure Centres, Zoos and Aquaria, Longman, 1994, pagg. 5-9.

www.tivoligardens.com/

- Tivoli Friheden - Aarhus (www.friheden.dk/)

- Tivoliland - Aalborg (www.tivoliland.dk)

- Varde Sommerland - Varde

\section{Finland}

www.themeparkcity.com/EURO_finn.htm www.germancoaster.de/parks-europa-finnland.html

- Alandsparken - Mariehamn

www.bellingham.fi/huvipuis/ukaland.htm

- Jukujukumaa hiekkasarkat

www.bellingham.fi/huvipuis/jukujuku.htm www.hiekkasarkat.fi/hinnsani.htm www.hiekkasarkat.fi/hiekka/eng/index.htm www.topcamping.net/eng/kalajoki.html

- Kesamaa - Punkaharju (www.lomaliitto.fi/kohteet/muut/kesamaa_valokuvia.htm)

- Linnanmaki - Helsinki (www.linnanmaki.fi/)

- Moominworld - Naantali (www.muumimaailma.fi/index.html)

www.publiscan.fi/b104e-1.htm

- Santapark - Rovaniemi (www.santapark.com)

www.bellingham.fi/huvipuis/uksanta.htm www.freizeitpark.de/(gal=gal_23\&psid=ab7e1a8b7f884edb52f97628b69f7edc) $/ \mathrm{mod} / \mathrm{gal}$ erie/detail.php www.virtualtourist.com $/ \mathrm{m} / \mathrm{tp} / 4 \mathrm{~d} 6 \mathrm{c} 9 /$

- Sarkanniemi - Tampere (www.sarkanniemi.fi/)

Tosetto Danilo, Parchi Ricreativi nel Mondo, Vol. 5 , Facto, Padova 1990, pagg. 198- 209. 
- Suomen Tivoli - (www.suomentivoli.fi/english/english_messages.html)

- Tykkimäen - Kouvola (www.tykkimaki.fi)

- Visualhti - Mikkeli

- Wasalandia (www.wasalandia.fi)

www.bellingham.fi/huvipuis/ukwasa.htm www.uwasa.fi/comm/cmc/multima/multima96/vaasa/vaasa/happy.htmlhttp://ql4pt.conte nt.parkscout.de/( $\mathrm{p}=349 \& \mathrm{l}=20 \& \mathrm{psid}=\mathrm{c} 501058 \mathrm{a} 6 \mathrm{bb} 9 \mathrm{e} 8 \mathrm{fa} 7 \mathrm{ca} 917 \mathrm{c} 49 \mathrm{a} 738 \mathrm{~b} 24) /$ show_park. php

\section{France}

http://perso.wanadoo.fr/loisirs60/idxparcs.html www.cci-oise.fr/polatouche/parc.html www.nomade.tiscali.fr/cat/sorties_spectacles/par_genre/loisirs_jeux/parcs_attractions/france/ nord_pas_calais/ www.oise60.com/jours/jdo112/parcs_jdo112.html www.themeparkcity.com/EURO_france.htm

- $\quad$ Base de Loisirs - Primpez

- Bagatelle - France (www.bagatelle.fr/)

- Disneyland Paris - Paris (www.disneylandparis.com/)

CERVER FRANCISCO ASENSIO, Landscape of recreation II: amusement parks, WatsonGuptill Pubns, Slipcase edition 1998, pagg. 52-57.

- Disney studios Paris - Paris

- France Miniature - Elancourt (www.franceminiature.com/anglais/presentation.htm)

www.atinternet.com/isa/voyage/mini.htm tourisme.voila.fr/villes/paris/fra/sit/elancour/frminiat/acc.htm

- Futurscope - Poitiers (www.futuroscope.com/)

MONORY RENÉ, The Futurscope, Harnessing the Future at Europe's Park of the Image, Editions du Mon iteur, Paris.

Tosetto Danilo, Parchi Ricreativi nel Mondo, Vol. 5 , Facto, Padova 1990, pagg. 2435.

TRÉBUCQ MATHILDE, La Nature du Futurscope, Les Editions du Futurscope, Haschette. www.futuroscope.fr/vivez_planete_futuroscope/carte/carte.htm

- Jardin d'Acclimation - Paris (www.jardindacclimatation.fr/)

www.gallica.bnf.fr/VoyagesEnAfrique/themes/T4a.htm www.paris.org/Kiosque/sep98/331clima.html 
www.bouture.com/jardindeseoul.htm

- La Villette - Paris

BARZILAY MARIANNe, HAYWARD CATHERINe, LOMBARD-VALENTINO LUCETTE, L'invention du parc: parc de la Villette: Paris, concours international 1982-1983, Graphite, Etablissement Public du Parc de la Villette 1984. Chaslin Francois, Parc de la Villette, Parigi, in "Domus" n. 817, Milano 1999, pagg. 8-17.

RogGero Mario F. (a cura di), Il parco e la città. Sette progetti italiani per Parigi-La Villette: Aymonino, BBPR, Dierna, GRAU, Pellegrin, Purini, Roggero, Alinea, Firenze 1986.

FABRIS LUCA MARIA FranCESCO, Il verde postindustriale, Liguori Editore, Napoli 1999.

- Le Pal - Dompierre Sur Bresbe (www.lepal.com/le-pal/)

- Puy du Fou - Les Epesses, Vendee (www.puydufou.com/)

www.vendee-terre-de-reussite.com/touristique/tourist_coeur.htm www.achus.net/achus/europa/parques/francia/puy_du_fou/

- Mer-de-Sable, Ermenonville (www.merdesable.fr/mer-de-sable/)

http://tourisme.voila.fr/villes/stdenis/fra/sit/ermenonv/mersable/acc.htm www.chantilly-tourisme.com/francais/loisirs parcs loisirs.php www.aventuriers.com/fr/france/picardie/60_dec_lamerdesable/index.shtml www.parisce.com/sable.htm

www.freeoise.free.fr/communes/lettree/ermenon/pglois.html

- Minifrance- Brignoles

Tosetto Danilo, Parchi Ricreativi nel Mondo, Vol. 5 , Facto, Padova 1990, pagg. 238- 245.

- Nigloland - Dolancourt (www.nigloland.fr/)

- OK Corral - Cuges-les-Pins (www.okcorral-france.com/)

- Parc Asterix - Plailly (http://www.parcasterix.fr)

Donin GianPIERo, Parchi, Biblioteca del Cenide, Cannitello (RC) 1999. Tosetto Danilo, Parchi Ricreativi nel Mondo, Vol. 5 , Facto, Padova 1990, pagg. 3655.

Wylson ANTHONy \& PATRICIA, Theme Parks, Leisure Centres, Zoos and Aquaria, Longman, 1994, pagg. 27-30.

www.chantilly-tourisme.com/francais/loisirs_parcs_loisirs.php

- Parc Saint Paul - Saint Paul (www.parcsaintpaul.com/) 
- Walibi Aquitaine - Aquitane

- Walibi Rhone-Alpes - Rhone Alpes

- Walibi Schtroumpf (ex Big-bang Schtroumpf)- Maizières-les-Metz

Tosetto Danilo, Parchi Ricreativi nel Mondo, Vol. 4 , Facto, Padova 1989, pagg. 2639.

\section{Germany}

www.germany-tourism.de/e/2411_2920.html www.freizeit-seiten-online.de/filmpark/

- Allgäu Skyline Park - Bad Wörishofen (www.skylinepark.de)

- Babelsberg Film Park - Potsdam

www.filmpark.de/

www.stageaccompany.com/referenc/babelsberg.html

- Bayern Park - Reisbach (www.bayern-park.de/)

- Bavaria Film Tour - Geiselgasteig (www.filmstadt.de/)

www.muenchen-tourist.de/englisch/stadtinformationen/erlebnis/muenchenstadtinformationen-sehenswuerdigkeiten-erlebnis_e_m.htm

- Churpfalzpark - Loifling (www.churpfalzpark.de/)

- Eifelpark - Gondorf (www.eifelpark.de/)

- Elspe - Festival - Lennenstadt Elspe (www.elspe.de/)

- Erlebnispark Forellenhof (www.erlebnispark-forellenhof.de/)

- Erlebnispark Steinau - Steinau (www.erlebnispark-steinau.de)

- Erlebnispark Tripsdrill - Cleebronn (www.tripsdrill.de/)

- Erse Park - Uetze (www.erse-park.de/index.html)

- Europa Park - Rust (www.europa-park.de/)

Wylson Anthony \& PAtricia, Theme Parks, Leisure Centres, Zoos and Aquaria, Longman, 1994, pagg. $34-38$.

- Fort Fun Abenteuerland - Wasserfall (www5.fortfun.de/)

- Frankisches Wunderland - Plech (www.wunderland.de/)

- Freizeit-Land Geiselwind - Geiselwind (www.freizeit-land.de/)

Tosetto Danilo, Parchi Ricreativi nel Mondo, Vol. 4 , Facto, Padova 1989, pagg. 116- 127. 
- Freizeitpark Lochmühle - Wehrheim (www.lochmuehle.de/)

- Freizeitpark Traumland - Sonnenbühl (www.e-biz.de/reisen/traumlan.htm)

- Hansa Park - Sierksdorf (www.hansapark.de/main.php)

Tosetto Danilo, Parchi Ricreativi nel Mondo, Vol. 4 , Facto, Padova 1989, pagg. 158- 171.

- Heide Park - Solatau (www.heidepark.de/)

Tosetto Danilo, Parchi Ricreativi nel Mondo, Vol. 3 , Facto, Padova 1988, pagg. 195- 2132.

- Holiday Park - Hassloch (www.holidaypark.de/)

Tosetto Danilo, Parchi Ricreativi nel Mondo, Vol. 4 , Facto, Padova 1989, pagg. 196- 211.

- Hollywood Park - Stukenbrock

- Jaderpark - Jaderberg (www.jaderpark.de/)

- Legoland Deutschland - Günzburg

- Maerchenwald im Isartal - Wolfratshausen

- Minidomm- Ratingen

Tosetto Danilo, Parchi Ricreativi nel Mondo, Vol. 4 , Facto, Padova 1989, pagg. 228- 235 .

- Panorama Park - Kirchhundem (www.panoramapark.de/)

- Phantasialand - Bruhlk (www.phantasialand.de/cgi-bin/xshcgi305?SFMCF=phantasia)

Tosetto Danilo, Parchi Ricreativi nel Mondo, Vol. 2 , Facto, Padova 1987, pagg 2335.

- Potts Park - Minden (www.potts-park.de/)

- Rasti-Land - Salzhemmendorf (www.rasti-land.de/)

- Schloss Beck Freizeitpark - Bottrop (www.schloss-beck.de/)

- Schloss Thurn - Heroldsbach (www.schloss-thurn.de/)

- Schwaben Park - Kaisersbach (www.schloss-thurn.de/)

- Serengeti Safaripark - Hodenhagen (www.schloss-thurn.de/)

- Spreepark - Berlin (www.spreepark.de/)

- Taunus Wunderland - Schlangenbad (www.taunuswunderland.de/standartneu/start.shtml)

- Tier- und Freizeitpark Thüle - Friesoythe-Thüle (www.tier-undfreizeitparkthuele.de/) 
- Tolk-Schau - Tolk (www.tolk-schau.de/)

- Warner Bros. Movie World (www.movieworld.de/)

Greece

- Aqualand - Corfu Water Park (www.aqualand.com.gr/)

www.parksmania.it/corfuwaterpark.htm

www.corfu-net.gr/aqualand/

- Waterland-Tessalonichi

www.waterland.gr/sen/default.htm

- Watermania - (www.waterland.gr/sen/default.htm)

www.vacation.forthnet.gr/

www.otenet.gr/waterland/

\section{Norway}

www.funguide.com/parks/park.pl?norway

- Kongeparken (hwww.kongeparken.no/ Ålgård)

http://www.touristmagazine.com/WN/kongeparken.html

- Kristiansand Dyreparken (www.dyreparken.com/)

- Lunds Tivoli (www.lundstivoli.no/)

- Telemark Sommarland - Bo

www.geocities.com/Heartland/Hills/1545/bo.html

- Tusenfryd - Oslo (www.tusenfryd.no/)

- Vikinglandet - Vinterbro

www.illustrert.no/kimsplace/Vikinglandet.html www.tripadvisor.com/ShowUrl-g190479-d206476-r251551-

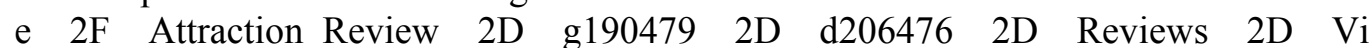
king Land Vikinglandet $\overline{2 \mathrm{D}}$ Oslo $2 \mathrm{E} \overline{\mathrm{h}}$ tml-Viking Land VikinglandetOslo.html www.awcoslo.org/Sections/LivingInOslo/things_to_do_in_and_around_oslo.htm

\section{Hungary}

- Vidam Park - Budapest, Hungary (www.vidampark.hu/) 


\section{Italy}

www.ituscany.it/sito/ITA_E/itiner/bambini/1bamb_e.htm

www.paesionline.it/search.asp?scat $=26$

- Adventure Golf Salt Lake - Levico terme (TN)

www.attentialpupo.com/tempoliberoevacanze/mappaparchi/adventure.htm

- Amusement Park - Padova (www.amusement.it)

www.ciaopadova.com/park/

- Bioparco - Roma (www.bioparco.it/)

www.romeyoung.com/italiano/spazio_verde/bioparco/

- Ciclilandia - Tirrenia (PI)

- Cowboyland - Voghera (www.cowboyland.it)

- Edenlandia - Napoli (www.edenlandia.it)

- Elbaland- Portoferraio (LI)

www.greenholiday.it/le_regioni/toscana/vita_e_cultura/parchi/elbaland

- Etnaland- Belpasso (CT) (www.parcozoo.it)

www.italyguide.com/parchi_italia/etnaland.htm

- Fantasilandia - Tirrenia (PI)

www.ituscany.it/sito/ITA_E/itiner/bambini/fanta_e.htm

- Fantasy World Minitalia - Capriate San Gervasio, Italy (www.fantasyworld.it/)

- Fiabilandia -Rivazzurra di Rimini

www.infoparchi.com/ipTematici/stories/storyReader $\$ 15$

- Gardaland - Verona (www.gardaland.it/it/home.html)

LUCAT MARCO, Gardaland progettazione e riprogettazione di un parco di "successo", in "Paesaggio Urbano", n. 5, Parchi di divertimento, Maggioli Editore, Rimini 1993, pagg. 30-35.

- Greenland- Limbiate (MI) (trovato nulla) 
- Gulliverlandia - Lignano Sabbiadoro

www.infoparchi.com/ipTematici/stories/storyReader $\$ 35$

- Idroscalo - Novero di Segrate ( MI)

TosetTo Danilo, Parchi Ricreativi nel Mondo, Vol. 2 , Facto, Padova 1987, pagg. 218- 225.

- Italia In Miniatura - Rimini (www.italiainminiatura.com/)

Tosetto Danilo, Parchi Ricreativi nel Mondo, Vol. 3 , Facto, Padova 1988, pagg. 278- 287.

- Le Caravelle- Ceriale, Savona

Tosetto Danilo, Parchi Ricreativi nel Mondo, Vol. 5 , Facto, Padova 1990, pagg. 280-289.

- Luneur - Roma (www.luneur.it/)

- Cavallino Matto-Magire' - Marina di Castagneto - Donoratico- (LI)

(www.cavallinomatto.it/)

www.infoparchi.com/ipTematici/stories/storyReader $\$ 20$

- Città della Domenica- Perugia

SANTONI ROBERTA, La città della domenica: da luogo per lo svago a luogo per gli animali, in "Paesaggio Urbano", n. 5, Parchi di divertimento, Maggioli Editore, Rimini 1993, pagg. 48-52.

- Magic World- Giugliano (NA) (www.magicworld.it)

www.ragazzinet.it/numero_019/Attualit\%C3\%A0/Parchi/parchi19.html

- Minitalia - Capriate San Gervasio (www.mirabilandia.it)

Tosetto Danilo, Parchi Ricreativi nel Mondo, Vol. 2 , Facto, Padova 1987, pagg. 274- 283.

- Mirabilandia - Savio (www.mirabilandia.it/). 
BERATI SANDRA, Mirabilandia. Temi e problemi dell'insediamento di un grande parco ricreativo, in "Paesaggio Urbano", n. 5, Parchi di divertimento, Maggioli Editore, Rimini 1993, pagg. 36-41.

- Movie Studios Park - Lazise (VR) (www.moviestudios.it)

www.corriere.it/speciali/moviestudio/index.shtml

- Nuovo Parco Lido, Lido di Ostia (RM)

- Palestina in Miniatura - Coriano (RN)

- Parco della Preistoria o Archeopark o- Rivolta d'Adda

Tosetto Danilo, Parchi Ricreativi nel Mondo, Vol. 5 , Facto, Padova 1990, pagg. 272- 279 .

- Safari park- Pombia (No) (www.safaripark.it/)

www.attentialpupo.com/tempoliberoevacanze/mappaparchi/safaripark.htm

- Sardegna in miniatura - Tuili (CA) (www.sardegnainminiatura.it)

- Villaggio Africano - Calcinate

\section{Netherlands}

- Attractiepark Slagharen - Slagharen (www.slagharen.org/)

- Beekse Bergen - Hilvarenbeek

Tosetto Danilo, Parchi Ricreativi nel Mondo, Vol. 2 , Facto, Padova 1987, pagg 123 $-133$.

- De Efteling - Kaatsheuvel (www.efteling.nl/docs.en/park/nav/index.html9

CERVER FRANCISCO ASENSIO, Landscape of recreation II: amusement parks, WatsonGuptill Pubns, Slipcase edition 1998, pagg. 86-97.

Tosetto Danilo, Parchi Ricreativi nel Mondo, Vol. 3 , Facto, Padova 1988, pagg. 2845.

VAnden Diepstraten H., De Efteling kroniek van een Sprookje, De Efteling B.V. Kaatsheuvel 2002.

Wylson Anthony \& PAtricia, Theme Parks, Leisure Centres, Zoos and Aquaria, Longman, 1994, 30-33.

- De Valkenier - Valkenburg (www.pretpark-de-valkenier.nl/)

- Drievliet - Rijswijk (www.drievliet.n1/)

- Duinrell - Wassenaar (www.duinrell.n1/)

- Hellendoorn (www.avonturenpark-hellendoorn.nl/) 
- Koningin Juliana Toren - Apeldoorn (www.julianatoren.n1/)

- Madurodam - Den Haag (www.madurodam.nl/default.asp?lng=en )

- Mordwijk Space Expo - Noordwijk

- Six Flags Holland

- Speelstad Oranje - Gemeente Midden (www.most.nl/oranje/)

\section{Portugal}

- Fantasia Park - Sintra, (www.fantasiapark.com/)

- Portugal do pequenitos - Coimbra

Tosetto Danilo, Parchi Ricreativi nel Mondo, Vol. 5 , Facto, Padova 1990, pagg. 246- 257.

www.regiaocentro.net/lugares/coimbra/parquesejardins/port_peqnitos/ www.fbb.pt/port_peq.htm

\section{Spain}

- Catalunia en miniatura - Barcellona (www.catalunyaenminiatura.com/indexeng.html)

- Cortylandia - Puerto Banús, Marbella (www.martinat.com/marbella/Cortylandia/)

- Isla Magica - Seville (www.islamagica.es/)

- Monte Igueldo - San Sebastián (www.tlm.unavarra.es/asignaturas/bi/bi98_99/bi40/ATRACIONES.HTML)

- Parque de Atracciones - Madrid

Tosetto Danilo, Parchi Ricreativi nel Mondo, Vol. 5 , Facto, Padova 1990, pagg. 186- 197.

- Terra Mitica - Benidorm (www.terramiticapark.com/)

- Tibidabo - Barcellona

CERVER FRANCISCO ASENSIO, Landscape of recreation II: amusement parks, WatsonGuptill Pubns, Slipcase edition 1998, pagg. 98-111.

- Tivoli World - Arroyo de la Miel (www.tivoli-atracciones.com/)

- Universal's Port Aventura - Salou (www.portaventura.es/)

- WB Movie World

\section{Sweden}

- Grona Lund's Tivoli - Stockholm (www.gronalund.com/) 
Tosetto Danilo, Parchi Ricreativi nel Mondo, Vol. 2 , Facto, Padova 1987, pagg 186- 193.

- Hassans Nöjesfältet - Åhus (www.hassans.se/)

- Liseberg Amusement Park (www.liseberg.se/intro.asp)

Tosetto Danilo, Parchi Ricreativi nel Mondo, Vol. 3 , Facto, Padova 1988, pagg. 148- 161.

- Ölands Djur \& Nöjespark - Färjestaden (www.olandsdjurpark.com/)

- Santaworld - Gesundaberget (www.santaworld.se)

\section{Switzerland}

- Labyrinthe Aventure- Evionnaz (www.labyrinthes.ch)

- Swiss Miniatur - Melide-Lugano (www.swissminiatur.ch)

\section{Great Britain}

- Adventure Island - Southend On Sea (www.adventureisland.co.uk/)

- Alton Towers (www.alton-towers.co.uk/)

- The American Adventure - Ilkeston

Tosetto Danilo, Parchi Ricreativi nel Mondo, Vol. 4 , Facto, Padova 1989, pagg. 4053.

- Bekonscot - Bucks

Tosetto Danilo, Parchi Ricreativi nel Mondo, Vol. 3 , Facto, Padova 1988, pagg. 216- 225 .

- Blackpool Pleasure Beach- Blackpool

Tosetto Danilo, Parchi Ricreativi nel Mondo, Vol. 3 , Facto, Padova 1988, pagg. 132- 147.

- Botton's Pleasure Beach - Skegness, England (www.pleasurebeachskegness.com/)

- Brean Leisure Park - Brean, England (www.brean.com/)

- Brighton Pier - Brighton, England (www.brightonpier.co.uk/) 
- Camelot Theme Park - Chorley, England (www.brightonpier.co.uk/)

Tosetto Danilo, Parchi Ricreativi nel Mondo, Vol. 4 , Facto, Padova 1989, pagg. 6675.

- Chessington World of Adventures, England (www.chessington.co.uk/)

- Clacton Pier - Clacton On Sea, England (www.clactonpier.co.uk/)

- Clarence Pier - Portsmouth, England (www.clarencepier.co.uk/)

- Crealy Park - Exeter, England (www.crealy.co.uk/)

- Drayton Manor - Birmingham, England (www.draytonmanor.co.uk/)

Tosetto Danilo, Parchi Ricreativi nel Mondo, Vol. 4 , Facto, Padova 1989, pagg. 144- 157.

- Fantasy Island - Skegness, England (www.fantasyisland.co.uk/)

- Great Yarmouth Pleasure Beach, England (www.pleasure-beach.co.uk/)

- Gulliver's Kingdom - Matlock (www.gulliversfun.co.uk/)

- Gulliver's Land - Milton Keynes, England (www.gulliversfun.co.uk/)

- Gulliver's World - Warrington, England (www.gulliversfun.co.uk/)

- Flambards Village Theme Park - Helston, , England (www.flambards.co.uk/intro.html)

- Flamingo Land - Malton, England (www.flamingoland.co.uk/)

- Harbour Park - Littlehampton, England (www.harbourpark.com/)

- Jorvik Center - York, England

Tosetto Danilo, Parchi Ricreativi nel Mondo, Vol. 5 , Facto, Padova 1990, 56- 65. Wylson ANTHONY \& PATRicia, Theme Parks, Leisure Centres, Zoos and Aquaria, Longman, 1994, pagg. 53-55.

- Legoland - Windsor (www.lego.com/legolandnew/windsor)

- Lightwater Valley - England (www.lightwatervalley.co.uk/home.htm)

Tosetto Danilo, Parchi Ricreativi nel Mondo, Vol. 4 , Facto, Padova 1989, pagg. 184- 195.

- Loudoun Castle - Galston, Scotland (www.loudouncastle.co.uk/)

- M\&D's Scotland's Theme Park - Motherwell, Scotland (www.scotlandsthemepark.com/)

- The Milky Way Adventure Park - Bideford, England (www.themilkyway.co.uk/)

- Oakwood - Pembrokeshire, Wales (www.oakwood-leisure.com/) 
- Paultons Park - Romsey, England (http://www.paultonspark.co.uk/index.html)

- Pleasure Island - Cleethorpes, England

- Pleasureland Southport - Southport, England (www.pleasure-island.co.uk/)

- Pleasurewood Hills Leisure Park - Lowestoft, England

- Sundown Adventureland - England (www.sundownadventureland.co.uk/)

- Thorpe Park - Surrey, England (www.thorpepark.co.uk/default.asp?flash=true)

Tosetto Danilo, Parchi Ricreativi nel Mondo, Vol. 5 , Facto, Padova 1990, pagg. 222- 235 .

- Walton Pier - Walton on the Naze, England (www.waltonpier.co.uk/)

http://storm-

cloud.eng.cse.dmu.ac.uk:8080/CoasterGrotto/ParkReviews/FantasyIsland/FantasyIsland.

jsp www.uk-tourist-

attractions.co.uk/Attractions/Attractions/Theme_Parks/Magical_World_Of_Fantasy_Isla nd.cwww.tour-britain.com/engarea22.html

- West Midland Safari \& Leisure Park - Bewdley, England (www.wmsp.co.uk/)

www.gtyarmouth.co.uk/

[Nota: nella SCHEDA ANALITICA in allegato non compaiono i parchi del presente elenco di cui non si è trovato materiale informativo.]

RIFERIMENTI BIBLIOGRAFICI per la stesura dell'elenco

"Lotus Navigatore", n. 2, Nuovi paesaggi, Electa, Milano2001.

"Paesaggio Urbano", n. 5, Parchi di divertimento, Maggioli Editore, Rimini 1993.

BARZILAY MARIANNE, HAYWARD CATHERINe, LOMBARD-VALENTINO LUCETTE, L'invention du parc: parc de la Villette: Paris, concours international 1982-1983, Graphite, Etablissement Public du Parc de la Villette 1984.

BÉDARIDA MARC, Tradizione francese e paradigma ecologico. Parc André Citroën, "Lotus international", n. 87, Electa, Milano1995, pagg. 7-31.

BurCKHARDT LuCIOUS, Tendenze attuali dell'arte dei giardini, in "Domus" n. 817, Milano 1999.

CERVER FRANCISCO ASENSIO, Landscape of recreation II: amusement parks, WatsonGuptill Pubns, Slipcase edition 1998, pagg. 98-111.

CERVER FrANCISCO ASENSIO, Theme and amusement parks, Atrium Internationa, Whitney Library of Designe, 1997.

CORTESI ISOTTA, Il parco pubblico_paesaggi 1985-2000, Federico Motta, Milano 2000.

Donin Gianfranco, Parchi, Biblioteca del Cenide, Cannitello (RC) 1999.

FABRIS LUCA MARIA FRANCESCO, Il verde postindustriale, Liguori Editore, Napoli 1999. 
FrIedBerg PAUL, et al., Il parco del XX secolo, "Casabella", n. 492, Electa, Milano1983, pagg. 12-23.

LANGLOIS GILleS-ANTOINE, Folies Tivolis et attractions. Les premieres parcs de loisirs parisiens, Délégation à l'action artistique de la ville de Paris, Paris 1991,

MINARDI EVERARDO, LUSETTI MARIALUISA (a cura di), I parchi del divertimento nella società del Loisir, Franco Angeli, Milano 1998.

Tosetto Danilo, Parchi Ricreativi nel Mondo, Vol. 1, Facto, Padova 1986.

Tosetto Danilo, Parchi Ricreativi nel Mondo, Vol. 2 , Facto, Padova 1987.

Tosetto Danilo, Parchi Ricreativi nel Mondo, Vol. 3 , Facto, Padova 1988.

Tosetto Danilo, Parchi Ricreativi nel Mondo, Vol. 4 , Facto, Padova 1989.

Tosetto Danilo, Parchi Ricreativi nel Mondo, Vol. 5 , Facto, Padova 1990.

Wylson, Antony \& PAtricia, Theme Parks, Leisure Centres, Zoos and Aquaria, Longman, 1994.

Siti web generali per la stesura dell'elenco

(sono esclusi quelli indicati in precedenza per i singoli parchi):

http://freizeitparkweb.de/html/links.html?page=no

http://guide.supereva.it/parchi_di_divertimento/link_ai_parchi_in_europa/

http://home.foni.net/ helenelangegymn/news_sptime25.htm

http://web.tiscali.it/no-redirect-tiscali/theparks/pks_europa/pks_tema_europa.htm

www.123world.com/amusement/

www.achus.net/achus/europa

www.ac-toulouse.fr/culture/citesparc.htm

www.aquarium-bbs.de/zoo/europa/zoo-euro.htm

www.attentialpupo.com/tempoliberoevacanze/mappaparchi/mappaparchi.htm

www.bambinopoli.it/scripts/bimbbase.exe?DET=1\&IDREC=904

www.chez.com/bienvenueatoulouse/cite12.jpg

www.cite-espace.com/english/index.htm

www.dreammingitaly.com/parchi.htm

www.funguide.com/

www.geocities.com/Colosseum/Sideline/7510/eurele.html

www.georgetown.edu/faculty/murphyd/netsearch/theme.html

www.germancoaster.de/parks-europa.html

www.girobimbi.it/parchi_divertimento/fr_rg_italia_div.html

www.infoparchi.com

www.kidzprintz.com/safetylinks/just4kids/themeparks.html

www.parksmania.it/cite_espace.htm

www.parksmania.it/eurpark.html

www.pegacity.it/viaggi/parksmania/waterpk.html

www.sordionline.com/parco parchi_europei.htm

www.sordionline.com/parco_parchi_italiani.htm

www.themeparkcity.com/EURO_index.htm

www.themeparkinsider.com/europe/

www.theparks.it/pks_europa/

www.virtualpark.org/parchi_eur.htm

www.vons.free.fr/toulouse/citeespace.html

www.zeal.com/category/preview.jhtml?cid=548667

www.zoo-ag.de/ausland.htm

www.zoo-archiv.de/slg_west.htm 


\section{- SCHEDA ANALITICA DEI PARCHI DEL DIVERTIMENTO EUROPEI \\ [vedi tavole in allegato]}

Lo scopo della stesura di una scheda analitica dei parchi del divertimento europei è quello di avere un quadro conoscitivo della situazione il più esaustivo possibile. La scheda deve consentire di scegliere alcuni parchi da sottoporre all'analisi del rapporto che intercorre fra elementi tematici e soluzioni spaziali, nei termini dell'architettura del paesaggio.

Nella PARTE I della ricerca si sono individuate le matrici culturali e le diverse modalità di tematizzazione, e si è visto come ad esse siano associati paesaggi con caratteri differenti, quanto a strumenti e modi di costruzione, e quanto ad esiti.

Si intende quindi individuare per ogni parco la matrice prevalente, la presenza o assenza di un tema principale, ed il ruolo che questo tema svolge. L'attribuzione della matrice prevalente e del ruolo del tema è di prima approssimazione, sia per il numero elevato dei parchi censiti (che non consente un approfondimento di dettaglio), sia perché ogni parco è una realtà complessa, subisce modifiche e sviluppi negli anni, cambi di proprietà e gestione, finalità ecc.

Per poter attribuire la matrice ed il ruolo del tema è necessario conoscere l'origine del parco, il territorio in cui si colloca, il tipo di attrazioni che offre e i temi che presenta; inoltre si tenta di conoscere - o intuire quando il materiale informativo risulta insufficiente -se c'è stata o meno una progettazione rivolta specificatamente al paesaggio nelle fasi di studio e di realizzazione del parco. Per cui si indagano i seguenti tematismi:

- COORDINATE GEOGRAFICHE

- ORIGINE STORICA

- contesto/PAESAGgio

- ATTRAZIONI

- TEMA

quindi conseguentemente:

- MATRICE (in base a quanto dedotto dalla "PARTE I.1" della ricerca)

- RUOLO DEL TEMA (in base a quanto dedotto dalla "PARTE I.2" della ricerca)

- PRogettazione Del PAESAGgio (c'è stata? Non c'è stata? A livello intuitivo dove non si hanno sufficienti dati).

\section{ALCUNI RISULTATI DALLA REDAZIONE DELLA SCHEDA ANALITICA}

Il quadro risultante dalla scheda dei parchi del divertimento europei è variegato e conferma la pluralità di modi organizzativi, di intenti, di riferimenti culturali che possono costituire il retroscena dei parchi del divertimento, tematici e non, messi in evidenza dall'indagine sulle matrici. Se si sottoponessero i dati raccolti ad analisi di tipo statistico probabilmente sarebbe possibile articolare le considerazioni in maniera maggiormente analitica e dare una lettura del quadro molto più approfondita. In questa sede vengono presentati alcuni aspetti emersi, utili per impostare le fasi successive della ricerca.

Per i parchi del divertimento non tematici o che presentano una miscellanea di temi, i modelli di riferimento (in alcuni casi si tratta proprio delle origini storiche) sono i pleasure gardens, e soprattutto gli amusement park americani sul tipo del Coney Island di New York. Quelli strettamente tematici (81 parchi) appaiono influenzati dal prototipo Disneyland (31 
parchi), riferimento riconoscibile nel tema proposto -mondo di personaggi di fumetti, favole, che sono diventati in qualche modo dei simboli- ma spesso anche nell'impianto planimetrico, definito, schematizzando, da un asse di accesso principale su cui si aprono, a forma di petali di fiore, le diverse aree tematiche, all'interno delle quali attrazioni, ristorazione, punti vendita sono tutti coerenti con il tema.

Dei parchi tematici tre sono antichi pleasure gerdens (la definizione viene usata in senso lato) ancora attualmente attivi: il Bakken nei pressi di Copenhagen, il Jardin d'Acclimatation al Bois de Boulogne di Parigi, l'Alton Towers in Inghilterra. Il Bakken nato per la trasformazione in struttura stabile degli accampamenti temporanei di artisti itineranti, in un località amena per la presenza di una fonte d'acqua curativa scoperta nel 1583, ancora oggi è rinomato per il suo festival annuale di Clowns. La maschera Pierrot è da tempi antichi il suo ospite più famoso e il suo simbolo. Il Jardin d'acclimatation, istituito da Napoleone III nel 1860, oggi si rivolge prevalentemente ad un pubblico di bambini, e vede allestiti, nel parco di alberi secolari, giochi a loro dedicati, per lo sviluppo delle capacità percettive e motorie, un teatro di marionette, l'exploradome con giochi interattivi, uno spazio per il circo, la stalla dei poni ed anche un'area somigliante, quanto ad attrazioni, ad un comune lunapark. Nel giardino ancora vengono piantate piante esotiche in sostituzione e completamento della vegetazione esistente. E' un parco urbano, e conserva il ruolo originario di luogo di incontro, di spettacolo, di divertimento, all'interno di un ambiente esotico in cui la città di Parigi è più che mai presente, sullo sfondo, nei profili dei palazzi e nella cultura dei suoi visitatori, prevalentemente locali. I giardini di Alton Towers vengono aperti al pubblico nel $1860 \mathrm{e}$ intorno al 1890 ospitano feste con spettacoli di animali esotici addomesticati; il disegno del paesaggio di Alton Towers risale al secolo XVIII e all'opera dei paesaggisti Thomas Allason e Robert Abrahams, ma attualmente l'organizzazione per aree tematiche del parco porta a pensare che il modello Disneyland abbia influenzato gli sviluppi di Alton Towers. Non si possiedono peraltro planimetrie o immagini che consentano di analizzare gli esiti della "modernizzazione" subita dal parco. Oltre ai tre antichi pleasure garden solo il parco Efteling (1952), in Olanda ha origini anteriori all'apertura del Disneyland californiano (1955): tutti gli altri parchi tematici vengono realizzati successivamente.

Fra i parchi tematici un tema ricorrente è la miniatura: i parchi che espongono modelli in scala di paesaggi, città, monumenti- non sono pochi (10 parchi sono "parchi in miniatura" veri e propri, altri 3 contengono fra le varie attrazioni anche ambienti in miniatura). Si identifica la matrice prevalente di questi parchi nelle esposizioni internazionali, per due motivi: innanzitutto alle esposizioni spesso venivano presentate miniature (si è detto della Golden Gate International Exposition di San Francisco del 1939 in cui vengono esposte stanze minuscole, perfettamente arredate con precisione di dettaglio), di architetture, ambienti, mezzi di trasporto ecc.; si è parlato inoltre, a proposito delle esposizioni internazionali, di paesaggi sintetici, in riferimento all'accostamento di ambienti caratteristici di luoghi diversi del mondo, in uno spazio e in un arco di tempo contratti, che è esattamente l'idea che sta dietro ai parchi miniature, portata ai termini estremi. All'interno dei parchi miniatura il tema ha da un lato un fine didattico e di valorizzazione di una cultura o di una nazione, quella di cui il parco vuole essere un riassunto - c'è un' Italia in miniatura e una Fantasy World Minitalia, una France miniature e una Minifrance, un Portugal do Pequenitos, Europa park ecc. - dall'altro la "collezione" di tanti luoghi in un unico luogo opera una deterritorializzazione e una dicronizzazione.

18 parchi hanno un tema che intende immettere in una "dimensione fantastica", e di questi 10 propongono un'immagine, di fantasia, che si impone come marchio: sono le caramelle e gli animali Bon Bon, i Moomin, personaggi di un disegnatore finlandese conosciuti a livello internazionale, i Lego, pezzi da costruzione per città giocattolo "fai da te", i personaggi del fumetto Asterix, Gulliver, catena di parchi inglesi; forse sarebbe opportuno considerare un marchio anche Santa Claus, che dal 1998 abita a Santapark, in Lapponia. 
Una diversità sostanziale rispetto al modello Disneyland, riscontrabile anche nei parchi tematici che per altri aspetti gli assomigliano molto, è la presenza diffusissima della componente spettacoli fra le attrazioni proposte - riscontrata in 35 parchi su 81 parchi tematici e in 75 su 191 parchi in totale (totale fra quelli di cui si è reperito materiale informativo) -. Sono spettacoli pirotecnici, di musica, di marionette, di acrobati, di artisti di strada, di varietà, di circo, di teatro. In alcuni parchi risultano essere le attrazioni principali, su cui punta la pubblicità in brochure, siti web ecc., e da cui deriva l'immagine del parco. La loro presenza dichiara che in Europa continua la tradizione dei pleasure gardens, dei jardins spectacle, delle forme di divertimento offerte dalla fiere, locali e internazionali. Rivela inoltre che è avvertito il legame fra divertimento ed evento particolare, diverso dal solito. Il circo, gli spettacoli di acrobati... vivono dello stupore che produce il partecipare a momenti di rischio, di pericolo, e sono quindi fra i migliori ingredienti per operare lo spaesamento dello spettatore. Disneyland in California non offre spettacoli, se si considera quale eccezione la Star Parade, sfilata giornaliera dei personaggi Disney più famosi, che passano, salutano, si intrattengono con i bambini. Disney nell'ideare il suo parco si oppone espressamente al clima di informalità, facilmente degenerante in malcostume, di Coney Island e dei comuni amusement park. Sicuramente lo spettacolo popolare rende l'atmosfera informale, ma soprattutto rappresenta all'interno del parco un elemento non completamente pianificabile, che ha margini di rischio, aspetto non gradito dalla Disney Company, la quale predilige forme di "teatro" completamente codificate, e intende mantenere sotto controllo il comportamento dei suoi visitatori - si è parlato di questo al paragrafo Fiction land, descrivendo i modi di composizione spaziali di Disneyland. Lo spettacolo che accade hic et nunc si preannuncia come un elemento importante al fine di non incorrere nell" "utopia noiosa" di cui dice Isabelle Auricoste, contribuisce a rendere unica l'esperienza della visita al parco. in particolare modo se l'hic è studiato, ovvero se il luogo che ospita lo spettacolo è in rapporto con le specifiche caratteriste del parco e del contesto del parco.

Fra i parchi "genericamente" del divertimento , che non presentano un tema coesivo, si evidenzia una percentuale elevata, concentrata in Inghilterra, di Pier e di complessi lungo la costa, che richiamano Coney Island, facenti parte della più vasta offerta turistica delle zone in cui sono localizzati, all'interno delle quali comunque si impongono quali maggiori attrazioni. Blackpool Pleasure Beach è uno di questi: il parco ha inizio nel 1896 ad opera di un ingegnere, William George Bean che è vissuto in America, ha lavorato precedentemente a Coney Island e vuole quindi tentare 1"'impresa" di costruire in Inghilterra un American style Amusement Park.

Alcune considerazioni possono essere fatte- oltre che riguardo al rapporto origine-matrice tema- anche sul rapporto che intercorre fra la realtà del parco ed il territorio in cui si colloca. Solo in 2 parchi tematici è leggibile in modo chiaro la volontà di valorizzare uno specifico contesto, quale movente per la realizzazione del parco e quale criterio guida per la scelta del tema: nel Futurescope a Poitiers e nel Mer de Sable di Ermenonville, entrambi in Francia.

Il parco Mer de Sable ha per tema il West d'America, scelto sfruttando la suggestione offerta da una distesa di dune di sabbia, particolarità geologica all'interno della Foresta di Ermenonville, che rimane compresa dentro il confine del parco - se ne parlerà maggiormente in dettaglio all'omonimo paragrafo.

Futurscope apre nel 1987 e viene completato in tutte le sue parti nel 1989. La scelta della sua localizzazione è dovuta alla volontà di conferire un'identità nuova alla regione Vienne e creare un centro d'attrazione che interrompa il "deserto francese" dovuto alla concentrazione di servizi e attività (opportunità) nel territorio di Parigi. Futurscope è quello che in Italia si definisce un "parco scientifico tecnologico", ovvero un parco in cui vengono insediate aziende, istituti di ricerca e attività culturali, che possono avere vantaggi dall'essere spazialmente vicini. Futurscope ha tre diverse aree tematiche: Distraction, Formation, Travail; è un parco ricreativo e didattico e un polo per la ricerca scientifica e tecnologica. Ogni sua componente viene progettata dichiarando, nelle tecnologie impiegate e nella 
forma, l'idealismo che muove tutta l'operazione di riqualificazione della Vienne e la costruzione del parco. L'architettura in particolare è pensata come un simbolo -"architecture évocatrice des forces de la vie"1 - e gli edifici si stagliano quali landmark nel territorio rurale adiacente (sono oggetti retorici, quasi di propaganda): Le Kinémax (1987) rappresenta nella forma di un cristallo tecnologico e fuori scala la genesi della terra; Le Pavillion $d u$ Futurscope (1987) è un sole -un'enorme sfera bianca- che sorge da un piano inclinato -in realtà si tratta di un volume architettonico- di vetro; Le Pavillion de la Communication (1988) è una goccia d'acqua, grande, come vista al microscopio; L'Omnimax (1990) è un meteorite, un prisma di vetro che contiene una sfera, incastro di forme primarie trasformate, da astratte, in corpi materiali ed abitabili; Le Pavillion de la Vienne (1994) è un gioco d'acqua e trasparenze che intende esprimere un'energia vitale. Allo stesso modo i giardini sono tematici e vogliono essere un compendio dei diversi ambienti naturali e dei diversi modi dell'arte dei giardini. L'ideologia accomuna la realizzazione di Futurscope agli eventi delle esposizioni universali del secolo precedente: il suo punto critico è la relazione fra valori che vengono proposti quali comunitari, di livello globale, e riqualificazione - marketing locale. Il parco vuole essere un luogo ideale di rappresentazione della condizione contemporanea, ribadendo di essere memore comunque della storia e della tradizione, mentre è tutto teso alla ricezione di una possibile civiltà del futuro. La proposta di Futurescope sembra, in una certa misura, simile a quella operata dal Parc de La Villette di Parigi (al contrario inserito all'interno del territorio urbano) se si considera che il tema del concorso di progettazione - e del parco- altro non era che la definizione del concetto e del ruolo del parco (pubblico) nella città e nella cultura contemporanea. Con La Villette si cerca "un nuovo tipo di spazio urbano", quale exemplum, definizione per la quale si guardava anche ad esempi di parchi del divertimento e tematici, come sottolineato da Silvie Brau nell'esporre il programma del parco ${ }^{2}$.

Parc de La Villette, come anche Futurscope, ha come tema il parco, perchè non solo, come sempre, la progettazione è occasione per una riflessione sul valore ideale e sul ruolo generale dell'oggetto progettato, ma il progetto vincitore di Tschumi passa dai concetti alle forme nello spazio senza sostanziali adeguamenti al contesto dato, conservando l'astrazione delle idee anche nella loro materializzazione in elementi costruiti - sono concetti/architetture le folies distribuite secondo la griglia ortogonale, il percorso sinuoso che ne spezza il rigore, l'asse principale segnato dalla pensilina quale spina dorsale e via diretta per raggiungere le varie funzioni del parco...).

Il concetto di "luogo ideale" quale tema del parco, soprattutto nei parchi realizzati o ripensati dagli anni novanta in poi, appare frequente, ed il parco tematico sembra porsi come una realtà estroversa in grado di caratterizzare l'offerta turistica più generale del contesto in cui si colloca e l'immagine dei luoghi (spesso non aderente affatto ai luoghi ma "imposta" prepotentemente), coinvolgendo un'intorno ampio, inteso anch'esso come parco. Esprime chiaramente questo fatto, a proposito della situazione italiana, Asterio Savelli:

\footnotetext{
“ Dalla sua realtà di isola, il parco muove alla conquista del territorio circostante, per gestirne le informazioni, per costruire legami con gli enti locali e con il mondo imprenditoriale, per creare imprese che agiscano all'esterno (sulla base della forza interna), per moltiplicarsi infine come modello". 3
}

“ Nato in uno spazio separato e protetto, il parco tende a proiettare all'esterno la sua logica selettiva interna, trasformando il territorio stesso in parco.",

\footnotetext{
${ }^{1}$ L'espressione è il titolo del paragrafo relativo all'architettura di Futurscope in TRÉBUCQ MATHILDE, La Nature $d u$ Futurscope, Les Editions du Futurscope, Vaschette, pag. 14-15.

2 In Silvie Barrau, Le programme du parc, in Marianne Barzilay, Catherine Hayward, Lucette Lombard-VAlentino, L'invention du parc: parc de la Villette: Paris, concours in ternational 1982-1983, Graphite, Etablissement Public du Parc de la Villette 1984, pag. 238.

3 ASterio SAVElli, Parco separato e territorio aperto: problemi di strategia nelle aree turistiche, in EVERARDO MinARDi, MARIAluisa LuSETti (a cura di), I parchi del divertimento nella società del Loisir, Franco Angeli, Milano 1998, pag. 20.
} 
Questa idea non è molto diversa da quella di parco-sistema di Bruno Sanguanini:

"Il parco sistema o "di quarta generazione" risponde all'idea di Super-Park, ovvero ad un sistema integrato di
una molteplicità di parchi speciali ed a tema: un sistema fatto da sottoinsiemi specialistici. Le novità sinora
prospettate rispondono a due formule: i parchi-giardino, a forte caratterizzazione ecologica, e i parchi high-tech,
dove prevalgono le recenti applicazioni tecno-scientifiche in materia telematica e virtual reality (...). In entrambi
i casi, ai nuovi artefatti e campi simbolici è chiesta la messa a regime di una sorta di "parco vivente", in grado di
ricreare i nuovi miti sociali legati alla "qualità della vita" ed all'immaginario collettivo."

Il rapporto con il territorio sembra in generale più esserci a livello funzionale, di programmazione delle attività e dell'offerta di servizi, che a livello paesaggistico. Questa è l'impressione che si avverte "sfogliando" i siti web e le brochure dei parchi.

La maggioranza dei parchi censiti è collocata in territorio extraurbano ed è ben collegata ai contesti urbani vicini dalla rete dei trasporti e soprattutto dalle arterie autostradali. Si trova inoltre in località turistiche o lungo itinerari turistici di rilievo. Il dati confermano le considerazioni di Alberto Abruzzese, facilmente comprensibili, riguardo alla localizzazione dei parchi del divertimento, per cui il territorio extraurbano è privilegiato - non solo per i motivi espressi nella PARTE I della ricerca, ovvero per il desiderio dei visitatori di $d i$ vertere dallo spazio quotidiano-urbano, ma anche perché il territorio extraurbano offre il vantaggio di consentire ai singoli soggetti-fruitori liberta di movimento e facilità di accesso e ai pianficatori una "semplificata" gestione dei flussi turistici:

\begin{abstract}
"Si tratta -stando alla modellistica che si è andata sviluppando in questi anni - di spazi all'aperto extraurbani per vocazione, tendenzialmente artificiali (...). L'esistenza di tali spazi attrezzati presuppone la cultura e la pratica dell'automobile, cioè non solo di una vasta e quotidiana agibilità delle distanze grazie a mezzi di trasporto leggeri, ma anche del bisogno personale di deviazione e sosta rispetto alle direzioni di marcia, di revoca dei regimi di transito. Collocati in zone rarefatte ma magnetiche, cruciali, perché attraversate da flussi intensi di popolazione, configurano una specifica meta del consumo collettivo o una sorta di tempo libero nel tempo "lasciato" libero tra una destinazione e l'altra, tra un lavoro e l'altro, tra un consumo e l'altro, garantendo l'incrocio di più opportunità per più pubblici.",
\end{abstract}

Solo 19 sono parchi urbani o periurbani e sono fra quelli di origine più antica; alcuni sono pleasure gardens che nel XIX sorgevano in luoghi a contatto con la città, ma esterni, ora inglobati all'interno in seguito all'espansione dell'urbanizzazione; alcuni di questi hanno ospitato Esposizioni Nazionali o Universali - al Prater di Vienna viene organizzata la Weltaustellung del 1873 (ad esempio) l'attuale Brupark di Brussels sorge nell'area dell'Exposition Universelle et Internazionale de Bruxelles del 1935 e dell'Expo '58, ed ha il suo simbolo nell'Atomium, architettura in acciaio che riproduce a scala gigante la struttura di una molecola, realizzata per l'Expo e poi, come la Tour Eiffel a Parigi, conservata quale presenza stabile e monumento.

I parchi all'interno dei contesti urbani si rivolgono prevalentemente a visitatori locali e hanno tematizzazioni "deboli" - probabilmente perché, utilizzando una distinzione operata da Mariano Sartore ${ }^{7}$ fra "parchi a consumo" e "parchi a visita", si tratta di spazi "a

\footnotetext{
4 Asterio Savelli, Op.cit., in Everardo Minardi, Marialuisa LuSEtTi (a cura di), I parchi del divertimento nella società del Loisir, Franco Angeli, Milano 1998, pag. 22.

${ }^{5}$ Bruno SANGUANini, Effetto benessere \& qualità della vita. A DisneyWorld, EuroDisney, Gardaland e altrove, in EVERARDo MinARDI, MARIALUISA LuSETTI (a cura di), I parchi del divertimento nella società del Loisir, Franco Angeli, Milano 1998, pag. 118-119.

${ }^{6}$ Alberto AbruZzese, I parchi del divertimento, in "Paesaggio Urbano", n. 5, Parchi di divertimento, Maggioli Editore, Rimini 1993, pagg.18-19.

${ }^{7}$ MARIANO SARTORE, Tipologie, geografia e problematiche dei parchi ricreativi in Italia, in "Paesaggio Urbano", n. 5, Parchi di divertimento, Maggioli Editore, Rimini 1993, pag. 28.

Sartore definisce: "I parchi a consumo si configurano peraltro come luoghi nei quali sono dominanti attività di relax, destinati ad essere frequentati periodicamente, a differenza dei parchi a visita dove le emozioni generate dalle attrazioni si esauriscono dopo le prime visite (...). Se la visita a questi ultimi si configura quindi come "evento", la frequentazione dei parchi a consumo potrebbe configurarsi come "pratica". Questa diversa natura dei
} 
consumo", che vogliono attirare per una frequentazione periodica e devono quindi svolgere i diversificati ruoli normalmente attribuiti allo spazio pubblico: offrire un divertimento "neutro", programmi duttili e vari.

All'interno del quadro offerto dalla scheda analitica vengono scelti tre parchi da sottoporre all'analisi semantica:

- Parc Asterix, Plailly, France (1989- 2002)

- Mer-de-Sable, Ermenonville, France (1962-2002)

- Efteling, Kaatsheuvel, Netherlands (1952-2002)

Essi vengono scelti perché hanno fra loro differenti matrici prevalenti, un diverso ruolo del tema, e soprattutto perché in essi è riscontrabile un "progetto di paesaggio" (vedi tabella seguente). Ad Efteling viene assegnata quale matrice prevalente il pleasure garden (anche se non è quella la sua origine storica, il parco risale alla metà del '900) perché lo studio del paesaggio si impone quale componente dominante del parco: Efteling è concepito come un giardino eccentrico e questo lo fa emergere fra gli altri parchi analizzati.

\begin{tabular}{|c|c|c|c|c|}
\hline & TEMA & MATRICE & RUOLO DEL TEMA & $\begin{array}{l}\text { PROGETTAZIONE DEL } \\
\text { PAESAGGIO }\end{array}$ \\
\hline Parc Asterix - Plailly, France & fumetto Asterix & Disneyland & MARCHIO_DIMENSIONE FANTASTICA & $\mathrm{x}$ \\
\hline Mer-de-Sable, Ermenonville, France & $\begin{array}{c}\text { West d'America } \\
\text { (principalmente)+ altri temi }\end{array}$ & amusement park & DETERRITORIALIZZAZIONE/SINGOLARIZZAZIONE & $\mathrm{x}$ \\
\hline Efteling - Kaatsheuvel, Netherlands & $\begin{array}{c}\text { Fiabe nordiche } \\
\text { (principalamente) + altri temi }\end{array}$ & pleasure garden & DIMENSIONE FANTASTICA & $\mathrm{x}$ \\
\hline
\end{tabular}

Come anticipato, Mer de Sable appare avere un ruolo del tema interessante da approfondire: da una parte il tema opera una deterritorializzazione, in quanto immette nella dimensione dell'Old West America, dall'altra rende singolare il "mare di sabbia" della Foresta di Ermenonville.

C'è un altro aspetto che guida la scelta dei tre parchi: Efteling, Mer de Sable, e Parc Asterix si impongono quali parchi originali rispetto al modello Disneyland (Parc Asterix assomiglia a Disneyland, ma presenta differenze sostanziali che si descrivono, in seguito, in dettaglio), per cui si vuole approfondire quali siano le componenti per cui si diversificano e se tali componenti possano essere elementi fertili, riproponibili anche per progettazioni future.

$\mathrm{Si}$ intuisce che dietro la realizzazione di questi parchi c'è una consapevolezza profonda della complessità dei ruoli che un parco è chiamato a svolgere, e che questi ruoli vengono tutelati e progettati, nonostante le logiche commerciali abbiano comunque parte rilevante nella pianficazione delle attività e nell'ideazione degli spazi.

due tipi di parco può riflettersi in tipi e bacini di domanda tra loro molto diversi: relativamente stabile e di origine locale (urbana o metropolitana) nei parchi a consumo, occasionale e di scala regionale o sovra-regionale negli altri. MARIANO SARTORE, Ibidem. 


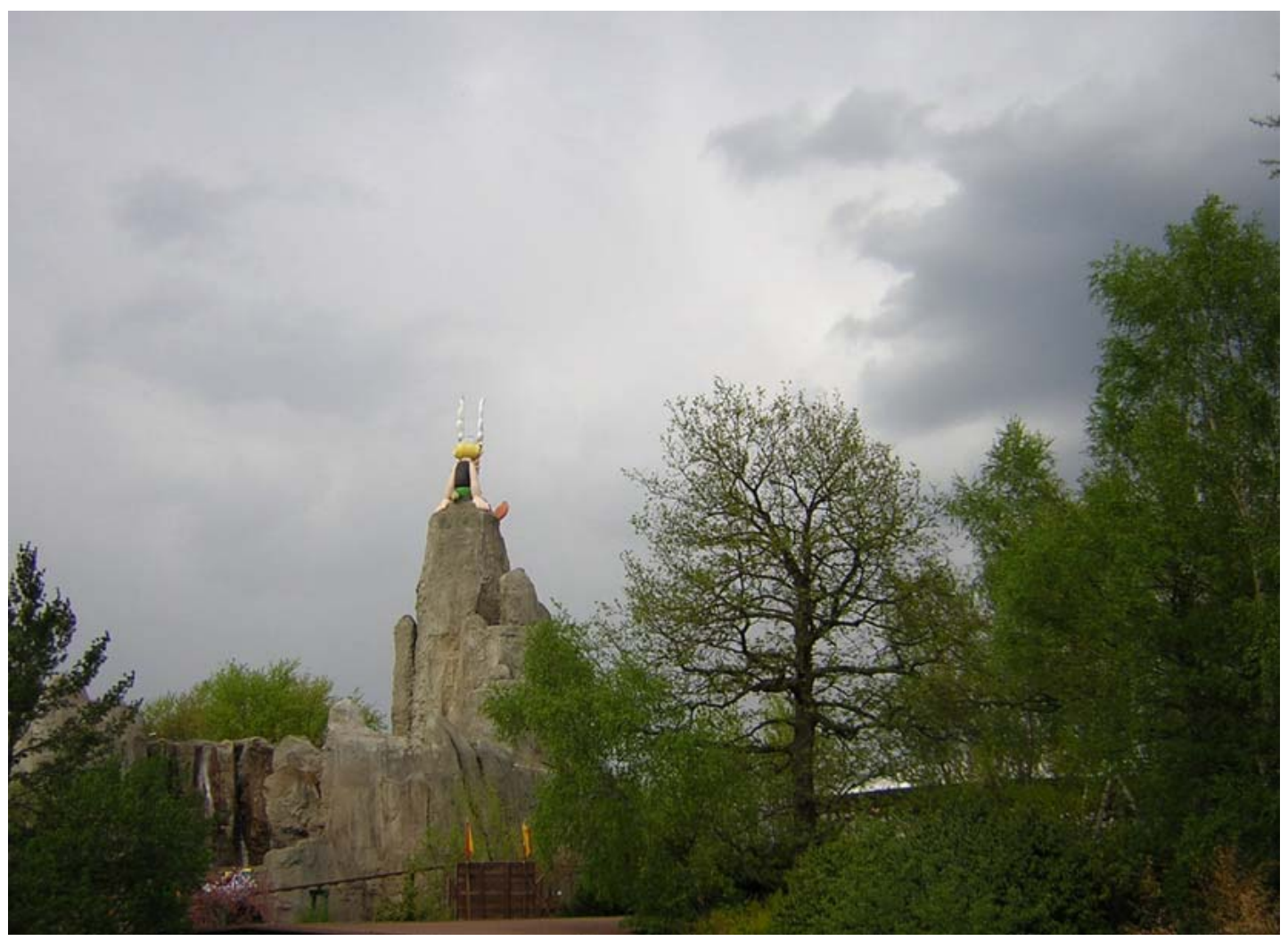

Fig. 1 La montagna di Asterix vista alle spalle. Enfatizzata dalla luce di una sera piovosa, l'immagine richiama un quadro romantico. Ha qualcosa dei paesaggi pittoreschi del XIX secolo.

Parc Asterix è un parco di realizzazione recente (1989), lungo l'autostrada A1 che da Parigi - da cui dista solo $30 \mathrm{~km}$ - giunge a Lille, all'interno della Foresta secolare di Ermenonville. La posizione è delle più favorevoli per il successo del parco. Evidenzia il fatto Langlois che vede in Parc Asterix un Tivoli contemporaneo, sottolineandone anche la seduzione esercitata dal marchio:

« Astérix a bénéficié de plusieurs factores favorables, dont il n'a sans doute pas encore turé tout le profit: une situation magnifique, en foret mais tout preès de l'A 1 ( la plus fréquentée des autoroutes), des études préliminaires très poussées, une image de marque d'emblèe séduisante. Comme Tivoli en 1795, Astérix a drainé jusqu'ici le plus grand nombre de clients ; sans doute parce qu'on lui a donné ce qu'il attendaient, dans un cadre ${ }^{\circ}$ la fois «naturel » et féerique. $»^{8}$

Ha come tema il fumetto Asterix creato da Rene Gosciny e Albert Uderzo. Uderzo è stato coinvolto nella progettazione del parco, coordinata dalla Duell Corporetion di Los Angeles a guida di un team di architetti e ingegneri francesi.

Sia il tema, sia la composizione spaziale e funzionale richiama il modello Disneyland, citato in maniera esplicita nella via centrale, di ingresso, la Via Antiqua, che vuole riprodurre caricaturalmente una strada dell'antica Roma: chiude la prospettiva della strada un montagna finta gigantesca, con seduto sulla cima un altrettanto gigantesco Asterix, benevolo e ammiccante, pronto a dare il benvenuto. La via Antiqua e l'Asterix enorme sono il

\footnotetext{
${ }^{8}$ Gilles-Antoine Langlois, Folies Tivolis et attractions. Les premiers parcs de loisirs parisiens, Délégation à l'action artistique de la ville de Paris, Paris 1991, pag. 67.
} 
corrispettivo della Main Street U.S.A che presenta sullo sfondo il castello della Bella Addormentata, a Disneyland in California (1955). Lo spirito del parco appare subito però molto diverso da quello del prototipo americano - Asterix in fondo non è Topolino, e soprattutto non è così serio. L'ironia che caratterizza il fumetto, viene rievocata dalle iscrizioni sui fronti dei negozi, da quelle di segnaletica, dai personaggi del fumetto (di plastica) che popolano il carosello, le sponde dei ruscelli artificiali, il villaggio dei Galli. Le iscrizioni non sono semplicemente uno strumento per l'ambientazione generale del parco, ma il loro contenuto è un commento quasi continuo al parco stesso, demistificatorio e sagace. Il paesaggio di Parc Asterix è filtrato dall'ironia su quanto il parco stesso propone al suo visitatore, comprendendo anche, fra gli aspetti su cui scherzare, l'insistente invito allo shopping e alla ristorazione lungo la Via Antiqua e la Rue de Paris e presente in ogni area tematica (All'ingresso dell'attrazione Les Espions de César, un percorso sopraelevato che consente di "spiare dall'alto" quello che accade nella Foresta dei Druidi si legge: "si vous échouez, ce n'est pas grave: vous sérez simplement jetés aux lions" e in un negozio di gadget: "sémaine du sarcophage: sur mésure; nombreux coloris").

Forse si deve a questa volontà di presa di distanza dal parco, proprio mentre si è dentro al parco, quella che sembra essere un'idea spaziale di notevole interesse: dall'area tematica Tonnerre de Zeus - non è altro che una bellissima montagna russa di legno montata in mezzo al bosco- si ha una vista di lontano delle altre zone tematiche, come se si fosse usciti dal parco. L'area è sotto il segno della grande statua di Zeus - vista da lontano appare come una statua greca (un bronzo di Riace), verde per ossidazione e concrezioni, mentre si mostra allo sguardo da vicino quale scherzo, (per l'espressione beffarda del viso e perché, passando sotto le sue gambe per entrare nella Tonnerre de Zeus, si vede che ha le mutande a fiori!?). Voltandosi e lasciando il grande Zeus alle proprie spalle, il resto del parco diventa uno sfondo distante, e la minuzia delle immagini, delle insegne, dei colori, viene tutta filtrata: rimangono appariscenti solo pochi landmark, la montagna di Asterix, il ristorante Arcimboldo, un castello antropomorfo. Immersi nella vegetazione che nasconde il "piano terra" ed il via-vai dei visitatori, essi sono come fabrique - architetture eccentriche in stili eterogenei e improbabili - in un dipinto o in un paesaggio pittoresco. Sono investiti dell'atmosfera malinconica della lontananza e dell'isolamento, che in realtà appartiene a chi guarda da fuori (rispetto al quadro, al paesaggio) e si esclude in questo modo dalla festa.

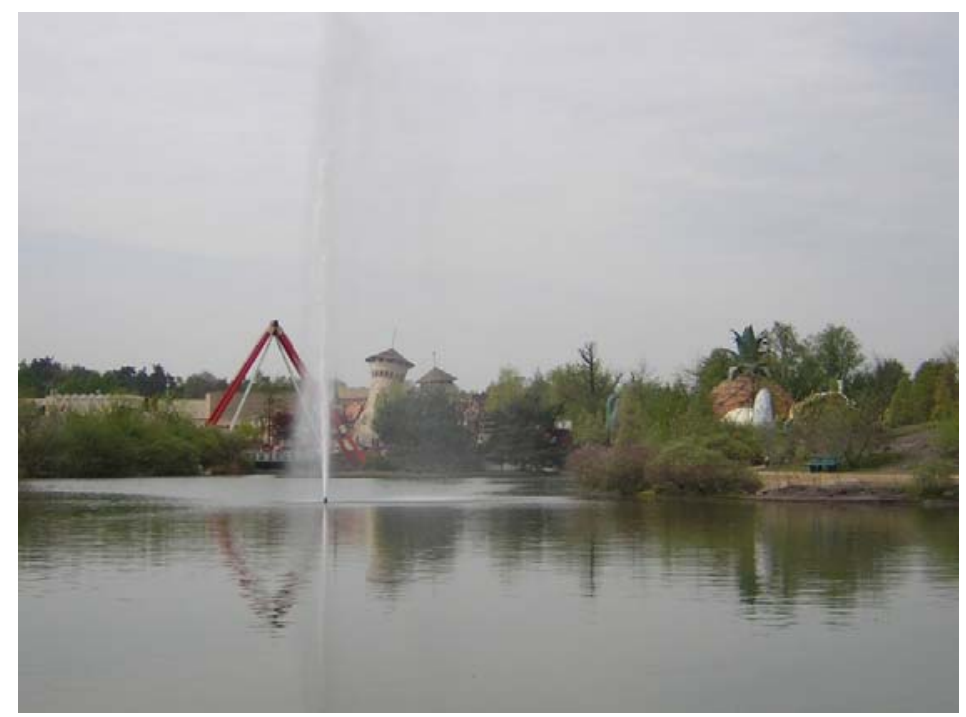

Fig. 2 Vista del parco dall'area tematica Tonnerre de Zeus sul lato opposto del lago: non è visibile la minuzia di insegne, immagini, colori e neppure il via-vai dei visitatori; appaiono solo pochi landmark, la Galère, il castello, il ristorante Arcimboldo di cui emerge la copertura a forma di ananas gigante, quali fabriques nel paesaggio. 


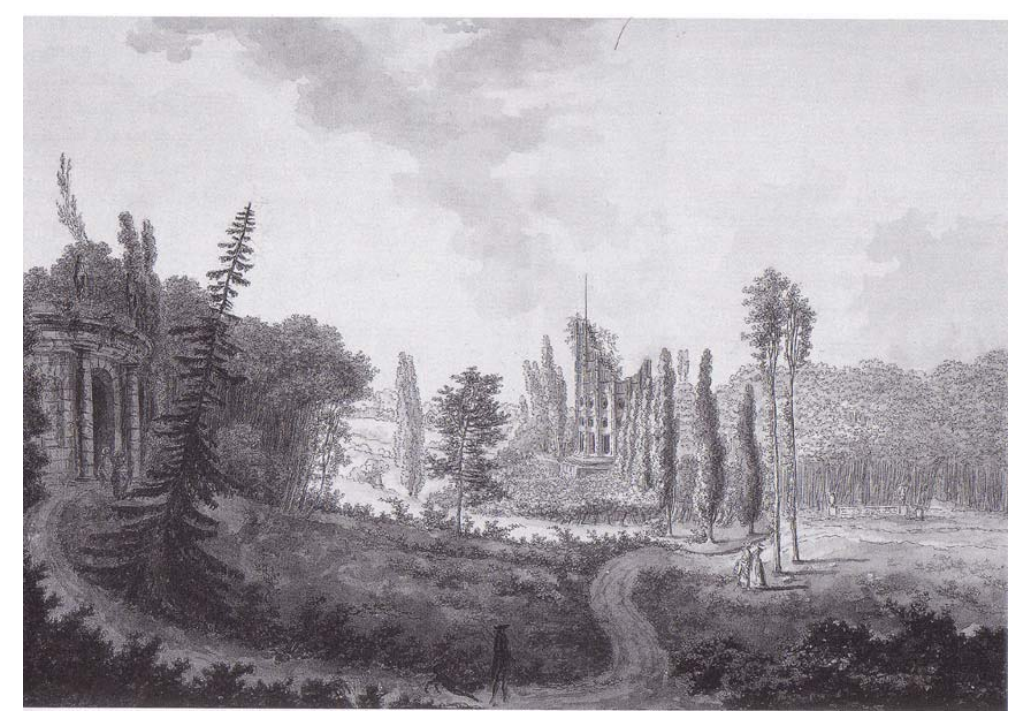

Fig. 3 Il Désert de Retze (fine XVIII sec.), con le fabriques del tempio di Pan e della Colonna gigante in rovina. Il disegno è attribuito al paesaggista carmontelle, progettista del parco.

In particolare il ristorante Arcimboldo ha un valore pittorico, riproducendo nelle tre dimensioni la bizzarria dei quadri di Giuseppe Arcimboldi (XVI sec.) ed enfatizzandola nelle dimensioni gigantesche dell'architettura. La frutta si amalgama agli alberi in una strana allegoria vegetale, fa apparire il bosco una miniatura e rimanda alla funzione dell'architettura, di ristorante, che si presenta come grande volto di Arcimboldi e allo stesso tempo come Bacco, il dio stravagante dei piaceri della tavola. L'Arcimboldo, così, è esattamente una fabrique e manifesta come il parco, pur seguendo le regole di Disneyland, si collochi all'interno della più antica tradizione dell'arte dei giardini francese, recuperando gli elementi a cui il XVIII secolo affidava il ruolo di catalizzatori del divertimento. A Parc Asterix il rapporto fra cultura di massa e raffinato senso estetico del paesaggio, è gestito attentamente mantenendo fra essi un'ambiguità continua.
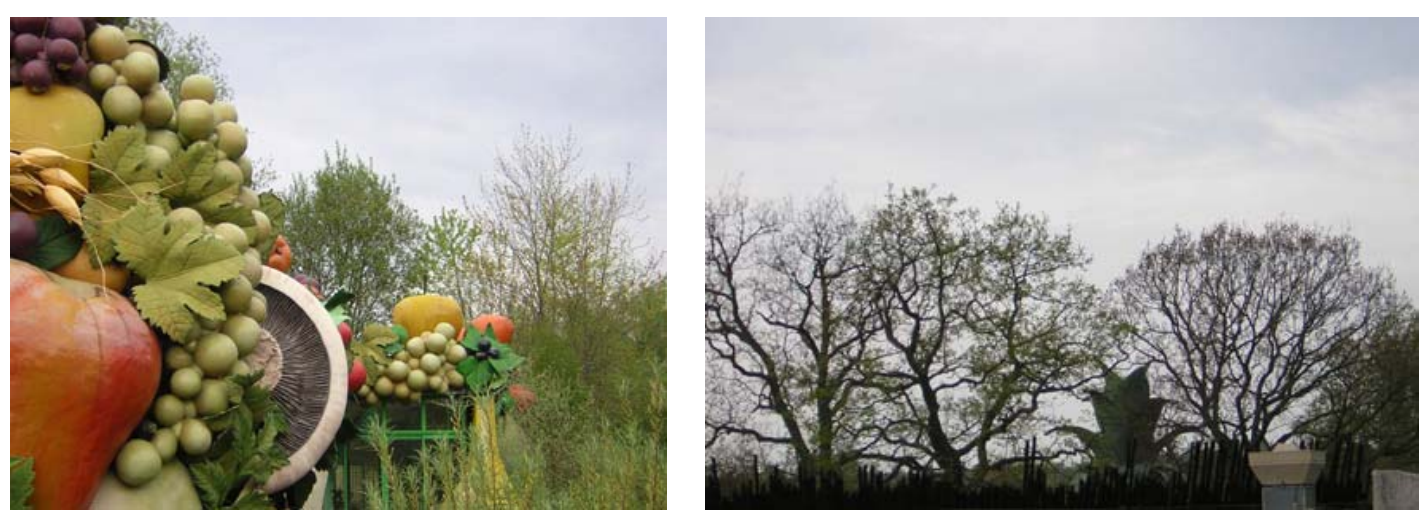

Fig. 4 Il ristorante Arcimboldo - in primo piano a dx e sullo sfondo a sx. E' un'architettura che collabora con la vegetazione nel suo intorno per creare un'immagine di fascino, quanto ad oggetti a confronto, colori e a rapporti di scala, fra gli elementi.

Una sensazione sempre di lontananza, si ha guardando lo spettacolo Main- basse sur La Joconde. Su uno sfondo da città industriale degli anni '20 ladri e poliziotti (acrobati) si inseguono contendendosi il quadro della Gioconda: la foresta subito fuori del parco completa la scenografia, vi si scolora contro il fumo di incendi simulati. Il tutto fa dimenticare completamente il tema principale del parco. 
Esistono molti luoghi in cui il visitatore è a contatto con il paesaggio naturale dell'intorno e molti in cui, anche se distante, è perfettamente visibile. Al contrario ci sono noccioli "duri" fortemente tematizzati, in cui insegne ed immagini scorrono molto fitte: sono la Via Antiqua e la Rue de Paris, la via di accesso al delfinario che prosegue fino al Tonnerre de Zeus, costeggiata da quinte architettoniche pseudo-città greca e da attrazioni dai rimandi mitologici le Vole d'Icare, L'Hydre de Lerne, la Rivière d'Elis. Lungo questo itinerario si apre un sentiero isolato che immette in un prato di forma circolare affacciato sul lago, ma estraniato da tutto. E' un altro momento in cui il tema cessa, anche se è evocato per creare un'aspettativa, con discrezione, da un masso collocato lungo il perimetro su cui una scritta recita: "Questo menhir attende Obelix".
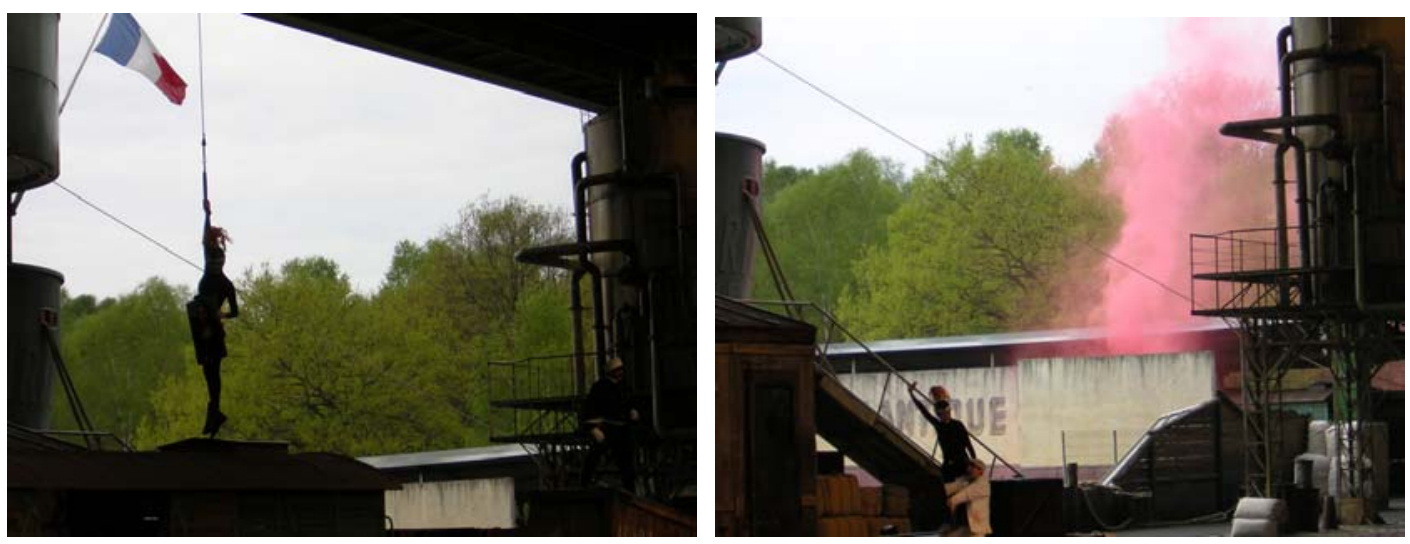

Fig. 5 Momenti dello spettacoli d'acrobati Main- basse sur La Joconde, che si svolge contro la foresta secolare di Ermenonville, fa dimenticare il tema principale del parco e annulla il confine fra il parco e il paesaggio al suo esterno.

C'è inoltre uno spazio particolare in cui i simboli si presentano ad un livello di astrazione, nella Foresta dei Druidi progettata da Isabelle Devin e Catherine Rannou'. Qui il disegno del giardino vuole assumere come propri elementi l'evocazione di effetti magici, i quali manifestano una relazione fra astuzia umana e poteri occulti della natura. Le finalità ludiche delle istallazioni sembrano un pretesto per ribadire che il giardino è sempre il luogo dell'interazione fra natura e arte, per cui il suo contenuto simbolico è di valore superiore, d'archetipo, rispetto a quello offerto dal tema contingente. Gli oggetti del giardino hanno forme evocative e allo stesso tempo astratte: il suolo è segnato da cerchi accostati l'uno all'altro ripetitivamente a creare una texture geometrica, sono tronchi interrati che richiamano la foresta; ci sono dei funghi su cui ci si può arrampicare, il loro colore è omogeneo, gli attacchi per salire sono distribuiti pittoricamente come punti variegati sulla superficie - non ci sono ne spore, ne qualsiasi altro dettaglio realistico che confermi si tratti di funghi; le altalene sono dei trepiedi di legno con un cavo che pende centrale ed un masso appeso al cavo su cui è possibile sedersi "a cavallina", di fianco si apre un sentiero che attraversa portali-pergola, avvolto da vapore acqueo fra cui si intravedono sospesi fra gli alberi delle figure di metallo e colori che no è possibile definire esattamente cosa siano.

\footnotetext{
${ }^{9}$ Progettiste anche del parco per bambini al Parc de La Villette di Parigi chiamato Il giardino delle dune.
} 

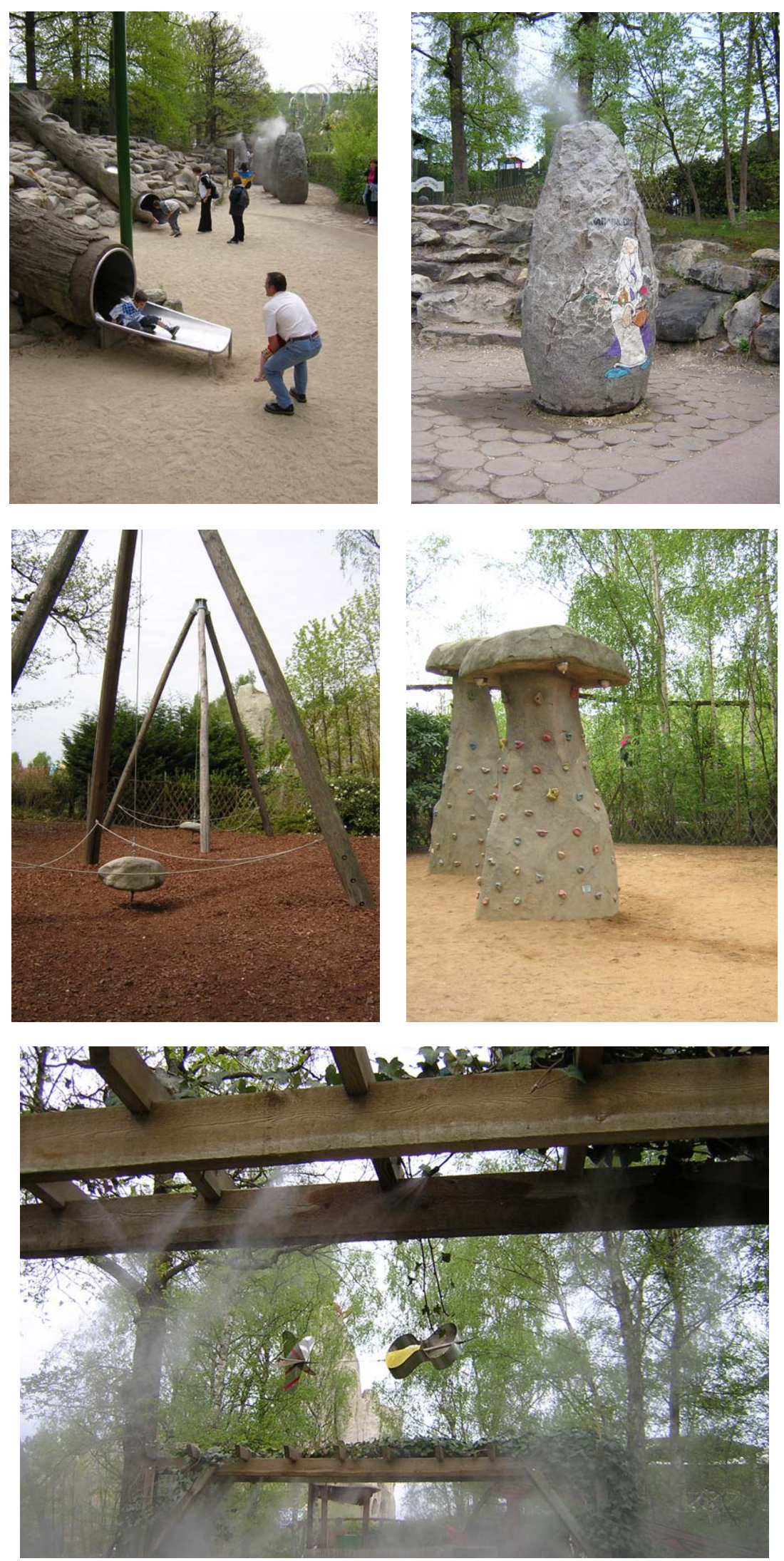

Fig. 6 Elementi evocativi ed allo stesso tempo astratti nella Foresta dei Druidi progettata da Isabelle Devin e Catherine Rannou; l'astrazione delle forme dichiara che il contenuto simbolico del giardino ha valore archetipico e trascende quello offerto dal tema contingente: il giardino si conferma quale luogo di interazione fra arte e natura. 


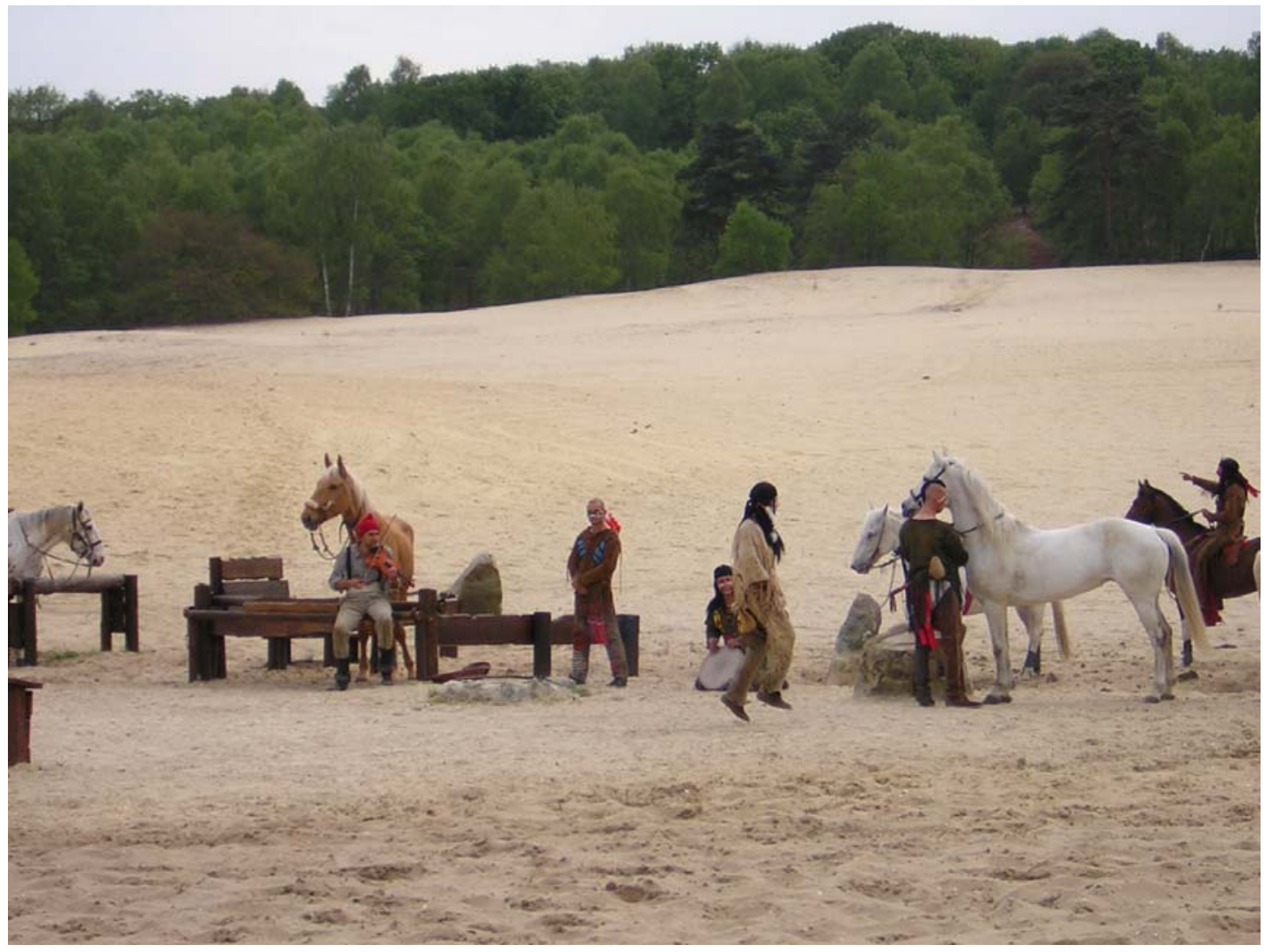

Fig. 7 paesaggio di Mer de Sable in un momento dello spettacolo Il figlio di Orso Nero. Sullo sfondo la foresta di Ermenonville.

Mer de Sable è un parco interno alla foresta di Ermenonville, a pochi chilometri da Parc Asterix, e vicino alla cittadina di Ermenonville a nord di Parigi, nota per essere stata la residenza del filosofo Roussoau e per la presenza del parco di Girarden, uno degli esempi più noti di pittoresco francese.

Mer de Sable non è immediatamente accessibile dall'autostrada A1, come al contrario è Parc Asterix, e per raggiungerlo si percorrono strade rurali fra campi incorniciati dalla foresta, poi si entra all'interno di questa e si gode della sua presenza intatta ai lati della via, in un paesaggio costante, seguendo le indicazioni, in piccolo, per Mer de Sable, il cui logo è un piccolo indiano d'America.

L'ingresso del parco è discreto, l'area di parcheggio è un prato di dimensioni limitate, ombreggiato dagli alberi della foresta che non sembra minimamente interrotta (il parco probabilmente si rivolge ad un pubblico locale, francese). Entrando si apre un viale, di fronte, costeggiato da alte siepi sempreverdi che inquadrano sullo sfondo una Tour Eiffel in miniatura. A destra è una ruota panoramica di piccole dimensioni e a sinistra una stazione ferroviaria. Non c'è nulla di straordinario, ma si intravede "il mare di sabbia" oltre la stazione.

"Il mare di sabbia" risale al XVIII secolo, tempo in cui le monache dell'Abbazia di Chaalis avevano disboscato la parte di foresta che era di loro proprietà, per venderla come terreno da pascolo, e hanno innescato inconsapevolmente il processo di desertificazione ed erosione che con il passare del tempo ha messo completamente a nudo la sabbia di cui è costituito il suolo dell'area. Nel 1963 " il mare di sabbia" diviene un parco d'attrazione gestito da una compagnia di acrobati-teatranti. 


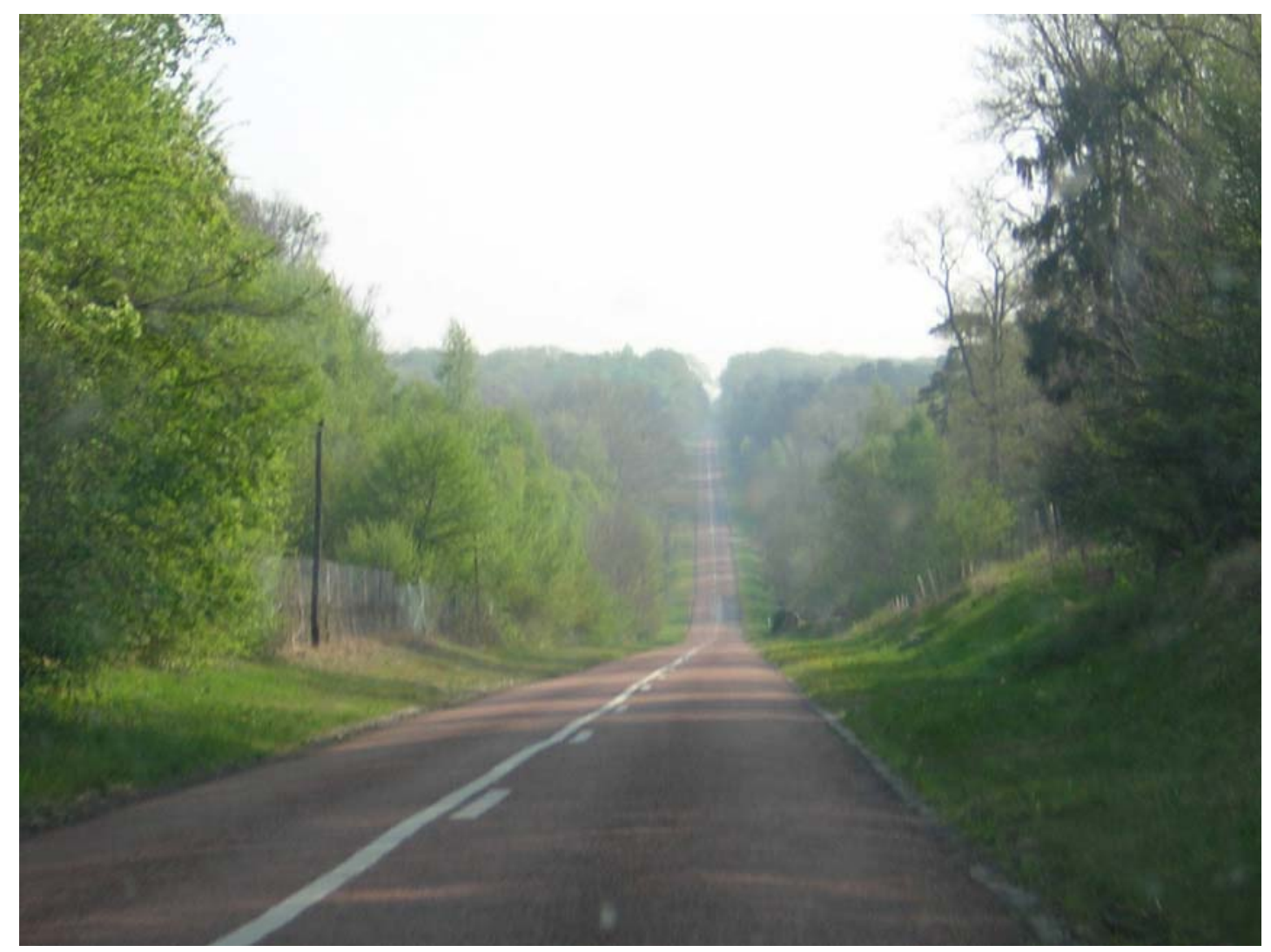

Fig. 8: strada nella Foresta di Ermenonville, che si percorre per giungere al parco Mer de Sable.

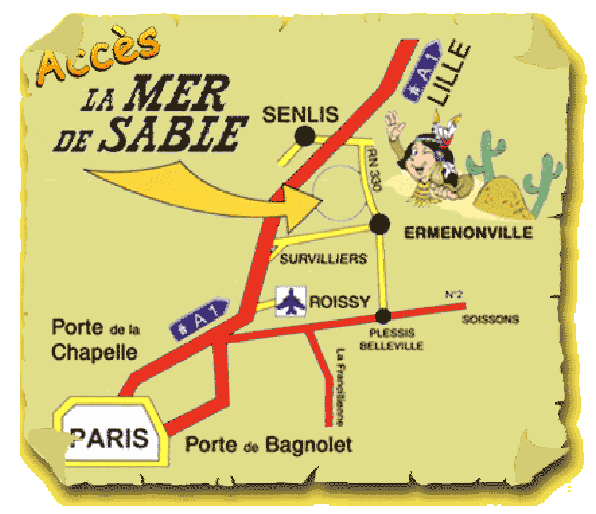

Fig. 9 Localizzazione di Mer de Sable, rappresentata nel sito web ufficiale del parco. Ancora prima di giungere al parco, la grafica delle indicazioni stradali discretamente invita ad un viaggio immaginario nel West d'America.

Il parco ha un tema prevalente, che è il mito del West America, dei suoi indiani e dei suoi Cow-boy. All'interno del parco sono leggibili tre aree molto distinte. Una è il vasto mare di sabbia: c'è un percorso principale che lo costeggia, vi si affaccia a tratti, in altri è chiuso da architetture-attrazioni il cui tema sfrutta come sfondo il deserto "naturale". Alla destra del percorso rimangono aree maggiormente eterogenee quanto a tema, meno studiate quanto a composizione spaziale, troppo dense di attrazioni diverse, che indeboliscono l'immagine complessiva del parco.

Il mare di sabbia al contrario resta quasi indisturbato: c'è solo una pista per motociclette nell'area adiacente la stazione ferroviaria, un trenino che nel primo tratto del proprio percorso corre a margine del deserto, poi lo attraversa; c'è una casa in legno stile far west 
con una cisterna, finta, per l'acqua, vicino alla casa. Il trenino consente un viaggio straniante, viaggio in cui risiede tutta la magia del parco Mer de Sable. La distesa di sabbia sembra immensa, anche se chiusa per tre lati dalla foresta. Guardandosi attorno non si vede nessun confine del parco e l'estensione stessa della foresta di Ermnonville caratterizza lo spazio, perché il suo colore cupo sullo sfondo ne dichiara la profondità.

Il binario percorre il limite fra foresta e sabbia, allontanando il più possibile dalla vista il resto del parco, che diviene come un miraggio fatto di poche architetture-simboli: ora la sagoma di un edificio orientale, ora la ruota panoramica, ora le pseudo-rocce del Train $d u$ Colorado. Il viaggio ha una sosta nell'area tematica Babagattaux, un po' strana: si entra dalla porta di un castello in rovina oltre la quale sono un giardino e un bar, fra gli alberi; un percorso si inoltra un po' avvicinando a scene di automi, animali animati e umanizzati che vivono nel bosco. Anche da questa area il confine del parco non è visibile perché il terreno a margine è modellato in rilevato.

L'aspetto interessante è la modalità di attribuzione del tema in rapporto al contesto specifico della foresta di Ermenonville e alla presenza della particolarità geologica del mare di sabbia. Quest'ultima sembra perfettamente intatta, nonostante sia all'interno di un parco d'attrazioni. E' accessibile solo salendo sul treno, a meno che il visitatore non decida di intraprendere una passeggiata a piedi (occorrerebbe troppo tempo, che verrebbe sottratto alle attrazioni del parco, nessuno lo fa). Il suo ruolo è di costituire l'immagine che catalizza lo spaesamento: mediante l'inserzioni di limitati elementi con funzione di simbolo - la casa del West, qualche roccia -è possibile fantasticare di essere in America al tempo degli indiani, pur riconoscendo di essere in quel punto unico della foresta di Ermenonville, che non viene stravolto, ma conservato e valorizzato nella sua capacità di stimolare un viaggio immaginario.

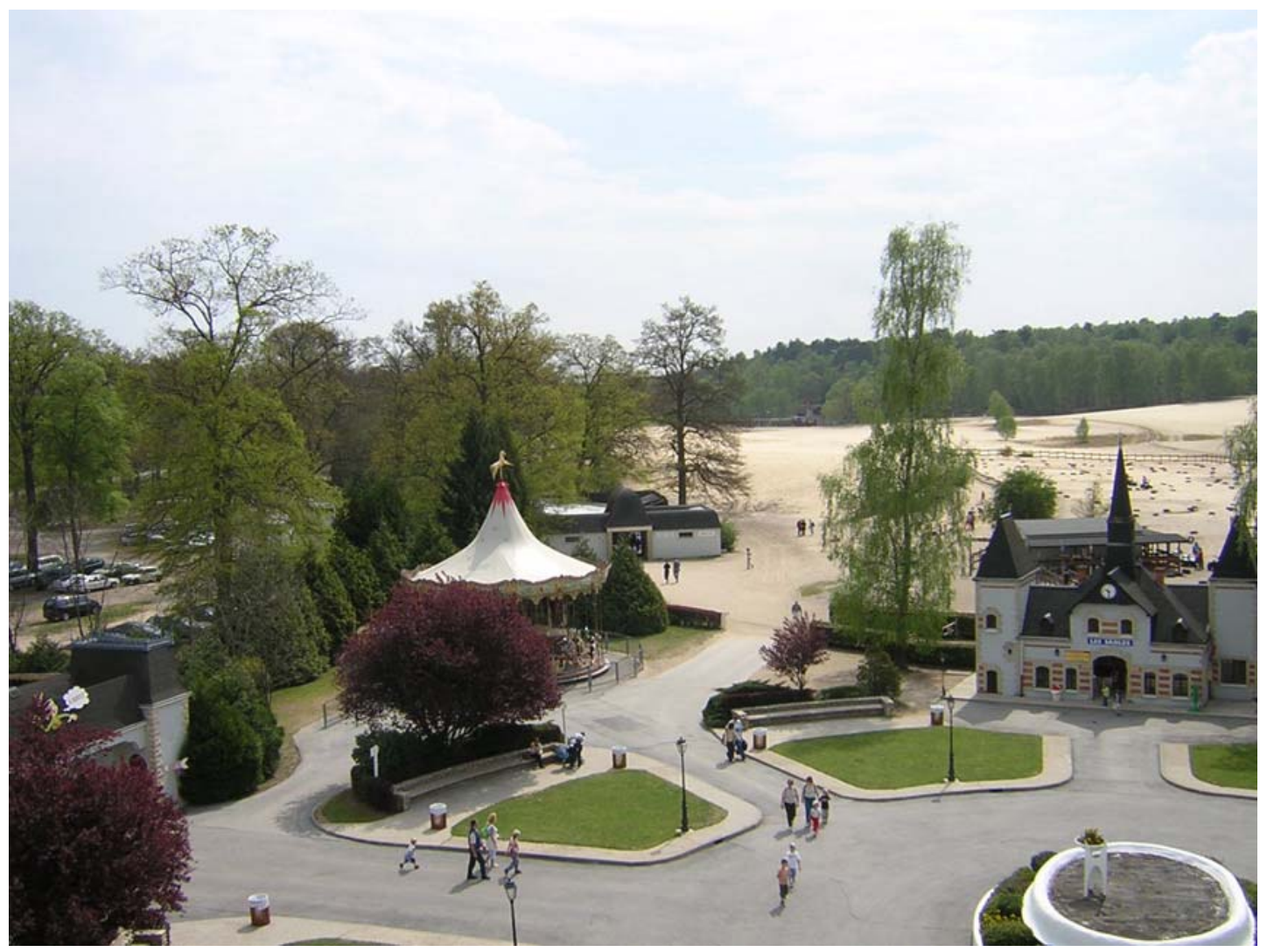

Fig. 10 Vista del deserto Mer de Sable dalla ruota panoramica del parco, nell'area di ingresso. 


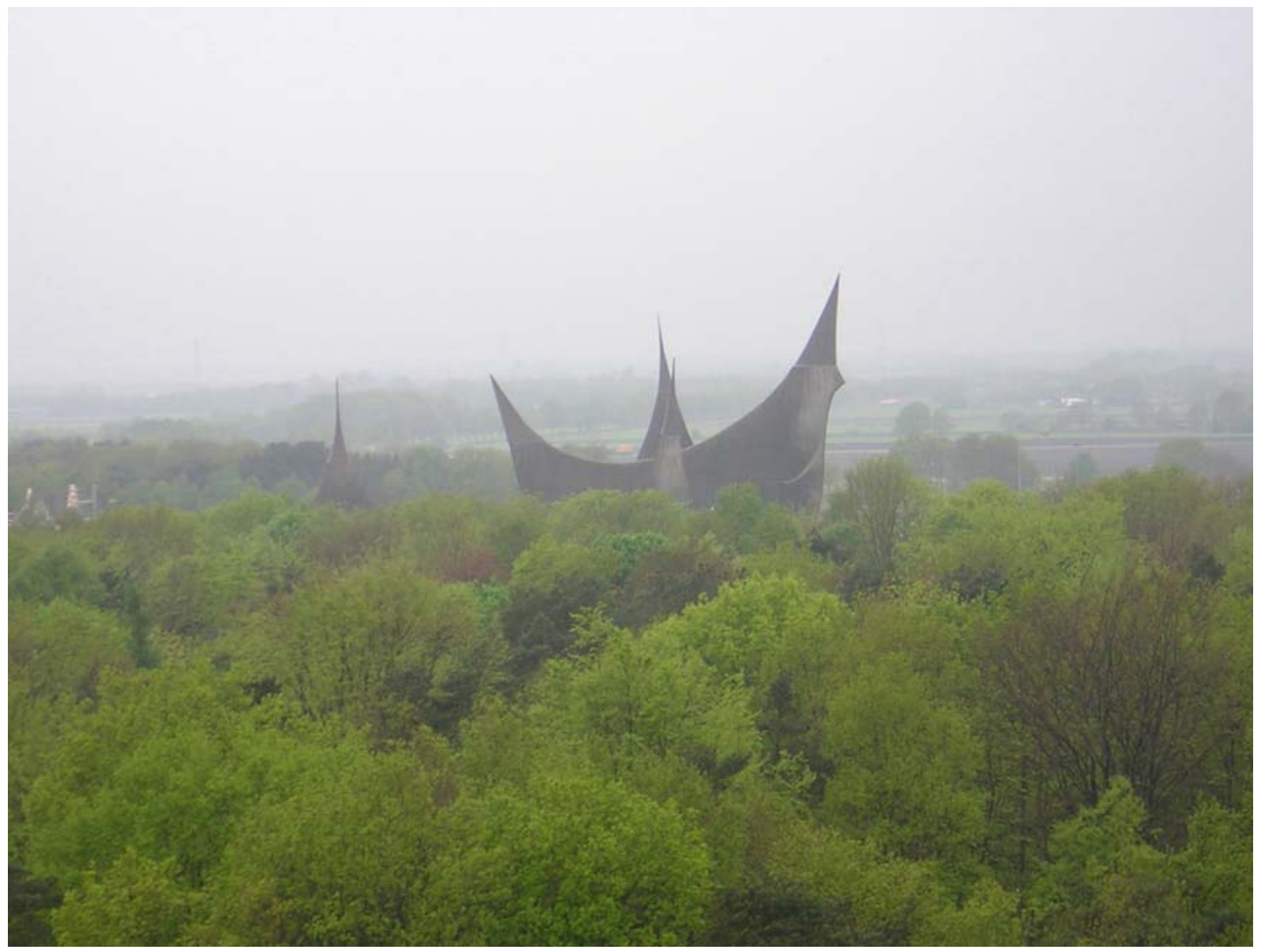

Fig. 11 Panorama dalla Pagoda Volante su Efteling e la campagna di Kaatsheuvel - come in alcuni momenti a Parc Asterix, non è percepibile un confine fra paesaggio e parco: i simboli del parco conferiscono nuovi significati al paesaggio, ne materializzano componenti inafferrabili quali i miti, le fiabe, percepiti collettivamente come l'anima del territorio.

Il parco di Efteling, in Olanda, ha il fascino di un parco a tema la cui storia comincia prima dell'inaugurazione "ufficiale" della serie dei parchi a tema, che si fa coincidere schematicamente e con un po' di ironia con l'apertura di Disneyland, nel 1955, in California. Efteling ha inizio nel 1950 con l'istituzione del Nature Park di Efteling, promossa dal sindaco della cittadina di Kaatsheuvel, nel luogo in cui negli anni '30 un cappellano del luogo aveva organizzato un centro sportivo-ricreativo per ragazzi. Nel 1952 il Nature Park ospita La foresta delle fiabe con scene animate di episodi tratti dalle più comuni fiabe nordiche: Biancaneve e i Sette Nani, La bella Addormentata nel Bosco, Hansel e Gretel ideate dallo scrittore Anton Pieck. E' considerato il primo parco tematico d'Europa.

Efteling risponde con una proposta unitaria a due esigenze fondamentali del momento ricreativo: il desiderio di stabilire un contatto con il mondo "naturale" - desiderio sentito con particolare urgenza nel territorio olandese, in cui la wilderness è quasi totalmente inesistente, e dove appare esserci è spesso stata ricreata artificialmente; la liberazione della fantasia, la sua traduzione in forme e presenze, reali o immaginarie, nel gioco, nell'esplorazione, nella visione contemplativa.

Anton Pieck, ideatore del parco, è scrittore e disegnatore di racconti, molto conosciuti in Olanda e rispondenti alle immagini che sembrano generate non da un singolo, ma dalla fantasia collettiva, con cui la popolazione si è figurata i misteri della natura, ha iconografato le proprie paure e i sogni. (Hertogenbosch, città del pittore Hieronymus Bosch - ca. 14501516 - interprete visionario dell'anima dei boschi, dista pochi chilometri da Kaatsheuvel). 
La foresta di Efteling ha un valore simbolico quanto il bosco del Sacro Bosco di Bomarzo (XVI sec.) - di cui si è detto a proposito del giardino manierista - in quanto genera il mito che vi viene rappresentato. Nel Villaggio dei Laaf ad Efteling la trasposizione dal disegno al paesaggio conserva la freschezza della visione fantastica pur nella fisicità dei propri materiali che ovviamente hanno una consistenza del tutto opposta rispetto a quella dei sogni. Per offrire al visitatore l'apparenza di essere in una città fantastica Walt Disney avvertì la necessità di fondare il parco assolutamente avulso dal territorio ospitante. I creatori di Efteling partirono dalla foresta esistente, elemento del parco naturale istituito nel 1950, sovrapponendovi un sistema di simboli che la specificano e ne fanno La foresta delle fiabe. Il paesaggio particolare di quel luogo costituisce il punto di partenza, non subisce annullamento ma piuttosto ulteriori assegnazioni di significato.

Ad Efteling le sponde lungo il lago del parco, sono ricoperte di bulbose che si estendono a tappeto fino alla linea in cui incomincia il bosco, con le proprie fioriture propongono un frammento di paesaggio olandese, reale e figurato. Il mondo della fiaba è il tema del parco : Efteling sembra dire che la fiaba è più vera - più affascinante- se racconta anche del paese in cui è stata immaginata e di cui è parte culturale. Il rapporto fra fiaba e paesaggio di Kaatsheuvel è simbiotico.

Nel 1987 viene istallata ad Efteling una nuova attrazione, la Pagoda Volante che grazie ad un braccio come quello di una gru, sembra totalmente sospesa in aria. La pagoda offre una vista panoramica su Efteling. Il contrasto fra le sue forme orientali ed il paesaggio tipicamente olandese, contro cui si staglia se la si guarda dalla gondola lungo il lago, denuncia che l'oggetto è strano -straniero- e ha compiuto in volo un lungo viaggio (da un regno di magia in quanto nessuna pagoda vola e dall'Oriente geografico, come dimostra lo stile della sua architettura).
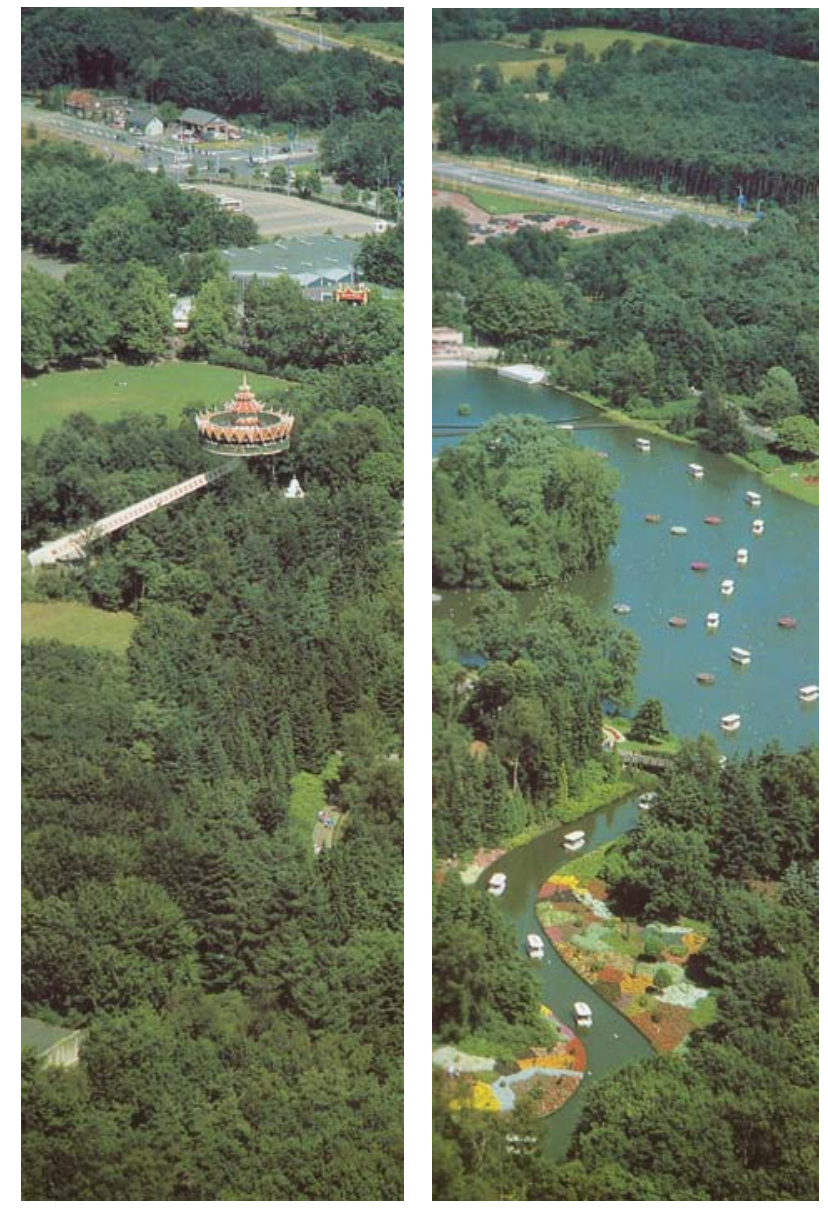

Fig. 12 la Pagoda Volante sul paesaggio di Efteling 
Nel tempo il parco subisce trasformazioni, con duttilità, in risposta al cambiamento dei modi di divertimento e ospita, oltre a punti di ristoro e commerciali, attrazioni tradizionali quali il Python, roller coaster, The Bob, pista artificiale da bob, il Pirana, fiume con rapide, costruite negli anni '80, e il Pegasus, roller coaster di legno, del 1991.

Tuttavia il carattere del parco non sembra essere stravolto, perché esse sono realizzate in posizione laterale, rispetto al nucleo, più magico, dove si svolge il racconto delle fiabe, e sono immerse nella vegetazione. Ma non si esaurisce la volontà che il parco sia il luogo della fiaba: nel 1986 viene realizzato il palazzo della fata Morgana affacciato su un lago che ne duplica l'immagine, con varietà di colori dal cielo e dall'acqua.

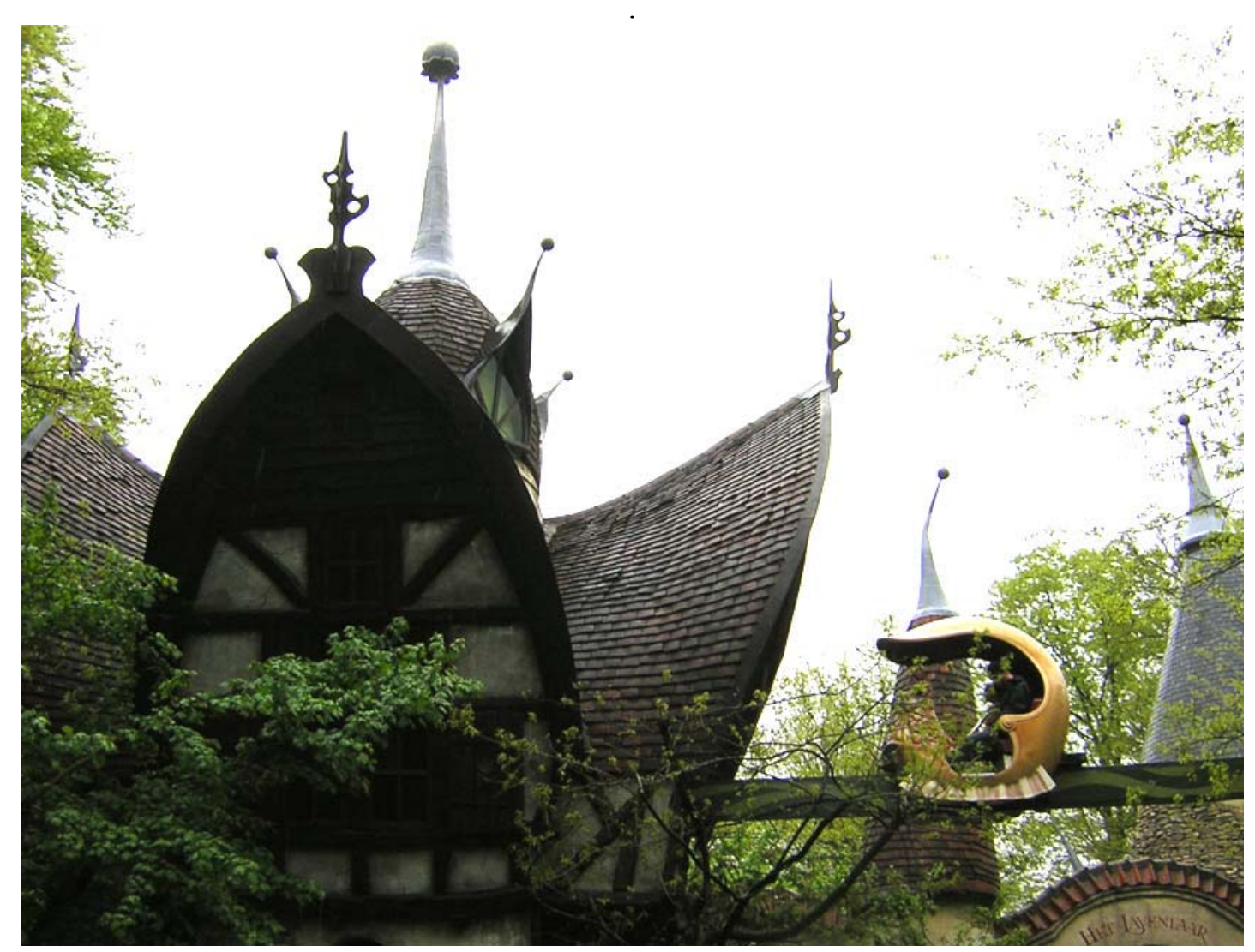

Fig. 13 Villaggio dei Laaf di Efteling.

Il Villaggio dei Laaf (1990), ideato da Ton van de Ven, creative director of landscape design di Efteling, è omogeneo con il paesaggio della foresta, come lo è la Foresta delle Fiabe: è fatto di case dalle forme ora morbide ora aguzze, fra le case sono giardini di erbe officinali e piccole piazze, sempre all'ombra della foresta e nella sua luce cupa; gli spazi vengono perlustrati dall'alto mediante una monorotaia che si insinua a nastro. E' animata dal medesimo spirito che abita l'architettura di ingresso al parco (1996) - della biglietteria e dei servizi - che si impone su tutto: sulla superficie a parcheggio all'esterno, quale enorme simbolo che accoglie in un benvenuto, ma soprattutto sulla foresta di Efteling: dalla foresta si intravede la copertura scurissima, affilarsi verso l'alto perdendo peso, come nel tentativo di sollevarsi in un volo (attorno ha il cielo aperto della pianura olandese). L'architettuta vista dalla foresta è solo sagoma e colore, ma nel minimalismo della sua immagine è in grado di evocare e di sintetizzare tutte le fiabe del parco, l'intero mondo figurativo allestito ad Efteling. Restituisce il mistero al paesaggio che ha ispirato quest'ultimo, mediante la 
materializzazione di componenti in realtà inesistenti -i miti - ma percepite come l'anima del territorio.

Deve essere la consapevolezza della complessità del paesaggio realizzato da Efteling a guidare la progettazione degli sviluppi del parco, nel tempo: è leggibile infatti la volontà di preservare delle aree prive di tematizzazione, il cui valore è quello di consentire di stare all'aperto, in un ambiente "naturale", anche attualmente nonostante la densità di attrazioni. Nei primi anni di apertura del parco la diversità delle attività possibili costituisce la caratteristica principale di Efteling, che offre campi sportivi, piscine, un lago per il canottaggio, l'area per i giochi, prati per il pic-nici, insieme alla Foresta della Fiabe. Oggi è un moderno parco tematico, ma la componente naturale è costantemente presente, come valore, tanto che a Efteling si può andare e tornare, nonostante l'ingresso sia a pagamento, anche semplicemente per un giro in gondola fra i bulbi del lago, o un "volo "sulla pagoda volante: l'unicità dell'esperienza non risiede esclusivamente nel brivido di una attrazione e nemmeno in un tema-marchio commerciale pervasivo, ma è, in maniera semplice, nella bellezza del luogo ${ }^{10}$.

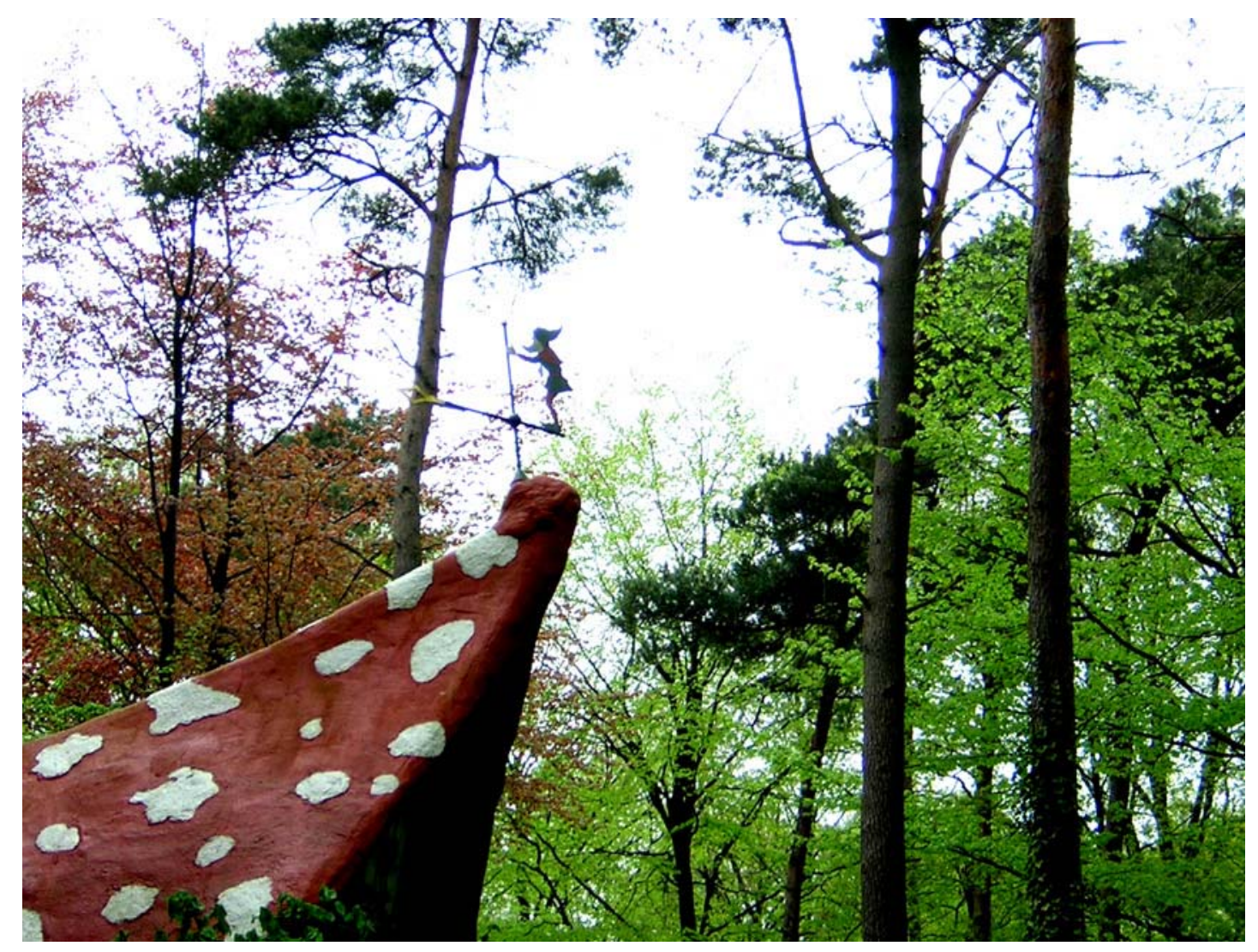

Fig. 4 Casetta a forma di fungo nella Foresta delle fiabe di Efteling.

\footnotetext{
${ }^{10} \mathrm{Nel} 1972$ Efteling vince il Pomme d'or, premio europeo per il turismo ricreativo, e nel 1992 viene dichiarato il parco dei divertimenti di più alta qualità nel mondo.
} 


\section{RIFERIMENTI BILIOGRAFICI}

ABruZZeSe Alberto, I parchi del divertimento, in "Paesaggio Urbano", n. 5, Parchi di divertimento, Maggioli Editore, Rimini 1993, pagg. 18-23.

BARRAU SILVIE, Le programme du parc, in BARZILAY MARIANNE, HAYWARD CATHERINE, LOMBARD-VALENTINO LUCETTE, L'invention du parc: parc de la Villette: Paris, concours in ternational 1982-1983, Graphite, Etablissement Public du Parc de la Villette 1984, pag. 238-239.

SANGUANINI BRUNO, Effetto benessere \& qualità della vita. A DisneyWorld, EuroDisney, Gardaland e altrove, in MINARDI EVERARDO, LUSETTI MARIALUISA (a cura di), I parchi del divertimento nella società del Loisir, Franco Angeli, Milano 1998, pagg. 86-127.

SAVElli ASTERIO, Parco separato e territorio aperto: problemi di strategia nelle aree turistiche, in MINARDI EVERARDO, LUSETTI MARIALUISA (a cura di), I parchi del divertimento nella società del Loisir, Franco Angeli, Milano 1998, pagg. 11-26.

TREBUCQ MATHILDE, La Nature du Futurscope, Les Editions du Futurscope, Vaschette.

\section{RIFERIMENTI BILIOGRAFICI per Parc Asterix}

Donin GianPIERO, Parchi, Biblioteca del Cenide, Cannitello (RC) 1999.

LANGloIs GILles-AnTOINE, Folies Tivolis et attractions. Les premiers parcs de loisirs parisiens, Délégation à l'action artistique de la ville de Paris, Paris 1991.

Tosetto Danilo, Parchi Ricreativi nel Mondo, Vol. 5 , Facto, Padova 1990, pagg. 36- 55.

Wylson Anthony \& PATRICIA, Theme Parks, Leisure Centres, Zoos and Aquaria, Longman, 1994, pagg. 27-30.

Siti Web:

www.parcasterix.fr

www.chantilly-tourisme.com/francais/loisirs_parcs_loisirs.php

\section{RIFERIMENTI BILIOGRAFICI PER Mer de Sable}

Siti Web

www.merdesable.fr/mer-de-sable

$\mathrm{http} / /$ tourisme.voila.fr/villes/stdenis/fra/sit/ermenonv/mersable/acc.htm

www.chantilly-tourisme.com/francais/loisirs_parcs_loisirs.php

www.aventuriers.com/fr/france/picardie/60_dec_lamerdesable/index.shtml

www.parisce.com/sable.htm

freeoise.free.fr/communes/lettree/ermenon/pglois.html

\section{RIFERIMENTI BIBLIOGRAFICI per Efteling}

CERVER Francisco ASENSIO, Landscape of recreation II: amusement parks, WatsonGuptill Pubns, Slipcase edition 1998, pagg. 86-97.

Tosetto Danilo, Parchi Ricreativi nel Mondo, Vol. 3 , Facto, Padova 1988, pagg. 28- 45. VANDEN DiePSTRATEN H., De Efteling kroniek van een Sprookje, De Efteling B.V. Kaatsheuvel 2002.

Wylson Anthony \& PAtricia, Theme Parks, Leisure Centres, Zoos and Aquaria, Longman, 1994, 30-33. 
Siti Web:

http://www.efteling.nl/docs.en/park/nav/index.html

\section{RIFERIMENTI ICONOGRAFICI}

Fig.1, 3-8, 10-11: fotografie di Enrica Dall'Ara.

Fig. 2: fotografia di Erik Cornelius, National Museum, Stockholm, in HuNT DiXON JOHN, The Picturesque Garden in Europe, Thames and Hudson, London 2002, pag. 125.

Fig. 9: www.merdesable.fr/mer-de-sable/

Fig. 12: CERVER FRANCISCO ASENSIO, Landscape of recreation II: amusement parks, Watson-Guptill Pubns, Slipcase edition 1998, pagg. 90-91. 


\section{- ANALISI SIMBOLO-SPAZIALE [vedi TAVV. I_1 e TAVV. II_2]}

I parchi Parc Asterix, Plailly, France (1989-2002), Mer de Sable, Ermenonville, France (1962-2002) ed Efteling, Kaatsheuvel, Netherlands (1952-2002), vengono sottoposti ad un'analisi che intende indagare il rapporto esistente fra il parco e il suo contesto ed il rapporto esistente, all'interno del parco, fra il tema, gli elementi materiali (vegetazione, architetture, percorsi) e la loro composizione spaziale. La finalità è quella di scomporre l'immagine complessiva del parco in elementi chiari e riuscire a scoprire in quali "punti" la progettazione è posta davanti a scelte che comportano esiti diversi, quanto a qualità del paesaggio e quanto alla possibilità di dare spazio al divertimento all'interno del parco: si vuole capire se ed in che modo il parco evita di essere un' "utopia noiosa".

Il metodo di rappresentazione e di analisi dei parchi scelti prende spunto dal lavoro svolto da Robert Venturi, Denise Scott Brown, Stephen Izenuor, per il paesaggio della Strip di Las Vegas, proposto dai suoi autori nelle edizioni successive di Learning from Las Vegas (1968 - 1976-1985). Il metodo d'analisi della Strip segue l'idea che essa sia costituita di simboli, piuttosto che di spazi tradizionalmente intesi -definiti da volumi architettonici- e che questa caratteristica sia dovuta al fatto che la Strip è costruita su misura per un utente che viaggia in automobile e deve essere sollecitato a fermarsi mediante segnali percepibili ad alta velocità. Quello che interessa al gruppo di Robert Venturi è rappresentare la Strip visualizzando il rapporto fra la scala dei simboli, la velocità di percezione e lo spazio fisico, anche comparando la Strip con altre situazioni spaziali (Fig. 1).

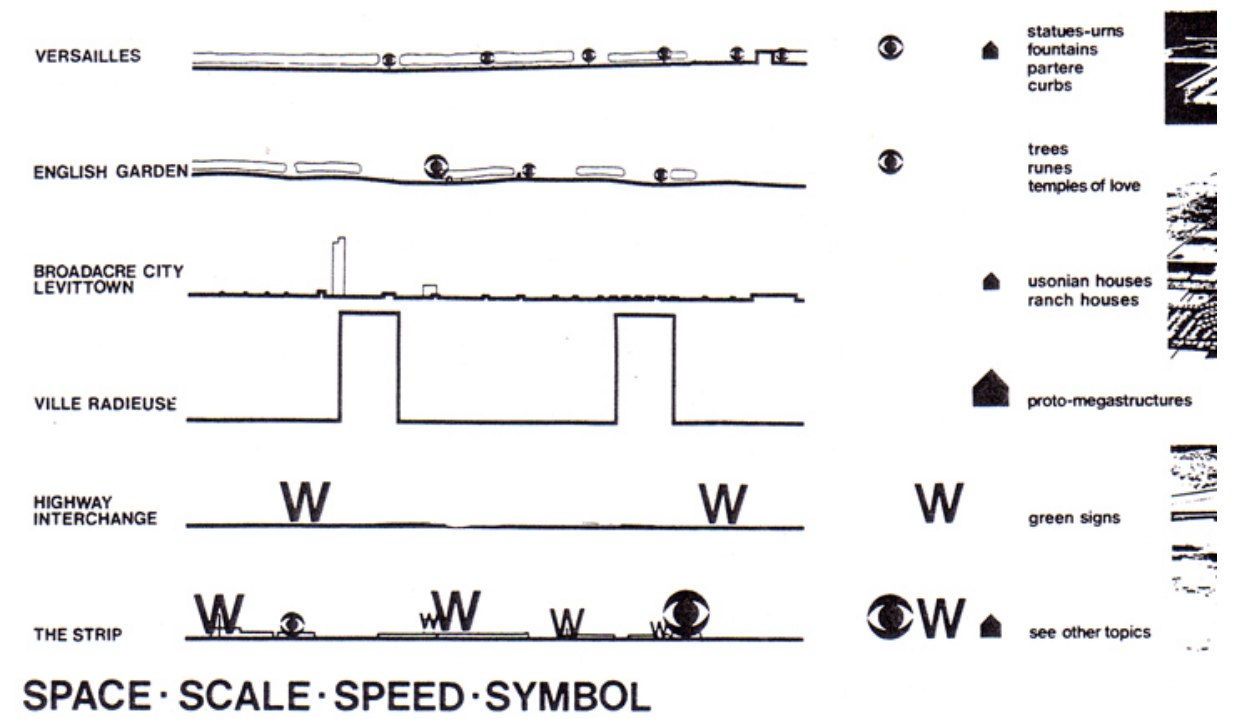

Fig. 1 esempio di tecnica rappresentativa da Robert Venturi: Analisi comparativa di spazi "vasti": il rapporto spazio/"scala" e il grado di simbolizzazione, dato da messaggio, architettura, ed elementi. E' interessante notare come Robert Venturi proponga una lettura dei giardini di Versailles e dei giardini inglesi, come luoghi strutturati dai simboli.

Il parco tematico condivide con la Strip un elevato grado di simbolizzazione e la comunicatività dei suoi elementi, componenti che appaiono prevalenti su ogni altro aspetto. Sia il parco tematico sia la Strip sono costituiti di "architettura di allusione" cosa che rende valida per entrambi la constatazione: 
"La tecniche di rappresentazione acquisite dall'architettura e dall'urbanistica tradizionali ostacolano la nostra comprensione di Las Vegas. (...) Le tradizionali tecniche di rappresentazione architettonica sono adatte per grandi, vasti oggetti isolati nello spazio, come gli edifici, ma non per oggetti "sottili" e "intensi" come le insegne". 1

Robert Venturi è consapevole di questa assonanza fra Las Vegas e i parchi a tema (la Strip potrebbe effettivamente essere interpretata come un esempio assoluto di parco tematico):

"Per l'architetto e l'urbanista, i confronti tra Las Vegas ed altri esempi di "luoghi di piacere" del mondo - come Marienbad, l'Alhambra, Xanadu e Disneyland, ad esempio - suggeriscono che ciò che è essenziale all'architettura dei luoghi-di-piacere sono la leggerezza, la qualità di essere un'oasi in un contesto forse ostile, un'intensificato simbolismo e la capacità di assorbire il visitatore per fargli assumere un nuovo ruolo: per tre giorni ci si può immaginare un centurione al Caesar Palace, un ranger al Frontier o un personaggio del jet-set al Riviera [casinò di Las Vegas], piuttosto che un commesso viaggiatore di Des Moines, Iowa, o un architetto di Haddonfield, New Jersey."

Ma esiste una sostanziale differenza fra un parco a tema e la Strip di Las Vegas, la modalità di percezione: il fruitore della Strip di Las Vegas era', negli anni '60-' 70 durante i quali viene approfondita l'analisi di Robert Venturi, l'automobilista che la percorreva ad elevata velocità e sostava eventualmente in un parcheggio, da cui accedeva poi ai "servizi" . Al contrario nel parco a tema il visitatore si muove a piedi ed è un esploratore che si insinua in ogni anfratto - restando sempre all'interno di percorsi obbligati. Egli cambia spesso mezzo di spostamento e spesso rimane fermo in coda in attesa di accedere alle attrazioni più affollate. Per cui il rapporto fra scala dei simboli e velocità di percezione è completamente diverso.

Inoltre lo spazio del parco a tema è molto articolato, non è una semplice "striscia", quindi la percezione non avviene solo per sequenze di immagini che scorrono lungo un asse: nel descriverlo è necessario rilevare cosa effettivamente sia visibile punto per punto -sempre evidenziando la velocità di percezione.

Per questo ai tematismi di Robert Venturi - architetture simboliche, insegne, immagini (grado di simbolizzazione) e rapporto spazio/scala - la seguente ricerca ne affianca di ulteriori: coni visuali, accelerazioni dovute a rides ad elevata velocità (tipo roller coaster), divagazioni ovvero presenza di attrazioni indoor, che si descrivono dettagliatamente in seguito.

Si sceglie di effettuare l'analisi mediante lo studio di sezioni significative per comprendere come lo spazio del parco sia scandito, indipendentemente dall'itinerario del visitatore - anche in considerazione del fatto che i percorsi sono obbligati ma non esiste un ordine, e neppure una direzione stabilita di percorrenza, anzi il visitatore è libero di fruire delle varie aree come crede. La rappresentazione in sezione è inoltre una rappresentazione "comoda" per evidenziare il ritmo spaziale fra i vari elementi, ovvero il rapporto di scala fra i simboli e lo spazio; la sezione è "comoda" anche perché si presta facilmente ad essere interpretata in forme diagrammatiche, a loro volta utili per l'individuazione di criteri progettuali.

Vengono sottoposte all'analisi semantica anche parchi ritenuti esempi significativi delle 5 matrici descritte nella PARTE I della ricerca: Pratolino, Firenze (XVI secolo) per il giardino manierista; Vauxhall, London (1660-1860) per i Pleasure gardens, folies, jardins spectacles; Il Midway Pleasance della Chiacago Columbian Exposition (1893) per le esposizioni universali; Santa Cruz Boardwalk, Santa Cruz, California U.S.A. (1908) per gli amusement parks americani e Disneyland, California, U.S.A. (1955).

In questo modo è possibile operare un confronto fra questi ed i parchi europei scelti.

\footnotetext{
${ }^{1}$ Robert Venturi , Denise Scott Brown, StePhen Izenuor, Imparando da Las Vegas. Il simbolismo dimenticato della forma architettonica, CLUVA editrice, 1985, pag. 29.

${ }^{2}$ Attualmente vasti marciapiedi e piazza sostituiscono i parcheggi antistanti i casinò e gli alberghi, invitando i visitatori al passeggio a piedi, cosa che modifica radicalmente la percezione di immagini e insegne lungo la Strip.
} 
L'analisi è così articolata:

Sezioni significative (comprensive del contesto prossimo al parco) con evidenziati:

- ELEMENTI:

PERCORSI (TRASVERSALI E LONGITUDINALI) E VISUALI

\section{ARCHITETTURA}

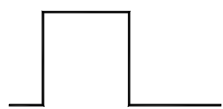

VEGETAZIONE :

FORESTE, MACCHIE (non tematiche)

ALBERI E ARBUSTI ISOLATI O COMPOSTI IN GIARDINO, AIUOLE

- SIMBOLI:

ARCHITTETTURA(*): edifici con valore di simbolo, tematizzati

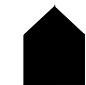

IMMAGINI(*): rappresentazioni, oggetti, figure tematizzati.

Es.: grande statua di Asterix all'ingresso del Parc Asterix in Francia

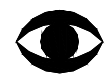

SCRITTE $(*)$ : insegne, pannelli informativi

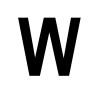

VEGETAZIONE TEMATICA: sempre se con valore di simbolo, finalizzata all'ambientazione tematica

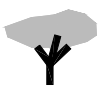


DIVAGAZIONI (e miniaturizzazione) :

Sono architetture tematiche - attrazioni indoor (al chiuso) - che rappresentano un tema dominante (ad esempio It's a small world a Disneyland), al cui interno il racconto si dipana in spazi di dimensioni contenute, dilatati spesso illusionisticamente mediante un accurato uso della luce e riduzione della scala degli oggetti. Al loro interno è un accumulo di simboli che realizza un'ambientazione. Questa può essere in coerenza con il tema generale dell'area tematica in cui l'architettura è collocata oppure rappresentare un'exfrasis: il visitatore viene immesso in un mondo descritto da un'immagine dettagliatissima, ricca e precisa come una miniatura.

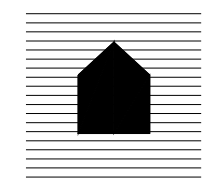

ACCELERAZIONI (nella visioni):

Sono operate da rides ad alta velocita', quali soprattutto i roller coaster (montagne russe). Esse creano all'improvviso un cambiamento di modalità di percezione degli spazi del parco - sono pertanto attrazioni che garantiscono il di-vertimento. Realizzano allo stesso tempo una tregua dal tema in quanto la velocità a cui si vedono le cose non consente di distinguere altro che scie di colori: l'ambientazione dell'attrazione è percepibile solo nei tratti iniziali in cui ci si muove lentamente.

C'è una descrizione molto efficace della particolare esperienza offerta dai roller coaster, dovuta al mutamento di velocità e quindi di percezione della scenografia del parco in relazione al contesto: Siegfried Kracauern (1889-1966), architetto e storico dell'architettura, racconta un viaggio sulle montagne russe del luna park di Halensee a Berlino che hanno come scenografia New York. ${ }^{3}$

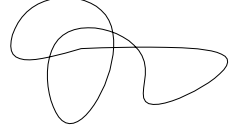

\section{SPETTACOLI}

Si è visto nella presentazione della scheda dei parchi del divertimento europei come i parchi europei spesso abbiano fra le attrazione proposte spettacoli di acrobati, di teatro, di musica e come in questo si differenzino dal modello Disneyland e conservino l'eredità dei pleasure gardens del Settecento e dell'Ottocento.

Gli spettacoli simbolizzano lo spazio che li accoglie limitatamente al tempo in cui avvengono: sembrano una componente interessante per comprendere il rapporto fra spazio e tematizzazione.

\footnotetext{
3 Siegfried Krakauer, Montagne russe-l'illusione metropolitana, in "Casabella" , n. 673/674, U.S.A., Architettura come spettacolo, Electa, Milano dicembre 1999- gennaio 2000, pag. 9. pubblicato per la prima volta col titolo Berg-und Talbahn il 14 Luglio 1928, in "Frankfurter Zeitung".
} 
Si evidenziano inoltre:

- RAPPORTI SPAZIALI E DI SCALA RISPETTO AI SIMBOLI (*)

Vengono espressi dalle dimensioni in cui $i$ simboli compaiono nella rappresentazione.

\section{- VELOCITA’ DI FRUIZIONE (*)}

- PRESENZA DI RELAZIONE FRA SIMBOLO E TERRITORIO ESTERNO AL PARCO.

ovvero si individuano gli elementi che evocano il genius loci, che rendono il parco un luogo radicato nel contesto in cui si colloca. la presenza di simboli che instaurano una relazione con il contesto rivela che il parco non è replicabile in altri luoghi, perché assume significato dal territorio in cui è costruito, espressamente.

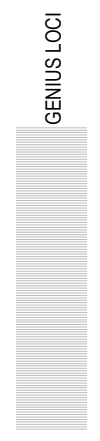

\section{- SPAZI IN CUI IL TEMA E' PRESENTE E SPAZI IN CUI IL TEMA E' ASSENTE e DIVERSITA' O COERENZA DI TEMI}

l'individuazione di questo rapporto fra presenza e assenza del tema viene effettuata mediante la redazione di un diagramma parallelo alla sezione; nel diagramma in cui vengono evidenziati in nero gli spazi non tematizzati ed in grigio quelli tematizzati. Quando i temi sono fra loro diversi e non coerenti vengono utilizzati grigi di tonalità differenti.

\section{presenza del tema}

assenza del tema

\section{IIIIHIII}

assenza del tema ma influenza del tema del parco sul modo di percepire lo spazio (ad es.: esistono luoghi nel giardino manierista di Bomarzo in cui sono assenti sculture e apparati iconografici, in cui il bosco non presenta simboli; esso stesso però assume un carattere simbolico perché richiama le selve incantate della letteratura cavalleresca, in virtù della narrazione che si svolge nel parco. Il tema è sia assente sia presente, in modo ambiguo).

(*): i tematisti segnalati con asterisco sono assunti da Robert Venturi, gli altri sono stati messi a punto in occasione della presente ricerca. 
La sezione viene descritta anche fotograficamente, per offrire un'immagine di supporto alla lettura del diagramma in relazione al paesaggio reale, e per visualizzare l'aspetto degli elementi-simboli.

Le sezioni elaborate sono dedotte dalle mappe informative dei parchi e da planimetrie pubblicate, non aggiornate e non corrispondenti allo stato attuale (nessun ente parco si è reso disponibile a fornire elaborati progettuali dettagliati). Mediante visite e rilievi fotografici nei parchi si è tentato di supplire alla carenza elaborati grafici esaustivi. 


\section{- INTERPRETAZIONE E IMPLICAZIONI PROGETTUALI}

\section{DIREZIONI PER LA PROGETTAZIONE DI UN PARCO DEL DIVERTIMENTO A TEMA}

[vedi TAV. II_3 in allegato].

Dalla PARTE I inerente le matrici e i processi di attribuzione del tema si arriva alla definizione di concetti che offrono primissime indicazioni su quali siano gli elementi cardine da tenere in considerazione nella fase di progettazione, capaci di direzionare verso la costruzione effettivamente di un parco -del divertimento e tematico- e non verso un'utopia noiosa (si fa sempre riferimento alla questione cristallizzata da Isabelle Auricoste: "Parchi o utopie noiose?"1 che guida l'intero percorso della presente ricerca).

Tali elementi cardine risultano essere:

\section{1) presenza (e non negazione del) CONTESTO}

Si è visto lungo il percorso di indagine sulle matrici culturali dei parchi del divertimento che il contesto viene ritenuto spesso accessorio, se non presenza da negare. In particolare sono le matrici amusement park americani e Disneyland che risultano indifferenti nei confronti del territorio in cui si insediano, fatta eccezione per gli aspetti che ineriscono a problemi di carattere logistico.

Anche il manuale Tourism and Recreation Development, The Architectural Press LTD, London 1977, relativo alla pianificazione di complessi turistici e ricreativi caratterizza il parco tematico (in relazione ad altri generi di parco maggiormente dipendenti dai valori paesaggistici e ambientali del sito) come un parco che non necessita di un contesto particolare se non in risposta a questioni di sostenibilità degli investimenti prima e di marketing poi. Si legge al suo interno riguardo ai requirements del parco tematico, a seguito del punto in cui si sottolinea l'importanza di una sua localizzazione vicina a intersezioni di arterie autostradali e a un "large excursion market":

"Site locations. Generally these are not critical. Large areas of land at low prices are essential, to offset the hight costs of development. Appreciation of land values around the site (by a factopr of 50 in some cases) for hotel, motel, and residentail development is an important benefit. The site will usuallly undergo major remodelling including excavations for lakes, ponds and waterways. Natural resources such as water, forests and rock outcrops, provide a basis for the layaut and landscaping.",2

L'indicazione contiene l'idea del valore dell'intorno del parco, ma il contesto appare come una cava da cui prelevare a basso costo i materiali per la costruzione. Non viene prospettata l'ipotesi di una sinergia in termini di collaborazione per la trasformazione del sito in un nuovo paesaggio, più complesso.

Il Disney Studio Park di recentissima apertura, adiacente a Disneyland Paris, per portare un esempio, ha su un lato, come margine-sfondo, un lembo di bosco che sicuramente esisteva già prima del parco, porzione naturale ad intervallo di una distesa di campi a seminativo come si incontrano costantemente nell'ampio territorio rurale, indisturbato dall'urbanizzazione, che caratterizza il paesaggio francese dell'interno. A parte lo sfruttare l'opportunità di avere una quantità di verde pronta all'uso e una barriera verso l'esterno

\footnotetext{
${ }^{1}$ Provocazione espressa in IsABelle Auricoste , Parchi o utopie mortali?, “ Ottagono” n. 99, Intrattenimento, 1991, pagg. 16-30, e in ISABELle AuRICOSTE, I parchi ricreativi in Europa: il divertimento e l'altrove, in Monique Mosser, Georges Teyssot, L'Architettura dei giardini d'Occidente- Dal Rinascimento al Novecento, Electa, Milano 1990, pagg. 479-490.

2 Manuel Baud-Bovy, Fred Lawson, Tourism and Recreation Development, The Architectural Press LTD, London 1977, pag. 107.
} 
sufficientemente alta per ribadire che il parco è un'enclosure, la Disney sembra non chiedere altro da questo bosco a contatto con il suo complesso ricreativo. A scala più ampia, se si osserva la carta degli intorni di Parigi, un cerchio perfetto definisce il centro dell'Eurodisney Resort: non è un diagramma, ma la forma reale del territorio destinato allo sviluppo ad opera della Disney Company, forma determinata fisicamente da una strada, che si pone quale bastione (i suoi lati sono infatti arginati dalla modellazione in rilevato del terreno e non si vede da dentro l'esterno) di una città ideale e per questo astratta - dal territorio, dal paesaggio fuori Parigi.

Non è quindi un'operazione banale focalizzare l'attenzione nuovamente sul rapporto con il territorio specifico in cui il parco si colloca.

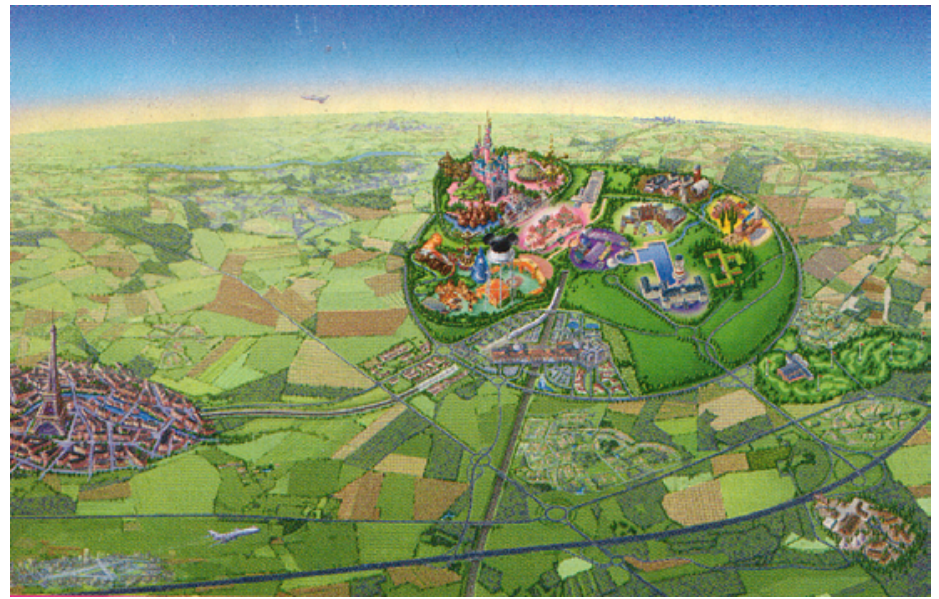

Fig. 1 Rappresentazione del Disneyland Resort- Paris in rapporto a Parigi ed al suo territorio circostante, che accompagna le spiegazioni di come raggiungere il Disneyland Resort nella brochure ufficiale. Ovviamente la scala di rappresentazione è falsata e il fine è di esprimere una gerarchia: Parigi è vicina (questo è vero), una volta raggiunto il Disneyland Resort, completo di tutto, è possibile anche, in giornata, fare una visita a Parigi. La prospettiva è ribaltata.

Nelle considerazioni sui risultati della Scheda Analitica (nel secondo capitolo della PARTE II) si è detto come il territorio extraurbano sia un territorio "privilegiato" per la locazione dei parchi del divertimento. Si è detto anche come sia leggibile nelle realizzazioni più recenti un nuovo contenuto utopico, un'accentuata forma di idealismo denunciata in primo luogo dai temi stessi che caratterizzano il parco (si sono portati l'esempio di La Villette e Futurscope in Francia, rispettivamente parco urbano e parco extraurbano), cui si accompagna una politica di apertura dei confini del parco. Si tratta di capire in termini di costruzione del paesaggio cosa comporti questa apertura, che appare - considerando i discorsi di Asterio Savelli $^{3}$ e di Bruno Sanguanini ${ }^{4}$ - sussistere più ad un livello funzionale e di immagine pubblicitaria che sul piano dell'attenzione ecologica reale e di un pensiero estetico sul paesaggio. Cosa implica pensare il parco dei divertimenti tematico come parte specializzata del proprio intorno (addirittura come catalizzatore delle sue trasformazioni )?

Forse la suggestione più bella, sul rapporto possibile fra spazio del divertimento (o magico, o di sogno) e contesto in cui vige invece il principio di realtà è offerta dalla descrizione del castello di Atlante nell'Orlando Furioso dell'Ariosto. Maurizio Calvesi individua tutti i legami esistenti fra il Sacro Bosco di Bomarzo (XVI sec.) ed il racconto del poema cavalleresco. Ma il paesaggio descritto dall'Ariosto nell'intorno del palazzo di Atlante è

\footnotetext{
${ }^{3}$ In Asterio SAVElli, Parco separato e territorio aperto: problemi di strategia nelle aree turistiche, in EVERARDo MinARDi, MARIALUISA LuSETti (a cura di), I parchi del divertimento nella società del Loisir, Franco Angeli, Milano 1998, pag. 20.

${ }^{4}$ In BRuno SANGUANINI, Effetto benessere \& qualità della vita. A DisneyWorld, EuroDisney, Gardaland e altrove, in EVERARDO MinARDI, MARIALUISA LuSETTI (a cura di), I parchi del divertimento nella società del Loisir, Franco Angeli, Milano 1998, pagg. 86-127.
} 
denso di idee anche per la progettazione dei parchi del divertimento contemporanei. Il castello del mago che mediante incantesimo intrattiene in "dolce pena" -nei piaceri ed in prigionia- i suoi ospiti è una "casa silvestra" che si staglia in "un gran prato" aperto fra "profonde selve". Il muro che delimita il territorio del mago Atlante è di vetro. La trasparenza del materiale è coerente con l'immaterialità dell'incantesimo che trasforma il luogo in un regno straordinario in cui sono soddisfatti tutti i desideri. Il muro è praticamente invisibile come l'incantesimo. Non avviene nessuna alterazione fisica del contesto, apparentemente non c'è soluzione di continuità fra il paesaggio silvestre e il paesaggio del palazzo di Atlante. Questo potenzia l'effetto di magia. Se nella progettazione del parco a tema si parte dall'immagine proposta, una prima soluzione può essere di lasciare che il contesto" prosegua" all'interno del parco. L'impressione di ingresso ad uno spazio altro varcando la soglia del parco è perseguibile mediante la semplice attribuzione di simboli sul contesto. Questo è il principio seguito dai realizzatori del parco di Efteling, in Olanda, il cui nucleo d'attrazione originario era dato dalla collocazione, all'interno della foresta di un Nature Park, istituito nell'area nel 1950, della Foresta delle Fiabe, ambientazioni tratte dalle fiabe nordiche più famose. Anche Mer de Sable ha confini «trasparenti » come il castello d'Atlante, fatta accezione per l'area d'ingresso : una volta all'interno non sono visibili i limiti perchè lo sguardo incontra prima, dalla radura di sabbia al centro, la cortina profonda di alberi della foresta di Ermenonville, che nasconde la rete di recinzione al suo interno.

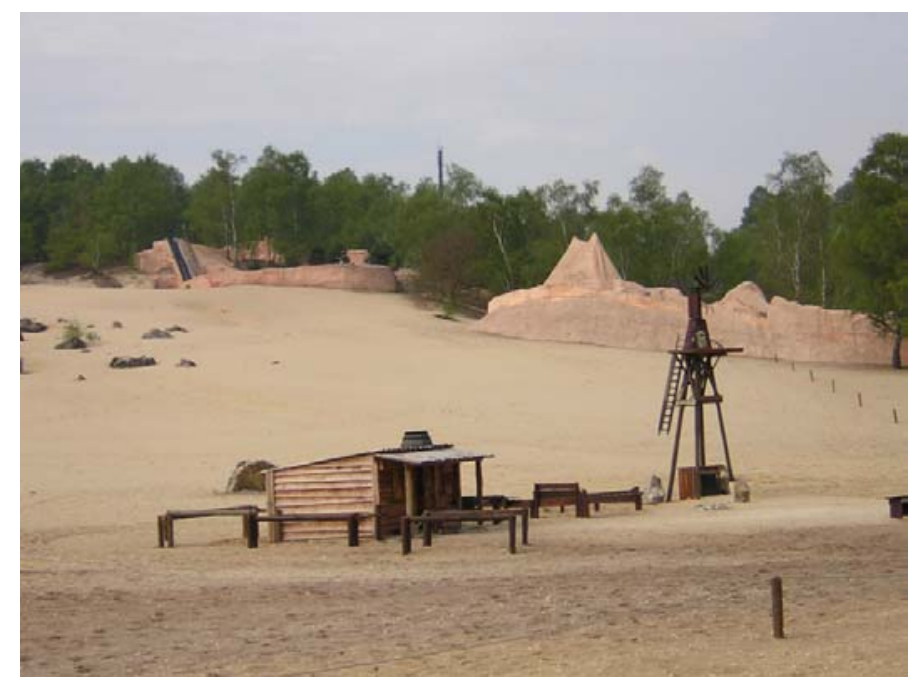

Fig. 2 La Mer de Sable (Ermenonville, France) - i confini non sono visibili e la Foresta di Ermenonville è presente all'interno del parco: i simboli istallati la trasfigurano in un paesaggio del west d'America, astraendo dal contesto e allo stesso tempo ospitandolo.

In questo modo è eluso il rischio di costruire un parco "replicabile" in altri contesti, in quanto il contesto è parte del parco stesso. Il di-vertimento è dato dai nuovi significati che $\mathrm{i}$ simboli conferiscono al contesto, consentendo di estraniarsi da esso. Il fascino di Mer de Sable risiede nel potere di simboli giustapposti al paesaggio delle dune della foresta di Ermenonville per cui all'improvviso capita di trovarsi nel deserto del west americano. La presenza del contesto può avvenire semplicemente garantendo punti di visibilità dell'esterno del parco in modo tale da istaurare un legame territoriale. (Si fa cenno a quanto sia strategico progettare questa possibilità all'interno dei parchi dei divertimenti più densi di temi e oggetti comunicativi, nell'analizzare gli esiti degli amusement park americani).

\footnotetext{
${ }^{5}$ Orlando Furioso, XII, 3-34.
} 


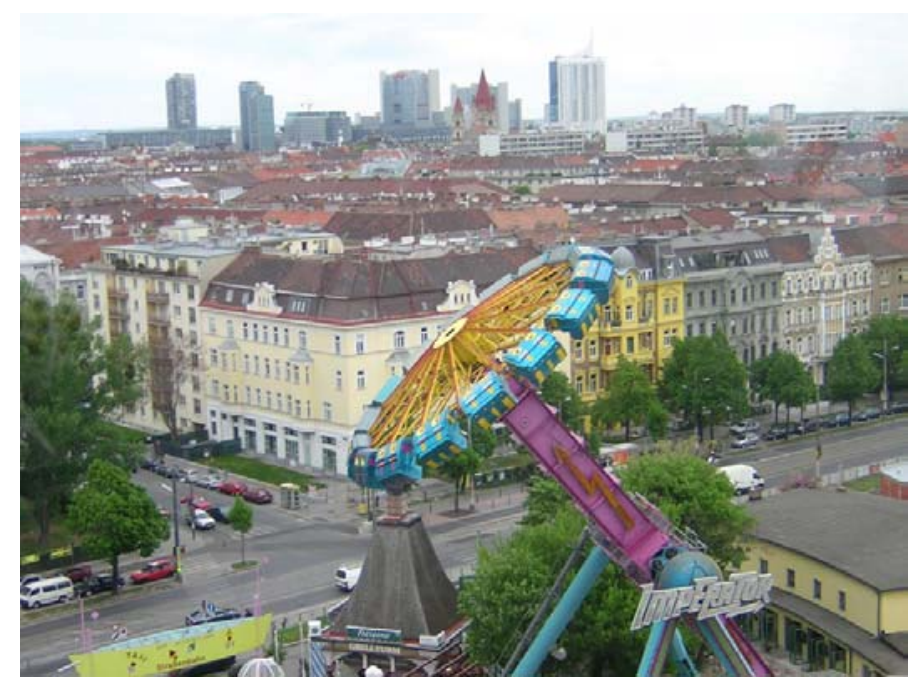

Fig. 3 Panorama di Vienna e attrazione del Prater in primo piano, dalla Riesenrad, ruota panoramica. Il paesaggio del parco ha come componente anche la città all'esterno ed è quindi radicato ad un territorio specifico.

Tonino Guerra non chiede ai suoi giardini di essere divertenti, tuttavia il suo modo di "attribuzione del tema" a partire dal contesto, è interessante e non troppo dissimile da quello riscontrato a la Mer de Sable: l'artista intende accentuare alcune componenti del paesaggio della sua terra fra Marche e Romagna, sono componenti presenti ma anche assenti e recuperabili esclusivamente materializzando un'immagine della memoria. Quindi Tonino Guerra colloca nel paesaggio pochi oggetti che sembrano coerenti con esso - i mucchi di sale nel giardino di Cervia, le casette colorate per gli uccelli a Pennabilli ecc.. In realtà gli oggetti sono interpretati, assunti come da un sogno o dai ricordi e per questo "assurdi", nelle dimensioni, o nel materiale, nei colori. Sono espressamente inventati. Inseriti nel paesaggio ordinario lo trasfigurano perché lo inglobano nella propria dimensione irreale. Il contesto fisicamente è inalterato (installare un totem, o un tronco di albero che diventa una fontana, o tappeti di ceramica su un prato, non costituisce ovviamente una modifica della struttura del territorio) ma i simboli lo rendono altro. Non c'è un reale motivo per cui nell'ideazione dei parchi del divertimento non si possa procedere nel medesimo modo.

\section{2) TREGUA (dal tema):}

La definizione di differenti modi di attribuzione del tema, oggetto del capitolo della PARTE I Tematizzazione e aura, unitamente ai paragrafi inerenti gli esiti dei modi di costruzione del paesaggio delle varie matrici, mettono in luce come il tema -quale percorso narrativo determinato, simbolizzazione che mira alla persuasione, pianificazione dei comportamenti del visitatore all'interno del parco- possa opporsi in modo rilevante alla libertà di interpretazione e all'elaborazione personale. Al contrario la presenza di questi fattori garantirebbe la possibilità di invenzione all'interno del parco, condizione necessaria per il di-vertimento nell'accezione descritta da Isabelle Auricoste, riportata ad introduzione della ricerca.

Allo stesso tempo il tema si rivela un modo per sottolineare l'esistenza di altre dimensioni rispetto a quelle sperimentate nei luoghi oridinari: il tema evoca e materializza luoghi e civiltà lontane, mondi di fantasia ecc., svolgendo un ruolo utile ai fini della costruzione di uno "spazio creativo", quale difinito in specifico al paragrafo omonimo all'interno di Pleasure gardens, folies, jardins spectacles, ma quale realizzato anche dagli esempi presentati di giardino manierista. I modi di attribuzione del tema di queste due matrici offrono un suggerimento: dare spazio a momenti di tregua dal tema, ovvero lasciare che il 
parco presenti spazi "neutri" e non smetta di essere un luogo anche di contemplazione, riposo, riflessione, tutti attributi consentiti dal lucus amoenus, concetto che quello di giardino da sempre sottende, ma che difficilmente si trova tradotto in discorso contemporaneo.

In un manuale pedagogico di fine Ottocento, Il giardino infantile di Pitagora Conti $(1892)^{6}$, è possibile leggere fra le righe un criterio di progettazione del giardino -nel caso specifico per l'infanzia ma anche, perché no, per gli spazi ricreativi in generale- che intende lasciare aperto il processo di invenzione dello spazio e delle attività possibili al suo interno.

Il giardino del titolo del testo è un giardino figurato: il libro intende presentare il metodo educativo froebelliano, all'epoca dell'autore diffusosi a livello internazionale ${ }^{7}$, e nuove direzioni per il suo perfezionamento, dedicando ampio spazio alla descrizione, anche mediante tavole grafiche, dei "doni froebelliani", ovvero oggetti-giochi, individuati da Froebel, funzionali all'educazione dei sensi e quindi alla coltivazione del "concetto del reale".

Esiste comunque anche un giardino fisico di cui tratta il paragrafo XIII del libro, che appare interessante. Il giardino proposto contiene aree tematizzate, come anche una sorta di zoning funzionale. Eppure tutti gli oggetti eccentrici contenuti al suo interno possono essere composti in modo arbitrario, assimilati per la creazione di nuove raffigurazioni. Il giardino descritto da Pitagora Conti è proprio lo spazio creativo di cui si è detto nell'indagine delle matrici del parco del divertimento tematico. Per prima cosa nelle premesse di apertura lo spazio del giardino si configura come "compendio della natura libera", presentando la suggestione delle foreste, risposta alla necessità di un contatto con le componenti del mondo naturale che la cultura non elimina, primitivo e selvaggio, sentita particolarmente nell'età infantile.

“ L'uomo per quanto civile, per quanto lontano dal suo stato primitivo, ha sempre in sé qualcosa di selvaggio. (...) L'impronta animale non si cancella con le seduzioni dell'arte, né con le elucubrazioni della scienza, e ad onta del tanto che s'è fatto per abituarci, specie nelle città, ad una vita artificiale, sentiamo ancora potentemente l'istinto antico e amiamo l'aria, la luce, il sole , i campi, il mare , i tramonti.

(...) questa tendenza verso una vita che la coltura e l'ingegno hanno in parte affievolito, se è potente negli adulti, è potentissima nei bambini, che sono i nostri cari selvaggi d'oggi." ${ }^{\circ}$

Il riferimento alla concezione storica di Vico che individua un parallelismo fra le attitudini dell'uomo agli esordi della storia e quelle dell'uomo nei primi anni della vita, proietta verso un ambiente in cui i fenomeni e le cose presenti divengono, per la particolare percezione dell'uomo-bambino, materia mitologica e quindi poetica nel senso etimologico del fare. Per questo, prima che nel testo venga descritto, il giardino può essere prefigurato come un luogo non ordinato e ornato, ma contenente qualcosa di caotico che attende ancora una cosmogonia. Essa avviene per una "corpulentissima fantasia" e quindi con una "maravigliosa sublimità". Successivamente all'interno del testo il disegno del giardino si delinea e presenta molte forme che miniaturizzano il sublime in grazioso ( il testo evidenzia l'empatia, in età infantile, per entrambi i caratteri). Deve essere, per esigenze igieniche, ben arieggiato e deve contenere un campo per gli esercizi ginnici. Allo stesso tempo ha viali con padiglioni o "chioschi adatti, con tutto quanto occorre per l'esecuzione dei lavorini" - qualcosa di

\footnotetext{
${ }^{6}$ Pitagora Conti, Il giardino infantile, Manuali Hoepli, Milano 1892. Il titolo fa pensare che si tratti di un manuale di giardinaggio per bambini o per spazi dedicati ai bambini. In realtà è un testo di pedagogia e giardino infantile traduce il termine kindergarten con cui il pedagogo tedesco Friedrich Froebel (1782-1852) definisce gli istituti per l'infanzia così come da lui teorizzati, a partire dalla concezione dei bambini simili a fiori che crescono in libertà in un giardino.

${ }^{7}$ Come riporta l'autore, il metodo fu applicato in Italia soprattutto in seguito ad una circolare del ministro Coppino, con data 17 settembre 1885 , “ in cui si consigliava l'aggregazione di un giardino froebelliano a tutte le scuole normali governative e la continuazione degli esercizi froebelliani nel corso inferiore delle scuole sperimentali".

${ }^{8}$ Pitagora Conti, Op.cit., Manuali Hoepli, Milano 1892, pag. 102.

${ }^{9}$ Da Giovan Battista Vico (1668-1744), Scienza nuova, II, I, 1 .
} 
analogo alle folies rosse di Tschiumi a La Villette che fungono da laboratori - e aiuole fiorite,

“(..) sarà anche la vista di facce nuove, di bimbi che si divertono allegramente, di prati e viali, di voci e canti, d'acqua e di nubi; (...) la vista di animali, (...) d'uccelli, di cani, di pecore, di anitre, di conigli, di colombi, di tortore, di tacchini, di pesciolini e magari anche di pavoni e cigni." 10

"I tappeti d'erba, l'acquario le gabbie e i gabbioni degli uccelli, il carosello, la vasca, la barchetta, il bagno, ecc." $" 11$

Inoltre per riprodurre quanto è possibile vivere nelle campagne, il giardino è anche orto. E' descritto, fra gli altri, uno spazio che dovrà servire alla coltivazione dei fiori e degli ortaggi e risponde al modo infantile di imparare a distinguere le forme, le proporzioni, le distanze, all'esigenza di formare alla pazienza e alla perseveranza e di indirizzare la loro operosità:

“ (...) Per imparare a leggere la natura i bambini più grandi cominciano dal lavorarla e per ciò nelle villeggiature si veggono i ricchi, come i contadini, correre pei campi e muovere la terra, spartirla, rincorrere le farfalle, e lungo i canali d'acqua costruir mulinelli e parate, pescare, scavare fosse, zappare vangare, costruire montagne, prendere attiva parte alla vendemmia, ai lavori dell'aia e godere un mondo nel far capriole sull'erba dei prati o sui mucchi di fieno! (...) per ciò si concede a ciascuno un pezzo di terra, almeno di un metro quadrato, in cui tutti possano divertirsi; piantare, spiantare, far solchi, siepi (...)."12

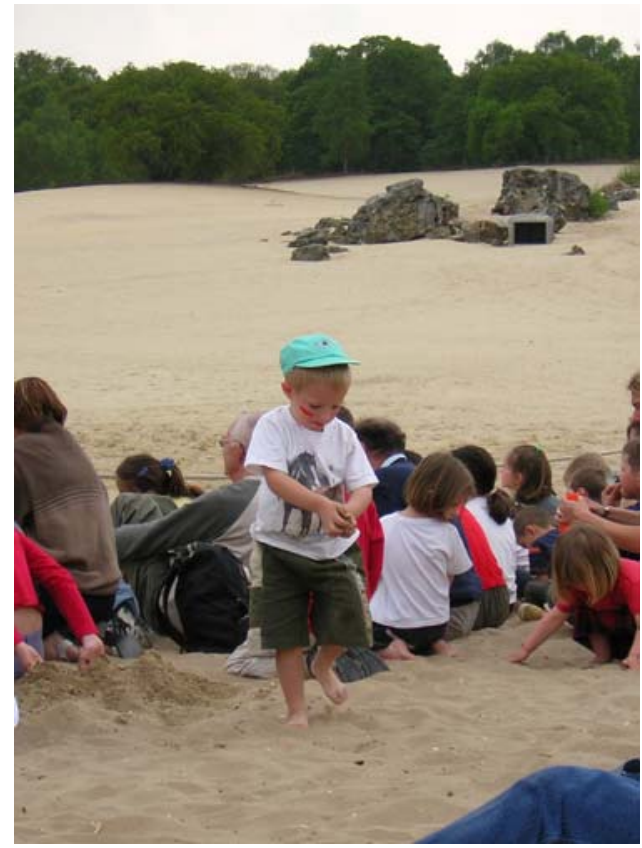

Fig. 4 Al Parco Mer de Sable (Ermenonville, France), durante uno spettacolo ad ambientazione western sul "mare di sabbia", un bambino si distrae e gioca a spostare la sabbia. Il parco consente la tregua dal tema per la presenza di ampi spazi duttili all'iniziativa personale dei suoi visitatori.

Nel brulichio delle azioni possibili e degli spazi rispondenti, il disegno del giardino risulterebbe già definitivamente strutturato, se non fosse per la possibilità di attribuirgli tutte le caratteristiche riferite genericamente all'ambiente educativo, presentate nei paragrafi precedenti il XIII, e di pensare che vi si svolgano molti dei giochi di costruzione/composizione legati ai "doni froebelliani", in quanto le attitudini e le necessità dei bambini richiedono e realizzano uno spazio di espressione, preferibilmente aperto.

\footnotetext{
${ }^{10}$ Pitagora Conti, Op. cit., Manuali Hoepli, Milano 1892, pag. 106.

${ }^{11}$ Pitagora Conti, Op. cit., Manuali Hoepli, Milano 1892, pag. 111.

${ }^{12}$ Pitagora Conti, Op .cit., Manuali Hoepli, Milano 1892, pag. 106.
} 
Trova conferma l'intuizione che l'aspetto del giardino sia in realtà difficilmente tangibile un magma di materie prime ${ }^{13}$ (Fig.4), ma anche un bazar di prodotti finiti dell' elaborazione culturale, comunque disponibili per l'invenzione e la manipolazione, offerti alla sensorialità - e che esso sia un luogo continuamente in potenza sotto l'azione demiurgica, coincidente con la progressiva acquisizione di esperienza da parte del bambino.

Per cui a informare il giardino sono: la tendenza a muoversi e a trasformare, a cambiare o "scomporre per ricomporre", quindi sperimentare che " ha un principio umile che sta nel moto fisico associato al movimento psichico, nel giuoco dei sensi" ed è "frivolo, perché l'esperimento è primitivo, non complesso", il piacere, l'interesse e l'azione, la rappresentazione più che la narrazione come modo di comunicazione, il canto e i movimenti coreografici, la educazione dei sensi, che " deve essere la causa del maggior numero possibile di piaceri”, come mezzi di informazione per giudicare la direzione, la distanza, il rumore, gli odori, i colori, i materiali ed elimina i confini fisici,

"L'uomo occupa poco spazio; ma coi sensi ingrandisce smisuratamente se stesso, rendendo meno pericolosa la sua debolezza. (...) Con gli occhi si giunge tutto intorno a distanze smisurate (...). Con l'udito si passa attraverso i muri; si sorpassa in un attimo la superficie estesa della terra, dei laghi e dei fiumi e si giunge così dove non si è",

l'immaginazione, un museo

"Ossia un mondo di cose, disposte non in una sala apposita; ma un po' dapertutto, atte a stimolare la curiosità (...). Una lanterna magica, un grande caleidoscopio, uno sterescopio con vedute adatte, la stanza della bambola (...); gingilli di vetro colorato e tutto ciò che splende, che luccica, che è bello, che è armonico, che è artistico (...); ma di quell'artistico primitivo (...). E un museo più bello, più ricco, più variato sarà lo stesso giardino, col suo bel cielo, col verde delle piante, coi vaghi fiori, con la sua continua varietà. Tali oggetti daranno occasione al giuoco e alla parola, rappresentazioni della realtà." 14

Il Jardin d'Acclimatation di Parigi (1860), ancora in attività quale parco ricreativo, assomiglia molto al giardino infantile ipotizzato da Pitagora Conti, non solo perché si rivolge anch'esso ai bambini. Al Jardin d' Acclimatation sono presenti cose che "splendono e che luccicano" e che hanno l'aspetto di materie prime disponibili ad essere plasmate creativamente. $\mathrm{Ci}$ sono inoltre giochi che rendono sperimentabile la molteplicità di modi di percezione visiva, variegando l'immagine del giardino: una piramide con struttura leggera per i movimenti acrobatici, gli specchi deformanti, un labirinto di canne muovendosi dentro e fuori dal quale il parco ora è presente, ora è negato. L'antico tema del giardino -la presenza dell'esotico- è sullo sfondo, nella vegetazione adulta, non commentata da cartelli didascalici con informazioni botaniche. E' un tono discreto: probabilmente pochi visitatori hanno la consapevolezza della rarità delle specie arboree e della loro provenienza, assorbono solo l'immagine risultante del giardino nella distrazione del gioco (Fig. 5) .

\footnotetext{
13 Fra le molteplici materie, nel testo ricorrono i ciottoli (fra gli oggetti del sedicesimo dono froebelliano), la sabbia, la terra, ed esiste un paragrafo, il XIV, dedicato a "La Plastica" che parla dei lavori di modellazione della creta. Vengono presi come esempi di attenzione all'esigenza infantile di interazione con lo spazio, in contrapposizione rispetto alla situazione italiana, Berlino - dove anche nei giardini pubblici "si concede ai bimbi un pezzo di terra in cui possano fare quel che vogliono" -, Londra - i cui cittadini che abitano vicino alle piazze ne prendono in affitto alcune porzioni per i loro bambini e li affidano ad "abili custodi" -, Parigi dove negli square, nei giardini, e nei grandi parchi, si permette ai bambini di ammucchiar sassolini, e di far pasticci con la polvere e la terra dei sentieri".

${ }^{14}$ Il giardino infantile sembra identificarsi come il luogo di relazione del "fanciullino" di G. Pascoli - Il fanciullino è stato pubblicato nel 1897 e nella redazione definitiva nel 1902 - che è " quello che parla alle bestie, agli alberi , ai sassi, alle nuvole, alle stelle; che popola l'ombra di fantasmi e il cielo di dei. Egli nell'interno dell'uomo serio sta ad ascoltare, ammirando, le fiabe e le leggende, e in quello dell'uomo pacifico fa echeggiare stridule fanfare di trombette e pive. (...) Egli ci fa perdere il tempo, quando andiamo per i fatti nostri, che ora vuol vedere la cinciallegra che canta, ora vuol cogliere il fiore che odora, ora vuol toccare la selce che riluce. E ciarla tanto senza chetarsi mai; e senza lui, non solo non vedremmo tante cose a cui non badiamo di solito, ma non potremmo nemmeno pensarle e ridirle, perché egli è l'Adamo che mette il nome a tutto ciò che vede e sente."
} 

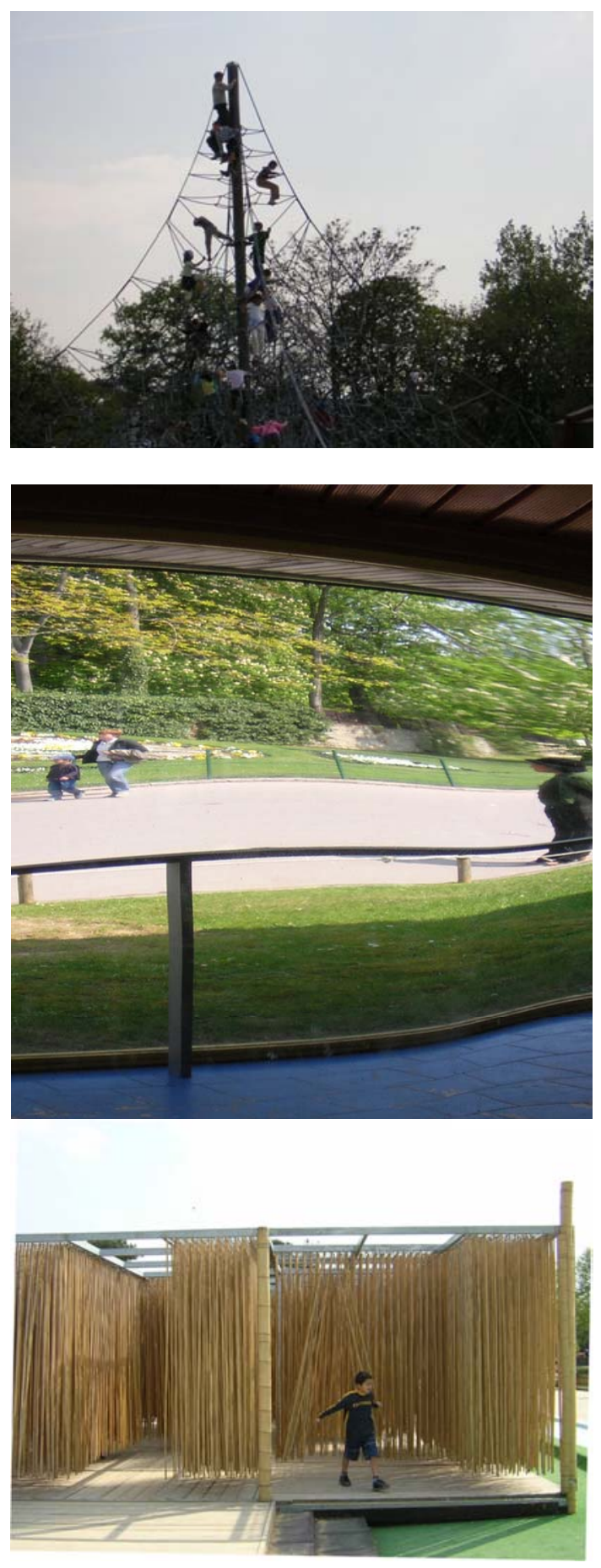

Fig. 5 Jardin d'Acclimatation, Parigi - giochi del parco, una piramide per le acrobazie, gli specchi deformanti, il labirinto di canne: l'aspetto del giardino cambia in relazione alle diverse percezioni consentite dai giochi.

Il giardino di Conti Pitagora non appare nemmeno molto diverso da quello manierista o dalle folies francesi del XVIII secolo, di cui si è sottolineato un aspetto: l'essere luoghi di affaccio a realtà non ancora esperienziate, nuove, in cui l'affaccio è consentito dalla presenza di elementi raccolti in forma di collezione, in ordine non casuale ma arbitrario, senza rigore, con curiosità, attenzione per i rapporti originali, mediante sperimentazione. E' necessario a lato della "collezione" avere un foglio bianco per fissare le idee che la collezione stessa suggerisce, scarabocchiare figure che sorgono per immaginazione, o pensare ad altro. Anche per fare avvenire gli incidenti di cui parla Michael Sorkin quali elementi fertili nello spazio 
urbano. Sorkin fa dipendere la probabilità di "incidenti" dal livello di mescolanza di attività diverse, ma si ritiene che essi siano soprattutto resi possibili dall'assenza di attività stabilite:

“ Un terzo aspetto positivo della fiera commerciale e dei parchi tematici potrebbe essere l'idea di mescolanza delle attività. Sebbene questa mescolanza sia spesso metaforica, è molto importante che queste strutture vengano progettate al di là della loro utilizzazione specifica. (...) Gli urbanisti razionalisti si battono contro gli "incidenti", contro le irregolarità che possono aver luogo nella città. (...) E tuttavia credo che abbiamo bisogno di cercare nella città di oggi tutti i mezzi possibili per creare "incidenti" nei termini di un rapporto più fecondo fra uso e forma." 15

La tregua dal tema si presenta come spazio vuoto rispetto al pieno simbolico e come spazio ampio rispetto all'accumulo-concentrato simbolico. Riguardo al parco di Pratolino si è detto dell'alternanza di luoghi circoscritti, gremiti di oggetti-simboli di dimensioni ridotte e fra loro composti in maniera ridondante, costituiti da grotte e gruppi scultorei, e di luoghi viceversa scarni - le aree di bosco dall'aspetto selviculturale ed il prato fra l'antico labirinto e la statua dell'Appennino.

Una composizione del tutto analoga è realizzata, probabilmente senza consapevolezza, al parco di Efteling in Olanda, dove un labirinto è collocato in posizione adiacente rispetto ad un prato libero, un tempo destinato ad area picnic (Fig. 6).
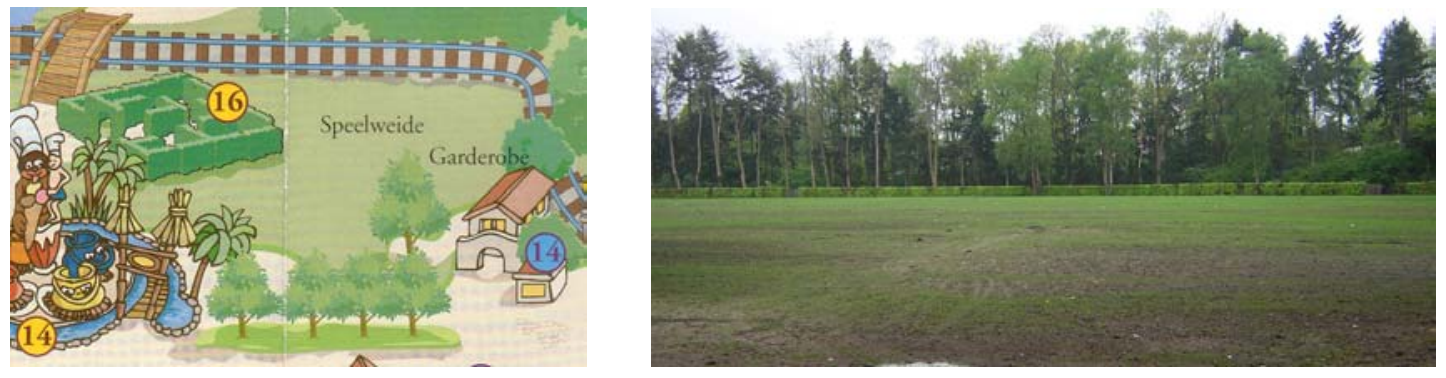

Fig. 6 Efteling (Olanda, 1952-2002), particolare della mappa attuale del parco in cui si vede il labirinto e il prato adiacente. Il labirinto in realtà è molto più piccolo paragonato alle dimensioni del prato adiacente. Il prato è visibile nella fotografia a destra, come ampio vuoto di tregua dal tema.

La tregua ad Efteling è frequentissima, intere porzioni del parco sono caratterizzate da sentieri o viali aperti all'interno della foresta, senza che si imponga nessun simbolo a dettare regole sul cosa fare (e come). Si può tornare a visitare il parco anche solo per compiere un giro sul lago e godere delle nuove fioriture di bulbi lungo le sponde, o per salire sulla pagoda volante, staccarsi da terra e vedere diventare minuscolo il parco, scomparire i simboli minuti che lo ambientano, aprirsi l'orizzonte a tutto l'intorno di foresta e campi. Efteling ripropone all'interno del parco tematico l'idea di affiancare parti tematizzate e parti "neutre" che è propria dei parchi pubblici europei del XIX secolo: Il Jardin d'Acclimatation di Parigi è una porzione tematizzata (specializzata) del Bois de Boulogne ed il Prater di Vienna è destinato a "luna-park", un tempo a circo, solo nella punta più vicina al centro della città, in tale modo si può fruire in alternanza del divertimento "spinto" e, al contrario, di un luogo di riposo, di passeggio e di tutto ciò che si richiede allo spazio aperto collettivo. Il Parc Citroen di Parigi manifesta questa consapevolezza: l'esito della progettazione di un parco che intende soddisfare la pluralità di esigenze di una comunità dipende dal rapporto proporzionale che sa stabilisce fra la piccola e la grande scala, fra l'esibizione di temi che focalizzino interesse e l'assenza di temi che invitino all'invenzione degli usi possibili. I giardini seriali tematici del paesaggista Gilles Clement dichiarano di avere alle spalle una tradizione che ha riflettuto costantemente su questo rapporto proporzionale. La dimensione ridotta, la posizione a latere

\footnotetext{
${ }^{15}$ Michael Sorkin , La tematizzazione della città, in "Lotus" n. 109, Il nuovo mondo allegorico. Sui parchi tematici, Electa, Milano 2001, pag. 8.
} 
, la distribuzione in serie ripropongono l'idea della "collezione", che è un momento di presa di conoscenza. Non li si può percorrere con sguardo distratto perché sono ricchi di elementi diversi. Vanno goduti al dettaglio. Nel prato immenso su cui è fondato il parco, e nel bosco adiacente, la visone può essere meno consapevole e il movimento nello spazio più "semplice". Prato e bosco costituiscono i "temi deboli" offerti dalla natura, grandi campiture in grado di assorbire gli eventi puntuali e fortemente tematici che vi si collocanoanalogamente a quanto descritto nell'indagare il rapporto fra spazio e simbolo all'interno del giardino manierista e delle folies settecentesche (Fig. 7).
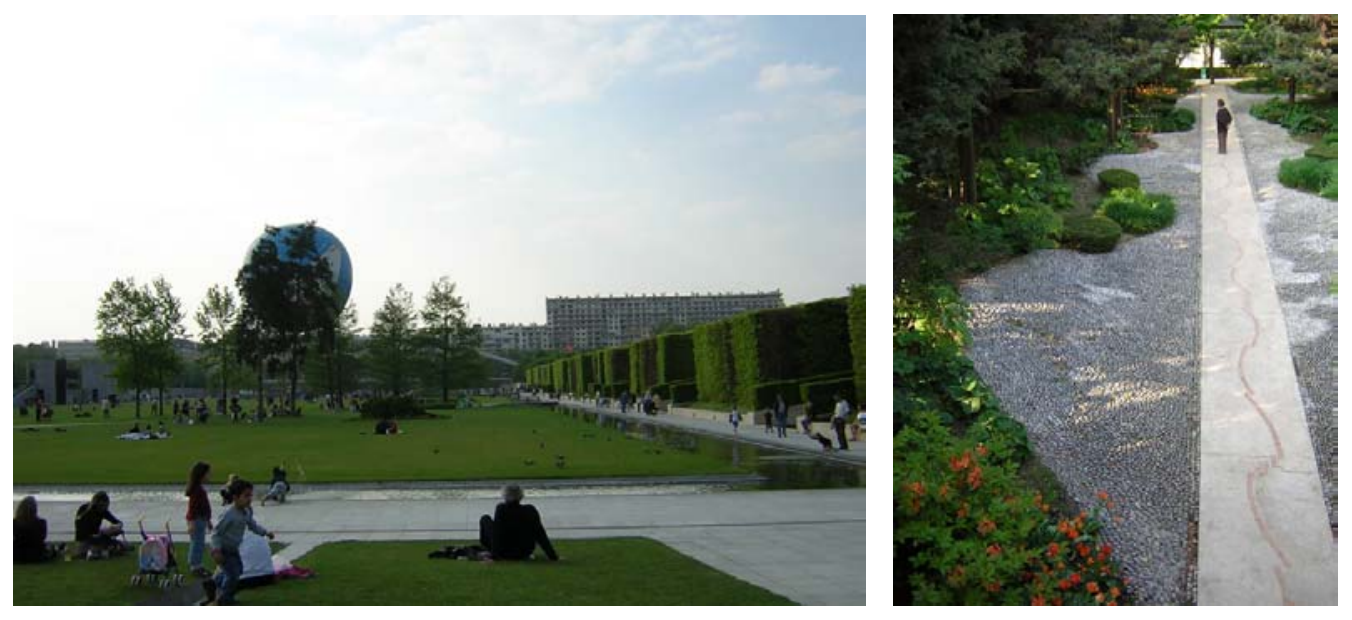

Fig. 7 Parc Citroen- Parigi : il vasto prato centrale e il jardin orange.

La tregua è anche realizzata dalla presenza di aperture prospettiche che immettono da uno spazio ricco di segnali, culturale, verso un altro "puro", naturale, aspetto di cui si è segnalata l'importanza nel descrivere gli esiti degli amusement parks americani. Si sono citati al proposito il centro commerciale al Fisherman's Wharf di San Francisco e il Santa Cruz Boardwalk, sempre in California. In queste occasioni la tregua in realtà coincide con la presenza del contesto.

Alla definizione di questi due elementi - presenza del contesto e tregua dal tema- si giunge operando la seguente schematizzazione, elaborata in forma di grafico nella TAV. II_ 3 allegata (si riportano anche, per chiarezza, brani di riferimento presentati in precedenza nella PARTE I):

\section{Il PARCO (1) DEL DIVERTIMENTO (2) TEMATICO (3) è:}

\section{(1) PARCO:}

"Nessun giardino potrebbe esistere altrove, se non nel territorio in cui si trova e da cui dipende. Si tratta di uno spazio materiale, di cui suolo, clima, topografia, e referenze culturali locali costituiscono determinanti essenziali." 16

- è in relazione ad un contesto e da esso dipendente

- consente un contatto con la natura ed è realizzato come sfida-collaborazione fra elementi culturali e naturali

- al suo interno i giardini sono in rapporto con l'insieme più vasto del parco e con il contesto

(2) del DIVERTIMENTO

\footnotetext{
${ }^{16}$ IsABelle Auricoste, Parchi o utopie mortali?, “ Ottagono”, n. 99, Intrattenimento, 1991, pagg. 19-20
} 
"Divertire (divertere) non significa forse distogliere, allontanare dall'uso comune, così come d'altronde distrarre (distrahere) rinvia all'azione di tirare in senso inverso? (...) sono al tempo stesso le metafore in termini di esperienza concreta che connettono l'altrove con la nozione di territorio situato. Il senso di estraneità risiede nella conoscenza vissuta delle diversità e dei confini tra due territori.",17

"Each Generic City has a waterfront, not necessarily with water - it can also be with desert, for istance - but at least an edge where it meets another conditions, as if a position of near escape is the best guarantee for its enjoyment." 18

- può avvenire in uno spazio allo stesso tempo vicino e separato rispetto ad un territorio urbanizzato determinato.

- esige un contatto con l'alterità (con l'esotico, con una dimensione misteriosa, fantastica),

esige novità

(3) TEMATICO sottoposto quindi ad un processo di tematizzazione che può avvenire:

- ponendo il contesto in primo piano, ovvero il contesto diviene il tema stesso e si conservano senza recisioni gli elementi fisici strutturanti il particolare territorio in cui il parco-giardino si colloca:

E' quanto avviene ad esempio nell'arte dei giardini di Ian Hamilton Finlay: l'iscrizione rende il paesaggio in cui viene posta un simbolo, sia perché costituisce un commento che lega il paesaggio ad un immagine letteraria, sia perché l'iscrizione diviene interna al paesaggio stesso in quanto elemento materiale - panche in pietra, lapidi, ecc.

La tematizzazione è in questo caso uno strumento retorico per la creazione di un'immagine artistica.

In altri casi il contesto è messo in primo piano a fini conoscitivi e didattici.

Questa modalità di attribuzione del tema è perfettamente compatibile con l'idea di parco: essendo il tema radicato e non "fuori terra" sicuramente la tematizzazione non produce uno spazio replicabile. Nel suo collocarsi in continuità con il territorio presente, sia per gli aspetti fisici sia per gli spetti semantici (il contesto è il tema), difficilmente è in grado di consentire il divertimento, perché non realizza ne' una separazione, ne' un'affaccio sul diverso, sull' altro, sullo sconosciuto ecc.. che appaiono sue condizioni.

La tematizzazione può avvenire in altro modo:

\section{- mediante estraniamento dal contesto}

L'estraniamento dal contesto è diversamente perseguito:

- tramite soluzione di continuità delle componenti strutturanti il territorio:

E' il modo di procedere della Disney Company che si è descritto. La tematizzazione viene preceduta ed è dipendente dalla creazione di una tabula rasa: non c'è memoria dei segni che caratterizzano il paesaggio circostante, ma nemmeno delle culture che li hanno prodotti. Non solo essi non entrano all'interno del parco come elementi regolatori, ma non sono neppure necessari al contorno, a fare da controparte alla nuova immagine del luogo.

Direzionare la progettazione in questo senso conduce molto probabilmente alla realizzazione di "utopie noiose". Sicuramente ci si colloca al di fuori del progetto di un parco.

\footnotetext{
${ }^{17}$ AURICOSTE ISABELLE, I parchi ricreativi in Europa: il divertimento e l'altrove, in MONIQUE MOSSER, GEORGES TEYssot, L'Architettura dei giardini d'Occidente- Dal Rinascimento al Novecento, Electa, Milano 1990, pag. 479.

${ }^{18}$ Rem KoolhaAs, La città generica, in S,M,L,XL, Taschen, 1997, pag. 1257.
} 
- tramite attribuzione di simboli al territorio:

In questo caso il territorio è compreso. E' il modo di attribuzione del tema proprio del giardino manierista, in particolare del Sacro Bosco di Bomarzo.

Il territorio è compreso soprattutto nel parco europeo di Efteling, come si evince dall'analisi semantica. Esso, come si è detto, nasce come Nature Park, ovvero come centro ricreativo interno ad un parco "naturale. Il parco naturale è dipendente dal territorio rurale circostante: il bosco che vi viene impiantato interrompe, assecondandone le geometrie, gli appezzamenti di terreno agricolo, in contrapposizione consapevole volume contro superficie, ricreazione contro produttività. Il bosco viene tematizzato dalle istallazioni della Foresta delle Fiabe. Così sul territorio rurale che esprime l'esigenza di essere completato dalla presenza della "naturalità", la fantasia (nordica, locale, collettiva) imbastisce un racconto, che è il mito del territorio stesso contenente un mondo misterioso, quale gene proprio.

Anche Mer de Sable di Ermenonville, in Francia, parte dal territorio per condurre ad un viaggio immaginario: pretesto per la tematizzazione western che lo caratterizza è l'esistenza della particolarità geologica di dune di sabbia, nella foresta secolare di Ermenonville, che in parte è interna al confine del parco: essa è la base su cui vengono posizionate poche architetture sufficienti a rendere la spiaggia ora un deserto dell'Old West America, ora d'Oriente - a seconda di quali simboli vengono intercettati dallo sguardo. Il contesto evoca altri contesti, per cui è possibile parlare di singolarizzazione e valorizzazione del contesto mediante l'astrazione da esso. La cosa è divertente. Viene enfatizzato il significato di paesaggio come icona relativa a un territorio. L'icona può essere anche dimentica del reale processo di formazione del luogo e divenire "fantastica", senza che vengano fisicamente cancellate le tracce che ne detengono la storia: il territorio a la Mer de Sable è sia conservato - fisicamente - sia annullato - ad opera dell'immagine del paesaggio risultante.

Questa direzione appare molto fertile perché, almeno nelle premesse, consente di creare un parco in senso proprio e utilizza il processo di tematizzazione ai fini del divertimento, nell'accezione di diversione da un territorio per affacciarsi su un altrove.

\section{RIFERIMENTI BIBLIOGRAFICI:}

Baud-Bovy Manuel, Lawson Fred, Tourism and Recreation Development, The Architectural Press LTD, London 1977.

PitAGORA CONTI, Il giardino infantile, Manuali Hoepli, Milano 1892

SANGUANINI BRUNO, Effetto benessere \& qualità della vita. A DisneyWorld, EuroDisney, Gardaland e altrove, in MINARDI EVERARDO, LUSETTI MARIALUISA (a cura di), I parchi del divertimento nella società del Loisir, Franco Angeli, Milano 1998, pagg. 86-127.

SAVElli ASTERIO, Parco separato e territorio aperto: problemi di strategia nelle aree turistiche, in MINARDI EVERARDO, LUSETTI MARIALUISA (a cura di), I parchi del divertimento nella società del Loisir, Franco Angeli, Milano 1998, pagg. 11-26.

Orlando Furioso, XII, 3-34.

\section{RIFERIMENTI ICONOGRAFICI}

Fig. 1: Disegno in una brochure ufficiale pubblicitaria del Disneyland Resort- Paris.

Fig. 2-7 : Fotografie di Enrica Dall'Ara. 
L'analisi operata prendendo a campioni esempi delle cinque matrici in una prima fase e tre realizzazioni europee contemporanee poi (vedi tavole II_1 e tavole II 2), porta all'individuazione di alcune linee guida per la progettazione della presenza del contesto e della tregua dal tema, e per la progettazione dei giardini, all'interno del parco del divertimento a tema (così come definito nel paragrafo precedente e in TAV. II_3):

\section{1 (presenza del) CONTESTO}

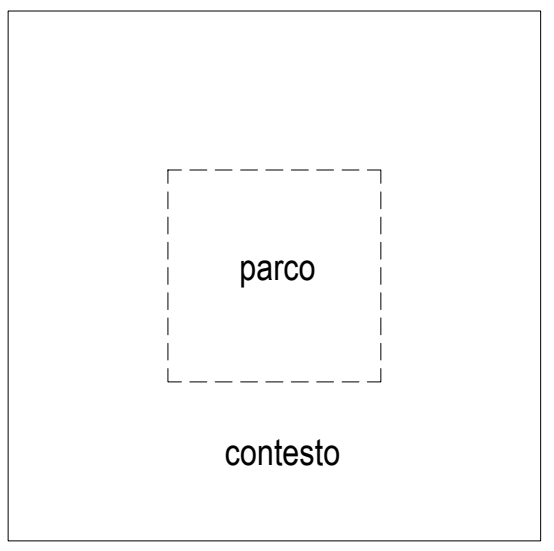

\subsection{CONTESTO DI VALORE}

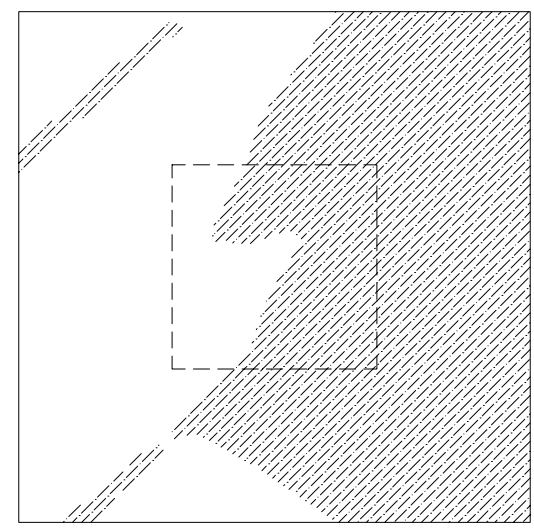

\section{Aspetti percettivi}

- qualità visiva all'esterno del parco:

dall'esterno visibilità di uno o limitati simboli con funzione di comunicazione, di segnalazione di un "passaggio" fra due luoghi differenti (landmark). 
In questo caso può non essere necessaria la visibilità del confine fisico, gli elementi strutturanti il paesaggio non necessitano di essere "recisi" perché il dentro ed il fuori del parco sono già resi territori differenti dalla simbolizzazione.
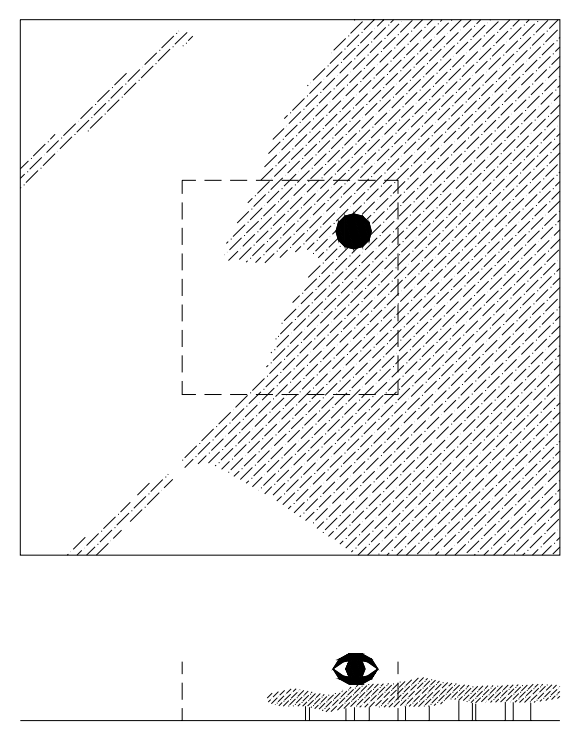

il confine fisico del parco, se si sceglie di renderlo visibile, deve costituire esso stesso un simbolo (landmark).

In questo caso gli elementi strutturanti il paesaggio non necessitano di essere "recisi" perché il dentro ed il fuori sono già resi territori differenti dalla simbolizzazione.

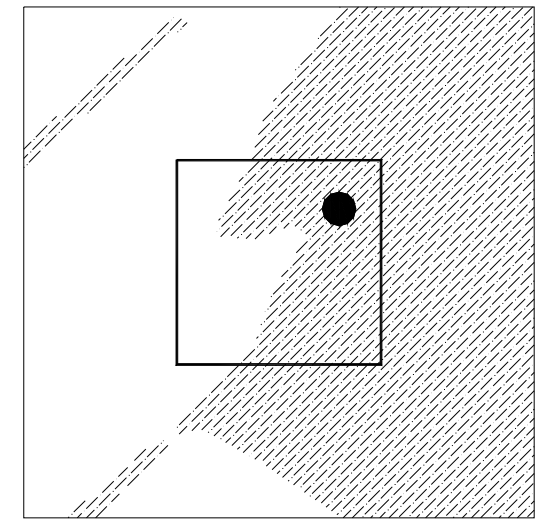

0

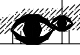

- qualità visiva all'interno del parco:

visibilità dall'interno del parco di brani del paesaggio circostante. In particolare il paesaggio nell'intorno può essere visto:

(1) inserendo attrazioni panoramiche 


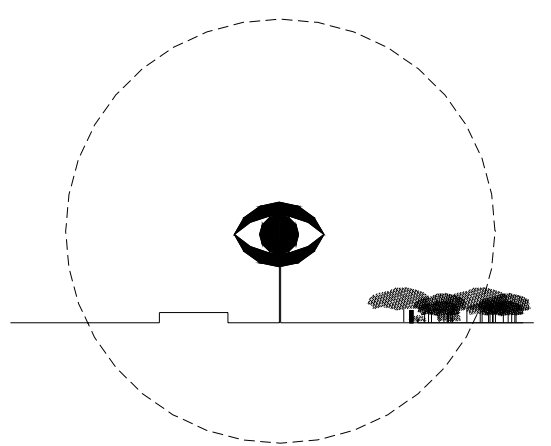

(2) posizionando rides quali i roller coaster - in cui nei tratti iniziali ci si muove a velocità lente ed ad altezze elevate - vicino ai confini con l'esterno,

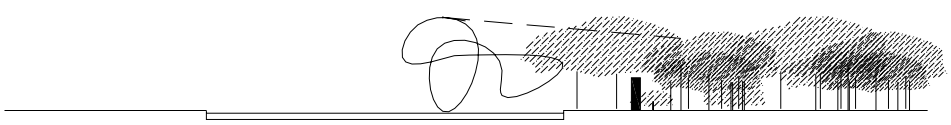

(3) posizionando i lunghi percorsi di attesa per l'ingresso alle varie attrazioni a contatto con il paesaggio circostante vicino ai confini, che devono essere in questo caso trasparenti, prestata attenzione a possibili problemi di impatto dovuto all'affluenza di massa.

[collabora anche alla progettazione della componente 2 TREGUA (DAL TEMA)]

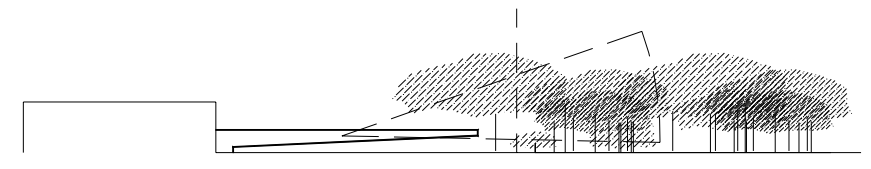

(4) attraverso un accurato studio prospettico che consenta di dilatare apparentemente lo spazio del parco e di inglobare luoghi in realtà esterni al confine e di qualità.

[collabora anche alla progettazione della componente 2 TREGUA (DAL TEMA)]

\section{Aspetti ecologici}

Il problema è di difficile schematizzazione e va indagato e risolto caso per caso.

Ad ogni modo:

- in fase di determinazione dell'estensione del parco è necessario mettere a bilancio necessità di ridurre al minimo la superficie di suolo da "sacrificare" alla costruzione del parco e necessità di spazio "aggiuntivo" per la realizzazione di connessioni ecologiche interne al parco.

- Si individuano elementi “disponibili” per la costruzione di connessioni ecologiche:

\section{aree a parcheggio}

(costituiscono fra l'altro una porzione molto consistente del parco, in alcuni esempi la superficie a parcheggio è pari a quella del parco vero e proprio)

Un esempio realizzato è il parcheggio progettato dei paesaggisti Desvigne e Dalnoky per le fabbriche Thomson a Guyancourt in Francia, in cui i fossi per lo scolo delle 
acque lungo le file di posti-auto divengono elementi di connessione ecologica fra le parti a gradiente di naturalità più elevato, agli estremi dell'area):

(1) spazi di divisione fra le corsie e le file di parcheggi

(2) alberature per l'ombreggiamento

(3) siepi o fasce alberate di costruzione dei confini del parcheggio

(4) siepi o fasce alberate di "indirizzo" ai percorsi pedonali di accesso al parco
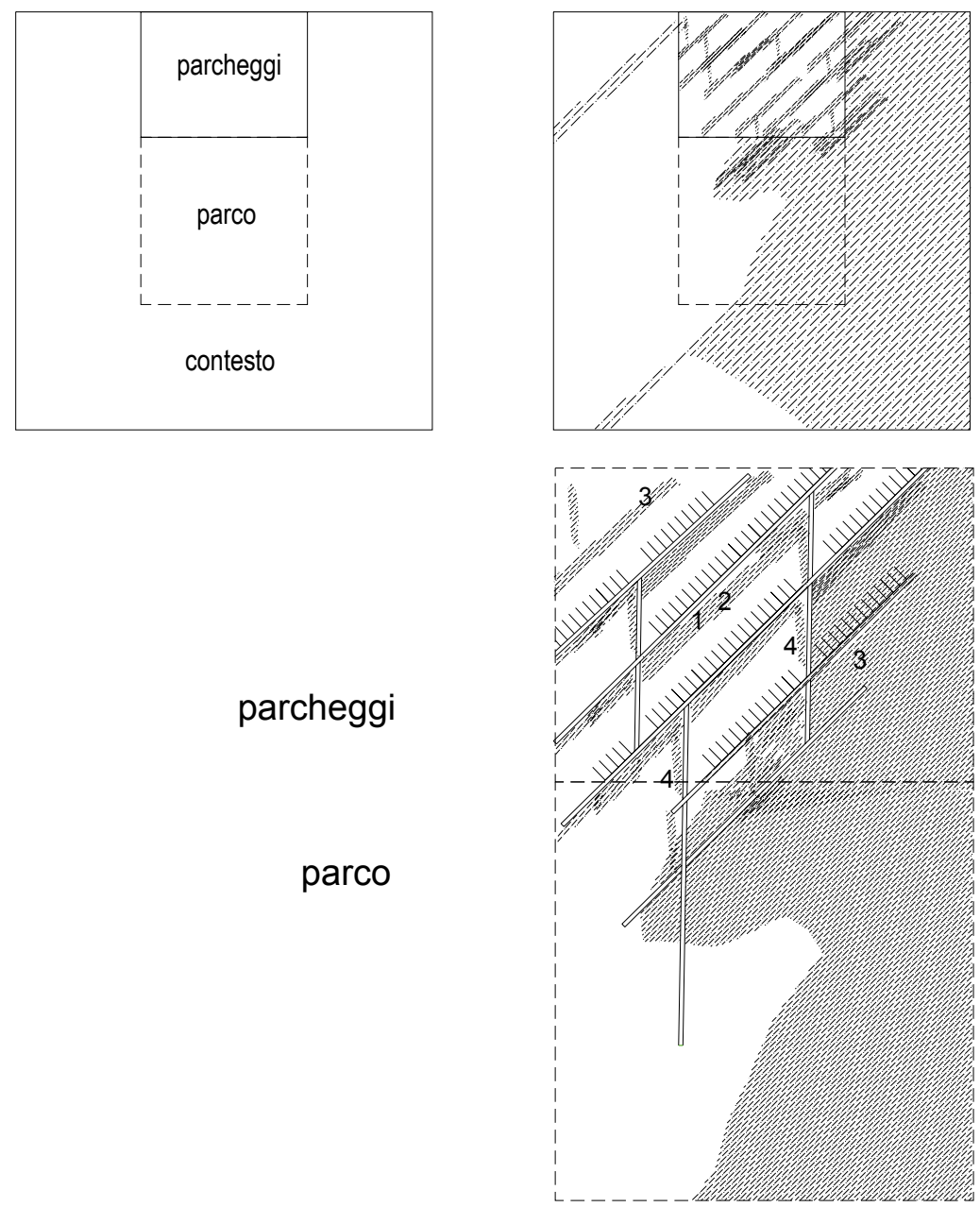

spazi di separazione delle diverse aree tematiche:

sono spazi che possono rimanere "indisturbati" se si direzionano i flussi dei visitatori in opportuni percorsi di collegamento. Normalmente sono spazi presenti nei parchi tematici, ma svolgono semplicemente la funzione di barriera visiva fra un'area e l'altra. Possono essere progettati in modo da costituire connessioni ecologiche fra interno ed esterno del parco e mitigare le cesure.

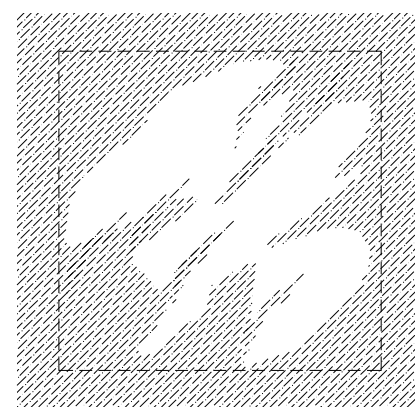




\section{Aspetti generali}

La necessità di ridurre al minimo la superficie di suolo da "sacrificare" alla costruzione del parco suggerisce le seguenti soluzioni:

Le attrazioni a più elevata infrastrutturazione possono venire concentrate in uno spazio relativamente "contratto", sfruttando l'eventuale possibilità di realizzare piani interrati e sopraelevati (questo espediente è già attualmente ricorrente) e offrire quanto più spazio aperto possibile.

[collabora anche alla progettazione della componente 2 TREGUA DAL TEMA]

conseguentemente:

- altre porzioni di territorio possono rientrare all'interno del parco ospitando attrazioni più soft e "reversibili", di minor impatto e con un maggiore grado di conservazione del paesaggio esistente. La tematizzazione sarà in questo contesto realizzata solamente attraverso l'inserimento di uno o pochi oggetti-simboli - es.: alcune rocce artificiali e una casetta di legno stile West-America tematizzano la Mer de Sable nell'omonimo parco di Ermenonville, France (la sezione seguente è parte la sezione del parco Mer de Sable di TAV. II_2.2a).

[collabora anche alla progettazione della componente 2 TREGUA DAL TEMA]

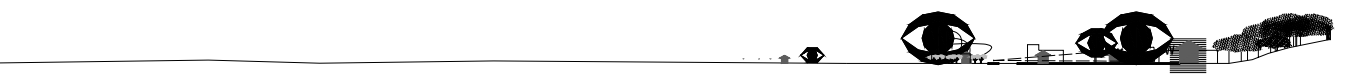

- nella parte maggiormente infrastrutturata e contratta è necessario più che altrove mantenere una composizione ordinata e un sistema di comunicazione/tematizzazione chiaro e coerente.

- nella parte maggiormente infrastrutturata e contratta è necessario più che altrove garantire un'elevata qualità estetica delle architetture e delle istallazioni.

\subsection{CONTESTO DA VALORIZZARE}

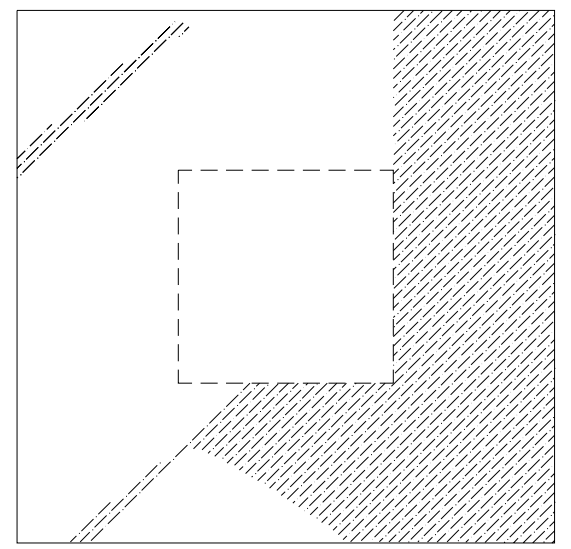

Il parco costituisce occasione per una riqualificazione dell'intorno, il più estesa possibile, interpretandone le esigenze: 


\section{Aspetti percettivi}

- qualità visiva all'esterno del parco:

il progetto deve inserirsi a completare e a potenziare il sistema di segni eventualmente già presenti, ma deboli, in quel preciso territorio, esplicitando l'artificialità dell'operazione e la consapevolezza dello stato quo ante. La visibilità di tali segni è una modalità di radicalizzazione del progetto.

Il parco ed i suoi confini dovranno essere realizzati in modo da costituire un oggetto estroverso ovvero $i$ cui elementi di qualità sono visibili dall'esterno e lo coinvolgono. L'idea viene rafforzata se si realizzano confini fisici "trasparenti" ovvero che non sembrino tali.

Es.: All'origine Efteling nasce come Nature park ovvero come area boscata di nuovo impianto finalizzata ad aumentare il "grado di presenza di natura" nel territorio rurale fra Tilburg, Breda ed Hentogerbosch, e ad ospitare allo stesso tempo attività sportive e ricreative.

Conseguentemente:

il passaggio dentro-fuori del parco verrà segnalato consentendo dall'esterno la visibilità di uno o limitati simboli con funzione di comunicazione (landmark).

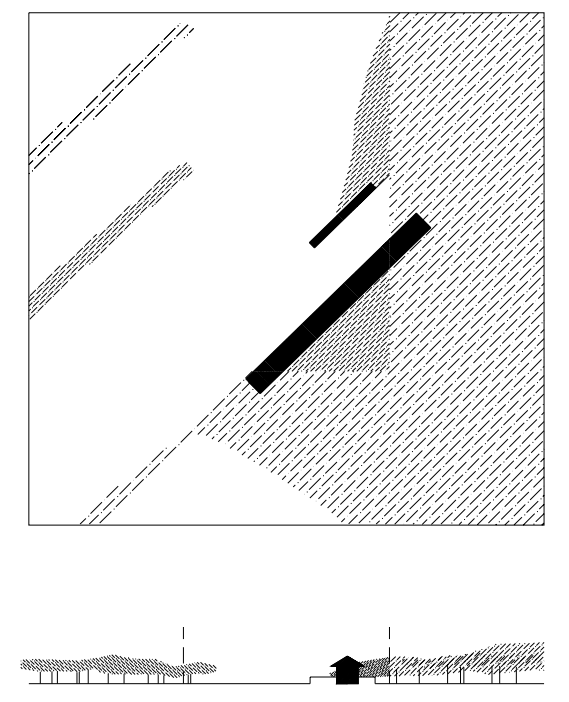

\section{Aspetti generali}

Non esistendo la necessità di ridurre al minimo la superficie di suolo, anzi volendo estendere i benefici del parco al maggior intorno possibile (compatibilmente ad esigenze di carattere economico):

Il parco può essere diffuso e contenere un elevato grado di naturalità per costituire nel senso più proprio un parco, unendo valori percettivi a valori ecologici.

[collabora anche alla progettazione della componente 2 TREGUA DAL TEMA] 
Per perseguire l'obiettivo di aumentare la connettività ecologica del contesto, valgono le indicazioni progettuali descritte per il parco in CONTESTO DI VALORE:
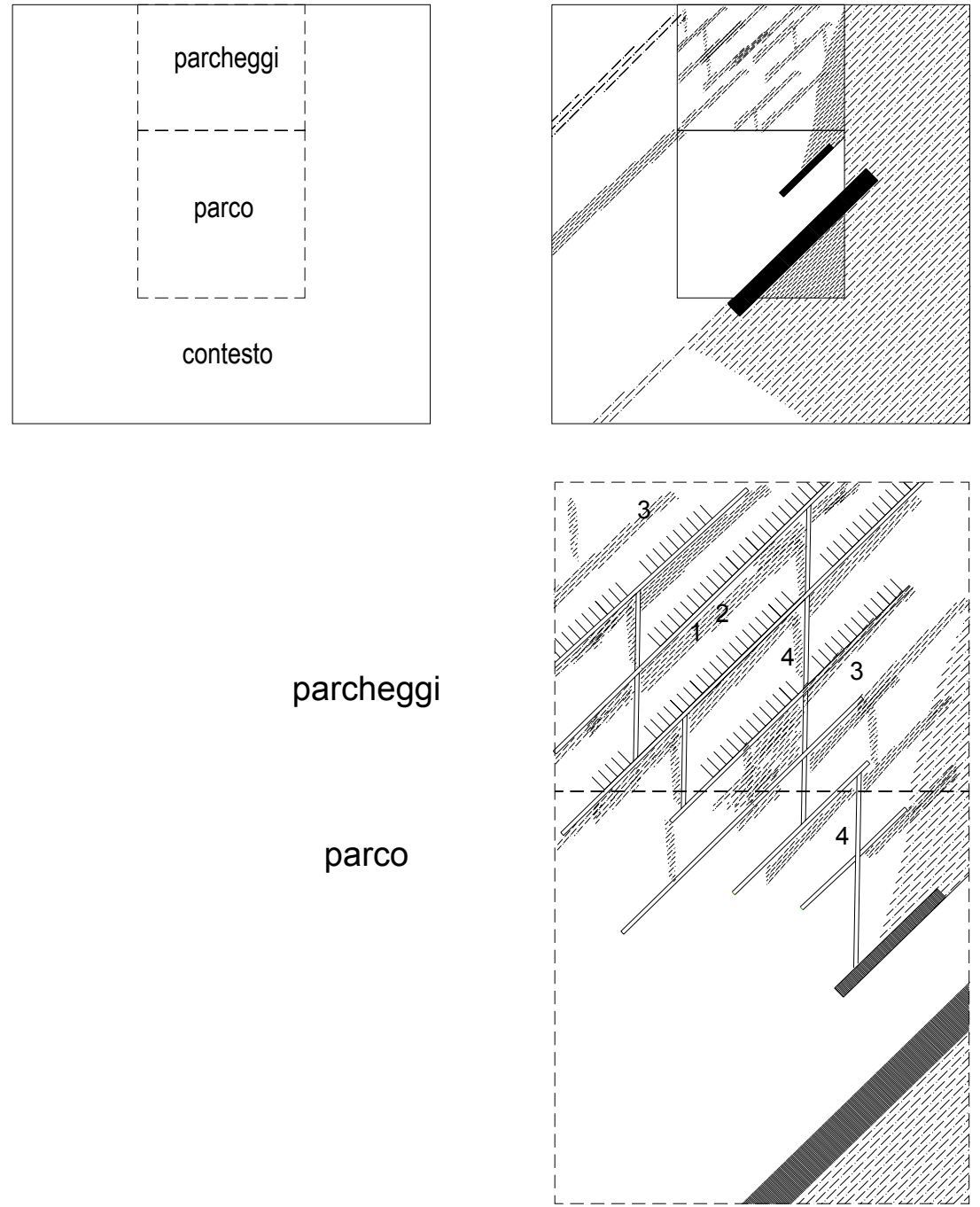

\section{TREGUA (dal tema)}

- Presenza nel parco di luoghi non tematizzati (parco neutro) per la sosta, il riposo, il gioco libero (la sezione seguente è parte della sezione del parco Efteling di TAV. II_2.3)

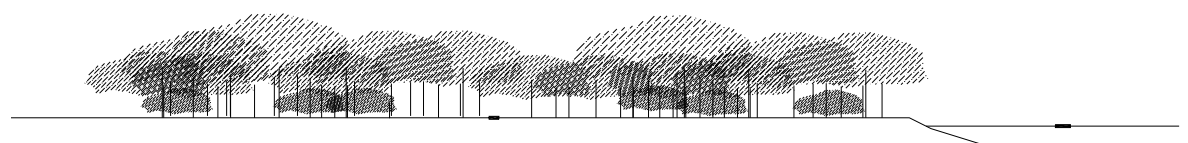

e/o in base alla disponibilità di spazio:

- Organizzazione planimetrica e prospettica per cui esistano punti del parco comunque tematizzati ma da cui si abbia una visione dei simboli delle altre aree del parco a 
distanza, rimpicciolendo percettivamente la loro scala e consentendone uno sguardo da lontano.

es.: esattamente in questo modo è progettata l'area Tonnerre de Zeus al Parc Asterix (la sezione seguente è tratta dalla sezione di Parc Asterix di TAV. II_2.1b); tutto il resto del parco appare lontano e segnato solo dalle istallazioni emblematiche, la visione è "pulita" dalla ridondanza di insegne e altro. Guardando il parco lontano, i simboli giganteschi dell'area rimangono alle spalle, per cui c'è tregua senza avere in realtà assenza di tematizzazione.

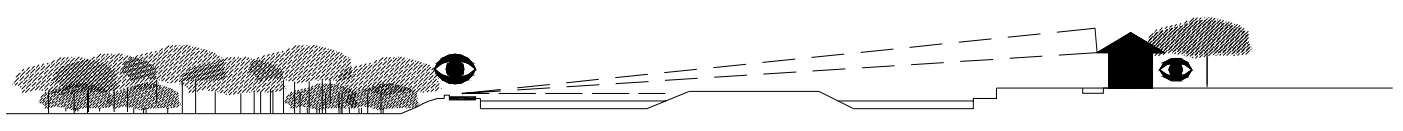

\section{- Presenza di luoghi neutri tematizzati solo temporaneamente ad opera e in occasione di spettacoli}

es.: la Mer de Sable nel parco omonimo: normalmente è un'estesa area di dune di sabbia, vuota, ma diventa tematizzata durante lo spettacolo che si tiene una volta al giorno "Il figlio di Orso Nero" (la sezione seguente è tratta dalla sezione di Mer de Sable di TAV. II_2.2a).

- Creazione di "opzioni prospettiche" all'interno dello spazio tematizzato: contemporaneamente possibilità di avere la prospettiva chiusa all'interno di uno spazio simbolico e, spostandosi di poco, di avere una prospettiva aperta verso luoghi non tematizzati.

E' possibile in particolare creare questa doppia opzione nelle aree tematiche che riproducono vie o spaccati di città, quali sono ad esempio la Via Antiqua e la Rue de Paris a Parc Asterix (Plailly- France).

L'espediente è importante soprattutto se l'ambientazione ospita, oltre che architetture, anche giardini tematici (al proposito si rimanda al punto Giardini).

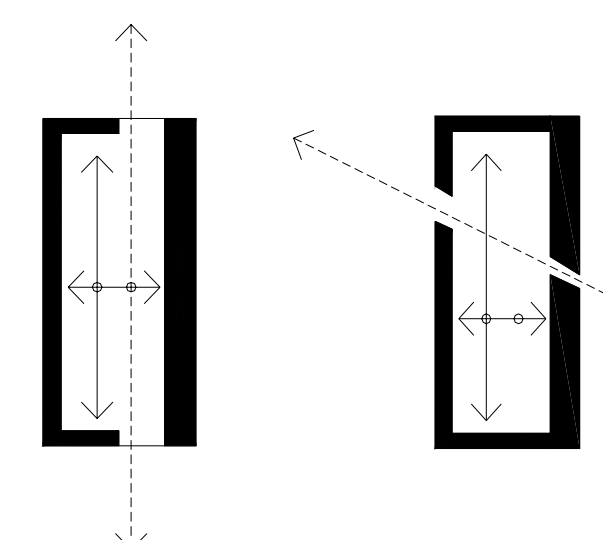

(in nero sono gli elementi-simboli: architetture, insegne, immagini, giardini tematici) 
3 GIARDINI (all'interno del parco)

All'interno dei parchi a tema, ancora più che in altri contesti, è necessario che il giardino sia un episodio d'arte e sia in un rapporto consapevole - e percepibile con chiarezza- con il paesaggio del contesto e con il tema del parco: possono contribuire alla presenza del contesto, alla creazione del tema o alla tregua dal tema.

I giardini non devono essere intesi quale casuale presenza di arbusti, composizioni floreali, come accade diffusamente nei contesti urbani in cui i "giardini" sono piuttosto aiuole: se così fosse costituirebbero un'intrusione di banalità all'interno di uno spazio che al contrario ha il fine di essere un luogo eccezionale. Da un lato non contribuirebbero alla costruzione del tema, dall'altro non creerebbero uno sfondo neutro contro cui i simboli possano a stagliarsi. [nei diagrammi seguenti in verde sono rappresentati gli elementi-simboli che creano il tema]

Il giardino deve essere:

- tematico, associato alle architetture, alle immagini alle insegne, al fine di costruire un ambiente tematico complessivo.

Oppure

- "neutro" nel senso di omogeneo rispetto al paesaggio, declinazione coerente di quest'ultimo, e slegato dal tema del parco.

\section{Ancora una volta è il contesto a suggerire indirizzi progettuali differenti:}

\section{In un CONTESTO DI VALORE}

Un paesaggio di valore, con un'identità già forte non necessita di inserzioni di elementi esotici, al contrario richiede la conservazione dei suoi elementi peculiari:

- tematico : possono essere progettati giardini tematici se il tema è anch'esso coerente con il paesaggio del contesto. (ad ess.: Foresta dei Druidi nella foresta di Ermenonville, del Parc Asterix, Plailly, France; Foresta delle Fiabe nella foresta di Kaaktsheuvel, del parco Efteling, Kaatsheuvel, Netherlands.)

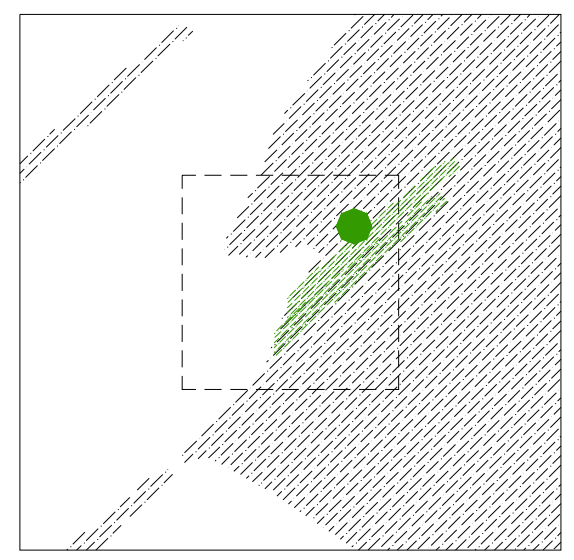


- "neutro": è più opportuno progettare giardini "neutri", se il tema del parco, o di una singola area tematica, non è coerente con il paesaggio del contesto; realizzare giardini tematici in questo caso implicherebbe interrompere, recidere, le caratteristiche peculiari del paesaggio esistente.

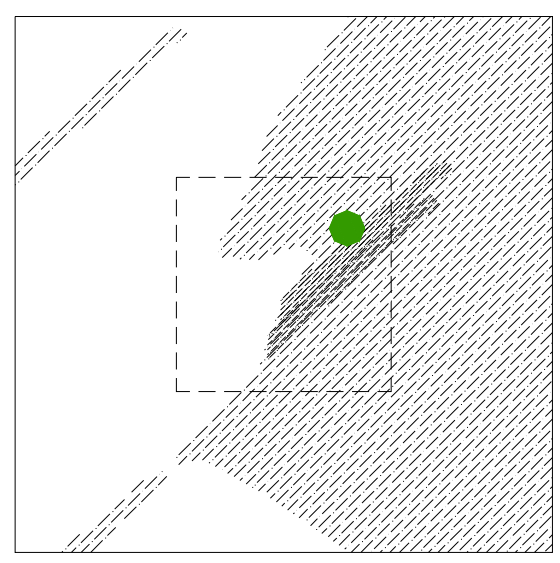

In un CONTESTO DA VALORIZZARE

- tematico, in contrasto con il paesaggio circostante, quindi "esotico" nell'accezione più ampia del termine.

Esistono due opzioni principali, che si descrivono a seguito. Per la scelta è ovviamente necessario comprendere e interpretare il "grado di debolezza" del contesto, ovvero verificare l'opportunità di progettare per inserzione di elementi altri rispetto al paesaggio esistente (1) o al contrario di "completare" questo ultimo con elementi omogenei (2).

(1) Il giardino tematico può coincidere con la totalità degli spazi verdi inseriti dal progetto, per conferire una nuova identità al paesaggio, esplicitando l'artificialità dell'operazione e dichiarando dove inizia e dove finisce il progetto.

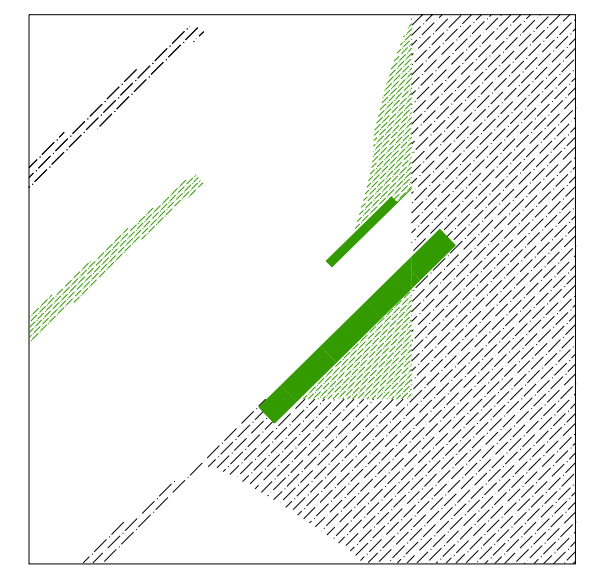

(2) Il giardino tematico può anche non coincidere con la totalità degli spazi verdi inseriti dal progetto: il progetto può da un lato, in modo quasi mimetico, completare e integrare il 
paesaggio esistente inserendo aree verdi omogenee rispetto ad esso, e dedicare porzioni limitate ai giardini tematici.

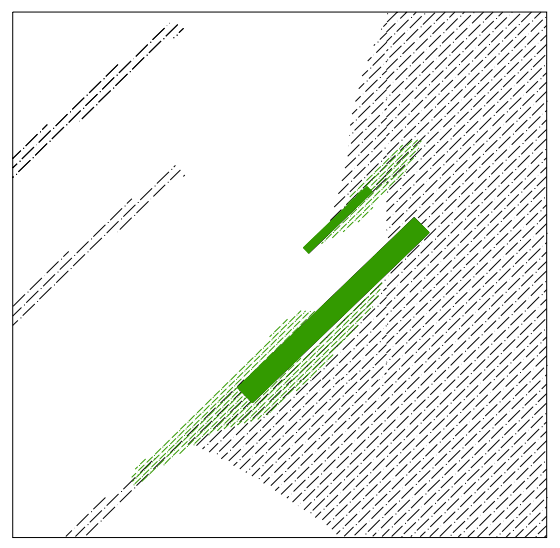

- "neutro" nel senso di omogeneo rispetto al paesaggio, derivazione coerente di quest'ultimo. I giardini diventano un modo per integrare e potenziare il paesaggio esistente, mediante l'utilizzo di elementi omogenei rispetto ad esso. Se il paesaggio esistente è molto debole, probabilmente questa soluzione non è la più opportuna: si rende necessario un progetto strong, per cui sarebbe preferibile che i giardini fossero tematizzati e non neutri, e collaborassero con gli altri elementi tematici al conferimento di un'immagine nuova al paesaggio.

Anche in questo caso sono possibili due opzioni:

(1) Il giardino "neutro" può coincidere con la totalità degli spazi verdi inseriti dal progetto esplicitando l'artificialità dell'operazione e dichiarando dove inizia e dove finisce il progetto.

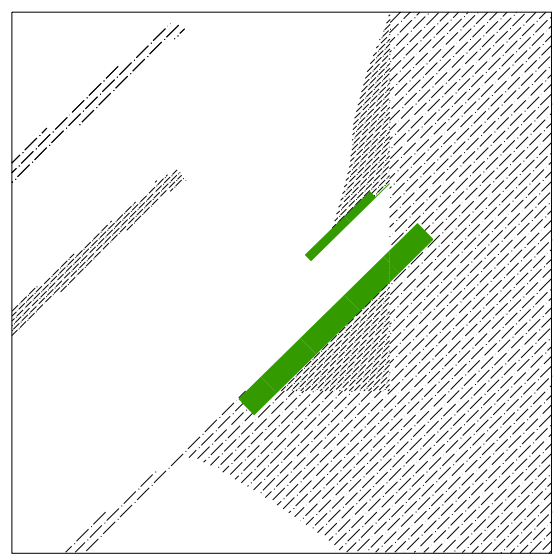

(2) Il giardino "neutro" può anche non coincidere con la totalità degli spazi verdi inseriti dal progetto: il progetto può da un lato, in modo quasi mimetico, completare e integrare il paesaggio esistente inserendo aree verdi omogenee rispetto ad esso, e dedicare porzioni limitate a giardino, comunque coerenti con il paesaggio. 


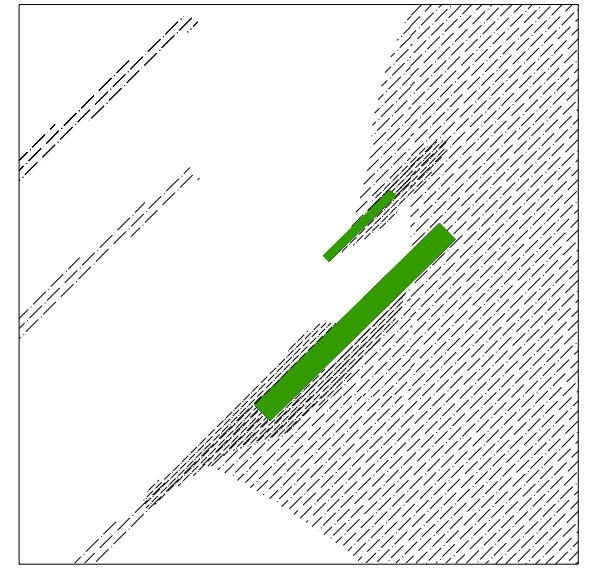

SINTETIZZANDO (paesaggio prima e dopo la progettazione del parco a tema, a scala territoriale):

CONTESTO DI VALORE

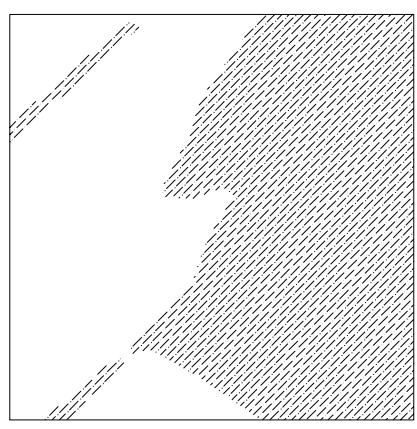

paesaggio esistente
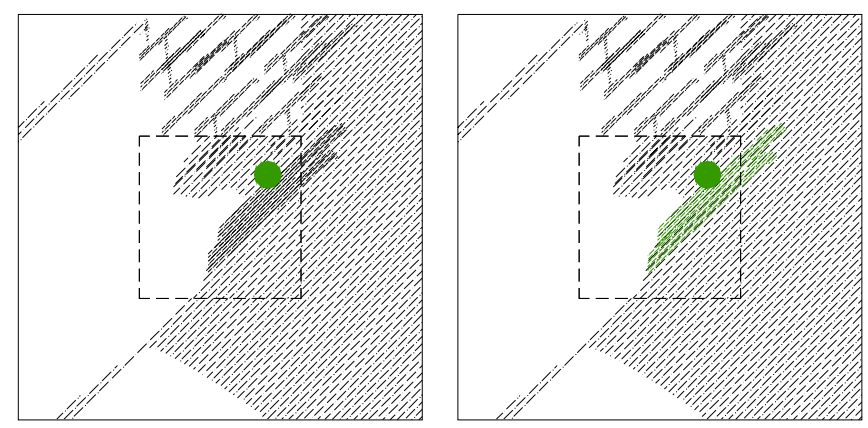

possibili paesaggi di progetto

CONTESTO DA VALORIZZARE

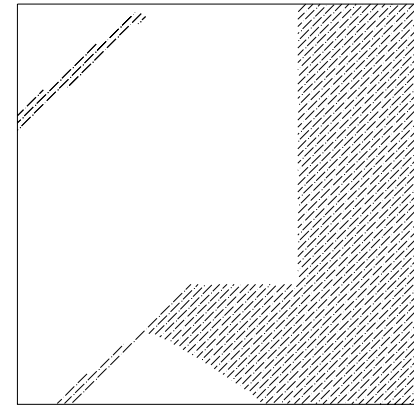

paesaggio esistente
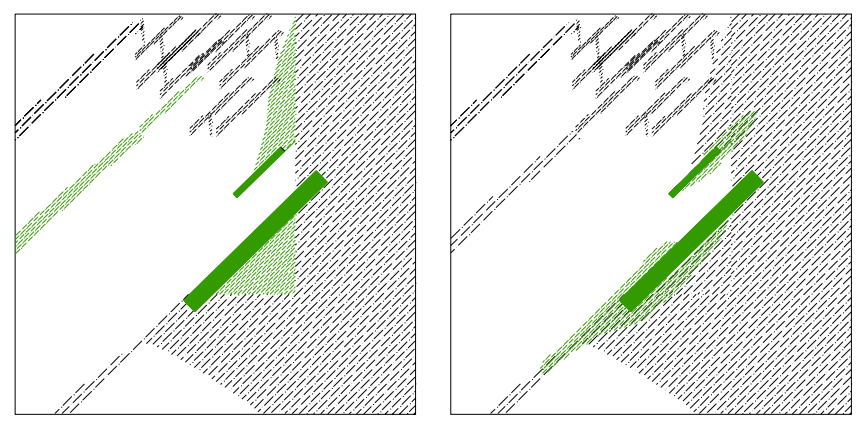

possibili paesaggi di progetto 


\section{BIBLIOGRAFIA GENERALE}

ACIDINI LUChINAT CRISTINA, L'Appennino del Giambologna: uomo , grotta, palazzo parte I, in AA.VV., Arte delle Grotte, Sagep Editrice, Genova 1987, pagg. 95- 100.

AIMONE LINDA, Le esposizioni universali, 1851-1900: il progresso in scena, Allemandi, Torino 1990.

Appelbaum Stanley, The New York World's Fair 1939/1940, Dover Publications, New York 1977.

AUgÉ MARC, Disneyland e altri nonluoghi, Bollati Boringhieri, Torino 2002.

AugÉ MARC, Nonluoghi, Elèuthera, Milano 1993.

AURICOSTE ISABELLE, I parchi ricreativi in Europa: il divertimento e l'altrove, in MONIQUE Mosser, GeORges TeYsSOT, L'Architettura dei giardini d'Occidente- Dal Rinascimento al Novecento, Electa, Milano 1990.

BANN STEPHEN, Giardino e arti visive. Arcadia, postclassico e "land art", in MOSSER Monique, Teyssot GeORGES, L'architettura dei giardini d'Occidente, Electa, Milano 1990, pagg. 491-502.

BANN STEPHEN, I giardini di Ian Hamilton Finlay, in Mosser MoniQue, TeYssot GEORGES, L'architettura dei giardini d'Occidente, Electa, Milano 1990, pagg. 518-520.

BANN STEPHEN, L'Arcadia di Finalay, "Lotus", 88, Electa, Milano 1996, pagg. 92-93.

BANN StePhen, Vivi ignorato: l'arte dei giardini di ian Hamilton Finlay, "Lotus", 88, Electa, Milano 1996, pagg. 94-131.

BARTHES Roland, Miti d'oggi, Einaudi, Torino 1974.

BARZILAY MARIANNE, HAYWARD CATHERINE, LOMBARD-VALENTINO LUCETTE, L'invention du parc: parc de la Villette: Paris, concours international 1982-1983, Graphite, Etablissement Public du Parc de la Villette 1984.

BAUd-BOvy MANUEL, LAWSON FRED, Tourism and Recreation Development, The Architectural Press LTD, London 1977.

BAUMAN ZYGMUNT, La società dell'incertezza, Il Mulino, Bologna 1999.

BECK GREGORY, Un atlante dei parchi tematici, in "Lotus" n. 109, Il nuovo mondo allegorico. Sui parchi tematici, Electa, Milano 2001, pagg. 104-117.

BÉDARIDA MARC, Tradizione francese e paradigma ecologico. Parc André Citroën, "Lotus international", n. 87, Electa, Milano 1995, pagg. 7-31.

BENJAMIN WALTER, L'opera d'arte nell'epoca della sua riproducibilità tecnica, Einaudi, Torino 1966 e 1991.

BONAZZI GABRIELE (a cura di), L'estetica nel pensiero contemporaneo (dal romanticismo al surrealismo), G. D’Anna, Firenze 1980.

Bonito Oliva ACHILle (a cura di), Avanguardia Transavanguardia, Electa, Milano 1982.

Bonito Oliva ACHILle, L'ideologia del Traditore - Arte, maniera, manierismo, Electa, Milano 1998.

BURCKHARDT LuCIOUS, Tendenze attuali dell'arte dei giardini, in "Domus" n. 817, Milano 1999.

CAlvesi Maurizio, Gli incantesimi di Bomarzo. Il Sacro Bosco tra arte e letteratura, Bompiani, Milano 2000.

CAPPIELlo Vito, Architettura contemporanea in Campania, "D'A", 13, pag. 34 e pagg. 3638.

CAPREtTini Gian PAOlo, Dal feeling allo spaesamento, in CASTELnOVi PAOLO, Il senso dle paesaggio, IRES Piemonte, Torino 2000.

CERVER Francisco ASENSIO, Landscape of Recreation II: Amusement Parks, (World of Environmental Design, V. 6), Watson-Guptill Pubns, Slipcase edition 1998.

CERVER FRANCISCO ASENSIO, Theme and amusement parks, Atrium International, Whitney Library of Design, 1997. 
Chaslin Francois, Parc de la Villette, Parigi, in "Domus" n. 817, Milano 1999.

Chiaramente Giovanni, Dall'apparenza alla visione, in "Ottagono", n. 99, giugno 1991, Intrattenimento, pagg. 31-37.

CHOAY FRANCOISE, L'allegoria del patrimonio, a cura di Ernesto d'Alfonso e Ilaria Valente, Officina Edizioni, 1995.

CONTI PITAGORA, Il giardino infantile, Manuali Hoepli, Milano 1892.

CORTESI ISOTTA, Il parco pubblico_paesaggi 1985-2000, Federico Motta, Milano 2000.

Cranz Galen, Il parco della riforma negli Stati Uniti, in MoniQue Mosser, GeORGES TEYSSOT, L'Architettura dei giardini d'Occidente- Dal Rinascimento al Novecento, Electa, Milano 1990, pagg. 462- 465.

CROISET PierRe Alain (a cura di), "Casabella", Il disegno degli spazi aperti, 597-598, Electa, Milano 1993.

De SolÀ Morales Ignasi, Il giardino Beaux -Arts, in Monique Mosser, GeORgeS TEYSSOT, L'Architettura dei giardini d'Occidente- Dal Rinascimento al Novecento, Electa, Milano 1990, pagg. 395- 404.

Desideri PaOlo, Ilardi Massimo (a cura di), Attraversamenti. I nuovi territori dello spazio pubblico, Costa \& Nolan, Genova 1997.

Desvigne Michel, Dalnoky Christine, Il ritorno del paesaggio, Federico Motta, Milano, 1996.

Donin GianPIERO, Parchi, Biblioteca del Cenide, Cannitello (RC) 1999.

ECo UMBerto, Dalla periferia dell'impero. Cronache da un nuovo medioevo, Bompiani, Milano 2003.

ECO UMBERTO, Travels in Hyperreality, Harcourt Brace Jovanovich, U.S.A. 1990.

EISNER D. MICHAEL (foreword by), Imagineering. A Behind the Dreams Look at Making the Magic Real, Disney Editions, New York 1996.

FABRIS LUCA MARIA FRANCESCO, Il verde postindustriale, Liguori Editore, Napoli 1999.

FIndlay John M., Magic Lands, Western City scapes and America Culture after 1940, University of California Press, Berkeley 1993.

FRAMPTON KENNETH, Storia dell'architettura moderna, Zanichelli, Bologna 1998.

FriedBerg PAUL, et al., Il parco del XX secolo, "Casabella", n. 492, Electa, Milano1983, pagg. 12-23.

GEUZE ADRIAAN, Nuovi parchi per nuove città, "Lotus international", 88, 1996, pagg. 5055.

GIANNINI RITA, GUERRA TONINO, URBINATI GIOVANNI , Il giardino pietrificato, Ramberti Arti Grafiche, Rimini.

GIANNINI RITA, Tonino Guerra e la sua valle, Raffaelli Editore, Rimini 1998.

Gravagnuolo BenedetTA, Architetture scolpite con la luce, "Controspazio", n. 6, Gangemi Editore, Roma 1994, pagg 34-36.

GRAVAGNUOlo BenEDETTA, Hortus Infinitus, “Architettura e Arte”, 3, 1998, pagg. 58-61.

GuERra TONINO, Con la poesia alle spalle, Edizioni Tipertì - Galleria Fabjbasaglia, Rimini 2001.

Guerra TONINO, Dizionario fantastico, Pietroneno Capitani, Rimini 2000.

GuERra TONINO, Il libro delle chiese abbandonate, Maggioli Editore, Rimini 1988.

Guerra Tonino, Il Polverone, Maggioli Editore, Rimini 1992.

GUERRA TONINO, Il Viaggio, Maggioli Editore, Rimini 1986.

HAMON PHILIPPE, Esposizioni: letteratura e architettura nel XIX secolo, Ed. It. Maurizio Giuffredi (a cura di), CLUEB, Bologna 1995.

HARGREAVES GEORGES, Furrows. The shapes of recycled landscape, in "Quaderns" n. 217, Barcelona 1997.

Hortus conclusus. Almanacco dell'Architettura Italiana, Electa, Milano 1993, pagg. 62-63.

Howett CATHERIN, Attrezzature ricreative negli Stati uniti( 1930-1940), in MONIQUE Mosser, GeORges Teyssot, L'Architettura dei giardini d'Occidente-Dal Rinascimento al Novecento, Electa, Milano 1990, pagg. 506-508.

Hunt DiXOn JHON, The Picturesque Garden in Europe, Thames and Hudson, London 2002. 
Hunt Dixon JHON, The theatres, gardens, and Gardens Theatres, in JHON DIXON HunT, Gardens and the Picturesque, The MIT Press, Cambridge (Mass.) 1992, pagg. 49-73.

HUNT DIXON JHON, Vauxhall and London Garden Theatres, Chadwyck-Healey in association with the Consortium for Drama and Media in Higher Educations, Cambridge 1985.

Hunt Dixon JHon, Willis PETER (edited by), The Genius of the Place. The English Landscape Garden 1620-1820, The MIT Press, Cambridge (Mass.) 1997.

HUNT JOHN DIXON, "Ut pictura poesis: il giardino e il pittoresco in Inghilterra (17101750), in MONIQUe Mosser, GeORges TeYsSOT, L'architettura dei giardini d'Occidente, Electa, Milano 1990, pagg. 227-237.

Il tappeto sospeso - fontana monumentale, catalogo, Maurizio Montanari, Ravenna 1997.

INGERSOLL RICHARD, Jampcut Urbanism, in "Casabella" n. 597-598, Electa, Milano 1993, pagg. 96-97

ISHERWOOD Robert M., Farce and fantasy. Popular entertainment in eighteenth-century Paris, Oxford University Press, Oxford 1986.

JACKSON JOHN B., Architettura vernacolare, in "Casabella" n. 680, Electa, Milano 2000.

JACKSON JOHN B., Discovering the Vernacular Landscape, Yale University Press, 1984

KASSON F. JoHn, Amusing the million. Coney Island at the turn of the Century, Hill \& Wang, New York 1978.

KIENAST DiETER, Un decalogo, "Lotus international”, n. 87, Electa, Milano 1995, pagg. 6365 .

Koening David \& Laura, Mouse under Glass: Secrets of Disney Animation \& Theme Parks, Bonaventure, 1997.

KoOlHaAs REM, The Generic City, in S,M,L,XL, Taschen, Germany 1997, pagg. 1239-1267. LANGloIs GILles-AnTOINE, Folies Tivolis et attractions. Les premiers parcs de loisirs parisiens, Délégation à l'action artistique de la ville de Paris, Paris 1991.

LiAO ALICE, Hortus Conclusus, in "www. Lightforum.com/magazine", 2000-2001.

LOCATELl ViTTORIO, Le architetture parlanti di Tonino Guerra, "АNАГКН" , n. 4, Alinea Editrice, Firenze 1993.

LYOTARD JEAN-FRANCOIS, la condizione postmoderna, Feltrinelli, Milano 1981.

Maling Karal Ann, Designing Disney's Theme Parks: The Architecture of Reassurance, Flammarion, Paris 1998.

MarCEllo FAgIOLO, Natura e artificio, Officina Edizioni, Roma 1997.

MARESCA PAOLA, Boschi sacri e giardini incantati, Angelo Pontecorboli Editore, Firenze1997.

MATTIE ERIK, World's Fair, Princeton Architectural Press, New York City 1998.

Mello PATRIZIA, Metamorfosi dello Spazio. Annotazioni sul divenire metropolitano, Bollati Boringhieri, Torino 2002.

MinARDi EVERARDO, LuSETTI MARIALUISA (a cura di), I parchi del divertimento nella società del Loisir, Franco Angeli, Milano 1998.

MOSSER MONIQUE, Le architetture paradossali ovvero piccolo trattato sulle "fabriques", in MoniQue Mosser, GeORges TeYssot, L'Architettura dei giardini d'Occidente- Dal Rinascimento al Novecento, Electa, Milano 1990, pagg. 259-276.

MotT TONY, Giardini e fotografia, in Monique Mosser, Georges Teyssot, L'architettura dei giardini d'Occidente, Electa, Milano 1990, pagg. 491-502.

MUNARI BRUNO, Fantasia, Universale Laterza, prima ed. 1977, Roma-Bari 1998, NeONATO FrancesCA, Un recinto sacro, “Acer”, n. 6, Il Verde Editoriale, Milano 2001, pagg.32-35.

NiCOLIN PIERLUigi, Architettura "light", in "Lotus" n. 105, 2000

ORVELl MiLES, After the machine. Visual arts and the erasing of cultural boundaries, University Press of Mississippi, USA 1995.

ORVELl MiLES, The real thing. Imitation and authenticity in American culture, 1880-1940, The University of North Carolina Press, Chapel Hill \& London 1989. 
PANSERA ANTY, VitTA MAURIZIO, Guida all'Arte contemporanea, Marietti, Casale Monferrato 1986.

PANZINI FRANCO (a cura di), Giardini di Tonino Guerra - Pennabilli (Pesaro) in Giardini delle Marche, Federico Motta, Milano 1998.

PANZINI FRANCO, Per i piaceri del popolo. L'evoluzione del giardino pubblico in Europa dalle origini al XX secolo, Zanichelli, Bologna 1997.

PIZZETTI IPPOLITO, Spazi rifiuto, spazi-scoria, spazi-scarto, "Casabella", n. 597-598, Electa, Milano1993, pagg. 96-97.

PIZZETTI IPPOLITO, Un parco è un parco è un parco è un parco. Il concorso de La Villette o nostalgia della piazza, "Spazio e Società", n. 25, 1984, pagg. 34-51.

PONTE ALESSANDRA, Il parco pubblico in Gran Bretagna e negli Stati Uniti. Dal genius loci al "genio della civilizzazione", in MONIQUe Mosser, GEORGES TEYSSOT, L'Architettura dei giardini d'Occidente- Dal Rinascimento al Novecento, Electa, Milano 1990, pagg. 369382.

PUPPI LIONELlo, Natura, artificio, inganno. Il giardino in Italia nel Cinquecento: temi e problemi, in MONIQUe MOSSER, GEORGES TEYSSOT, L'Architettura dei giardini d'Occidente- Dal Rinascimento al Novecento, Electa, Milano 1990.

RAFFESTIN Claude, Il paesaggio introvabile, in PAOlo CASTELnOVI (a cura di), "Il valore del paesaggio", Contributi al seminario internazionale, Torino, 9 giugno 2000.

REBORI STEPHEN, Disney and the Development of Vacation Destination Resorts ( $\mathrm{Cpl}$ Bibliography, No 320).

REBORI STEPHEN, The Influence of Disney Entertainment Parks on Architecture and Development (Cpl Bibliography, 321).

REBORI STEPHEN, Theme \& Entertainment Parks: Planning, design, Development \& Management, CPL Bibliographies, 1995.

Rizzo Givlio G. (a cura di), Roberto Burle Marx. Il giardino del Novecento, introduzione di Lionello Puppi, catalogo della mostra, Pistoia 28 ottobre 1992-10 gennaio 1993, Cantini, FIRENZE 1992.

RoCCA AlESSANDRO, L'interperiferica di Chemetov, "Lotus International", n.84, Electa, Milano1995, pagg. 89-101.

Roggero Mario F. (a cura di), Il parco e la città. Sette progetti italiani per Parigi-La Villette: Aymonino, BBPR, Dierna, GRAU, Pellegrin, Purini, Roggero, Alinea, Firenze 1986.

ROLAND Michel MARIANNE, Scenografia e prospettiva nei giardini francesi del Settecento, in Monique Mosser, Georges Teyssot, L'Architettura dei giardini d'Occidente- Dal Rinascimento al Novecento, Electa, Milano1990, pagg. 239-248.

Sleiter Rossella, L'orto di Tonino, "Il Venerdì di Repubblica" del 14 luglio 1989.

SORKIN MICHAEL (edited by), Variations on a theme park. The new American city and the and of public space, Hill and Wang, New York 1999, pagg. 205-232.

STANTON JEFFREY, Early Coney Islands History (1609-1880), Early Coney island History (1881-1903), Amusement Industry, Coney Island - Rides \& Shows List, Coney island Transportation, in www.naid.sppsr.ucla.edu/coneyisland/histart.htm

TAgLIOLINI Alessandro, Storia del giardino italiano, La casa Usher, Firenze 1988.

TESSARI ROBERTO, Il mercato delle Maschere, in RoBerTo AlOnge, GUIDO DAVICO BONINO (diretta da), Storia del teatro moderno e contemporaneo, volume primo, La nascita del teatro moderno. Cinquecento- Seicento, Einaudi, Torino, 2000, pag. 119.

TESTA FAUSTO, Spazio e allegoria nel giardino manierista, La Nuova Italia, Firenze 1991.

Tosetto Danilo, Parchi Ricreativi nel Mondo, Vol. 1, Facto, Padova 1986.

Tosetto Danilo, Parchi Ricreativi nel Mondo, Vol. 2 , Facto, Padova 1987.

Tosetto Danilo, Parchi Ricreativi nel Mondo, Vol. 3 , Facto, Padova 1988, pagg. $28-45$.

Tosetto Danilo, Parchi Ricreativi nel Mondo, Vol. 4 , Facto, Padova 1989.

Tosetto Danilo, Parchi Ricreativi nel Mondo, Vol. 5 , Facto, Padova 1990, pagg. 36- 55.

VANDEN DiePSTRaten H., De Efteling kroniek van een Sprookje, De Efteling B.V.

Kaatsheuvel 2002. 
VENTURI ROBERT, Complessità e contraddizioni nell'architettura, Dedalo, Bari 1980.

Venturi Robert, Scott Brown Denise, IzenUor StePhen, Imparando da Las Vegas. Il simbolismo dimenticato della forma architettonica, CLUVA editrice, 1985.

VERGINE LEA, L'arte in trincea - Lessico delle tendenze artistiche 1960- 1990, Skira, Milano 1996.

VÉRIN HÉLÈN, La tecnologia nel parco: ingegneri e giardinieri nella Francia del Seicento, in Monique Mosser, GeOrges Teyssot, L'Architettura dei giardini d'Occidente- Dal Rinascimento al Novecento, Electa, Milano1990, pagg. 131-142.

VEZZOSI ALESSANDRO (a cura di), Il concerto di statue, Alinea, Firenze 1986.

Vezzosi Alessandro (a cura di), Il giardino d'Europa. Pratolino come modello nella cultura europea, Mazzotta, Firenze 1986.

Walker Peter, Simo Melanie, Invisible Gardens. The search for Modernism in the American Landscape, The MIT Press, Cambridge, Massachusetts 1996.

WYLSON ANTHONY \& PATRICIA, Theme Parks, Leisure Centres, Zoos and Aquaria, Longman, 1994.

ZANGHERI LUIGI, Pratolino, il giardino delle meraviglie, Firenze 1987.

ZANGHERI LUIGI, Naturalia $e$ curiosa nei giardini del Cinquecento, in MONIQUE Mosser, GEORgES TEYSSOT, L'Architettura dei giardini d'Occidente- Dal Rinascimento al Novecento, Electa, Milano 1990, pagg. 55-64.

ZANGHERI LUIGI, Suggestioni e fortuna dei teatrini d'automi. Pratolino come una Broadway manierista, in "Quaderni di teatro", Vii, 25, 1984, pagg. 78-84.

ZANGHERI LUIGI, Tra abilità e tecnologia. l'acqua nelle grotte del manierismo, in AA.VV., Arte delle Grotte, Sagep Editrice, Genova 1987, pagg. 9- 14.

Numeri monografici di riviste, attinenti al tema:

"Casabella", n. 673/674, U.S.A., Architettura come spettacolo, Electa, Milano dicembre 1999- gennaio 2000.

"Domus", n. 787, Milano 1996.

"Lotus Navigatore", n. 2, Nuovi paesaggi, Electa, Milano2001.

"Lotus" n. 109, Il nuovo mondo allegorico. Sui parchi tematici, Electa, Milano 2001.

"Ottagono", n. 99, Intrattenimento, giugno 1991.

"Paesaggio Urbano", n. 5, Parchi di divertimento, Maggioli Editore, Rimini 1993.

Siti web:

A History of the fair in www. xroads.virginia.edu/ MA96/WCE/title

freeoise.free.fr/communes/lettree/ermenon/pglois.html

Hortus Conclusus, in www. Comune .benevento.it/Turismo/ Hortus.

Hortus Conclusus, in www. Francescomorante.it/pag_3/318ab.

$\mathrm{http} / / /$ freizeitparkweb.de/html/links.html?page=no

http://guide.supereva.it/parchi_di_divertimento/link_ai_parchi_in_europa/

$\mathrm{http} / / /$ home.foni.net/ helenelangegymn/news_sptime 25 .htm

http://jacketmagazine.com/15/rash-iv-finlay.html

http://stuartcollection.ucsd.edu/finlay/bio.html

http://stuartcollection.ucsd.edu/finlay/unda6.gif

http://tourisme.voila.fr/villes/stdenis/fra/sit/ermenonv/mersable/acc.htm

http://web.tiscali.it/no-redirect-tiscali/theparks/pks_europa/pks_tema_europa.htm

http://www.efteling.nl/docs.en/park/nav/index.html

I luoghi dell'anima in WWW.montefeltro.net/pennabilli.htm

Interactive Guide to the World's Columbian Exposition in

www.users.vnet.net/schulman/Columbian/columbian.html

Le Jardin d'Acclimatation, in www.jardindacclimatattion.fr/pages/histoireJardin

Mimmo Paladino, in www. Francescomorante.it/pag_3/318a.

Post modern, in www. Francescomorante.it/pag_3/318.

Post modern, in www. Francescomorante.it/pag_3/318. 
The Chicago Columbian Exposition in www.chicagohs.org/history/expo.html The Chicago Columbian Exposition of 1893 in

www.park.org/Pavilions/WorldExpositions/chicago.text.html

The story of Bakken, in www.bakken.dk/engelsk $/ \mathrm{htm} / \mathrm{sider} / \mathrm{history}$

The World's Columbian Exposition - Chicago 1893 in

www.chicagohs.org/AOTM/May98/may98fact3

The World's Columbian Exposition in 1893 in www.columbus.gl.iit.edu/

www. tivoligardens.com

www.123world.com/amusement/

www.achus.net/achus/europa

www.aquarium-bbs.de/zoo/europa/zoo-euro.htm

www.attentialpupo.com/tempoliberoevacanze/mappaparchi/mappaparchi.htm

www.aventuriers.com/fr/france/picardie/60_dec_lamerdesable/index.shtml

www.bambinopoli.it/scripts/bimbbase.exe?DET $=1 \&$ IDREC $=904$

www.chantilly-tourisme.com/francais/loisirs parcs_loisirs.php

www.chantilly-tourisme.com/francais/loisirs_parcs_loisirs.php

www.chez.com/bienvenueatoulouse/cite12.jpg

www.cichw.net/pmvaux $2 . \mathrm{html}$

www.dreammingitaly.com/parchi.htm

www.dundee.ac.uk/transcript/volume2/issue2_1/finlay.htm

www.funguide.com/

www.geocities.com/Colosseum/Sideline/7510/eurele.html

www.georgetown.edu/faculty/murphyd/netsearch/theme.html

www.germancoaster.de/parks-europa.html

www.girobimbi.it/parchi_divertimento/fr_rg_italia_div.html

www.infoparchi.com/ipTematici/

www.kidzprintz.com/safetylinks/just4kids/themeparks.html

www.merdesable.fr/mer-de-sable/

www.parcasterix.fr

www.parisce.com/sable.htm

www.parksmania.it/eurpark.html

www.pegacity.it/viaggi/parksmania/waterpk.html

www.sordionline.com/parco_parchi_europei.htm

www.sordionline.com/parco_parchi_italiani.htm

www.themeparkcity.com/EURO_index.htm

www.themeparkinsider.com/europe/

www.theparks.it/pks_europa/

www.victorianlondon.org/entertainment/vauxhallgardens.htm

www.virtualpark.org/parchi_eur.htm

www.zeal.com/category/preview.jhtml?cid $=548667$

www.zoo-ag.de/ausland.htm

www.zoo-archiv.de/slg_west.htm 


\section{RIFERIMENTI ICONOGRAFICI NELLE TAVOLE}

TAV. I_1.1a:

Planimetria da Pianta dei due Barchi, Viali, Fontane e Fabbriche della Real Villa di Pratolino di B.S. Sgrilli (1742) e da un rilievo eseguito nel 1962

in Monique Mosser, Georges Teyssot, L'Architettura dei giardini d'Occidente- Dal Rinascimento al Novecento, Electa, Milano 1990, pag. 23.

particolari della "Veduta dell'intero parco di Pratolino da Sud", anteriore al 1590, in S. Vitale, "Ad Annales Sardiniae", Firenze 1639, in VeZzosi AlesSANDRO (a cura di), Il concerto di statue, Alinea, Firenze 1986, pag. 104.

"Il viale degli Zampilli" o "delle fontane" oppure "dell'Arcobaleno", o "d'Iride", di Stefano Della Bella, incisione c. 1653,

in VezZOSi AlessANDro (a cura di), Il concerto di statue, Alinea, Firenze 1986, pag. 129.

Il "Mugnone" del Giambologna a Pratolino,

in VezZosi Alessandro (a cura di), Il concerto di statue, Alinea, Firenze 1986, pag. 109.

"Planimetria delle grotte della Villa di Pratolino" di Heinrich Shickardt, c.1600., in VezZOSI AlessandRo (a cura di), Il concerto di statue, Alinea, Firenze 1986, pag. 111.

"La Reale Villa di Pratolino" disegno di Giuseppe Zocchi, anteriore al 1744, in VeZzosi AlessANDRO (a cura di), Il concerto di statue, Alinea, Firenze 1986, pag. 107.

Sezione longitudinale dell'Appennino

in VeZZOSI ALESSANDRO (a cura di), Il concerto di statue, Alinea, Firenze 1986, pag. 74.

statua di "Giove" di Baccio Bandinelli, c. 1552,

in VeZzosi AlESSANDRO (a cura di), Il concerto di statue, Alinea, Firenze 1986, pag. 103.

TAV. I_1.1b:

Planimetria da Pianta dei due Barchi, Viali, Fontane e Fabbriche della Real Villa di Pratolino di B.S. Sgrilli (1742) e da un rilievo eseguito nel 1962

in Monique Mosser, Georges Teyssot, L'Architettura dei giardini d'Occidente- Dal Rinascimento al Novecento, Electa, Milano 1990, pag. 23.

"Grotta di Cupido a Pratolino", di Heinrich Shickardt ,1600

in VEZzOSI ALESSANDRO (a cura di), Il concerto di statue, Alinea, Firenze 1986, pag. 134.

Particolare della "Veduta dell'intero parco di Pratolino da Sud", anteriore al 1590

in S. Vitale, "Ad Annales Sardiniae”, Firenze 1639,

in Vezzosi Alessandro (a cura di), Il concerto di statue, Alinea, Firenze 1986, pag. 104.

Ricostruzione della fontana dell'Ammannati a Pratolino, veduta frontale, disegno di Marcella Giuntini e Ricciarda Malaspina, in VeZZOSI AlesSANDRO (a cura di), Il concerto di statue, Alinea, Firenze 1986, pag. 31. 
TAV. I_1.2a:

Pianta di Vauxhall del 1826

in PANZINI FRANCO, Per i piaceri del popolo. L'evoluzione del giardino pubblico in Europa dalle origini al XX secolo, Zanichelli, Bologna 1997, pag. 103.

veduta dei Vauxhall Gardens, c. 1751

in PANZINI FRANCO, Per i piaceri del popolo. L'evoluzione del giardino pubblico in Europa dalle origini al XX secolo, Zanichelli, Bologna 1997, pag. 101.

TAV. I_1.2b:

Pianta di Vauxhall del 1826

in PANZINI FRANCO, Per i piaceri del popolo. L'evoluzione del giardino pubblico in Europa dalle origini al XX secolo, Zanichelli, Bologna 1997, pag. 103.

Vauxhall Gardens (1784), disegno di Thomas Rowlandson, Victoria and Albert Royal Museum

in www.wwnorton.com/nael/18century/topic_1/illustrations/imvauxhall.htm

particolare della veduta dei Vauxhall Gardens, c. 1751

in PANZINI FRANCO, Per i piaceri del popolo. L'evoluzione del giardino pubblico in Europa dalle origini al XX secolo, Zanichelli, Bologna 1997, pag. 101.

TAV. I_1.2c:

Pianta di Vauxhall del 1826

in PANZINI FRANCO, Per i piaceri del popolo. L'evoluzione del giardino pubblico in Europa dalle origini al XX secolo, Zanichelli, Bologna 1997, pag. 103.

Grand South Walk,

in www.ggdc.org/mp\%20-aavauxhall_gardens.htm

TAV.I_1.3:

Planimetria del Midway Pleasance e fotografie in http://columbus.gl.iit.edu/

TAV.I_1.4:

Mappa della Santa Cruz Boardwalk

in www.beachboardwalk.com/03_directions_wmap.html

Fotografie di Enrica Dall'Ara

TAV.I_1.5:

Planimetria di Disneyland

in EISNER D. MICHAEL (foreword by), Imagineering. A Behind the Dreams Look at Making the Magic Real, Disney Editions, New York 1996, pag. 15. 
Fotografie di Enrica Dall'Ara

Studio comparativo delle altezze dei castelli Disney per Anaheim, Orlando, e Disneyland Paris, disegno di Ahmad Safari

in MaLing Karal ANN, Designing Disney's Theme Parks: The Architecture of Reassurance, Flammarion, Paris 1998, pag. 91- illustrazione 77.

Tomorrowland, disegno di Herbert Ryman, 1954 in

in MALING KARAL ANN, Designing Disney's Theme Parks: The Architecture of Reassurance, Flammarion, Paris 1998, pag. 67- illustrazione 50.

TAVV. II_2.1:

Mappe in www.parcasterix.fr

Fotografie di Enrica Dall'Ara

TAVV. II_2.2:

Mappe in www.merdesable.fr/mer-de-sable

Fotografie di Enrica Dall'Ara

TAVV. II_2.3:

Mappa ufficiale di Efteling, 2003

Fotografie di Enrica Dall'Ara 
SCHEDA ANALITICA DEI PARCHI DEL DIVERTIMENTO EUROPEI in grigio: $\mathrm{i}$ parchi tematici

nelle ATTRAZIONI
"kid"." giochi per bambin

lkid": : giochi per bambini
family tun: atrazioni ier tutta la famiglia
Ferris Wheel: ruota panoramica

PARCO

COORDINATE GEOGRAFICHE

ORIGINE STORICA

CONTESTO/PAESAGGIO

area ATTRAZIONI

TEMA

MATRICE

RUOLO DEL TEMA

PROGETTA-
ZIONE DEL
PAESAGGIO

\begin{tabular}{|c|c|c|c|c|c|c|c|c|c|}
\hline Familienland Erlebnispark & Mühau, Austria & 1 & Iocallta alpina- Triolo & ' & indes - 200 okid" & $\begin{array}{l}\text { miscellanea (singole atrrazioni } \\
\text { debolmente) }\end{array}$ & amusement park & SCENOGRAFICO DEBOLMENTE & (?) \\
\hline Minimundus & Klagenfurt, Austria & 1976 & $\begin{array}{l}\text { in Carinzia, in territionio extraurbano, Ingo lautostrada } \\
\text { A2, a Nord di Klagenfurt }\end{array}$ & $26.000 \mathrm{mq}$ & 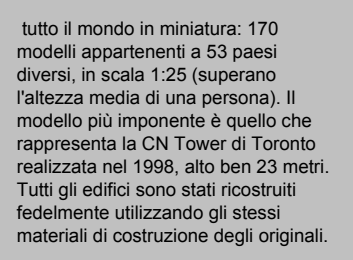 & tutto il mondo in mianiatura & esposizioni universali & $\begin{array}{l}\text { DETERRITORIALZZZAZIONE/ } \\
\text { DIACRONIZZAZIONE }\end{array}$ & $\mathbf{x}$ \\
\hline Prater Park & Vienna, Austria & 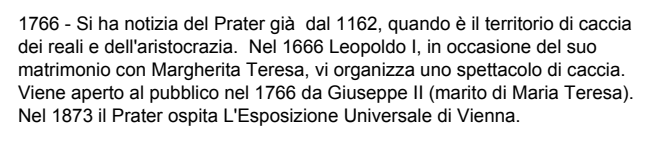 & $\begin{array}{l}\text { parco urbano di Vienna, in unisola del Danubio con } \\
\text { foreste, giardini, vasti prati }\end{array}$ & & $\begin{array}{l}\text { ridese eatrrazioni varie (rra cuil a Ferris } \\
\text { Wheel e caroselli- - sale per spettacoli- } \\
\text { tetnnis club - museo sulla storia del } \\
\text { parco. }\end{array}$ & miscellanea ( singole attrazioni) & $\begin{array}{l}\text { pleasure garden - esposizioni } \\
\text { universali }\end{array}$ & "AFFACCIO" & $\mathrm{x}$ \\
\hline \multirow[t]{2}{*}{ Styrassic } & Bad Glichenberg, Austria & 1999 (?) & I & $50.000 \mathrm{ma}$ & percorsis nella foresta con dinosauri & dinosauri & (?) & DIDATTICO & $X(?)$ \\
\hline & Belgium & & & & & & & & \\
\hline Bellewaerde & Westhoek, Belgium & 1990 - catena SIX FLAGS in Europa. & 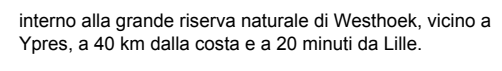 & ' & rides e atrrazioni varie - zoolrisenva & $\begin{array}{l}\text { miscellanea ( singole atrrazioni } \\
\text { debolmente) }\end{array}$ & amusement park & SCENOGRAFICO DEBOLMENTE & (?) \\
\hline Bobbejaanland & Lichtaart, Belgium & $\begin{array}{l}1975 \text { - realizzato per iniziaitiva del cantante Bobbejaan Schoepen, dove } \\
\text { inizialmente era solo una palude. }\end{array}$ & 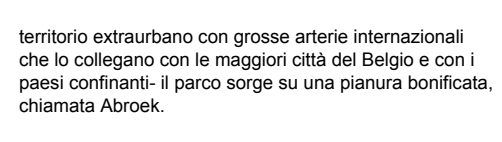 & ' & 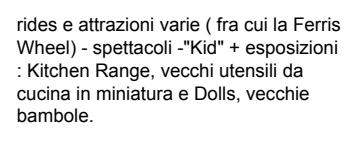 & miscellanea ( singole attrazioni) & $\begin{array}{l}\text { amusement park - esposizioni } \\
\text { universali }\end{array}$ & SCENOGRAFICO DEBOLMENTE & $x$ \\
\hline Boudewijnpark & Brugge, Belgium & 1 & 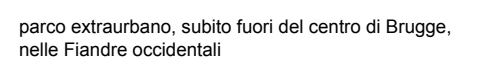 & ' & 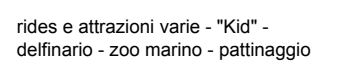 & 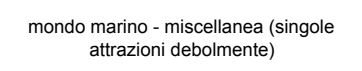 & amusement park & SCENOGRAFICO DEBOLMENTE & (?) \\
\hline Brupark & Brusseles, Belgium & 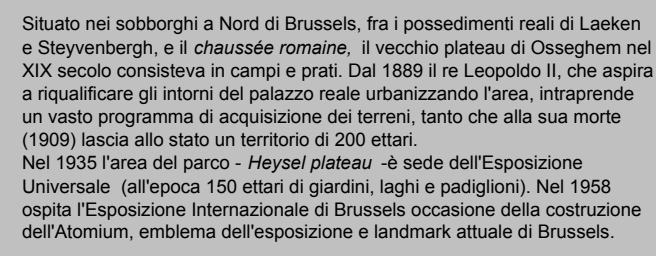 & parco urbano oi Brusseles. & ' & attrazioni varie & $\begin{array}{l}\text { per aree tematiche: : The } \\
\text { Village, Atomium, coeade } \\
\text { (parco acquatico, Mini Europe } \\
\text { (Europa in miniatura), Kinepolis } \\
\text { (multisala cinema) }\end{array}$ & esposizioni universali & $\begin{array}{l}\text { DIDATTICOI ESOTICO- } \\
\text { DETERRITORIALZZZAZIONE }\end{array}$ & $\mathbf{x}$ \\
\hline Monde Sauvage Safari Deignè & Aywaille, Belgium & 1 & , & $86.000 \mathrm{ma}$ & 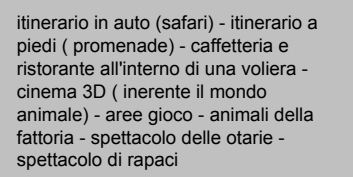 & regno animale & 200 & $\begin{array}{l}\text { DIDATTICOI ESOTICO- } \\
\text { DETERRITORIALIZZAZIONE }\end{array}$ & $X(?)$ \\
\hline
\end{tabular}




\begin{tabular}{|c|c|c|c|c|c|c|}
\hline Plopsaland & Adinkerke-De Panne, Belgium & 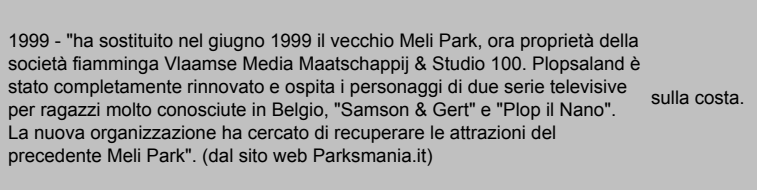 & 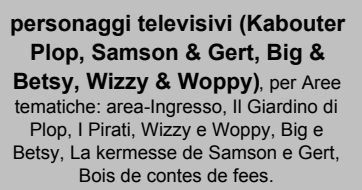 & $\begin{array}{l}\text { amusement park - } \\
\text { Disneyland }\end{array}$ & MARCHIO / DIMENSIONE FANTASTICA & $X(?)$ \\
\hline
\end{tabular}

Telecoo

$$
\text { Stavelot, Beglium }
$$

\section{Waterworld}

Agia Napa, Belgium

1996

parco acquatico

mondo (mitologico) dell' acqua:
Antica Grecia

amusement park -
Disneyland

SINGOLARIZZAZIONEI
DIACRONIZZAIIONE

Denmark

\begin{tabular}{|c|c|c|c|c|c|c|c|c|}
\hline Bakken & Klampenborg, Denmark & 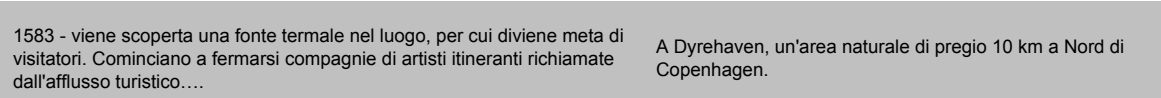 & 1 & 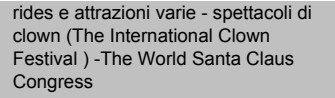 & Pierrot, Clowns & pleasure garden & "AFFACCIO" & $\mathbf{x}$ \\
\hline BonBon-Land & Holme-OIstrup, Denmark & 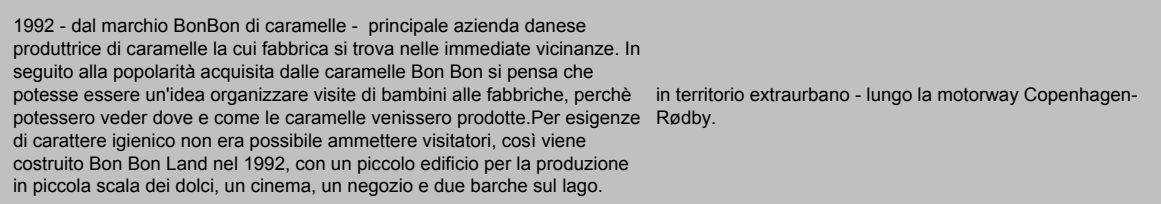 & $110.000 \mathrm{mq}$ & 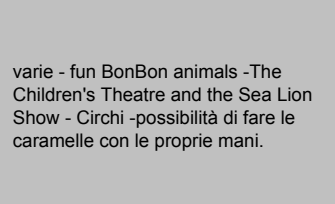 & caramelle BON BON & $\begin{array}{l}\text { amusement park - } \\
\text { Disneyland }\end{array}$ & MARCHOO / DIMENSIONE FANTASTICA & $\mathbf{x}$ \\
\hline
\end{tabular}



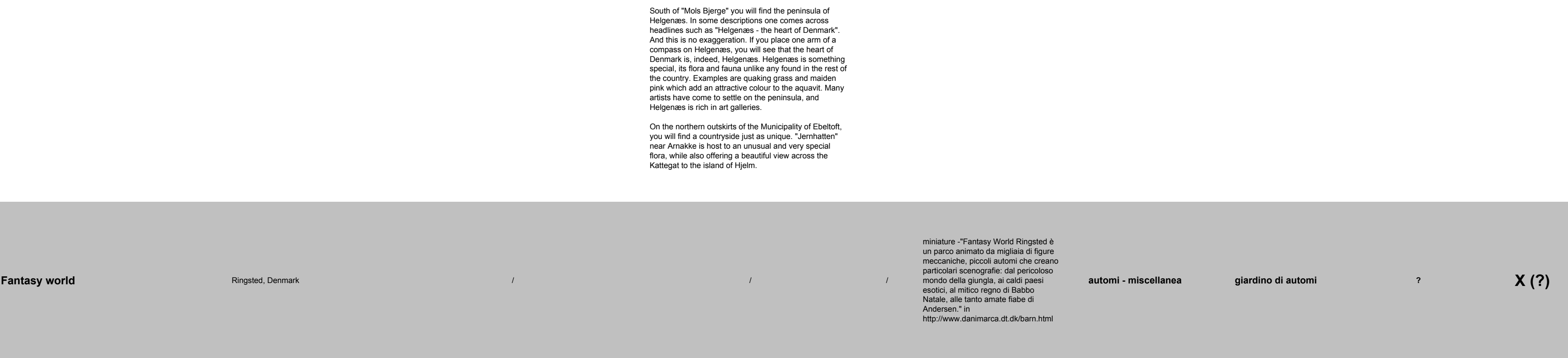

Fårup Acquapark e Sommerland

Saltum, Denmark

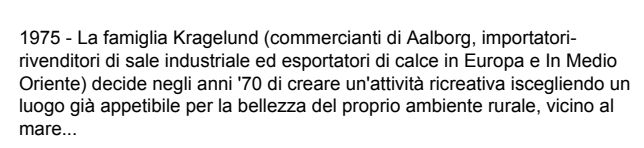

Fanily - Action - Aquapark $\quad \begin{gathered}\text { miscellanea (singole attrazioni } \\ \text { debolmonente) }\end{gathered}$

amusement park

$X(?)$

\begin{tabular}{|c|c|c|c|c|c|c|c|c|}
\hline Legoland & Billund, Denmark & 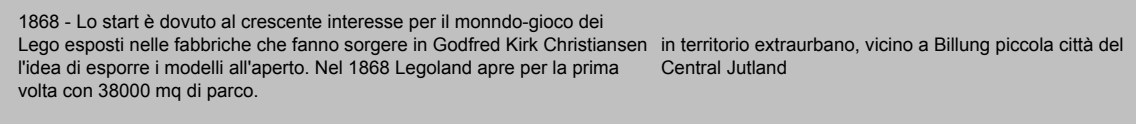 & $100.000 \mathrm{mq}$ & rides e attrazioni varie - circo & 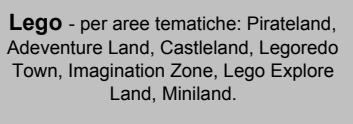 & Disneyland & MARCHIO / DIMENSIONE FANTASTICA & $\mathbf{x}$ \\
\hline Sommerland Sjælland & Nykabing, Denmark & 1 & 1 & $\begin{array}{l}\text { ridese eatrazionivarie - spe } \\
\text { cui i circo) }\end{array}$ & $\begin{array}{l}\text { per aree tematicice: Miniland, } \\
\text { Actionland, Vandland, } \\
\text { Amazonas, Western, }\end{array}$ & Disneyland & 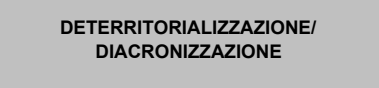 & (?) \\
\hline
\end{tabular}

Sommerland Syd

Tinglev, Denmark

rides e attrazioni vart

miscellanea (singole atrazion
debolmente)

amusement park

(?)

Tivoli

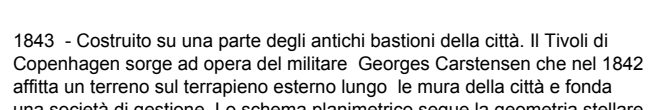

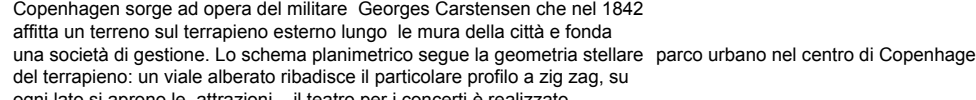

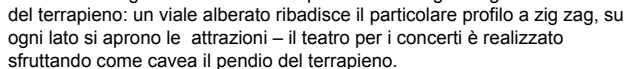

rides eatrrazioni varie (fra cui una
Feris Wheel)- septacoli (Rockmusik
Pantomime Theater

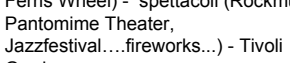

esotico

pleasure garden

"AFFACCIO" 
Tivoli Friheden

Aarhus, Denmark

Tivoliland

Kesamaa Punkaharju

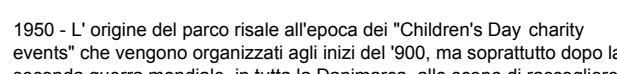

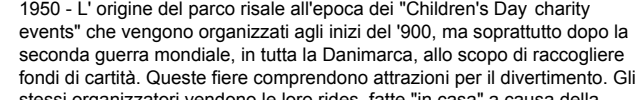

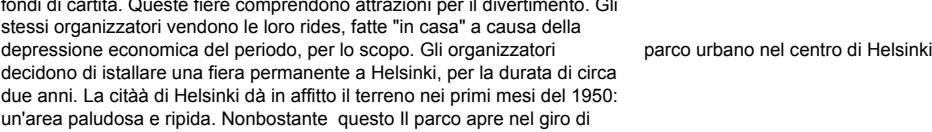

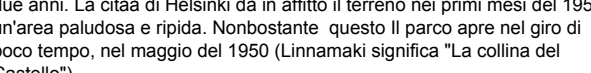

ad Aarhus, sulla costa Est, seconda citita, quanto a
dimensioni, della Danimarca, con una particolare

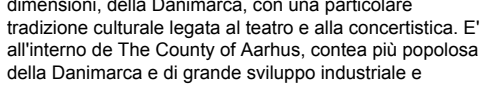

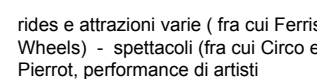

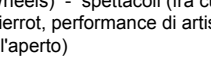

miscellanea (singole attrazioin

pleasure garden

parco urbano nel centro di A
industriale, centro turisitio.

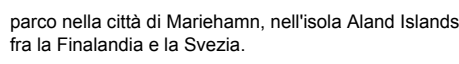

sulla costa, in una locelitat turisicica molto popolare rides eattrazioni varie - family fun -
accuatico

miscellanea ( singole attrazioni)

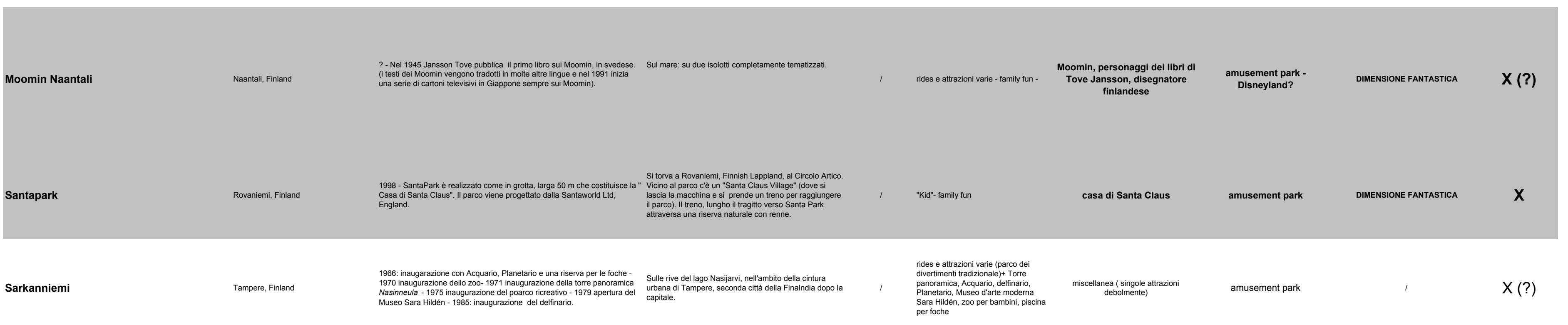




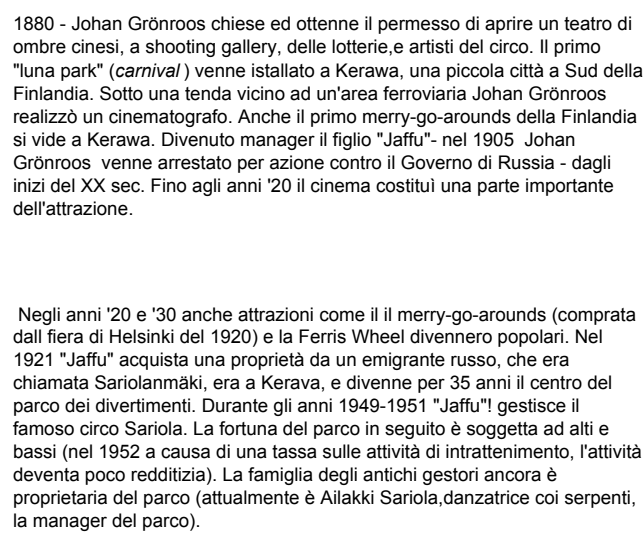

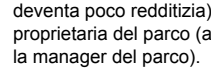

France

\section{Bagatelle}

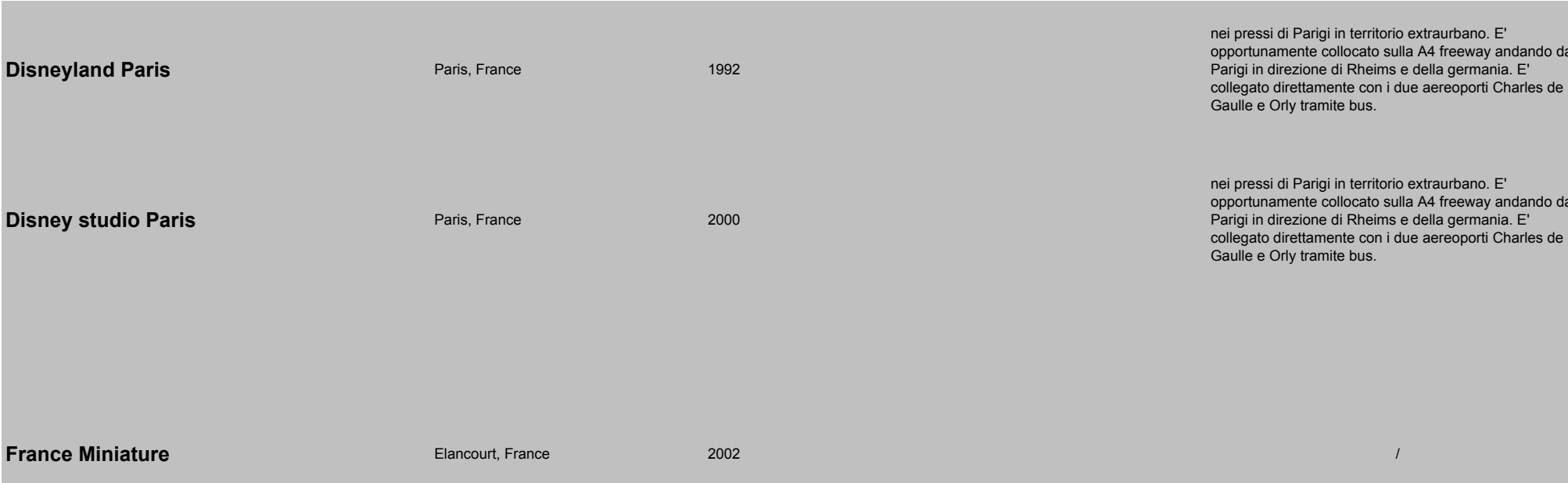

ridese attrazioni varie- - spettacoli (fra
cuic icico) - 200

miscellanea ( singole attrazioni)

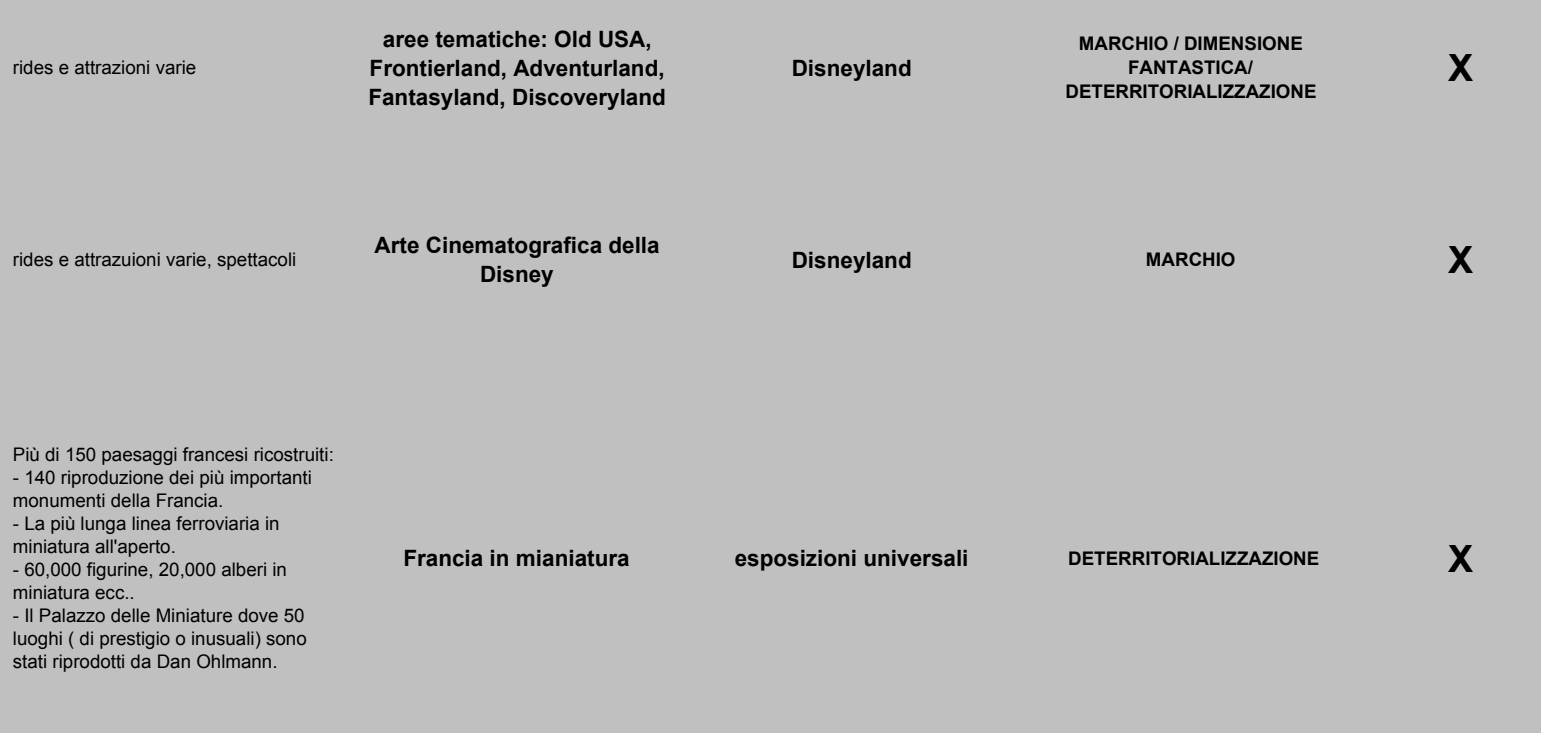




\begin{tabular}{|c|c|c|c|c|c|c|c|c|c|}
\hline PARCO & COORDINATE GEOGRAFICHE & ORIIINE STORICA & CONTESTOIPAESAGGIO & area & ATTRAZIONI & ТЕМА & MATRICE & RUOLO DEL TEMA & $\begin{array}{l}\text { PROGETTA- } \\
\text { ZlONDEEL } \\
\text { PAESAGGIO }\end{array}$ \\
\hline Futurscope & Politers, Vereme, France & 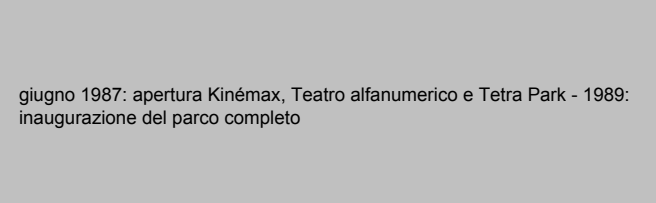 & 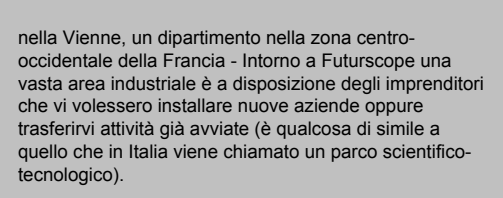 & 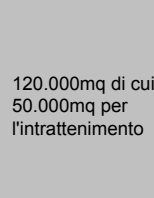 & 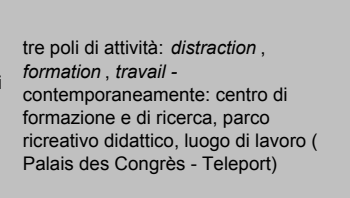 & Civiltà del futuro & esposizioni universali & DDAATICOOV VALORZZZAZIONE & $\mathrm{x}$ \\
\hline Jardin d'Acclimation & Paris, France & 1860- per popera di Napolobere III & 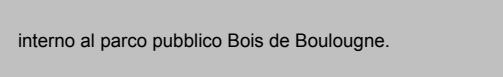 & , & 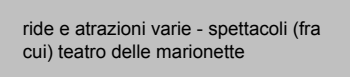 & esotico & pleasure garden & AfFACCIO & $x$ \\
\hline La Villette & Paris & 1997 & parco ubanondif Parigi & $2983000 \mathrm{ma}$ & 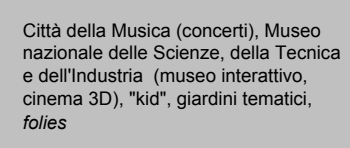 & $\begin{array}{l}\text { Città della Musica e Città della } \\
\text { Scienza }\end{array}$ & $\begin{array}{c}\text { pleasure garden- - } \\
\text { esposizioni universali }\end{array}$ & & $x$ \\
\hline Le Pal & Dompiere Sur Bresso, France & 1 & 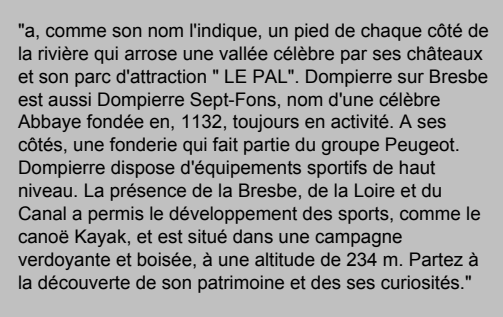 & 更 & 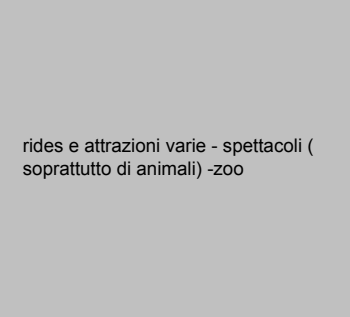 & 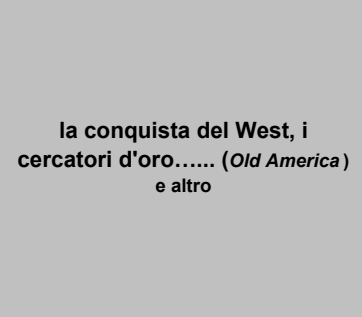 & $\begin{array}{c}\text { amusement park- } \\
\text { Disseyland? }\end{array}$ & $\begin{array}{l}\text { SCENOGBAFICOO } \\
\text { DEETERRTORRALIZZAZIONE }\end{array}$ & $x(?)$ \\
\hline Mer-de-Sable & Emenononile, France & 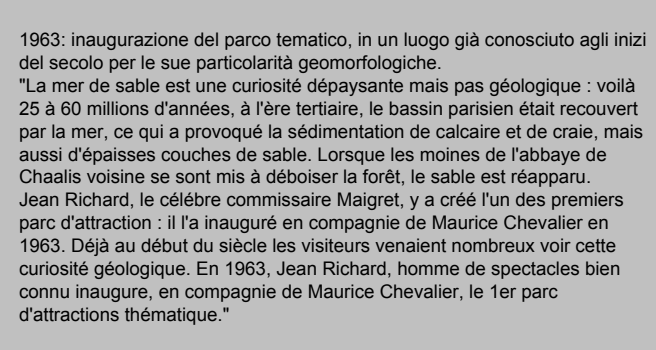 & nat & , & 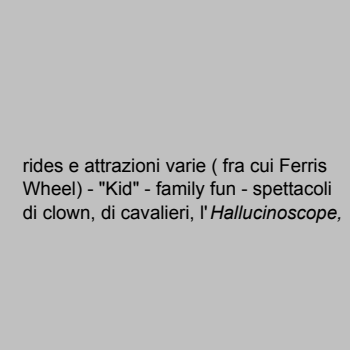 & $\begin{array}{l}\text { Western-Indiani } \\
\text { (principalmente) a tatri temi }\end{array}$ & amusement park & 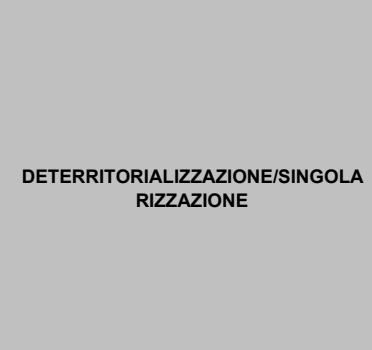 & $x$ \\
\hline Minifrance & Bigronoses Fance & 1998 & tra Aixe Frejus. & $20000 \mathrm{ma}$ & 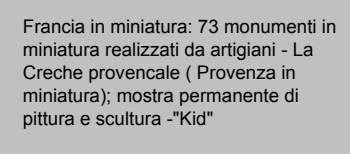 & Francia in miniatura & esposizioni universali & 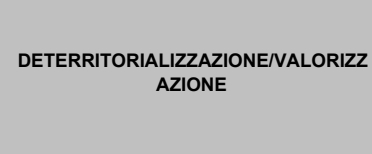 & $x$ \\
\hline Nigloland & Doancout, France & , & 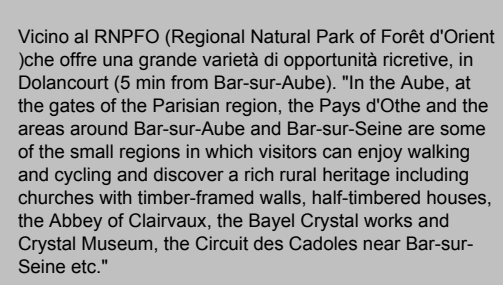 & $180.000 \mathrm{ma}$ & 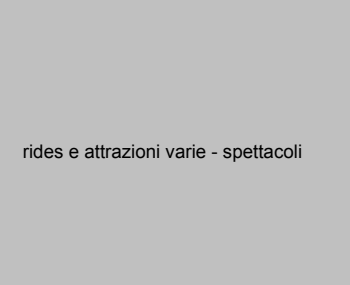 & 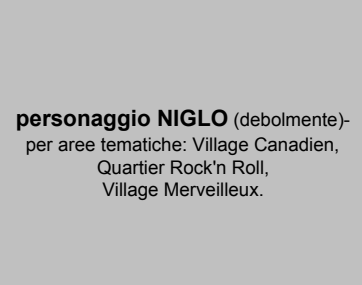 & amusement park. & DEtERRRTORRALZZZAZIONE & $x(?)$ \\
\hline
\end{tabular}




\begin{tabular}{|c|c|c|c|c|c|c|c|c|c|}
\hline PARCO & COORDINATE GEOGRAFICHE & ORIGINE STORICA & CONTESTO/PAESAGGIO & area & ATTRAZIONI & TEMA & MATRICE & RUOLO DEL TEMA & $\begin{array}{l}\text { PROGETTA- } \\
\text { ZlONE DEL } \\
\text { PAESAGGIO }\end{array}$ \\
\hline OK Corral & Cuges-les-Pins, France & 1 & 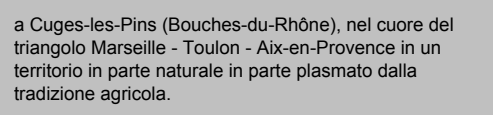 & 1 & rides e attrazioni varie - spettacoli & West & amusement park & DETERRITORIALIZZAZIONE & $X(?)$ \\
\hline Parc Asterix & Plailly, France & 1989 & allinterno di una foresta secolare di 155 ettari. & 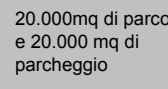 & 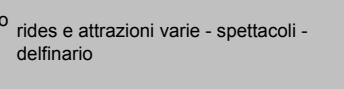 & fumetto Asterix & Disneyland & MARCHIO - DIMENSIONE FANTASTICA & $\mathbf{x}$ \\
\hline Parc Saint Paul & Saint Paul, France & 1 & territorio extraurbano & 1 & 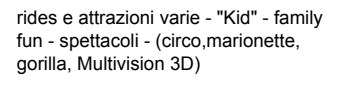 & $\begin{array}{l}\text { miscellanea (singole attrazioni } \\
\text { debolmente) }\end{array}$ & amusement park? & 1 & ' \\
\hline Puy du Fou & Les Epesses, Vendee & 1 & 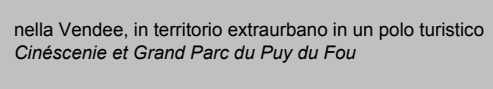 & $400.000 \mathrm{mq}$ & $\begin{array}{l}\text { rides e attrzzioni varie - numerosi } \\
\text { spettacoli }\end{array}$ & $\begin{array}{l}\text { la Storia (anche fantastica) } \\
\text { della Francia }\end{array}$ & amusement park & $\begin{array}{l}\text { SCENNGRAAICOI } \\
\text { DETERITORALIZAZIONEIIACRONI } \\
\text { ZZAZIONE }\end{array}$ & $X(?)$ \\
\hline Walibi Aquitaine & France & 1975/2001 - Six Flags in Europa & $\begin{array}{l}\text { Vicina a Agen, area a verde di piü di i } 30 \text { etara, dominata } \\
\text { da un castello del XVVIII. }\end{array}$ & $1.000 .000 \mathrm{mq}$ (?) & rides e attrazioni varie & nessuno & amusement park & 1 & $X(?)$ \\
\hline Walibi & Rhone-Alpes - France & 1975/2001 - Six Flags sin Europa & 1 & 1 & rides e attrazioni varie & nessuno & amusement park & ' & $X(?)$ \\
\hline $\begin{array}{l}\text { Walibi Schtroumpf (ex-Big Bang } \\
\text { Schtroumpf) }\end{array}$ & Mäizirers-les-Metz, France & 1989 apre il Big Bang Schtroumpf- 2001: Six Flags in Europa & 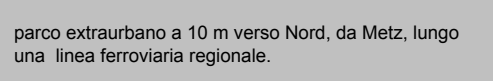 & $260.000 \mathrm{mq}$ & $\begin{array}{l}\text { rides eatrazioni varie (fra cui tore } \\
\text { panoramica) }\end{array}$ & fiaba de Puffi & amusement park & DIMENSIONE FANTASTICA & $X(?)$ \\
\hline
\end{tabular}

Allgäu Skyline Park

Bad Wörshofen

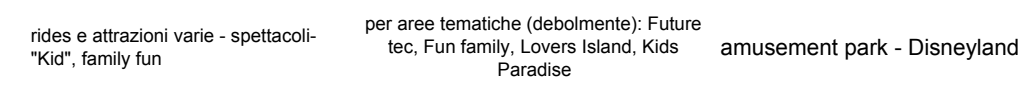

$(?)$

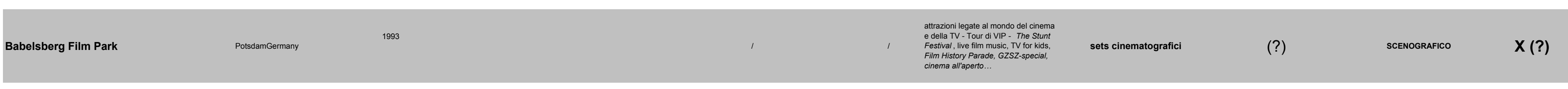

Bayern Park 


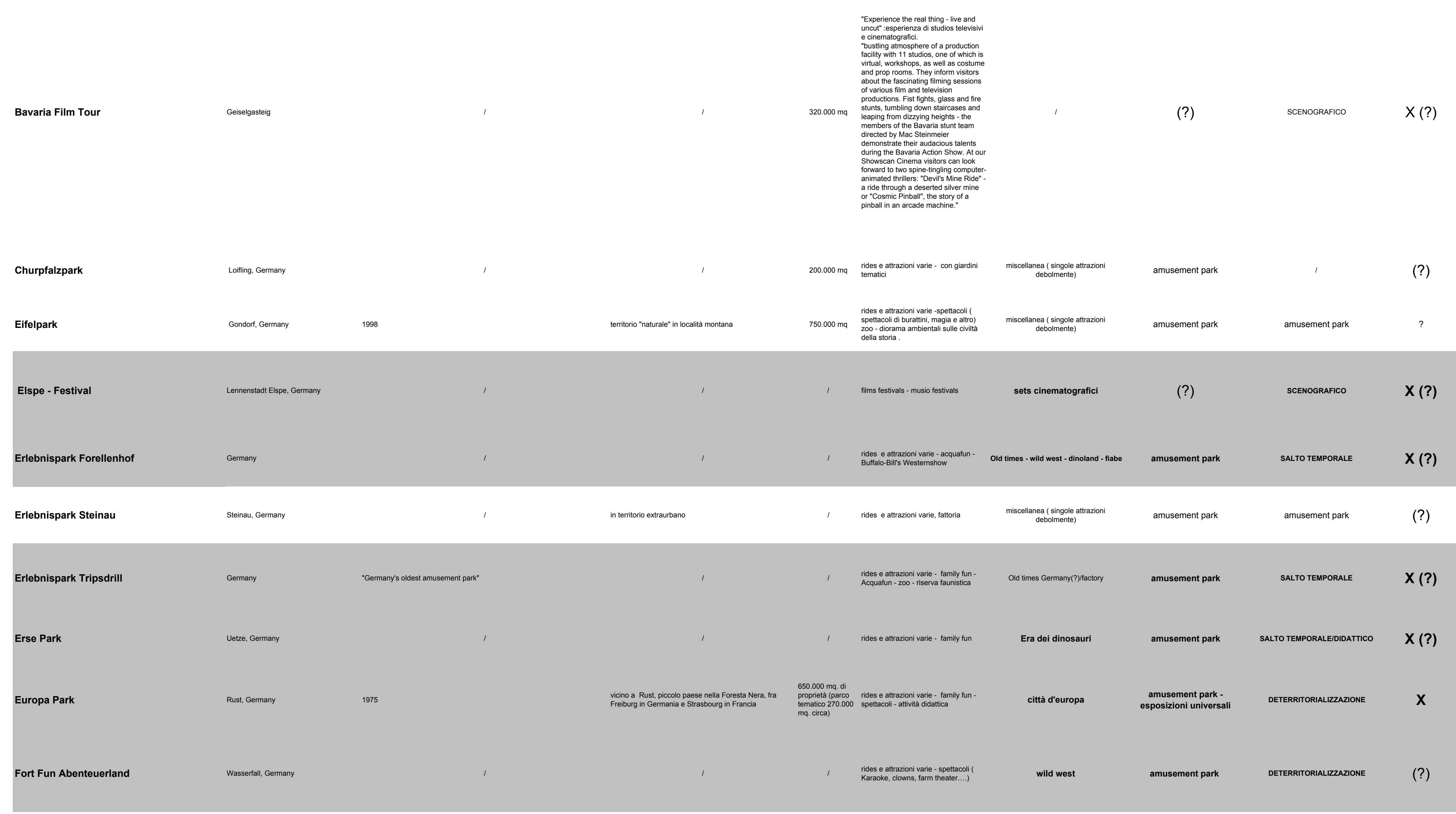




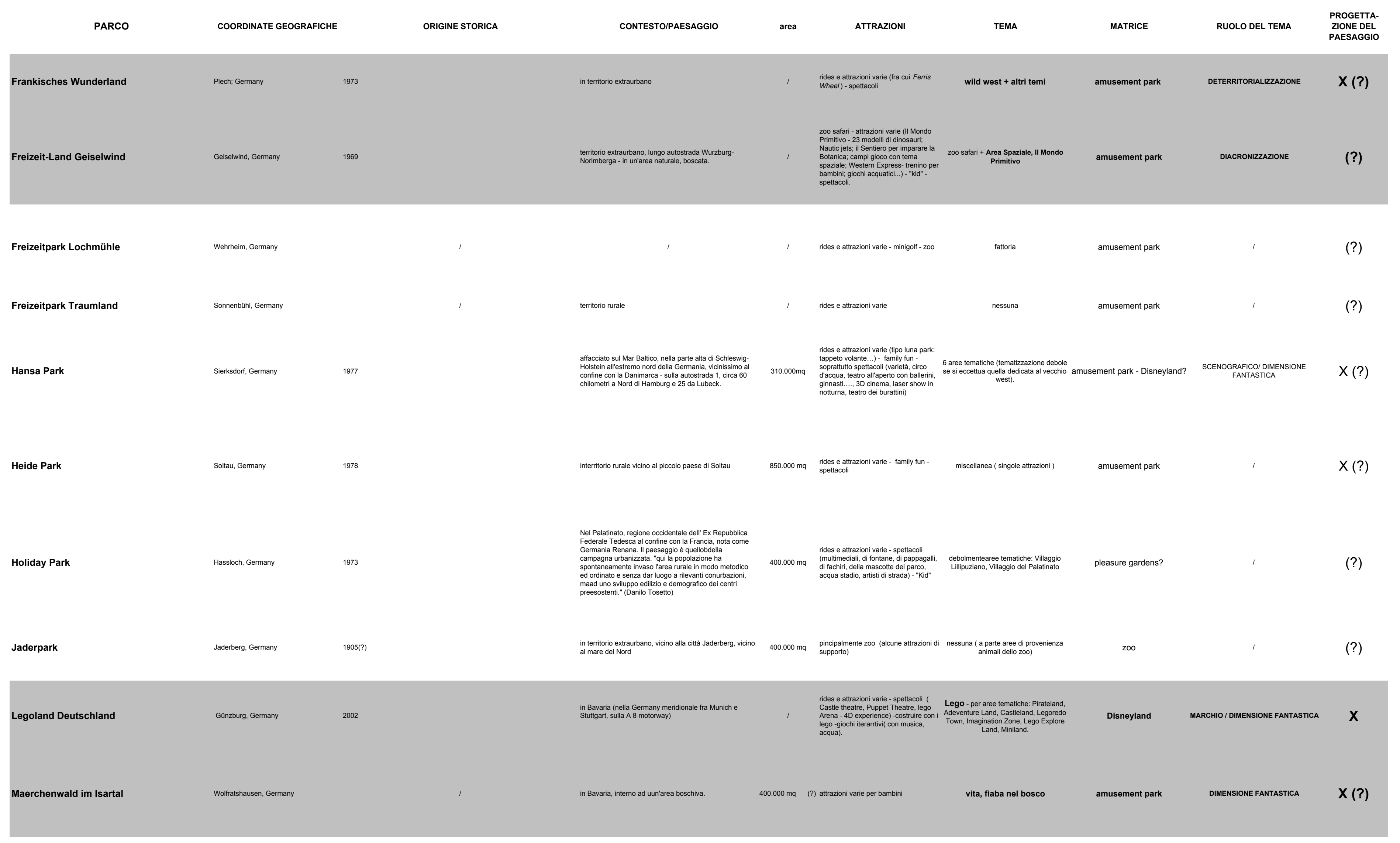




\begin{tabular}{|c|c|c|c|c|c|c|c|c|c|}
\hline PARCO & COORDINATE GEOGRAFICHE & ORIGINE STORICA & CONTESTOIPAESAGGIO & area & ATTRAZIONI & TEMA & MATRICE & RUOLO DEL TEMA & $\begin{array}{l}\text { PROGETTA- } \\
\text { ZIONE DEL } \\
\text { PAESAGGIO }\end{array}$ \\
\hline Minidomm & Ratingen, Germany & 1967 & $\begin{array}{l}\text { Sorge vicino al raccordo tra lautostrada Dusseldorf, } \\
\text { Essen e Muhheim. In territorio extraurbano. }\end{array}$ & I & 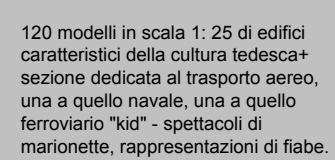 & $\begin{array}{l}\text { Edifici itipici della Germania in } \\
\text { miniatura }\end{array}$ & esposizioni universali & $\begin{array}{l}\text { DETERRITORIALIZZAZIONENALORIZZ } \\
\text { AZIINE }\end{array}$ & $\mathbf{x}$ \\
\hline
\end{tabular}

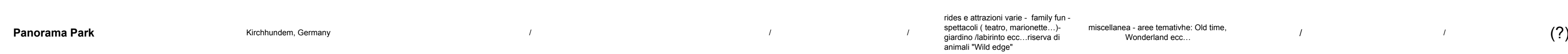

\begin{tabular}{|c|c|c|c|c|c|c|c|c|c|}
\hline Phantasialand & Bruihl, Gemmany & 1967 & in un luogo dove prima era una miniera ad il ignite & $280.000 \mathrm{mq}$ & 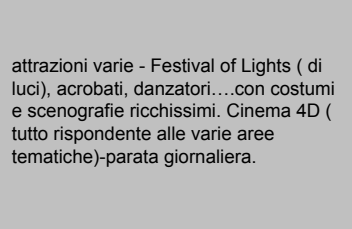 & 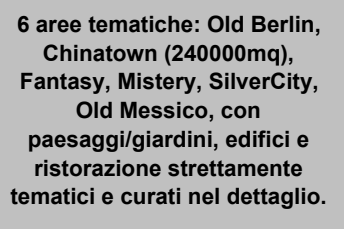 & $\begin{array}{l}\text { pleasure gardens - } \\
\text { Disneyland }\end{array}$ & DIMENSIONE FANTASTICA & $X(?)$ \\
\hline
\end{tabular}

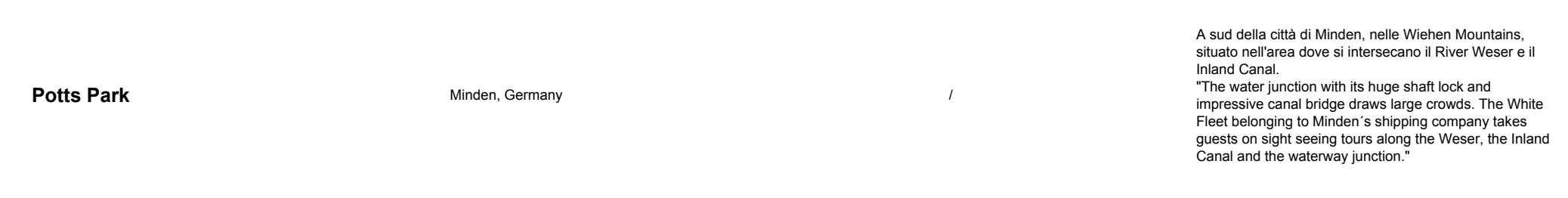

\begin{tabular}{|c|c|c|c|c|c|c|c|c|}
\hline Rasti-Land & Sazhemmendorf, Germany & ' & ' & 1 & attrazioni varie & miscellanea & amusement park & 1 \\
\hline Schloss Beck Freizeitpark & Bottrop, Germany & trassirmazione parco della villa Scholss Thurne in parco dei divertimenti & parco di villa Schloss Thurn & ' & attrazioni varie, zoo & miscellanea & amusement park & ' \\
\hline Schloss Thurn & Heroldsbach, Germany & $\begin{array}{l}\text { 1975: trastromazione parco della villa Scholss Thume in parco dei } \\
\text { divertimenti }\end{array}$ & parco di villa Schloss Thurn & 1 & 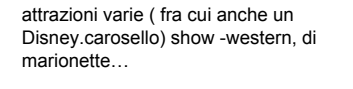 & miscellanea & amusement park & 1 \\
\hline Schwaben Park & Kaisersbach, Germany & 1 & in territorio extraurbano & ' & 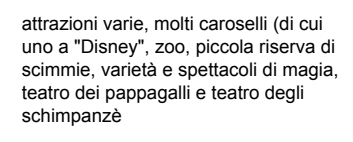 & miscellanea & pleasure gardens & I \\
\hline
\end{tabular}

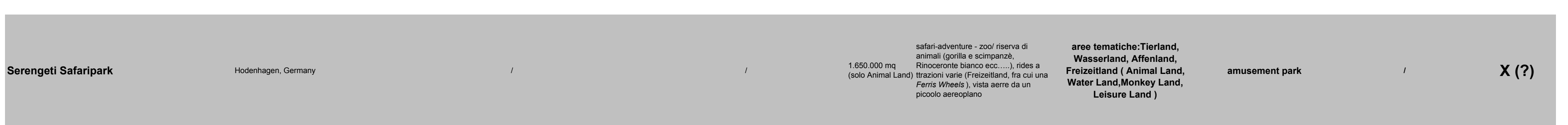




\begin{tabular}{|c|c|c|c|c|c|c|c|c|c|}
\hline PARCO & COORDINATE GEOGRAFICHE & ORIGINE STORICA & CONTESTO/PAESAGGIO & area & ATTRAZIONI & TEMA & MATRICE & RUOLO DEL TEMA & $\begin{array}{l}\text { PROGETTA- } \\
\text { ZZONEDEL } \\
\text { PAESAGGIO }\end{array}$ \\
\hline Tier- und Freizeitpark Thüle & Friesoyyhe-Thüle, Germany & ' & in territorio extraurbano & 1 & zoo - parco ded idivertimentif & nessuno & amusement park & 1 & (?) \\
\hline Tolk-Schau & Tolk, Germany & I & in territorio extraurbano & $300.000 \mathrm{mq}$ & 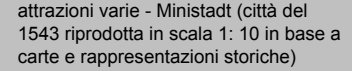 & $\begin{array}{l}\text { singole a atrazioni- Ministadt } \\
\text { Schleswig }\end{array}$ & esposizioni universali & DIDATTICO/ DIACRONIZZAZZIONE & $X(?)$ \\
\hline \multirow[t]{2}{*}{ Warner Bros. Movie World } & Bottrop-Kirchhellen, Germany & 1996 & 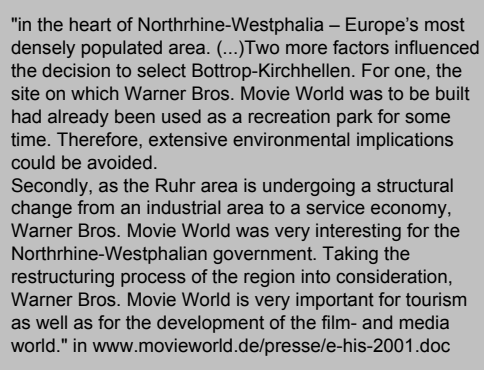 & 1 & 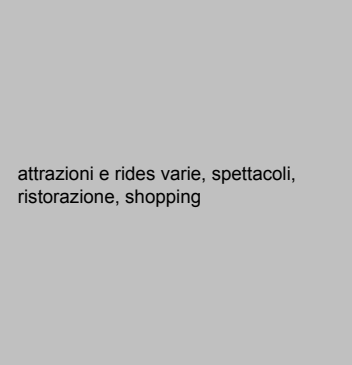 & 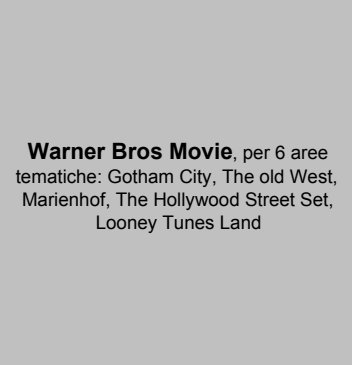 & Disneyland & MARCHIO & $X(?)$ \\
\hline & Greece & & & & & & & & \\
\hline Aqualand - Corfú Water park & Agios loannis, Cortü, Greece & 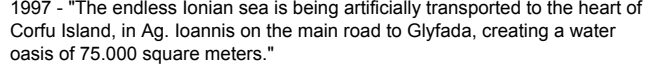 & 1 & 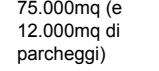 & $\begin{array}{l}\text { acquatitco + attrazionie rides varie out- } \\
\text { of-water (sala giochi, mini cars...) }\end{array}$ & nessuno & (?) & 1 & (?) \\
\hline & Norway & & & & & & & & \\
\hline Kongeparken & Noway & 1 & 1 & 1 & 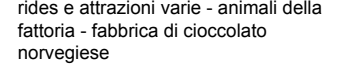 & ' & (?) & 1 & (?) \\
\hline Kristiansand Dyreparken & Noway & 1 & 1 & ' & $\begin{array}{l}\text { Aquatitishows, oncerts, carousel, } \\
\text { stage shows, zoo }\end{array}$ & miscellanea (singole attrazioni) (?) & (?) & 1 & (?) \\
\hline Lunds Tivoli & Noway & 1895 & ' & 1 & 1 & 1 & pleasure gardens & (?) & (?) \\
\hline Telemark Sommarland & Bo, Noway & , & allinterno di un'area a foresta & I & 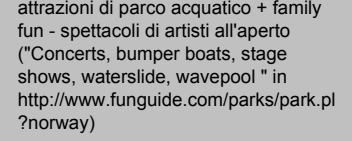 & 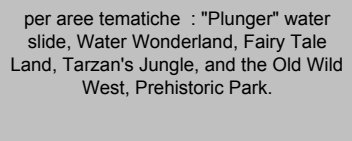 & $\begin{array}{l}\text { amusement park - } \\
\text { Disneyland }\end{array}$ & DETERRITORIALIZZAZIONE & (?) \\
\hline Tusenfryd & Oslo, Norway & 1 & 1 & 1 & 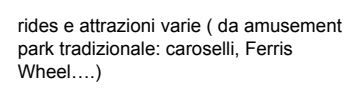 & miscellanea (singole attrazioni) & & & \\
\hline
\end{tabular}




\begin{tabular}{|c|c|c|c|c|c|c|c|c|c|}
\hline PARCO & COORDINATE GEOGRAFICHE & ORIGIIE STORICA & CONTESTOPAAESAGGIO & area & ATtraZion & темА & MATRICE & RUOLO DEL TEMA & 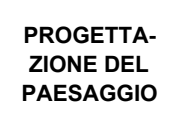 \\
\hline Vikinglandet & Vinetero, Noway & , & 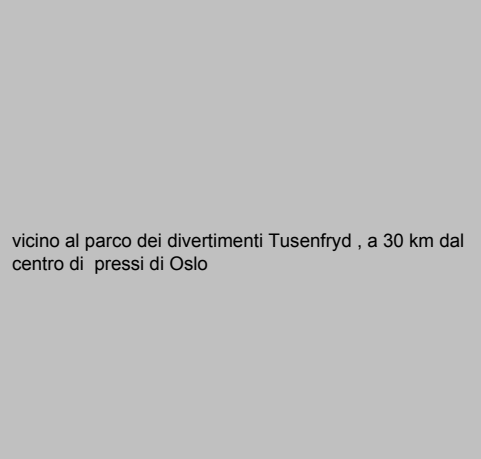 & $20.000 \mathrm{ma}$ & 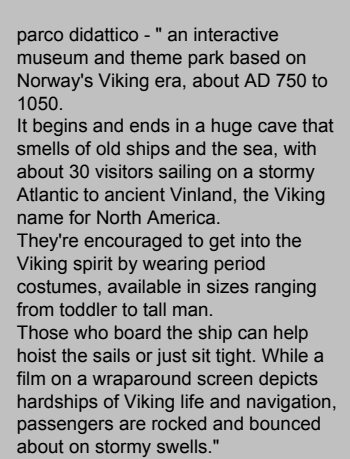 & $\begin{array}{l}\text { era dei Vichinghi in Norvegia } \\
\text { (about AD } 750 \text { to } 1050)\end{array}$ & $\begin{array}{c}\text { amusement park - } \\
\text { esposizioni universali }\end{array}$ & DIDATTICO & X (?) \\
\hline
\end{tabular}

Hungary

\begin{tabular}{|c|c|c|c|c|c|c|c|}
\hline Vidam Park & Budapest, Hungary & primi anni del $X X$ sec. & parco urbano odi Budapest, adiacente i parco Varosiliget & $64.800 \mathrm{mq}$ & 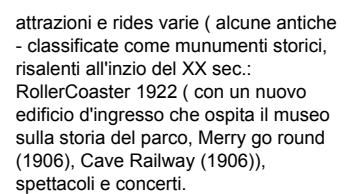 & miscellanea (singole attrazioni) & $\begin{array}{l}\text { pleasure gardens? Amusement } \\
\text { park? }\end{array}$ \\
\hline
\end{tabular}

Italy

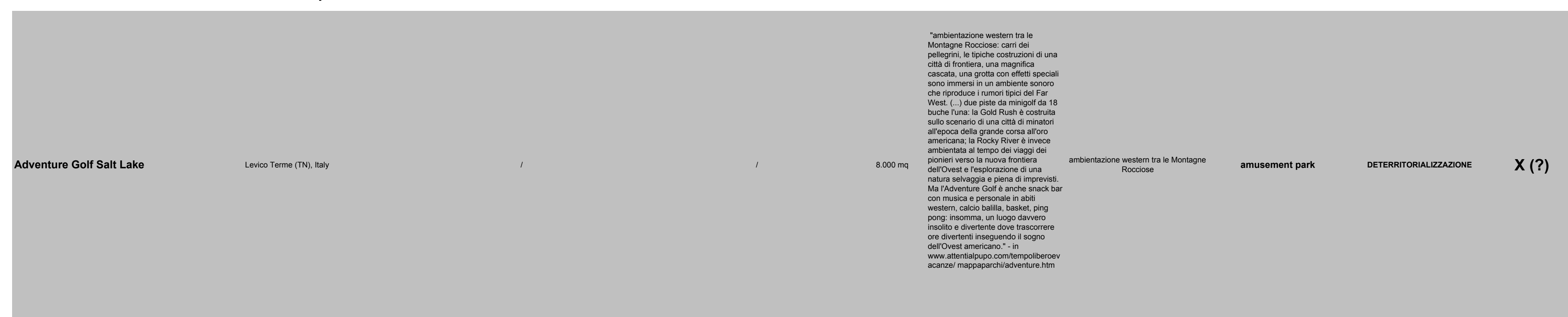


Città della Domenica

\begin{tabular}{|c|c|c|c|c|c|c|c|c|c|}
\hline Cowboyland & Voghera, taly & ?- dallestate 2001 apre l'area tematica Cowboyland $(35.000 \mathrm{mq})$ & & $50.000 \mathrm{ma}$ & attrazioni varie (con tema westerm) & West America & amusement park & DETERRITORALLIZZZZIONE & $X(?)$ \\
\hline Edenlandia & Napoli, ttaly & metà degli anni '60 & $\begin{array}{l}\text { parco urbano alle porte dei Campi Flegrei, vicino al } \\
\text { giadrino botanico e alla Mostra d'oltremare. }\end{array}$ & $50.000 \mathrm{mq}$ & 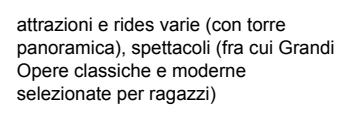 & miscellanea ( singole attrazioni) & amusement park & 1 & $X(?)$ \\
\hline Etnaland -Parco Zoo Sicilia & Belpasso, (CT) taly & 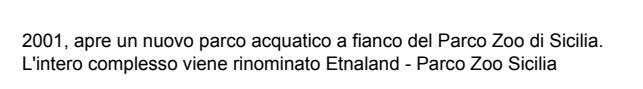 & ai piedid dellEna & $\begin{array}{l}100.000 \mathrm{mq} \text { (solo } \\
\text { parco accuatioco) }\end{array}$ & $\begin{array}{l}\text { Parco dei Dinosauri Accuapark + } \\
\text { Parco Zoo }\end{array}$ & per aree tematiche (in sviluppo) & amusement park & SCENOGRAFICO & (?) \\
\hline Fantasilandia & Tirenia (PI), taly & ' & 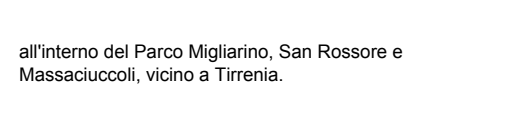 & $15.000 \mathrm{mq}$ & 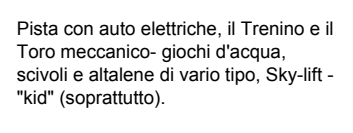 & miscellanea & amusement park & 1 & (?) \\
\hline Fantasy World Minitalia & Capriate San Gervasio & 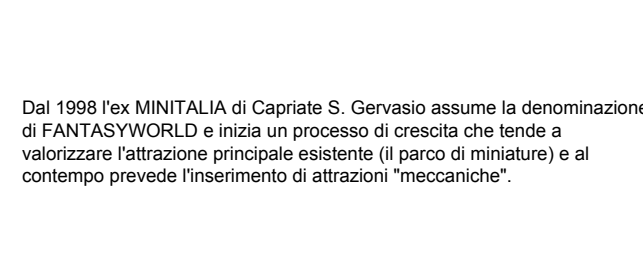 & territiori extraurbano, Lungo lautostrada Bergamo-Milano & 1 & 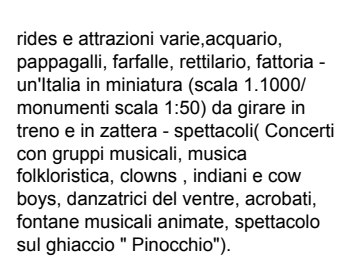 & $\begin{array}{l}\text { Miscellanea a (singole attrazioni) } \\
\text { compress unthlalia in miniautura }\end{array}$ & $\begin{array}{l}\text { amusement park- esposizioni } \\
\text { universali }\end{array}$ & $\begin{array}{l}\text { DIMENSIONE FANTASTICA- } \\
\text { DETERRITORALLZZZZIONE }\end{array}$ & $\mathrm{x}$ \\
\hline Fiabilandia & Rivazzura di Rimini & 1965 & territorio extraurbano & $120.000 \mathrm{mq}$ & attrazioni varie- -family tun- didatitica. & miscellanea (singole attrazioni) & amusement park & SCENOGRAFICO & $X(?)$ \\
\hline Gardaland & Verona, ttaly & 1975 & territorio extraurbano, sul lago di Garda & $240.000 \mathrm{mq}$ & $\begin{array}{l}\text { rides e eatrazioni varie, spettacoli, } \\
\text { shopping, attivitá didatica }\end{array}$ & per aree tematiche (miscellanea) & $\begin{array}{l}\text { amusement park - } \\
\text { Disneyland }\end{array}$ & 1 & $\mathbf{x}$ \\
\hline
\end{tabular}




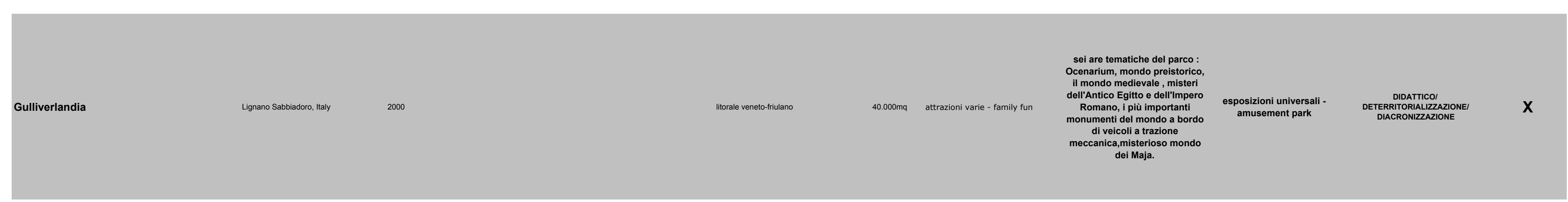

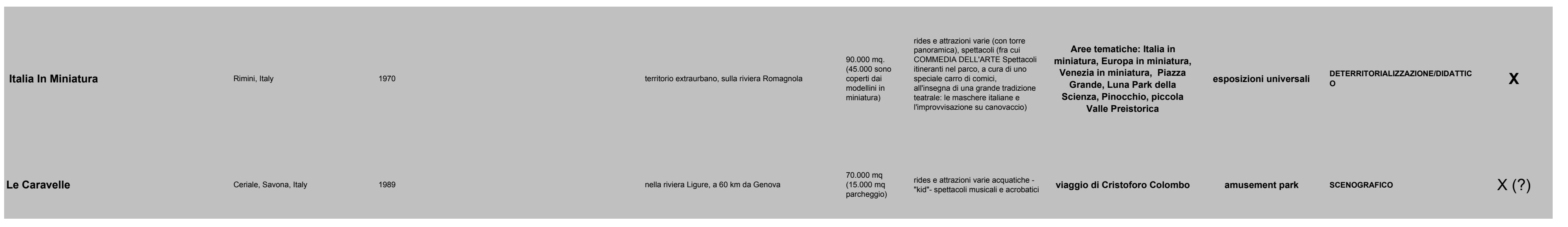

\begin{tabular}{|c|c|c|c|c|c|c|c|c|c|}
\hline Luneur & Roma, taly & 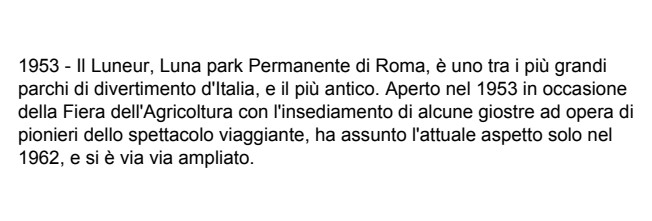 & parco urbano interno al parco del quartiere Eur & $70.000 \mathrm{ma}$ & 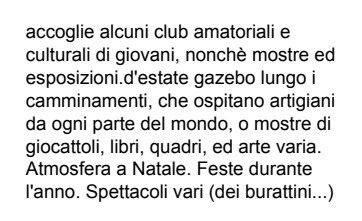 & miscellanea (singole attrazioni) & $\begin{array}{l}\text { esposizioni universali- } \\
\text { aususement park }\end{array}$ & I & $X(?)$ \\
\hline Magic World & Giugliano (NA), ttaly & 1990 parco accuatico - 1998 parco dei divertimenti & 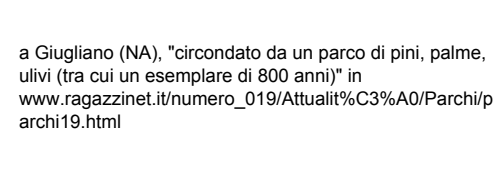 & $300.000 \mathrm{mq}$ & 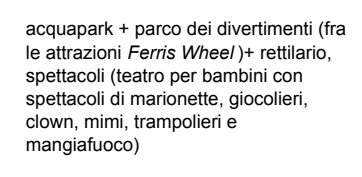 & miscellanea (singole attrazioni) & amusement park & 1 & $X(?)$ \\
\hline Minitalia & Capratet San Gevasio, taly & 1972 & $\begin{array}{l}\text { in territorio extraurbano, in un paesino in provincia } \\
\text { disergamo }\end{array}$ & $180.000 \mathrm{mq}$ & $\begin{array}{l}\text { laghi, monti icc.). - areana teatro- } \\
\text { parco giochi. }\end{array}$ & Italia in miniatura & esposizioni universali & $\begin{array}{l}\text { DETERRITORIALZZZAZIONENALORIZZZ } \\
\text { AIINE }\end{array}$ & $\mathbf{x}$ \\
\hline Mirabilandia & Savio, taly & 1992 (dal 1997 nuova societă proprietaria) & $\begin{array}{l}\text { paraco extraurbanon nele apcro della Standiana, sula } \\
\text { Strada Statale Adriatia }\end{array}$ & 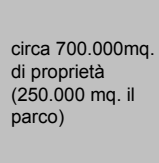 & $\begin{array}{l}\text { ridese eatrazion ivare (fra cui cinema } \\
40 \text { ), spettacoli, shopping, area picnic }\end{array}$ & 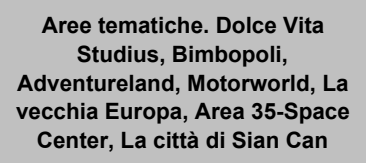 & $\begin{array}{l}\text { amusement park - } \\
\text { Disneyland }\end{array}$ & $?$ & $\mathbf{x}$ \\
\hline
\end{tabular}




\begin{tabular}{|c|c|c|c|c|c|c|c|c|c|}
\hline PARCO & COORDINATE GEOGRAFICHE & ORIGINE STORICA & CONTESTOIPAESAGGIO & area & ATTRAZIONI & ТЕМА & MATRICE & RUOLO DEL TEMA & 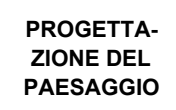 \\
\hline Movie Studios Park & lazise (NR), taly & 2002 & Lagodi Garda & $100.000 \mathrm{ma}$ & 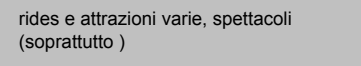 & sets cinematografici & (?) & SCENoGBafico & $X(?)$ \\
\hline Zoosafari Fasanolandia & Fasano, aly & 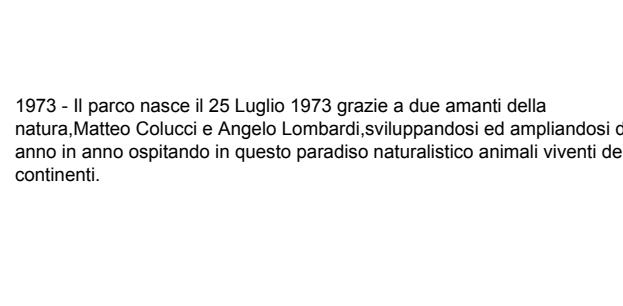 & 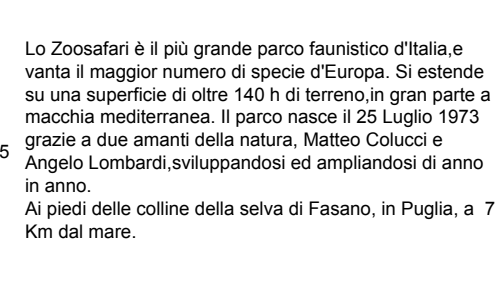 & $\begin{array}{c}1.400 .000 m 9 \\
\text { (Z200sarari) } \\
7\end{array}$ & 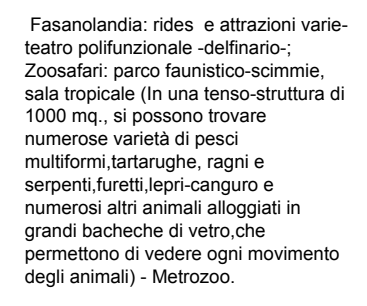 & Zoosatari- Fasonodanda & amusement park & 1 & $X(?)$ \\
\hline Parco della Preistoria & Rivota daAdda (CR), taly & 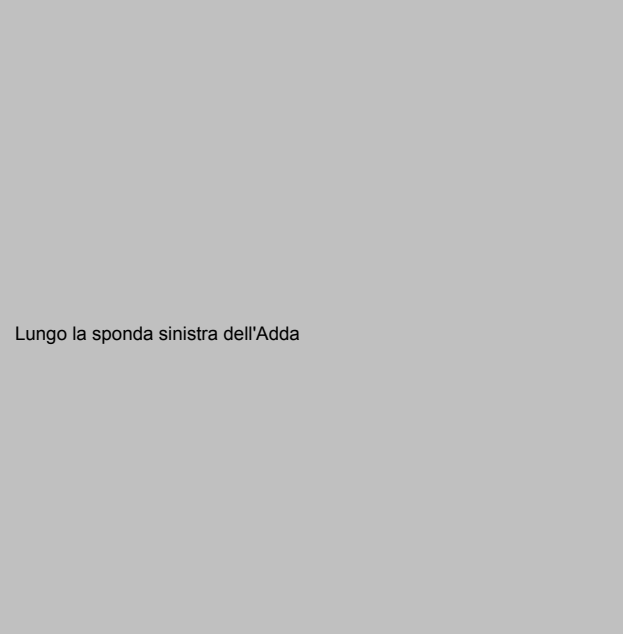 & 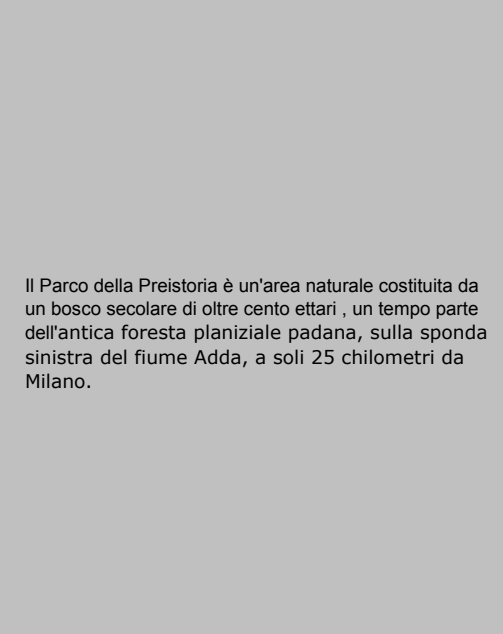 & $1.000 .000 \mathrm{mq}$ & 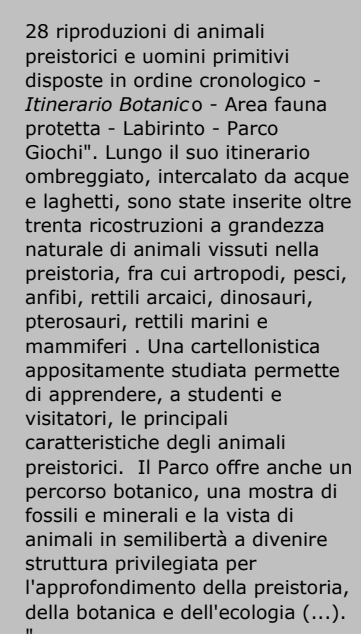 & mondo della preistoria & esposizioni universali? & DidATtICO & $x$ \\
\hline Safari park & Ponbia (No), tay & 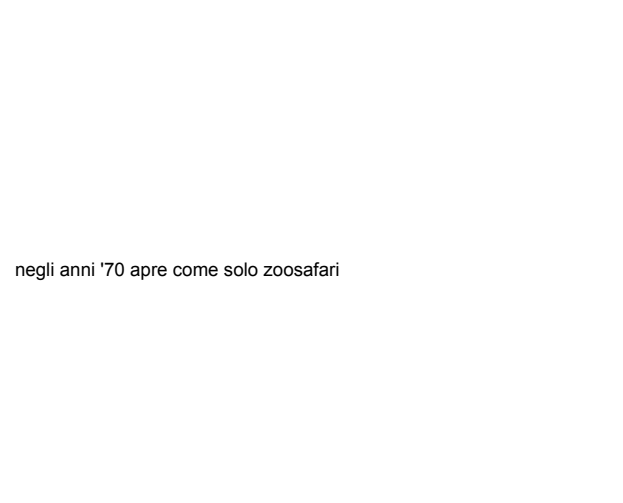 & & $400.000 \mathrm{ma}$ & 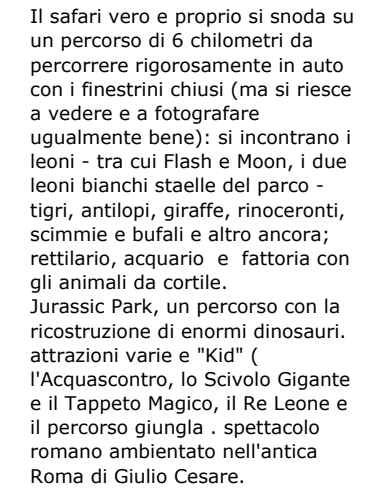 & 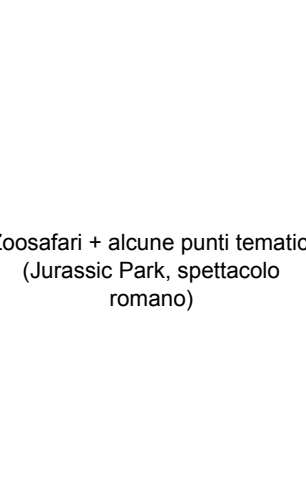 & zoo e a ausement park & ' & $X(?)$ \\
\hline Sardegna in miniatura & Tuili (ca), taly & 2000 & 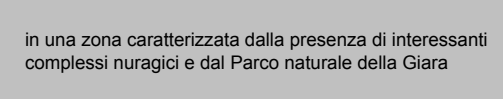 & $30.000 \mathrm{ma}$ & 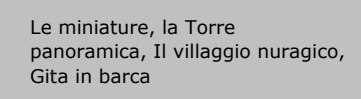 & Sardegna in miniatura & esposizioni universali & $\begin{array}{l}\text { DETERRrToRIALZZZZZIONENALORIZZ } \\
\text { AZIONEE }\end{array}$ & $x$ \\
\hline
\end{tabular}


PARCO

COORDINATE GEOGRAFICHE

ORIGINE STORICA

CONTESTO/PAESAGGIO
ATTRAZIONI

TEMA

MATRICE

RUOLO DEL TEMA

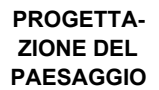

Netherlands

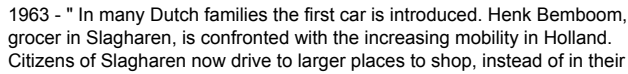

Attractiepark Slagharen

Slagharen, Netherands

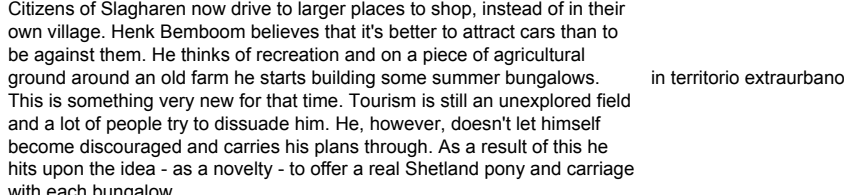

dies e eatrazioni varie + Pony park

miscellanea (singole attrazioni) a parte
Main Streat

amusement park

(?)

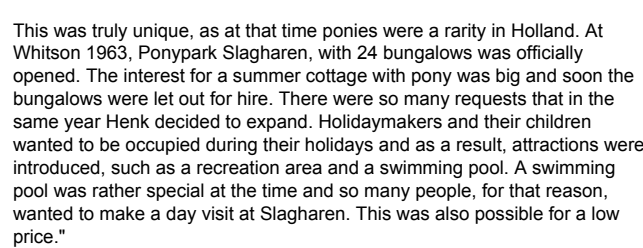

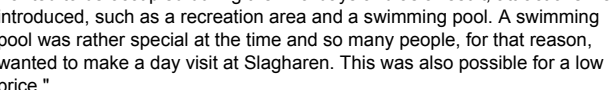

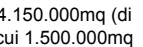

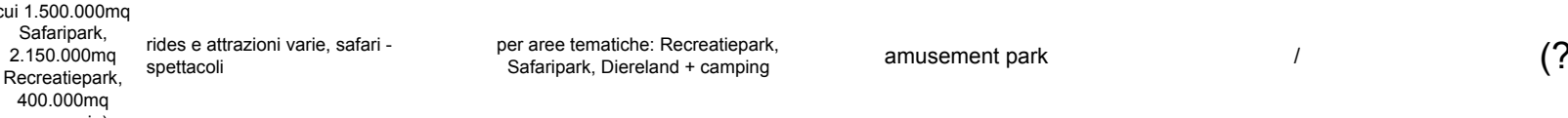

Beekse Bergen

Hilvarenbeek. Netherands

1964 amusement park-1968 sataripark

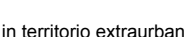

\section{De Valkenier}

Drievliet

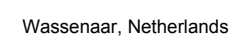

Duinrell

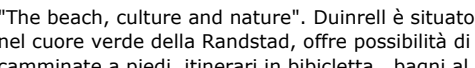

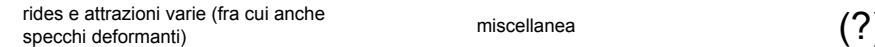

ridese eatrazioni varie, "Kid", Family
fun, spettacoil

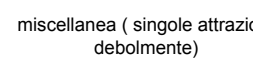

amusement park
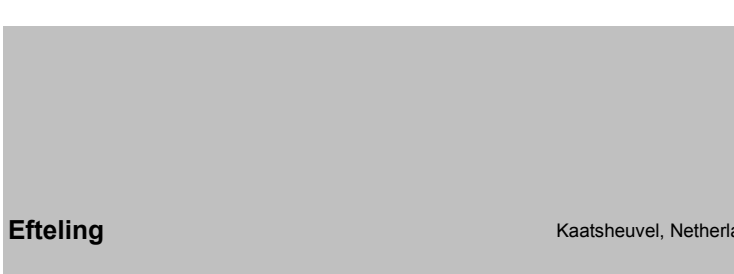

Kaatsheuve, Netherlands

1952

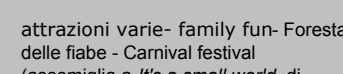

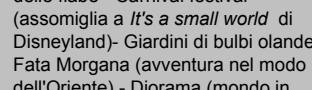

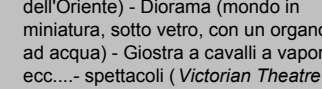

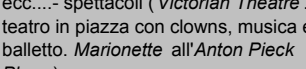

Fabe nordiche: Fain Tala Forest.
People of Laaf + altrit temi

pleasure gardens

DIMENSIONE FANTASTICA

$x$

Hellendoorn

Netherlands

1939

Koningin Juliana Toren rides e attrazioni varie (frac cui un
giardinin labirinto, Broadway
Theator

miscellanea (

varie nessuno

(?) 


\begin{tabular}{|c|c|c|c|c|c|c|c|c|c|}
\hline PARCO & COORDINATE GEOGRAFICHE & ORIGINE STORICA & CONTESTOIPAESAGGIO & area & ATTRAZIONI & TЕMA & MATRICE & RUOLO DEL TEMA & $\begin{array}{l}\text { PROGETTA- } \\
\text { ZIONE DEL } \\
\text { PAESAGGIO }\end{array}$ \\
\hline Madurodam & Den Haag & 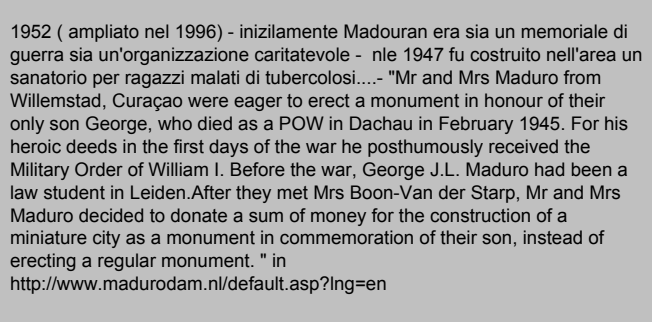 & 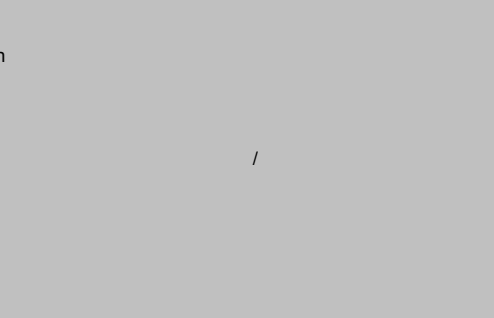 & 1 & 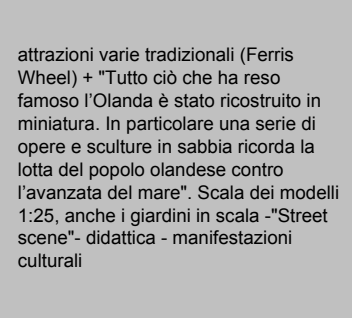 & Olanda in miniatura & esposizioni universali & $\begin{array}{l}\text { DIDATTICOI } \\
\text { DETERRIRORIALIZZAZZIONE }\end{array}$ & $\mathbf{x}$ \\
\hline Six Flags Holland & Netherands & 2000 & in territiorio extrautrano & ' & $\begin{array}{l}\text { rides eatrazioni varie - family fun - } \\
\text { "kid" }\end{array}$ & nessuno & amusement park & ' & (?) \\
\hline \multirow[t]{2}{*}{ Speelstad Oranje } & Gemeente Midden, Netherlands & 1 & in territorio extraurbano & 1 & $\begin{array}{l}\text { ridese eatrazioni varie - family fun - } \\
\text { "Kid" }\end{array}$ & nessuno & amusement park & 1 & (?) \\
\hline & Portugal & & & & & & & & \\
\hline \multirow[t]{2}{*}{ Portugal do pequenitos } & Coimbra, Portugal & 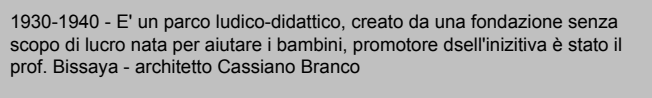 & parco urbanodella citità di Coimbra & 1 & 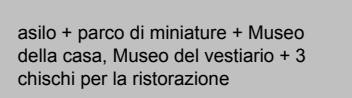 & $\begin{array}{l}\text { Portogallo in miniatura (per } \\
\text { aree tematiche) }\end{array}$ & (?) & DIDATTICO/ DIACRONIZZAZIIONE & $x$ \\
\hline & Spain & & & & & & & & \\
\hline Catalunia en miniatura & Barcellona, Spain & , & , & , & $\begin{array}{l}\text { Oltre a Cataturiai in miniatura, } \\
\text { spsetcacol per bambini }\end{array}$ & Catalunia in miniatura & & & \\
\hline Cortylandia & Puerto Banús, Marbella, Spain & & interno al Centro Comercial Costa Marbella & 1 & $\begin{array}{l}\text { ridese eatrazioni varie - family fun - } \\
\text { "Kid" }\end{array}$ & nessuno & (?) & 1 & (?) \\
\hline Isla Magica & Seville, Spain & ' & parco urbanon nel cuore di Sevilla & , & 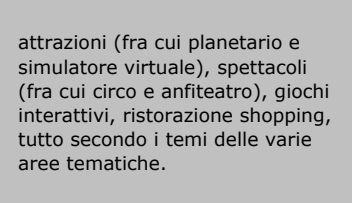 & 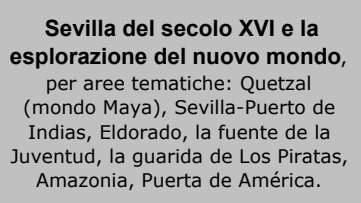 & $\begin{array}{l}\text { Disneyland - esposizizoni } \\
\text { internazionali }\end{array}$ & $\begin{array}{l}\text { DETERRITORIALIZZAZIONEDIACRONI } \\
\text { ZZAZZIONE }\end{array}$ & $X(?)$ \\
\hline Monte Igueldo & San Sebastiann, Spain & 1912 & 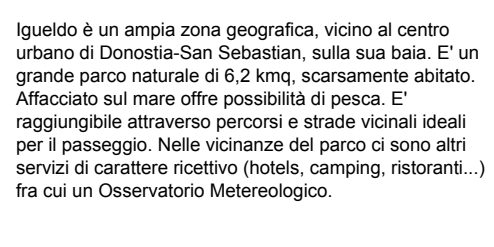 & 1 & 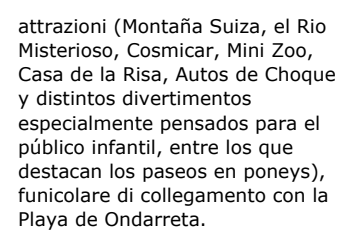 & nessuno & amusement park & 1 & (?) \\
\hline Parque de Atracciones & Madrid, Spain & 1969 & 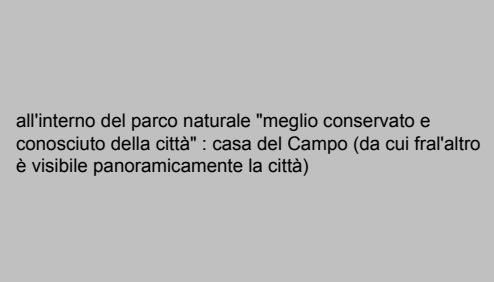 & $\begin{array}{l}20.0000 \mathrm{ma} \mathrm{di} \\
\text { 10.000mg } \\
\text { occupat da at } \\
\text { verdi }\end{array}$ & 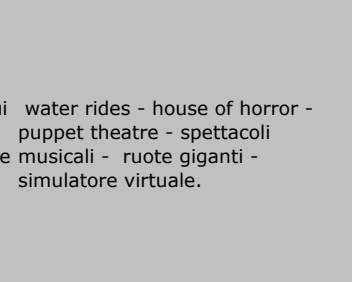 & $\begin{array}{l}6 \text { Aree tematiche : Naturaleza } \\
\text { (Nature), Maquinismo } \\
\text { (Automation), Tranquilidad } \\
\text { (Tranquility), Infantiti (linfants), } \\
\text { Gran Avenida (Grand Avenue) } \\
\text { and Trispace Virtual (Virtual } \\
\text { Wrild). (al loro interno le } \\
\text { attrazioni hanno temi diversi, } \\
\text { quanto ad ambientazione) }\end{array}$ & $\begin{array}{l}\text { amusement park - } \\
\text { Disneyland }\end{array}$ & SCENOGRAFICO DEBOLMENTE & (?) \\
\hline
\end{tabular}




\begin{tabular}{|c|c|c|c|c|c|c|c|c|c|}
\hline PARCO & COORDINATE GEOGRAFICHE & ORIGINE STORICA & CONTESTOIPAESAGGIO & area & ATTRAZIONI & TEMA & MATRICE & RUOLO DEL TEMA & $\begin{array}{l}\text { PROGETTA- } \\
\text { ZIONE DEL } \\
\text { PAESAGGIO }\end{array}$ \\
\hline Terra Mitica & Camino de Moralet, Benidorm, Spain & , & , & , & $\begin{array}{l}\text { attrazioni, gicchi interattivi, } \\
\text { ristorazione estopopping secondo i } \\
\text { temi delle varie aree }\end{array}$ & $\begin{array}{l}\text { civiltà delle origini, per aree } \\
\text { tematiche: Grecia, Roma, Egitto } \\
\text {,Iberia, Las Islas }\end{array}$ & Disneyland & $\begin{array}{l}\text { DETERRITORIALIZZAZIONEDDACRONI } \\
\text { ZZAZIONE }\end{array}$ & $X(?)$ \\
\hline Tibidabo & Barcellona, Spain & 1988 - La storia del parco inizia nei primi anni del XX secolo. De 1988 & 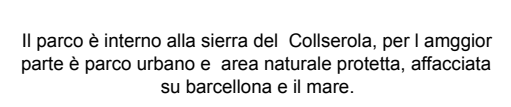 & $49.500 \mathrm{mq}$ & $\begin{array}{l}\text { rides e attrazion varie (molto } \\
\text { panoramiche) }\end{array}$ & misecellanea (singole attrazioni) & (?) & I & (?) \\
\hline Tivoli World & Arroyo de la Miel, Spain & , & , & , & $\begin{array}{l}\text { attrazioni (frac cui ruota), teatro, } \\
\text { spettacoli, ristorazione }\end{array}$ & miscellanea ( singole attrazioni) & scenografico & I & $\mathrm{x}$ \\
\hline Universal's Port Aventura & Salou, Spain, Spain & , & Nella Costa Daurada del Mediterraneo & , & $\begin{array}{l}\text { attrazioni, gicchi interertivi, } \\
\text { ristorazione enshopping secondo i } \\
\text { temi delle varie aree }\end{array}$ & $\begin{array}{l}5 \text { aree tematiche: Far West, } \\
\text { Messico, China, Polynnesia, } \\
\text { mediterrania }\end{array}$ & Disneyland & $\begin{array}{l}\text { DETERRITORIALIZZAZIONEDIACRONI } \\
\text { ZZZZZIONE }\end{array}$ & $X(?)$ \\
\hline Movie World & Madrid, Spain & 2002 & lungol a costa, vicino a Cardifie & , & 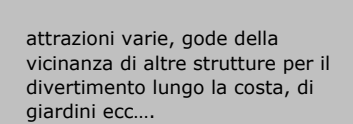 & movie world & amusement park & MARCHIO & $X(?)$ \\
\hline
\end{tabular}

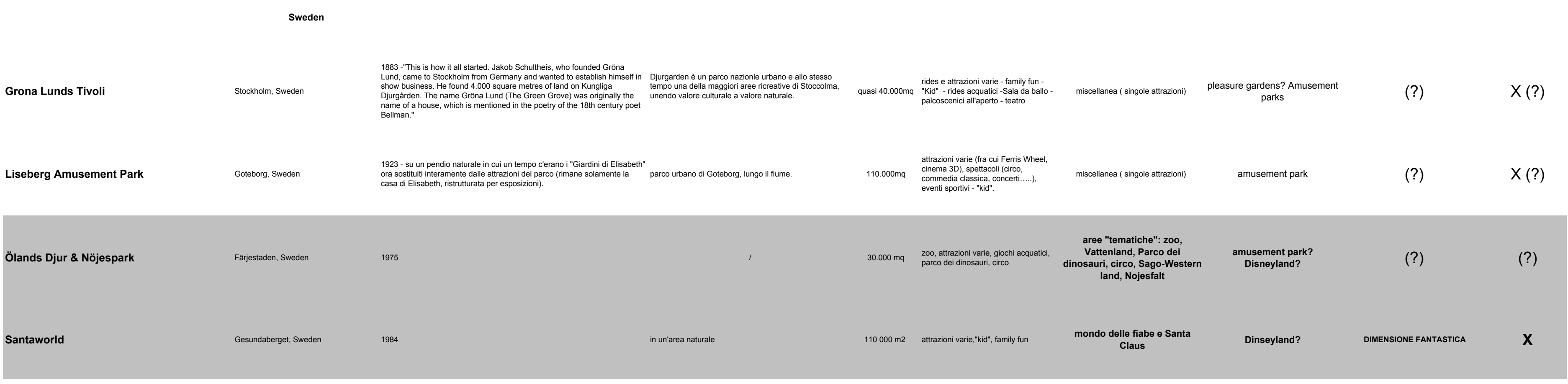




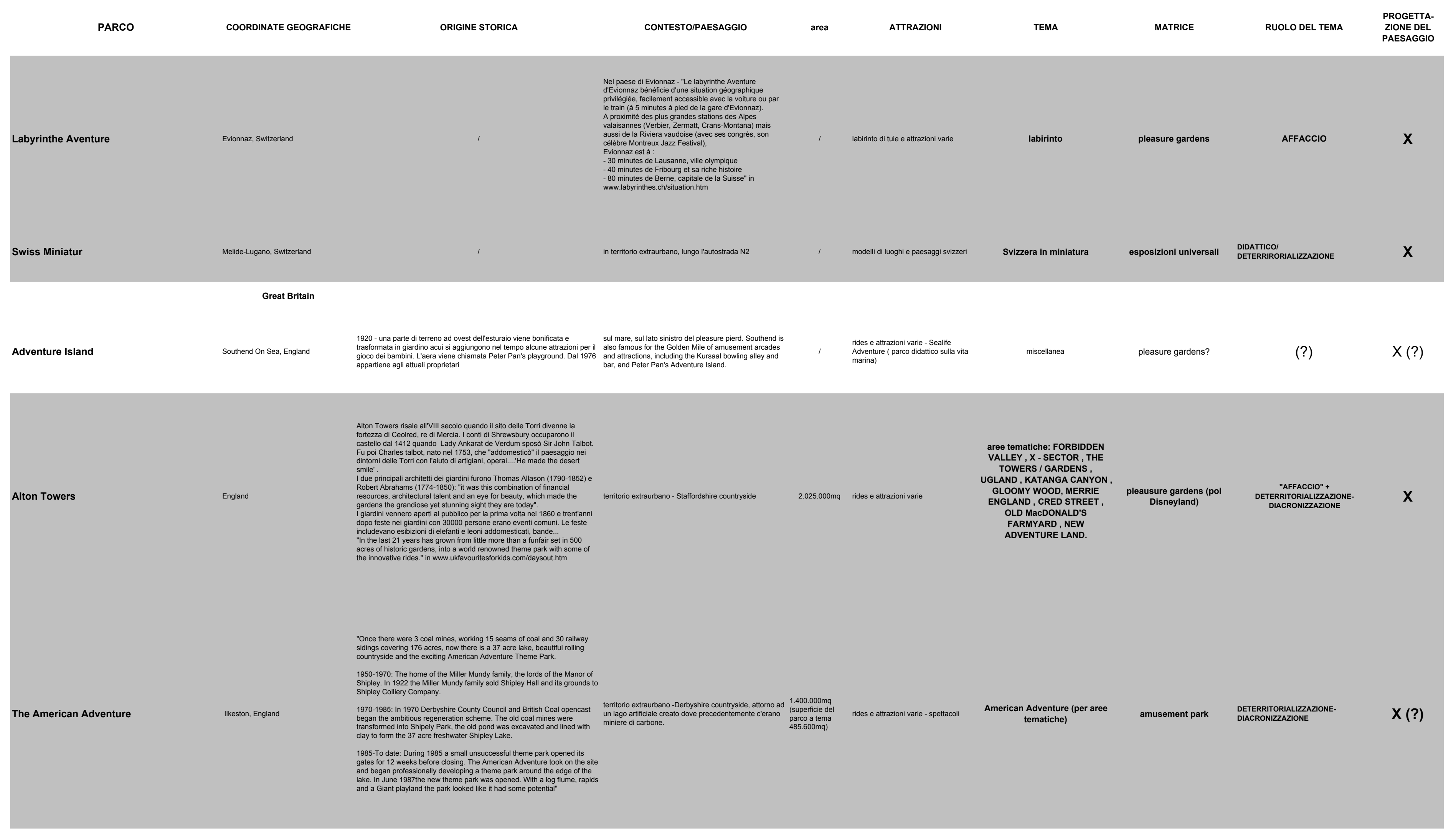




\begin{tabular}{|c|c|c|c|c|c|c|c|c|c|}
\hline PARCO & COORDINATE GEOGRAFICHE & ORIGINE STORICA & CONTESTOIPAESAGGIO & area & ATTRAZIONI & TEMA & MATRICE & RUOLO DEL TEMA & $\begin{array}{l}\text { PROGETTA- } \\
\text { ZIONE DEL } \\
\text { PAESAGGI }\end{array}$ \\
\hline Bekonscot & Bucks & 1929 & , & $6.070 \mathrm{ma}$ & $\begin{array}{l}75 \text { modell in miniatura a ie edfici tipicic i } \\
\text { un viliaggio rurale inglese }\end{array}$ & $\begin{array}{l}\begin{array}{l}\text { villaggio rurale inglese in } \\
\text { miniataura }\end{array} \\
\text {. }\end{array}$ & esposizioni universali & $\begin{array}{l}\text { DETERRITORIALIZZAZIONENALORIZZ } \\
\text { AZIONE }\end{array}$ & $\mathrm{x}$ \\
\hline Blackpool Pleasure Beach & Blackpool, England & 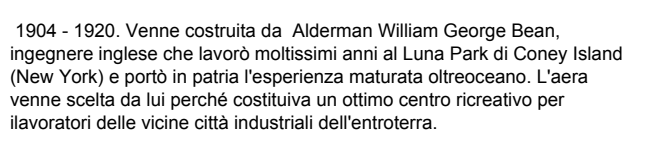 & sulla costa & $170.000 \mathrm{mq}$ & 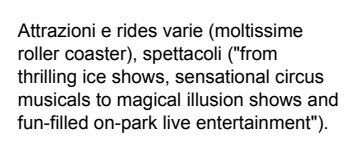 & nessuno & amusement park & 1 & $X(?)$ \\
\hline Botton's Pleasure Beach & Skegness, England & 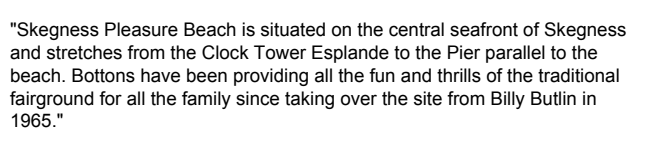 & sul mare in località turistica (Costa est, Skegness) & 1 & $\begin{array}{l}\text { attrazioni varie (fra cuil simulatore } \\
\text { virtuale, Ferris Wheel) }\end{array}$ & $\begin{array}{l}\text { miscellanea ( singole attrazioni, } \\
\text { debolmente) }\end{array}$ & amusement park & ' & (?) \\
\hline Brean Leisure Park & Brean, England & ' & $\begin{array}{l}\text { sul mare in I localltà turisitica ( M5 South West, Junction } \\
\text { 22) }\end{array}$ & , & attrazioni varie & nessuna & amusement park & ' & (?) \\
\hline Brighton Pier & Brighton, England & 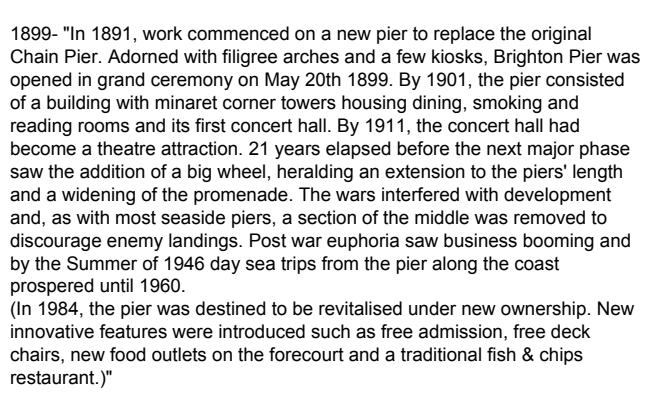 & sul mare a Brighton & , & attrazioni varie, spettacoli, istorazione is & sclalanea (singole attrazioni, debolments & amusement park & ' & (?) \\
\hline Camelot Theme Park & Chorley, England & $\begin{array}{l}\text { 1984- - dove esisteva un antico convento di Benededtinin, attualmente hotel } \\
\text { del parco. }\end{array}$ & 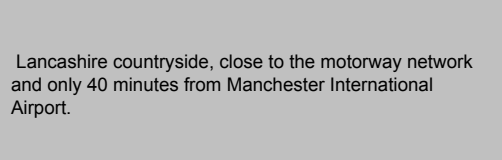 & 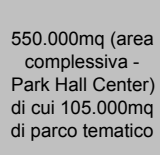 & $\begin{array}{l}\text { Attrazioni e rides varie, spettacoli, } \\
\text { tattria }\end{array}$ & $\begin{array}{l}\text { Leggenda di Camelot utteriore } \\
\text { divisione in aree tematiche }\end{array}$ & Disneyland & DIMENSIONE FANTASTICA & $X(?)$ \\
\hline Clacton Pier & Clacton On Sea, England & 1 & 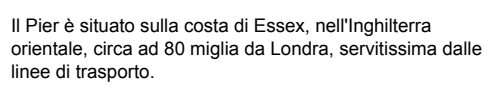 & $26.325 \mathrm{mq}$ & attrazioni varie, spettacoli, istororazione & $\begin{array}{l}\text { miscellanea ( singole attrazioni, } \\
\text { debolmente) }\end{array}$ & amusement park & 1 & ? \\
\hline Clarence Pier & Portsmouth, England & 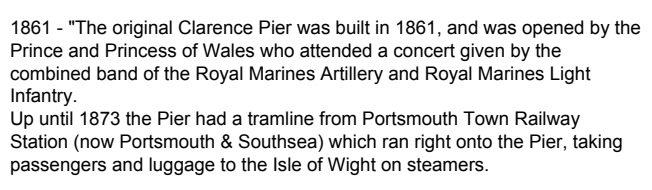 & sul mare, Southsea, Portsmouth, Hampshire & , & $\begin{array}{l}\text { rides, sale piochie un'rearea a tema per } \\
\text { bambini iriates Petés }\end{array}$ & nessuna eccetto Priate Pete's & pleasure gardens? & 1 & $X(?)$ \\
\hline
\end{tabular}


In 1905 the Pier was extended to accomodata increased boat trafifi. Further
extensions were made in 1932 when a café, sun deck and concourse hall

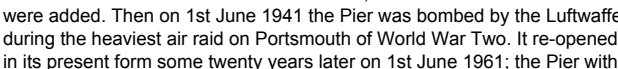

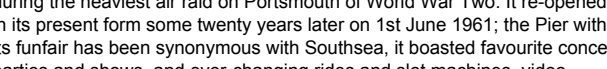

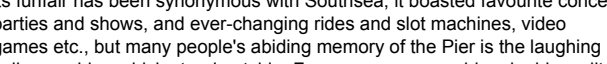

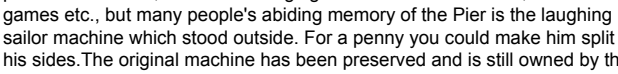

Crealy Park

Exeter, England

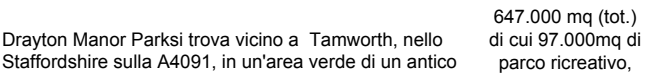

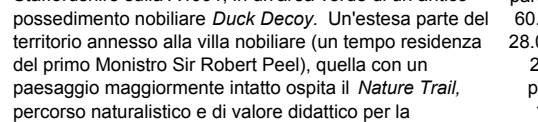

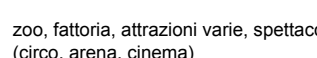
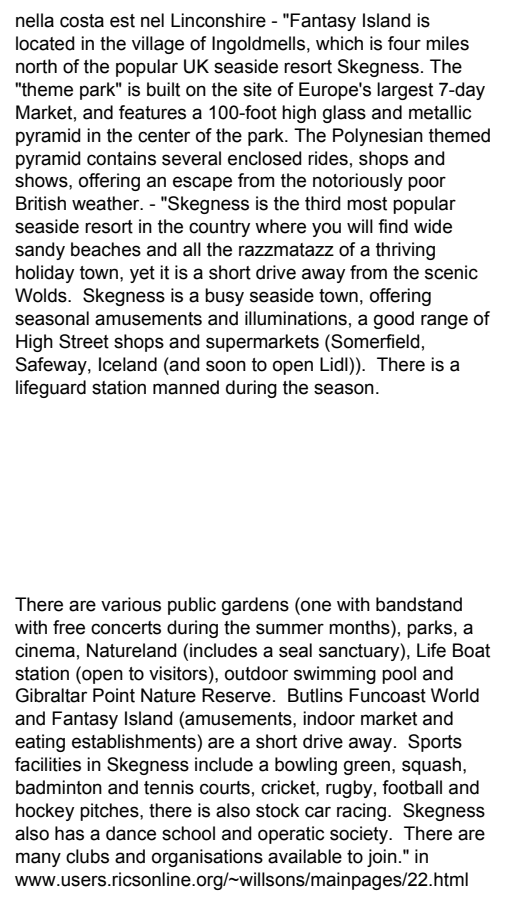

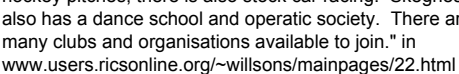

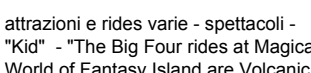

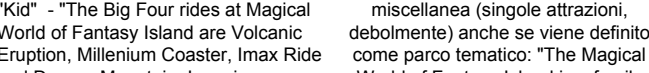
nind Dragon Mountain. Imaxis an Word of Fantasy Istand is is fam

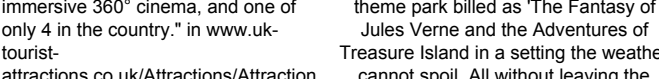

Disneyland? 


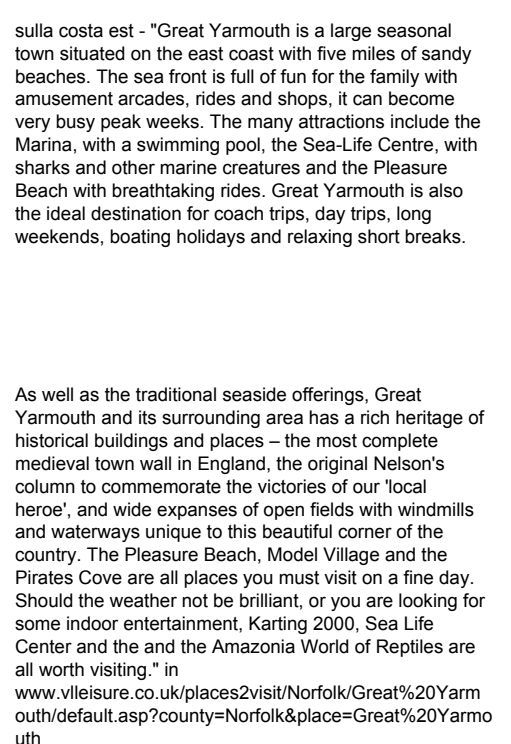

\begin{tabular}{|c|c|c|c|c|c|c|c|c|c|}
\hline Gulliver's Kingdom & Mattock, England & 1 & 1 & 1 & $\begin{array}{l}\text { ridese attrazioni varie - family fun - } \\
\text { "Kid" }\end{array}$ & 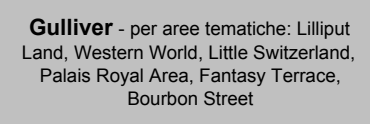 & Disneyland & MARCHIOIIIMENSIONE FANTASTICA & $X(?)$ \\
\hline Gulliver's Land & Milton Keynes, England & 1 & ' & ' & $\begin{array}{l}\text { ridese eatrazioni varie (fra cui Ferris } \\
\text { Wheel)-fanily tun - "Kid" }\end{array}$ & 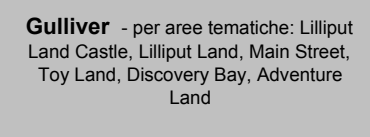 & Disneyland & MARCHIOIIMENSIONE FANTASTICA & $X(?)$ \\
\hline Gulliver's World & Warrington, England & ' & 1 & 1 & 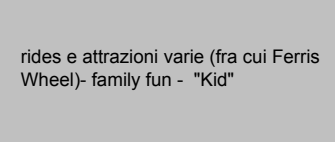 & 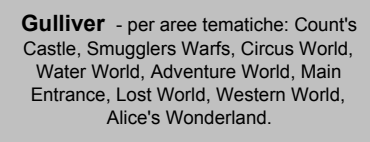 & Disneyland & MARCHIOIIMENSIONE FANTASTICA & $X(?)$ \\
\hline Flambards Village Theme Park & Hestson, England & ' & , & , & $\begin{array}{l}\text { ividse eatrazioni varie - villaggio } \\
\text { vituriano }\end{array}$ & villaggio vittoriano & amusement park & DIDATTICO? IIACRONIZZAZZIONE & $X(?)$ \\
\hline Flamingo Land & Matton, England & ' & nel North Yorkshire parkland & $1.520 .000 \mathrm{mac}$ & $\begin{array}{l}\text { rides e attrazioni varie-spettacoli- } \\
\text { zoo }\end{array}$ & ' & (?) & (?) & (?) \\
\hline Harbour Park & Littlehampton, England & 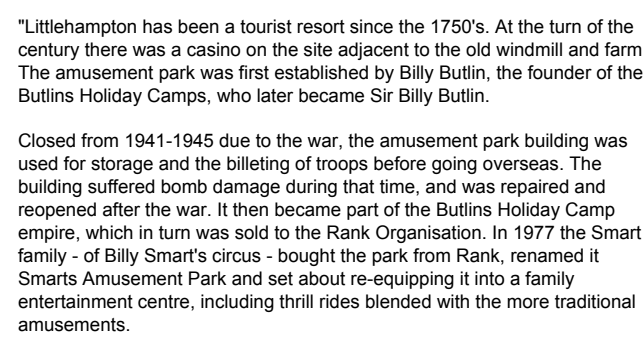 & 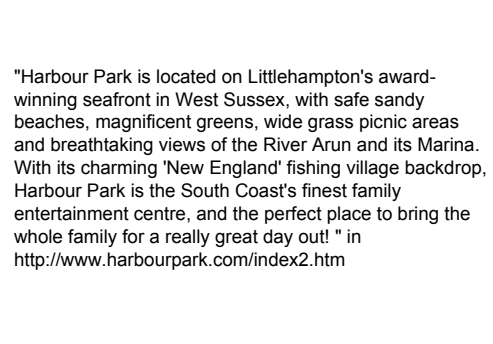 & 1 & $\begin{array}{l}\text { rides e eatrazioni varie - "Kid" - } \\
\text { didaticica (?) }\end{array}$ & 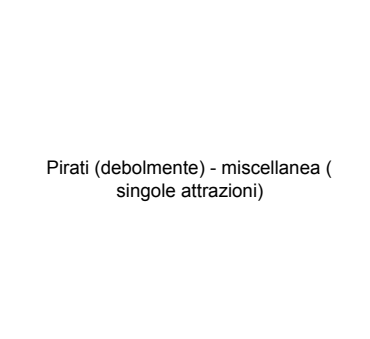 & amusement park & SCENOGRAFICO & $X(?)$ \\
\hline
\end{tabular}




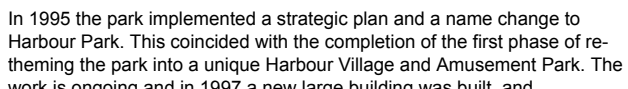

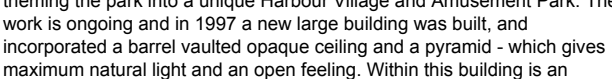

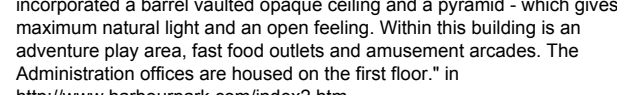

\begin{tabular}{|c|c|c|c|c|c|c|c|c|c|}
\hline Jorvik Viking Centre & York & 1984 & $\begin{array}{l}\text { II centro it trova nella zona del Coppergate shopping } \\
\text { center }\end{array}$ & ' & 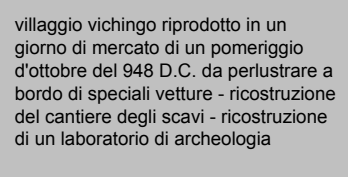 & villaggio vichingo & $\begin{array}{l}\text { esposizioni internazionali - } \\
\text { amusement park }\end{array}$ & DIDATTICO-VALORIZZAZIONE & $\mathbf{x}$ \\
\hline Legoland & Windor, England & 1 & a due miglia dal centro della città di W Windsor & 1 & 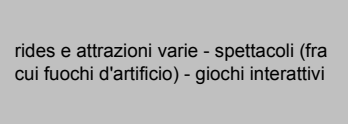 & 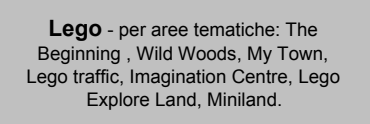 & Disneyland & MARCHO / DIMENSIONE FANTASTICA & $\mathbf{x}$ \\
\hline Lightwater Valley & England & 1 & 1 & 1 & rides e attrazioni varie & 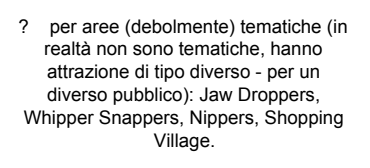 & Disneyland? & 1 & (?) \\
\hline Loudoun Castle & Galston, Scotland & 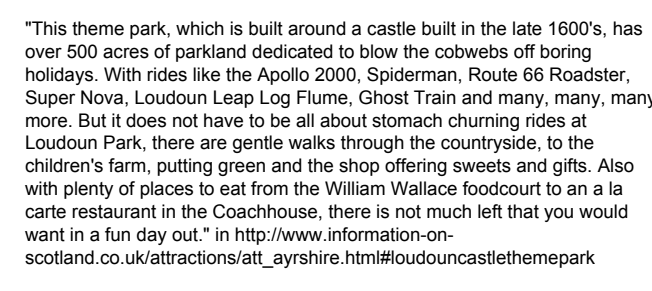 & 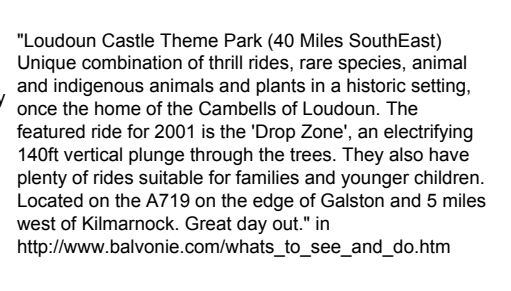 & 1 & 1 & (2) & pleasure gardens? & 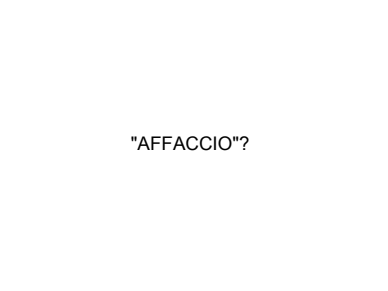 & $X(?)$ \\
\hline M\&D's Scotland's Theme Park & Motherwell, Scottand & ' & ' & , & 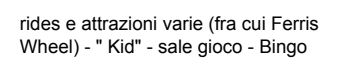 & nessuna & (?) & (?) & (?) \\
\hline The Milky Way Adventure Park & Bideford, England & ' & ' & 1 & rides eatrazioni varie -zoo di uccelli & $\begin{array}{c}\text { per aree : Clone Zone, , Time } \\
\text { Warp, Droid Destryyoers, Sports } \\
\text { hall ecc.... }\end{array}$ & $\begin{array}{l}\text { amusement park - } \\
\text { Disneyland? }\end{array}$ & SCENOGRAFICO ...... & $X(?)$ \\
\hline Oakwood & Pembrokessire, Wales & 1 & ' & , & , & , & (?) & (?) & (?) \\
\hline Paultons Park & Romsey, England & ' & 1 & ' & rides e altrazioniv varie -"kid". & miscellanea & (?) & (?) & (?) \\
\hline Pleasure Island & Cleethorpes, England & 1 & 1 & , & 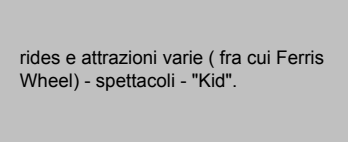 & $\begin{array}{c}\text { per aree tematiche: White } \\
\text { Knuckle Valley, Kiddies } \\
\text { Kindom, Spain Africa, Marocco, } \\
\text { Old England }\end{array}$ & $\begin{array}{l}\text { amusement park - } \\
\text { Disneyland? }\end{array}$ & $\begin{array}{l}\text { DETERRITRRIALZZZZIONE- } \\
\text { DIACRONIZZAZIONEE }\end{array}$ & $X(?)$ \\
\hline
\end{tabular}


PARCO

COORDINATE GEOGRAFICHE

ORIGINE STORIC

CONTESTO/PAESAGGIO

are

ATTRAZIONI

TEMA

MATRICE

RUOLO DEL TEMA

PROGETTA-
ZIONE DEL
PAESAGGIO

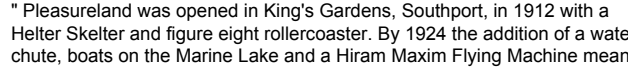

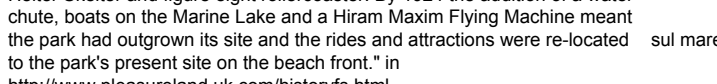

rides eatrazioni varie (tra cui 5 punti
panoramici)

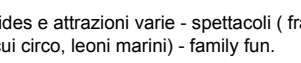

nessuna

amusement park

amusement park

miscellanea

\begin{tabular}{|c|c|c|c|c|c|c|c|c|c|}
\hline Thorpe Park & Staines Road, Chertsey, Surry, England & 1979 & 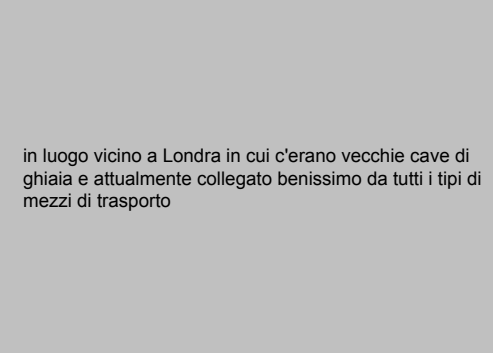 & 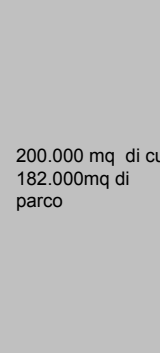 & 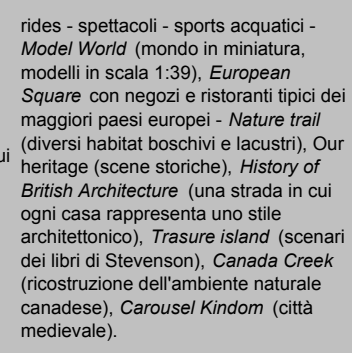 & 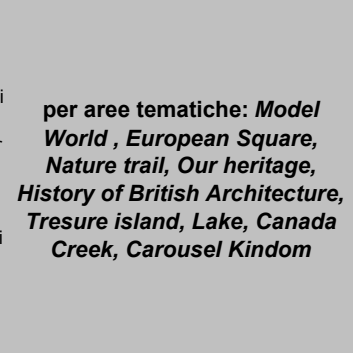 & $\begin{array}{l}\text { amusement park - esposizioni } \\
\text { universali }\end{array}$ & $\begin{array}{l}\text { i DETERRITORIALIZZZZZIONENALORIZZ } \\
\text { AZIONE }\end{array}$ & $\mathbf{x}$ \\
\hline Walton Pier & Watton on the Naze, England & ' & , & I & 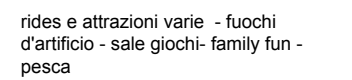 & nessuna & amusement park & ' & (?) \\
\hline West Midland Safari \& Leisure Park & Bewdley, England & I & situato nelle campagne dello Worcestershire & $\begin{array}{c}810.000 \mathrm{ma} \\
\text { (totale) }\end{array}$ & 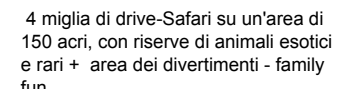 & & (?) & ' & (?) \\
\hline
\end{tabular}

(?)

?)

